\title{
Compilation of Laboratory Scale Aluminum Wash and Leach Report Results
}

\section{S. J. Harrington}

Washington River Prolection Solutions, LLC

Richland, WA 99352

U.S. Department of Energy Contract DE-AC27-08RV14800

$\begin{array}{ll}\text { EOT/ECN: } & \text { WC: } \\ \text { Cost Center: } & \text { Charge Code: } \\ \text { E\&R Code: } & \text { Total Pages: } 134\end{array}$

Key Words: Aluminum; Leach; Caustic; Laboratory Data; Hanford; Tank Waste; Boehmite; Oibbsite; Aluminosilicate: Cancrinite.

Abstract: This teport compiles and analyzes all known wash and caustic leach laboralory studies. As further data is produced, this report will be updated. Included are aluminum mineralogical analysis restlsts as twell as a summation of the wash and leach procedures and results. Of the 177 undergroumd storage tanks at Hanford, information was only available for five individual double-sheil tanks, forty-one individual single-shell tanks (e.g. thirty-nine 100 stries and two 200 series tanks), and twelve grouped tank wastes, Seven of the individual single-shell tank studies provided data for the percent of aluminum removal as a function of time for various causlic concentrations and leaching temperatures. It was deternined that in most cases increased leaching temperature, caustic concentration, and leaching time leads to increased dissolution of leachable eluminum solids.

TRADEMAFK DISCLAIMEA. Roference hergin to any specilic commencial produch, proctst, or service by trade name, trademark, manufacturer, or Ghemise, does not recessarily constilute or imphy ite endorsement, recommendation, or favoing by the Uhited Steles Cowernment or any agency thareot or its condrackors or subxcomiractors.
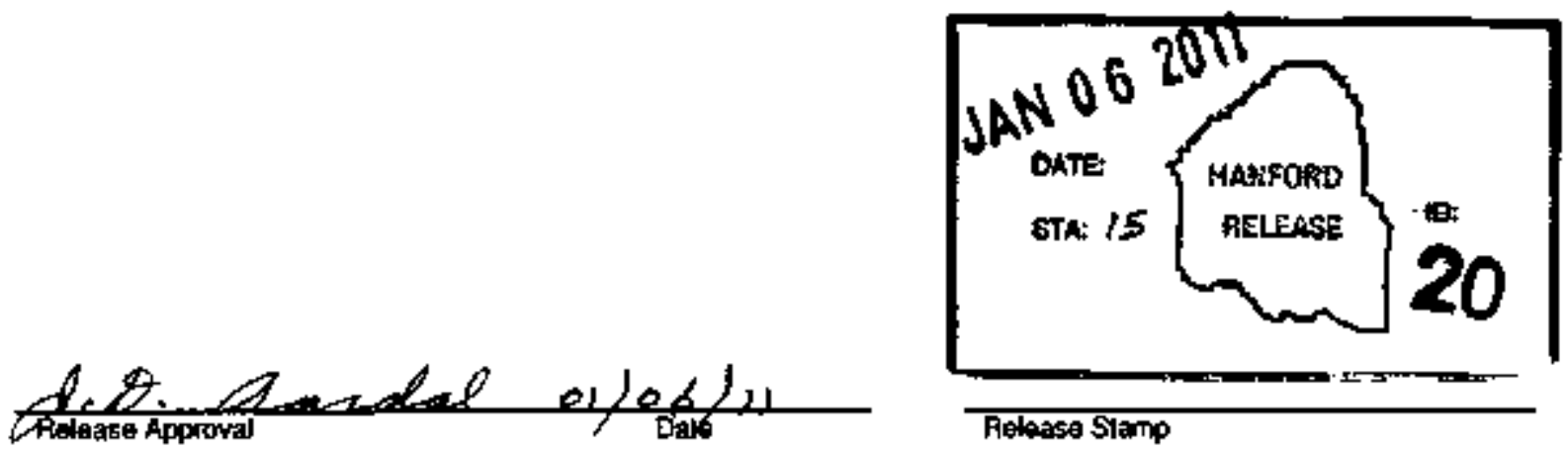

\section{Approved For Public Release}




\section{Compilation of Laboratory Scale Aluminum Wash and Leach Report Results}

Author

\section{S. J. Harrington}

Washington River Protection Solutions, LLC

Date Published

January 2011

Prepared for the U.S. Department of Energy

Assistant Secretary for Environmental Management

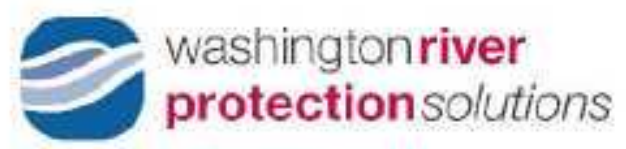

P.O. Box 850

Richland, Washington

Contractor for the U.S. Department of Energy

Office of River Protection under Contract DE-AC27-08RV14800 
RPP-RPT-46791

Revision 0

\section{EXECUTIVE SUMMARY}

This report summarizes published laboratory results for aluminum removal through inhibited water wash and caustic leaching tests performed on Hanford tank wastes. The dat a compilation and evaluation provided in this document is part of an analysis described in RPP-PLAN-46002, Wash and Leach Factor Work Plan. The objective of this report is to analyze the available data and ease the process of determining what information is available. As more test results are published. this compilation will be updated to incorporate the new information.

The laboralory studies currently available provide data on the effects of temperature, caustic concentration, and leaching time on the dissolution of aluminum from archived radioactive tank waste samples. A total of 39 laboratory reports are compiled. In particular. information is provided for five individual double-shell tanks, forty-one individual single-shell tanks (e.g. thirty-nine 100 series and two 200 series tanks), and twelve grouped lank wastes. Of these, only three of the individual double-shell, twelve of the individual single-shell, and seven of the composite grouped tank analyses were performed withiu the most recent proposed operation temperature range $\left(80\right.$ to $90^{\circ} \mathrm{C}$ ) for the Waste Treatment Plan1 caustic leaching to be performed at the Pretreatment Facility. Of these, only three individual single-shell tank samples and one composite tank waste sample were analyzed without inhibited water washing: a technique that is not planned for use at the Pretreatment Facility. Seven of the individual single-shell tank studies performed within the appropriate temperature range provided concentration versus time data for aluminum leaching at various temperatures and caustic concentrations. This information, provided in the Appendix A3,0, can be used to evaluate how changes in temperature. caustic concentration, and leaching time could affect the extent of aluminum leaching for these waste samples. Overall. increases in these three parameters result in improvements to the removal of aluminum from the solid phase in most cases. Analyses of the solid phases present after inhibited water washing and caustic leaching show that there are three aluminum phases present in the majority of tauk waste analyses. Of these. gibbsite was most easily renoved. whereas boehmite and aluminosilicates/cancrinites were the most dominant aluminum mineral phases present after leaching. 


\section{TABLE OF CONTENTS}

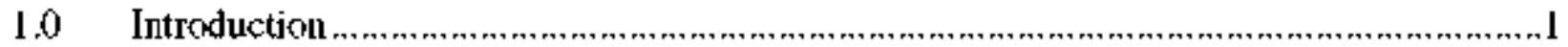

2.0 Aluminum Caustic Leaching Laboratory Studies ................................................

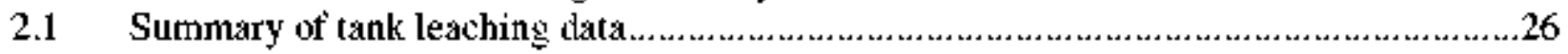

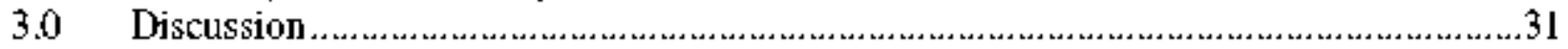

4.0 References

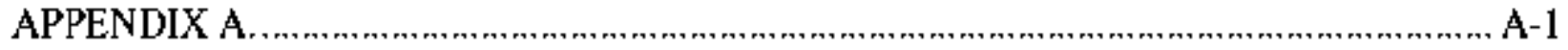

A1.0 Single- and Double-Shell Tanks with Laboratory Wash and Leach Information ......... A-2

A2.0 Single- and Double-Shell Tanks without Laboratory Wash and Leach Information ... A-68

A3,0 Single- and Double-Shell Tank Laboratory Concentration versus Time Information. A-76

A4.0 Appendix References

A-87

\section{LIST OF FIGURES}

Figure 1-1. Reprint of Aluminosilicate solubility at $25^{\prime \prime} \mathrm{C}$ figure from ORNLTM-1999/263,....4 Figure A3-1. Compilation of Laboratory Data for Percent Aluminum Removal versus Leaching

Time A-79

Figure A3-2. All caustic leaching analyses for Tank 241-B-101. A- 80

Figure A3-3. All caustic leaching analyses for Tank 241-BX-1 10. ............................. A-80

Figure A3-4. All caustic leaching analyses for Tank 241-BX-112 ................................. A-81

Figure A3-5. All caustic leaching analyses for Tank 241-C-102. ............................... A-81

Figure A3-6. All caustic leaching analyses for Tank 241-S-101 ......................................... A-82

Figure A3-7. All caustic leaching analyses for Tank 241-\$-110 ...................................... A-82

Figure A3-8. All caustic leaching analyses for Tank 241-T-110................................. A-83

Figure A3-9. Caustic leaching using $1 \mathrm{M} \mathrm{NaOH}$ at $60^{\circ} \mathrm{C}$.............................................

Figure A3-10. Caustic leaching using $3 \mathrm{M} \mathrm{NaOH}$ at $60^{\prime \prime} \mathrm{C}$.............................................. A-84

Figure A3-11. Caustic leaching using $1 \mathrm{M} \mathrm{NaOH}$ at $80^{\circ} \mathrm{C}$.............................................. 84

Figure $\mathrm{A} 3-12$. Caustic leaching using $3 \mathrm{M} \mathrm{NaOH}$ at $80^{\prime \prime} \mathrm{C}$. .............................................

Figure A3-13. Caustic leaching using $1 \mathrm{M} \mathrm{NaOH}$ at $100^{\circ} \mathrm{C}$..................................... A-85

Figure A3-14. Caustic leaching using $3 \mathrm{M} \mathrm{NaOH}$ at $100^{\circ} \mathrm{C}$............................................. A-86

\section{LIST OF TABLES}

Table 1-1. Solid Waste Types Found in the BBI Calculation Detail Report, as Described in RPP8847 (3 sheets). ........................................................................................................

Table 2-1. Aluniuum mineralogical data as described in individual laboratory studies. Included is information prior to leaching, after washing, and after caustic leaching (19 sheets)......7

Table 2-2. DSTs and SSTs with Available Laboratory Scale Aluminum Leaching Data (4 sheets).

Table 2-3. SSTs Analyzing the Effects of T and [OH] on Aluninum Leaching (2 sheets)........30 
Table A1-1. SSTs and DSTs Wash and Leach Information for Aluminum, Part 1. (38 sheets) A-3 Table A1-2. SSTs and DSTs Wash and Leach Information for Aluminum. Part 2. (27 sheets)A-41 Table A2-1. SSTs and DSTs with no wash and leach information available for Aluminum. \& sheets) A- 68

Table A3-1. Caustic Leaching Aluminum Concentration versus Time Data (2 sheets). A-77 
RPP-RPT-46791

Revision 0

\section{Abbreviations and Acronyms}

$\sim$

$\#$

IC

$2 \mathrm{C}$

Al

am

BARD

BBI

$\mathrm{BiPO}_{4}$

$\mathrm{CC}$.

$\mathrm{Cr}$

$\mathrm{cr}$

CW

DN/PD

DST

EB

ECN

EDS

$\mathrm{FeCN}$

FY

HDPE

HLW

ID

IX

LAW

$\mathrm{Na}$

NA

N/A

NP

$\mathrm{NaOH}$

OWW

$\mathrm{PO}_{4}$

PTF

PUREX

REDOX

rpm

RT

SEM

SORWT

SST

$T$

TBP approximalely

number

first decontamination cycle from bismuth phosphate process at $B$ aud $T$ plants

second decontamination cycle from bismuth phosphate process at B and $T$ plants aluninum

amorphous

Flowsheet Bases, Assumptions, and Requirements Document

Best Basis Inventory

Bismuth Phosphate

Complex Concentrate

Chromium

crystalline

Cladding Waste

Dilute Non-complexed/PUREX neutralized cladding removal waste (NCRW) solids or sludge remaining after decanting

Double-shell tank

Evaporator bottoms

Engineering Change Notice

Energy dispersive spectroscopy

Ferrocyanide

fiscal year

high-density polyethylene

High-level Waste

identification

ion exchange

Low-activity Waste

sodium

ungrouped waste type

not applicable

not performed

sodium bydroxide (caustic)

Organic wash waste

phosphate

Pretreatment Facility

plutonium-uranium extraction

reduction-oxidation

rotations per ninute

room temperature

scanning electron microscopy

Sort on Radioactive Waste Type

Single-shell tank

temperature

Tributyl phosphate 
TEM transmission electron microscopy

TRU transuranic

UDS undissolved solids

Wf with

WRPS Washinglon River Protection Solutions

WTP Waste Treatment and Immobilization Plant

XRD X-ray diffraction

\title{
Units
}

${ }^{\circ} \mathrm{C}$

g

hr

ug

uL

um

$\mathrm{mL}$

min

M

$\%$

wt

\author{
degrees Celsius \\ gram \\ hour \\ microgram \\ microliter \\ micromeler; micron \\ milliliter \\ minute \\ molefiter \\ percent \\ weight
}


RPP-RPT-46791

Revision 0

\subsection{INTRODUCTION}

One of the primary missions at the Hanford site is to manage the 53 million gallons of radioactive waste stored in the 149 single-shell and 28 double-shell underground storage tanks. This includes reducing the worker, public, and environmental risk of contamination by ensuring tank integrity is maintained, ensuring safe working and handling practices, and preparing to transfer and dispose of the waste at the Waste Treatment and Immobilization Plant (WTP) currently being constructed.

The underground storage tanks, which are grouped into 18 tank farms, store wastes generated from the production of nuclear materials which began in 1944. These tanks range in volume from 55 thousand to one million gallon capacity. The waste categories still held within these tanks include aqueous supernatants and solid saltcakes and sludge. These waste categories are subsequently grouped by waste types representative of the processes which produced them. The solid waste types are provided in Table 1-1 below with information from RPP-8847, Best-Basis Inventory Template Composition of Common Tank Waste Layers.

Table 1-1. Solid Waste Types Found in the BBI Calculation Detail Report, as Described in RPP-8847 ( 3 sheets).

\begin{tabular}{|l|l|}
\hline \multicolumn{1}{|c|}{ Waste Type } & \multicolumn{1}{c|}{ Waste Type Process Description } \\
\hline 1C (solid) & $\mathrm{BiPO}_{4}$ first cycle decontamination waste and coating waste (1944 to 1949 and 1950 to 1956). \\
\hline 1CFeCN (solid) & $\begin{array}{l}\text { Ferrocyanide sludge from in-plant scavenging of 1C waste (without coating waste) from T } \\
\text { plant that was transferred to 241-TY tank farm (October 1954 to 1956). }\end{array}$ \\
\hline 224-1 (solid) & Lanthanum fluoride process 224 building waste (1944 to 1948). \\
\hline $224-2$ (solid) & Lanthanum fluoride process 224 building waste (1949 to 1956). \\
\hline 2C (solid) & $\begin{array}{l}\text { BiPO } \text { second cycle decontamination waste (1944 to 1949 and 1950 to 1956) with low } \\
\text { activity cell 5-6 drainage waste (June 1951 to 1956). }\end{array}$ \\
\hline A1-SltCk (solid) & $\begin{array}{l}\text { Saltcake from the first 242-A evaporator campaign using 241-A-102 as the feed tank (1977 } \\
\text { to 1980). }\end{array}$ \\
\hline A2-SitSlr (solid) & $\begin{array}{l}\text { Saltcake from the second 242-A evaporator campaign using 242-AW-102 as the feed tank } \\
\text { (1981 to 1988). }\end{array}$ \\
\hline AR (solid) & $\begin{array}{l}\text { Water washed PUREX sludge entrained in decants of recovered sludge or the water washes } \\
\text { of this sludge and the solids remainmg after acidification (1967 to 1976). }\end{array}$ \\
\hline B (solid) & $\begin{array}{l}\text { B Plant high-activity waste- Rare earth (RE) fission products, recovered current acid waste } \\
\text { (CAW), solvent wash waste, and any solution containing high activity including cask station } \\
\text { receipts, cell drainage containing product spills, etc. (1963 to 1972). }\end{array}$ \\
\hline BL (solid) & $\begin{array}{l}\text { B Plant low-activity waste - 1AW solvent extraction waste stream (which includes } \\
\text { complexants added for solvent extraction), the 1CP/organic wash waste during PUREX } \\
\text { acidified sludge (PAS) processing, and insoluble solids remaining after treatment of solids } \\
\text { centrifuged from CAW feed (i.e. acid leached and water washed PUREX HLW sludge). Cell } \\
\text { drainage and Waste Encapsulation Storage Facility (WESF) transfers with low radionuclide } \\
\text { content (1967 to 1976). }\end{array}$ \\
\hline Saltcake from the 242-B evaporator operation (1951 to 1954). \\
\hline B-SltCk (solid)
\end{tabular}


RPP-RPT-46791

Revision 0

Table 1-1. Solid Waste Types Found in the BBI Calculation Detail Report, as Described in RPP-8847 ( 3 sheets).

\begin{tabular}{|c|c|}
\hline Waste Type & Waste Type Process Description \\
\hline BY-SltCk (solid) & Saltcake from in-tank solidification in 241-BY farm (1965 to 1974), \\
\hline CWP1 (solid) & PUREX cladding waste from aluminum clad fuel (1956 to 1960). \\
\hline CWP2 (solid) & PUREX cladding waste from aluminum clad fuel (1961 to 1972). \\
\hline CWR1 (solid) & REDOX cladding waste from aluminum clad fuel (1952 to 1960). \\
\hline CWR2 (solid) & REDOX cladding waste from aluminum clad fuel (1961 to 1966). \\
\hline CWZr1 (solid) & PUREX (and REDOX) zirconium cladding waste (1968 to 1972). \\
\hline CWZt2 (solid) & PUREX zirconium cladding waste (1983 to 1989 ). \\
\hline $\mathrm{DE}$ (solid) & $\begin{array}{l}\text { Diatomaceous earth added to tanks } 241-\mathrm{BX}-102,241-\mathrm{SX}-113,241-\mathrm{TX}-116,241-\mathrm{TX}-117 \text {, } \\
241-\mathrm{TY}-106 \text {, and } 241-\mathrm{U}-104 .\end{array}$ \\
\hline HS (solid) & Hot Semiworks strontium and RE purification waste (1961 to 1968). \\
\hline MW1 (solid) & $\mathrm{BiPO}_{4}$ Metal Waste (1944 to 1949). \\
\hline MW2 (solid) & $\mathrm{BiPO}_{4}$ Metal Waste (1950 to 1956$)$. \\
\hline $\mathrm{NA}(\mathrm{SltCk})$ & Mixture of saltcakes from tank transfers and retrievals. \\
\hline NA (Sludge) & Mixture of sludge material from tank transfers and retrievals. \\
\hline P1 (solid) & PUREX high-level waste (1956 to 1962 ). \\
\hline P2 (solid) & PUREX high-level waste (1963 to 1967$)$. \\
\hline P3AZ1 (solid) & PUREX high-level waste to AZ-101 (1983 to March 13, 1986). \\
\hline P3AZ2 (solid) & PUREX high-level waste to AZ-102 (March 13, 1986 to 1990). \\
\hline PFeCN (solid) & $\begin{array}{l}\text { Ferrocyanide sludge from TBP in-plant scavenged supernatant and co-disposed TBP sludge } \\
\text { (1954 to 1955). }\end{array}$ \\
\hline PL2 (solid) & PUREX organic wash waste and non-boiling waste (1983 to 1988). \\
\hline $\begin{array}{l}\text { Portland Cement } \\
\text { (solid) }\end{array}$ & Portland cement added to tank 241-BY-105. \\
\hline R1 (solid) & REDOX high-level waste (1952 to 1958). \\
\hline $\mathrm{R} 2$ (solid) & REDOX high-level waste (1959 to 1966 ). \\
\hline R-SltCk (solid) & Salteake from self-concentration in 241-S and 241-SX tank farms ( 1952 to 1966 ). \\
\hline S1-SltCk (solid) & $\begin{array}{l}\text { Salteake from the first } 242-S \text { evaporator campaign using } 241-S-102 \text { as a feed tank (1973 to } \\
1976 \text { ). }\end{array}$ \\
\hline S2-SltSlr (solid) & $\begin{array}{l}\text { Saltcake from the second } 242-S \text { evaporator campaign using } 241-S Y-102 \text { as a feed tank (1977 } \\
\text { to } 1980 \text { ). }\end{array}$ \\
\hline SRR (solid) & $\begin{array}{l}\text { High-activity waste from B Plant processing of PAS, solids centrifuged from AR vault feed, } \\
\text { strontium purification wastes after solvent extraction, RE carrier precipitation or ion } \\
\text { exchange rework, and other solutions containing activity including cask station receipts, cell } \\
\text { drainage containing product spills, WESF returns unsuitable for rework, and crude RE } \\
\text { disposal (1969 to 1985). }\end{array}$ \\
\hline T1-SltCk (solid) & Saltcake from the $242-\mathrm{T}$ evaporator (1951 to 1955 ). \\
\hline T2-SltCk (solid) & Saltcake from the last 242-T evaporator campaign (1965 to 1974). \\
\hline TBP (solid) & Tributyl phosphate process waste (1952 to 1957$)$. \\
\hline
\end{tabular}


RPP-RPT-46791

Revision 0

Table 1-1. Solid Waste Types Found in the BBI Calculation Detail Report, as Described in RPP-8847 (3 sheets).

\begin{tabular}{|l|l|}
\hline \multicolumn{1}{|c|}{ Waste Type } & \multicolumn{1}{c|}{ Waste Type Process Description } \\
\hline TFeCN (solid) & $\begin{array}{l}\text { Ferrocyanide sludge from supernatant scavenging in the 244-CR Vault (1955 to 1958) } \\
\text { consisting of Tributyl phosphate (TBP) supernatant and the comingled supernatants from } \\
\text { other wastes stored in the same tanks. }\end{array}$ \\
\hline TH1 (solid) & Thoria process wastes (1966). \\
\hline Z(solid) & PFP waste (1974 to 1988). \\
\hline
\end{tabular}

In preparation for operation of the WTP, the solid and liquid wastes stored in the tank farms will be separated into two feed types: High-level waste (HLW) and Low-activity waste (LAW). The LAW will be made up of salt solutions containing minimal undissolved solids, whereas the remainder of the radioactive waste will be processed as HLW. In order to reduce the volume of HLW, as well as costs, a Pretreatment Facility (PTF) will be utilized to separate out some of the more soluble non-radioactive species. This facility will implement processes such as caustic leaching to remove aluminum at elevated temperature, oxidative leaching to remove chromium at ambient temperature, ultra filtration solids/liquid concentration, and slurry washing to remove soluble species [24590-WTP-RPT-PT-02-005, Flowsheet Bases, Assumptions, and Requirements Document (BARD), Rev. 5].

Here, we compile previously documented laboratory data gathered while investigating the removal efficiency of aluminum from tank waste solids through washing and caustic leaching. This is part of the work described in RPP-PLAN-46002, Wash and Leach Factor Work Plan. Aluminum concentration has been estimated to be as high as 30 weight percent (wt \%) of the sludge material in certain tanks, as detailed in the Best Basis Inventory (BBI) Calculation Detail (WRPS, 2010), When a significant portion of aluminum is removed through caustic leaching, the loading of solid oxides in the HLW glass will be reduced resulting in a reduction of the volume of HLW glass formed.

Removal of aluminum from tank waste through inhibited water washing and caustic leaching has been tested in laboratory settings. The available mineralogical and leaching data has been used in conjunction with other reports (e.g. RPP-RPT-47306, Waste Type Analysis for Aluminum Leachability Estimates of All Non-Retrieved Hanford Tank Waste and RPP-RPT-46618, Hanford Waste Mineralogy Reference Report) to group the aluminum solid species into three categories:

(1) Easily leachable aluminum (e.g. gibbsite, dawsonite, nordstrandite, etc.);

(2) Slow leaching/kinetically controlled aluminum (e.g. boehmite); and

(3) Non-leachable/refractory aluminum (e.g. aluminosilicates and cancrinites).

Examples of the chemical structures documented for the non-leachable/refractory aluminum include aluminosilicates $\left(\mathrm{H}_{12} \mathrm{Al}_{12} \mathrm{Si}_{36} \mathrm{O}_{96}\right)$, sodium aluminosilicates hydrate $\left[\left(\mathrm{Na}_{2} \mathrm{O}\right)_{1.31} \mathrm{Al}_{2} \mathrm{O}_{3}\left(\mathrm{SiO}_{2}\right)_{2.01}\left(\mathrm{H}_{2} \mathrm{O}\right)_{2}\right]$, cancrinite $\left[\left(\mathrm{Na}_{7.14} \mathrm{Al}_{6} \mathrm{Si}_{7.08} \mathrm{O}_{26.73}\left(\mathrm{H}_{2} \mathrm{O}\right)_{4.87}\right)\right.$ or $\left.\left(\mathrm{Na}_{8}\left(\mathrm{AlSiO}_{4}\right)_{6}\left(\mathrm{CO}_{3}\right)\left(\mathrm{H}_{2} \mathrm{O}\right)_{2}\right)\right]$, hydroxycancrinite $\left(1.06 \mathrm{Na}_{2} \mathrm{O} \cdot \mathrm{Al}_{2} \mathrm{O}_{3} \cdot 1.6 \mathrm{SiO}_{2} \cdot 1.6 \mathrm{H}_{2} \mathrm{O}\right)$, and nitrate cancrinite $\left(\mathrm{Na}_{9.16}\left(\mathrm{Al}_{6} \mathrm{Si}_{6} \mathrm{O}_{24}\right)\left(\mathrm{NO}_{3}\right)_{2.16}\left(\mathrm{H}_{2} \mathrm{O}\right)_{1.62}\right)$ as provided in RPP-RPT-46618 as well as later in this report. 
RPP-RPT-46791

Revision 0

The three aluminum categories described above have been designed to predict the extent of aluminum solids removal that will be possible during caustic leaching at the PTF. The leachable solids (e.g. gibbsite and, to a small extent, boehmite) dissolve in caustic to form the aluminate ion $\left[\mathrm{Al}(\mathrm{OH})_{4}^{-}\right]$, as shown in Equations 1.1 and 1.2, whereas the insoluble solids (e.g. aluminosilicates, cancrinites, etc) are not easily leached into the aqueous phase with the addition of caustic.

$$
\begin{aligned}
& \text { boehmiteAlOOH}+\mathrm{H}_{2} \mathrm{O}+\mathrm{OH} \underset{k_{-1}}{\stackrel{k_{1}}{\longrightarrow}} \mathrm{A}(\mathrm{OH})_{4}^{-} \\
& \text {gibbsite: } \mathrm{Al}(\mathrm{OH})_{3}+\mathrm{OH}^{-} \underset{\mathrm{k}_{-2}}{\stackrel{\mathrm{k}_{2}}{\rightleftarrows}} \mathrm{Al}(\mathrm{OH})_{4}^{-}
\end{aligned}
$$

As described in ORNL/TM-1999/263, Prevention of Solids Formation: Results of the FY 1999 Studies, the formation of aluminosilicates is a counterintuitive process. Even though both aluminum and silicon have increased solubilities as $\mathrm{pH}$ is increased, when both elements are present their solubility drops drastically resulting in the precipitation of aluminosilicates. This trend is shown in Figure 1 of ORNL/TM-1999/263, provided below as Figure 1-1.

Figure 1-1. Reprint of Aluminosilicate solubility at $25^{\circ} \mathrm{C}$ figure from ORNL/TM-1999/263.

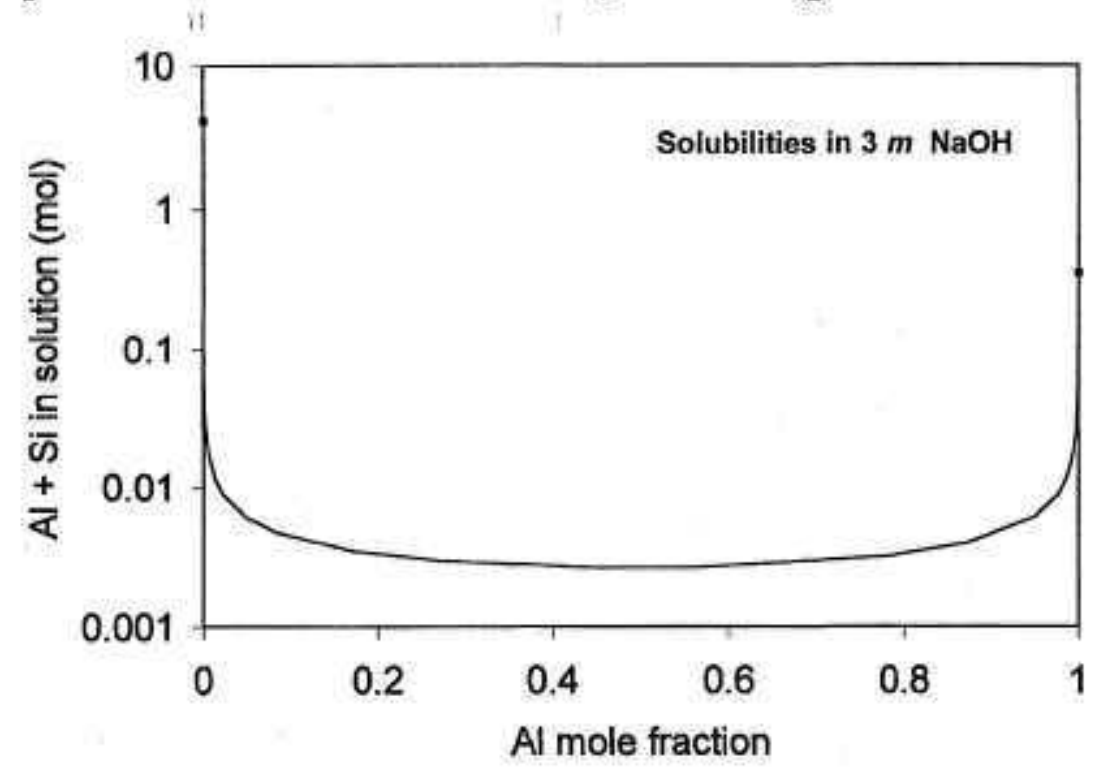

The increased $\mathrm{pH}$ during caustic leaching would increase the potential for aluminosilicate precipitation, provided that both aluminum and silicon ions are present in solution. This was observed in the caustic leaching of solids from tank 241-BX-112 in PNNL-12026, Washing and Caustic Leaching of Hanford Tank Sludge: Results of FY 1998 Studies. Even after performing several wash cycles with the tank 241-BX-112 solids, results of mineralogical analysis indicated the formation of aluminosilicates during leaching, with an increase in formation as temperature was increased.

Many of the 'wash and leach' studies analyzed in this document were performed on archived core and auger samples. For the studies which identified the material used, analyses were performed on samples from one core, composites of many different core samples from the same 
tank. or even combinations of tank wastes that fit into specific tank waste groups. Some of these waste samples had been in storage for up to $15+$ years and had dried out. Therefore they were reconstituted using water, inhibited water $(0.01 \mathrm{M} \mathrm{NaOH}+0.01 \mathrm{M} \mathrm{NaNO})$, or a low concentration (0.01 $\mathrm{M}$ to $0.1 \mathrm{M} \mathrm{NaOH}$ ) caustic solution in order to obtain a slurry $\mathrm{pH}$ near the original measured value. Temperatures used for caustic leaching performance analyses were between room tenperature (RT) and 100 degrees Celsius ("C.); the majority of which were performed at $100^{\circ} \mathrm{C}$ (see Table A1-1 and Table A1-2 in the Appendix).

An important aspect for this investigation is 10 determine how much of the information from the awailable laboratory analyses is still directly pertinent to the current operational parameters for the PTF at the WTP. To do this, we used the latest revision to 24590-WTP-RPT-PT-02-005 which is a compilation of information describing how the WTP will be run and the operational parameters which will be used for the various irealment provesses. The laboratory studies which analyzed inhibited water washiug and caustic leaching at $100^{\prime \prime} \mathrm{C}$ were acceptable according to revision 4 of 24590-WTP-RPT-PT-02-005. Revision 4 allowed for caustic leaching temperatures to lange from 85 to $100^{\circ} \mathrm{C}$. In Jevision 5 of 24590 -WTP-RPT-PT-02-005, the PTF operational couditions were adjusted, the most noticeable beiug (a) the renoval of the preliminary inhibited water washing and (b) caustic leaching temperatures adjusted to a range of 80 to $90^{\circ} \mathrm{C}$. These were impotant changes, as inhibited water washing was pelformed in neally all previously performed laboratory studies, with the majority of caustic leaching tests performed at $100^{\circ} \mathrm{C}$. Since both of these conditions are not within the scope of the curtent operational plan for the PTF, uncertainties in the relevancy of the laboratory results currently available for aluminum leaching exist.

A majority of the laboratory data currently available are from tests performed with preliminary inhibited water washing and caustic leaching outside of the current acceptable temperature range ( 80 to $90^{\circ} \mathrm{C}$ ). Even though this information is not directly relevant to the current WTP PTF operational parameters, important characteristics can be derived from the available dala. These characteristics include the effects of leaching time on aluminum dissolution, the quantities of easily leachable aluminum solids. chemical and physical properties of the various solids currently in the as-received tank waste samples, the types of solids formed during leaching. etcetera.

This seport compiles all of the currently available laboratory data available to date. Due to the numerous documents available with pertinent information on this subject matter, all of the existing laboratory results are incorporated within this docunent in order to provide one reference that contains all of the available data. As further analytical resulis are documented, the new information will be added.

\subsection{ALUMINUM CAUSTIC LEACHING LABORATORY STUDIES}

Thirty-nine reports have been generated to date to aualyze the effects of inhibited water washing and high temperature caustic leaching on the removal of aluminum from tank waste samples. Significant iuformation concerning aluminum removal has been conpiled into Table Al-1 and 
RPP-RPT-46791

Revision 0

Table A1-2 in the appendix of this report, with mineralogy data provided in Table 2-1. The information in Table 2-1 is based solely on information contained in the referenced report and not on any other known information. Similarly, the mineralogical information in the table is based on the analytical results from the referenced report which may not have observed a specific aluminum mineral phase even though aluminum was found in the solids.

Some of the caustic leaching laboratory studies identify aluminum mineral phases present in the as-received and treated materials, as well as analyze the effects of temperature, caustic concentration, and leaching time on the extent of aluminum leaching. Included in Table A1-1 and Table A1-2 is a brief description of the processes that generated the waste found in the individual tanks. These processes have been included to indicate where the aluminum in the specific wastes originated (as referenced in Table 1-1). Understanding these processes provides insight into the conditions (e.g. temperature) at which the aluminum in a waste was exposed to in the past. This information has previously been used to develop trends in the types of aluminum solids present in untested tanks (see RPP-RPT-47306).

Additional mineralogy information was obtained from PNNL-13394, Status Report on Phase Identification in Hanford Tank Sludges, and PNWD-3300 (WTP-RPT-076), Identification of Washed Solids from Hanford Tanks 241-AN-102 and 241-AZ-101 with X-Ray Diffraction, Scanning Electron Microscopy, and Light-Scattering Particle Analysis, with additional insight into the waste sources stored in the various tanks obtained from PNL-9814, The Sort On Radioactive Waste Type Model: A Method to Sort Single-Shell Tanks Into Characteristic Groups, and WHC-EP-0625, Hanford Site Waste Storage Tank Information Notebook. Information from these reports is also included in Table 2-1. Tanks that have not had leaching studies performed up to this point are detailed in Table A2-1.

Aluminum mineral phases were observed using techniques such as transmission electron microscopy (TEM), scanning electron microscopy (SEM), energy dispersive spectroscopy (EDS), and X-ray diffraction (XRD). These analytical techniques were used to determine which aluminum species were dominant prior to and following caustic leaching. As shown in Table $2-1$, three aluminum minerals were found to be in a majority of either the removed species due to caustic leaching or still mostly present after caustic leaching. The species dissolved in a majority of the studies was gibbsite (17 studies), since it was observed in the as-received solids but not in the remaining leached solids. The other two, various aluminosilicates (including cancrinites; 16 studies) and boehmite ( 8 studies), were in a majority of the studies as still present following caustic leaching. The observed reactions to the leaching events for these three mineral phases, which were in a majority of the tank waste samples analyzed, justifies the three solid aluminum mineral categories that have previously been identified in RPP-RPT-47306: (1) easily leachable aluminum (e.g. gibbsite, dawsonite, nordstrandite, etc.); (2) slow leaching/kinetically controlled aluminum (e.g. boehmite); and (3) non-leachable/refractory aluminum (e.g. aluminosilicates). 
Table 2-1. Aluminum mineralogical data as described in individual laboratory studies. Included is information prior to leaching, after washing, and after caustic leaching (19 sheets).

\begin{tabular}{|c|c|c|c|c|c|}
\hline Tank & $\begin{array}{l}\text { Leaching } \\
\text { Temp. }\left({ }^{\circ} \mathrm{C}\right)\end{array}$ & $\begin{array}{l}\text { Lab Study } \\
\text { Reference }\end{array}$ & $\begin{array}{l}\text { Waste type } \\
\text { (WRPS, 2010). }\end{array}$ & Waste Sources. & $\begin{array}{l}\text { Aluminum minerals determined in as- } \\
\text { received, washed, or caustic leached } \\
\text { solids. }\end{array}$ \\
\hline $241-\mathrm{AN}-102$ & None & $\begin{array}{l}\text { PNWD-3300 } \\
\text { (WTP-RPT- } \\
076 \text { ) }\end{array}$ & $\begin{array}{l}\text { A2-SitSlr } \\
\text { (solid) and NA } \\
\text { (liquid). }\end{array}$ & Complexant Concentrate (CC) Waste, & $\begin{array}{l}\text { Washed solids: boehmite, zeolite, sodium } \\
\text { aluminate, and diaspore. }\end{array}$ \\
\hline $241-\mathrm{AN}-104$ & $100^{\circ} \mathrm{C}$ & PNNL-11636 & $\begin{array}{l}\text { A2-SitSlr } \\
\text { (liquid and } \\
\text { solid). }\end{array}$ & $\begin{array}{l}\text { Primarily "salt cake", characterized as } \\
\text { Double-shell slurry feed. } \\
\text { Double-shell Slurry Feed. }\end{array}$ & $\begin{array}{l}\text { Fine particles (likely sodium aluminate) and } \\
\text { Al and Si species with clay-like (or film- } \\
\text { like) morphologies. Amorphous (am) } \\
\text { aluminosilicate (AISi). } \\
\text { AlSi (am). } \\
\text { AlSi (am) in as-received; none identified in } \\
\text { leached sludge using TEM. }\end{array}$ \\
\hline 241-AW-105 & None & PNNL-13394 & $\begin{array}{l}\text { CWZr2 (solid), } \\
\text { PL2 (solid), and } \\
\text { NA (liquid). }\end{array}$ & $\begin{array}{l}\text { Primarily Neutralized Cladding Removal } \\
\text { Waste } \\
\text { Dilute Non-complexed/PUREX } \\
\text { neutralized cladding removal waste } \\
\text { (NCRW) solids or sludge remaining } \\
\text { after decanting (DN/PD). }\end{array}$ & $\begin{array}{l}\text { As-received sludge: gibbsite and crystalline } \\
\text { (cr) AlSi. } \\
\text { Leached sludge: AlSi (cr) identified using } \\
\text { TEM/SEM/XRD. }\end{array}$ \\
\hline 241-AZ-101 & 80 to $85^{\circ} \mathrm{C}$ & $\begin{array}{l}\text { PNWD-3206 } \\
\text { (WTP-RPT- } \\
\text { (043) }\end{array}$ & $\begin{array}{l}\text { NA (sludge) } \\
\text { and P3AZ1 } \\
\text { (liquid and } \\
\text { solid). }\end{array}$ & - & $\begin{array}{l}\text { In washed solids: Gibbsite, boehmite, and } \\
\mathrm{Al}-\mathrm{Zr}-\mathrm{Fe} \text { phase. }\end{array}$ \\
\hline $\begin{array}{l}241-\mathrm{AZ}-101 \text { and } \\
241-\mathrm{AZ}-102\end{array}$ & 80 to $85^{\circ} \mathrm{C}$ & PNNL-11580 & - & Aging. $^{\top}$ & $\begin{array}{l}\text { None identified in as-received; analysis not } \\
\text { performed for leached sludge using SEM. }\end{array}$ \\
\hline $241-\mathrm{AZ}-102$ & 80 to $85^{\circ} \mathrm{C}$ & $\begin{array}{l}\text { PNWD-3045 } \\
\text { (BNFL-RPT- } \\
\text { 038) }\end{array}$ & $\begin{array}{l}\text { NA (sludge), } \\
\text { P3AZ2 (solid), } \\
\text { PL2 (solid), } \\
\text { SRR (solid), } \\
\text { NA Lower } \\
\text { (liquid), and } \\
\text { NA Upper } \\
\text { (liquid). }\end{array}$ & - & $\begin{array}{l}\text { AISi (am) in as-received; none identified as } \\
\text { present in leached sludge using SEM. }\end{array}$ \\
\hline
\end{tabular}


Table 2-1. Aluminum mineralogical data as described in individual laboratory studies. Included is information prior to leaching, after washing, and after caustic leaching (19 sheets).

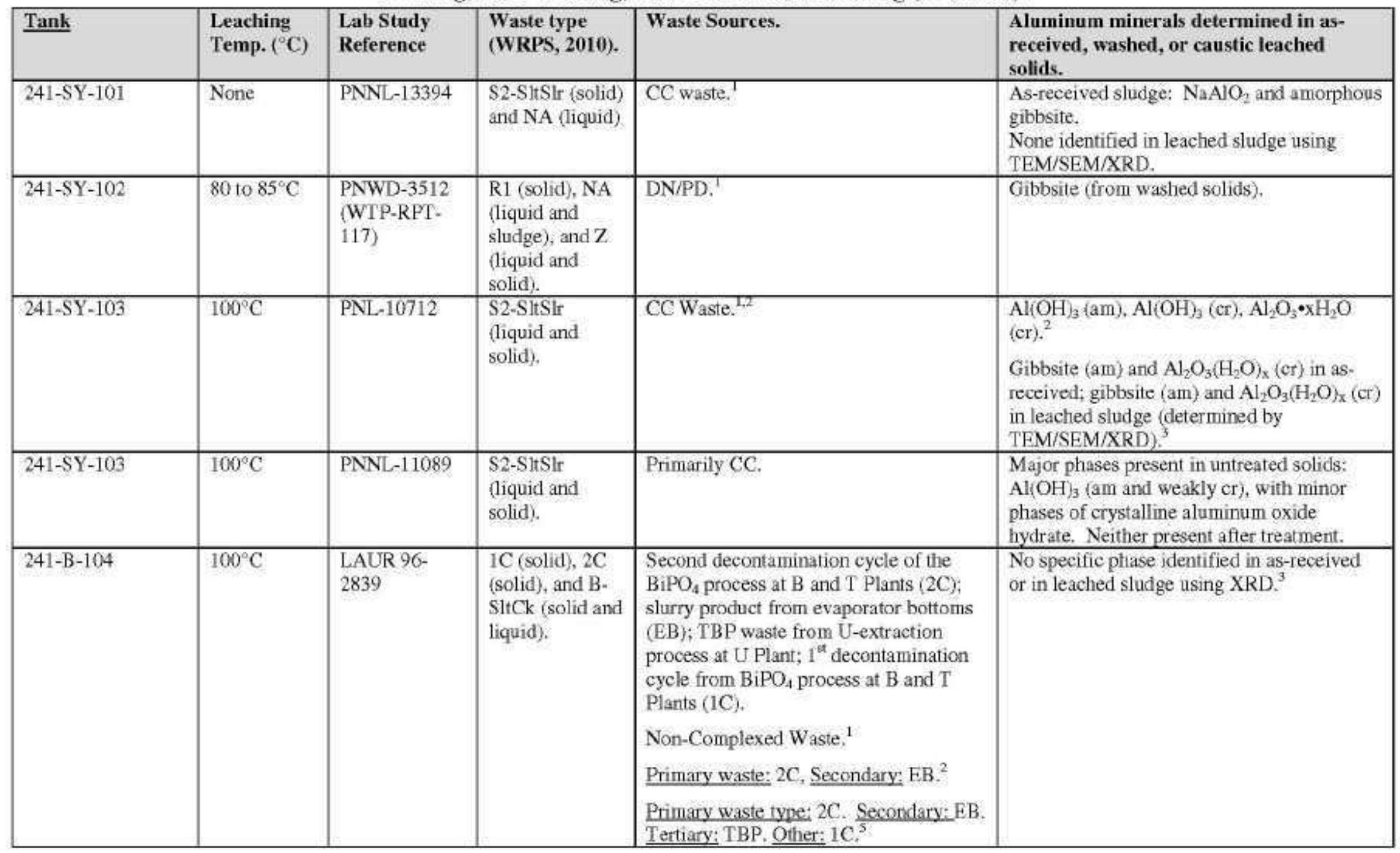


Table 2-1. Aluminum mineralogical data as described in individual laboratory studies. Included is information prior to leaching, after washing, and after caustic leaching (19 sheets).

\begin{tabular}{|c|c|c|c|c|c|}
\hline Tank & $\begin{array}{l}\text { Leaching } \\
\text { Temp. }\left({ }^{\circ} \mathrm{C}\right)\end{array}$ & $\begin{array}{l}\text { Lab Study } \\
\text { Reference }\end{array}$ & $\begin{array}{l}\text { Waste type } \\
\text { (WRPS, 2010). }\end{array}$ & Waste Sources. & $\begin{array}{l}\text { Aluminum minerals determined in as- } \\
\text { received, washed, or caustic leached } \\
\text { solids. }\end{array}$ \\
\hline $241-B-106$ & $100^{\circ} \mathrm{C}$ & $\begin{array}{l}\text { LAUR 97- } \\
2889\end{array}$ & $\begin{array}{l}\text { 1C (solid), TBP } \\
\text { (solid, and B- } \\
\text { SltCk (liquid). }\end{array}$ & $\begin{array}{l}\text { 1C, TBP from U-extraction process at U } \\
\text { Plant, lab waste from the } 300 \text { area. } \\
\text { Non-Complexed Waste. } \\
\text { Primary waste: } 1 C \text {. Secondary: TBP. } \\
\text { Primary waste type: } 1 \mathrm{C} \text {. Secondary: } \\
\text { TBP. Tertiary: Hanford Laboratory } \\
\text { Operations. Other: mixture of several } \\
\text { miscellaneous wastes. }\end{array}$ & $\begin{array}{l}\text { No specific phase identified in as-received } \\
\text { or in leached sludge using XRD. }\end{array}$ \\
\hline $241-B-110$ & $100^{\circ} \mathrm{C}$ & PNL-9387 & $\begin{array}{l}2 \mathrm{C} \text { (solid), B } \\
\text { (solid), and } \\
\text { CSR (liquid). }\end{array}$ & $\begin{array}{l}2 \mathrm{C} \text { in } 40-50 \text { to recover \& purify } \mathrm{Pu} \\
\text { from irradiated fuel, adjusted to high pH; } \\
\text { fission product waste produced in } \\
\text { campaigns ('60s) to isolate fission } \\
\text { products; ion exchange waste from B } \\
\text { Plant Cs recovery process. } \\
\text { Non-Complexed Waste. } \\
\text { Primary waste: } 2 \mathrm{C} \text {. Secondary: High- } \\
\text { level B Plant waste from bottom of } \\
\text { Section } 5 \text {. }^{2} \\
\text { Primary waste type: } 2 \mathrm{C} \text {. Sccondary: } \\
\text { high-level B Plant waste. Tertiary: } \\
\text { Fission Products Waste. Other: IX } \\
\text { waste. }\end{array}$ & $\begin{array}{l}\text { Sodium AlSi hydrate in as-received; none in } \\
\text { leached sludge (determined by SEM/XRD). }{ }^{3}\end{array}$ \\
\hline $241-B-111$ & $100^{\circ} \mathrm{C}$ & PNL-10712 & $\begin{array}{l}2 \mathrm{C} \text { (solid), B } \\
\text { (solid), and } \\
\text { CSR (liquid). }\end{array}$ & $\begin{array}{l}\text { Non-Complexed Waste. } \\
\text { Primary waste: } 2 \text { C, Secondary: High- } \\
\text { level B Plant waste from bottom of } \\
\text { Section } 5^{2} \\
\text { Primary waste type: } 2 \text { C. Secondary. } \\
\text { high-level B Plant waste. Tertiary: } \\
\text { Fission Products Waste. Other: IX } \\
\text { waste. }\end{array}$ & $\begin{array}{l}\text { AISi (cr) } \\
\text { AlSi (cr) in as-received; AlSi (cr) in } \\
\text { leached sludge (determined by } \\
\text { TEM/SEM/XRD). }\end{array}$ \\
\hline
\end{tabular}


Table 2-1. Aluminum mineralogical data as described in individual laboratory studies. Included is information prior to leaching, after washing, and after caustic leaching (19 sheets).

\begin{tabular}{|c|c|c|c|c|c|}
\hline Tank & $\begin{array}{l}\text { Leaching } \\
\left.\text { Temp. ( }{ }^{\circ} \mathrm{C}\right)\end{array}$ & $\begin{array}{l}\text { Lab Study } \\
\text { Reference }\end{array}$ & $\begin{array}{l}\text { Waste type } \\
\text { (WRPS, 2010). }\end{array}$ & Waste Sources. & $\begin{array}{l}\text { Aluminum minerals determined in as- } \\
\text { received, washed, or caustic leached } \\
\text { solids. }\end{array}$ \\
\hline $241-B-111$ & $100^{\circ} \mathrm{C}$ & PNNL-11089 & $\begin{array}{l}2 \mathrm{C} \text { (solid), } \mathrm{B} \\
\text { (solid), and } \\
\text { CSR (liquid). }\end{array}$ & $\begin{array}{l}\text { Primarily } 2 \mathrm{C} \text {, with secondary waste } \\
\text { from HLW from Tank } 5-6 \text { at B Plant. }\end{array}$ & $\begin{array}{l}\text { Minor phase in untreated and treated solids: } \\
\text { AlSi (cr). }\end{array}$ \\
\hline $241-\mathrm{B}-202$ & $100^{\circ} \mathrm{C}$ & $\begin{array}{l}\text { LAUR 95- } \\
2070\end{array}$ & $224-2$ (solid). & $\begin{array}{l}\text { Final decontamination and concentration } \\
\text { stage of } \mathrm{BiPO}_{4} \text { process. } \\
\text { Primary waste: Lanthanum fluoride } \\
\text { decontamination waste. } \\
\text { Primary waste type: Lanthanum fluoride } \\
\text { decontamination waste. }\end{array}$ & $\begin{array}{l}\text { No specific phase identified in as-received } \\
\text { or in leached sludge using XRD. }\end{array}$ \\
\hline 241-BX-103 & $100^{\circ} \mathrm{C}$ & $\begin{array}{l}\text { LAUR 97 } \\
2889\end{array}$ & $\begin{array}{l}\text { CWP2 (solid), } \\
\text { TBP (solid), } \\
\text { and CWP2 } \\
\text { (liquid). }\end{array}$ & $\begin{array}{l}\text { TBP from U-extraction process at U } \\
\text { Plant, CW produced at PUREX plant } \\
\text { from dissolution of Zr or Al fuel } \\
\text { cladding, organic solvent wash waste } \\
\text { (OWW) from PUREX. } \\
\text { Non-Complexed Waste. } \\
\text { Primary waste type: TBP. Secondary: } \\
\text { CW. Tertiary: OWW. Other: mixture of } \\
\text { several miscellaneous wastes. }\end{array}$ & $\begin{array}{l}\text { Gibbsite in as-received sample. } \\
\text { Gibbsite in as-received; none in leached } \\
\text { sludge (determined by XRD). }\end{array}$ \\
\hline 241-BX-105 & $100^{\circ} \mathrm{C}$ & $\begin{array}{l}\text { LAUR 95: } \\
2070\end{array}$ & $\begin{array}{l}\text { BY-\$ltCk } \\
\text { (solid), CWP2 } \\
\text { (solid), MW1 } \\
\text { (solid), TBP } \\
\text { (solid), and } \\
\text { CWP2 (liquid). }\end{array}$ & $\begin{array}{l}\text { Frst waste received was TBP from TBP } \\
\text { uranium extraction process at U plant: } \\
\text { PUREX plant dissolution of zirconium } \\
\text { or aluminum fuel cladding; ion exchange } \\
\text { (IX) waste from cesium recovery } \\
\text { process at B plant; Last was EB } \\
\text { consisting of a slurry product from } \\
\text { evaporators. } \\
\text { Non-Complexed Waste. } \\
\text { Primary waste: TBP, Secondary: CW. } \\
\text { Primary waste type: TBP. Secondary: } \\
\text { CW. Tertiary: LX waste. Other: EB. }\end{array}$ & $\begin{array}{l}\text { As-received XRD: } \mathrm{Al} \text { hydroxide compound } \\
\left.\text { (Gibbsite } \mathrm{Al}(\mathrm{OH})_{3}\right) \text {. } \\
\text { Treated XRD: } \mathrm{Al} \text { hydroxide compound } \\
\left.\text { (Gibbsite } \mathrm{Al}(\mathrm{OH})_{3}\right) \text {. } \\
\text { Gibbsite in as-received; gibbsite in leached } \\
\text { sludge (determined by XRD). }\end{array}$ \\
\hline
\end{tabular}


Table 2-1. Aluminum mineralogical data as described in individual laboratory studies. Included is information prior to leaching, after washing, and after caustic leaching (19 sheets).

\begin{tabular}{|c|c|c|c|c|c|}
\hline Tank & $\begin{array}{l}\text { Leaching } \\
\text { Temp. }\left({ }^{\circ} \mathrm{C}\right)\end{array}$ & $\begin{array}{l}\text { Lab Study } \\
\text { Reference }\end{array}$ & $\begin{array}{l}\text { Waste type } \\
\text { (WRPS, 2010). }\end{array}$ & Waste Sources. & $\begin{array}{l}\text { Aluminum minerals determined in as- } \\
\text { received, washed, or caustic leached } \\
\text { solids. }\end{array}$ \\
\hline $241-B X-107$ & $100^{\circ} \mathrm{C}$ & PNL-10712 & $1 C$ (solid). & $\begin{array}{l}\text { Non-Complexed Waste. } \\
\text { Primary waste: } 1 C \text {, Secondary: TBP. } \\
\text { Primary waste type: 1C. Secondary: } \\
\text { TBP. Tertiary: CW. Other: IX waste, }\end{array}$ & $\begin{array}{l}\mathrm{Al}(\mathrm{OH})_{3} \text { (am), } \mathrm{Al}(\mathrm{OH})_{3} \text { (cr), } \mathrm{AlPO}_{4}, \mathrm{AlSi} \\
\text { (am), AlSi (cr) } \\
\mathrm{AlPO4}, \mathrm{Al}(\mathrm{OH})_{3}(\mathrm{am}) \text {, and } \mathrm{AlSi} \text { (cr and am) } \\
\text { in as-received; AlSi (cr, am) in leached } \\
\text { sludge (determined by TEM/SEM } / \text { XRD), }\end{array}$ \\
\hline 241-BX-107 & $100^{\circ} \mathrm{C}$ & PNNL-11089 & 1C (solid). & $\begin{array}{l}\text { Primarily } 1 \mathrm{C} \text {, with secondary waste } \\
\text { from the TBP uranium extraction } \\
\text { process at U Plant. }\end{array}$ & $\begin{array}{l}\text { Major phases present in untreated tank } \\
\text { solids: } \mathrm{AlPO}_{4} \text {, AlSi (cr and am), and } \\
\text { aluminum hydroxide (am). AlSi (cr and am) } \\
\text { present after treatment. }\end{array}$ \\
\hline 241-BX-109 & $100^{\circ} \mathrm{C}$ & $\begin{array}{l}\text { LAUR 96- } \\
2839\end{array}$ & TBP (solid). & $\begin{array}{l}\text { TBP from U-extraction process at } \mathrm{U} \\
\text { Plant; waste produced at PUREX Plant } \\
\text { from dissolution of } \mathrm{Zr} \text { or } \mathrm{Al} \text { fuel } \\
\text { cladding; } 1 \mathrm{C} \text {; IX waste from } \mathrm{Cs} \\
\text { recovery process at B Plant. } \\
\text { Non-Complexed Waste. } \\
\text { Primary waste: TBP. Secondary: CW. } \\
\text { Primary waste type: TBP. Secondary: } \\
\text { CW. Tertiary: } 1 \mathrm{C} \text {. Other: } \mathrm{IX} \text { waste. }\end{array}$ & $\begin{array}{l}\text { Nordstrandite }[\mathrm{Al}(\mathrm{OH}) 3] \text { in non-treated } \\
\text { waste sample. } \\
\text { Nordstrandite in as-received; none in } \\
\text { leached sludge (determined by XRD). }\end{array}$ \\
\hline $241-\mathrm{BX}-112$ & $\begin{array}{l}\text { Multiple } \\
\text { Leaching } \\
\text { Temperatures }\end{array}$ & PNNL-12026 & $\begin{array}{l}1 \mathrm{C} \text { (solid) and } \\
1 \mathrm{C} 2 \text { (liquid). }\end{array}$ & $\begin{array}{l}\text { Primary waste: } 1 \mathrm{C} \text {. Secondary waste: } \\
\text { EB. } \\
\text { Non-Complexed Waste. } \\
\text { Primary waste type: } 1 \mathrm{C} \text {. Secondary: EB. } \\
\text { Tertiary: CW. Other: IX. }\end{array}$ & $\begin{array}{l}\text { AlSi formed during leach step - increase w/ } \\
\text { increasing temperature (T). Washed solids } \\
\text { contained significant concentrations of } \\
\text { aluminum phosphates. }\end{array}$ \\
\hline
\end{tabular}


Table 2-1. Aluminum mineralogical data as described in individual laboratory studies. Included is information prior to leaching, after washing, and after caustic leaching (19 sheets).

\begin{tabular}{|c|c|c|c|c|c|}
\hline$\underline{\text { Tank }}$ & $\begin{array}{l}\text { Leaching } \\
\text { Temp. }\left({ }^{\circ} \mathrm{C}\right)\end{array}$ & $\begin{array}{l}\text { Lab Study } \\
\text { Reference }\end{array}$ & $\begin{array}{l}\text { Waste type } \\
\text { (WRPS, 2010). }\end{array}$ & Waste Sources. & $\begin{array}{l}\text { Aluminum minerals determined in as- } \\
\text { received, washed, or caustic leached } \\
\text { solids. }\end{array}$ \\
\hline 241-BY-104 & $100^{\circ} \mathrm{C}$ & PNNL-11278 & $\begin{array}{l}\text { BY-S1tCk } \\
\text { (liquid and } \\
\text { solid) and } \\
\text { PFeCN (solid). }\end{array}$ & $\begin{array}{l}\text { Primary: TBP extraction process waste } \\
\text { and FeCN-scavenged waste, Secondary: } \\
\text { EB and in-tank solidification. } \\
\text { Non-Complexed Waste. } \\
\text { Primary Waste: TBP and FeCN- } \\
\text { scavenged. Secondary: EB and in-tank } \\
\text { solidification. } \\
\text { Primary waste type: TBP/FeCN- } \\
\text { scavenged waste. Secondary: EB/in- } \\
\text { tank solidification. Tertiary: CW. } \\
\text { Other: IX waste. }\end{array}$ & $\begin{array}{l}\text { Untreated: aluminum oxide and } \mathrm{Si} / \mathrm{Al} \text { clay } \\
\text { material }(\mathrm{am}) \text {. Treated aluminum oxide. } \\
\mathrm{Al}_{2} \mathrm{O}_{3} \cdot \mathrm{xH}_{2} \mathrm{O}(\mathrm{cr}), \mathrm{AlSi} \text { (am). } \\
\left(\mathrm{Al}_{2} \mathrm{O}_{3}\right)_{\mathrm{x}}-\left(\mathrm{H}_{2} \mathrm{O}\right)_{\mathrm{y}} \text { and } \mathrm{AlSi}(\mathrm{am}) \text { in as- } \\
\text { received; }\left(\mathrm{Al}_{2} \mathrm{O}_{3}\right)_{\mathrm{x}}-\left(\mathrm{H}_{2} \mathrm{O}\right)_{\mathrm{y}} \text { in leached sludge } \\
\text { (determined by TEM). }\end{array}$ \\
\hline 241-BY-108 & $100^{\circ} \mathrm{C}$ & PNNL-11636 & $\begin{array}{l}\text { BY-SltCk } \\
\text { (liquid and } \\
\text { solid) and } \\
\text { PFeCN (solid). }\end{array}$ & $\begin{array}{l}\text { Non-Complexed Waste. } \\
\text { Primary Waste: TBP and FeCN- } \\
\text { scavenged, Secondary: EB and in-tank } \\
\text { solidification. }{ }^{2} \\
\text { Primary waste type: TBP/FeCN- } \\
\text { scavenged waste. Secondary: EB/in- } \\
\text { tank solidification. Tertiary: 1C. Other: } \\
\text { CW }\end{array}$ & $\begin{array}{l}\text { No spocific Al mineral phase determined by } \\
\text { TEM. }^{3}\end{array}$ \\
\hline 241-BY-110 & $100^{\circ} \mathrm{C}$ & PNNL-11278 & $\begin{array}{l}\text { BY-SltCk } \\
\text { (liquid and } \\
\text { solid) and } \\
\text { PFeCN (solid). }\end{array}$ & $\begin{array}{l}\text { Primary: TBP extraction process waste } \\
\text { and FeCN-scavenged waste, Secondary: } \\
\mathrm{EB} \text { and In-tank solidification. } \\
\text { Non-Complexed Waste. } \\
\text { Primary Waste: TBP and FeCN- } \\
\text { scavenged, Secondary: EB and in-tank } \\
\text { solidification. } \\
\text { Primary waste type. TBP/FeCN- } \\
\text { scavenged waste. Secondary: EB/in- } \\
\text { tank solidification. Tertiary: 1C. Other: } \\
\text { CW }{ }^{5}\end{array}$ & $\begin{array}{l}\text { No specific Al mineral phase determined by } \\
\text { TEM. }\end{array}$ \\
\hline
\end{tabular}


Table 2-1. Aluminum mineralogical data as described in individual laboratory studies. Included is information prior to leaching, after washing, and after caustic leaching (19 sheets).

\begin{tabular}{|c|c|c|c|c|c|}
\hline Tank & $\begin{array}{l}\text { Leaching } \\
\left.\text { Temp. ( }{ }^{\circ} \mathrm{C}\right)\end{array}$ & $\begin{array}{l}\text { Lab Study } \\
\text { Reference }\end{array}$ & $\begin{array}{l}\text { Waste type } \\
\text { (WRPS, 2010). }\end{array}$ & Waste Sources. & $\begin{array}{l}\text { Aluminum minerals determined in as- } \\
\text { received, washed, or caustic leached } \\
\text { solids. }\end{array}$ \\
\hline $241-\mathrm{C}-104$ & $\begin{array}{l}\text { Multiple } \\
\text { Leaching } \\
\text { Temperatures }\end{array}$ & $\begin{array}{l}\text { ORNL TM- } \\
13500\end{array}$ & $\begin{array}{l}\text { CWP1 (solid) } \\
\text { and NA } \\
\text { (sludge). }\end{array}$ & $\begin{array}{l}\text { High aluminum, low phosphorous and } \\
\text { bismuth. } \\
\text { High phosphorous sludge with moderate } \\
\text { concentrations of alumimum and } \\
\text { chromium. Waste types include PUREX } \\
\text { aluminum waste and zirconium CW; CC } \\
\text { Waste. }\end{array}$ & $\begin{array}{l}\text { No specific phase identified in as-received } \\
\text { or in leached sludge using XRD. }\end{array}$ \\
\hline $241-\mathrm{C}-105$ & $100^{\circ} \mathrm{C}$ & $\begin{array}{l}\text { LAUR 97- } \\
2889\end{array}$ & $\begin{array}{l}\text { CWP1 (solid) } \\
\text { and TBP } \\
\text { (solid). }\end{array}$ & $\begin{array}{l}\text { TBP waste from U-extraction process at } \\
\text { U plant, Sr wash of PUREX waste in } \\
\text { AR vault, waste from dissolution of } \mathrm{Zr} \\
\text { or Al fuel cladding at PUREX, high- } \\
\text { activity neutralized acid waste generated } \\
\text { by PUREX. } \\
\text { Non-Complexed Waste. } \\
\text { Primary waste: TBP. Secondary: } \\
\text { Particulates from Sr wash of PUREX } \\
\text { wastes in the AR vault. }{ }^{2} \\
\text { Primary waste type: TBP. Secondary: } \\
\text { particulates from Sr wash of PUREX } \\
\text { wastes in AR-vault. Tertiary: CW. } \\
\text { Other: neutralized acid waste. }\end{array}$ & $\begin{array}{l}\text { Gibbsite in as-received samples. } \\
\mathrm{Al}(\mathrm{OH}) 3(\mathrm{cr}) \text {. } \\
\text { Gibbsite in as-received; none in leached } \\
\text { sludge (determined by XRD). }\end{array}$ \\
\hline $241-\mathrm{C}-106$ & $100^{\circ} \mathrm{C}$ & PNNL-11381 & $\begin{array}{l}\text { NA (liquid and } \\
\text { sludge). }\end{array}$ & $\begin{array}{l}\text { Non-Complexed Waste. } \\
\text { Primary waste: Sr leached sludge, } \\
\text { Secondary: Particulates from Sr wash of } \\
\text { PUREX wastes in the AR vault. } \\
\text { Primary waste type: Sr sludge. } \\
\text { Secondary: particulates from Sr wash of } \\
\text { PUREX wastes in the AR-vault. } \\
\text { Tertiary: neutralized acid waste. Other: } \\
\text { TBP. }\end{array}$ & $\begin{array}{l}\text { Untreated sludge solids: aluminum } \\
\text { hydroxide (am) and AlSi (am). After } \\
\text { leaching (solids): predominantly AlSi (am). } \\
\text { Al(OH) (am), AlSi (am). } \\
\text { Gibbsite (am) and AlSi (am) in as-received; } \\
\text { AlSi (am) in leached sludge (determined by } \\
\text { TEM). }\end{array}$ \\
\hline
\end{tabular}


Table 2-1. Aluminum mineralogical data as described in individual laboratory studies. Included is information prior to leaching, after washing, and after caustic leaching (19 sheets).

\begin{tabular}{|c|c|c|c|c|c|}
\hline$\underline{\text { Tank }}$ & $\begin{array}{l}\text { Leaching } \\
\left.\text { Temp. ( }{ }^{\circ} \mathrm{C}\right)\end{array}$ & $\begin{array}{l}\text { Lab Study } \\
\text { Reference }\end{array}$ & $\begin{array}{l}\text { Waste type } \\
\text { (WRPS, 2010). }\end{array}$ & Waste Sources. & $\begin{array}{l}\text { Aluminum minerals determined in as- } \\
\text { received, washed, or caustic leached } \\
\text { solids. }\end{array}$ \\
\hline $241-\mathrm{C}-107$ & $100^{\circ} \mathrm{C}$ & PNNL-11278 & $\begin{array}{l}\text { 1C (solid), } \\
\text { CWP2 (solid), } \\
\text { and SRR } \\
\text { (solid). }\end{array}$ & $\begin{array}{l}\text { Primary: 1C. Secondary: CW. } \\
\text { Dilute Complexed Waste. } \\
\text { Primary waste type: } 1 \text {. } \\
\text { sludge. Tecondary: Sr } \\
\text { Other: IX waste. }\end{array}$ & $\begin{array}{l}\text { Untreated and Treated solids: Si/Al clay } \\
\text { material (am) and aluminum oxide. } \\
\mathrm{Al}_{2} \mathrm{O}_{3} \cdot \mathrm{xH}_{2} \mathrm{O}(\mathrm{cr}), \mathrm{AlSi} \text { (am). } \\
\left(\mathrm{AJ}_{2} \mathrm{O}_{3}\right)_{x}-\left(\mathrm{H}_{2} \mathrm{O}\right)_{y} \text { and } \mathrm{AlSi} \text { (am) in as- } \\
\text { received; }\left(\mathrm{Al}_{2} \mathrm{O}_{3}\right)_{x} \cdot\left(\mathrm{H}_{2} \mathrm{O}\right)_{y} \text { and } \mathrm{AlSi} \text { (am) in } \\
\text { leached sludge (determined by TEM). }\end{array}$ \\
\hline 241-C-109 & $100^{\circ} \mathrm{C}$ & PNL-9387 & IC (solid). & $\begin{array}{l}\text { TBP U extraction process operated at U } \\
\text { Plant (wastes treated w/ } \mathrm{FeCN} \text { to } \\
\text { scavenge } 137 \text {-Cs from supernatant } \\
\text { solution); } 1 \mathrm{C} ; \mathrm{Al} \text { \&/or } \mathrm{Zr} \mathrm{CW} \text {; IX waste } \\
\text { from B Plant } \mathrm{Cs} \text { recovery process. } \\
\text { Non-Complexed Waste, } \\
\text { Primary waste: TBP and FeCN- } \\
\text { scavenged waste, Secondary: } 1 \mathrm{C}^{2} \\
\text { Primary waste type: TBP/FeCN- } \\
\text { scavenged waste. Secondary: } 1 \mathrm{C} \text {. } \\
\text { Tertiary: CW. Other: IX waste. }\end{array}$ & $\begin{array}{l}\text { Gibbsite in as-received; gibhsite, boehmite, } \\
\text { and nickel aluminum oxide in leached. } \\
\text { sludge (determined by SEM/XRD). }\end{array}$ \\
\hline
\end{tabular}


Table 2-1. Aluminum mineralogical data as described in individual laboratory studies. Included is information prior to leaching, after washing, and after caustic leaching (19 sheets).

\begin{tabular}{|c|c|c|c|c|c|}
\hline Tank & $\begin{array}{l}\text { Leaching } \\
\text { Temp. }\left({ }^{\circ} \mathrm{C}\right)\end{array}$ & $\begin{array}{l}\text { Lab Study } \\
\text { Reference }\end{array}$ & $\begin{array}{l}\text { Waste type } \\
\text { (WRPS, 2010). }\end{array}$ & Waste Sources. & $\begin{array}{l}\text { Aluminum minerals determined in as- } \\
\text { received, washed, or caustic leached } \\
\text { solids. }\end{array}$ \\
\hline $241-\mathrm{C}-112$ & $100^{\circ} \mathrm{C}$ & PNL-9387 & $\begin{array}{l}\text { 1C (solid), } \\
\text { CWP1 (solid), } \\
\text { CWP2 (solid). } \\
\text { HS (solid), and } \\
\text { TFeCN (solid). }\end{array}$ & $\begin{array}{l}\text { TBP U extraction process operated at } \mathrm{U} \\
\text { Plant (wastes treated w/ FeCN to } \\
\text { scavenge } 137 \text {-Cs from supernatant } \\
\text { solution); } 1 \mathrm{C} \text {; Al \&lor } \mathrm{Zr} \mathrm{CW} \text {; IX waste } \\
\text { from B Plant Cs recovery process. } \\
\text { Non-Complexed Wastc. } \\
\text { Primary waste: TBP and FeCN- } \\
\text { scavenged waste. Secondary: } 1 \mathrm{C} .^{2} \\
\text { Primary waste type: TBP/FeCN- } \\
\text { scavenged waste. Secondary: } 1 \mathrm{C} \text {. } \\
\text { Tertiary: CW. Other: IX waste. }\end{array}$ & $\begin{array}{l}\mathrm{Al}(\mathrm{OH}) 3 \text { (cr) } \\
\text { Gibbsite in as-received; none identified in } \\
\text { leached sludge (determined by SEM/XRD). }\end{array}$ \\
\hline $241-5-101$ & $100^{\circ} \mathrm{C}$ & PNNL-11636 & $\begin{array}{l}\text { S1-SltCk } \\
\text { (liquid and } \\
\text { solid), S2-SliSlr } \\
\text { (liquid and } \\
\text { solid), and NA } \\
\text { (sludge), }\end{array}$ & $\begin{array}{l}\text { Non-Complexed Waste. } \\
\text { Primary Waste: High-Level REDOX, } \\
\text { Secondary: EB. } \\
\text { Primary waste type: High-level REDOX } \\
\text { waste. Secondary: EB. Tertiary: IX } \\
\text { waste. Other: mixture of several } \\
\text { miscellaneous wastes. }\end{array}$ & $\begin{array}{l}\text { Bochmite was the predominant solid phase; } \\
\text { leached solids contained AlSi (am) and rod- } \\
\text { like aluminum oxyhydroxide (diaspore). } \\
\mathrm{Al}(\mathrm{O}) \mathrm{OH} \text { (boehmite), } \mathrm{Al}(\mathrm{O}) \mathrm{OH} \text { (diaspore), } \\
\mathrm{Al}(\mathrm{OH}) 3 \text { (cr), AlSi (am). } \\
\text { Boehmite in as-received; boehmite, AlSi } \\
\text { (am), and diaspore in leached sludge } \\
\text { (determined by TEM). }\end{array}$ \\
\hline $241-5-104$ & $100^{\circ} \mathrm{C}$ & $\begin{array}{l}\text { LALR 95- } \\
2070\end{array}$ & $\begin{array}{l}\text { R-SltCk (liquid } \\
\text { and solid), } \\
\text { CWR1 (solid), } \\
\text { and R1 (solid). }\end{array}$ & $\begin{array}{l}\text { Primary waste: High level waste from } \\
\text { the REDOX process; Non-Complexed } \\
\text { Waste. }{ }^{1}\end{array}$ & $\begin{array}{l}\text { As-received XRD: two } \mathrm{Al} \text { oxide-hydroxide } \\
\text { compounds }[\mathrm{AlO}(\mathrm{OH}) \text { and } \\
\left.\mathrm{Al}_{45} \mathrm{O}_{45}(\mathrm{OH})_{45} \mathrm{Cl}\right] \text {. } \\
\text { Treated } \mathrm{XRD}: \mathrm{Al} \text { oxide-hydroxide } \\
\text { compound }[\mathrm{AlO}(\mathrm{OH})] \text {. }\end{array}$ \\
\hline $241-S-104$ & $100^{\circ} \mathrm{C}$ & PNNL-11089 & $\begin{array}{l}\text { R-SltCk (liquid } \\
\text { and solid), } \\
\text { CWR1 (solid), } \\
\text { and RI (solid). }\end{array}$ & $\begin{array}{l}\text { Primarily reduction oxidation (REDOX) } \\
\text { process HLW. }\end{array}$ & $\begin{array}{l}\text { Boehmite present in treated and untreated } \\
\text { tank solids. }\end{array}$ \\
\hline
\end{tabular}


Table 2-1. Aluminum mineralogical data as described in individual laboratory studies. Included is information prior to leaching, after washing, and after caustic leaching (19 sheets).

\begin{tabular}{|c|c|c|c|c|c|}
\hline Tank & $\begin{array}{l}\text { Leaching } \\
\text { Temp. }\left({ }^{\circ} \mathrm{C}\right)\end{array}$ & $\begin{array}{l}\text { Lab Study } \\
\text { Reference }\end{array}$ & $\begin{array}{l}\text { Waste type } \\
\text { (WRPS, 2010). }\end{array}$ & Waste Sources. & $\begin{array}{l}\text { Aluminum minerals determined in as- } \\
\text { received, washed, or caustic leached } \\
\text { solids. }\end{array}$ \\
\hline $241-S-104$ & $100^{\circ} \mathrm{C}$ & PNNL-11636 & $\begin{array}{l}\text { R-SltCk (liquid } \\
\text { and solid), } \\
\text { CWR1 (solid), } \\
\text { and R1 (solid). }\end{array}$ & $\begin{array}{l}\text { Non-Complexed Waste. } \\
\text { Primary Waste: High-Level REDOX }{ }^{2}\end{array}$ & $\begin{array}{l}\text { Boehmite (AlOOH) indicated as the } \\
\text { predominant phase present. Some clay, } \\
\text { some iron-containing particles, and some } \\
\text { other minor phases were also observed. } \\
\mathrm{Al}(\mathrm{O}) \mathrm{OH} \text { (boehmite). (PNL-10712) } \\
\text { Boehmite in as-received; boehmite and AISi } \\
\text { (am) in leached sludge (determined by } \\
\text { TEM). }\end{array}$ \\
\hline $241-5-107$ & $100^{\circ} \mathrm{C}$ & PNNL-11278 & $\begin{array}{l}\text { S1-SltCk } \\
\text { (solid), S2- } \\
\text { S1tSlr (solid), } \\
\text { T2-SitCk } \\
\text { (solid), CWR1 } \\
\text { (solid), CWR2 } \\
\text { (solid), CWZr1 } \\
\text { (solid), and R1 } \\
\text { (solid). } \\
\end{array}$ & $\begin{array}{l}\text { Primary: High-level REDOX process } \\
\text { waste. Secondary: EB. } \\
\text { Non-Complexed Waste. } \\
\text { Primary Waste: High-Level REDOX, } \\
\text { Secondary EB. }{ }^{2}\end{array}$ & $\begin{array}{l}\text { Untreated and Treated solids: boehmite and } \\
\mathrm{Si} / \mathrm{Al}(\mathrm{am}) \text { clay material. } \mathrm{Al}(\mathrm{O}) \mathrm{OH} \\
\text { (boehmite). AlSi (am) } \\
\text { Bochmite and AlSi (am) in as-received; } \\
\text { boehmite and AlSi (am) in leached sludge } \\
\text { (determined by TFM). }\end{array}$ \\
\hline $241-S-110$ & $\begin{array}{l}\text { Multiple } \\
\text { Leaching } \\
\text { Temperatures }\end{array}$ & PNNL-13702 & $\begin{array}{l}\text { S1-Sitek } \\
\text { (liquid and } \\
\text { solid), CWR1 } \\
\text { (solid), and R1 } \\
\text { (solid). }\end{array}$ & $\begin{array}{l}\text { Non-Complexed Waste. } \\
\text { Primary waste type: High-level REDOX } \\
\text { waste. Secondary: EB. Tertiary: } \\
\text { mixture of several miscellaneous } \\
\text { wastes. }\end{array}$ & $\begin{array}{l}\text { XRD analysis of washed solids indicated } \\
\text { crystalline forms of } \mathrm{Al} \text { were } 10-20 \% \\
\text { gibbsite and } 80-90 \% \text { boehmite. }\end{array}$ \\
\hline
\end{tabular}


Table 2-1. Aluminum mineralogical data as described in individual laboratory studies. Included is information prior to leaching, after washing, and after caustic leaching (19 sheets).

\begin{tabular}{|c|c|c|c|c|c|}
\hline$\underline{\text { Tank }}$ & $\begin{array}{l}\text { Leaching } \\
\text { Temp. }\left({ }^{\circ} \mathrm{C}\right)\end{array}$ & $\begin{array}{l}\text { Lab Study } \\
\text { Reference }\end{array}$ & $\begin{array}{l}\text { Waste type } \\
\text { (WRPS, 2010). }\end{array}$ & Waste Sources. & $\begin{array}{l}\text { Aluminum minerals determined in as- } \\
\text { received, washed, or caustic leached } \\
\text { solids. }\end{array}$ \\
\hline $241-S-110$ & $\begin{array}{l}\text { Caustic and } \\
\text { Oxidative } \\
\text { Leaching } \\
\text { Performed }\end{array}$ & PNNL-14018 & $\begin{array}{l}\text { S1-Sltck (liquid } \\
\text { and solid), } \\
\text { CWR1 (solid). } \\
\text { and R1 (solid). }\end{array}$ & $\begin{array}{l}\text { Primarily REDOX waste, with } \\
\text { secondary stream from EB and tertiary } \\
\text { stream composed as a mixture of several } \\
\text { miscellaneous wastes. } \\
\text { From cluster analysis, contains saltcake } \\
\text { from } 242 \mathrm{~A} \text { campaign ( } 1976-1980 \text { and } \\
1981-1988 \text { ), saltcake from the } 242-\mathrm{B} \\
\text { operation (1951-1953), saltcake from the } \\
242-\mathrm{S} \text { campaign (1973-1976), saltcake } \\
\text { from self condensation, REDOX waste, } \\
\text { and saltcake from the } 242 \text {-S campaign } \\
\text { (1977-1980). } \\
\text { Non-Complexed Waste. }\end{array}$ & $\begin{array}{l}\text { XRD analysis of washed sludge shows both } \\
(80-90 \%) \text { boehmite and }(10-20 \%) \text { gibbsite } \\
\text { present. }\end{array}$ \\
\hline $241-S-111$ & $100^{\circ} \mathrm{C}$ & PNNL-11636 & $\begin{array}{l}\text { S1-Sltck (liquid } \\
\text { and solid), } \\
\text { CWR1 (solid). } \\
\text { and R1 (solid). }\end{array}$ & $\begin{array}{l}\text { Non-Complexed Waste.' } \\
\text { Primary Waste: High-Level REDOX, } \\
\text { Secondary: EB. }\end{array}$ & $\begin{array}{l}\text { Leached solids major phases were bayerite } \\
{[\mathrm{Al}(\mathrm{OH}) 3] \text { and boehmite }[\mathrm{AlOOH}]} \\
\mathrm{Al}(\mathrm{O}) \mathrm{OH} \text { (boehmite), } \mathrm{Al}(\mathrm{OH}) 3(\mathrm{cr})^{2} \\
\text { Bayerite and boehmite in leached sludge } \\
\text { (determined by TEM). }\end{array}$ \\
\hline 241-SX-101 & 80 to $85^{\circ} \mathrm{C}$ & $\begin{array}{l}\text { PNWD-3512 } \\
\text { (WTP-RPT. } \\
\text { 117) }\end{array}$ & $\begin{array}{l}\text { R-SitCk (liquid } \\
\text { and solid), S1- } \\
\text { SltCk (liquid } \\
\text { and solid,, S2- } \\
\text { Sitslr (liquid } \\
\text { and solid), and } \\
\text { R1 (solid). }\end{array}$ & Dilute Complexed Waste. $^{1}$ & $\begin{array}{l}\text { "Some sort of } \mathrm{Al}(\mathrm{OH})_{3} \text { is postulated from } \\
\text { washed solids analysis." }\end{array}$ \\
\hline
\end{tabular}


Table 2-1. Aluminum mineralogical data as described in individual laboratory studies. Included is information prior to leaching, after washing, and after caustic leaching (19 sheets).

\begin{tabular}{|c|c|c|c|c|c|}
\hline$\underline{\text { Tank }}$ & $\begin{array}{l}\text { Leaching } \\
\text { Temp. }\left({ }^{\circ} \mathrm{C}\right)\end{array}$ & $\begin{array}{l}\text { Lab Study } \\
\text { Reference }\end{array}$ & $\begin{array}{l}\text { Waste type } \\
\text { (WRPS, 2010). }\end{array}$ & Waste Sources. & $\begin{array}{l}\text { Aluminum minerals determined in as- } \\
\text { received, washed, or caustic leached } \\
\text { solids. }\end{array}$ \\
\hline 241-SX-108 & $100^{\circ} \mathrm{C}$ & PNNL-11278 & $\begin{array}{l}\text { R1 (solid) and } \\
\text { R2 (solid). }\end{array}$ & $\begin{array}{l}\text { Primary: High-level RED OX process } \\
\text { waste. } \\
\text { Non-Complexed Waste. } \\
\text { Primary waste: High level REDOX }{ }^{2}\end{array}$ & 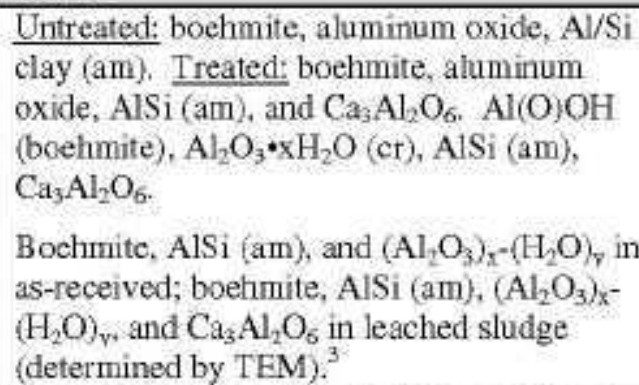 \\
\hline $241-5 X-113$ & $100^{\circ} \mathrm{C}$ & $\begin{array}{l}\text { LAUR 97- } \\
2889\end{array}$ & $\begin{array}{l}\mathrm{DE} \text { (solid) and } \\
\mathrm{R} 1 \text { (solid). }\end{array}$ & $\begin{array}{l}\text { Non-Complexed Waste. } \\
\text { Primary waste: High level REDOX, } \\
\text { Secondary: Diatomaceous earth } \\
\text { Primary waste type: high-level REDOX } \\
\text { waste. Secondary: diatomaceous earth. }\end{array}$ & $\begin{array}{l}\text { No specific phase identified in as-received } \\
\text { or in leached sludge using XRD. }\end{array}$ \\
\hline $241-\mathrm{T}-104$ & $100^{\circ} \mathrm{C}$ & $\begin{array}{l}\text { LAUR 95- } \\
2070\end{array}$ & 1C (solid), & $\begin{array}{l}1 \mathrm{C}, \\
\text { Non-Complexed Waste. }\end{array}$ & $\begin{array}{l}\text { In as received: } \mathrm{Na}_{2} \mathrm{Fe}_{2} \mathrm{Al}_{1}\left(\mathrm{PO}_{43}\right)_{3}- \\
\text { Ferrowyllieite and } \mathrm{Bi}_{24} \mathrm{Al}_{2} \mathrm{O}_{39}-\text { Aluminum } \\
\text { Bismuth Oxide. } \\
\text { In treated: } 2 \text { types of sodium AlSi. }\end{array}$ \\
\hline
\end{tabular}


Table 2-1. Aluminum mineralogical data as described in individual laboratory studies. Included is information prior to leaching, after washing, and after caustic leaching (19 sheets).

\begin{tabular}{|c|c|c|c|c|c|}
\hline Tank & $\begin{array}{l}\text { Leaching } \\
\text { Temp. }\left({ }^{\circ} \mathrm{C}\right)\end{array}$ & $\begin{array}{l}\text { Lab Study } \\
\text { Reference }\end{array}$ & $\begin{array}{l}\text { Waste type } \\
\text { (WRPS, 2010). }\end{array}$ & Waste Sources. & $\begin{array}{l}\text { Aluminum minerals determined in as- } \\
\text { received, washed, or caustic leached } \\
\text { solids. }\end{array}$ \\
\hline $241-\mathrm{T}-107$ & $100^{\circ} \mathrm{C}$ & $\begin{array}{l}\text { LAUR 95- } \\
2070\end{array}$ & $\begin{array}{l}\text { 1C (solid), } \\
\text { CWP2 (solid), } \\
\text { and TBP } \\
\text { (solid). }\end{array}$ & $\begin{array}{l}\text { Three significant types of waste: IC, } \\
\text { PUREX CW, and TBP from the TBP } \\
\text { uranium-extraction process at U Plant. } \\
\text { Non-Complexed Waste. } \\
\text { Primary waste: } 1 \mathrm{C} \text {. Secondary: CW. } \\
\text { Primary waste type: TBP/FeCN- } \\
\text { scavenged waste. Secondary: } 1 \mathrm{C} \text {. } \\
\text { Tertiary: CW. Other: IX waste. }\end{array}$ & $\begin{array}{l}\text { In as received; gibbsite. In treated: sodium } \\
\text { aluminum carbonate, albite, sodium } \\
\text { aluminum nitrate silicon, berlinite. } \\
\text { Gibbsite in as-received; AlSi ( } \mathrm{cr} \text { ) and } \\
\mathrm{Al}\left(\mathrm{PO}_{4} \text { ) in leached sludge (determined by }\right. \\
\mathrm{XRD)} \text { ? }\end{array}$ \\
\hline $241-\mathrm{T}-111$ & $100^{\circ} \mathrm{C}$ & PNNL-11089 & $\begin{array}{l}\text { 224-2 (solid) } \\
\text { and } 2 \mathrm{C} \text { (solid). }\end{array}$ & $\begin{array}{l}\text { Primary: } 2 \mathrm{C} \text {. Secondary: Lanthanum } \\
\text { Fluoride Decontamination waste from } \\
\mathrm{BiPO}_{4} \text { Process. } \\
\text { Non-Complexed Waste. } \\
\text { Primary waste: } 2 \mathrm{C} \text {, Secondary: } \\
\text { Lanthanum fluoride decontamination. } \\
\text { Primary waste type: } 2 \mathrm{C} \text {. Secondary: } \\
\text { lanthanum fluoride decontamination } \\
\text { waste. }^{5}\end{array}$ & $\begin{array}{l}\text { No specific phase identified in as-received } \\
\text { or in leached sludge using } \\
\text { TEM/SEM/XRD? }\end{array}$ \\
\hline
\end{tabular}


Table 2-1. Aluminum mineralogical data as described in individual laboratory studies. Included is information prior to leaching, after washing, and after caustic leaching (19 sheets).

\begin{tabular}{|c|c|c|c|c|c|}
\hline Tank & $\begin{array}{l}\text { Leaching } \\
\text { Temp. }\left({ }^{\circ} \mathrm{C}\right)\end{array}$ & $\begin{array}{l}\text { Lab Study } \\
\text { Reference }\end{array}$ & $\begin{array}{l}\text { Waste type } \\
\text { (WRPS, 2010). }\end{array}$ & Waste Sources. & $\begin{array}{l}\text { Aluminum minerals determined in as- } \\
\text { received, washed, or caustic leached } \\
\text { solids. }\end{array}$ \\
\hline 241-TY-104 & $100^{\circ} \mathrm{C}$ & $\begin{array}{l}\text { LAUR 96- } \\
2839\end{array}$ & $\begin{array}{l}\text { 1CFeCN } \\
\text { (solid), TBP } \\
\text { (solid), and DW } \\
\text { (liquid). }\end{array}$ & $\begin{array}{l}\text { TBP waste from U-extraction process at } \\
\text { U Plant; } 1 \mathrm{C} \text { which had been FeCN } \\
\text { scavenged; decontamination waste wash } \\
\text { solution from equipment } \\
\text { decontamination efforts at T Plant } \\
\text { (mostly dilute } \mathrm{NaNO}_{2} \text { solution). } \\
\text { Non-Complexed Waste. } \\
\text { Primary waste: TBP. Secondary: } 1 \mathrm{C} \text { and } \\
\text { FeCN-scavenged waste. }{ }^{2} \\
\text { Primary waste type: TBP Secondary: } \\
\text { 1C/FeCN-scavenged waste. Tertiary: } \\
\text { decontamination waste. Other: mixture } \\
\text { of several miscellaneous wastes/high- } \\
\text { level REDOX waste. }\end{array}$ & $\begin{array}{l}\text { No specific phase identified in as-received } \\
\text { or in leached sludge using XRD. }\end{array}$ \\
\hline 241-U-108 & $\begin{array}{l}\text { Caustic and } \\
\text { Oxidative } \\
\text { Leaching } \\
\text { Performed }\end{array}$ & PNNL-14019 & $\begin{array}{l}\text { S1-SltCk } \\
\text { (liquid and } \\
\text { solid), S2-SitSlr } \\
\text { (liquid and } \\
\text { solid), and } \\
\text { CWR2 (solid). }\end{array}$ & $\begin{array}{l}\text { Tank waste group } 17 \text {. Non-Complexed } \\
\text { Waste. }{ }^{1}\end{array}$ & $\begin{array}{l}\text { Two } \mathrm{Al} \text { species found in washed tank } \\
\text { sludge: gibbsite, } \mathrm{Al}(\mathrm{OH})_{3} \text {, and an } \mathrm{AlSi} \text {, } \\
\text { Hydroxycancrinite, } \\
\left(\mathrm{Na}_{2} \mathrm{O}_{166}\left(\mathrm{Al}_{2} \mathrm{O}_{3}\right)\left(\mathrm{SiO}_{2}\right)_{16}\left(\mathrm{H}_{2} \mathrm{O}_{16}\right.\right.\end{array}$ \\
\hline $241-\mathrm{U}-110$ & $100^{\circ} \mathrm{C}$ & PNL 9387 & $\begin{array}{l}\text { 1C (solid), } \\
\text { CWR1 (solid), } \\
\text { and R1 (solid). }\end{array}$ & $\begin{array}{l}\text { Non-Complexed Waste. } \\
\text { Primary waste: } 1 C \text {. Secondary: CW. } \\
\text { Primary waste type: } 1 \mathrm{C} \text {. Secondary: } \\
\text { CW. Tertiary: high-level REDOX } \\
\text { waste. Other: Laboratory waste from } \\
222-S \text { building. }\end{array}$ & $\begin{array}{l}\text { Gibbsite and boehmite in as-received } \\
\text { (determined by SEM/XRD). }{ }^{3}\end{array}$ \\
\hline $\begin{array}{l}\text { Group } 1 \text { Bismuth } \\
\text { Phosphate Sludge } \\
\text { (1C and } 2 \mathrm{C} \text { )- } \\
\text { Tanks } 241-\mathrm{B}-104, \\
241-\mathrm{BX}-112 \text { and } \\
241-\mathrm{T}-104\end{array}$ & $\begin{array}{l}\text { Multiple } \\
\text { Leaching } \\
\text { Temperatures }\end{array}$ & $\begin{array}{l}\text { PNNL-17992 } \\
\text { (WTP-RPT- } \\
166)\end{array}$ & - & $\begin{array}{l}\text { Composite of } 31 \text { samples. } 93 \% \text { from B- } \\
104,4 \% \text { from BX-112, and } 3 \% \text { from T- } \\
104 \text {. Solids passed through } 3.2 \mathrm{~mm} \\
\text { sieve. Additional } 742.97 \mathrm{~g} \text { DI added } \\
\text { during compositing. }\end{array}$ & $\begin{array}{l}\text { Washed solids: Probable phase is } \\
\text { ammonium aluminum hydrogen phosphate } \\
\left(\mathrm{NH}_{4} \mathrm{AlHP} \mathrm{O}_{10}\right) \text {. Possible phases present } \\
\text { include boehmite and vauxite } \\
{\left[\mathrm{FeAl}_{2}\left(\mathrm{PO}_{4}\right)_{2}(\mathrm{OH})_{2} \cdot 6 \mathrm{H}_{2} \mathrm{O}\right] \text {. Leached solids: }} \\
\text { Bochmite and possible berlinite }(\mathrm{AIFO})_{4} \text {. }\end{array}$ \\
\hline
\end{tabular}


Table 2-1. Aluminum mineralogical data as described in individual laboratory studies. Included is information prior to leaching, after washing, and after caustic leaching (19 sheets).

\begin{tabular}{|c|c|c|c|c|c|}
\hline Tank & $\begin{array}{l}\text { Leaching } \\
\text { Temp. }\left({ }^{\circ} \mathrm{C}\right)\end{array}$ & $\begin{array}{l}\text { Lab Study } \\
\text { Reference }\end{array}$ & $\begin{array}{l}\text { Waste type } \\
\text { (WRPS, 2010). }\end{array}$ & Waste Sources. & $\begin{array}{l}\text { Aluminum minerals determined in as- } \\
\text { received, washed, or caustic leached } \\
\text { solids. }\end{array}$ \\
\hline $\begin{array}{l}\text { Group } 2 \text { Bismuth } \\
\text { Phosphate Saltcake } \\
\text { (BY and T } \\
\text { saltcake)-Tanks } \\
241-\mathrm{BX}-110,241 \text { - } \\
\text { BX-111, 241-BY- } \\
104,241-\mathrm{BY}-105 \text {, } \\
241-\mathrm{BY}-107,241 \text { - } \\
\text { BY-108, 241-BY- } \\
109,241-\mathrm{BY}-110 \text {, } \\
241-\mathrm{BY}-112,241- \\
\mathrm{T}-108,241-\mathrm{T}-109 \\
241-\mathrm{TX}-104, \text { and } \\
241-\mathrm{TX}-113 \text {. }\end{array}$ & $\begin{array}{l}\text { Multiple } \\
\text { Leaching } \\
\text { Temperatures }\end{array}$ & $\begin{array}{l}\text { PNNL-17992 } \\
\text { (WTP-RPT- } \\
166 \text { ) }\end{array}$ & - & $\begin{array}{l}\text { Composite of } 41 \text { samples from } 13 \text { tanks: } \\
7.1 \% \text { from BX-110, } 11.6 \% \text { from BX- } \\
111.5 .9 \% \text { from BY-104, } 4 \% \text { from BY- } \\
105,10.4 \% \text { from BY-107, } 24.9 \% \text { from } \\
\text { BY }-108,11 \% \text { from BY }-109,8.2 \% \text { from } \\
\text { BY }-110,2.4 \% \text { from BY }-112,1 \% \text { from } \\
\text { T-108, } 1.8 \% \text { from T-109, } 4 \% \text { from TX- } \\
104 \text {, and } 7.6 \% \text { from TX-113. Total of } \\
1966 \text { g solids passed through } 3.2 \text { mm } \\
\text { sieve. Additional } 2973.6 \text { g of water } \\
\text { added. Homogenized sample allowed } \\
\text { settling over the weckend to allow } \\
\text { separation of settled solids for sampling. }\end{array}$ & $\begin{array}{l}\text { Washed solids: gibbsite }(\mathrm{cr}) \text { and cancrinite } \\
{\left[\mathrm{cr} ; \mathrm{Na}_{2}(\mathrm{AlSiO})_{6}\left(\mathrm{NO}_{3} \mathrm{H}_{7}\left(\mathrm{H}_{2} \mathrm{O}\right)_{234}\right] \text {, also }\right.} \\
\text { AlSi (possibly cancrinite) determined using } \\
\text { TEM. } \\
\frac{\text { Leached solids: cancrinite }(\mathrm{cr})}{\left[\mathrm{Na}_{7.14} \mathrm{Al}_{6} \mathrm{Si}_{7.08} \mathrm{O}_{26}\left(\mathrm{H}_{2} \mathrm{O}\right)_{487}\right] .}\end{array}$ \\
\hline $\begin{array}{l}\text { Group } 3 \text { PUREX } \\
\text { Cladding Waste } \\
\text { Sludge - Tanks } \\
241-\text { C-103, 241-B- } \\
109,241-\text { C-105, } \\
241-\mathrm{B}-108,241-\mathrm{C} \text { - } \\
104 \text {, and } 241-\mathrm{BY} \text { - } \\
109 \text {. }\end{array}$ & $\begin{array}{l}\text { Multiple } \\
\text { Leaching } \\
\text { Temperatures }\end{array}$ & $\begin{array}{l}\text { PNNL-18054 } \\
\text { (WTP-RPT- } \\
\text { 167) }\end{array}$ & - & $\begin{array}{l}\text { Composite of } 15 \text { samples from } 6 \text { tanks: } \\
51 \% \text { from B Y-109, } 11 \% \text { from B-108, } \\
14 \% \text { from C-105, } 11 \% \text { from B-109, } 10 \% \\
\text { from C- } 104 \text {, and } 3 \% \text { from C- } 103 \text {. Total } \\
\text { of } 509.23 \mathrm{~g} \text { from original samples } \\
\text { transferred to composite. Total } \\
\text { composite slurry weight transferred } \\
\text { (including transfer water) was } 947.245 \\
\mathrm{~g} \text {. This was passed through a stainless } \\
\text { steel strainer to ensure no large pieces of } \\
\text { foreign material in composite. Total } \\
\text { sample weight was } 1016.81 \mathrm{~g} \text { after } 11 \\
\text { days. }\end{array}$ & $\begin{array}{l}\text { Washed solids: } 87.6 \text { wt } \% \text { gibbsite and }-5.5 \\
\text { wt\% Nitrate Cancrinite } \\
{\left[\mathrm{Na}_{8.16}\left(\mathrm{Al}_{6} \mathrm{Si}_{6} \mathrm{O}_{24}\right)\left(\mathrm{NO}_{3}\right)_{2.16}\left(\mathrm{H}_{2} \mathrm{O}\right)_{162]}\right] \text { and/or }} \\
\mathrm{Hydroxycancrinite} \\
{\left[1.06 \mathrm{Na}_{2} \mathrm{O} \cdot \mathrm{Al}_{2} \mathrm{O}_{3} \cdot 1.6 \mathrm{SiO}_{2} \cdot 1.6 \mathrm{H}_{2} \mathrm{O}\right] .} \\
\text { Leached solids: no specific aluminum } \\
\text { species found. }\end{array}$ \\
\hline
\end{tabular}


Table 2-1. Aluminum mineralogical data as described in individual laboratory studies. Included is information prior to leaching, after washing, and after caustic leaching (19 sheets).

\begin{tabular}{|c|c|c|c|c|c|}
\hline Tank & $\begin{array}{l}\text { Leaching } \\
\text { Temp. }\left({ }^{\circ} \mathrm{C}\right)\end{array}$ & $\begin{array}{l}\text { Lab Study } \\
\text { Reference }\end{array}$ & $\begin{array}{l}\text { Waste type } \\
\text { (WRPS, 2010). }\end{array}$ & Waste Sources. & $\begin{array}{l}\text { Aluminum minerals determined in as- } \\
\text { received, washed, or caustic leached } \\
\text { solids. }\end{array}$ \\
\hline $\begin{array}{l}\text { Group 4 REDOX } \\
\text { Cladkling Waste } \\
\text { Sludge - Tanks } \\
241-U-105,241-U- \\
201,241-\mathrm{U}-202 \text {, } \\
241-\mathrm{U}-203 \text {, and } \\
241-\mathrm{U}-204 \text {. }\end{array}$ & $\begin{array}{l}\text { Multiple } \\
\text { Leaching } \\
\text { Temperatures }\end{array}$ & $\begin{array}{l}\text { PNNL-18054 } \\
\text { (WTP-RPT- } \\
167 \text { ) }\end{array}$ & - & $\begin{array}{l}\text { Composite of } 14 \text { samples from } 5 \text { tanks: } \\
32 \% \text { from U-202, } 34 \% \text { from U- } 105 \text {. } \\
17 \% \text { from U-204, } 13 \% \text { from U-201. and } \\
4 \% \text { from U-203. Total of } 572.02 \mathrm{~g} \text { from } \\
\text { original samples transferred to } \\
\text { composite. Total composite slurry } \\
\text { weight transferred (including transfer } \\
\text { water) was } 666.02 \mathrm{~g} \text {. This was passed } \\
\text { through a stainless steel strainer to } \\
\text { ensure no large pieces of foreign } \\
\text { material in composite. Total sample } \\
\text { weight was } 1055.14 \mathrm{~g} \text { after } 4 \text { days. }\end{array}$ & $\begin{array}{l}\text { Washed solids: } 91.2 \mathrm{wt} \% \text { gibbsite (cr), } 1.5 \\
\text { wt\% sodium aluminum silicate hydrate } \\
{\left[\left(\mathrm{Na}_{2} \mathrm{O}_{1,31} \mathrm{Al}_{2} \mathrm{O}_{3}\left(\mathrm{SiO}_{2}\right)_{2} \text { or }\left(\mathrm{H}_{2} \mathrm{O}\right)_{65}\right] \text {, and }\right.} \\
0.73 \text { wt } \% \text { boehmite (possible) determined } \\
\text { using XRD. TEM showed presence of } \\
\text { boehmite, gibbsite, and cancrinite (Al:Si } \\
\text { ratio of } 1: 1=\text { zeolitic aluminosilicate). } \\
\text { Leached solids: tests not performed, but } \\
\text { nearly completely dissolved } \mathrm{AI} \text { (<20\% } \\
\text { remained). }\end{array}$ \\
\hline $\begin{array}{l}\text { Group } 5 \text { REDOX - } \\
\text { Tanks } 241-S-101 \text {, } \\
241-S-110,241-S- \\
107 \text {, and } 241-S X- \\
103 \text {. }\end{array}$ & $\begin{array}{l}\text { Multiple } \\
\text { Leaching } \\
\text { Temperatures }\end{array}$ & $\begin{array}{l}\text { PNNL-17368 } \\
\text { (WTP-RPT. } \\
\text { 157) }\end{array}$ & - & $\begin{array}{l}1283 \mathrm{~g} \text { from } 241-\mathrm{S}-101,397 \mathrm{~g} \text { from } 241- \\
\mathrm{S}-110,213 \mathrm{~g} \text { from } 241-\mathrm{S}-107 \text {, and } 78 \mathrm{~g} \\
\text { from } 241-\mathrm{SX}-103 \text {. Composite of } 39 \\
\text { different archived samples. Solids } \\
\text { passed through } 1 / 8 \text { inch stainless steel } \\
\text { sieve, with } 4 \text { L DI added to solids to } \\
\text { homogenize. }\end{array}$ & $\begin{array}{l}\text { Washed solids XRD; dominantly boehmite } \\
\text { ( } 73.8 \% \text { of mass fraction) with minor gibbsite } \\
\text { (5.7\% of mass fraction) with the average } \\
\text { gibbsite to boehmite phase ratio being } 0.077 \text {. } \\
\text { Washed and leached solids XRD: Boehmite } \\
(15 \mathrm{wt} \% \text { ) and Sodium AlSi ( } 9 \mathrm{wt} \% \text {; } \\
\left.\mathrm{NaSiAlO}_{4}\right) \text {. }\end{array}$ \\
\hline $\begin{array}{l}\text { Group } 6 \text { S-Saltcake } \\
\text { - Tanks 241-SX- } \\
106,241-S Y-103 \text {, } \\
241-S X-105,241- \\
\text { SX-102, 241-S- } \\
111,241-U-108 \text {, } \\
241-U-103 \text {, and } \\
241-\text { S-106. }\end{array}$ & $100^{\circ} \mathrm{C}$ & $\begin{array}{l}\text { PNNL-17368 } \\
\text { (WTP-RPT- } \\
157 \text { ) }\end{array}$ & - & $\begin{array}{l}965.3 \mathrm{~g} \text { from SX-106, } 227,3 \mathrm{~g} \text { from SY- } \\
103,151.7 \mathrm{~g} \text { from } \mathrm{SX}-105,131.5 \mathrm{~g} \text { from } \\
\mathrm{SX}-102,76.2 \mathrm{~g} \text { from } \mathrm{S}-111,55.1 \mathrm{~g} \text { from } \\
\mathrm{U}-108,53.4 \mathrm{~g} \text { from U-103, } 30.3 \mathrm{~g} \text { from } \\
\mathrm{S}-106 . \text { Composite of } 28 \text { different } \\
\text { archived samples. Solids passed through } \\
1 / 8 \text { inch stainless steel sieve to remove } \\
\text { large particles }(-5.7 \mathrm{~g} \text { ). Added DI to } \\
\text { make total volume } \sim 3 \mathrm{~L} \text {. }\end{array}$ & $\begin{array}{l}\text { Washed solids XRD: dominantly gibbsite } \\
(39.4 \mathrm{wt} \%) \text {, with minor boehmite }(8.9 \mathrm{wt} \%) \text {, } \\
\text { cancrinite }(6.7 \mathrm{wt} \%) \text {, and aluminum oxide. } \\
\text { The material was dominated by aluminum. } \\
\text { Washed and leached solids XRD: } \\
\text { dominantly boehmite, followed by } \\
\text { hydroxycancrinite } \\
\left(1.06 \mathrm{Na}_{2} \mathrm{O} \cdot \mathrm{Al}_{2} \mathrm{O}_{3} \cdot 1.6 \mathrm{SiO}_{2} \cdot 1.6 \mathrm{H}_{2} \mathrm{O}\right) \text { and } \\
\text { gibbsite; also calcium aluminum oxide } \\
\left(\mathrm{CaAl}_{2} \mathrm{O}_{4}\right) \text { seen in one peak. }\end{array}$ \\
\hline
\end{tabular}


Table 2-1. Aluminum mineralogical data as described in individual laboratory studies. Included is information prior to leaching, after washing, and after caustic leaching (19 sheets).

\begin{tabular}{|c|c|c|c|c|c|}
\hline Tank & $\begin{array}{l}\text { Leaching } \\
\text { Temp. }\left({ }^{\circ} \mathrm{C}\right)\end{array}$ & $\begin{array}{l}\text { Lab Study } \\
\text { Reference }\end{array}$ & $\begin{array}{l}\text { Waste type } \\
\text { (WRPS, 2010). }\end{array}$ & Waste Sources. & $\begin{array}{l}\text { Aluminum minerals determined in as- } \\
\text { received, washed, or caustic leached } \\
\text { solids. }\end{array}$ \\
\hline $\begin{array}{l}\text { Group } 7 \text { TBP } \\
\text { Waste Sludge- } \\
\text { Tanks } 241-\mathrm{BX}-109 \\
\text { and } 241-\mathrm{B}-106 .\end{array}$ & $\begin{array}{l}\text { Multiple } \\
\text { Leaching } \\
\text { Temperatures }\end{array}$ & $\begin{array}{l}\text { PNNL-18119 } \\
\text { (WTP-RPT- } \\
169 \text { ) }\end{array}$ & - & $\begin{array}{l}\text { Composite of } 33 \text { samples from } 2 \text { tanks. } \\
92 \% \text { from BX-109and } 8 \% \text { from B- } 106 \text {. } \\
\text { Total of } 1479.16 \mathrm{~g} \text { from original samples } \\
\text { transferred to composite. A total of } \\
779.7 \mathrm{~g} \text { DI added during compositing. } \\
\text { This was passed through a stainless steel } \\
\text { strainer to ensure no large pieces of } \\
\text { foreign material in composite. }\end{array}$ & $\begin{array}{l}\text { Washed solids: Zeolite }\left[\mathrm{NaAlSiO}{ }_{4}\left(\mathrm{H}_{2} \mathrm{O}\right)_{1.1}\right] \text {, } \\
\text { Threadgoldite }\left[\mathrm{Al}\left(\mathrm{UO}_{2}\right)_{2}\left(\mathrm{PO}_{4}\right)_{2}(\mathrm{OH})\left(\mathrm{H}_{2} \mathrm{O}\right)_{8}\right] \text {, } \\
\text { and Gibbsite }\left[\mathrm{Al}(\mathrm{OH})_{3}\right] \text {. } \\
\text { Leached solids: a sodium aluminophosphate } \\
\text { phase and } \mathrm{Al}-\mathrm{Zr} \text { - } \mathrm{U} \text { region determined by } \\
\text { TEM analysis. }\end{array}$ \\
\hline $\begin{array}{l}\text { Group } 8 \text { FeCN } \\
\text { Waste Sludge- } \\
\text { Tanks 241-BY- } \\
110,241-\text { BY-108, } \\
241-\text { BY-105, 241- } \\
\text { BY-104, and } 241 \text { - } \\
\text { BY-106. }\end{array}$ & $60^{\circ} \mathrm{C}$ & $\begin{array}{l}\text { PNNL-18120 } \\
\text { (WTP-RPT- } \\
170 \text { ) }\end{array}$ & - & $\begin{array}{l}\text { Composite of } 30 \text { samples from } 5 \text { tanks. } \\
45 \% \text { from BY- } 110,22 \% \text { from BY-108, } \\
21 \% \text { from BY-105, } 10 \% \text { from BY-104, } \\
\text { and } 2 \% \text { from BY-106. Total of } 1358.8 \mathrm{~g} \\
\text { from original samples transferred to } \\
\text { composite. A total of }-960 \mathrm{~g} \text { DI added } \\
\text { during compositing. This was passed } \\
\text { through a sieve (collect objects with } \\
\text { greater than } 3 \text { mm diameter) to ensure no } \\
\text { large pieces of foreign material in } \\
\text { composite. No significant sample was } \\
\text { trapped in sieve. }\end{array}$ & $\begin{array}{l}\text { Washed solids: Gibbsite, Sodium } \\
\text { Aluminum lron Oxide (possible) } \\
{\left[\mathrm{Na}_{2} \mathrm{Al}_{0.5} \mathrm{Fe}_{9} \mathrm{O}_{15}\right] \text {, Hydroxycancrinite }} \\
{\left[1.06 \mathrm{Na}_{2} \mathrm{O} \cdot \mathrm{Al}_{2} \mathrm{O}_{3} \cdot 1.6 \mathrm{SiO}_{2} \cdot 1.6 \mathrm{H}_{2} \mathrm{O}\right] \text {, and }} \\
\text { Ammonium Aluminum Hydrogen Phosphate } \\
\text { Hydrate (possible) } \\
{\left[\mathrm{NH}_{4} \mathrm{AlH}_{2}\left(\mathrm{PO}_{4}\right)_{2} \cdot 0.5 \mathrm{H}_{2} \mathrm{O} \text {. }\right.} \\
\text { Leached solids: } 63 \text { wt\% Al removed. All } \\
\text { Gibbsite dissolved. Hydroxycancrinite } \\
\text { determined by SEM-EDS. }\end{array}$ \\
\hline
\end{tabular}


Table 2-1. Aluminum mineralogical data as described in individual laboratory studies. Included is information prior to leaching, after washing, and after caustic leaching (19 sheets).

\begin{tabular}{|c|c|c|c|c|c|}
\hline Tank & $\begin{array}{l}\text { Leaching } \\
\text { Temp. ( } \mathrm{C} \text { ) }\end{array}$ & $\begin{array}{l}\text { Lab Study } \\
\text { Reference }\end{array}$ & $\begin{array}{l}\text { Waste type } \\
\text { (WRPS, 2010). }\end{array}$ & Waste Sources. & $\begin{array}{l}\text { Aluminum minerals determined in as- } \\
\text { received, washed, or caustic leached } \\
\text { solids. }\end{array}$ \\
\hline $\begin{array}{l}\text { Mixture of Group } 5 \\
\text { and Group } 6 \text { waste. } \\
31.6 \text { wt } \% \text { Group } 5 \\
\text { Slurry + } 16.8 \text { wt } \% \\
\text { Group } 6 \text { slurry + } \\
51.6 \text { wt } \% \text { Group } 6 \\
\text { supernatant - Tanks } \\
241-S-101,241-S- \\
110,241-S-107 \text {, } \\
\text { and } 241-S X-103 \\
\text { from Group } 5 \text { and } \\
241-S X-106,241- \\
\text { SY-103, } 241-S X- \\
\text { I05, } 241-S X-102 \text {, } \\
241-S-111,241-U- \\
108,241-U-103 \text { - } \\
\text { and } 241-S-106 \\
\text { from Group } 6 \text {. }\end{array}$ & $100^{\circ} \mathrm{C}$ & $\begin{array}{l}\text { PNNL-18007 } \\
\text { (WTP-RPT- } \\
\text { 171) }\end{array}$ & - & $\begin{array}{l}\text { Group } 5 \text { solids contained } 33 \mathrm{wt} \% \mathrm{Al} \text {, } \\
\text { with } ~ 90 \text { wt } \% \text { Al in the form of } \\
\text { boehmite. The individual tank waste } \\
\text { material sources, compositions, and } \\
\text { physical properties are described in } \\
\text { PNNL-17368 above. }\end{array}$ & 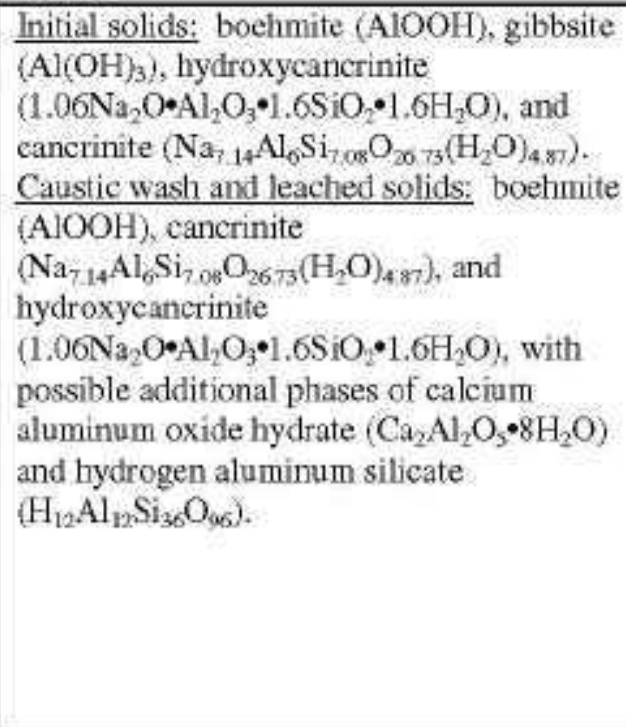 \\
\hline
\end{tabular}


Table 2-1. Aluminum mineralogical data as described in individual laboratory studies. Included is information prior to leaching, after washing, and after caustic leaching (19 sheets).

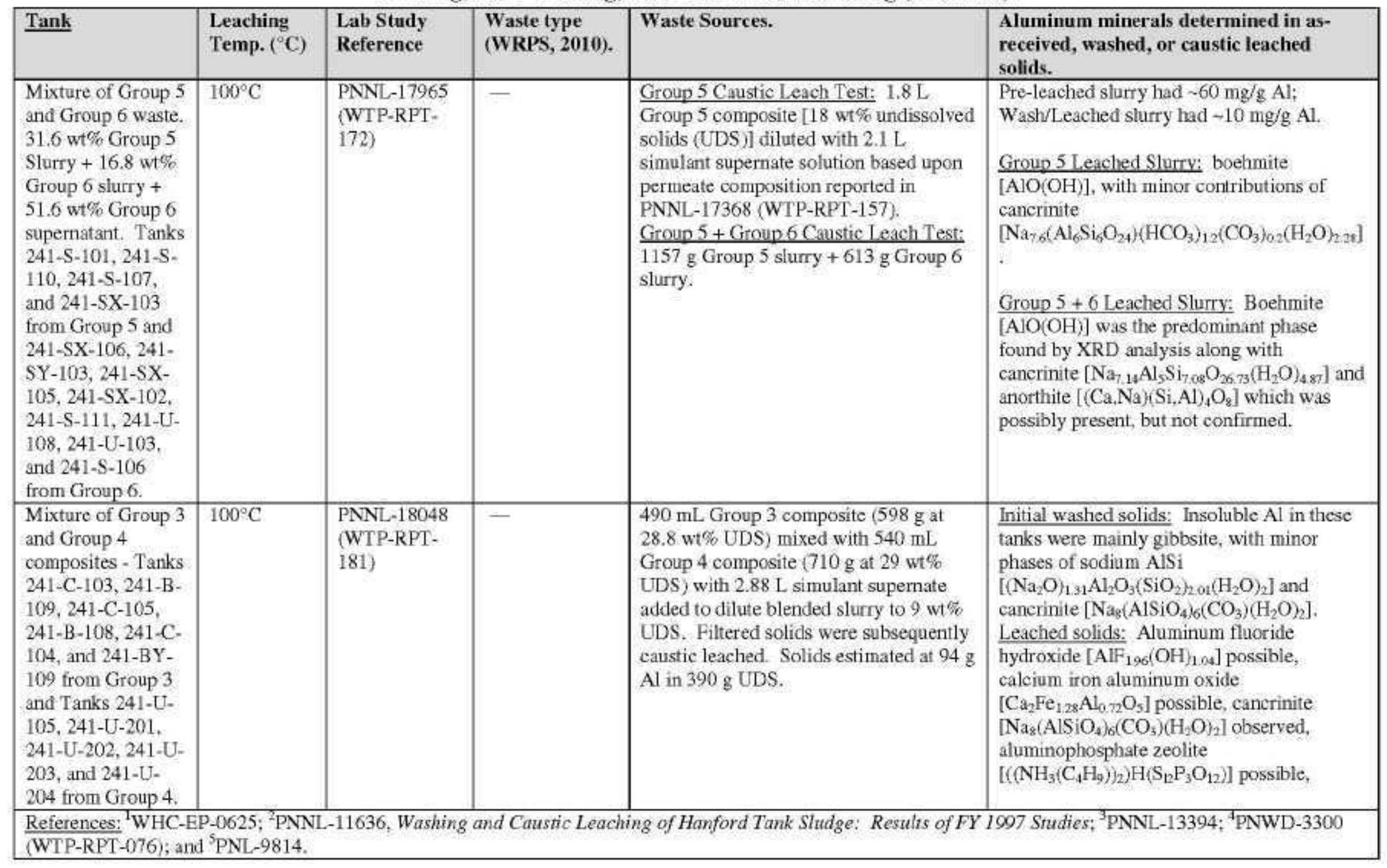


RPP-RPT-46791

Revision 0

\subsection{SUMMARY OF TANK LEACHING DATA}

As shown in Table A1-1 and Table A1-2 of Appendix A1.0, a total of 5 double-shell tanks (DSTs), 41 single-shell tanks (SSTs), and 12 composite mixtures of tank wastes have had water washing and caustic leaching analyses performed to determine the extent of aluminum dissolution from their respective solid phases. A brief summary of the tanks and the conditions of their analyses are provided in Table $2-2$ below. The remaining 108 SSTs and 23 DSTs have not had aluminum leaching tests performed to our knowledge.

Table 2-2. DSTs and SSTs with Available Laboratory Scale Aluminum Leaching Data (4 sheets).

\begin{tabular}{|c|c|c|c|c|}
\hline SST/DST & Tank number ${ }^{1}$ & $\begin{array}{l}\text { Temperatures } \\
\text { studied; }{ }^{\circ} \mathrm{C}\end{array}$ & $\begin{array}{l}\text { Caustic or } \\
\text { Caustic and } \\
\text { Oxidative } \\
\text { leaching }\end{array}$ & Reference(s) \\
\hline DST & 241-AN-104 & $100^{\circ} \mathrm{C}$ & caustic & PNNL-11636 \\
\hline DST & 241-AZ-101 & $85^{\circ} \mathrm{C}$ & caustic & $\begin{array}{l}\text { PNWD-3206 } \\
\text { (WTP-RPT-043) }\end{array}$ \\
\hline DST & $241-A Z-101+241-A Z-102$ & $80^{\circ} \mathrm{C}$ & caustic & PNNL-11580 \\
\hline DST & $241-\mathrm{AZ}-102$ & $85^{\circ} \mathrm{C}$ & caustic & $\begin{array}{l}\text { PNWD-3045 } \\
\text { (BNFL-RPT-038) }\end{array}$ \\
\hline DST & 241-SY-102 & $85^{\circ} \mathrm{C}$ & caustic & $\begin{array}{l}\text { PNWD-3512 } \\
\text { (WTP-RPT-117) }\end{array}$ \\
\hline DST & 241-SY-103 & $100^{\circ} \mathrm{C}$ & caustic & $\begin{array}{l}\text { PNL-10712 and } \\
\text { PNNL-11089 }\end{array}$ \\
\hline SST & $241-B-101$ & $\begin{array}{l}60^{\circ} \mathrm{C} \text { and } 100 \\
{ }^{\circ} \mathrm{C}\end{array}$ & caustic & PNNL-12026 \\
\hline SST & $241-B-104$ & $100^{\circ} \mathrm{C}$ & caustio & LAUR 96-2839 \\
\hline SST & 241-B-106 & $100^{\circ} \mathrm{C}$ & caustic & LAUR 97-2889 \\
\hline SST & $241-\mathrm{B}-110$ & $100^{\circ} \mathrm{C}$ & caustic & $\begin{array}{l}\text { PNL-9387 and } \\
\text { Internal Letter } \\
9404238\end{array}$ \\
\hline SST & $241-\mathrm{B}-111$ & $100^{\circ} \mathrm{C}$ & caustic & $\begin{array}{l}\text { PNL-10712 and } \\
\text { PNNL-11089 }\end{array}$ \\
\hline SST & $241-\mathrm{B}-201$ & $100^{\circ} \mathrm{C}$ & caustic & PNL-10078 \\
\hline SST & $241-\mathrm{B}-202$ & $100^{\circ} \mathrm{C}$ & caustic & LAUR $95-2070$ \\
\hline SST & 241-BX-103 & $100^{\circ} \mathrm{C}$ & caustic & LAUR 97-2889 \\
\hline SST & 241-BY-105 & $100^{\circ} \mathrm{C}$ & caustic & LAUR $95-2070$ \\
\hline SST & 241-BY-107 & $100^{\circ} \mathrm{C}$ & caustic & $\begin{array}{l}\text { PNL-10712 and } \\
\text { PNNL-11089 }\end{array}$ \\
\hline SST & 241-BX-109 & $100^{\circ} \mathrm{C}$ & caustic & LAUR 96-2839 \\
\hline SST & 241-BX-110 & $\begin{array}{l}60^{\circ} \mathrm{C}, 80^{\circ} \mathrm{C} \text {, } \\
\text { and } 95^{\circ} \mathrm{C}\end{array}$ & caustic & PNNL-12026 \\
\hline SST & $241-\mathrm{BX}-112$ & $\begin{array}{l}60^{\circ} \mathrm{C}, 80^{\circ} \mathrm{C} \text {, } \\
\text { and } 100^{\circ} \mathrm{C}\end{array}$ & caustic & PNNL-12026 \\
\hline SST & 241-BY-104 & $100^{\circ} \mathrm{C}$ & caustic & PNNL-11278 \\
\hline
\end{tabular}

\footnotetext{
${ }^{1}$ Multiple tank names indicate a composite was made with samples from all of the tanks indicated.
} 
RPP-RPT-46791

Revision 0

Table 2-2. DSTs and SSTs with Available Laboratory Scale Aluminum Leaching Data (4 sheets).

\begin{tabular}{|c|c|c|c|c|}
\hline SST/DST & Tank number ${ }^{1}$ & $\begin{array}{l}\text { Temperatures } \\
\text { studied; }{ }^{\circ} \mathrm{C}\end{array}$ & $\begin{array}{l}\text { Caustic or } \\
\text { Caustic and } \\
\text { Oxidative } \\
\text { leaching }\end{array}$ & Reference(s) \\
\hline$\overline{\text { SST }}$ & 241-BY-108 & $100^{\circ} \mathrm{C}$ & caustic & PNNL-11636 \\
\hline SST & $241-B Y-110$ & $1000^{\circ} \mathrm{C}$ & caustic & PNNL-11278 \\
\hline SST & 241-C-102 & $\begin{array}{l}60^{\circ} \mathrm{C} \text { and } 100 \\
{ }^{\circ} \mathrm{C}\end{array}$ & caustic & PNNL-12026 \\
\hline SST & 241-C-103 & $100^{\circ} \mathrm{C}$ & caustic & $\begin{array}{l}\text { PNL-10712 and } \\
\text { PNNL-11089 }\end{array}$ \\
\hline SST & 241-C-104 & $\begin{array}{l}50^{\circ} \mathrm{C}, 80^{\circ} \mathrm{C} \text {, } \\
85^{\circ} \mathrm{C}, 93^{\circ} \mathrm{C} \text {, } \\
\text { and } 100^{\circ} \mathrm{C}\end{array}$ & caustic & $\begin{array}{l}\text { LAUR 97-2889, } \\
\text { ORNL/TM-13500, } \\
\text { PNWD-3024 } \\
\text { (BNFL-RPT-030), } \\
\text { and PNWD-3027 } \\
\text { (BNFL-RPT-021) }\end{array}$ \\
\hline SST & $241-\mathrm{C}-105$ & $\begin{array}{l}70^{\circ} \mathrm{C} \text { and } \\
100^{\circ} \mathrm{C}\end{array}$ & caustic & $\begin{array}{l}\text { LALR } 97-2889 \text { and } \\
\text { ORNLTM- } 13500\end{array}$ \\
\hline SST & $241-C-106$ & $\begin{array}{l}85^{\circ} \mathrm{C} \text { and } \\
100^{\circ} \mathrm{C}\end{array}$ & caustic & $\begin{array}{l}\text { PNWD-3013 } \\
\text { (BNFL-RPT-017), } \\
\text { PNNL-11381, and } \\
\text { PNNL-11432 }\end{array}$ \\
\hline SST & 241-C-107 & $\begin{array}{l}70^{\circ} \mathrm{C} \text { and } \\
100^{\circ} \mathrm{C}\end{array}$ & caustic & $\begin{array}{l}\text { ORNLTM-13500, } \\
\text { LAUR 96-2839, and } \\
\text { PNNL-11278 }\end{array}$ \\
\hline SST & $241-\mathrm{C}-108$ & $100^{\circ} \mathrm{C}$ & caustic & LAUR $95-2070$ \\
\hline SST & $241-C-109$ & $100^{\circ} \mathrm{C}$ & caustic & PNL-9387 \\
\hline SST & $241-\mathrm{C}-112$ & $100^{\circ} \mathrm{C}$ & caustic & PNL-9387 \\
\hline SST & $241-S-101$ & $\begin{array}{l}70^{\circ} \mathrm{C}, 95^{\circ} \mathrm{C} \text {, } \\
93^{\circ} \mathrm{C} \text {, and } \\
100^{\circ} \mathrm{C}\end{array}$ & canstic & $\begin{array}{l}\text { PNNL-12026, } \\
\text { PNNL-11636, } \\
\text { ORNL/TM-13500, } \\
\text { and ORNL/TM- } \\
13655\end{array}$ \\
\hline SST & $241-S-104$ & $\begin{array}{l}67^{\circ} \mathrm{C}, 70^{\circ} \mathrm{C} \text {, } \\
80^{\circ} \mathrm{C} \text {, and } \\
100^{\circ} \mathrm{C}\end{array}$ & caustic & $\begin{array}{l}\text { ORNLITM-13500, } \\
\text { PNL-10712, LAUR } \\
95-2070 \text {, PNNL- } \\
11089 \text {, and PNNL- } \\
11636\end{array}$ \\
\hline SST & $241-S \cdot 107$ & $100^{\circ} \mathrm{C}$ & caustic & $\begin{array}{l}\text { PNNL-11278 and } \\
\text { PNNL-12010 }\end{array}$ \\
\hline SST & $241-S-110$ & $\begin{array}{l}60^{\circ} \mathrm{C}, 80^{\circ} \mathrm{C} \text {, } \\
\text { and } 100^{\circ} \mathrm{C}\end{array}$ & caustic & PNNL-13702 \\
\hline SST & $241-S-110$ & $\begin{array}{l}30^{\circ} \mathrm{C}, 80^{\circ} \mathrm{C} \text {, } \\
\text { and } 85^{\circ} \mathrm{C}\end{array}$ & $\begin{array}{l}\text { Caustic and } \\
\text { oxidative }\end{array}$ & PNNL-14018 \\
\hline SST & $241-S-111$ & $100^{\circ} \mathrm{C}$ & caustic & PNNL-11636 \\
\hline SST & 241-SX-101 & $85^{\circ} \mathrm{C}$ & caustic & $\begin{array}{l}\text { PNWD-3512 } \\
\text { (WTP-RPT-117) }\end{array}$ \\
\hline SST & $241-S X-108$ & $100^{\circ} \mathrm{C}$ & caustic & PNNL-11278 \\
\hline SST & $241-S X-108$ & $80^{\circ} \mathrm{C}$ & $\begin{array}{l}\text { Caustic and } \\
\text { oxidative }\end{array}$ & PNNL-11908 \\
\hline SST & $241-S X-113$ & $\begin{array}{l}70^{\circ} \mathrm{C}, 80^{\circ} \mathrm{C} \text {, } \\
\text { and } 100^{\circ} \mathrm{C}\end{array}$ & caustic & $\begin{array}{l}\text { LAUR } 97-2889 \text { and } \\
\text { ORNL/TM-13500 }\end{array}$ \\
\hline
\end{tabular}


RPP-RPT-46791

Revision 0

Table 2-2. DSTs and SSTs with Available Laboratory Scale Aluminum Leaching Data (4 sheets).

\begin{tabular}{|c|c|c|c|c|}
\hline SST/DST & Tank number ${ }^{1}$ & $\begin{array}{l}\text { Temperatures } \\
\text { studied; }{ }^{\circ} \mathrm{C}\end{array}$ & $\begin{array}{l}\text { Caustic or } \\
\text { Caustic and } \\
\text { Oxidative } \\
\text { leaching }\end{array}$ & Reference(s) \\
\hline SST & 241-T-104 & $\begin{array}{l}\text { RT, } 60^{\circ} \mathrm{C} \text {, } \\
95^{\circ} \mathrm{C} \text {, and } \\
100^{\circ} \mathrm{C}\end{array}$ & caustic & $\begin{array}{l}\text { ORNLTM-13660, } \\
\text { PNL-10712, PNNL. } \\
11089 \text {, and LAUR } \\
95-2070\end{array}$ \\
\hline SST & 241-T-107 & $100^{\circ} \mathrm{C}$ & caustic & LAUR 95-2070 \\
\hline SST & $241-\mathrm{T}-110$ & $\begin{array}{l}60^{\circ} \mathrm{C}, 80^{\circ} \mathrm{C} \text {, } \\
\text { and } 100^{\circ} \mathrm{C}\end{array}$ & caustic & PNNL-13956 \\
\hline SST & $241-T-111$ & $100^{\circ} \mathrm{C}$ & caustic & $\begin{array}{l}\text { PNL-10712 and } \\
\text { PNNL-11089 }\end{array}$ \\
\hline SST & 241-TY-104 & $100^{\circ} \mathrm{C}$ & caustic & LALR $96-2839$ \\
\hline SST & 241-U-108 & $\begin{array}{l}\mathrm{RT}, 80^{\circ} \mathrm{C} \\
30^{\circ} \mathrm{C} \text {, and } 85^{\circ} \mathrm{C}\end{array}$ & $\begin{array}{l}\text { Caustic and } \\
\text { oxidative }\end{array}$ & $\begin{array}{l}\text { PNNL-11908 and } \\
\text { PNNL-14019 }\end{array}$ \\
\hline SST & $241-\mathrm{U}-109$ & $\mathrm{RT}$ and $80^{\circ} \mathrm{C}$ & $\begin{array}{l}\text { Caustic and } \\
\text { oxidative }\end{array}$ & PNNL-11908 \\
\hline SST & $241-\mathrm{U}-110$ & $100^{\circ} \mathrm{C}$ & caustic & $\begin{array}{l}\text { PNL-9387, PNL- } \\
10078 \text {, and PNNL- } \\
11779\end{array}$ \\
\hline SST's & $\begin{array}{l}241-\mathrm{B}-104,241-\mathrm{BX}-112 \text {, and } \\
241-\mathrm{T}-104 \cdot\left(\mathrm{BiPO}_{4} \text { sludge }\right. \\
\text { waste) }\end{array}$ & $\begin{array}{l}40^{\circ} \mathrm{C}, 60^{\circ} \mathrm{C} \text {, } \\
\text { and } 80^{\circ} \mathrm{C}\end{array}$ & caustic & $\begin{array}{l}\text { PNNL-17992 } \\
\text { (WTP-RPT-166) }\end{array}$ \\
\hline SSTs & $\begin{array}{l}\text { 241-BX-110, 241-BX-111, 241- } \\
\text { BY-104, 241-BY-105, 241-BY- } \\
107,241-\mathrm{BY}-108,241-\mathrm{BY}-109 \text {, } \\
241-\mathrm{BY}-110,241-\mathrm{BY}-112,241- \\
\text { T-108, 241-T-109, 241-TX-104. } \\
\text { and } 241-\mathrm{TX}-113 .\left(\mathrm{BiPO}_{4}\right. \\
\text { Saltcake waste) }\end{array}$ & $\begin{array}{l}60^{\circ} \mathrm{C}, 80^{\circ} \mathrm{C} \text {, } \\
\text { and } 100^{\circ} \mathrm{C}\end{array}$ & caustic & $\begin{array}{l}\text { PNNL-17992 } \\
\text { (WTP-RPT-166) }\end{array}$ \\
\hline SSTs & $\begin{array}{l}241-\mathrm{C}-103,241-\mathrm{B}-109,241-\mathrm{C}- \\
105,241-\mathrm{B}-108,241-\mathrm{C}-104, \text { and } \\
241-\mathrm{BY}-109 . \text { (PUREX Cladding } \\
\text { waste sludge) }\end{array}$ & $\begin{array}{l}60^{\circ} \mathrm{C}, 80^{\circ} \mathrm{C} \text {, } \\
\text { and } 100^{\circ} \mathrm{C}\end{array}$ & caustic & $\begin{array}{l}\text { PNNL-18054 } \\
\text { (WTP-RPT-167) }\end{array}$ \\
\hline SSTs & $\begin{array}{l}\text { 241-U-105, 241-U-201, 241-U- } \\
\text { 202, 241-U-203, and } 241-\mathrm{U}- \\
\text { 204. (REDOX Cladding waste } \\
\text { sludge) }\end{array}$ & $\begin{array}{l}60^{\circ} \mathrm{C} .80^{\circ} \mathrm{C} \text {, } \\
\text { and } 100^{\circ} \mathrm{C}\end{array}$ & caustic & $\begin{array}{l}\text { PNNL-18054 } \\
\text { (WTP-RPT-167) }\end{array}$ \\
\hline SSTs & $\begin{array}{l}241-\mathrm{S}-101,241 \text {-S-110, } 241-\mathrm{S}- \\
107 \text {, and } 241-\mathrm{SX}-103 \text {. (REDOX } \\
\text { Sludge waste) }\end{array}$ & $\begin{array}{l}80^{\circ} \mathrm{C}, 90^{\circ} \mathrm{C} \text {, } \\
\text { and } 100^{\circ} \mathrm{C}\end{array}$ & caustic & $\begin{array}{l}\text { PNNL-17368 } \\
\text { (WTP-RPT-157) }\end{array}$ \\
\hline SSTs & $\begin{array}{l}241-\mathrm{SX}-106,241-\mathrm{SY}-103,241- \\
\text { SX-105, 241-SX-102, 241-S- } \\
111,241-\mathrm{U}-108,241-\mathrm{U}-103 \text {, } \\
\text { and } 241-\mathrm{S}-106 . \text { (S-Salteake } \\
\text { waste) }\end{array}$ & $100^{\circ} \mathrm{C}$ & caustic & $\begin{array}{l}\text { PNNL-17368 } \\
\text { (WTP-RPT-157) }\end{array}$ \\
\hline SSTs & $\begin{array}{l}\text { 241-BX-109 and 241-B-106. } \\
\text { (TBP waste) }\end{array}$ & $\begin{array}{l}40^{\circ} \mathrm{C}, 60^{\circ} \mathrm{C} \text {, } \\
\text { and } 80^{\circ} \mathrm{C}\end{array}$ & caustic & $\begin{array}{l}\text { PNNL-18119 } \\
\text { (WTP-RPT-169) }\end{array}$ \\
\hline SSTs & $\begin{array}{l}\text { 241-BY-110, 241-BY-108, 241- } \\
\text { BY-105, 241-BY-104, and 241- } \\
\text { BY-106. (FeCN Tank Sludge) }\end{array}$ & $60^{\circ} \mathrm{C}$ & caustic & $\begin{array}{l}\text { PNNL-18120 } \\
\text { (WTP-RPT-170) }\end{array}$ \\
\hline
\end{tabular}


RPP-RPT-46791

Revision 0

Table 2-2. DSTs and SSTs with Available Laboratory Scale Aluminum Leaching Data (4 sheets).

\begin{tabular}{|c|c|c|c|c|}
\hline SST/DST & Tank number ${ }^{1}$ & $\begin{array}{l}\text { Temperatures } \\
\text { studied; }{ }^{\circ} \mathrm{C}\end{array}$ & $\begin{array}{l}\text { Caustic or } \\
\text { Caustic and } \\
\text { Oxidative } \\
\text { leaching }\end{array}$ & Reference(s) \\
\hline SSTs & $\begin{array}{l}\text { 241-S-101, 241-S-110, 241-S- } \\
107,241-\mathrm{SX}-103,241-\mathrm{SX}-106, \\
241-\mathrm{SY}-103,241-\mathrm{SX}-105,241- \\
\text { SX-102, 241-S-111, 241-U-108, } \\
241-\mathrm{U}-103 \text {, and } 241-\mathrm{S}-106 . \\
\text { (REDOX Sludge and S-Saltcake } \\
\text { waste mixture) }\end{array}$ & $100^{\circ} \mathrm{C}$ & caustic & $\begin{array}{l}\text { PNNL-18007 } \\
\text { (WTP-RPT-171) }\end{array}$ \\
\hline SSTs & $\begin{array}{l}\text { 241-S-101, 241-S-110, 241-S- } \\
107,241-S X-103,241-S X-106, \\
241-S Y-103,241-S X-105,241- \\
\text { SX-102, 241-S-111, 241-U-108, } \\
241-U-103 \text {, and } 241-S-106 . \\
\text { (REDOX Sludge and S-Saltcake } \\
\text { waste mixture) }\end{array}$ & $100^{\circ} \mathrm{C}$ & caustic & $\begin{array}{l}\text { PNNL-17965 } \\
\text { (WTP-RPT-172) }\end{array}$ \\
\hline SSTs & $\begin{array}{l}\text { 241-C-103, 241-B-109, 241-C- } \\
105,241-\mathrm{B}-108,241-\mathrm{C}-104, \\
241-\mathrm{BY}-109,241-\mathrm{U}-105,241- \\
\text { U-201, 241-U-202, 241-U-203, } \\
\text { and } 241-\mathrm{U}-204 . \text { (PUREX and } \\
\text { REDOX Cladding waste sludge } \\
\text { mixture) }\end{array}$ & $100^{\circ} \mathrm{C}$ & caustic & $\begin{array}{l}\text { PNNL-18048 } \\
\text { (WTP-RPT-181) }\end{array}$ \\
\hline
\end{tabular}

As can be seen in Table 2-2, twenty-four of the SSTs, two of the DSTs, and four composite groups have had caustic leach tests performed only at $100^{\circ} \mathrm{C}$, which is outside the currently acceptable temperature range for the PTF at the WTP. Also, of all of the caustic leach tests performed, only eight SSTs and three of the composite groups did not perform a wash step prior to caustic leaching (see Table A1-1). Of the remaining aluminum leaching analyses, three of the DSTs, twelve of the SSTs, and seven of the composite tank waste tests have been performed in a temperature range relevant to the current PTF plan, albeit with water washing performed (24590WTP-RPT-PT-02-005, Rev. 5). Only the DST composite and three SSTs (241-C-104, 241-S104, and 241-SX-113) were within the currently appropriate temperature range and had not had water washing performed prior to caustic leaching.

Washing of the solids prior to leaching has been performed in many 'wash and leach' tests up to several times prior to caustic leaching. This resulted in the rehydration of potentially dehydrated solids which had been in archives for sometimes over a decade, and was necessary in order to begin leaching at a state (e.g. hydroxide concentration) similar to the original tank waste sample in some cases. Secondary consequences of the wash step included (1) rehydration of solid species, (2) reduction in the overall ionic strength, (3) reduction in solid and/or aqueous concentrations of soluble constituents such as aluminum, chromium, silica, phosphates, and cesium, and (4) reduced potential of certain precipitate formation, including aluminosilicates as was described in ORNL/TM-1999/263. The washing step seemed to be a benefit in some instances, while not in others. This can be observed in Table A1-1 which indicates that some 
washing steps resulted in large solids dissolution while others only had a small $(\leq 1 \%)$ or no reduction in solids concentration.

Of the 12 SSTs with laboratory studies performed within the relevant temperature range, ten tanks were analyzed to determine the affects of different temperatures and caustic concentration on the extent of leaching, as detailed in Table 2-3. Seven of these provided information for the dissolution of aluminum over time, with data provided at individual sample times to show the progression of leaching. This information is provided in Table A3-1, with a plot of all of the percent aluminum leached versus time data depicted in Figure A3-1. More specific plots for individual tanks or temperatures are provided in Figure A3-2 through Figure A3-14.

Table 2-3. SSTs Analyzing the Effects of $\mathrm{T}$ and $\left[\mathrm{OH}^{-}\right]$on Aluminum Leaching (2 sheets).

\begin{tabular}{|c|c|c|c|c|c|}
\hline SST & Temperature & $\begin{array}{l}\text { Caustic } \\
\text { concentration } \\
(\mathrm{NaOH})\end{array}$ & $\begin{array}{l}\text { Total time for } \\
\text { Caustic leaching }\end{array}$ & $\%$ All leached & Reference \\
\hline \multirow{4}{*}{$241-B-101$} & $60^{\circ} \mathrm{C}$ & $1 \mathrm{M}$ & $168 \mathrm{hr}$ & 56 & \multirow{4}{*}{ PNNL- 12026} \\
\hline & $60^{\circ} \mathrm{C}$ & $3 \mathrm{M}$ & $168 \mathrm{hr}$ & 63 & \\
\hline & $100^{\circ} \mathrm{C}$ & $1 \mathrm{M}$ & $168 \mathrm{hr}$ & 59 & \\
\hline & $100^{\circ} \mathrm{C}$ & $3 \mathrm{M}$ & $168 \mathrm{hr}$ & 62 & \\
\hline \multirow{6}{*}{$241-\mathrm{BX}-110$} & $60^{\circ} \mathrm{C}$ & $1 \mathrm{M}$ & $168 \mathrm{hr}$ & 95 & \multirow{6}{*}{ PNNL-12026 } \\
\hline & $60^{\circ} \mathrm{C}$ & $3 \mathrm{M}$ & $168 \mathrm{hr}$ & 99 & \\
\hline & $80^{\circ} \mathrm{C}$ & $1 \mathrm{M}$ & $168 \mathrm{hr}$ & 98 & \\
\hline & $80^{\circ} \mathrm{C}$ & $3 \mathrm{M}$ & $168 \mathrm{hr}$ & 97 & \\
\hline & $95^{\circ} \mathrm{C}$ & $1 \mathrm{M}$ & $168 \mathrm{hr}$ & 99 & \\
\hline & $95^{\circ} \mathrm{C}$ & $1 \mathrm{M}$ & $168 \mathrm{hr}$ & 99 & \\
\hline \multirow{6}{*}{$241-B X-112$} & $60^{\circ} \mathrm{C}$ & $1 \mathrm{M}$ & $168 \mathrm{hr}$ & 64 & \multirow{6}{*}{ PNNL-12026 } \\
\hline & $60^{\circ} \mathrm{C}$ & $3 \mathrm{M}$ & $168 \mathrm{hr}$ & 69 & \\
\hline & $80^{\circ} \mathrm{C}$ & $1 \mathrm{M}$ & $168 \mathrm{hr}$ & 56 & \\
\hline & $80^{\circ} \mathrm{C}$ & $3 \mathrm{M}$ & $168 \mathrm{hr}$ & 65 & \\
\hline & $100^{\circ} \mathrm{C}$ & $1 \mathrm{M}$ & $168 \mathrm{hr}$ & 53 & \\
\hline & $100^{\circ} \mathrm{C}$ & $3 \mathrm{M}$ & $168 \mathrm{hr}$ & 61 & \\
\hline \multirow{4}{*}{$241-C-102$} & $60^{\circ} \mathrm{C}$ & $1 \mathrm{M}$ & $72 \mathrm{hr}$ & 27 & \multirow{4}{*}{ PNNL-12026 } \\
\hline & $60^{\circ} \mathrm{C}$ & $3 \mathrm{M}$ & $72 \mathrm{hr}$ & 95 & \\
\hline & $100^{\circ} \mathrm{C}$ & $1 \mathrm{M}$ & $72 \mathrm{hr}$ & 20 & \\
\hline & $100^{\circ} \mathrm{C}$ & $3 \mathrm{M}$ & $72 \mathrm{hr}$ & 95 & \\
\hline \multirow{3}{*}{$241-C-104$} & $50^{\circ} \mathrm{C}$ & $4 \mathrm{M}$ & $65 \mathrm{hr}$ & 39.7 & \multirow{3}{*}{$\begin{array}{l}\text { ORNL TM- } \\
13500\end{array}$} \\
\hline & $80^{\circ} \mathrm{C}$ & $4 \mathrm{M}$ & $65 \mathrm{hr}$ & 28.7 & \\
\hline & $93^{\circ} \mathrm{C}$ & $4 \mathrm{M}$ & $65 \mathrm{hir}$ & 90.2 & \\
\hline \multirow{4}{*}{$241-S-101$} & $70^{\circ} \mathrm{C}$ & $1 \mathrm{M}$ & $168 \mathrm{hr}$ & 70 & \multirow{4}{*}{ PNNL- 12026} \\
\hline & $70^{\circ} \mathrm{C}$ & $3 \mathrm{M}$ & $168 \mathrm{hr}$ & 63 & \\
\hline & $95^{\circ} \mathrm{C}$ & $1 \mathrm{M}$ & $168 \mathrm{hr}$ & 88 & \\
\hline & $95^{\circ} \mathrm{C}$ & $3 \mathrm{M}$ & $168 \mathrm{hr}$ & 90 & \\
\hline \multirow{4}{*}{$241-S-101$} & $70^{\circ} \mathrm{C}$ & $1 \mathrm{M}$ & $168 \mathrm{hr}$ & 78.4 & \multirow{4}{*}{$\begin{array}{l}\text { ORNL TM- } \\
13655\end{array}$} \\
\hline & $70^{\circ} \mathrm{C}$ & $3 \mathrm{M}$ & $168 \mathrm{hr}$ & 64.9 & \\
\hline & $95^{\circ} \mathrm{C}$ & $1 \mathrm{M}$ & $168 \mathrm{hr}$ & 89 & \\
\hline & $95^{\circ} \mathrm{C}$ & $3 \mathrm{M}$ & $168 \mathrm{hr}$ & 91.5 & \\
\hline $241-8-104$ & $67^{\circ} \mathrm{C}$ & $3.8 \mathrm{M}$ & $24 \mathrm{hr}$ & 98.5 & ORNL TM- \\
\hline
\end{tabular}


RPP-RPT-46791

Revision 0

Table 2-3. SSTs Analyzing the Effects of T and [OH'] on Aluminum Leaching ( 2 sheets).

\begin{tabular}{|c|c|c|c|c|c|}
\hline SST & Temperature & $\begin{array}{l}\text { Caustic } \\
\text { concentration } \\
(\mathrm{NaOH})\end{array}$ & $\begin{array}{l}\text { Total time for } \\
\text { Caustic leaching }\end{array}$ & $\%$ Al leached & Reference \\
\hline & $70^{\circ} \mathrm{C}$ & $3.99 \mathrm{M}$ & $21 \mathrm{hr}$ & 20.8 & \multirow[t]{3}{*}{13500} \\
\hline & $70^{\circ} \mathrm{C}$ & $6.33 \mathrm{M}$ & $21 \mathrm{hr}$ & 26.6 & \\
\hline & $80^{\circ} \mathrm{C}$ & $6.33 \mathrm{M}$ & $126 \mathrm{hr}$ & 96.3 & \\
\hline \multirow{9}{*}{$241-5-110$} & $60^{\circ} \mathrm{C}$ & $1 \mathrm{M}$ & $168 \mathrm{hr}$ & 39 & \multirow{9}{*}{ PNNL- 13702} \\
\hline & $60^{\circ} \mathrm{C}$ & $3 \mathrm{M}$ & $168 \mathrm{hr}$ & 47 & \\
\hline & $60^{\circ} \mathrm{C}$ & $5 \mathrm{M}$ & $168 \mathrm{hr}$ & 50 & \\
\hline & $80^{\circ} \mathrm{C}$ & $1 \mathrm{M}$ & $168 \mathrm{hr}$ & 69 & \\
\hline & $80^{\circ} \mathrm{C}$ & $3 \mathrm{M}$ & $168 \mathrm{hr}$ & 91 & \\
\hline & $80^{\circ} \mathrm{C}$ & $5 \mathrm{M}$ & $168 \mathrm{hr}$ & 96 & \\
\hline & $100^{\circ} \mathrm{C}$ & $1 \mathrm{M}$ & $168 \mathrm{hr}$ & 91 & \\
\hline & $100^{\circ} \mathrm{C}$ & $3 \mathrm{M}$ & $168 \mathrm{hr}$ & 100 & \\
\hline & $100^{\circ} \mathrm{C}$ & $5 \mathrm{M}$ & $168 \mathrm{hr}$ & 100 & \\
\hline \multirow{2}{*}{$241-\mathrm{SX}-113$} & $70^{\circ} \mathrm{C}$ & $6.33 \mathrm{M}$ & $21 \mathrm{hr}$ & 51 & \multirow{2}{*}{$\begin{array}{l}\text { ORNL TM- } \\
13500\end{array}$} \\
\hline & $80^{\circ} \mathrm{C}$ & $6.33 \mathrm{M}$ & $126 \mathrm{hr}$ & 78.7 & \\
\hline \multirow{9}{*}{$241-T-110$} & $60^{\circ} \mathrm{C}$ & $0.6 \mathrm{M}$ & $168 \mathrm{hr}$ & 27 & \multirow{9}{*}{ PNNL-13956 } \\
\hline & $60^{\circ} \mathrm{C}$ & $2.5 \mathrm{M}$ & $168 \mathrm{hr}$ & 42 & \\
\hline & $60^{\circ} \mathrm{C}$ & $4 \mathrm{M}$ & $168 \mathrm{hr}$ & 59 & \\
\hline & $80^{\circ} \mathrm{C}$ & $0.6 \mathrm{M}$ & $168 \mathrm{hr}$ & 53 & \\
\hline & $80^{\circ} \mathrm{C}$ & $2.5 \mathrm{M}$ & $168 \mathrm{hr}$ & 67 & \\
\hline & $80^{\circ} \mathrm{C}$ & $4 \mathrm{M}$ & $168 \mathrm{hr}$ & 83 & \\
\hline & $100^{\circ} \mathrm{C}$ & $0.6 \mathrm{M}$ & $168 \mathrm{hr}$ & 50 & \\
\hline & $100^{\circ} \mathrm{C}$ & $2.5 \mathrm{M}$ & $168 \mathrm{hr}$ & 75 & \\
\hline & $100^{\circ} \mathrm{C}$ & $4 \mathrm{M}$ & $168 \mathrm{hr}$ & 77 & \\
\hline
\end{tabular}

The extent of aluminum removal due to inhibited water washing and/or caustic leaching varied considerably depending upon the temperature, caustic concentration, and leaching time. Other important parameters which affect the extent of aluminum dissolution include the solid to solution ratio, the aluminum mineralogy, initial aqueous and solid chemistry, and the degree of mixing. A kinetic model, which takes these factors into consideration, is currently being developed.

\subsection{DISCUSSION}

Due to the chemical variability within the 177 radioactive storage tanks, laboratory studies have been performed on samples to determine the extent of aluminum leaching possible through inhibited water washing and caustic leaching. In this report we have compiled the available information on aluminum removal from tank waste solids from a total of thirty-nine laboratory studies (see Section A1.0 of the appendix). This collection summarizes the results for aluminum removal and the conditions used for their analyses. 
Within these thitty-nine laboratory reports, a total of 5 DSTs, 41 SSTs, and 12 composite tank waste samples were investigaled for aluminum leachability. Of these, only 3 of the DSTs, 12 of the SSTs, and 7 of the composite tank studies were performed within the nost recent acceptable operation temperature range of the WTP PTF. Seven of the SSTs were studied within the current acceptable temperature range, providing concentration versus time data for aluminum leaching. This information (shown in Figure A3-1 through Figure A3-14) can be used as an indication for how changes in temperature and caustic concentration could affect aluminum leaching on prewashed solids during caustic leaching of these waste samples.

An initial trend observed in Figure A3-2 through Figure A3-8 was that an increase in the caustic concentration in nearly all analyses increased the percent of aluminum removal. This is evident due to the need for free hydroxide for both gibbsite and boehmite dissolution. as shown in Equations 1.1 and 1.2. Both of these reactions will only proceed to a point where the saturation limit of aluminate is reached in solution. Therefore. the solid to solution ratio and aluminate concentration initially in solution are also inportant pieces of information. yet are not always provided during these analyses. Caustic is also necessary for the remoyal of other nonradioactive components that linit $\mathrm{HLW}$ loading, including chronium ( $\mathrm{Cr}$ ) and phosphate ( $\mathrm{PO}_{4}$ ) (24590-WTP-RPT-PT-02-005, Rev. 5). Therefore, additional caustic will be necessaly to ensure aderuate concentrations for dissolution of not only aluminum, but also the other bulk soluble nonradioactive species during this step in the PTF.

Gibbsite has been found to be easily leachable in caustic due to its favorable kinetics. whereas boehmite dissolution is more rate limited, leading to slower dissolution [LBL-21482. Thermochemical properties of gibbsite, bayerite, boehmite, diaspore, and the aluminate ion between $\left(0\right.$ and $350^{\circ} \mathrm{C}$. "Dissolution/precipitation kinetics of boehmite and gibbsite: Application of a pH-relaxation technigue to study near-equilibrium rates" (Benezeth et al. 2008), ${ }^{2}$ Comprebensive Model of Synthetic Bayer Liquors. Part 3. Sodium Aluminate Solutions and the Solubility of Gibbsite and Boehnite" (Konigsberger et al. 2006), "Studies on the Gibbsite to Boebmite Transition" (Ruff el al., 2008), and "Boehmite Actual Waste Dissolution Studjes" (Snow et al., 2008)]. Aluminosilicate solids. as previously described, are more 'refractcy," meaning that they have less of a tendency to dissolve with the addition of caustic and will remain as solids in most cases. These three mineral phases account for the majority of the aluminum species observed within these tanks, leading to a reinforcement of the theory that the majority of the aluminum can be grouped into three categories: (1) easily leachable (e.g. gibbsite, dawsonite, etc.), (2) slow leaching/kinetically controlled (e.g. boehmite), and (3) nou-leachable/ refractory (e.g. aluminosilicates and cancrinites).

It is evident from this compilation, there is a lack of information concerning the effects of unwashed, caustic leached actual waste samples within the acceptable temperature range of $80^{\circ} \mathrm{C}$ to $90^{\circ} \mathrm{C}$ (as detailed in 24590-WTP-RPT-PT-02-005. Rev. 5). From the 46 individual SSTs and DSTs with laboratory data available, only three analyses were performed with no inhibited water wash prior to caustic leaching between $80^{\circ} \mathrm{C}$ and $90^{\circ} \mathrm{C}$. These were for SSTs 241-C-104, 241-S104 , and $241-\mathrm{SX}-113$ (all documented in ORNL TM-13500). Of the remaining conposite studies, only the 241-AZ-101 + 241-AZ-102 analysis described in PNNL-11580 was performed without water washing and leached in the temperature range of $80-90^{\circ} \mathrm{C}$. All of the remaining 
laboratory studies were either performed by washing the solids with inhibited water prior to caustic leaching, or performing caustic leaching at a temperature outside the cutrent acceptable range.

Preliminary washing was found to remove soluble aluminum species from the solids prior to caustic leaching, and in some cases (e.g. that for tanks 24I-AN-104 and 241-BY-110) dissolved over 90 percent of the solid aluminum present in the sample. Then again, preliminary washing sometimes had little to no affect on the solids concentration or even increased the amount of aluminum in solid form. Even though this process will not be used al the PTF, it does provide an indication of the fraction of solid aluminum which is in an easily leachable form for these tanks. It does not indicate, however. the extent of inhibition that will occur due to aluminum saturation limits.

The removal of easily leachable aluminum during inhibited water washing reduces the solid aluminum concentration by transferring it to the aqueous phase. After washing in the referenced laboratory analyses, this aqueous fraction is typically removed by centrifugation and decanting. The aluninate ion in solution is therefore removed, allowing further dissolution of aluminum from the remaining solids. If the aluminate ion concentration in solution were not removed, the initial aqueous aluminate would have to be considered during caustic leaching. This is due to its influence on the reactions shown in Equations 1.1 and 1.2, and its inhibitory effect when the aluminate ion concentration reaches its saturation point. The initial aluninate ion concentration in solution will reduce the total amount of solid aluminum which could be dissolved. Measurements of both the aqueous and solid phase soluble aluminum are therefore key aspects in determining how much aluminum can be removed through high temperature caustic leaching at the WTP PTF prior to vitrification.

Results of aluminum leaching varied between the thirty-nine laboratory studies. In a majority, increases in the caustic concentration in solution, leaching time, and leaching temperature resulted in higher removal of aluminum from the solid phase. Also, the major aluminum solid species found within the wastes initially or following inhibited water washing and/or caustic leaching were gibbsite, bohmite, and aluminosilicales/cancrinites. Gibbsite was found to be easily soluble, being removed from the solid phase in many cases, whereas boehnite and aluminosilicates/cancrinites were found to remain in the solid phase after caustic leaching. These three solid aluminum phases will be used in further analyses to deternine the potential extent of aluminum removal due to caustic leaching. 
RPP-RPT-46791

Revision 0

\subsection{REFERENCES}

24590-WTP-RPT-PT-02-005. Rev. 4, Flowsheet Bases. Asstmptions, and Requirements, Bechtel, Richland, Washington.

24590-WTP-RPT-PT-02-005. Rev. 5, Flowsheet Bases, Assumptions, and Requirements, Bechtel, Richland, Washington.

75764-PCS95-086, 1995, "Caustic Washing of Sludge Samples from Tank 241-AZ-102." (intemal memorandum from D. L. Herting to G. T. MacLean, September 29). Westinghouse Hanford Company, Richland, Washinglon.

9404238. 1994, "Transmittal of Third Quarterly Report for Sludge Treatment Extraction Task Entitled "Sludge Treatment and Extraction Technology Development: Radionuclide Separations":" (intemal letter from L. K. Holton to K. A. Gasper, June 28). Battelle, Pacific Northwest Division. Richland, Washington.

Benezeth, P, D. A. Palmer, and D. J. Wesolowski, 2008, "Dissolution/precipitation kinetics of bohmite and gibbsite: Application of a pH-relaxation technique to study nearequilibrium rates." Geochimica et Cosmochimica Acta, Vol. 72, Issue 10, pp. 2429-2453.

Konigsberger, E.. P. M. May. and G. Hefter, 2006, "Comprehensive Model of Synthetic Bayer' Liquors. Part 3. Sodium Aluninate Solutions and the Solubility of Gibbsite and Boebmite," Monatshefte fiar Chemie / Chemical Monthly, Vol. 137, No. 9. pp. 1139-1149.

LAUR 95-2070. 1995. Sludge Washing and Alkaline Leaching Tests on Actual Hanford Tank Siudge: A Status Report, Los Alamos National Laboratory, Los Alamos, New Mexico.

LAUR 96-2839, 1996, Studge Washing and Alkaline Leaching Tests on Actual Hanford Tank Sludge: FY 1996 Results, Los Alamos National Laboratory, Los Alamos. New Mexico.

LAUR 97-2889, 1997, Sludge Washing and Alkaline Leaching Tests on Actual Hanford Tank Sludge: FY 1997 Results. Los Alamos National Laboratory, Los Alamos. New Mexico.

LBL-21482. 1988. Thermochemical properties of gibbsite, bayerite, boehmite, diaspore, and the aluminate ion between 0 and $350^{\circ} \mathrm{C}$, Lawrence Berkeley Laboratory. Berkeley, California.

ORNLTM-1999/263, 2000, Prevention of Solids Formation: Results of the FY 1999 Shudies, Oak Ridge National Laboratory. Oak Ridge. Tennessee.

ORNLTM-13500, 1998, Caustic Leaching of Sludges from Selected Hanford Tanks, Oak Ridge National Laboratory, Oak Ridge, Tennessee.

ORNLTM-13655, 1998, Water Washes and Caustic Leaches of Sludge from Hanford Tank S101 and Water Washes of Shdze from Hanford Tank C-103, Oak Ridge National Laboratory. Oak Ridge. Tennessee.

ORNLTM-13660, 1998, Status Report ost Solid Controt in Leachates, Oak Ridge Nationa] Laboratory, Oak Ridge, Tennessee. 
RPP-RPT-46791

Revision 0

PNL-9387. 1994. Sludge Treatment and Extraction Technology Development: Results of FY 1993 Studies, Pacific Northwes1 Laboratory, Richland, Washington.

PNL-9814, 1994, The Sort On Radioactive Waste Type Model: A Method to Sort Single-Shell Tanks lno Characteristic Groups, Rev. 2. Pacific Northwest Laboratory, Richland. Washinglon.

PNL-10078. 1994. Washing and Alkaline Leaching of Hatford Tark Sludges: A Status Report. Pacific Northwest Laboratory, Richland, Washing1on.

PNL-10712, 1995, Washing and Caustic Leaching of Hanford Tank Sludges: Results of FY 1995 Studies, Pacific Northwest Laboratory, Richland, Washington.

PNNL-1 1089, 1996, The Chemistry of Sludge Washing and Caustic Leaching Processes for Selected Harfford Tank Wastes, Pacific Northwest National Laboratory, Richland, Washing1on.

PNNL-11278. 1996. Washing and Caustic Leaching of Hanford Tank Sludges: Results of FY 1996 Studies, Pacific Northwest National Laboratory, Richland, Washington.

PNNL-11381, 1996, Washing and Caustic Leaching of Hanford Tank C.106 Sludge, Pacific Northwest National Laboratory, Richland. Washington.

PNNL-1 1432, 1997, Bench-Scale Enhanced Siudge Washing and Gravity Setting of Hanford Tank C-106 SIudge. Pacific Northwest National Laboratory. Richland, Washington.

PNNL-1 1580, 1997, Caustic Leaching of Composite AZ-101/AZ-102 Hanford Tank Sludge, Pacific Northwest National Laboratory, Richland, Washington.

PNNL-1 1636, 1997, Washing and Caustic Leaching of Hanford Tant Shdge: Results of FY 1997 Studies, Pacific Northwest National Laboratory, Richland, Washington.

PNNL-11779, 1997, Leaching of Iron from Hanford Tank Studge: Results of FY 1997 Studies. Pacific Northwest National Laboratory, Richland, Washington.

PNNL-1 1908, 1998, Oxidarive Alkaline Dissolution of Chromium from Hanford Tank Sludges: Results of FY 98 Studies. Pacific Nothwest National Laboratory. Richland. Washington.

PNNL-12010, 1998, Bench-Scale Enhanced Sludge Washing and Gravity Setting of Hanford Tank s. 167 Sludge, Pacific Northwest National Laboratory. Richland, Washington.

PNNL-12026, 1998, Washing and Caustic Leaching of Hanford Tank Sludge: Results of FY 1998 Studies, Pacific Northwest National Laboratory, Richland, Washington.

PNNL-13394, 2000, Status Report on Phase Identification in Hanford Tank Studges. Pacific Northwest National Laboratory, Richland, Washington.

PNNL-13702, 2001, Caustic Leaching of Hanford Tank \$-110 Sladge, Pacific Nothwest National Laboratory, Richland, Washington.

PNNL-13956, 2002, Canstic Leaching of Hanford Tank T.110 Sludge, Pacific Northwest National Laboratory, Richland, Washington.

PNNL-14018, 2002, Alkaline Leaching of Key, Nont-Radicactive Contponents from Simulants and Hanford Tank Studge 241-S-110: Results of FYO1 Studies. Pacific Nothwest National Laboratory, Richland, Washing1on. 
RPP-RPT-46791

Revision 0

PNNL-14019, 2002, Selective Leadhing of Chromium from Hanford Tartk Shdge 241-U-108. Pacific Northwest National Laboratory, Richland, Washington.

PNNL-17368 (WTP-RPT-157). 2008, Characterization and Leach Testing for REDOX Sludge and S-Saltcake Actual Waste Sample Composites. Pacific Northwest National Laboratory, Richland, Washington.

PNNL-17965 (WTP-RPT-172), 2009. Filtration and Leach Testing for REDOX Sludge and SSaltcake Actual Waste Sample Composites. Pacific Northwest National Laboratory. Richland, Washington.

PNNL-17992 (WTP-RPT-166), 2009, Characterization, Leaching, and Filtration Testing for Bismuth Phosphate SIndge (Group 1) and Bismuth Phosphate Sahtcake (Group 2) Actual Waste Sample Composites, Pacific Northwest National Laboratory, Richland, Washinglon.

PNNL-18007 (WTP-RPT-171), 2009. Laboratory Demonstration of the Pretreatment Process with Caustic and Oxidative Leaching Ising Actual Hanford Tank Waste, Pacific Northwest National Laboratory. Richland, Washington.

PNNL-18048 (WTP-RPT-181), 2009. Filtration and Leach Testing for PUREX Cladding Studge and REDOX Cladding Sludge Achal Waste Sample Composites, Pacific Northwest National Laboratory, Richland, Washington.

PNNL-18054 (WTP-RPT-167), 2009, Characterization and Leach Testing for PUREX Cladding Waste Sludge (Group 3) and REDOX Cladding Waste Sludge (Growp 4) Actual Waste Sample Composites, Pacific Northwest National Laboratory, Richland, Washington.

PNNL-18119 (WTP-RPT-169), 2009, Characterization, Leaching, and Filtration Testing for Tributy Phosphate (IBP, Group 7) Actual Waste Sample Conposites, Pacific Northwest National Laboratory, Richland, Washington.

PNNL-18120 (WTP-RPT-170), 2009, Characterization, Leaching, and Filtration Testing of Fenrocyanide Tatk Situdge (Group 8) Actual Waste Composite. Pacific Northwest National Laboratory, Richland, Washinglon.

PNWD-3013 (BNFL-RPT-017), 2000, C-1/6 High-Level Waste Solids: Washing/Leaching and Solubility Versus Temperature Studies, Battelle. Richland, Washington.

PNWD-3024 (BNFL-RPT-030), 2000, Characterization, Washing, Leaching, and Filtration of C-104 Sindge. Battelle, Richland. Washington.

PNWD-3027 (BNFL-RPT-021), 2000, C-104 High-Level Waste Solids: Washing/Leaching and Solubility Versus Temperature Studies, Battelle. Richland, Washington.

PNWD-3045 (BNFL-R PT-038), 2000, Characterization, Washing, Leaching, and Fihtration of AZ-102 Siludge, Battelle, Richland, Washington.

PNWD-3206 (WTP-RPT-043), 2003, Filtration, Washing, and Caustic Leaching of Hanford Tank AZ-101 Sludge, Battelle, Richland, Washington.

PNWD-3300 (WTP-RPT-076), 2003, Identification of Washed Solids from Hanford Tanks 2-1. AN-102 atd 241-AZ-101 with X-Ray Diffractiont, Scanting Electron Microscopy, atd Light-Scattering Particle Analysis, Batlelle, Richland, Washington. 
RPP-RPT-46791

Revision 0

PNWD-3512 (WTP-RPT-117), 2004, Oxidative-Alkaline Leaching of Washed 241-SY-102 and 241-SX-101 Tank Sludges, Battelle, Richland, Washington.

RPP-8847, 2007, Best-Basis Inventory Template Compositions of Common Tank Waste Layers, CH2M Hill Hanford Group, Inc., Richland, Washington.

RPP-PLAN-46002, 2010, Wash and Leach Factor Work Plan, Rev. 0, Washington River Protection Solutions, LLC, Richland, Washington.

RPP-RPT-46618, 2010, Hanford Waste Mineralogy Reference Report, Rev. 2, Washington River Protection Solutions, LLC, Richland, Washington.

RPP-RPT-47306, 2010, Waste Type Analysis for Aluminum Leachability Estimates of All NonRetrieved Hanford Tank Wastes, Washington River Protection Solutions, LLC, Richland, Washington.

Ruff, T. J., R. K. Toghiani, L. T. Smith, and J. S. Lindner, 2008, "Studies on the Gibbsite to Boehmite Transition," Separation Science and Technology, Vol. 43, pp. 2887-2899.

Snow, L. A., G. J. Lumetta, S. Fiskum, and R. A. Peterson, 2008, "Boehmite Actual Waste Dissolution Studies," Separation Science and Technology, Vol. 43, pp. 2900-2916.

WHC-EP-0625, 1993, Hanford Site Waste Storage Tank Information Notebook, Westinghouse Hanford Company, Richland, Washington.

WRPS, 2010, Tank Waste Information Network System (TWINS), Queried 06/2010 (FY10 Q3), [Data, Data source selection forms, Best Basis Inventory, Best Basis Inventory Calculation Detail, All tanks, Analyte $=\mathrm{Al}$, waste phase $=$ sludge , Internet at http:/twins.pnl.gov/twinsdata/forms/BuildQuery.aspx?SourceName=bb published.dbo.p calc detail\&whatsnew=Best+Basis+Inventory, Washington River Protection Solutions, LLC, Richland, Washington. 
RPP-RPT-46791

Revision 0

APPENDIX A.

INFORMATION FROM LABORATORY STUDIES

A-1 
RPP-RPT-46791

Revision 0

\section{A1,0 SINGLE- AND DOUBLE-SHELL TANKS WITH LABORATORY WASH AND LEACH INFORMATION}

Pertinent information gathered from laboratory reports have been compiled here to provide all of the known data concerning the effects of washing and caustic leaching on the renoval of aluminum from actual waste solids. This report analyzes 39 of these reports. This information is detailed in Table A1-1 and Table A1-2 below. These two tables are meant to be placed side-by-side to provide pertinent information gathered from the individual reports. 
Table A1-1. SSTs and DSTs Wash and Leach Information for Aluminum, Part 1. (38 sheets)

\begin{tabular}{|c|c|c|c|c|c|c|c|c|c|c|c|c|c|}
\hline Tank & $\begin{array}{c}\text { Lab Study } \\
\text { Reference(s) }\end{array}$ & $\begin{array}{l}\text { Year of } \\
\text { Sampling } \\
\text { Event }\end{array}$ & Test type & $\begin{array}{l}\text { Sample } \\
\text { color }\end{array}$ & Waste Sources & Sample ID & $\mathrm{NaOH}$ Wash Procedure & $\begin{array}{l}\text { Initial Al in } \\
\text { Untreated } \\
\text { Solids }\end{array}$ & $\begin{array}{l}\text { Final Al in } \\
\text { Washed } \\
\text { solids }\end{array}$ & $\begin{array}{l}\text { Al measured in } \\
\text { Wash Solution }\end{array}$ & $\begin{array}{c}\% \\
\text { recovery }\end{array}$ & comments & Caustic Leach \#1 Procedure \\
\hline DSTs & - & - & - & - & - & - & - & - & - & - & - & - & - \\
\hline 241-AN-104 & $\begin{array}{l}\text { PNNL- } \\
11636\end{array}$ & FY97 & $\begin{array}{l}\text { Caustic } \\
\text { leaching. }\end{array}$ & - & \begin{tabular}{|l} 
Primarily "salt cake", \\
characterized as Double- \\
shell slurry feed. \\
Double-shell Slurry \\
Feed. \\
\end{tabular} & $\begin{array}{l}\text { 222-S Lab ID } \\
\text { S96T005974; Jar \# } \\
\text { 12039; Core \# } 164\end{array}$ & $\begin{array}{l}\text { Wash } 3 \text { times by adding } 10 \\
\text { ml } 0.01 \mathrm{M} \text { NaOH to } 4.702 \mathrm{~g} \\
\text { slurry m mix } 1 \mathrm{hr} \text { at } 100^{\circ} \mathrm{C} ; \\
\text { cool; centrifuge; decant; } \\
\text { repeat w/ solidis portion. }\end{array}$ & $791 \mathrm{ug} / \mathrm{mL}$ & $30.4 \mathrm{ug} / \mathrm{mL}$ & $951 \mathrm{ug} / \mathrm{mL}$ & $99 \%$ & $\begin{array}{l}\text { Component } \\
\text { amounts in washed } \\
\text { and untreated } \\
\text { solids normalized } \\
\text { to amount of } \mathrm{Fe} \\
\text { present }\end{array}$ & $\begin{array}{l}\text { Add } 4.5 \mathrm{ml} \text { of } 10 \mathrm{M} \mathrm{NaOH} \text { to } \\
21.485 \mathrm{~g} \text { sluryy; mix } 5 \mathrm{hr} \text { at } 100^{\circ} \mathrm{C} \text {; } \\
\text { cool; centrifuge; decant. }\end{array}$ \\
\hline 241-AZ-101 & $\begin{array}{l}\text { PNWD- } \\
\text { 3206 (WTP- } \\
\text { RPT-043) }\end{array}$ & 2000 & $\begin{array}{l}\text { Crossflow } \\
\text { filtration, } \\
\text { water } \\
\text { washing } \\
\text { and } \\
\text { caustic } \\
\text { leaching }\end{array}$ & $\begin{array}{l}\text { Material } \\
\text { was very } \\
\text { cohesivel } \\
\text { adhesive } \\
\text { in nature } \\
\text { (pg. } 2.8 \text { ) }\end{array}$ & - & $\begin{array}{l}\text { See Table } 2.1 \text { of } \\
\text { WTP-RPT-048, } \\
\text { Chemical Analysis } \\
\text { and Physical } \\
\text { Property Testing of } \\
\text { 241-AZ-101 Tank } \\
\text { Waste- } \\
\text { Supernatant and } \\
\text { Centrifuged Solids. } \\
\end{array}$ & $\begin{array}{l}\text { Following initial filtration } \\
\text { of } 4312.5 \mathrm{~g} \text { composite } \\
\text { sample }(7.6 \mathrm{wt} \% \\
\text { undissolved solids), } \\
\text { performed two washes using } \\
\text { batch additions of } 1 \mathrm{~L} 0.01 \mathrm{M} \\
\mathrm{NaOH} \text { at } 25 \pm 5^{\circ} \mathrm{C} \text { and } \\
\text { removing permeate by } \\
\text { filtration. }\end{array}$ & $\begin{array}{l}95000 \text { ug } \\
\mathrm{AV} / \mathrm{g} \text { slurry or } \\
5325 \text { ug } \\
\mathrm{Al} / \mathrm{mL} \\
\text { supernate. }\end{array}$ & $\begin{array}{l}186000 \text { ug } \\
\text { Al/g slurry }\end{array}$ & $\begin{array}{l}9 \% \text { removed in } \\
\text { wash. Wash } 1 \\
\text { permeate: } \\
2350 \text { ug } \\
\text { Al/mL. Wash } \\
2 \text { permeate: } \\
1210 \text { ug } \\
\mathrm{Al} / \mathrm{mL} \text {. }\end{array}$ & - & $\begin{array}{l}\text { Physical properties } \\
\text { (ie. density and } \\
\text { particle size) and } \\
\text { rheological data } \\
\text { available in report. }\end{array}$ & $\begin{array}{l}\text { Combine } \mathrm{NaOH} \text { with washed slury } \\
\text { to } 3 \mathrm{M} \mathrm{OH} \text { by adding } \mathrm{NaOH} \text { (1120 } \\
\mathrm{g} \text { of } 3 \mathrm{M} \mathrm{NaOH} \text { and } 645 \mathrm{~g} 9 \mathrm{M} \\
\mathrm{NaOH} \text { added), heat to } 85^{\circ} \mathrm{C} \text {, and } \\
\text { mix for } 8 \text { hr. Desvater by filtration } \\
\text { at } 25^{\circ} \mathrm{C} \text {. }\end{array}$ \\
\hline $\begin{array}{l}241-A Z-101 \\
+241-A Z- \\
102\end{array}$ & $\begin{array}{l}\text { PNNL- } \\
11580\end{array}$ & FY96 & $\begin{array}{l}\text { Lab scale } \\
\text { caustic } \\
\text { leaching: } \\
\text { Six } \\
\text { leaches } \\
\text { performed } \\
\text { at } 80^{\circ} \mathrm{C} \text { in } \\
\text { scries, } \\
\text { each } 5 \\
\text { hours } \\
\text { long. }\end{array}$ & - & Aging ${ }^{1}$ & - & - & $6 \mathrm{wt} \% \mathrm{Al}$ & - & - & - & $\begin{array}{l}\text { Combined } 24 \mathrm{~g} \\
\text { (16.1 mL.) } \\
\text { 'reconstituted' AZ- } \\
102 \text { with } 9.55 \mathrm{~g} \\
\text { (5.9 mL) AZ-101. } \\
\text { Add } 67.8 \mathrm{~g} \text { water, } \\
\text { mixed and remove } \\
2.924 \mathrm{~g} \text { slurry, then } \\
\text { performed } 6 \\
\text { caustic leaches and } \\
3 \text { wash steps. } \\
\\
\text { Reconstituted' } \mathrm{AZ} \text { - } \\
102 \text { sludge: add } \\
\text { equal weight water } \\
\text { to dried sludge. }\end{array}$ & 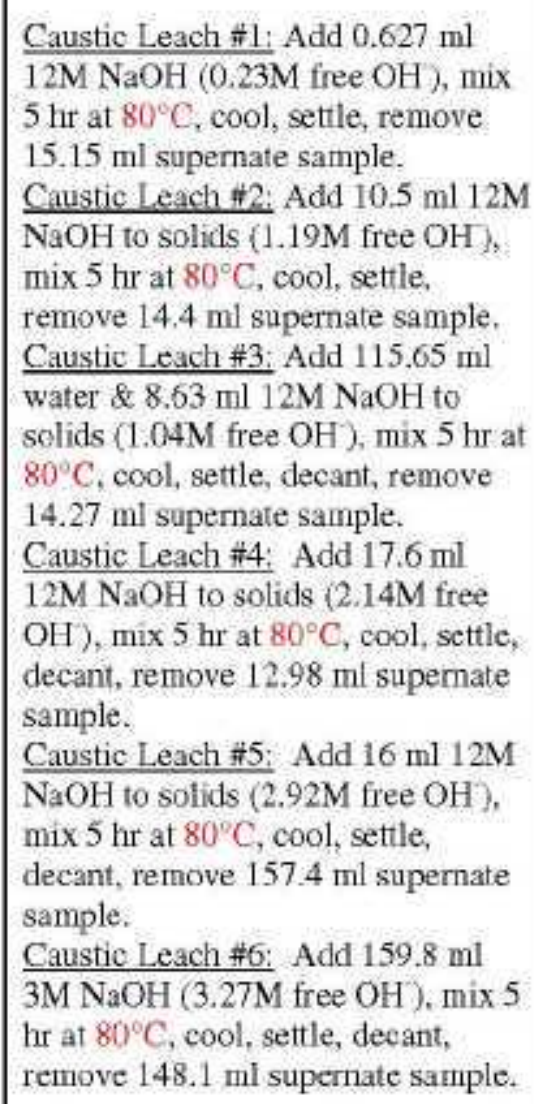 \\
\hline
\end{tabular}

${ }^{2}$ Percent recovery, in most cases, is a comparison of measured concentrations using two methods: (1) summation of the individual solid and liquid samples following the laboratory study and (2) a direct analysis of the total concentration in the initial sample. 
Table A1-1. SSTs and DSTs Wash and Leach Information for Aluminum, Part 1. (38 sheets)

\begin{tabular}{|c|c|c|c|c|c|c|c|c|c|c|c|c|c|}
\hline Tank & $\begin{array}{c}\text { Lab Study } \\
\text { Reference(s) }\end{array}$ & $\begin{array}{c}\text { Year of } \\
\text { Sampling } \\
\text { Event }\end{array}$ & Test type & $\begin{array}{l}\text { Sample } \\
\text { color }\end{array}$ & Waste Sources & Sample ID & $\mathrm{NaOH}$ Wash Procedure & $\begin{array}{l}\text { Initial } \mathrm{Al} \text { in } \\
\text { Untreated } \\
\text { Solids }\end{array}$ & $\begin{array}{l}\text { Final Al in } \\
\text { Washed } \\
\text { solids }\end{array}$ & $\begin{array}{l}\mathrm{Al} \text { measured in } \\
\text { Wash Solution }\end{array}$ & $\begin{array}{c}\% \\
\text { recovery }\end{array}$ & comments & Caustic Leach \#1 Procedure \\
\hline 241-AZ-102 & $\begin{array}{l}75764- \\
\text { PCS } 95-086\end{array}$ & 1994 & $\begin{array}{l}\text { Sludge } \\
\text { water } \\
\text { washing/ } \\
\text { caustic } \\
\text { washing }\end{array}$ & - & - & - & $\begin{array}{l}\text { Sludge sample (labeled } 102- \\
\text { AZ-4) was agitated until } \\
\text { homogeneous and allowed } \\
\text { to settle } 24 \text { hrs. The siudge } \\
\text { was agitated again, with } 25 \\
\mathrm{~mL} \text { aliquots transferred to } 4 \\
\text { centrifuge cones, with } \\
\text { weights and volumes } \\
\text { recorded. After centifuging } \\
3 \text { hrs, the supernatant was } \\
\text { decanted. Water or caustic } \\
\text { solution (1, } 2 \text {, or } 3 \mathrm{M} \text { ) was } \\
\text { added to each cone to } \\
\text { perform a wash of the solids } \\
\text { to remove soluble species. } \\
\text { Cones were tumbled for } 24 \\
\text { hrs at ambient T and } \\
\text { subsequently centrifuged for } \\
1 \text { hr. The water wash and } 1 \\
\text { M Caustic wash cones broke } \\
\text { during centrifuging, losing } \\
\text { all supernate and some } \\
\text { solids. }\end{array}$ & 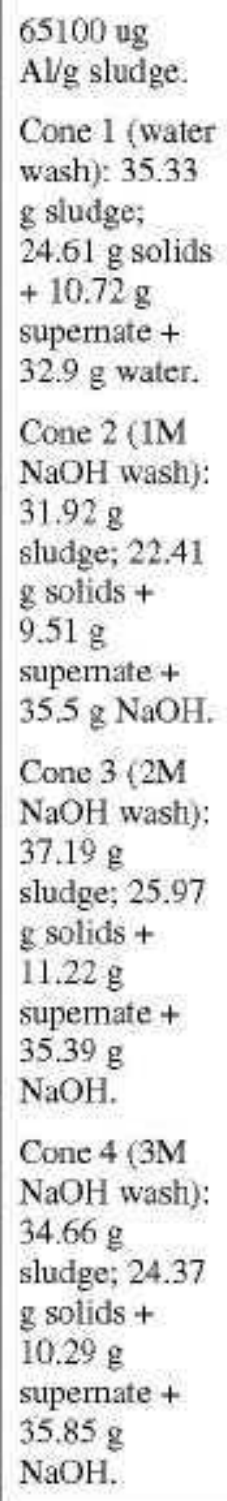 & $\begin{array}{l} \\
\\
\\
\text { Cone 1: } \\
6.468 \mathrm{~g} \\
\text { Al/100 g } \\
\text { solids. } \\
\text { Cone 2: } \\
5.597 \mathrm{~g} \mathrm{Al} / \\
100 \mathrm{~g} \text { solids. } \\
\text { Cone 3: } \\
2.007 \\
\text { (4.057 } \\
\text { All 100 g } \\
\text { solids. } \\
\text { Cone } 4: \\
3.803 \mathrm{~g} \text {. Al/ } \\
100 \mathrm{~g} \text { solids. }\end{array}$ & $\begin{array}{l}\text { Al in washed } \\
\text { supcrnate: } \\
\text { Cone } 1: 0.018 \\
\mathrm{~g} / 100 \mathrm{~g} \\
\text { sludge. } \\
\text { Cone } 2: 0.095 \\
\mathrm{~g} / 100 \mathrm{~g} \\
\text { sludge. } \\
\text { Cone } 3: 0.131 \\
\mathrm{~g} / 100 \mathrm{~g} \\
\text { sludge. } \\
\text { Cone } 4: 0.155 \\
\mathrm{~g} / 100 \mathrm{~g} \\
\text { sludge. } \\
\text { Al in } \\
\text { interstitial } \\
\text { liquid: } \\
\text { Cone } 1: 0.005 \\
\mathrm{~g} / 100 \mathrm{~g} \\
\text { sludge. } \\
\text { Cone } 2: 0.025 \\
\mathrm{~g} / 100 \mathrm{~g} \\
\text { sludge. } \\
\text { Cone } 3: 0.042 \\
\mathrm{~g} / 100 \mathrm{~g} \\
\text { sludge. } \\
\text { Cone } 4: 0.049 \\
\mathrm{~g} / 100 \mathrm{~g} \\
\text { sludg. }\end{array}$ & - & - & - \\
\hline
\end{tabular}

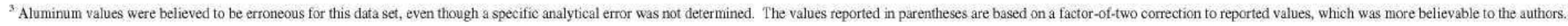


Table A1-1. SSTs and DSTs Wash and Leach Information for Aluminum, Part 1. (38 sheets)

\begin{tabular}{|c|c|c|c|c|c|c|c|c|c|c|c|c|c|}
\hline Tank & $\begin{array}{l}\text { Lab Study } \\
\text { Reference(s) }\end{array}$ & $\begin{array}{c}\text { Year of } \\
\text { Sampling } \\
\text { Event }\end{array}$ & Test type & $\begin{array}{l}\text { Sample } \\
\text { color }\end{array}$ & Waste Sources & Sample ID & $\mathrm{NaOH}$ Wash Procedure & $\begin{array}{l}\text { Initial Al in } \\
\text { Untreated } \\
\text { Solids }\end{array}$ & $\begin{array}{l}\text { Final Al in } \\
\text { Washed } \\
\text { solids }\end{array}$ & $\begin{array}{l}\text { Al measured in } \\
\text { Wash Solution }\end{array}$ & $\begin{array}{c}\% \\
\text { recovery }\end{array}$ & comments & Caustic Leach \#1 Procedure \\
\hline 241-AZ-102 & $\begin{array}{l}\text { PNWD- } \\
3045 \\
\text { (BNFL- } \\
\text { RPT-038) }\end{array}$ & $\begin{array}{l}1999 . \\
2000\end{array}$ & $\begin{array}{l}\text { Crossflow } \\
\text { filtration, } \\
\text { water- } \\
\text { washing, } \\
\text { and } \\
\text { caustic- } \\
\text { leaching }\end{array}$ & - & - & $\begin{array}{l}\text { Four jars - sample } \\
\# 17165=153.53 \mathrm{~g} ; \\
\# 17166=134.15 \mathrm{~g} \text {; } \\
\# 17167=128.85 \mathrm{~g} \text {; } \\
\# 17168=145.32 \mathrm{~g} \text {. } \\
\text { Total of } 538.9 \mathrm{~g} \\
\text { composite sample } \\
\text { mixed for } 29.42 \mathrm{hr} \\
\text { and allowed to } \\
\text { settle } 90 \mathrm{hr} \text {. } \\
\text { Material was very } \\
\text { cohesive/adhesive, } \\
\text { being hard to } \\
\text { transfer and } \\
\text { leaving a thick } \\
\text { clinging layer on } \\
\text { tools and vessels. } \\
\text { Also formed strong } \\
\text { agglomererations: } \\
\text { easy to break apart } \\
\text { when allowed to sit } \\
\text { for a few days, } \\
\text { difficult when } \\
\text { allowed to sit for } \\
\text { weeks. Material } \\
\text { also entrained air } \\
\text { after filtration in } \\
\text { quantities that } \\
\text { increased the } \\
\text { sample volume by } \\
\text { a third. }\end{array}$ & $\begin{array}{l}\text { Following initial filtration } \\
\text { of sample performed three } \\
\text { washes using } 0.01 \mathrm{M} \mathrm{NaOH} \\
\text { at } 25^{\circ} \mathrm{C} \text {. Washing solution } \\
\text { added, followed by removal } \\
\text { of an equal amount of liquid } \\
\text { as permeate using the } \\
\text { crossflow filter. }\end{array}$ & $\begin{array}{l}1.25 \mathrm{E}+5 \mathrm{ug} \\
\mathrm{A} / \mathrm{g} \text { dry } \\
\text { solids }\end{array}$ & - & $\begin{array}{l}\text { Wash 1: } 146 \\
\text { ug Al/mL. } \\
\text { Wash 2: } 108 \\
\text { ug Al/mL. } \\
\text { Wash 3: } 93 \text { ug } \\
\text { Al/mL. }\end{array}$ & $\begin{array}{l}\text { Following } \\
\text { Wash: } \\
1.85 \mathrm{E}+5 \\
\text { ug Al/g } \\
\text { dry solids. } \\
\text { Wash } \\
\text { efficiency } \\
=2.5 \%\end{array}$ & $\begin{array}{l}\text { Physical properties } \\
\text { (i.e. density and } \\
\text { particle size) and } \\
\text { rheological data } \\
\text { available in report. }\end{array}$ & $\begin{array}{l}\mathrm{Combine} \mathrm{NaOH} \text { with washed siurry } \\
\text { to } 3 \mathrm{M} \mathrm{OH} \text { by adding } 609.16 \mathrm{~g} \text { of } \\
7.37 \mathrm{M} \mathrm{NaOH} \text {, heat to } 85^{\circ} \mathrm{C} \text {, and } \\
\text { mix for } 8 \mathrm{hr} \text {. Dewater by filtration. }\end{array}$ \\
\hline
\end{tabular}


Table A1-1. SSTs and DSTs Wash and Leach Information for Aluminum, Part 1. (38 sheets)

\begin{tabular}{|c|c|c|c|c|c|c|c|c|c|c|c|c|c|}
\hline Tank & $\begin{array}{l}\text { Lab Study } \\
\text { Reference(s) }\end{array}$ & $\begin{array}{c}\text { Year of } \\
\text { Sampling } \\
\text { Event }\end{array}$ & Test type & $\begin{array}{l}\text { Sample } \\
\text { color }\end{array}$ & Waste Sources & Sample ID & $\mathrm{NaOH}$ Wash Procedure & $\begin{array}{l}\text { Initial Al in } \\
\text { Untreated } \\
\text { Solids }\end{array}$ & $\begin{array}{l}\text { Final Al in } \\
\text { Washed } \\
\text { solids }\end{array}$ & $\begin{array}{l}\text { Al measured in } \\
\text { Wash Solution }\end{array}$ & $\begin{array}{c}\% \\
\text { recovery }\end{array}$ & comments & Caustic Leach \#1 Procedure \\
\hline 241-SY-102 & $\begin{array}{l}\text { PNWD- } \\
3512 \text { (WTP. } \\
\text { RPT-117) }\end{array}$ & $\begin{array}{l}\text { Sample } \\
\text { shipped } \\
\text { to } \\
\text { PNWD } \\
\text { in July } \\
2003\end{array}$ & $\begin{array}{l}\text { Lab scale } \\
\text { caustic } \\
\text { and } \\
\text { oxidative } \\
\text { leaching }\end{array}$ & - & DN/PD. ${ }^{1}$ & $\begin{array}{l}\text { Mixture of solids } \\
\text { and liquids from } \\
\text { two cores (284 \& } \\
286 \text { ), } 11 \text { jars, and } \\
11 \text { sample } \mathrm{Ds} \text { (see } \\
\text { end of row for } \\
\text { details) }\end{array}$ & $\begin{array}{l}\text { Combine all samples in } \\
\text { single } 2 \mathrm{~L} \text { jar; mix, transfer } \\
\text { portion into separate } 50 \mathrm{~mL} \\
\text { centrifugation cone. Wash } \\
2 \text { times w/ } 0.01 \mathrm{M} \mathrm{NaOH} \text { so } \\
\text { total volume }=50 \mathrm{~mL} \text {. } \\
\text { Centrifuge and separate } \\
\text { solidiliquid. Transfer solid } \\
\text { to } 50 \mathrm{~mL} \text { centrifuge bottles } \\
\text { and contact approximately } 5 \\
\text { times (until bulk of color } \\
\text { removed) with fresh } 0.01 \mathrm{M} \\
\text { NaOH w/ solutionssolid } \\
\text { ratio of approximately } 5: 1 \text {. } \\
\text { Decant supernate after each } \\
\text { contact and discard. } \\
\text { Prepare final slurry by } \\
\text { adding a portion of } 0.01 \mathrm{M} \\
\text { NaOH to washed, } \\
\text { centrifuged solids. Remove } \\
\text { two weighed aliquots of } \\
\text { stirred suspension and dry to } \\
\text { constant tw at } 105{ }^{\circ} \mathrm{C} \text {. } \\
\text { Repeat above procedure to } \\
\text { obtain enough solid for } \\
\text { analysis. }\end{array}$ & - & $\begin{array}{l}171000 \mathrm{ug} \\
\mathrm{Al} / \mathrm{g} \text { dried } \\
\text { solids }\end{array}$ & - & - & - & 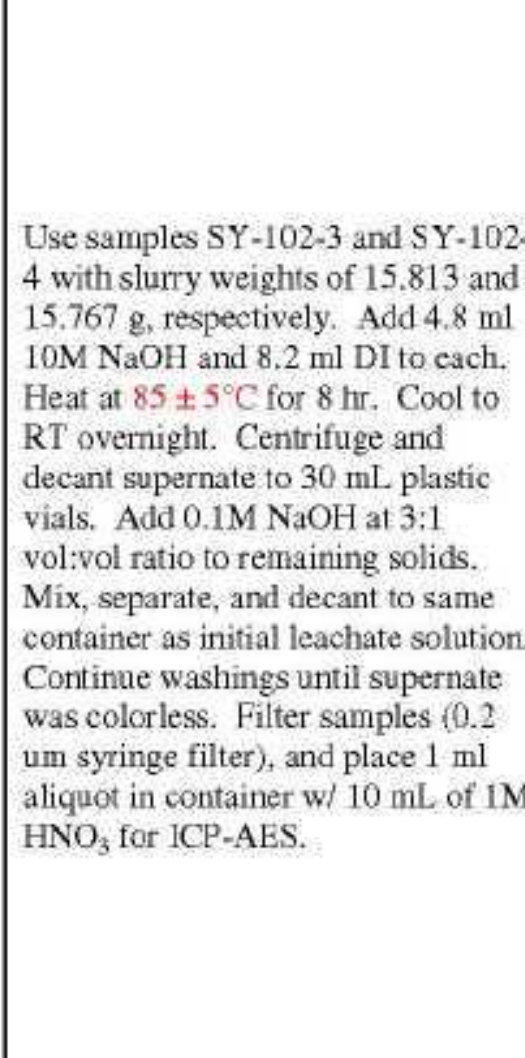 \\
\hline 241-SY-103 & PNL-10712 & FY95 & $\begin{array}{l}\text { Lab scale } \\
\text { caustic } \\
\text { leaching }\end{array}$ & - & $\mathrm{CC}^{1,2}$ & $\begin{array}{l}\text { Composite of } \\
\text { segments } 10 \\
\text { through } 14 \text { from } \\
\text { Core 62 (222-S Jar } \\
\# 6590)\end{array}$ & $\begin{array}{l}\text { Add } 40.2 \mathrm{~g} \text { water to } 10.2 \mathrm{~g} \\
\text { SY103-1, mix, and sample. } \\
\text { Use } 1.11 \mathrm{~g} \text { slurry: Wash } 3 \\
\text { times by adding } 5 \text { ml } 0.01 \\
\text { M NaOH/0.01 } / \mathrm{NaNO}_{2} \text { to } \\
\text { slurry; mix } 0.5 \mathrm{hr} \text { at } \mathrm{RT} \text {; } \\
\text { centrifuge; decant; repeat w/ } \\
\text { solids portion. Dry } \\
\text { remaining solids at } 80^{\circ} \mathrm{C} \\
\text { (0.016 g solid remains) }\end{array}$ & - & - & - & - & $\begin{array}{l}\text { Retrieval Wash } \\
\text { prior to caustic } \\
\text { leach: add } 0.43 \mathrm{~g} \\
1 \mathrm{M} \mathrm{NaOH} / 1 \mathrm{M} \\
\mathrm{NaNO}_{2} \text { to } 6.05 \mathrm{~g} \\
\text { dry solids in slurry. } \\
\text { Mix } 1 \mathrm{~h} \text { at } 100^{\circ} \mathrm{C} \text {, } \\
\text { cool, measure } \\
\text { settling, centrifuge, } \\
\text { decant. }\end{array}$ & $\begin{array}{l}\text { Add } 1.3 \mathrm{~g} \text { water and } 1.6 \mathrm{~mL} \text { of } 10 \mathrm{M} \\
\mathrm{NaOH} \text { to } 4 \mathrm{~mL} \text { centrifuged solids; } \\
\mathrm{mix} 5 \mathrm{hr} \text { at } 100^{\circ} \mathrm{C} \text {; cool; measure } \\
\text { settling; centrifuge; decant }\end{array}$ \\
\hline 241-SY-103 & $\begin{array}{l}\text { PNNL-- } \\
11089\end{array}$ & FY95 & $\begin{array}{l}\text { Sludge } \\
\text { washing } \\
\text { and } \\
\text { caustic } \\
\text { leaching }\end{array}$ & - & Primarily CC. & Core \# 62 & $\begin{array}{l}\text { Add water and inhibited } \\
\text { water to sludge sample to } \\
\text { get } 2.3 \text { wt's solids and } \\
0.01 \mathrm{M} \mathrm{NaOH} / \mathrm{NaNO}_{2} \text {. Mix } \\
\text { at } 100^{\circ} \mathrm{C} \text { for } 1 \mathrm{hr} \text {. Cool, } \\
\text { centrifuge, and decant. }\end{array}$ & - & - & $\begin{array}{l}9 \% \text { recovery } \\
\text { of } \mathrm{Al}\end{array}$ & - & - & $\begin{array}{l}\text { Caustic leach of solids: Add } \mathrm{NaOH} \\
\text { to get } \sim 8 \text { wt\% solids and a final } \\
\mathrm{NaOH} \text { of } 2.2 \mathrm{M} \text {. Mix at } 100^{\circ} \mathrm{C} \text { for } 5 \\
\text { hr. Cool, centrifuge, decant. }\end{array}$ \\
\hline
\end{tabular}


Table A1-1. SSTs and DSTs Wash and Leach Information for Aluminum, Part 1. (38 sheets)

\begin{tabular}{|c|c|c|c|c|c|c|c|c|c|c|c|c|c|}
\hline Tank & $\begin{array}{l}\text { Lab Study } \\
\text { Reference(s) }\end{array}$ & $\begin{array}{c}\text { Year of } \\
\text { Sampling } \\
\text { Event }\end{array}$ & Test type & $\begin{array}{l}\text { Sample } \\
\text { color }\end{array}$ & Waste Sources & Sample ID & $\mathrm{NaOH}$ Wash Procedure & $\begin{array}{l}\text { Initial } \mathrm{Al} \text { in } \\
\text { Untreated } \\
\text { Solids }\end{array}$ & $\begin{array}{l}\text { Final Al in } \\
\text { Washed } \\
\text { solids }\end{array}$ & $\begin{array}{l}\text { Al measured in } \\
\text { Wash Solution }\end{array}$ & $\begin{array}{c}\% \\
\text { recovery }\end{array}$ & comments & Caustic Leach \#1 Procedure \\
\hline SSTs & - & - & - & - & - & - & - & - & - & - & - & - & - \\
\hline 241-B-101 & $\begin{array}{l}\text { PNNL- } \\
12026\end{array}$ & FY98 & $\begin{array}{l}\text { Lab scale } \\
\text { caustic } \\
\text { leaching: } \\
\text { compare } \\
60 \text { and } \\
100^{\circ} \mathrm{C} \\
\text { cusstic } \\
\text { leaching } \\
\text { over } 168 \\
\text { hr. }\end{array}$ & - & $\begin{array}{l}\text { Primary: BB; } \\
\text { Secondary: CW. } \\
\text { Non-Complexed Waste. } \\
\text { Primary: CW. } \\
\text { Secondary: EB. } \\
\text { Tertiary: B Plant low- } \\
\text { level waste. }\end{array}$ & $\begin{array}{l}\text { Composite mixture } \\
\text { of two segments } \\
\text { from Core } \# 90 \text { and } \\
\text { two segments from } \\
\text { Core } \# 91\end{array}$ & $\begin{array}{l}\text { Mix } 50.15 \mathrm{~g} \text { B-101 } \\
\text { composite with } 100 \mathrm{~mL} 0.1 \\
\text { M NaOH in } 225 \mathrm{~mL} \text { bottle; } \\
\text { stir } 30 \mathrm{~min} \text { at RT; centrifuge } \\
\text { at } 1200 \mathrm{G} \text { for } 15 \mathrm{~min} \\
\text { decant. Repeat wash with } \\
\text { NaOH for } 7 \text { total wash } \\
\text { cycles. }\end{array}$ & $\begin{array}{l}3.04 \text { wt } \% \text { Al; } \\
30378 \text { ug } \\
\mathrm{Al} / \mathrm{g} \text { solids }\end{array}$ & $\begin{array}{l}82615 \text { ug } \\
\mathrm{Al} / \mathrm{g} \text { washed } \\
\text { solids; } \\
1148348 \mathrm{ug} \\
\mathrm{Al}\end{array}$ & $\begin{array}{l}473 \text { ug Al/mL } \\
\text { or } 375089 \text { ug } \\
\text { Al }\end{array}$ & $\begin{array}{l}25 \% \mathrm{Al} \\
\text { removed }\end{array}$ & $\begin{array}{l}\text { Shows time versus } \\
\text { concentration } \\
\text { graph for leaching } \\
\text { at different } \\
\text { temperature and } \\
\mathrm{NaOH} \\
\text { concentrations. } \\
\text { Diluted washed } \\
\text { solids w/ } 50 \mathrm{~mL} \text {. DI } \\
\& \text { stir } 30 \text { min. } \\
\text { Distribute } \sim 15 \mathrm{~g} \\
\text { aliquots between } 5 \\
\text { bottles }\end{array}$ & 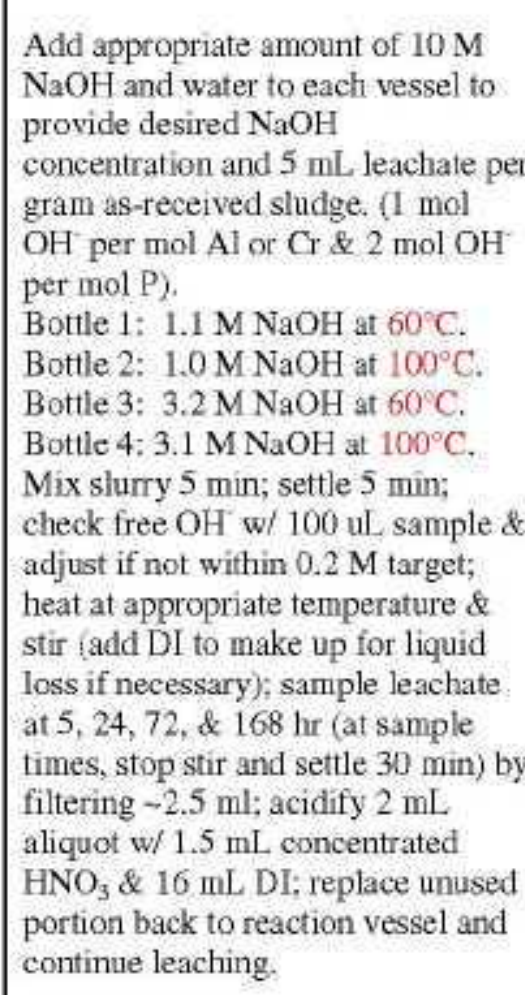 \\
\hline $241-B-104$ & $\begin{array}{l}\text { LAUR 96- } \\
2839\end{array}$ & FY96 & $\begin{array}{l}\text { Lab scale } \\
\text { caustic } \\
\text { leaching }\end{array}$ & $\begin{array}{l}\text { reddish- } \\
\text { brown } \\
\text { thin paste }\end{array}$ & $\begin{array}{l}\text { 2C: slurry product from } \\
\text { evaporators (EB); TBP } \\
\text { waste from U-extraction } \\
\text { process at U Plant; 1C. } \\
\text { Non-Complexed Waste. }{ }^{1} \\
\text { Primary: } 2 \mathrm{C} \text {, Secondary: } \\
\text { EB. } \\
\text { Primary: 2C. Secondary: } \\
\text { EB. Tertiary: TBP. } \\
\text { Other: } 1 C^{5} \text {. }\end{array}$ & - & $\begin{array}{l}\text { Mix } 13.54 \mathrm{~g} \text { wet sludge with } \\
27 \mathrm{~mL} \text {, water. With } 4.8 \mathrm{~g} \\
\text { slurry, wash } 3 \text { times by } \\
\text { adding } 10 \mathrm{~mL} \text { of } 0.01 \mathrm{M} \\
\mathrm{NaOH} \text {; mix for } 1 \mathrm{hr} \text { at } \\
100^{\circ} \mathrm{C} \text {; cool; centrifuge; } \\
\text { decant; repeat wash with } \\
\text { solids. Dry solids a } 105^{\circ} \mathrm{C} \text {, } \\
\text { Produced } 0.148 \mathrm{~g} \text { or } 9.45 \mathrm{wt} \\
\text { \% insoluble solids }\end{array}$ & $\begin{array}{l}0.3 \mathrm{wt} \% \mathrm{Al} ; \\
2118.03 \mathrm{ug} \\
\mathrm{Al} ; 14349.8 \\
\text { ug Al/g solids }\end{array}$ & $94 \% \mathrm{Al}$ & $\begin{array}{l}140.67 \mathrm{ug} \mathrm{Al} ; \\
4.91 \mathrm{ug} \mathrm{Al} / \mathrm{mL}\end{array}$ & $\begin{array}{l}6 \% \mathrm{Al} \\
\text { removed } \\
\text { by simple } \\
\text { wash }\end{array}$ & - & $\begin{array}{l}\text { Add } 10 \mathrm{M} \mathrm{NaOH} \text { and water to } 10.97 \\
\mathrm{~g} \text { mixed wet sludge (5.76 g dry } \\
\text { sludge) to get } \sim 5 \mathrm{wt} \% \text { solids and } \\
\text { final } \mathrm{NaOH} \text { of }-2 \mathrm{M}(4 \mathrm{~mL} \text {. of } 10 \mathrm{M} \\
\mathrm{NaOH}) \text {; mix } 5 \mathrm{hr} \text { at } 100^{\circ} \mathrm{C} \text {; cool; } \\
\text { measure settling; centrifuge; decant. }\end{array}$ \\
\hline
\end{tabular}


Table A1-1. SSTs and DSTs Wash and Leach Information for Aluminum, Part 1. (38 sheets)

\begin{tabular}{|c|c|c|c|c|c|c|c|c|c|c|c|c|c|}
\hline Tank & $\begin{array}{c}\text { Lab Study } \\
\text { Reference(s) }\end{array}$ & $\begin{array}{l}\text { Year of } \\
\text { Sampling } \\
\text { Event }\end{array}$ & Test type & $\begin{array}{l}\text { Sample } \\
\text { color }\end{array}$ & Waste Sources & Sample ID & $\mathrm{NaOH}$ Wash Procedure & $\begin{array}{l}\text { Initial Al in } \\
\text { Untreated } \\
\text { Solids }\end{array}$ & $\begin{array}{l}\text { Final Al in } \\
\text { Washed } \\
\text { solids }\end{array}$ & $\begin{array}{l}\text { Al measured in } \\
\text { Wash Solution }\end{array}$ & $\begin{array}{c}\% \\
\text { recovery }\end{array}$ & comments & Caustic Leach \#1 Procedure \\
\hline 241-B-106 & $\begin{array}{l}\text { LAUR 97- } \\
2889\end{array}$ & FY97 & $\begin{array}{l}\text { Labscale } \\
\text { caustic } \\
\text { leaching }\end{array}$ & $\begin{array}{l}\text { reddish- } \\
\text { brown } \\
\text { liquid } \\
\text { paste; } \\
\text { dried } \\
\text { material: } \\
\text { tannish- } \\
\text { brown in } \\
\text { color; dry } \\
\text { treated } \\
\text { material: } \\
\text { dark } \\
\text { brown in } \\
\text { color } \\
\end{array}$ & $\begin{array}{l}\text { 1C, TBP from U- } \\
\text { extraction process at U } \\
\text { Plant, lab waste from the } \\
300 \text { area. } \\
\text { Non-Complexed Waste } \\
\text { Primary: } 1 \mathrm{C}, \text { Secondary: } \\
\text { TBP. }^{2} \\
\text { Primary: } 1 \mathrm{C} \text {. Secondary: } \\
\text { TBP. Tertiary: Hanford } \\
\text { Laboratory Operations. } \\
\text { Other: mixture of several } \\
\text { miscellaneous wastes. } \\
\end{array}$ & $\begin{array}{l}\text { Composite of } \\
\text { sludge from } \\
\text { segments } 1 \& 2.2 \\
\text { from cores } 93 \text { and } \\
94(222-5 \text { lab ID } \\
\text { S96T001862, jar } \\
\text { \#9794) }\end{array}$ & $\begin{array}{l}\text { Mix } 11.27 \mathrm{~g} \text { wet sludge with } \\
22.5 \mathrm{~mL} \text { water. With } 4.47 \mathrm{~g} \\
\text { slurry, wash } 3 \text { times by } \\
\text { adding } 10 \mathrm{~mL} \text { of } 0.01 \mathrm{M} \\
\mathrm{NaOH} ; \text { mix for } 1 \mathrm{hr} \text { at } \\
100^{\circ} \mathrm{C} ; \text { corl; centrifuge; } \\
\text { decant; repeat with solids. } \\
\text { Dry solids at } 105^{\circ} \mathrm{C} \text {. } 8.55 \\
\text { wt } \% \text { insoluble solids, } 33.1 \\
\mathrm{~mL} \text { supernate) }\end{array}$ & 0.7 wt $\% \mathrm{Al}$ & $\begin{array}{l}23858 \text { ug } \\
\mathrm{Al} / \mathrm{g}, 3061 \\
\mathrm{ug} \text { Al }(72 \% \\
\mathrm{Al} \\
\text { remaining) }\end{array}$ & $\begin{array}{l}35.7 \mathrm{ug} \mathrm{Al} / \mathrm{ml} \text {, } \\
1181.67 \text { ug Al } \\
(28 \% \mathrm{Al} \\
\text { removed) }\end{array}$ & \begin{tabular}{|l}
$28 \%$ \\
removed \\
$(4242.59$ \\
ug Al)
\end{tabular} & - & $\begin{array}{l}\text { Mix } 11.27 \mathrm{~g} \text { wet sludge w/ } 22.5 \mathrm{~mL} \text { L } \\
\text { water. Subsample to get } 8.8 \mathrm{~g} \text { wet } \\
\text { sludge (3.53 g dry sludgc). Add } \\
7.23 \mathrm{~mL} \text { of } 10 \mathrm{M} \mathrm{NaOH} \text { and water } \\
(2.1 \mathrm{wt} \% \text { solids and } \sim 2 \mathrm{M} \mathrm{NaOH} \text { ). } \\
\text { Mix } 5 \mathrm{hr} \text { a } 100^{\circ} ; \text { cool; measure } \\
\text { settling; centrifuge; decant. } \\
\text { Measured } 26.08 \mathrm{~mL} \text { supernate w/ } \\
2.33 \mathrm{M} \text { free } \mathrm{OH}, 9.5 \mathrm{~mL} \text { settled } \\
\text { solids, and } 4.1 \mathrm{~mL} \text { centrifuged } \\
\text { solids. }\end{array}$ \\
\hline 241-B-110 & PNL-9387 & FY93. & $\begin{array}{l}\text { Lab scale } \\
\text { caustic } \\
\text { and acid } \\
\text { leaching }\end{array}$ & - & $\begin{array}{l}2 \mathrm{C} \text { in } 1940 \text { is to recover \& } \\
\text { purify Pu from irradiated } \\
\text { fuel, adjusted to high } \\
\text { pH; fission product } \\
\text { waste produced in } \\
\text { campaigns (60s) to } \\
\text { isolate fission products; } \\
\text { IX from B Plant Cs } \\
\text { recovery process. } \\
\text { Non-Complexed Waste }{ }^{1} \text {. } \\
\text { Primary waste: } 2 \mathrm{C} \text {. } \\
\text { Secondary; High-level B } \\
\text { Plant waste from bottom } \\
\text { of Section 5. } \\
\text { Primary waste type: } 2 \mathrm{C} \text {. } \\
\text { Secondary: high-level B } \\
\text { Plant waste. Tertiary: } \\
\text { Fission Products Waste. } \\
\text { Other: IX. }\end{array}$ & $\begin{array}{l}\text { A composite of the } \\
5 \text { segments } \\
\text { obtained in the } \\
1989 \text { Core } 1 \text {. Cl } \\
\text { Composite } 90 \text { - } \\
4316 \text { ) }\end{array}$ & $\begin{array}{l}\text { Mix } 5.15 \mathrm{~g} \mathrm{Cl} \text { composite } \\
\text { with } 14.5 \mathrm{~mL} \text { of } 0.1 \mathrm{M} \\
\mathrm{NaOH} \text {. With } 2.2 \mathrm{~g} \text { slurry, } \\
\text { stir } 1 \text { hr at } 100^{\circ} \mathrm{C} ; \\
\text { centrifuge; decant. Add } 2 \\
\mathrm{~mL} \text { of } 0.1 \mathrm{M} \mathrm{NaOH} \text { to } \\
\text { solids; mix } 1 \mathrm{hr} \text { at } 100^{\circ} \mathrm{C} \text {; } \\
\text { centrifuge; decant; add } 5 \\
\mathrm{~mL} \text { water to solids; stir } 0.5 \\
\text { hr at RT; centrifuge; decant; } \\
\text { add } 1 \mathrm{~mL} \text { water to solids; } \\
\text { stit } 0.5 \mathrm{~h} \text { hat } \mathrm{RT} \text {; save } 0.13 \mathrm{~g} \\
\text { slurry for analysis }\end{array}$ & $\begin{array}{l}1.5 \mathrm{E}-3 \text { to } 7 \mathrm{E}- \\
4 \mathrm{~g} \mathrm{Al} / \mathrm{g} \\
\text { sludge }\end{array}$ & $\begin{array}{l}\text { Less than or } \\
\text { equal to } \\
27 \% \mathrm{Al} \\
\text { remaining }\end{array}$ & - & - & $\begin{array}{l}\text { Sludge washing } \\
\text { and acid } \\
\text { dissolution } \\
\text { experiment done as } \\
\text { well - not included } \\
\text { here. }\end{array}$ & $\begin{array}{l}\text { Add } 0.1 \mathrm{~mL} \text {. water and } 0.3 \mathrm{~mL} \text { of } 10 \\
\mathrm{M} \mathrm{NaOH} \text { to slurry (Effectively } \\
2.3 \mathrm{M} \text { NaOH;) mix } 5 \mathrm{hr} \text { at } 100^{\circ} \mathrm{C} \text {; } \\
\text { centrifuge; decant. Addd } 1 \mathrm{~mL} 3 \mathrm{M} \\
\mathrm{NaOH} \text { to solids; mix } 5 \mathrm{hr} \text { at } 100^{\circ} \mathrm{C} \text {; } \\
\text { centrifuge; decant. Add } 5 \mathrm{~mL} \text { water } \\
\text { to solids; mix } 0.5 \mathrm{hr} \text { at RT; } \\
\text { centrifuge; decant. Add } 1 \mathrm{~mL} \text { water } \\
\text { to solids; mix } 0.5 \mathrm{hr} \text { at RT; take } \\
0.05 \mathrm{~g} \text { slurry subsample; centrifuge; } \\
\text { decant. ( } 8.1 \mathrm{~mL} \text { sludge leach } \\
\text { solution) }\end{array}$ \\
\hline 241-B-110 & $\begin{array}{l}\text { Internal } \\
\text { Letter } \\
9404238\end{array}$ & 1989 & $\begin{array}{l}\text { Lab scale } \\
\text { wash, } \\
\text { caustic } \\
\text { leach, and } \\
\text { acid } \\
\text { dissolutio } \\
\text { n }\end{array}$ & - & $\begin{array}{l}\text { Primary waste: } 2 \text { C. } \\
\text { Other waste: fission } \\
\text { product waste produced } \\
\text { in campaigns (in the } \\
1960 \text { ) to isolate various } \\
\text { fission products, and IX } \\
\text { from B Plant Cs } \\
\text { recovery process. }\end{array}$ & $\begin{array}{l}\text { A } 9.34 \text { gram wet } \\
\text { composite of cores } \\
1,2,3 \text {, and } 4 \text { form } \\
\text { the } 1989 \text { sampling } \\
\text { event. }\end{array}$ & $\begin{array}{l}\text { Mix } 9.34 \mathrm{~g} \text { wet sludge } \\
\text { composite with three times } \\
\text { its weight in water for } 1 \mathrm{hr} \\
\text { at RT using an orbital } \\
\text { shaker. Allow the sludge to } \\
\text { settle } 20 \mathrm{hr} \text { after mixing. } \\
\text { Decant wash liquor, replace } \\
\text { with fresh water, and repeat } \\
\text { the process twice. }\end{array}$ & $\begin{array}{l}\text { Between } \\
2.1 \mathrm{E}-3 \text { and } \\
2.2 \mathrm{E}-3 \mathrm{~g} \mathrm{Al} / \mathrm{g} \\
\text { sludge }\end{array}$ & $\begin{array}{l}\geq 94 \% \\
\text { remaining }\end{array}$ & $\leq 6 \%$ removed & - & $\begin{array}{l}\text { Acid dissolution of } \\
\text { the solids was } \\
\text { performed after } \\
\text { caustic leaching } \\
\text { and final wash of } \\
\text { solids. This is not } \\
\text { included in this } \\
\text { table. }\end{array}$ & $\begin{array}{l}\text { Add } 8 \mathrm{~mL} \text { water and } 9 \mathrm{~mL} \text { of } 10 \mathrm{M} \\
\mathrm{NaOH} \text { to solids. Mix, using a } \\
\text { magnetic stirrer, for } 5 \mathrm{hr} \text { at } 100^{\circ} \mathrm{C} \text {. } \\
\text { Cool, then settle and decant liquid. }\end{array}$ \\
\hline
\end{tabular}


Table A1-1. SSTs and DSTs Wash and Leach Information for Aluminum, Part 1. (38 sheets)

\begin{tabular}{|c|c|c|c|c|c|c|c|c|c|c|c|c|c|}
\hline Tank & $\begin{array}{l}\text { Lab Study } \\
\text { Reference(s) }\end{array}$ & $\begin{array}{l}\text { Year of } \\
\text { Sampling } \\
\text { Event }\end{array}$ & Test type & $\begin{array}{l}\text { Sample } \\
\text { color }\end{array}$ & Waste Sources & Sample ID & $\mathrm{NaOH}$ Wash Procedure & $\begin{array}{l}\text { Initial Al in } \\
\text { Untreated } \\
\text { Solids }\end{array}$ & $\begin{array}{l}\text { Final Al in } \\
\text { Washed } \\
\text { solids }\end{array}$ & $\begin{array}{l}\mathrm{Al} \text { measured in } \\
\text { Wash Solution }\end{array}$ & $\begin{array}{c}\% \\
\text { recovery }\end{array}$ & comments & Caustic Leach \#1 Procedure \\
\hline 241-B-111 & PNL-10712 & FY95 & $\begin{array}{l}\text { Lab scale } \\
\text { caustic } \\
\text { leaching }\end{array}$ & - & $\begin{array}{l}\text { Non-Complexed Waste. } \\
\text { Primary: 2C. Secondary: } \\
\text { High-level B Plant waste } \\
\text { from bottom of Section } \\
5^{2} \\
\text { Primary: 2C. Secondary: } \\
\text { high-level B Plant waste. } \\
\text { Tertiary: Fission } \\
\text { Products Waste. Other: } \\
\text { IX. }^{5}\end{array}$ & $\begin{array}{l}\text { Composite sample } \\
\text { comprised of } 2.65 \\
\mathrm{~g} \text { Core } 29 \\
\text { composite } 1 \\
\text { [Analytical Lab } \\
\text { Operations \#93- } \\
04314], 1.96 \mathrm{~g} \\
\text { Core } 29 \text { composite } \\
2 \text { [ALO \#93- } \\
\text { 04315], } 2.64 \mathrm{~g} \\
\text { Core } 30 \text { composite } \\
1 \text { [ALO } \$ 93- \\
\text { 043222], and } 2.57 \mathrm{~g} \\
\text { Core } 30 \text { composite } \\
2 \text { [ALO \$93- } \\
04323 \text { ] } \\
\end{array}$ & $\begin{array}{l}\text { Add } 39.1 \mathrm{~g} \text { water to } 9.67 \mathrm{~g} \\
\mathrm{~B} 111-1 \text {, mix, and then } \\
\text { sample. Wash } 3 \text { times by } \\
\text { adding } 5 \mathrm{ml} \text { of } 0.01 \mathrm{M} \\
\mathrm{NaOH} / 0.01 \mathrm{M} \mathrm{NaNO}_{2} \text { to } \\
1.05 \mathrm{~g} \text { slurry; mix } 0.5 \mathrm{hr} \text { at } \\
\text { RT; centrifuge, decant; } \\
\text { repeat w/ solids portion. } \\
\text { Dry remaining solids at } \\
80^{\circ} \mathrm{C}(0.021 \mathrm{~g} \text { solid } \\
\text { remains) }\end{array}$ & - & - & - & - & $\begin{array}{l}\text { Retrieval Wash } \\
\text { prior to caustic } \\
\text { leach: Add } 2.14 \mathrm{~g} \\
\text { water and } 0.39 \mathrm{~g} \\
1 \mathrm{M} \mathrm{NaOH} / 1 \mathrm{M} \\
\mathrm{NaNO} 2 \text { to } 4.03 \mathrm{~g} \\
\text { dry solids in slurry. } \\
\text { Mix } 1 \mathrm{~h} \text { at } 100^{\circ} \mathrm{C} \text {, } \\
\text { cool, measure } \\
\text { settling, centrifuge, } \\
\text { decant. }\end{array}$ & $\begin{array}{l}\text { Add } 0.4 \mathrm{~g} \text { water and } 2.8 \mathrm{~g} 10 \mathrm{M} \\
\mathrm{NaOH} \text { to } 4 \mathrm{~mL} \text { centrifuged solids; } \\
\text { mix } 5 \text { tri at } 100^{\circ} \mathrm{C} \text {; cool; measure } \\
\text { settling; centrifuge; decant }\end{array}$ \\
\hline 241-B-111 & $\begin{array}{l}\text { PNNL-- } \\
11089\end{array}$ & FY95 & $\begin{array}{l}\text { Sludge } \\
\text { washing } \\
\text { and } \\
\text { caustic } \\
\text { leaching. }\end{array}$ & - & $\begin{array}{l}\frac{\text { Primarily 2C. }}{\text { Secondary HLW from }} \\
\text { Tank 5-6 at B Plant. }\end{array}$ & Core \#29 and 30 & $\begin{array}{l}\text { Add water and inhibited } \\
\text { water to sludge sample to } \\
\text { get }-2.3 \mathrm{wt} \mathrm{S}^{\circ} \text { solids and } \\
0.01 \mathrm{M} \mathrm{NaOH} / \mathrm{NaNO}_{2} \text { in } \\
\text { solution. Mix at } 100^{\circ} \mathrm{C} \text { for } \\
1 \mathrm{hr} \text {. Cool, centrifuge, and } \\
\text { decant. } \\
\end{array}$ & - & - & - & - & - & $\begin{array}{l}\text { Caustic leach of solids: } \mathrm{Add} \mathrm{NaOH} \\
\text { to get } \sim 8 \text { wt } \text { solids and a final } \\
\mathrm{NaOH} \text { of } 2.3 \mathrm{M} \text {. Mix at } 1000^{\circ} \mathrm{C} \text { for } 5 \\
\text { hr. Cool, centrifuge, decant. }\end{array}$ \\
\hline 241-B-201 & PNL-10078 & FY94 & $\begin{array}{l}\text { Caustic } \\
\text { leaching }\end{array}$ & - & $\begin{array}{l}\text { Primary waste: } \\
\text { Lanthanum fluoride } \\
\text { decontamination waste. } \\
2,5\end{array}$ & $\begin{array}{l}\text { Two composite } \\
\text { samples which } \\
\text { represented } 2 \text { core } \\
\text { samples (26 and } \\
27 \text { ). Two dry } \\
\text { composite analyses } \\
\text { from cores } 26 \text { and } \\
27 \text {, and one wet } \\
\text { core } 26 \text { composite } \\
\text { (untreated) }\end{array}$ & $\begin{array}{l}\text { Drv cores: Add } 5 \mathrm{~mL} \text { of } 0.1 \\
\mathrm{M} \mathrm{NaOH} \& \text { mix at } 100^{\circ} \mathrm{C} \\
\text { for } 1 \mathrm{hr} \text {, centrifuge, decant. } \\
\text { Add } 2 \mathrm{~mL} 0.1 \mathrm{M} \mathrm{NaOH} \& \\
\text { mix at } 100^{\circ} \mathrm{C} \text { for } 1 \mathrm{hr} \text {, } \\
\text { centrifuge, decant. Add } 5 \\
\text { mL water \& mix at RT for } \\
0.5 \mathrm{hr} \text {, centrifuge, decant; } \\
\text { dry at } 80^{\circ} \mathrm{C} \text {. } \\
\text { Wet core } 26 \text { : same as dry } \\
\text { cores, but after } 3^{\text {trd }} \text { wash do } \\
\text { not dry. }\end{array}$ & - & - & $\begin{array}{l}\frac{\text { Core 26: }}{1 \% \text { Al }} \\
\text { dissolved } \\
\text { Core 27: } 0 \text { 0. } \\
2 \% \text { Al } \\
\text { dissolved }\end{array}$ & - & - & $\begin{array}{l}\text { Drv cores: Add } 6 \mathrm{~mL} \text { of } 3 \mathrm{M} \mathrm{NaOH} \\
\text { to solids from wash, mix at } 100^{\circ} \mathrm{C} \\
\text { for } 5 \mathrm{hr} \text {, centrifuge, decant. Add } 5 \\
\mathrm{~mL} 3 \mathrm{MM} \mathrm{NaOH} \mathrm{to} \mathrm{solids,} \mathrm{mix} \mathrm{at} \\
100^{\circ} \mathrm{C} \text { for } 5 \mathrm{hr} \text {, centrifuge, decant. } \\
\text { Add } 5 \mathrm{~mL} \text { water to solids, mix at } \\
\text { RT for } 0.5 \mathrm{hr} \text {, centrifuge, decant. } \\
\text { Wet core } 26: \text { Add } 1 \text { mL } 3 \mathrm{M} \\
\text { NaOH to solids from wash, mix at } \\
100^{\circ} \mathrm{C} \text { for } 5 \mathrm{hr} \text {, centrifuge, decant; } \\
\text { repeat. Add } 5 \mathrm{~mL} \text { water to solids, } \\
\text { mix at RT for } 0.5 \mathrm{hr} \text {, centrifuge, } \\
\text { decant. } \\
\end{array}$ \\
\hline 241-B-202 & $\begin{array}{l}\text { LAUR 95- } \\
2070\end{array}$ & 1995 & $\begin{array}{l}\text { Lab scale } \\
\text { caustic } \\
\text { leaching }\end{array}$ & $\begin{array}{l}\text { thick } \\
\text { black } \\
\text { paste }\end{array}$ & $\begin{array}{l}\text { Final decontamination } \\
\text { and concentration stage } \\
\text { of } \mathrm{BiPO}_{4} \text { process. } \\
\text { Primary waste: } \\
\text { Lanthanum fluoride } \\
\text { decontamination waste. } \\
2,5\end{array}$ & - & $\begin{array}{l}\text { Add } 4.2 \mathrm{~mL} \text { water to get } \\
-2.3 \mathrm{wt} \% \text { solids. Add } \\
\mathrm{NaOH} / \mathrm{NaNO}_{2} \text { to } 0.01 \mathrm{M} \\
(0.37 \mathrm{~mL}) \text {, mix at } 100^{\circ} \mathrm{C} \text { for } \\
1 \mathrm{hr}, \text { cool, measure settling, } \\
\text { centrifugc, decant }(-22.3 \\
\mathrm{mL} \text { settled solids/ } \sim 10 \mathrm{~mL} \\
\text { centrifuged solids) }\end{array}$ & - & - & $\begin{array}{l}\text { Less than } 4.25 \\
\text { ug Al/mL } \\
\text { (Less than } \\
2.89 \% \mathrm{Al} \\
\text { removed) }\end{array}$ & - & - & $\begin{array}{l}\text { Add } 2 \mathrm{~mL} \text { of } 10 \mathrm{M} \mathrm{NaOH} \text { to get }-8 \\
\text { wt } 5 \text { solids and final NaOH of }-3 \\
\mathrm{M} \text {, mix at } 100^{\circ} \mathrm{C} \text { for } 5 \mathrm{hr} \text {, cool, } \\
\text { measure settling, centrifuge, decant } \\
\text { (Vol. settled solids } \rightarrow 9 \mathrm{~mL} \text {; Vol. } \\
\text { centrifuged solids } \sim 4.1 \mathrm{~mL} \text { ) }\end{array}$ \\
\hline
\end{tabular}


Table A1-1. SSTs and DSTs Wash and Leach Information for Aluminum, Part 1. (38 sheets

\begin{tabular}{|c|c|c|c|c|c|c|c|c|c|c|c|c|c|}
\hline Tank & $\begin{array}{l}\text { Lab Study } \\
\text { Reference(s) }\end{array}$ & $\begin{array}{l}\text { Year of } \\
\text { Sampling } \\
\text { Event }\end{array}$ & Test type & $\begin{array}{l}\text { Sample } \\
\text { color }\end{array}$ & Waste Sources & Sample ID & $\mathrm{NaOH}$ Wash Procedure & $\begin{array}{l}\text { Initial Al in } \\
\text { Untreated } \\
\text { Solids }\end{array}$ & $\begin{array}{l}\text { Final Al in } \\
\text { Washed } \\
\text { solids }\end{array}$ & $\begin{array}{l}\text { Al measured in } \\
\text { Wash Solution }\end{array}$ & $\begin{array}{c}\% \\
\text { recovery }\end{array}$ & comments & Caustic Leach \#1 Procedure \\
\hline 241-BX-103 & $\begin{array}{l}\text { LAUR 97- } \\
2889\end{array}$ & FY97 & $\begin{array}{l}\text { Lab scale } \\
\text { caustic } \\
\text { leaching }\end{array}$ & $\begin{array}{l}\text { Wet dark } \\
\text { brown } \\
\text { thick paste }\end{array}$ & $\begin{array}{l}\text { TBP from uranium } \\
\text { extraction process at U } \\
\text { Plant, CW produced at } \\
\text { PUREX plant from } \\
\text { dissolution of } \mathrm{Zr} \text { or } \mathrm{Al} \\
\text { fuel cladding, and OWW } \\
\text { from PUREX. } \\
\text { Non-Complexed Waste. } \\
\text { Primary: TBP. } \\
\text { Sccondary. CW. } \\
\text { Tertiary: OWW. Other: } \\
\text { mixture of several } \\
\text { miscellaneous wastes. }\end{array}$ & $\begin{array}{l}\text { Composite of } \\
\text { sludge from } \\
\text { portions of } \\
\text { segment 2, core } 86 \\
\text { (222-S Lab II } \\
\text { S96T005277, jar \# } \\
11629)\end{array}$ & $\begin{array}{l}\text { Mix } 10.05 \mathrm{~g} \text { wet sludge with } \\
20.1 \mathrm{~mL} \text { water then } \\
\text { subsample } 4.82 \mathrm{~g} \text {. Wash } 3 \\
\text { times by adding } 10 \mathrm{mLL} 0.01 \\
\text { M NaOH; mix } 1 \mathrm{hr} \text { at } \\
100^{\circ} \mathrm{C} \text {; cool; centrifuge; } \\
\text { decant. Obtain } 32.94 \mathrm{~mL} \\
\text { supernate. Dry solids at } \\
105^{\circ} \mathrm{C} \text { to obtain } 55.7 \mathrm{wt} \% \\
\text { insoluble solids. }\end{array}$ & $21 \mathrm{wt} \% \mathrm{Al}$ & $\begin{array}{l}244437.2 \mathrm{ug} \\
\mathrm{Al} / \mathrm{g}, \\
218991 \mathrm{ug} \\
\mathrm{Al} \text { (99\% } \mathrm{Al} \\
\text { remaining) }\end{array}$ & \begin{tabular}{|l}
62.055 ug \\
Al/mL, \\
2044.09 ug Al \\
(1\% Al \\
removed)
\end{tabular} & - & - & 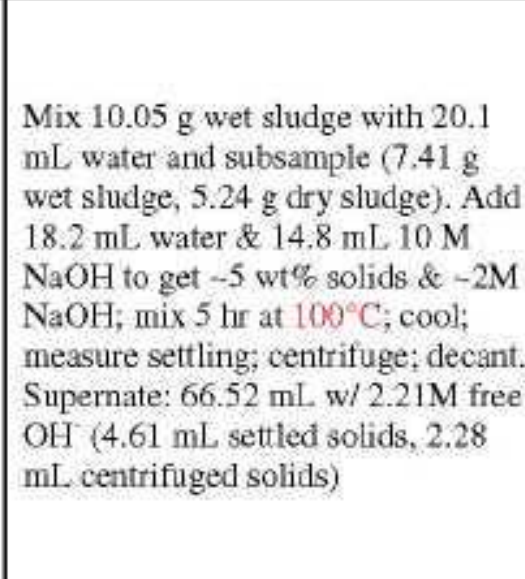 \\
\hline 241-BX-105 & $\begin{array}{l}\text { LAUR 95- } \\
2070\end{array}$ & 1995 & $\begin{array}{l}\text { Lab scale } \\
\text { caustic } \\
\text { leaching }\end{array}$ & $\begin{array}{l}\text { Composit } \\
\text { e sludge- } \\
\text { wet } \\
\text { yellow- } \\
\text { white } \\
\text { material }\end{array}$ & $\begin{array}{l}\text { First waste received was } \\
\text { TBP from TBP uranium } \\
\text { extraction process at U } \\
\text { plant; PUREX plant } \\
\text { dissolution of Zr or Al } \\
\text { fuel cladding; IX waste } \\
\text { from cesium recovery } \\
\text { process at B plant; Last } \\
\text { was EB consisting of a } \\
\text { slurry product from } \\
\text { cvaporators. } \\
\text { Non-Complexed Waste. }{ }^{1} \\
\text { Primary: TBP. } \\
\text { Secondary: CW. } \\
\text { Primary: TBP. } \\
\text { Secondary. CW. } \\
\text { Tertiary: IX. Other: } \\
\text { EB. }\end{array}$ & - & $\begin{array}{l}\text { Add } 49.5 \mathrm{~mL} \text { water to } \\
12.381 \mathrm{~g} \text { wet sludge. With } \\
1.0971 \mathrm{~g} \text { slurry, add } \\
\mathrm{NaOH} / \mathrm{NaNO}_{2} \text { to } 0.01 \mathrm{M}(5 \\
\mathrm{mL}) \text {, mix at RT for }>0.5 \mathrm{hr} \text {, } \\
\text { centrifuge, decant, and } \\
\text { repest } 2 \text { more times. Dry } \\
\text { solids a } 80^{\circ} \mathrm{C}(0.1329 \mathrm{~g} \\
\text { solids: } 60.5 \text { wt } \% \text { insoluble } \\
\text { solids) }\end{array}$ & - & - & - & - & $\begin{array}{l}\text { Detection limit = } \\
4.25 \mathrm{ug} \mathrm{Al} / \mathrm{mL} \text {; } \\
541.13 \mathrm{ug} \mathrm{Al} / \mathrm{g}\end{array}$ & 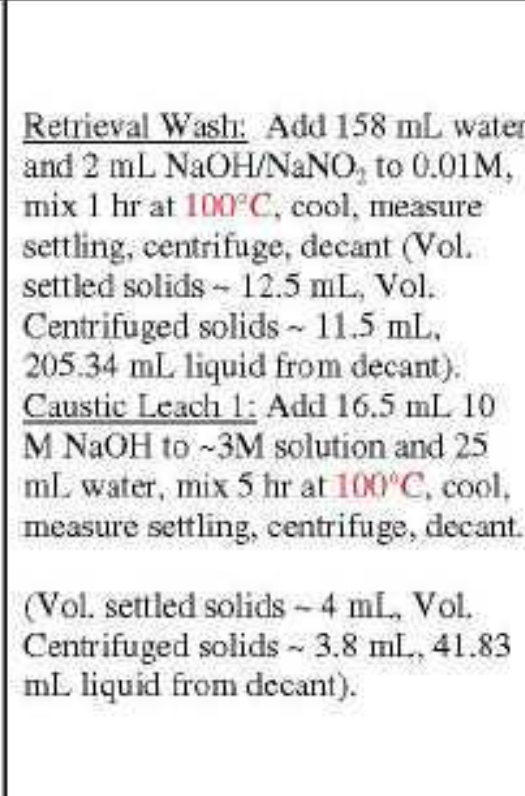 \\
\hline 241-BX-107 & PNL-10712 & FY95 & $\begin{array}{l}\text { Lab scale } \\
\text { caustic } \\
\text { leaching }\end{array}$ & - & $\begin{array}{l}\text { Non-Complexed Waste. }{ }^{1} \\
\text { Primary: } 1 \mathrm{C} . \text { Secondary: } \\
\text { TBP. }^{2} \\
\text { Primary: } 1 \mathrm{C} \text { Secondary: } \\
\text { TBP. Tetriary: CW. } \\
\text { Other: IX. }\end{array}$ & $\begin{array}{l}\text { Composite from } \\
\text { core } 41 \text { (222-S Jar } \\
\# 3941)\end{array}$ & $\begin{array}{l}\text { Add } 37 \mathrm{~g} \text { water to } 10.01 \mathrm{~g} \\
\text { B 107-2, mix, and then } \\
\text { sample. Wash } 3 \text { times by } \\
\text { adding } 5 \text { ml } 0.01 \mathrm{M} \\
\text { NaOH } 0.01 \mathrm{M} \mathrm{NaNO} \text { to } \\
1.08 \text { g slurry; mix } 0.5 \mathrm{hr} \text { at } \\
\text { RT; centrifuge; decant; } \\
\text { repeat w/ solids portion. } \\
\text { Dry remaining solids at } \\
80^{\circ} \mathrm{C}(0.041 \text { g solid } \\
\text { remains) }\end{array}$ & - & - & - & - & \begin{tabular}{|l|} 
Retrieval Wash \\
prior to caustic \\
leach: add $36 \mathrm{~g}$ \\
water and $0.67 \mathrm{~g}$ \\
$1 \mathrm{M} \mathrm{NaOH} / 1 \mathrm{M}$ \\
$\mathrm{NaNO}_{2}$ to $4.89 \mathrm{~g}$ \\
dry solids in slurry. \\
Mix 1 hr at $1000^{\circ} \mathrm{C}$, \\
cool, measure \\
settling, centrifuge, \\
decant.
\end{tabular} & $\begin{array}{l}\text { Add } 4.4 \mathrm{~g} \text { water and } 6.6 \mathrm{~g} \text { of } 10 \mathrm{M} \\
\mathrm{NaOH} \text { to } 6.1 \mathrm{~mL} \text { centrifuged solids; } \\
\text { mix } 5 \mathrm{hr} \text { at } 100^{\circ} \mathrm{C} ; \text { cool; measure } \\
\text { settling; centrifuge; decant }\end{array}$ \\
\hline
\end{tabular}


Table A1-1. SSTs and DSTs Wash and Leach Information for Aluminum, Part 1. (38 sheets

\begin{tabular}{|c|c|c|c|c|c|c|c|c|c|c|c|c|c|}
\hline Tank & $\begin{array}{c}\text { Lab Study } \\
\text { Reference(s) }\end{array}$ & $\begin{array}{l}\text { Year of } \\
\text { Sampling } \\
\text { Event }\end{array}$ & Test type & $\begin{array}{l}\text { Sample } \\
\text { color }\end{array}$ & Waste Sources & Sample ID & $\mathrm{NaOH}$ Wash Procedure & $\begin{array}{l}\text { Initial Al in } \\
\text { Untreated } \\
\text { Solids }\end{array}$ & $\begin{array}{l}\text { Final Al in } \\
\text { Washed } \\
\text { solids }\end{array}$ & $\begin{array}{l}\text { Al measured in } \\
\text { Wash Solution }\end{array}$ & $\begin{array}{c}\% \\
\text { recovery }\end{array}$ & comments & Caustic Leach \#1 Procedure \\
\hline 241-BX-107 & $\begin{array}{l}\text { PNNL- } \\
11089\end{array}$ & FY95 & $\begin{array}{l}\text { Sludge } \\
\text { washing } \\
\text { and } \\
\text { caustic } \\
\text { leaching }\end{array}$ & - & $\begin{array}{l}\text { Primarily 1C, with } \\
\text { secondary waste from } \\
\text { the TBP uranium } \\
\text { extraction process at U } \\
\text { Plant. }\end{array}$ & Core \# 41 & $\begin{array}{l}\text { Add water to sludge sample } \\
\text { to get } \sim 2.3 \text { wt\% solids, as } \\
\text { well as inhibited water } \\
\left(\mathrm{NaOH} / \mathrm{NaNO}_{2} \text { ) to } 0.01 \mathrm{M} \text {. }\right. \\
\mathrm{Mix} \text { at } 100^{\circ} \mathrm{C} \text { for } 1 \mathrm{hr} \text {. } \\
\mathrm{Cool} \text { centrifuge, and } \\
\text { decant. }\end{array}$ & - & - & $\begin{array}{l}\text { 1\% } \mathrm{Al} \\
\text { removed }\end{array}$ & - & - & $\begin{array}{l}\text { Caustic leach of solids: Add } \mathrm{NaOH} \\
\text { to get }-8 \mathrm{wt} \% \text { solids and a final } \\
\mathrm{NaOH} \text { of } 2.0 \mathrm{M} \text {. Mix at } 100 \mathrm{C}^{\mathrm{C}} \text { for } 5 \\
\text { hr. Cool, centrifuge, decant. }\end{array}$ \\
\hline 241-BX-109 & $\begin{array}{l}\text { LAUR 96- } \\
2839\end{array}$ & FY96 & $\begin{array}{l}\text { Lab scale } \\
\text { caustic } \\
\text { leaching }\end{array}$ & $\begin{array}{l}\text { reddish- } \\
\text { brown } \\
\text { clay } \\
\text { material }\end{array}$ & $\begin{array}{l}\text { TBP from U-extraction } \\
\text { process at U Plant; waste } \\
\text { produced at PUREX } \\
\text { Plant from dissolution of } \\
\text { ZZ or Al fuel cladding; } \\
\text { 1C; IX waste from Cs } \\
\text { recovery process at B } \\
\text { Plant. } \\
\text { Non-Complexed Waste. }{ }^{1} \\
\text { Primary: TBP. } \\
\text { Secondary: CW: } \\
\text { Primary: TBP. } \\
\text { Secondary. CW. } \\
\text { Tertiary: 1C. Other: IX. }\end{array}$ & - & $\begin{array}{l}\text { Mix } 7.23 \mathrm{~g} \text { wet sludge with } \\
14 \mathrm{~mL} \text { water; with } 4.62 \mathrm{~g} \\
\text { slurry, wash } 3 \text { times by } \\
\text { adding } 10 \mathrm{~mL} \text { of } 0.01 \mathrm{M} \\
\mathrm{NaOH} \text {; mix for } 1 \mathrm{hr} \text { at } \\
100^{\circ} \mathrm{C} \text {; cool; centrifuge; } \\
\text { decant; repcat with solids } \\
\text { portion. Dry solids at } \\
105^{\circ} \mathrm{C} \text { to get } 0.132 \mathrm{~g} \text { dry } / \\
8.58 \text { wt } \% \text { insoluble solids }\end{array}$ & $\begin{array}{l}0.1 \mathrm{wt} \text { \% } \mathrm{Al} ; \\
397.71 \text { ug Al; } \\
3017.6 \mathrm{ug} \\
\mathrm{Al} / \mathrm{g}\end{array}$ & $\begin{array}{l}39 \% \mathrm{Al} \\
\text { remaining }\end{array}$ & $\begin{array}{l}625.58 \text { ug } \mathrm{Al} ; \\
19.231 \text { ug } \\
\mathrm{Al} / \mathrm{mL}\end{array}$ & $\begin{array}{l}61 \% \\
\text { removed } \\
\text { by simple } \\
\text { wash }\end{array}$ & - & $\begin{array}{l}\text { Add } 10 \mathrm{M} \mathrm{NaOH} \text { and water to } 4.73 \\
\mathrm{~g} \text { mixed wet sludge }(2.37 \mathrm{~g} \text { dry } \\
\text { sludge) to get } \sim 5 \mathrm{wt} \% \text { solids and } \\
\text { final } \mathrm{N} \text { a OH of }-2 \mathrm{M} \text {; mix } 5 \mathrm{hr} \text { at } \\
100{ }^{\circ} \mathrm{C} \text {; cool, measure settling; } \\
\text { centrifuge; decant. }\end{array}$ \\
\hline
\end{tabular}


Table A1-1. SSTs and DSTs Wash and Leach Information for Aluminum, Part 1. (38 sheets)

\begin{tabular}{|c|c|c|c|c|c|c|c|c|c|c|c|c|c|}
\hline Tank & $\begin{array}{c}\text { Lab Study } \\
\text { Reference(s) }\end{array}$ & $\begin{array}{c}\text { Year of } \\
\text { Sampling } \\
\text { Event }\end{array}$ & Test type & $\begin{array}{l}\text { Sample } \\
\text { color }\end{array}$ & Waste Sources & Sample ID & $\mathrm{NaOH}$ Wash Procedure & $\begin{array}{l}\text { Initial Al in } \\
\text { Untreated } \\
\text { Solids }\end{array}$ & $\begin{array}{l}\text { Final Al in } \\
\text { Washed } \\
\text { solids }\end{array}$ & $\begin{array}{l}\text { Al measured in } \\
\text { Wash Solution }\end{array}$ & $\begin{array}{c}\% \\
\text { recovery }\end{array}$ & comments & Caustic Leach \#1 Procedure \\
\hline $241-\mathrm{BX}-112$ & $\begin{array}{l}\text { PNNL- } \\
12026\end{array}$ & FY98 & $\begin{array}{l}\text { Lab scale } \\
\text { caustic } \\
\text { leaching: } \\
\text { compare } \\
60,80, \\
\text { and } 100^{\circ} \mathrm{C} \\
\text { caustic } \\
\text { leaching } \\
\text { for total of } \\
168 \mathrm{hr} \text {. }\end{array}$ & - & $\begin{array}{l}\text { Primary: } 1 \mathrm{C} . \text { Secondary: } \\
\text { EB. } \\
\text { Non-Complexed Waste. } \\
\text { Primary: } 1 \mathrm{C} . \text { Secondary: } \\
\text { EB. Tertiary. CW. } \\
\text { Other: } \mathrm{IX}^{\mathrm{S}}\end{array}$ & $\begin{array}{l}\text { A mixture of } \\
\text { segment } 3 \text { from } \\
\text { core } 118 \text { and } \\
\text { segment } 1 \text { from } \\
\text { core } 119 \text {, with } \\
\text { segment } 3 \\
\text { providing most of } \\
\text { the sample (actual } \\
\text { proportions } \\
\text { undocumented) }\end{array}$ & $\begin{array}{l}\text { Mix }-100 \mathrm{~mL} \text { centrifuged } \\
\text { solids and } \sim 100 \mathrm{~mL} \text { liquid } \\
\text { supermate in } 2000 \mathrm{~mL} \text { bottle } \\
\text { stirred } 30 \text { min; centrifuge } 30 \\
\text { min at } 1200 \mathrm{G} \text {; decant. } \\
\text { Replace decanted liquid w/ } \\
0.1 \mathrm{M} \text { NaOH to } 200 \mathrm{~mL} \\
\text { mark; repeat for } 7 \text { total } \\
\text { wash cycles. }\end{array}$ & $\begin{array}{l}3.8 \mathrm{wt} \% \mathrm{Al} ; \\
38000 \mathrm{ug} \\
\mathrm{A} 1 \mathrm{~g} ; \\
3157800 \mathrm{ug} \\
\mathrm{Al}(1.25 \mathrm{~g} \\
\mathrm{Al} / \mathrm{g} \mathrm{Fe})\end{array}$ & $\begin{array}{l}63000 \mathrm{ug} \\
\mathrm{Al} / \mathrm{g} ; \\
2431800 \mathrm{ug} \\
\mathrm{Al}(1.17 \mathrm{~g} \\
\mathrm{Al} / \mathrm{ge})\end{array}$ & $\begin{array}{l}191 \mathrm{ug} \mathrm{Al} / \mathrm{mL} ; \\
140194 \mathrm{ug} \mathrm{Al}\end{array}$ & $\begin{array}{l}5 \% \mathrm{Al} \\
\text { removed; } \\
81 \% \mathrm{Al} \\
\text { mass } \\
\text { recovery } \\
\text { (6\% Al } \\
\text { removed) }\end{array}$ & $\begin{array}{l}\text { Shows time versus } \\
\text { concentration } \\
\text { graph for leaching } \\
\text { at different temp } \\
\text { and NaOH } \\
\text { concentrations. } \\
\text { Dilute washed } \\
\text { sludge to } 200 \mathrm{~mL} \\
\text { w/ DI \& stir. } \\
\text { Distribute }-8 \mathrm{~mL} \\
\text { aliquots between } \\
\text { seven } 60 \mathrm{~mL} \\
\text { bottles \& one } 25 \\
\text { mL glass vial } \\
\text { (dried at105. } \\
\text { obtain solids } \\
\text { measurement) }\end{array}$ & 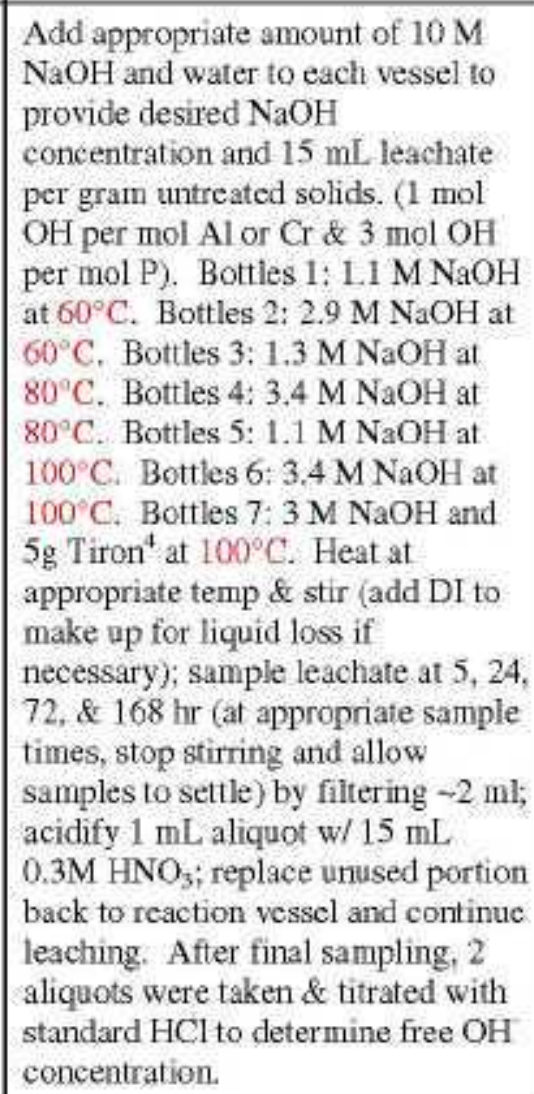 \\
\hline 241-BY-104 & $\begin{array}{l}\text { PNNL- } \\
11278\end{array}$ & FY96 & $\begin{array}{l}\text { Lab scale } \\
\text { caustic } \\
\text { leaching }\end{array}$ & - & 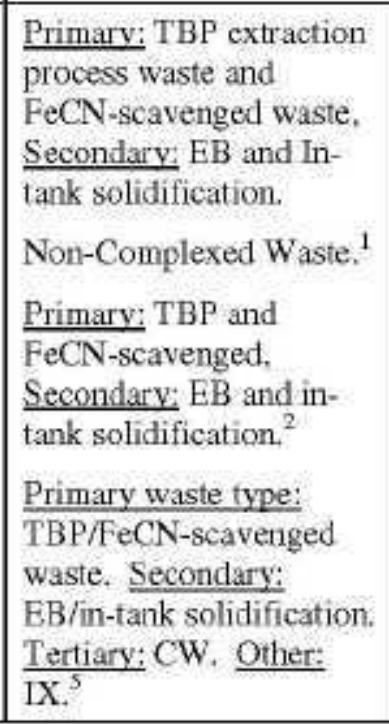 & $\begin{array}{l}\text { Core 116: Core } \\
\text { composite (222-S } \\
\text { LABCORE sample } \\
\text { \#S96T000371) }\end{array}$ & $\begin{array}{l}\text { Mix } 7.588 \mathrm{~g} \text { sample with } \\
15.36 \mathrm{~g} \text { water. With } 4.83 \mathrm{~g} \\
\text { slurry add } 10 \mathrm{~mL} 0.01 \mathrm{M} \\
\mathrm{NaOH} \text {. Mix at } 100^{\circ} \mathrm{C} \text { for } 1 \\
\text { hr, cool, centrifuge, and } \\
\text { decant. Repeat for a total of } \\
3 \text { washes. Dry solids after } \\
\text { washes at } 105 \mathrm{C} .1 .622 \mathrm{~g} \\
\text { sludge initially in sample, } \\
0.042 \mathrm{~g} \text { washed solids, } 2.7 \\
\text { wt\% insoluble solids in } \\
\text { sludge. }\end{array}$ & $1.9 \mathrm{wt} \% \mathrm{Al}$ & - & - & $\begin{array}{l}65 \% \mathrm{Al} \\
\text { removed }\end{array}$ & $\begin{array}{l}\text { An initial wash was } \\
\text { performed on two } \\
\text { sludge samples, } \\
\text { with the solids and } \\
\text { slurry mixed for } \\
\text { use with saustic } \\
\text { leach test. Sample } \\
\text { 1: } 25.27 \mathrm{~g} \text { sludge - } \\
\text { wash } 3 \text { times. } \\
\text { Sample 2: } 26.47 \mathrm{~g} \\
\text { sludge - wash } 3 \\
\text { times. For wash, } \\
\text { add } 24 \mathrm{~mL} 0.01 \mathrm{M} \\
\mathrm{NaOH,} \text { mix at RT } \\
\text { for } 0.5 \mathrm{hr}, \\
\text { centrifuge, decant. } \\
\text { and repeat. } \\
\end{array}$ & $\begin{array}{l}\text { After mixing washed solids and } \\
\text { sludge from both wash samples, } \\
\text { centrifuge and decant. With solids } \\
\text { add } 7.2 \mathrm{~mL} 10 \mathrm{M} \mathrm{NaOH} \text { and water } \\
\text { to a total volume of } 22 \mathrm{~mL}(3 \mathrm{wt} \% \\
\text { solids). mix at } 100^{\circ} \mathrm{C} \text { for } 5 \mathrm{hr} \text {, cool. } \\
\text { measure settling, centrifuge, and } \\
\text { decant. } \\
19.9 \mathrm{~mL} \text { decanted liquid, } 1.4 \mathrm{~mL} \text {. } \\
\text { interstitial liquid, } 2.6 \mathrm{M} \mathrm{OH} \text {. }\end{array}$ \\
\hline
\end{tabular}

${ }^{4}$ Tiron is also known as 4,5-dihydroxy-1,3-benzenedisulfonate. 
Table A1-1. SSTs and DSTs Wash and Leach Information for Aluminum, Part 1. (38 sheets

\begin{tabular}{|c|c|c|c|c|c|c|c|c|c|c|c|c|c|}
\hline Tank & $\begin{array}{c}\text { Lab Study } \\
\text { Reference(s) }\end{array}$ & $\begin{array}{l}\text { Year of } \\
\text { Sampling } \\
\text { Event }\end{array}$ & Test type & $\begin{array}{l}\text { Sample } \\
\text { color }\end{array}$ & Waste Sources & Sample ID & $\mathrm{NaOH}$ Wash Procedure & $\begin{array}{l}\text { Initial Al in } \\
\text { Untreated } \\
\text { Solids }\end{array}$ & $\begin{array}{l}\text { Final Al in } \\
\text { Washed } \\
\text { solids }\end{array}$ & $\begin{array}{l}\text { Al measured in } \\
\text { Wash Solution }\end{array}$ & $\begin{array}{c}\% \\
\text { recovery }\end{array}$ & comments & Caustic Leach \#1 Procedure \\
\hline 241-BY-108 & $\begin{array}{l}\text { PNNL- } \\
11636\end{array}$ & FY97 & $\begin{array}{l}\text { Lab scale } \\
\text { caustic } \\
\text { leaching }\end{array}$ & - & $\begin{array}{l}\text { Non-Complexed Waste. } \\
\text { Primary: TBP and } \\
\text { FeCN-scavenged, } \\
\text { Secondary: EB and in. } \\
\text { tank solidification. } \\
\text { 2. } \\
\text { Primary: TBP/FeCN- } \\
\text { seavenged waste. } \\
\text { Secondary. EB/in-tank } \\
\text { solidification. Tertiary: } \\
\text { 1C. Other: CW? }\end{array}$ & $\begin{array}{l}\text { 222-S Lab ID } \\
\text { \$96T002035; Jar \# } \\
10527 ; \text { composite } \\
\text { of segment } 4 \text { from } \\
\text { Core } 99\end{array}$ & $\begin{array}{l}\text { Wash } 3 \text { times by adding } 10 \\
\text { ml } 0.01 \mathrm{M} \mathrm{NaOH} \text { to } 4.891 \mathrm{~g} \\
\text { slurry; mix } 1 \mathrm{hr} \text { at } 100^{\circ} \mathrm{C} \text {; } \\
\text { cool; centrifuge; decant; } \\
\text { repeat w/ solids portion. }\end{array}$ & $\begin{array}{l}12600 \text { ug } \\
\mathrm{Al} / \mathrm{g}\end{array}$ & $\begin{array}{l}5993 \text { ug } \mathrm{Al} \\
(37 \% \mathrm{Al})\end{array}$ & $\begin{array}{l}10055 \text { ug Al } \\
(63 \% \mathrm{Al})\end{array}$ & $111 \% \mathrm{Al}$ & - & $\begin{array}{l}\text { Add } 7.586 \mathrm{ml} 10 \mathrm{M} \mathrm{NaOH} \text { to } \\
36.672 \mathrm{~g} \text { slurry, mix } 5 \mathrm{hr} \text { at } 100^{\circ} \mathrm{C} \text {; } \\
\text { cool; gravity settle; decant }\end{array}$ \\
\hline $241-B Y-110$ & $\begin{array}{l}\text { PNNL- } \\
11278\end{array}$ & FY96 & $\begin{array}{l}\text { Lab scale } \\
\text { caustic } \\
\text { leaching }\end{array}$ & - & $\begin{array}{l}\text { Primary: TBP extraction } \\
\text { process waste and } \\
\text { FeCN-scavenged waste, } \\
\text { Secondary: EB and In- } \\
\text { tank solidification. } \\
\text { Non-Complexed Waste. } \\
\text { Primary: TBP and } \\
\text { FeCN-scavenged, } \\
\text { Secondary: EB and in- } \\
\text { tank solidification. } \\
\text { Primary: TBP/FeCN- } \\
\text { scavenged waste. } \\
\text { Secondary: EB /in-tank } \\
\text { solidification. Tertiary: } \\
\text { 1C. Other: CW. } \\
\end{array}$ & $\begin{array}{l}\text { Core 113: Core } \\
\text { composite (222-S } \\
\text { LABCORE sample } \\
\text { \#\$96T000497) }\end{array}$ & $\begin{array}{l}\text { Mix } 8.802 \mathrm{~g} \text { sample with } \\
17.27 \mathrm{~g} \text { water. With } 4.85 \mathrm{~g} \\
\text { slurry add } 10 \mathrm{~mL} 0.01 \mathrm{M} \\
\mathrm{NaOH} \text {. Mix at } 100^{\circ} \mathrm{C} \text { for } 1 \\
\mathrm{hr} \text {, cool, centrifuge, and } \\
\text { decant. Repeat for a total of } \\
3 \text { washes. Dry solids after } \\
\text { washes a } 105^{\circ} \mathrm{C} .1 .639 \mathrm{~g} \\
\text { sludge initially in sample, } \\
0.134 \mathrm{~g} \text { in washed solids, } \\
\text { and } 8.2 \text { wt\% insoluble } \\
\text { solids in sludge. }\end{array}$ & $\begin{array}{l}3.4 \mathrm{wt} \% \mathrm{Al} ; \\
\text { Summation: } \\
4.28 \mathrm{E}+4 \mathrm{ug} \\
\text { Al/g; Direct: } \\
3.37 \mathrm{E}+4 \mathrm{ug} \\
\mathrm{Al} / \mathrm{g} ; 127 \% \\
\mathrm{Al} \text { recovery }\end{array}$ & $\begin{array}{l}21750 \text { ug } \\
\mathrm{Al} / \mathrm{mL} ; 2915 \\
\text { ug } \mathrm{Al}(6 \\
\text { wt\% } \mathrm{Al})\end{array}$ & $\begin{array}{l}1407 \text { ug } \\
\mathrm{Al} / \mathrm{mL} ; 42351 \\
\text { ug Al } 494 \mathrm{wt} \% \\
\mathrm{Al})\end{array}$ & $\begin{array}{l}94 \% \text { Al } \\
\text { removed } \\
\text { Total } \\
\text { mass = } \\
45265 \text { ug } \\
\text { Al }\end{array}$ & - & $\begin{array}{l}\text { Wt. Sludge Solids in sample }=4.01 \\
\text { g. Addd } 4 \mathrm{~mL} \text { 10 M NaOH and } \\
\text { water to a total volume of } 20 \mathrm{~mL} \\
\left(2.3 \text { wt } \% \text { solids), mix at } 100^{\circ} \mathrm{C} \text { for }\right. \\
\text { hr, cool, measure settling, } \\
\text { centrifuge, and decant. } 16.5 \mathrm{~mL} \\
\text { decanted liquid, } 1.7 \mathrm{~mL} \text { interstitial } \\
\text { liquid, and } 2.6 \mathrm{M} \mathrm{OH} \text {. }\end{array}$ \\
\hline 241-C-102 & $\begin{array}{l}\text { PNNL- } \\
12026\end{array}$ & FY98 & $\begin{array}{l}\text { Lab scale } \\
\text { caustic } \\
\text { leaching: } \\
\text { compare } \\
60 \text { and } \\
100^{\circ} \mathrm{C} \\
\text { caustic } \\
\text { leaching } \\
\text { at } 1 \text { or } 3 \mathrm{M} \\
\mathrm{NaOH}\end{array}$ & $\begin{array}{l}\text { dry } \\
\text { greenish- } \\
\text { brown } \\
\text { powder }\end{array}$ & $\begin{array}{l}\text { Primary: CW. } \\
\text { Secondary waste from } \\
\text { TBP extraction process. } \\
\text { Dilute Complexed } \\
\text { Waste. } \\
\text { Primary: CW. } \\
\text { Secondary: TBP. } \\
\text { Tertiary: OWW: }\end{array}$ & $\begin{array}{l}\text { Auger sample } \\
\text { taken in August of } \\
1995 \text { (represents } \\
\text { top portion of } \\
\text { sludge). }\end{array}$ & $\begin{array}{l}\text { Add } 25.55 \mathrm{~g} \text { composite } \\
\text { sample to } 50 \mathrm{~mL} 0.1 \mathrm{M} \\
\mathrm{NaOH} \text {, stir } 30 \text { min at RT, } \\
\text { remove } 2 \mathrm{~mL} \text { slurry, } \\
\text { centrifuge } 15 \text { min at } 1200 \\
\text { G, decant. Add } 100 \mathrm{~mL} 0.1 \\
\mathrm{M} \text { NaOH to solids, stir } 30 \\
\text { min at RT, centrifuge } 15 \\
\text { min at } 1200 \mathrm{G} \text {, decant. } \\
\text { Repeat for total of } 3 \text { washes. } \\
\text { Wash solution: } 224 \mathrm{~mL} \text {. } \\
\text { (231.5g solution. } 1.0329 \\
\mathrm{~g} / \mathrm{mL} \text { ). }\end{array}$ & $\begin{array}{l}10.95 \mathrm{wt} \% \\
\text { Al: } 109542 \\
\text { ug Al/g: } \\
2711153 \text { ug } \\
\text { Al }\end{array}$ & $\begin{array}{l}189297 \text { ug } \\
\mathrm{Al} / \mathrm{g} \text {; } \\
3180186 \text { ug } \\
\mathrm{Al} \\
\text { ( } \# 1=611429 \\
\text { ug } \mathrm{Al} ; \\
\# 2=670111 \\
\text { ug Al; } \\
\# 3=620894 \\
\text { ug Al: } \\
\# 4=569783 \\
\text { ug Al) }\end{array}$ & $\begin{array}{l}53.35 \mathrm{ug} \\
\mathrm{Al} / \mathrm{mL} ; 11950 \\
\mathrm{ug} \mathrm{Al}\end{array}$ & $\begin{array}{l}0.4 \% \mathrm{Al} \\
\text { removed; } \\
118 \% \mathrm{Al} \\
\text { mass } \\
\text { recovery; } \\
16.36 \mathrm{~g} \\
\mathrm{Al} / \mathrm{g} \mathrm{Fe} \mathrm{in} \\
\text { washed } \\
\text { solids }=- \\
10 \% \mathrm{Al} \\
\text { removal }\end{array}$ & 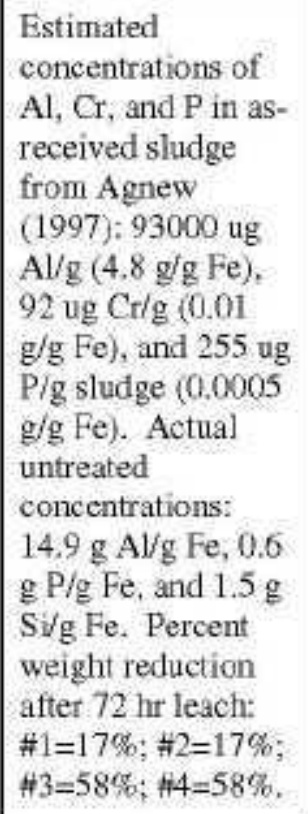 & 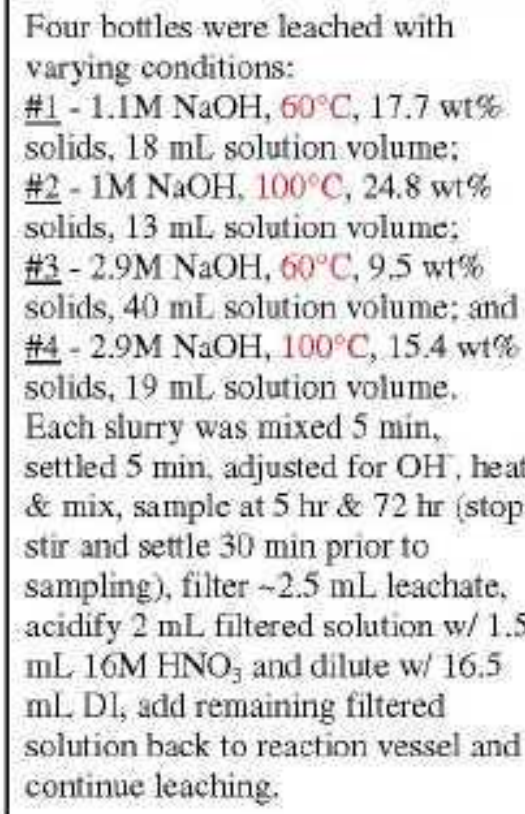 \\
\hline
\end{tabular}


Table A1-1. SSTs and DSTs Wash and Leach Information for Aluminum, Part 1. (38 sheets

\begin{tabular}{|c|c|c|c|c|c|c|c|c|c|c|c|c|c|}
\hline Tank & $\begin{array}{c}\text { Lab Study } \\
\text { Reference(s) }\end{array}$ & $\begin{array}{l}\text { Year of } \\
\text { Sampling } \\
\text { Event }\end{array}$ & Test type & $\begin{array}{l}\text { Sample } \\
\text { color }\end{array}$ & Waste Sources & Sample ID & $\mathrm{NaOH}$ Wash Procedure & $\begin{array}{l}\text { Initial } \mathrm{Al} \text { in } \\
\text { Untreated } \\
\text { Solids }\end{array}$ & $\begin{array}{l}\text { Final Al in } \\
\text { Washed } \\
\text { solids }\end{array}$ & $\begin{array}{l}\text { Al measured in } \\
\text { Wash Solution }\end{array}$ & $\begin{array}{c}\% \\
\text { recovery }\end{array}$ & comments & Caustic Leach \#1 Procedure \\
\hline $241-\mathrm{C}-103$ & PNL-10712 & FY95 & $\begin{array}{l}\text { Lab scale } \\
\text { caustic } \\
\text { leaching }\end{array}$ & - & $\begin{array}{l}\text { Non-Complexed Waste. } \\
\text { Primary: Sr leached } \\
\text { sludge. Secondary: } \\
\text { Particulates from Sr } \\
\text { wash of PUREX wastes. } \\
\text { in the AR vault. } \\
\text { Primary: Sr sludge. } \\
\text { Pecondary: particulates } \\
\text { Srom Sr wash of PUREX } \\
\text { wastes in the AR-vault. } \\
\text { Tertiary: neutralized acid } \\
\text { waste. Other: TBP/CW. }\end{array}$ & $\begin{array}{l}\text { Composite sample } \\
\text { of segments } 2-4 \\
\text { from core } 63 \text { (222- } \\
\text { S Jar \#6770). }\end{array}$ & $\begin{array}{l}\text { Wash } 3 \text { times by adding } 5 \\
\text { ml } 0.01 \mathrm{M} \text { NaOH/0.01 M } \\
\mathrm{NaNO}_{2} \text { to } 0.21 \mathrm{~g} \\
\text { sludge } 0.82 \mathrm{~g} \text { water; mix } 0.5 \\
\text { hr at } \mathrm{RT} ; \text { centrifuge; decant; } \\
\text { repeat w/ solids portion. } \\
\text { Dry remaining solids at } \\
80^{\circ} \mathrm{C}(0.027 \mathrm{~g} \text { solid } \\
\text { remains). }\end{array}$ & - & - & - & - & $\begin{array}{l}\text { Retrieval Wash } \\
\text { prior to caustic } \\
\text { leach: add } 23.3 \mathrm{~g} \\
\text { water and } 0.86 \mathrm{~g} \\
1 \mathrm{M} \mathrm{NaOH} / 1 \mathrm{M} \\
\mathrm{NaNO}_{2} \text { to } 6.3 \mathrm{~g} \mathrm{dry} \\
\text { solids in slurry. } \\
\text { Mix lhr at } 100^{\circ} \mathrm{C} \text {, } \\
\text { cool, measure } \\
\text { settling, centrifuge, } \\
\text { decant. }\end{array}$ & $\begin{array}{l}\text { Add } 2.6 \mathrm{~g} \text { water and } 4.4 \mathrm{~g} 10 \mathrm{M} \\
\mathrm{NaOH} \text { to } 6.7 \mathrm{~mL} \text { centrifuged solids; } \\
\text { mix } 5 \text { hr at } 100 \mathrm{C} \text {; cool; measure } \\
\text { settling; centrifuge; decant. }\end{array}$ \\
\hline 241-C-103 & $\begin{array}{l}\text { PNNL- } \\
11089\end{array}$ & FY95 & $\begin{array}{l}\text { Sludge } \\
\text { washing } \\
\text { and } \\
\text { caustic } \\
\text { leaching }\end{array}$ & - & $\begin{array}{l}\text { Primarily sludge feed for } \\
\text { the Sr extraction process } \\
\text { at B Plant, with } \\
\text { secondary waste from } \\
\text { particulates from the } \mathrm{Sr} \\
\text { wash of PUREX wastes } \\
\text { in AR Vault. }\end{array}$ & Core \# 63 & $\begin{array}{l}\text { Add water to sludge sample } \\
\text { to get } \sim 2.3 \text { wt\% solids, as } \\
\text { well as inhibited water } \\
\left(\mathrm{NaOH} / \mathrm{NaNO}_{2}\right) \text { to } 0.01 \mathrm{M} \text {. } \\
\text { Mix at } 100^{\circ} \mathrm{C} \text { for } 1 \mathrm{hr} \text {. } \\
\mathrm{Cool} \text {, centrifuge, and } \\
\text { decant. }\end{array}$ & - & - & - & - & - & $\begin{array}{l}\text { Caustic leach of solids: Add } \mathrm{NaOH} \\
\text { to get } \sim 8 \text { wt } \% \text { solids and a final } \\
\mathrm{NaOH} \text { of } 0.6 \mathrm{M} \text {. Mix at } 100^{\circ} \mathrm{C} \text { for } 5 \\
\text { hr. Cool, centrifuge, decant. }\end{array}$ \\
\hline 241-C-103 & $\begin{array}{l}\text { ORNL/TM- } \\
13655\end{array}$ & 1998 & $\begin{array}{l}\text { Lab scale } \\
\text { caustic } \\
\text { leaching }\end{array}$ & - & $\begin{array}{l}\text { Group XX waste based } \\
\text { on the Sort on } \\
\text { Radioactive Waste Type } \\
\text { (SORWT) model, with } \\
\text { the only other tank in } \\
\text { this group being C-106. } \\
\text { Primary waste is Sr- } \\
\text { leached sludge; next } \\
\text { largest fraction of HLW } \\
\text { is suspended particulates } \\
\text { from strontium washes } \\
\text { of the PUREX wastes. } \\
\text { Also has a separate layer } \\
\text { of organic liquid. } \\
\text { Non-Complexed Waste. }\end{array}$ & $\begin{array}{l}\text { WHC 222-S lab \# } \\
\text { S97M000283, jar \# } \\
\text { 13633 }\end{array}$ & $\begin{array}{l}\text { Wash } 4 \text { times by adding } \\
120.95 \mathrm{~g} \text { inhibited water } \\
(0.01 \mathrm{M} \text { NaOH/NaNO} \text { ) to } \\
83.19 \mathrm{~g} \text { sludge, wash at } \mathrm{RT} \\
\text { for } 143 \mathrm{hr} \text {, centrifuge } 20 \\
\text { min at } 4500 \text { rotations per } \\
\text { minute (rpm), decant. Add } \\
116.78 \mathrm{~g} \text { inthibited water to } \\
\text { solids, mix } 22 \mathrm{hr} \text { at RT, } \\
\text { centrifuge, decant. Add } \\
126.7 \mathrm{~g} \text { inhibited water to } \\
\text { solids, mix } 29 \mathrm{hr} \text { at RT, } \\
\text { centrifuge, decant. Add } \\
113.11 \mathrm{~g} \text { inhibited water, } \\
\text { mix } 2 \mathrm{hr} \text { at } 97^{\circ} \mathrm{C} \text {, centrifuge, } \\
\text { decant. }\end{array}$ & $\begin{array}{l}\text { From Table } \\
2.1: 4.56 \mathrm{E}+3 \\
\mathrm{~kg} \\
\mathrm{~A} / 3.14 \mathrm{E}+5 \\
\mathrm{~kg} \text { total } \\
\text { sludge mass }\end{array}$ & - & - & - & $\begin{array}{l}\text { No measurements } \\
\text { of Al in wash } \\
\text { solutions. Only } \\
\text { measured the } \\
\text { following: Cs-137 } \\
\text { activity and } \\
\text { conductance of } \\
\text { wash solutions and } \\
\text { wet weights of } \\
\text { centrifuged solids. }\end{array}$ & No caustic leach performed. \\
\hline
\end{tabular}


Table A1-1. SSTs and DSTs Wash and Leach Information for Aluminum, Part 1. (38 sheets)

\begin{tabular}{|c|c|c|c|c|c|c|c|c|c|c|c|c|c|}
\hline Tank & $\begin{array}{c}\text { Lab Study } \\
\text { Reference(s) }\end{array}$ & $\begin{array}{c}\text { Year of } \\
\text { Sampling } \\
\text { Event }\end{array}$ & Test type & $\begin{array}{l}\text { Sample } \\
\text { color }\end{array}$ & Waste Sources & Sample ID & $\mathrm{NaOH}$ Wash Procedure & $\begin{array}{l}\text { Initial Al in } \\
\text { Untreated } \\
\text { Solids }\end{array}$ & $\begin{array}{l}\text { Final Al in } \\
\text { Washed } \\
\text { solids }\end{array}$ & $\begin{array}{l}\text { Al measured in } \\
\text { Wash Solution }\end{array}$ & $\begin{array}{c}\% \\
\text { recovery }\end{array}$ & comments & Caustic Leach \#1 Procedure \\
\hline $241-C-104$ & $\begin{array}{l}\text { LAUR } 97 \text { - } \\
2889\end{array}$ & FY97 & $\begin{array}{l}\text { Lab scale } \\
\text { caustic } \\
\text { leaching }\end{array}$ & $\begin{array}{l}\text { reddish- } \\
\text { brown; } \\
\text { dried } \\
\text { material } \\
\text { was dark } \\
\text { brown }\end{array}$ & $\begin{array}{l}\text { CW produced at PUREX } \\
\text { Plant from dissolution of } \\
\text { Zr or Al fuel cladding, } \\
\text { OWW from PUREX, Sr } \\
\text { wash of PUREX waste } \\
\text { in AR vault, sludge feed } \\
\text { for Sr extraction process } \\
\text { at B Plant. } \\
\text { CC! } \\
\text { Primary: CW. } \\
\text { Secondary: OWW from } \\
\text { PUREX. }\end{array}$ & $\begin{array}{l}\text { Composite of } \\
\text { sludge made from } \\
\text { portions of } \\
\text { segments } 1,2, \& \text { \& } 3 \\
\text { from core } 165 \\
\text { (2222-S lab ID } \\
\text { S96T005188, jar \# } \\
11620 \text { ) }\end{array}$ & $\begin{array}{l}\text { Mix } 11.76 \mathrm{~g} \text { wet sludge with } \\
23.5 \mathrm{~mL} \text { water; with } 4.7 \mathrm{~g} \text { of } \\
\text { this slurry. Wash } 3 \text { times by } \\
\text { adding } 10 \mathrm{~mL} 0.01 \mathrm{M} \\
\mathrm{NaOH} \text {; mix for } 1 \mathrm{hr} \text { at } \\
100^{\circ} \mathrm{C} ; \text { cool; centrifuge; } \\
\text { decant; repeat with solids. } \\
\text { Dry solids at } 105^{\circ} \mathrm{C} \text { to } \\
\text { obtain } 28.13 \mathrm{wt} \text { \% insoluble } \\
\text { solids } \& 32.68 \mathrm{~mL} \\
\text { supemate. }\end{array}$ & $6.3 \mathrm{wt} \% \mathrm{Al}$ & $\begin{array}{l}92793 \text { ug } \\
\mathrm{Al} / \mathrm{g}, 41247 \\
\text { ug Al (91\% } \\
\mathrm{Al})\end{array}$ & \begin{tabular}{|l|}
129.15 ug \\
$\mathrm{Al} / \mathrm{mL}$, \\
4220.62 ug $\mathrm{Al}$ \\
$(9 \% \mathrm{Al})$
\end{tabular} & - & - & 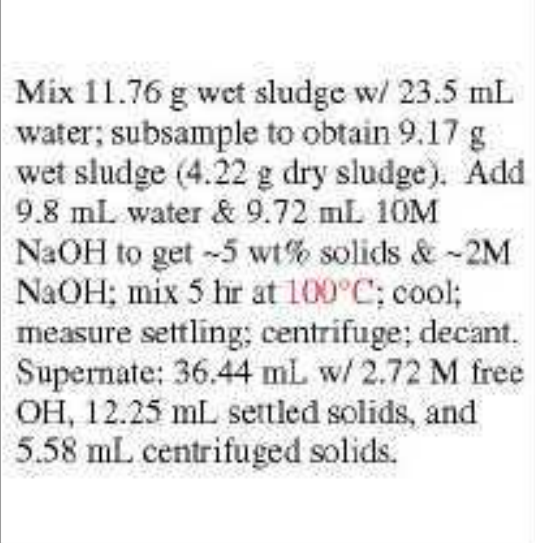 \\
\hline 241-C-104 & $\begin{array}{l}\text { PNWD- } \\
3027 \\
\text { (BNFL- } \\
\text { RPT-021) }\end{array}$ & 1999 & $\begin{array}{l}\text { Lab scale } \\
\text { caustic } \\
\text { leaching }\end{array}$ & - & $\mathrm{CC}^{1}$ & $\begin{array}{l}\text { Sample received in } \\
14 \text { glass jars and } \\
\text { composited as per } \\
\text { test plan BNFL- } \\
\text { 29953-031. } \\
\text { Composite was } \\
\text { mixed for lhr } 20 \\
\text { min at } 34^{\circ} \mathrm{C} \text { prior } \\
\text { to sub-sampling. } \\
\text { Composite sample } \\
\text { made of the } \\
\text { following (sample } \\
\text { \#/weight in grams): } \\
16273 / 150.046 ; \\
16274 / 157.638 ; \\
16275 / 176.435 ; \\
162767157.212 ; \\
162771162.65 ; \\
16278 / 164.872 ; \\
16279 / 149.645 ; \\
16280 / 141.802 ; \\
16281 / 142.608 ; \\
162821 / 160.345 ; \\
162831159.172 ; \\
16284 / 160.251 ; \\
16285 / 147.301 \text {; } \\
\text { and } \\
16286 / 151.652 \text {. }\end{array}$ & $\begin{array}{l}\text { Filter sample through } 0.45 \\
\text { um nylon filter membrane. } \\
\text { Transfer filtered solids to } \\
125 \mathrm{~mL} \text { HDPE bottle, with } \\
\text { residual solids transferred } \\
\text { using } 0.01 \mathrm{M} \mathrm{N} \mathrm{NOH} \text {. Fill } \\
\text { bottle to capacity with } 0.01 \\
\mathrm{M} \mathrm{NaOH} \text {. Heat and stir at } \\
85^{\circ} \mathrm{C} \text { for } 16.5 \mathrm{hr} \text {, filter with } \\
0.45 \text { um nylon filtration } \\
\text { unit. Transfer solids back to } \\
\text { bottle esing } 0.01 \mathrm{M} \mathrm{NaOH} \text {, } \\
\text { filling to capacity }(123 \mathrm{~g} \\
\text { slurry). Heat and stiir for } \\
22.5 \mathrm{hr} \text {, then filter. Repeat } \\
\text { transfer and heat for } 24 \mathrm{hr} \text {, } \\
\text { and then filter. Analyze } \\
\text { composite sample of three } \\
\text { wash solutions. }\end{array}$ & $\begin{array}{l}49461 \text { ug Al } \\
\text { (??? Read } \\
\text { from Table } \\
8 ? ? ?)\end{array}$ & $\begin{array}{l}\text { Mean value } \\
\text { from KOH } \\
\text { fusion = } \\
146000 \text { ug } \\
\text { Alg gry } \\
\text { solid; Mean } \\
\text { value from } \\
\mathrm{Na}_{2} \mathrm{O}_{2} \\
\text { Fusion }= \\
157500 \text { ug } \\
\text { Al/g dry } \\
\text { solid. Total } \\
\text { weight = } \\
2178963 \text { ug } \\
\text { Al. }\end{array}$ & $\begin{array}{l}126 \mathrm{ug} \mathrm{Al} / \mathrm{g}, \\
36901 \mathrm{ug} \mathrm{Al}\end{array}$ & $\begin{array}{l}25 \mathrm{Al} \\
\text { removed }\end{array}$ & $\begin{array}{l}\text { Begin wash with } \\
50.8765 \mathrm{~g} \text { aliquot } \\
\text { ( } 44.8 \mathrm{~g} \text { as-received } \\
\mathrm{C}-104 \text { sample). } \\
\text { Affer } 1^{\mathrm{a}} \text { washh, had } \\
100.13 \mathrm{~g} \text { filtrate } \\
\text { and } 41.64 \mathrm{~g} \text { solids. } \\
\text { After } 2^{\text {sd }} \text { wash, had } \\
82.79 \mathrm{~g} \text { washing } \\
\text { solution and } 40.49 \\
\mathrm{~g} \text { wet solids. Third } \\
\text { wash produced } \\
93.11 \mathrm{~g} \text { washing } \\
\text { solution and } 48.55 \\
\mathrm{~g} \text { wet solids. After } \\
\text { evaposation of } \\
\text { water at } 80^{\circ} \mathrm{C}, \\
\text { dried overnight at } \\
105^{\circ} \mathrm{C} \text { (after } 3^{\text {rd }} \\
\text { wash), had } 14.3589 \\
\mathrm{~g} \text { dry washed } \\
\text { solids. }\end{array}$ & $\begin{array}{l}\text { Filter } 45.8422 \mathrm{~g} \text { ( } 40.4 \mathrm{~g} \text { as-received) } \\
\text { sample through a } 0.45 \text { um nylon } \\
\text { filter membrane. Transfer filtered } \\
\text { solids to HDPE bottle using } 3 \mathrm{M} \\
\mathrm{NaOH} \text {, filling bottle to capacity } \\
\text { yielding } \sim 140 \mathrm{~g} \text { slury. Heat } \& \text { stir } \\
\text { for } 21.5 \mathrm{hr} \text { at } 85^{\circ} \mathrm{C} \text {. Filter hot using } \\
\text { a } 0.45 \mathrm{um} \text { nylon filtration unit. } \\
\text { ( } 98.84 \mathrm{~g} \text { filtrate; } 41.47 \mathrm{~g} \text { wet solids). }\end{array}$ \\
\hline
\end{tabular}


Table A1-1. SSTs and DSTs Wash and Leach Information for Aluminum, Part 1. (38 sheets)

\begin{tabular}{|c|c|c|c|c|c|c|c|c|c|c|c|c|c|}
\hline Tank & $\begin{array}{l}\text { Lab Study } \\
\text { Reference(s) }\end{array}$ & $\begin{array}{l}\text { Year of } \\
\text { Sampling } \\
\text { Event }\end{array}$ & Test type & $\begin{array}{l}\text { Sample } \\
\text { color }\end{array}$ & Waste Sources & Sample ID & $\mathrm{NaOH}$ Wash Procedure & $\begin{array}{l}\text { Initial } \mathrm{Al} \text { in } \\
\text { Untreated } \\
\text { Solids }\end{array}$ & $\begin{array}{l}\text { Final Al in } \\
\text { Washed } \\
\text { solids }\end{array}$ & $\begin{array}{l}\text { Al measured in } \\
\text { Wash Solution }\end{array}$ & $\begin{array}{c}\% \\
\text { recovery }\end{array}$ & comments & Caustic Leach \#1 Procedure \\
\hline 241-C-104 & $\begin{array}{l}\text { PNWD- } \\
3024 \\
\text { (BNFL- } \\
\text { RPT-030) }\end{array}$ & 1999 & $\begin{array}{l}\text { Filtration, } \\
\text { water- } \\
\text { washing, } \\
\text { and } \\
\text { caustic- } \\
\text { leaching } \\
\text { tests }\end{array}$ & - & $\begin{array}{l}\text { Primary; CW. } \\
\text { Secondary: OWW. } \\
\text { Tertiary; particulates } \\
\text { from Sr wash of PUREXX } \\
\text { wastes in the AR-vault. } \\
\text { Other: Strmixed waste. }\end{array}$ & 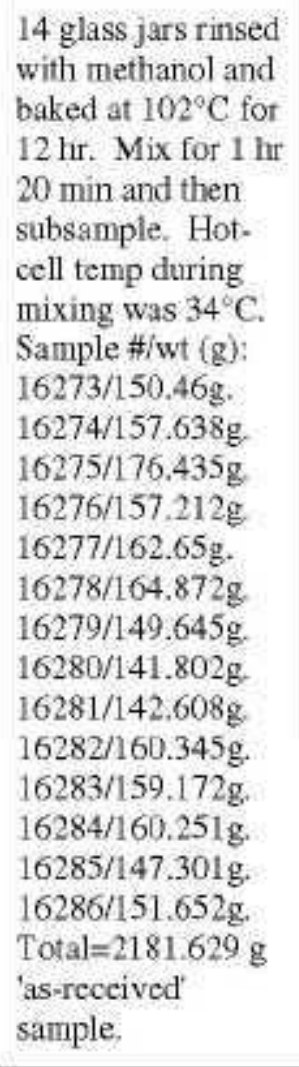 & $\begin{array}{l}\text { After initial filtration, wash } \\
\text { at } 25^{\circ} \mathrm{C} \text { (add } 715 \mathrm{~g} 0.01 \mathrm{M} \\
\mathrm{NaOH} 7 \text { times) and filter, } \\
\text { collecting three samples for } \\
\text { analysis. Add } 641.11 \mathrm{~g} \\
\text { additional inhibited water to } \\
\text { reconstitute slurry for } \\
\text { leaching. }\end{array}$ & $\begin{array}{l}125000 \text { ug } \\
\mathrm{Al} / \mathrm{g}(7.4 \mathrm{E}+7 \\
\text { ug Al) in } \\
\text { initial slurry } \\
\text { sample; } 207 \\
\text { ug Al/mL } \\
\text { (40674 ug Al) } \\
\text { in initial } \\
\text { permeate }\end{array}$ & $\begin{array}{l}\text { Slurry prior } \\
\text { to leaching: } \\
155667 \mathrm{ug} \\
\mathrm{Al} / \mathrm{g} \text {. }\end{array}$ & 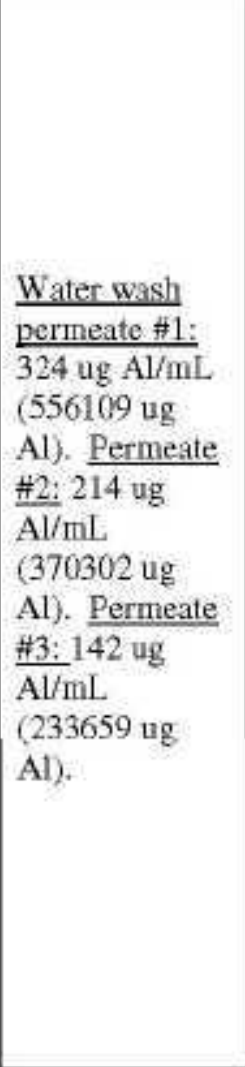 & $\begin{array}{l}2.4 \% \mathrm{Al} \\
\text { removed }\end{array}$ & $\begin{array}{l}\text { Slurry samples } \\
\text { measured on a dry } \\
\text { basis (water } \\
\text { removed by drying } \\
\text { at } 105^{\circ} \text { C). } \\
\text { Permeate samples } \\
\text { measured on a wet } \\
\text { basis. Physical } \\
\text { properties (i.e. } \\
\text { density and particle } \\
\text { size) and rheology } \\
\text { data available in } \\
\text { report. }\end{array}$ & $\begin{array}{l}\text { Combine slurry with } 2899.26 \mathrm{~g} 3 \mathrm{M} \\
\mathrm{NaOH} \text { (2M NaOH concentration). } \\
\text { Heat to } 85^{\circ} \mathrm{C} \text { and mix } 8 \mathrm{hr} \text {. } \\
\text { Dewater via filtration. }\end{array}$ \\
\hline 241-C-104 & $\begin{array}{l}\text { ORNLTM- } \\
13500\end{array}$ & 1998 & $\begin{array}{l}\text { Lab scale } \\
\text { caustic } \\
\text { leaching: } \\
\text { compare } \\
50,80, \\
\text { and } 93^{\circ} \mathrm{C} \\
\text { caustic } \\
\text { leaching } \\
\text { at } 3.99 \mathrm{M} \\
\mathrm{NaOH}\end{array}$ & - & $\begin{array}{l}\text { High aluminum, low } \\
\text { phosphorous and } \\
\text { bismuth. } \\
\text { High phosphorous } \\
\text { sludge with moderate } \\
\text { concentrations of } \\
\text { aluminum and } \\
\text { chromium. } \\
\text { Waste types include } \\
\text { PUREX aluminum waste } \\
\text { and } \mathrm{Zt} C W \text {. } \\
\mathrm{CC}^{1}\end{array}$ & $\begin{array}{l}\text { Two bottles used to } \\
\text { perform three leach } \\
\text { tests. For leach } \\
\text { tests } 1 \text { \& } 3: \text { slurry- } \\
\text { like material w/ } \\
\text { water content of } \\
63.5 \text { wt } \% \text { water, } \\
\text { contained small } \\
\text { piece of hard } \\
\text { material } \\
\text { resembling } \\
\text { concrete. For leach } \\
\text { test } 2 \text { : usual } \\
\text { consistency wl } \\
\text { water content of } \\
43.7 \text { wt } \% \text {. }\end{array}$ & - & 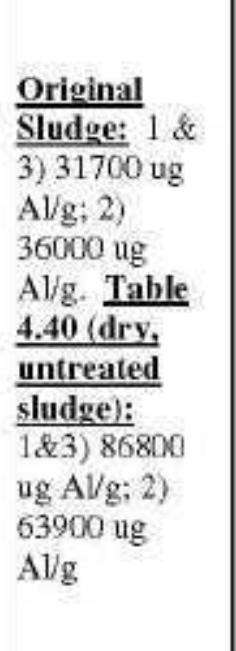 & - & - & - & - & 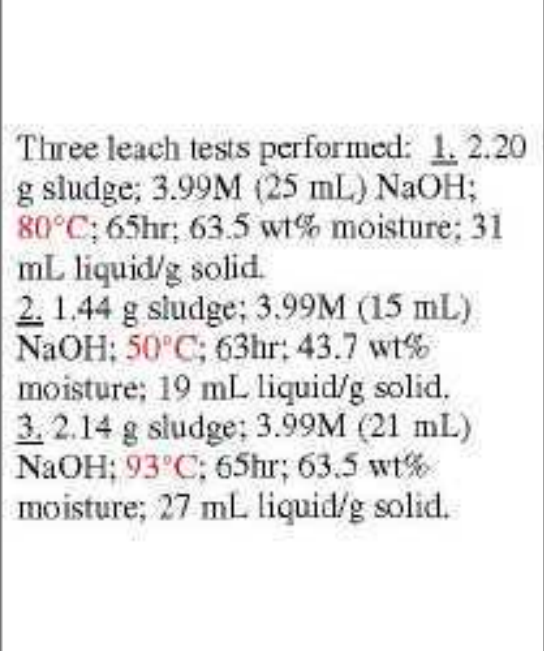 \\
\hline
\end{tabular}


Table A1-1. SSTs and DSTs Wash and Leach Information for Aluminum, Part 1. (38 sheets)

\begin{tabular}{|c|c|c|c|c|c|c|c|c|c|c|c|c|c|}
\hline Tank & $\begin{array}{l}\text { Lab Study } \\
\text { Reference(s) }\end{array}$ & $\begin{array}{l}\text { Year of } \\
\text { Sampling } \\
\text { Event }\end{array}$ & Test type & $\begin{array}{l}\text { Sample } \\
\text { color }\end{array}$ & Waste Sources & Sample ID & $\mathrm{NaOH}$ Wash Procedure & $\begin{array}{l}\text { Initial } \mathrm{Al} \text { in } \\
\text { Untreated } \\
\text { Solids }\end{array}$ & $\begin{array}{l}\text { Final Al in } \\
\text { Washed } \\
\text { solids }\end{array}$ & $\begin{array}{l}\mathrm{Al} \text { measured in } \\
\text { Wash Solution }\end{array}$ & $\begin{array}{c}\% \\
\text { recovery }\end{array}$ & comments & Caustic Leach \#1 Procedure \\
\hline 241-C-105 & $\begin{array}{l}\text { LAUR 97- } \\
2889\end{array}$ & FY97 & $\begin{array}{l}\text { Lab scale } \\
\text { caustic } \\
\text { leaching }\end{array}$ & $\begin{array}{l}\text { reddish- } \\
\text { brown; } \\
\text { dried } \\
\text { material } \\
\text { was light } \\
\text { brown }\end{array}$ & $\begin{array}{l}\text { TBP waste from U- } \\
\text { extraction process at U } \\
\text { plant, Sr wash of } \\
\text { PUREX waste in AR } \\
\text { vault, waste from } \\
\text { dissolution of Zr or Al } \\
\text { fuel cladding at PUREX, } \\
\text { high-activity neutralized } \\
\text { acid waste generated by } \\
\text { PUREX. } \\
\text { Non-Complexed Waste. } \\
\text { Primary: TBP. } \\
\text { Secondary: Particulates } \\
\text { from Sr wash of PUREX } \\
\text { wastes in the AR vault. } \\
\text { Primary: TBP. } \\
\text { Secondary: particulates } \\
\text { from Sr wash of PUREX } \\
\text { wastes in AR-valt. } \\
\text { Tertiary: CW. Other: } \\
\text { neutralized acid waste. }\end{array}$ & $\begin{array}{l}\text { Portions of } \\
\text { segment } 3 \text { of core } \\
72(222-S \text { lab ID } \\
\text { S96T001826, jar } \\
\# 9765)\end{array}$ & $\begin{array}{l}\text { Mix } 6.71 \mathrm{~g} \text { wet sludge with } \\
11.4 \mathrm{~mL} \text { water. With } 4.99 \mathrm{~g} \\
\text { slurry, wash } 3 \text { times by } \\
\text { adding } 10 \mathrm{~mL} \text {. } 0.01 \mathrm{M} \\
\mathrm{NaOH} \text {; mix } 1 \mathrm{hr} \text { at } 100^{\circ} \mathrm{C} \text {; } \\
\text { cool; centrifuge; decant; } \\
\text { repeat with solids. Dry } \\
\text { solids at } 105^{\circ} \mathrm{C} \text {. } 75.5 \mathrm{wt} \text { \% } \\
\text { insoluble solids \& } 32.97 \mathrm{~mL} \\
\text { supenate. }\end{array}$ & $27.2 \mathrm{wt} \% \mathrm{Al}$ & $\begin{array}{l}314925 \text { ug } \\
\text { Al/g, } \\
398853 \text { ug } \\
\text { Al }\end{array}$ & $\begin{array}{l}52.5 \mathrm{ug} \\
\mathrm{Al} / \mathrm{mL}, \\
1683.68 \text { ug Al }\end{array}$ & $\begin{array}{l}\text { Sum: } \\
\text { 3.16E-1\% } \\
\text { Al; Direct: } \\
\text { 2.72E-1\% } \\
\text { Al; Mass } \\
\text { recovery: } \\
115.9 \%\end{array}$ & - & 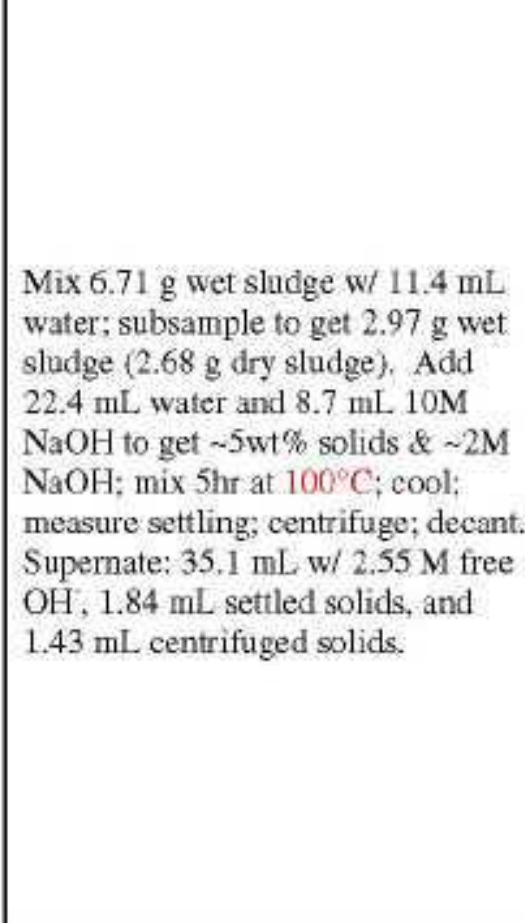 \\
\hline 241-C-105 & $\begin{array}{l}\text { ORNL/TM- } \\
13500\end{array}$ & 1998 & $\begin{array}{l}\text { Lab scale } \\
\text { caustic } \\
\text { leaching }\end{array}$ & - & $\begin{array}{l}\text { Contains high } \\
\text { concentrations of } \\
\text { aluminum. Waste types } \\
\text { include TBP uranium } \\
\text { recovery and sludge } \\
\text { wash waste from Sr } \\
\text { extraction process. } \\
\text { Non-Complexed Waste }\end{array}$ & - & - & $\begin{array}{l}\text { Original } \\
\frac{\text { Sludge: }}{2470000} \text { ug } \\
\text { Al/g (276000 } \\
\text { ug Al/g from } \\
\text { Table 4.27) }\end{array}$ & - & - & - & - & $\begin{array}{l}1.51 \mathrm{~g} \text { sludge; } 6.33 \mathrm{M}(15 \mathrm{~mL}) \\
\mathrm{NaOH} ; 70^{\circ} \mathrm{C} ; 22 \mathrm{hr} ; 4.7 \mathrm{wt} \% \\
\text { moisture; } 10 \mathrm{~mL} \text { liquid/g solid. }\end{array}$ \\
\hline 241-C-106 & $\begin{array}{l}\text { PNNL- } \\
11381\end{array}$ & FY96 & $\begin{array}{l}\text { Lab scale } \\
\text { caustic } \\
\text { leaching. }\end{array}$ & - & $\begin{array}{l}\text { Non-Complexed Waste. }{ }^{1} \\
\text { Primary: Sr leached } \\
\text { sludge, Secondary: } \\
\text { Particulates from Sr } \\
\text { wasto of PUREX wastes } \\
\text { in the AR vault. } \\
\text { Primary: Sr sludge. } \\
\text { Pecondary. particulates } \\
\text { from Sr wash of PUREX } \\
\text { wastes in the AR-vault. } \\
\text { Tertiary: neutralized acid } \\
\text { waste. Other: TBP. }\end{array}$ & $\begin{array}{l}17 \text { "grab" samples } \\
\text { homogenized prior } \\
\text { to testing and } \\
\text { analysis }\end{array}$ & $\begin{array}{l}\text { Mix } 28.8 \mathrm{~g} \text { water into } 15.14 \\
\text { g sludge. Using } 4.45 \mathrm{~g} \\
\text { slurry, wash } 3 \text { times by } \\
\text { adding } 10 \mathrm{~mL} 0.01 \mathrm{M} \\
\mathrm{NaOH} \text { to slurry, mix } 1 \text { hr at } \\
100^{\circ} \mathrm{C} \text {, cool, centrifuge, } \\
\text { decant. Repeat w/ solids } \\
\text { portion. For } 3 \mathrm{rd} \text { wast, add } \\
10.1 \mathrm{~mL} \text {. NaH. After } 3 \mathrm{rd} \\
\text { wash, dry solids at } 105^{\circ} \mathrm{C} \text {. }\end{array}$ & $\begin{array}{l}1.529 \mathrm{~g} \\
\text { sludge; } 0.587 \\
\text { g solids in } \\
\text { initial } \\
\text { unwashed } \\
\text { sample. } 4.8 \\
\text { wt's Al in } \\
\text { untreated } \\
\text { sludge solids. }\end{array}$ & $\begin{array}{l}0.243 \mathrm{~g} \\
\text { washed } \\
\text { solids } \\
\text { remaining, } \\
86400 \mathrm{ug} \\
\mathrm{Al} / \mathrm{g} ; 20995 \\
\mathrm{ug} \text { Al }(76 \% \\
\mathrm{Al})\end{array}$ & $\begin{array}{l}31.8 \mathrm{~mL} \text { wash } \\
\text { solution } \\
\text { produced at } \\
\mathrm{pH}=11.1 \text {. } \\
205 \mathrm{ug} \mathrm{Al} / \mathrm{mL} ; \\
6519 \mathrm{ug} \mathrm{Al} \\
(24 \% \mathrm{Al})\end{array}$ & $\begin{array}{l}\text { Concentra } \\
\text { tion in } \\
\text { initial } \\
\text { solids: } \\
\text { Sum }= \\
4.69 \mathrm{E}+04 \\
\text { ug Al/g; } \\
\text { Direct }= \\
4.85 \mathrm{E}+04 \\
\text { ug Al/g. } \\
97 \% \text { Al } \\
\text { recovery. }\end{array}$ & $\begin{array}{l}\text { (16 wt\% insoluble } \\
\text { solids in sludge) }\end{array}$ & $\begin{array}{l}\text { After mixing } 28.8 \mathrm{~g} \text { water to } 15.14 \\
\mathrm{~g} \text { sludge, begin leach of } 4.76 \mathrm{~g} \\
\text { sludge solids Add } 10 \mathrm{M} \mathrm{NaOH} \\
(10.6 \mathrm{~mL} \text { ) and water to a total } \\
\text { volume of } 45 \mathrm{~mL} \text { ( } 49.4 \mathrm{~g} \text { slurry; } 3.3 \\
\text { wt } \mathrm{s} \% \text { solids). Mix } 5 \mathrm{hr} \text { at } 100^{\circ} \mathrm{C} \text {. } \\
\text { Cool, settle, and decant off liquid. } \\
\text { Liquid: } 31.6 \mathrm{~mL} \text { with } 2.6 \mathrm{M} \mathrm{OH} \text {. } \\
\text { Solids: } 12.7 \mathrm{~g} \text { shirry with } 9.6 \mathrm{~mL} \\
\text { interstitial liquid. }\end{array}$ \\
\hline
\end{tabular}


Table A1-1. SSTs and DSTs Wash and Leach Information for Aluminum, Part 1. (38 sheets

\begin{tabular}{|c|c|c|c|c|c|c|c|c|c|c|c|c|c|}
\hline Tank & $\begin{array}{c}\text { Lab Study } \\
\text { Reference(s) }\end{array}$ & $\begin{array}{c}\text { Year of } \\
\text { Sampling } \\
\text { Event }\end{array}$ & Test type & $\begin{array}{l}\text { Sample } \\
\text { color }\end{array}$ & Waste Sources & Sample ID & $\mathrm{NaOH}$ Wash Procedure & $\begin{array}{l}\text { Initial Al in } \\
\text { Untreated } \\
\text { Solids }\end{array}$ & $\begin{array}{l}\text { Final Al in } \\
\text { Washed } \\
\text { solids }\end{array}$ & $\begin{array}{l}\mathrm{Al} \text { measured in } \\
\text { Wash Solution }\end{array}$ & $\begin{array}{c}\% \\
\text { recovery }\end{array}$ & comments & Caustic Leach \#1 Procedure \\
\hline $241-\mathrm{C}-106$ & $\begin{array}{l}\text { PNNL- } \\
11432\end{array}$ & 1997 & $\begin{array}{l}\text { Lab scale } \\
\text { caustic } \\
\text { leaching }\end{array}$ & - & Non-Complexed Waste. ${ }^{1}$ & $\begin{array}{l}20 \text { "grab" sample } \\
\text { bottles combined to } \\
\text { make composite } \\
\text { sample. Mixed } \\
3074 \mathrm{~g} \text { sludge with } \\
1965 \mathrm{~g} \text { DI (minus } \\
35 \mathrm{~mL} \text { spilled). } \\
\text { Mix } 2 \text { days ( 9.8 } \\
\text { wt } \% \text { solids). } 5 \\
\text { samples ( } 100 \mathrm{~g} \text { ) } \\
\text { sent for analysis ( } 4 \text { ) } \\
\text { or archive (1) }\end{array}$ & - & - & - & - & - & - & $\begin{array}{l}\text { Add }-2340 \mathrm{~g} 10 \mathrm{M} \mathrm{NaOH} \text { to }-4494 \\
\mathrm{~g} \text { slurry in sludge receipt tank (lost } \\
-584 \mathrm{~g} \text { to evaporation), }-3.4 \mathrm{M} \\
\mathrm{NaOH} \text { in solution; measured } 2.7 \mathrm{M} \\
\text { free } \mathrm{OH} \text { by titration. Mix } \& \text { heat to } \\
100^{\circ} \mathrm{C} \text {. Mix for } 5 \mathrm{hr} \text { at } 100^{\circ} \mathrm{C} \text {. } \\
\text { Transfer to sludge settler and } \\
\text { gavity settle at } 85^{\circ} \mathrm{C} \text { (lost }-306 \mathrm{~g} \text { to } \\
\text { evaporation). } 5.9 \mathrm{wt} \% \mathrm{c} \text { caustic- } \\
\text { insoluble solids. Sample superate. } \\
\text { Decant } 2578 \mathrm{~mL} \text { supernate. Add } \\
3286 \mathrm{~g} 3 \mathrm{M} \mathrm{MaOH} \text { to re-suspend } \\
\text { solids (spill } 450 \mathrm{~mL} \text { when } \\
\text { recirculating to rinse settler. Made } \\
\text { up by adding } 503 \mathrm{~g} \text { caustic.) Rinse, } \\
\text { transfer to sludge receipt tank. }\end{array}$ \\
\hline
\end{tabular}


Table A1-1. SSTs and DSTs Wash and Leach Information for Aluminum, Part 1. (38 sheets)

\begin{tabular}{|c|c|c|c|c|c|c|c|c|c|c|c|c|c|}
\hline Tank & $\begin{array}{c}\text { Lab Study } \\
\text { Reference(s) }\end{array}$ & $\begin{array}{l}\text { Year of } \\
\text { Sampling } \\
\text { Event }\end{array}$ & Test type & $\begin{array}{l}\text { Sample } \\
\text { color }\end{array}$ & Waste Sources & Sample ID & $\mathrm{NaOH}$ Wash Procedure & $\begin{array}{l}\text { Initial } \mathrm{Al} \text { in } \\
\text { Untreated } \\
\text { Solids }\end{array}$ & $\begin{array}{c}\text { Final Al in } \\
\text { Washed } \\
\text { solids }\end{array}$ & $\begin{array}{l}\text { Al measured in } \\
\text { Wash Solution }\end{array}$ & $\begin{array}{c}\% \\
\text { recovery } y^{2}\end{array}$ & comments & Caustic Leach \#1 Procedure \\
\hline $241-C-106$ & $\begin{array}{l}\text { PNWD- } \\
3013 \\
\text { (BNFL- } \\
\text { RPT-017) }\end{array}$ & 2000 & $\begin{array}{l}\text { Lab scale } \\
\text { caustic } \\
\text { leaching }\end{array}$ & - & Non-Complexed Waste. ${ }^{1}$ & $\begin{array}{l}\text { Portion of } \\
\text { homogenized C- } \\
106 \text { initial } \\
\text { composite material } \\
\text { prepared from } 20 \\
\text { grab samples } \\
\text { delivered to PNNL. } \\
\text { in June 1996. Used } \\
60 \mathrm{~g} \text { sub-sample of } \\
\text { this homogenized } \\
\text { dry material for } \\
\text { tests. Data from } \\
\text { sample analusis is } \\
\text { not easily } \\
\text { correlated to } \\
\text { individual samples } \\
\text { from three } \\
\text { procedures used in } \\
\text { this study. }\end{array}$ & 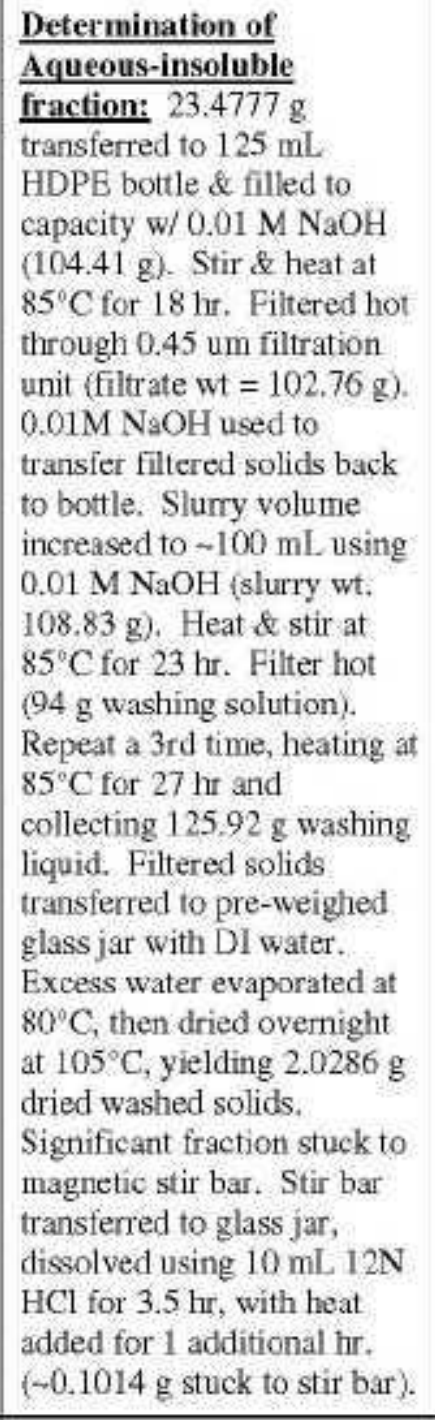 & - & - & $\begin{array}{l}14 \% \text { removed } \\
\text { by wash }\end{array}$ & - & $\begin{array}{l}\text { Solubility versus } \\
\text { temperature test: } \\
10.1157 \mathrm{~g} \text { aliquot } \\
\text { transferred to } 60 \\
\mathrm{~mL} \mathrm{HDPE} \text { bottle. } \\
50 \mathrm{~mL} \text { ( } 49.43 \mathrm{~g} \text { ) } \\
0.1 \mathrm{M} \mathrm{NaOH} \text { added } \\
\text { to bottle and } \\
\text { capped. Sample } \\
\text { heated \& stirred at } \\
30^{\circ} \mathrm{C} \text { for } 19 \mathrm{hr}+3 \\
\text { hr. Two } 4 \mathrm{~mL} \\
\text { aliquots taken \& } \\
\text { immediately } \\
\text { filtered using } \\
\text { preheated } 0.45 \text { um } \\
\text { nylon syringe filter } \\
\text { for analysis. T } \\
\text { increased to } 40^{\circ} \mathrm{C} \\
\text { and stirred for } 18.5 \\
\text { hr. Sample again } \\
\text { (same as done after } \\
\text { first step). } \\
\text { Thereased T again } \\
\text { to } 50^{\circ} \mathrm{C} \text { and stin for } \\
24 \mathrm{hr} \text {. Sample and } \\
\text { filter again. }\end{array}$ & $\begin{array}{l}\text { late } \\
24.7022 \mathrm{~g} \text { aliquot transferred to } 125 \\
\mathrm{~mL} \mathrm{HDPE} \text { bottle. } 108.08 \mathrm{~g} \mathrm{3} 3 \mathrm{M} \\
\mathrm{NaOH} \text { added. Heat } \& \text { stir at } 85^{\circ} \mathrm{C} \\
\text { for } 20 \mathrm{hr} \text {. Filter while still hot using } \\
0.45 \text { um nylon filtration unit. } \\
\text { Filtrate wt }=104.07 \mathrm{~g} \text {. Leachate } \\
\text { solution sample analyzed. }\end{array}$ \\
\hline 241-C-107 & $\begin{array}{l}\text { LAUR } 96- \\
2839 \text {. }\end{array}$ & FY95 & $\begin{array}{l}\text { Lab scale } \\
\text { caustic } \\
\text { leaching }\end{array}$ & $\begin{array}{l}\text { wet dark } \\
\text { red } \\
\text { material }\end{array}$ & $\begin{array}{l}\text { 1C; waste produced at } \\
\text { PUREX Plant from } \\
\text { dissolution of } \mathrm{Zr} \text { or } \mathrm{Al} \\
\text { fucl cladding; sludge } \\
\text { feed from Sr extraction } \\
\text { process at B Plant. } \\
\text { Dilute Complexed } \\
\text { Waste. } \\
\text { Primary: } 1 \mathrm{C} \text {. Secondary. } \\
\mathrm{CW}^{2}\end{array}$ & - & $\begin{array}{l}\text { Mix } 11.0187 \mathrm{~g} \text { wet sludge } \\
\text { with } 44 \text { mL water. With } \\
\text { ImL of this sample, wash } 3 \\
\text { times by adding } 5 \mathrm{~mL} 0.01 \\
\text { M NaOH/NaNO } 0 \text {; mix for } \\
0.5 \mathrm{hr} \text { at RT; centrifuge; } \\
\text { decant; Repeat with solids. } \\
\text { Dry solids at } 80^{\circ} \mathrm{C}=0.0409 \\
\mathrm{~g} \text { or } 19.4 \text { wt } \% \text { insoluble } \\
\text { solids }\end{array}$ & $10.5 \mathrm{wt} \leqslant \mathrm{Al}$ & - & - & $\begin{array}{l}2 \% \\
\text { removed } \\
\text { by simple } \\
\text { wash }\end{array}$ & $\begin{array}{l}\text { Retrieval wash: } \\
\text { mix } 10.2464 \mathrm{~g} \text { wet } \\
\text { sludge }(4.6122 \mathrm{~g} \\
\text { dry) with } 42.3 \mathrm{~mL} \\
\text { water and } 0.85 \mathrm{~mL} \\
0.01 \mathrm{M} \\
\mathrm{NaOH} / \mathrm{NaNO}_{2}, \text { mix } \\
1 \text { hr at } 100^{\circ} \mathrm{C} \text {, cool, } \\
\text { measure settling, } \\
\text { centrifuge, decant } \\
\text { and send solids to } \\
\text { caustic leach } 1 \text {. }\end{array}$ & $\begin{array}{l}\text { Add } 4.6 \mathrm{~mL} 10 \mathrm{M} \mathrm{NaOH} \text { and } 3.3 \\
\text { mL water to solids (14.5 mL settled } \\
\text { solids } 6.4 \mathrm{~mL} \text { centrifuged solids): } \\
\text { mix } 5 \text { hr at } 100^{\circ} \mathrm{C} \text {; cool; measure } \\
\text { settling; centrifuge; decant. }\end{array}$ \\
\hline
\end{tabular}


Table A1-1. SSTs and DSTs Wash and Leach Information for Aluminum, Part 1. (38 sheets)

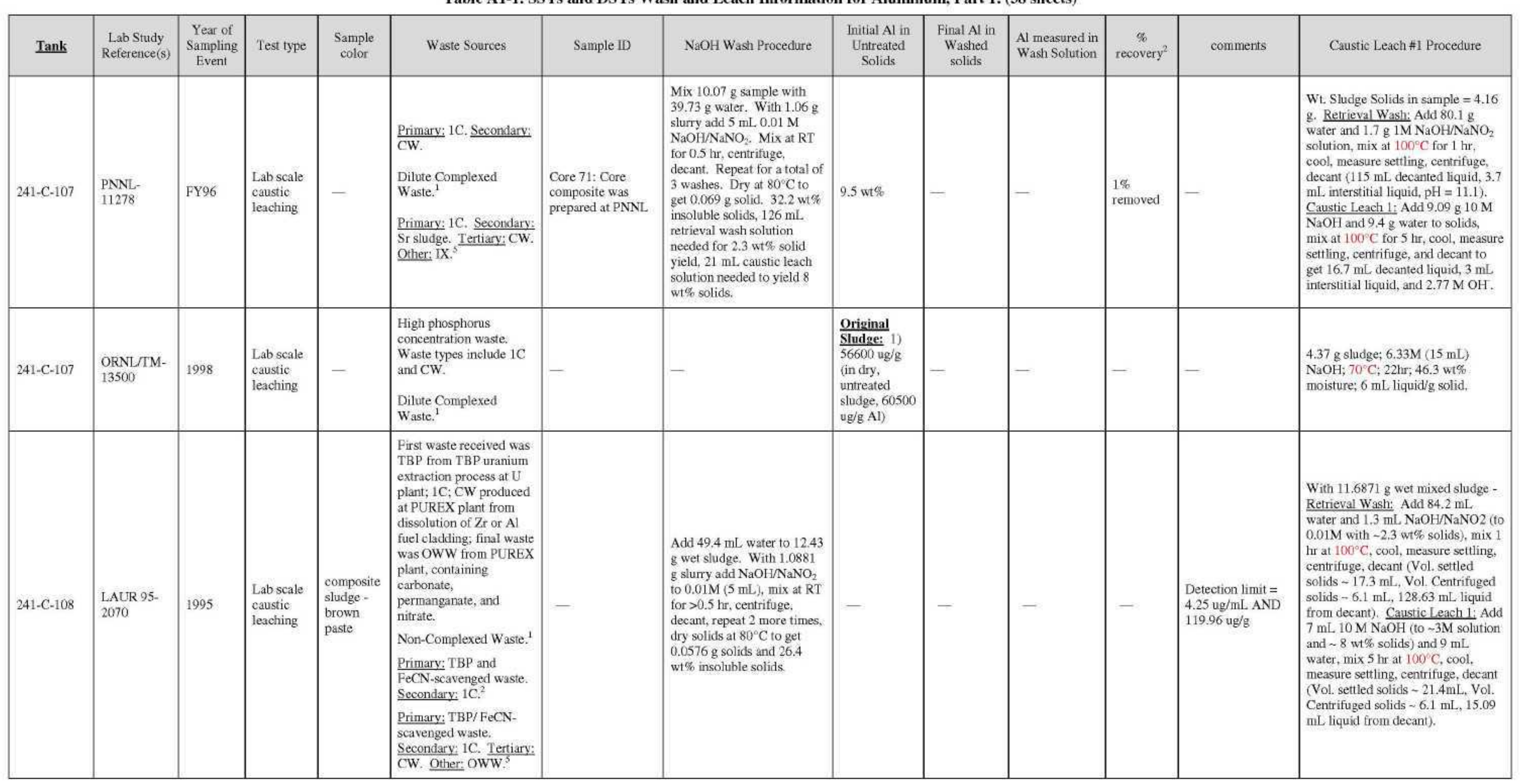


Table A1-1. SSTs and DSTs Wash and Leach Information for Aluminum, Part 1. (38 sheets

\begin{tabular}{|c|c|c|c|c|c|c|c|c|c|c|c|c|c|}
\hline Tank & $\begin{array}{l}\text { Lab Study } \\
\text { Reference(s) }\end{array}$ & $\begin{array}{c}\text { Year of } \\
\text { Sampling } \\
\text { Event }\end{array}$ & Test type & $\begin{array}{l}\text { Sample } \\
\text { color }\end{array}$ & Waste Sources & Sample ID & $\mathrm{NaOH}$ Wash Procedure & $\begin{array}{l}\text { Initial } \mathrm{Al} \text { in } \\
\text { Untreated } \\
\text { Solids }\end{array}$ & $\begin{array}{l}\text { Final Al in } \\
\text { Washed } \\
\text { solids }\end{array}$ & $\begin{array}{l}\text { Al measured in } \\
\text { Wash Solution }\end{array}$ & $\begin{array}{c}\% \\
\text { recovery }\end{array}$ & comments & Caustic Leach \#1 Procedure \\
\hline $241-C-109$ & PNL-9387 & FY93 & $\begin{array}{l}\text { Lab scale } \\
\text { caustic } \\
\text { leaching }\end{array}$ & $\begin{array}{l}\text { A portion } \\
\text { of the } \\
\text { composite } \\
\text { sample of } \\
\text { Core } 47 \\
\text { taken in } \\
1992 .\end{array}$ & 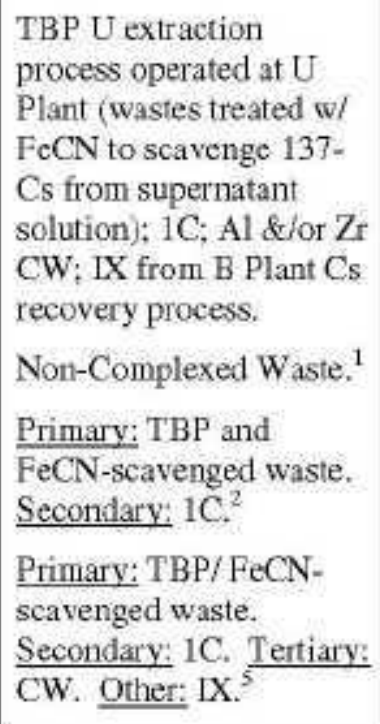 & $\begin{array}{l}\text { Core } 47 \text { taken from } \\
\text { C-109 in } 1992 \text {. }\end{array}$ & $\begin{array}{l}\text { Mix } 2 \mathrm{~g} \text { sludge w/ } 6 \mathrm{~mL} \\
0.1 \mathrm{M} \mathrm{NaOH} \text {. With } 2.16 \mathrm{~g} \\
\text { slury, mix } \mathrm{hr} \text { at } 100^{\circ} \mathrm{C} \text {; } \\
\text { centrifuge; decant; add } 2 \\
\mathrm{~mL} 0.1 \mathrm{M} \mathrm{NaOH} \text { to solids: } \\
\text { mix } 1 \mathrm{Hr} \text { at } 100^{\circ} \mathrm{C} \text {, } \\
\text { centrifuge; decant; add } 5 \\
\mathrm{~mL} \text { water to solids; mix } 0.5 \\
\text { hr at RT; centrifuge; decant; } \\
\text { dry at } 50^{\circ} \mathrm{C}(0.16 \mathrm{~g} \text { dry } \\
\text { sludge). }\end{array}$ & $\begin{array}{l}5.4 \mathrm{E}-2 \mathrm{~g} / \mathrm{g} \\
\text { sludge }\end{array}$ & - & $8 \% \mathrm{Al}$ & - & $\begin{array}{l}\text { Sludge washing } \\
\text { and acid } \\
\text { dissolution } \\
\text { experiment done as } \\
\text { well, but not noted } \\
\text { here. }\end{array}$ & $\begin{array}{l}\text { Add } 1 \mathrm{~mL} \text { water to dry solids from } \\
\text { wash (save } 0.12 \mathrm{~g} \text { slurry for } \\
\text { analysis;; add } 0.39 \mathrm{~mL} 10 \mathrm{M} \mathrm{NaOH} \\
\text { (effectively } 3 \mathrm{M} \mathrm{NaOH} \text {; mix } 6 \mathrm{hr} \text { at } \\
100^{\circ} \mathrm{C} \text {; centrifuge; decantl; add } 1 \mathrm{~mL} \\
3 \mathrm{M} \mathrm{NaOH} \text { to solids; mix } 5.5 \mathrm{hr} \text { at } \\
100^{\circ} \mathrm{C} \text {; centrifuge; decant; add } 5 \mathrm{~mL} \\
\text { water to solids; mix } 0.5 \mathrm{hr} \text { at } \mathrm{RT} ; \\
\text { centrifuge; decant; add } 1 \mathrm{~mL} \text { water } \\
\text { to solids; mix } 0.5 \mathrm{hr} \text { at } \mathrm{RT} \text {; take } 0.6 \\
\mathrm{~g} \text { slurry sample for particle analysis; } \\
\text { centrifuge; decant; dry solids at } \\
50^{\circ} \mathrm{C} \text { to obtain } 0.07 \mathrm{~g} \text { dry sludge. } \\
7.3 \mathrm{~mL} \text { sludge leach solution. }\end{array}$ \\
\hline $241-\mathrm{C}-112$ & PNL-9387 & FY93 & $\begin{array}{l}\text { Lab scale } \\
\text { oaustic } \\
\text { leaching }\end{array}$ & $\begin{array}{l}\text { A } \\
\text { composite } \\
\text { of } 2 \\
\text { segments } \\
\text { obtained } \\
\text { in the } \\
1992 \text { Core } \\
36 \text { diluted } \\
\text { with } 3 \\
\text { parts } \\
\text { water (by } \\
\text { wt.). }\end{array}$ & 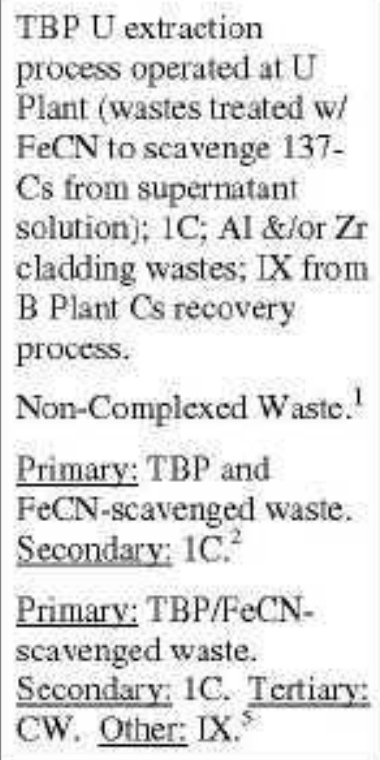 & - & $\begin{array}{l}\mathrm{Mix} 2 \mathrm{~g} \mathrm{C} 36 \text { composite w/ } 6 \\
\mathrm{~mL} 0.1 \mathrm{M} \mathrm{NaOH} \text {. Mix } 2.3 \mathrm{~g} \\
\text { slurry for } 1 \mathrm{hr} \text { at } 100^{\circ} \mathrm{C} \text {; } \\
\text { centrifuge; decant; add } 2 \mathrm{~mL} \\
0.1 \mathrm{M} \mathrm{NaOH} \text {; mix } 1 \mathrm{hr} \text { at } \\
100^{\circ} \mathrm{C} \text {; centrifuge; decant; } \\
\text { add } 5 \mathrm{~mL} \text { water; mix } 0.5 \mathrm{hr} \\
\text { at } \mathrm{RT} \text {; centrifuge; decant } \\
\text { (total } 8.2 \mathrm{~mL} \text { sludge wash } \\
\text { solution); dry at } 50^{\circ} \mathrm{C}(0.35 \\
\mathrm{g} \text { dity sludge). }\end{array}$ & $\begin{array}{l}\text { 4.5E-3 g Al/g } \\
\text { sludge }\end{array}$ & - & $34 \%$ & - & $\begin{array}{l}\text { Sludge washing } \\
\text { and acid } \\
\text { dissolution } \\
\text { experiment done as } \\
\text { well, but not } \\
\text { reported here. }\end{array}$ & 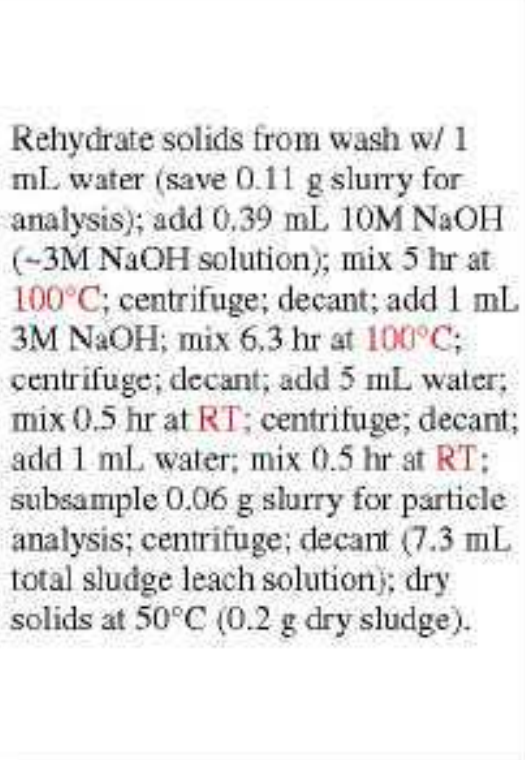 \\
\hline
\end{tabular}


Table A1-1. SSTs and DSTs Wash and Leach Information for Aluminum, Part 1. (38 sheets)

\begin{tabular}{|c|c|c|c|c|c|c|c|c|c|c|c|c|c|}
\hline Tank & $\begin{array}{c}\text { Lab Study } \\
\text { Reference(s) }\end{array}$ & $\begin{array}{l}\text { Year of } \\
\text { Sampling } \\
\text { Event }\end{array}$ & Test type & $\begin{array}{l}\text { Sample } \\
\text { color }\end{array}$ & Waste Sources & Sample ID & $\mathrm{NaOH}$ Wash Procedure & $\begin{array}{l}\text { Initial Al in } \\
\text { Untreated } \\
\text { Solids }\end{array}$ & $\begin{array}{l}\text { Final Al in } \\
\text { Washed } \\
\text { solids }\end{array}$ & $\begin{array}{l}\text { Al measured in } \\
\text { Wash Solution }\end{array}$ & $\begin{array}{c}\% \\
\text { recovery }\end{array}$ & comments & Caustic Leach \#1 Procedure \\
\hline $241-S-101$ & $\begin{array}{l}\text { PNNL- } \\
12026\end{array}$ & FY98 & $\begin{array}{l}\text { Lab scale } \\
\text { caustic } \\
\text { leaching: } \\
\text { multiple } \\
\text { samples } \\
\text { taken at } \\
\text { two } \\
\text { temperatu } \\
\text { res }(70 \\
\left.\text { and } 95^{\circ} \mathrm{C}\right) \\
\text { and two } \\
{[0 \mathrm{H}](1} \\
\text { and } 3 \mathrm{M})\end{array}$ & - & $\begin{array}{l}\text { Primary: High-level } \\
\text { REDOX process waste. } \\
\text { Secondary: EB. } \\
\text { Non-Complexed Waste. }\end{array}$ & $\begin{array}{l}222-S \text { lab ID } \\
\text { S96T005965; Jar \# } \\
11720 ; \text { Composite } \\
\text { made from } \\
\text { segments } 5 \text { through } \\
9 \text { of core number } \\
137 \text {. }\end{array}$ & 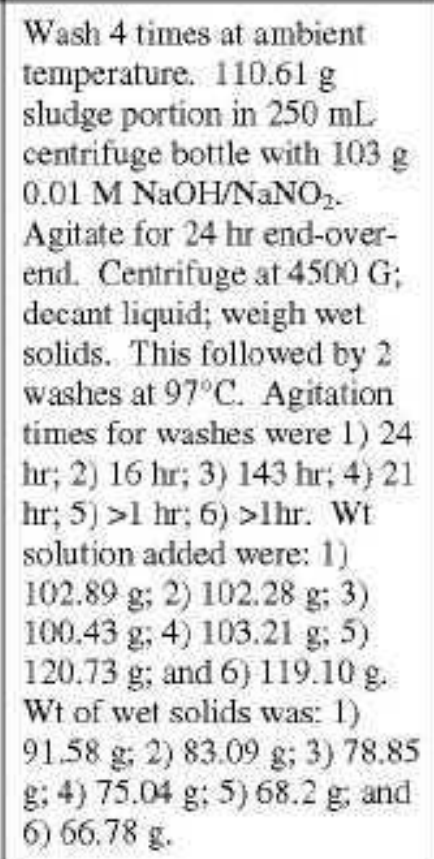 & $\begin{array}{l}9.41 \mathrm{wt} \% \\
9.41 \mathrm{E}+4 \mathrm{ug} / \mathrm{g}\end{array}$ & $\begin{array}{l}1.04 \mathrm{E}+5 \\
\mathrm{ug} / \mathrm{g} ; \\
9.27 \mathrm{E}+6 \text { ug } \\
\mathrm{Al}\end{array}$ & $\begin{array}{l}\text { 1.4E+3 ug/mL; } \\
1.14 \mathrm{E}+6 \text { ug } \mathrm{Al}\end{array}$ & $\begin{array}{l}11 \% \\
\text { removed }\end{array}$ & - & 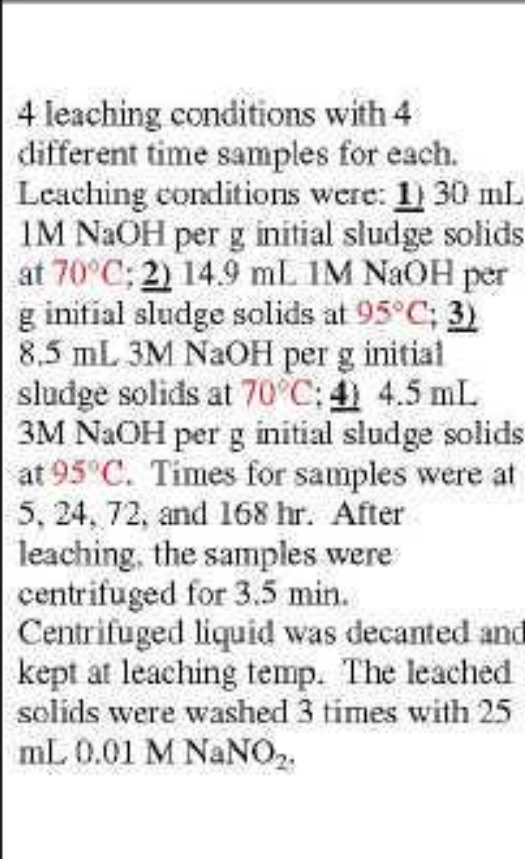 \\
\hline 241-S-101 & $\begin{array}{l}\text { PNNL- } \\
11636\end{array}$ & FY97 & $\begin{array}{l}\text { Lab scale } \\
\text { caustic } \\
\text { leaching. }\end{array}$ & - & $\begin{array}{l}\text { Non-Complexed Waste. }{ }^{1} \\
\text { Primary: High-Level } \\
\text { REDOX, Secondary: } \\
\text { BB. } \\
\text { Primary: High-level } \\
\text { REDOX waste. } \\
\text { Secondary: EB. } \\
\text { Tertiary: IX. Other: } \\
\text { mixture of several } \\
\text { miscellaneous wastes. }\end{array}$ & $\begin{array}{l}\text { 222-S Lab ID } \\
\text { S96T003486; Jar \# } \\
\text { 10519; sludge }\end{array}$ & $\begin{array}{l}\text { Wash } 3 \text { times by adding } 10 \\
\mathrm{ml} 0.01 \mathrm{M} \mathrm{NaOH} \text { to } 3.120 \mathrm{~g} \\
\text { slurry; mix } 1 \text { hr at } 100^{\circ} \mathrm{C} ; \\
\text { cool; centrifuge; decant; } \\
\text { repeat w/ solids portion. }\end{array}$ & $147000 \mathrm{ug} / \mathrm{g}$ & 87338 ug & 11578 ug & $101 \%$ & - & $\begin{array}{l}\text { Add } 6.87 \mathrm{ml} 10 \mathrm{M} \mathrm{NaOH} \text { to } 18.705 \\
\mathrm{~g} \text { slurry; mix } 5 \mathrm{hr} \text { at } 100^{\circ} \mathrm{C} \text {; cool; let } \\
\text { sit overnight; centrifuge }-20 \text {-ml } \\
\text { portion and transfer solids back to } \\
\text { vessel. }\end{array}$ \\
\hline 241-S-101 & $\begin{array}{l}\text { ORNL } / \text { TM- } \\
13500\end{array}$ & 1998 & $\begin{array}{l}\text { Lab scale } \\
\text { caustic } \\
\text { leaching }\end{array}$ & - & $\begin{array}{l}\text { High chromium } \\
\text { concentration waste. } \\
\text { (High Al sample). } \\
\text { Waste type is REDOX } \\
\text { waste. } \\
\text { Non-Complexed Waste. }\end{array}$ & - & - & $\begin{array}{l}\text { Original } \\
\text { Sludge had } \\
86300 \text { ug/g } \\
\text { (in dry, } \\
\text { untreated } \\
\text { sludge, } \\
127000 \text { ug/g } \\
\text { Al). }\end{array}$ & - & - & - & - & $\begin{array}{l}\text { One leach test performed with the } \\
\text { following conditions: } 1.90 \mathrm{~g} \\
\text { sludge; } 3.99 \mathrm{M}(21 \mathrm{~mL}) \mathrm{NaOH} \\
93^{\circ} \mathrm{C} ; 6 \mathrm{hir} ; 31.8 \text { wt\% moisture; } 16 \\
\mathrm{~mL} \text { liquid/g solid. }\end{array}$ \\
\hline
\end{tabular}


Table A1-1. SSTs and DSTs Wash and Leach Information for Aluminum, Part 1. (38 sheets)

\begin{tabular}{|c|c|c|c|c|c|c|c|c|c|c|c|c|c|}
\hline Tank & $\begin{array}{c}\text { Lab Study } \\
\text { Reference(s) }\end{array}$ & $\begin{array}{l}\text { Year of } \\
\text { Sampling } \\
\text { Event }\end{array}$ & Test type & $\begin{array}{l}\text { Sample } \\
\text { color }\end{array}$ & Waste Sources & Sample ID & $\mathrm{NaOH}$ Wash Procedure & $\begin{array}{l}\text { Initial Al in } \\
\text { Untreated } \\
\text { Solids }\end{array}$ & $\begin{array}{l}\text { Final Al in } \\
\text { Washed } \\
\text { solids }\end{array}$ & $\begin{array}{l}\text { Al measured in } \\
\text { Wash Solution }\end{array}$ & $\begin{array}{c}\% \\
\text { recovery }\end{array}$ & comments & Caustic Leach \#1 Procedure \\
\hline 241-S-101 & $\begin{array}{l}\text { ORNL/TM- } \\
13655\end{array}$ & 1998 & $\begin{array}{l}\text { Lab scale } \\
\text { caustic } \\
\text { leaching: } \\
\text { multiple } \\
\text { samples } \\
\text { taken at } \\
\text { two } \\
\text { temperatu } \\
\text { res }(70 \\
\text { and } 95^{\circ} \mathrm{C} \text { ) } \\
\text { and two } \\
{[\mathrm{OH}](1} \\
\text { and } 3 \mathrm{M} \text { ). }\end{array}$ & - & $\begin{array}{l}\text { Group } 1 \text { waste based on } \\
\text { the SORWT model. } \\
\text { Largest fraction of HLW } \\
\text { is from REDOX process } \\
\text { (formed sludge } \\
\text { accumulation); second } \\
\text { largest is EB (formed } \\
\text { extremely hard salt } \\
\text { cake). } \\
\text { Non-Complexed Waste.! }\end{array}$ & $\begin{array}{l}\text { WHC 222-S lab \# } \\
\text { S96T005965, jar \# } \\
11720\end{array}$ & 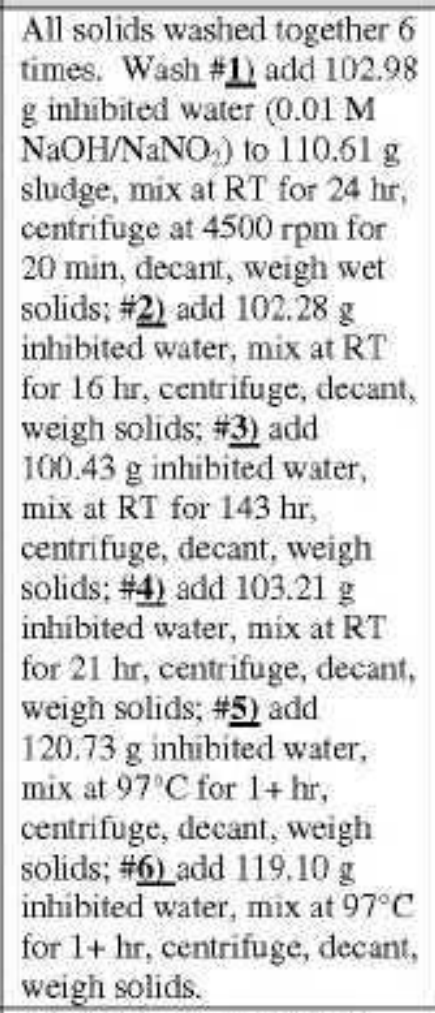 & $\begin{array}{l}\text { From Table } \\
\frac{\text { 2.1. }}{\mathrm{kg}} 2.37 \mathrm{E}+5 \\
\text { Al/2.67E+6 } \\
\mathrm{kg} \text { total } \\
\text { sludge mass, } \\
\text { From Table } \\
\frac{4.1}{4.1}: 9.41 \mathrm{E}+4 \\
\mathrm{ug} \mathrm{Al} / \mathrm{g} \\
\text { untreated } \\
\text { solids } \\
\text { (calculated by } \\
\text { sum of wash } \\
\text { solution + } \\
\text { washed solids } \\
\text { divided by } \\
\text { total mass of } \\
\text { S-101 sample } \\
\text { used). }\end{array}$ & $\begin{array}{l}\frac{\text { From Table }}{4.1: 1.04 \mathrm{E}+5} \\
\text { ug Al/g } \\
\text { washed } \\
\text { solids; } \\
9.27 \mathrm{E}+6 \text { ug } \\
\text { Al total. }\end{array}$ & $\begin{array}{l}\text { From Table } \\
\frac{4.1: 1.40 \mathrm{E}+3}{\text { ug Al/mL }} \\
\text { wash solution; } \\
1.14 \mathrm{E}+6 \text { ug } \mathrm{Al} \\
\text { total. }\end{array}$ & $\begin{array}{l}11 \% \\
\text { removed }\end{array}$ & $\begin{array}{l}\text { Weights of wet } \\
\text { solids from } 6 \\
\text { washes: } 191.58 \\
\mathrm{~g} ; 2) 83.09 \mathrm{~g} ; 3) \\
78.85 \mathrm{~g} ; 4) 75.04 \mathrm{~g} ; \\
5,68.2 \mathrm{~g} ; \text { and } 6 \text { ) } \\
66.78 \mathrm{~g} \text {. }\end{array}$ & 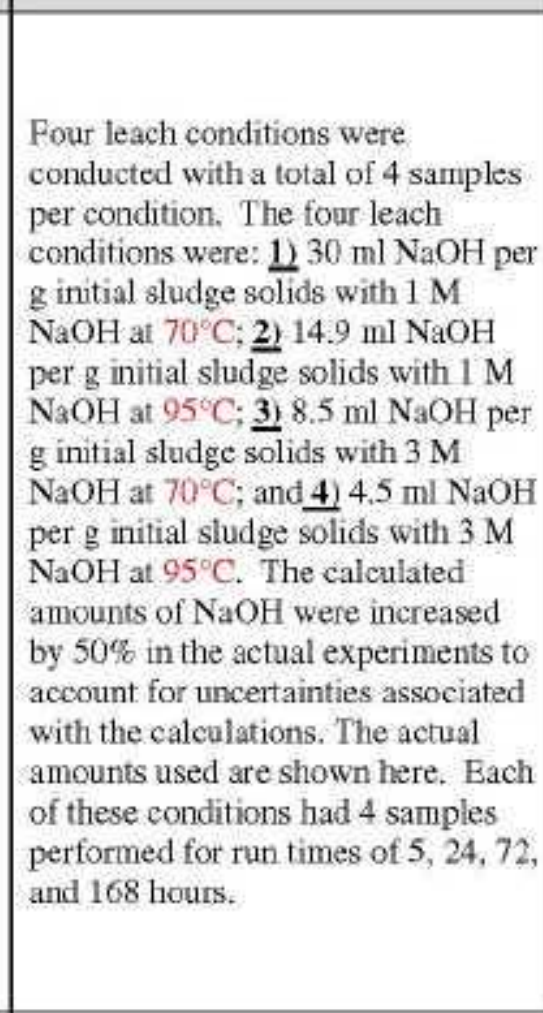 \\
\hline 241-S-104 & PNL-10712 & FY95 & $\begin{array}{l}\text { Lab scale } \\
\text { caustic } \\
\text { leaching }\end{array}$ & - & $\begin{array}{l}\text { Non-Complexed Waste. }{ }^{1} \\
\text { Primary waste type was } \\
\text { high-level REDOX } \\
\text { waste. }\end{array}$ & $\begin{array}{l}\text { Composite of } \\
\text { segments } 4 \text { and } 5 \\
\text { from core } 43(222- \\
\text { S Jar \#6588) }\end{array}$ & $\begin{array}{l}\text { Add } 34.2 \mathrm{~g} \text { water to } 7.9 \mathrm{~g} \\
\text { S104-1, mix and sample. } \\
\text { Using } 1.12 \mathrm{~g} \text { slurry: Wash } \\
3 \text { times by adding } 5 \mathrm{ml} 0.01 \\
\mathrm{M} \mathrm{NaOH} 0.01 \mathrm{M} \mathrm{NaNO} \text { to } \\
\text { slurry; mix } 0.5 \mathrm{hr} \text { at } \mathrm{RT} ; \\
\text { centrifuge; decant; repeat } \\
\text { with solids portion. Dry } \\
\text { remaining solids at } 80^{\circ} \mathrm{C} \\
(0.09 \mathrm{~g} \text { solid remains). }\end{array}$ & - & - & - & - & $\begin{array}{l}\text { Retrieval Wash } \\
\text { prior to caustic } \\
\text { leach: add } 99.2 \mathrm{~g} \\
\text { water and } 1.5 \mathrm{~g} \mathrm{IM} \\
\mathrm{NaOH} / \mathrm{M} \mathrm{NaNO} \\
\text { to } 6.1 \mathrm{~g} \text { dry solids } \\
\text { in slury. Mix } \mathrm{lh} \\
\text { at } 100^{\circ} \mathrm{C} \text {, cool. } \\
\text { measure settling, } \\
\text { centrifuge, decant. }\end{array}$ & $\begin{array}{l}\text { Add } 9.3 \mathrm{~g} \text { of } 10 \mathrm{M} \mathrm{NaOH} \text { to } 17 \mathrm{~mL} \\
\text { centrifuged solids; mix } 5 \mathrm{hr} \text { at } \\
100^{\circ} \mathrm{C} \text {; cool; masure settling; } \\
\text { centrifuge; decant: }\end{array}$ \\
\hline $241-5-104$ & $\begin{array}{l}\text { LAUR } 95 \text { - } \\
2070\end{array}$ & 1995 & $\begin{array}{l}\text { Lab scale } \\
\text { caustic } \\
\text { leaching }\end{array}$ & $\begin{array}{l}\text { A } \\
\text { composite } \\
\text { sample } \\
\text { consisting } \\
\text { of ddy tan } \\
\text { material. }\end{array}$ & $\begin{array}{l}\text { Primary waste type was } \\
\text { high level waste from the } \\
\text { REDOX process. } \\
\text { Non-Complexed Waste. }\end{array}$ & - & $\begin{array}{l}\text { Add } 25.8 \mathrm{~mL} \text { water to } \\
6.4603 \mathrm{~g} \text { wet sludge. With } \\
1.1157 \mathrm{~g} \text { slurry add } \\
\mathrm{NaOH} / \mathrm{NaNO} \text { to } 0.01 \mathrm{M}(5 \\
\mathrm{mL}) \text {, mix at RT for } 0.5 \mathrm{hr} \text {, } \\
\text { centrifuge, decant, repeat } 2 \\
\text { more times, dry solids at } \\
80^{\circ} \mathrm{C}(0.0822 \mathrm{~g} \text { solids; } 36.8 \\
\text { wt } \% \text { insoluble solids). }\end{array}$ & - & - & - & - & $\begin{array}{l}\text { Detection limit = } \\
4.25 \mathrm{ug} / \mathrm{mL} \text { AND } \\
124.85 \mathrm{ug} / \mathrm{g} \text {. }\end{array}$ & 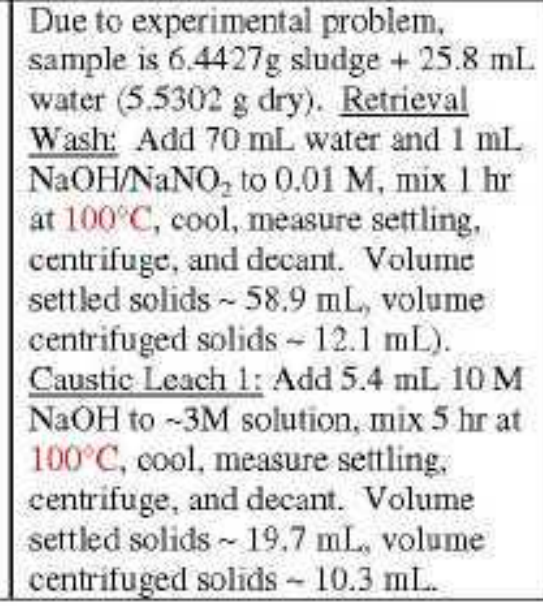 \\
\hline
\end{tabular}


Table A1-1. SSTs and DSTs Wash and Leach Information for Aluminum, Part 1. (38 sheets)

\begin{tabular}{|c|c|c|c|c|c|c|c|c|c|c|c|c|c|}
\hline Tank & $\begin{array}{c}\text { Lab Study } \\
\text { Reference(s) }\end{array}$ & $\begin{array}{l}\text { Year of } \\
\text { Sampling } \\
\text { Event }\end{array}$ & Test type & $\begin{array}{l}\text { Sample } \\
\text { color }\end{array}$ & Waste Sources & Sample ID & $\mathrm{NaOH}$ Wash Procedure & $\begin{array}{l}\text { Initial } \mathrm{Al} \text { in } \\
\text { Untreated } \\
\text { Solids }\end{array}$ & $\begin{array}{l}\text { Final Al in } \\
\text { Washed } \\
\text { solids }\end{array}$ & \begin{tabular}{|l|}
$\mathrm{Al}$ measured in \\
Wash Solution
\end{tabular} & $\begin{array}{c}\% \\
\text { recovery }\end{array}$ & comments & Caustic Leach \#1 Procedure \\
\hline $241-S-104$ & $\begin{array}{l}\text { PNNL-- } \\
11089\end{array}$ & FY95 & $\begin{array}{l}\text { Sludge } \\
\text { washing } \\
\text { and } \\
\text { caustic } \\
\text { leaching }\end{array}$ & - & $\begin{array}{l}\text { Primarily REDOX } \\
\text { process HLW. }\end{array}$ & Core \# 43 & $\begin{array}{l}\text { Add water to sludge sample } \\
\text { to get }-2.3 \text { wt } \% \text { solids, as } \\
\text { well as inhibited water } \\
\left(\mathrm{NaOH} / \mathrm{NaNO}_{2} \text { ) to } 0.01 \mathrm{M} \text {. }\right. \\
\mathrm{Mix} \text { at } 100^{\circ} \mathrm{C} \text { for } 1 \mathrm{hr} \text {. } \\
\mathrm{Cool} \text {, centrifuge, and } \\
\text { decant. }\end{array}$ & - & - & $3 \%$ recovered & - & - & $\begin{array}{l}\text { Caustic leach of solids: Add } \mathrm{NaOH} \\
\text { to get }-8 \text { wt } \% \text {, solids and a final } \\
\mathrm{NaOH} \text { of } 3.8 \mathrm{M} \text {. Mix at } 1000^{\circ} \mathrm{C} \text { for } 5 \\
\text { hr. Cool, centrifuge, decant. }\end{array}$ \\
\hline 241-S-104 & $\begin{array}{l}\text { PNNL- } \\
11636\end{array}$ & FY97 & $\begin{array}{l}\text { Lab scale } \\
\text { caustic } \\
\text { leaching. }\end{array}$ & - & $\begin{array}{l}\text { Non-Complexed Waste. } \\
\text { Primarily high-level } \\
\text { REDOX waste. }\end{array}$ & - & $\begin{array}{l}\text { Wash } 3 \text { times by adding } 10 \\
\mathrm{ml} 0.01 \mathrm{M} \mathrm{NaOH} \text { to } 9.01 \mathrm{~g} \\
\text { slurry; mix } 1 \mathrm{hr} \text { at } 100^{\circ} \mathrm{C} \text {; } \\
\text { cool centrifguge; decant; } \\
\text { repeat } w / \text { solids portion. }\end{array}$ & $153000 \mathrm{ug} / \mathrm{g}$ & $307063.5 \mathrm{ug}$ & 5871 ug & $128 \%$ & $\begin{array}{l}\text { Solution density = } \\
1.18 \mathrm{~g} / \mathrm{mL}, 1.685 \mathrm{~g} \\
\text { sludge }(1.595 \mathrm{~g} \\
\text { solids) initially in } \\
\mathrm{B} 1 ; 0.807 \mathrm{~g} \\
\text { washed solids in } \\
\text { final analysis. } \\
\text { Sample B1 has } \\
9.01 \mathrm{~g} \text { slurry (see } \\
\text { Fig. 3.1). }\end{array}$ & $\begin{array}{l}\text { Add } 13.3 \mathrm{mll} 10 \mathrm{M} \mathrm{NaOH} \text { and } 10 \mathrm{ml} \\
0.01 \mathrm{M} \mathrm{NaOH} \text { to } 54.938 \mathrm{~g} \text { slurry; } \\
\text { mix } 5 \mathrm{hr} \text { at } 100^{\circ} \mathrm{C} \text {; cool; centrifuge; } \\
\text { take a } 0.5 \mathrm{ml} \text { sample; mix for } 70 \mathrm{hr} \\
\text { at } 100^{\circ} \text {; c cool; centrifuge; take a } \\
0.5 \mathrm{ml} \text { sample; mix } 136 \mathrm{hr} \text { at } 100^{\circ} \mathrm{C} \text {; } \\
\text { cool; centrifuge; decant. }\end{array}$ \\
\hline 241-S-104 & $\begin{array}{l}\text { ORNL/TM- } \\
13500\end{array}$ & 1998 & $\begin{array}{l}\text { Lab scale } \\
\text { caustic } \\
\text { leaching } \\
\text { at three } \\
\text { temperatu } \\
\text { res }(67, \\
70 \text {, and } \\
\left.80^{\circ} \mathrm{C}\right) \text { and } \\
\text { two }[\mathrm{OH}] \\
(3.8,3.99 \text {, } \\
\text { and } 6.33 \\
\text { M) }\end{array}$ & - & $\begin{array}{l}\text { REDOX waste } \\
\text { characterized by average } \\
\text { aluminum concentration } \\
\text { and high chromium } \\
\text { concentration. } \\
\text { Non-Complexed Waste. }\end{array}$ & - & - & $\begin{array}{l}\text { 1-6) } 140000 \\
\text { ug/g }\end{array}$ & - & - & - & - & 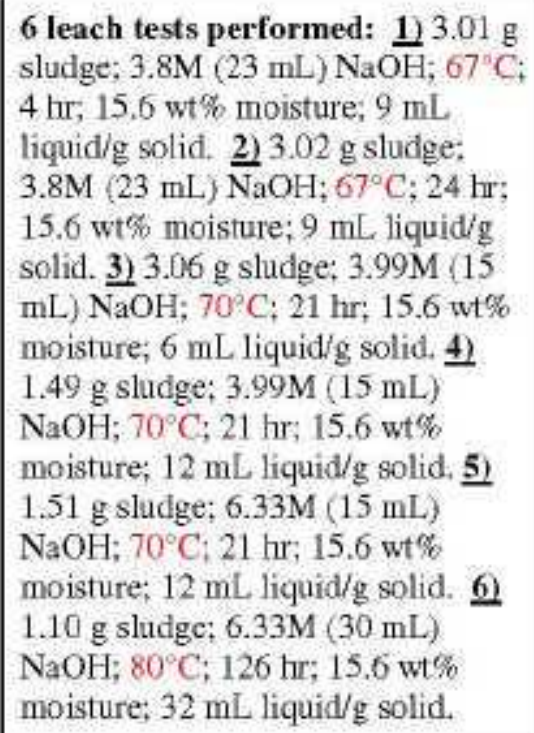 \\
\hline $241-$ S-107 & $\begin{array}{l}\text { PNNL- } \\
11278\end{array}$ & FY96 & $\begin{array}{l}\text { Lab scale } \\
\text { caustic } \\
\text { leaching }\end{array}$ & - & $\begin{array}{l}\text { Primary: High-level } \\
\text { REDOX process waste. } \\
\text { Secondary: EB. } \\
\text { Non-Complexed Waste. } \\
\text { Primary: High-Level } \\
\text { REDOX; Secondary: } \\
\text { EB. }\end{array}$ & $\begin{array}{l}\text { Cores 105, } 110, \\
\text { and } 111: \\
\text { Composite of three } \\
\text { core composites } \\
\text { (222-S LABCORE } \\
\text { sample numbers } \\
\text { S95T003158, } \\
\text { \$95T003159, and } \\
\text { S95T003164). }\end{array}$ & $\begin{array}{l}\text { Mix } 8.393 \mathrm{~g} \text { sample with } \\
15.5 \mathrm{~g} \text { water. With } 4.75 \mathrm{~g} \\
\text { slurry add } 10 \mathrm{~mL} 0.01 \mathrm{M} \\
\text { NaOH. Mix at } 100^{\circ} \mathrm{C} \text { for } 1 \\
\text { hr, cool, centrifuge, and } \\
\text { decant. Repeat for a total of } \\
3 \text { washes. Dry solids after } \\
\text { wash } \# 3 \text { at } 105^{\circ} \mathrm{C} \text {. Had } \\
1.668 \mathrm{~g} \text { sludge initially in } \\
\text { sample, } 0.622 \mathrm{~g} \text { of washed } \\
\text { solids, and } 37 \text { w } \% \text { \% } \\
\text { insoluble solids in sludge. }\end{array}$ & $\begin{array}{l}20.5 \text { wt } \% \\
\text { Summation: } \\
2.02 \mathrm{E}+5 \\
\text { ugg: Direct: } \\
2.05 \mathrm{E}+5 \text { ug/g }\end{array}$ & \begin{tabular}{|l}
303000 \\
ug/g: \\
188466 ug \\
(92 wt $\%$ )
\end{tabular} & $\begin{array}{l}522 \mathrm{ug} / \mathrm{mL} \text {; } \\
17017 \mathrm{ug}(8 \\
\text { wt\%) }\end{array}$ & $\begin{array}{l}8 \% \\
\text { removed }\end{array}$ & - & $\begin{array}{l}\text { Weight of sludge solids in sample } \\
\text { was } 3.4 \mathrm{~g} \text {. Addd } 8.5 \mathrm{mLL}, 10 \mathrm{M} \mathrm{NaOH} \\
\text { and water to a total volume of } 37 \\
\mathrm{~mL} \text { (1.8 wtos solids), mix at } 100^{\circ} \mathrm{C} \\
\text { for } 5 \mathrm{hr} \text {, cool, measure settling, } \\
\text { centrifuge, and decant. Measured } \\
29.8 \mathrm{~mL} \text { decanted liquid, } 4.2 \mathrm{~mL} \\
\text { interstitial liquid, and } 2.1 \mathrm{M} \mathrm{OH} \text {. }\end{array}$ \\
\hline
\end{tabular}


Table A1-1. SSTs and DSTs Wash and Leach Information for Aluminum, Part 1. (38 sheets)

\begin{tabular}{|c|c|c|c|c|c|c|c|c|c|c|c|c|c|}
\hline Tank & $\begin{array}{c}\text { Lab Study } \\
\text { Reference(s) }\end{array}$ & $\begin{array}{l}\text { Year of } \\
\text { Sampling } \\
\text { Event }\end{array}$ & Test type & $\begin{array}{l}\text { Sample } \\
\text { color }\end{array}$ & Waste Sources & Sample ID & $\mathrm{NaOH}$ Wash Procedure & $\begin{array}{l}\text { Initial Al in } \\
\text { Untreated } \\
\text { Solids }\end{array}$ & $\begin{array}{l}\text { Final Al in } \\
\text { Washed } \\
\text { solids }\end{array}$ & $\begin{array}{l}\text { Al measured in } \\
\text { Wash Solution }\end{array}$ & $\begin{array}{c}\% \\
\text { recovery }\end{array}$ & comments & Caustic Leach \#1 Procedure \\
\hline 241-S-107 & $\begin{array}{l}\text { PNNL- } \\
12010\end{array}$ & FY98 & $\begin{array}{l}\text { Lab scale } \\
\text { caustic } \\
\text { leaching: } \\
\text { performed } \\
\text { extended } \\
\text { caustic } \\
\text { leach at } \\
80^{\circ} \mathrm{C} \text {. }\end{array}$ & - & $\begin{array}{l}\text { Non-Complexed Waste. }{ }^{1} \\
\text { Primary: High-level } \\
\text { REDOX waste. } \\
\text { Secondary: EB. } \\
\text { Tertiary: CW. Other: } \\
\text { IX/mixture of several } \\
\text { miscellaneous wastes. }\end{array}$ & $\begin{array}{l}11 \text { Containers from } \\
222-\mathrm{S} \text { Laboratory } \\
\text { combined (using } \\
205 \mathrm{~g} \text { DI water and } \\
\text { mixer) to make } \\
\text { homogeneous } \\
\text { sludge sample. } \\
\text { Average solids } \\
\text { wt } \%=67.5 ; \\
\text { Average sludge } \\
\text { bulk density }= \\
1.815 \mathrm{~g} / \mathrm{mL} \text {. }\end{array}$ & 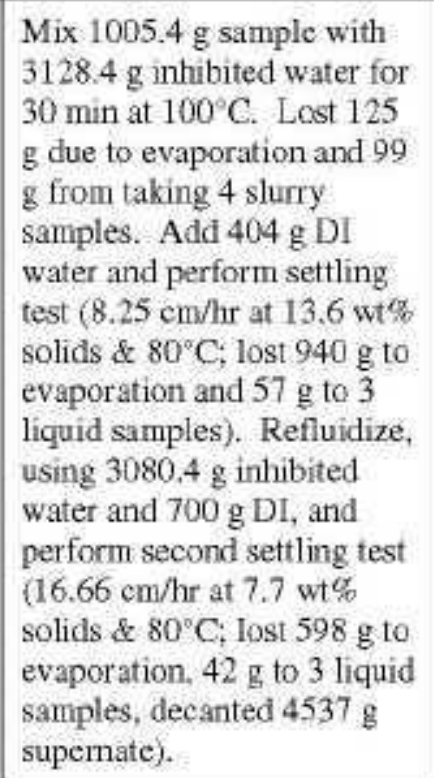 & - & - & $\begin{array}{l}799 \text { ug/g, } \\
4.74 \mathrm{E}+6 \text { ug Al } \\
(2.5 \%)\end{array}$ & - & $\begin{array}{l}\text { Performed many } \\
\text { settling tests and } \\
\text { particle size } \\
\text { analyses. Data } \\
\text { available within } \\
\text { doeument. }\end{array}$ & 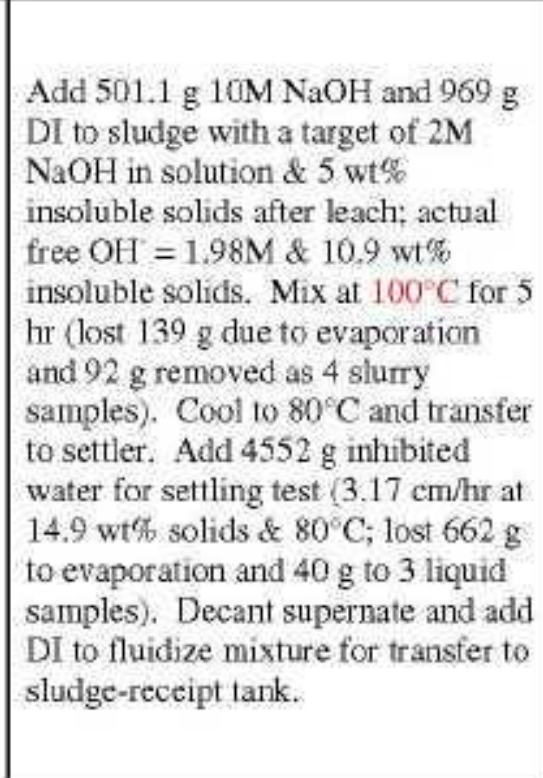 \\
\hline $241-S-110$ & $\begin{array}{l}\text { PNNL- } \\
13702\end{array}$ & FY01 & $\begin{array}{l}\text { Lab scale } \\
\text { caustic } \\
\text { leaching: } \\
\text { multiple } \\
\text { samples } \\
\text { taken at } \\
\text { three } \\
\text { temperatu } \\
\text { res }(60 \text {, } \\
80 \text {, and } \\
\left.100^{\circ} \mathrm{C}\right) \\
\text { and two } \\
{[\mathrm{OH}](\sim 1,} \\
-3 \text {, and } \\
\sim 5 \mathrm{M})\end{array}$ & - & $\begin{array}{l}\text { Non-Complexed Waste. }{ }^{1} \\
\text { Primary: High-level } \\
\text { REDOX waste. } \\
\text { Secondary: EB. } \\
\text { Tertiary: mixture of } \\
\text { several miscellaneous } \\
\text { wastes. }\end{array}$ & 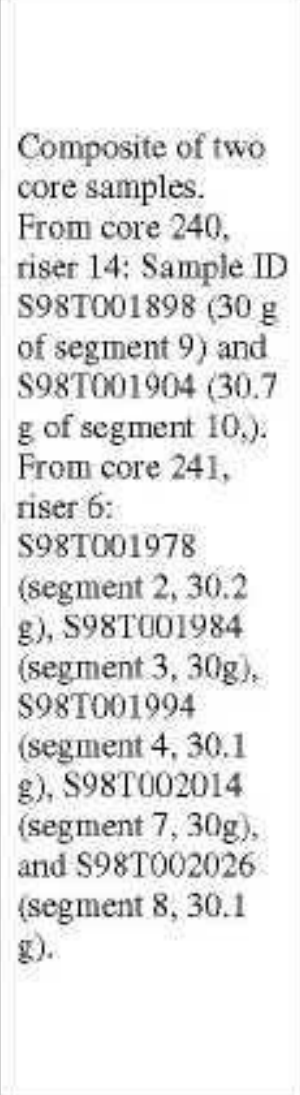 & $\begin{array}{l}\text { Transfer } 211 \mathrm{~g} \text { composite to } \\
500 \mathrm{~mL} \text { HDPE bottle with } \\
0.01 \mathrm{M} \mathrm{NaOH} \text {, adding to } \\
\text { total vol. of } 400 \mathrm{~mL} \text {. Shake } \\
\text { overnight. Stand } 2 \text { hr. Split } \\
\text { between } 2 \text { centrifuge bottles } \\
\text { and centrifuge } 20 \text { min at } \\
1200 \mathrm{G} \text {. Decant wash liquor } \\
\text { and save. Transfer solids to } \\
500 \mathrm{~mL} \text {. bottle and repeat for } \\
\text { second wash. For third } \\
\text { wash, conduct in centrifuge } \\
\text { bottles. Add } 0.01 \mathrm{M} \mathrm{NaOH} \\
\text { to each centrifuge bottle to } \\
\text { yield } 175 \mathrm{~mL} \text { total volume. } \\
\text { Shake overnight \& } \\
\text { centrifuge. Decant wash } \\
\text { liquor and combine all } \\
\text { together. }\end{array}$ & - & $\begin{array}{l}\text { Total mass } \\
\text { of washed } \\
\text { solids = } \\
74.96 \mathrm{~g} . \\
3.25 \mathrm{E}+5 \mathrm{ug} \\
\mathrm{Al} / \mathrm{g} \text {. } \\
24362000 \\
\text { ug Al }\end{array}$ & $\begin{array}{l}\text { Total mass of } \\
\text { combined } \\
\text { wash solution } \\
=764.7 \mathrm{~g} \text {. } \\
1410 \mathrm{ug} \mathrm{Al} / \mathrm{g} \text {. } \\
1077863 \mathrm{ug} \\
\mathrm{Al} \text {. }\end{array}$ & $\begin{array}{l}4 \% \\
\text { removed }\end{array}$ & - & 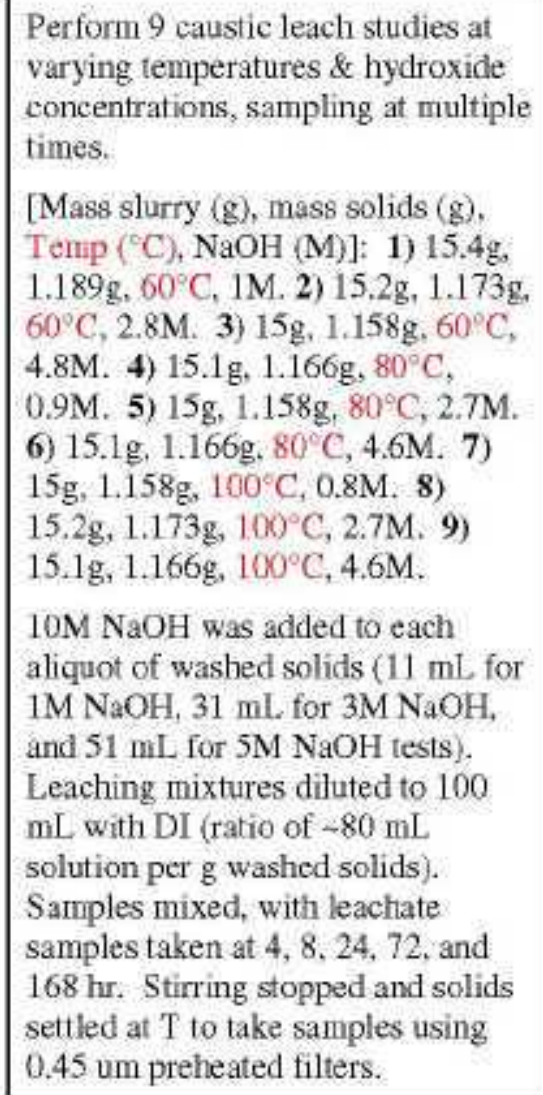 \\
\hline
\end{tabular}


Table A1-1. SSTs and DSTs Wash and Leach Information for Aluminum, Part 1. (38 sheets)

\begin{tabular}{|c|c|c|c|c|c|c|c|c|c|c|c|c|c|}
\hline Tank & $\begin{array}{l}\text { Lab Study } \\
\text { Reference(s) }\end{array}$ & $\begin{array}{l}\text { Year of } \\
\text { Sampling } \\
\text { Event }\end{array}$ & Test type & $\begin{array}{l}\text { Sample } \\
\text { color }\end{array}$ & Waste Sources & Sample ID & $\mathrm{NaOH}$ Wash Procedure & $\begin{array}{l}\text { Initial } \mathrm{Al} \text { in } \\
\text { Untreated } \\
\text { Solids }\end{array}$ & $\begin{array}{l}\text { Final Al in } \\
\text { Washed } \\
\text { solids }\end{array}$ & $\begin{array}{l}\text { Al measured in } \\
\text { Wash Solution }\end{array}$ & $\begin{array}{c}\% \\
\text { recovery }\end{array}$ & comments & Caustic Leach \#1 Procedure \\
\hline $241-S-110$ & $\begin{array}{l}\text { PNNL- } \\
14018\end{array}$ & FY01 & $\begin{array}{l}\text { Lab scale } \\
\text { caustic } \\
\text { plus } \\
\text { oxidant } \\
\text { leaching }\end{array}$ & - & $\begin{array}{l}\text { Primarily REDOX } \\
\text { waste, with secondary } \\
\text { stream from EB and } \\
\text { tertiary stream composed } \\
\text { as a mixture of several } \\
\text { miscellaneous wastes. } \\
\text { From cluster analysis, } \\
\text { contains saltcake from } \\
242 \mathrm{~A} \text { campaign (1976- } \\
1980 \text { and } 1981-1988 \text { ), } \\
\text { saltcake from the } 242-\mathrm{B} \\
\text { operation (1951-1953), } \\
\text { saltcake from the 242-S } \\
\text { campaign (1973-1976), } \\
\text { saltcake from self } \\
\text { condensation, REDOX } \\
\text { waste, and saltcake from } \\
\text { the 242-S campaign } \\
\text { (1977-1980). } \\
\text { Non-Complexed Waste. }\end{array}$ & $\begin{array}{l}\text { Composite of two } \\
\text { core samplcs. } \\
\text { From core 240, } \\
\text { riser 14: Sample ID } \\
\text { S98T001898 (30 g } \\
\text { from segment 9) } \\
\text { and s98T001904 } \\
\text { (30.7 g from } \\
\text { segment 10j. From } \\
\text { core 241, riser 6: } \\
\text { S98T001978 } \\
\text { (segment 2, 30.2 } \\
\text { g) S98T001984 } \\
\text { (segment 3, 30g), } \\
\text { S98T001994 } \\
\text { (segment 4, 30.1), } \\
\text { S98T002014 } \\
\text { (segment 7, 30g), } \\
\text { and S98T002026 } \\
\text { (scgment 8,30.1 } \\
\text { g). }\end{array}$ & $\begin{array}{l}\text { Transfer } 211 \mathrm{~g} \text { composite to } \\
500 \mathrm{~mL} \text { HDPE bottle. } \\
\text { Contact } 3 \text { times with fresh } \\
\text { portions of } 0.01 \mathrm{M} \mathrm{NaOH} \text {, } \\
\text { with the supemate removed } \\
\text { after each contact. Final } \\
\text { slurry prepared by adding } \\
\text { final portion of } 0.01 \mathrm{M} \\
\text { NaOH to washed solids. } \\
\text { Two weighed aliquots were } \\
\text { removed and dried at } 105^{\circ} \mathrm{C} \\
\text { to constant wt. }(7.72 \mathrm{wt} \% \\
\text { insoluble solids). }\end{array}$ & - & $\begin{array}{l}325000 \text { ug } \\
\mathrm{Al} / \mathrm{g} \text { dried } \\
\text { solids }\end{array}$ & - & - & $\begin{array}{l} \\
\\
\text { Study mostly } \\
\text { concerning Cr } \\
\text { removal using } \\
\text { various oxidants, } \\
\text { temperatures, and } \\
\text { OH } \\
\text { concentrations. }\end{array}$ & 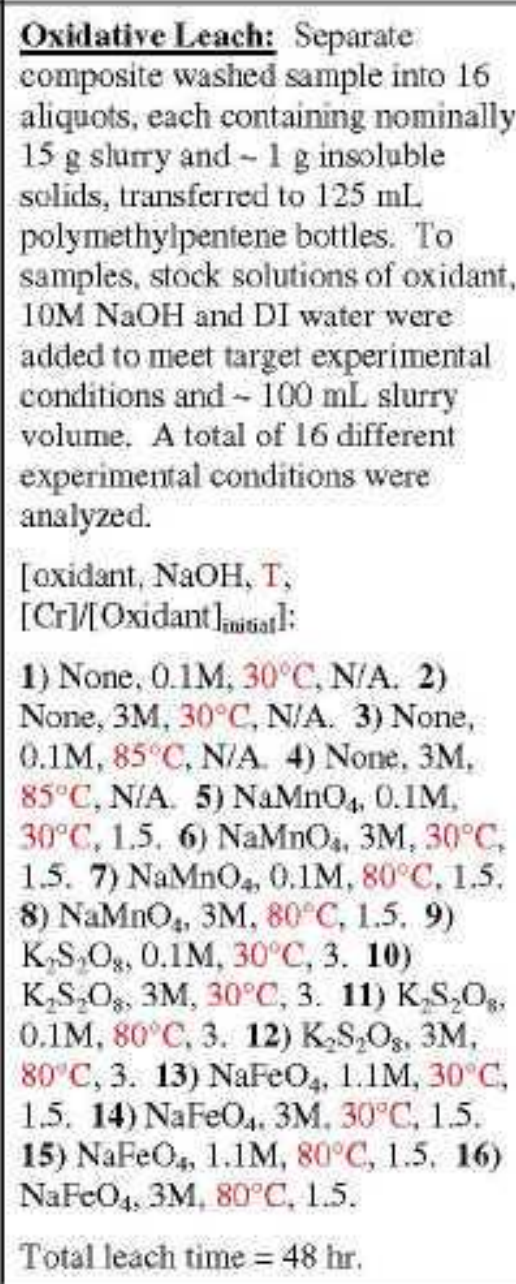 \\
\hline 241-S-111 & $\begin{array}{l}\text { PNNL- } \\
11636\end{array}$ & FY97 & $\begin{array}{l}\text { Lab scale } \\
\text { caustic } \\
\text { leaching }\end{array}$ & - & $\begin{array}{l}\text { Non-Complexed Waste. }{ }^{1} \\
\text { Primary: High-Level } \\
\text { REDOX, Secondary: } \\
\text { EB. }^{2.5}\end{array}$ & $\begin{array}{l}\text { 222-S Lab ID } \\
\text { S96T005106; Jar \# } \\
\text { 11150; sludge } \\
\text { received Dec. } 5 \text {, } \\
1996 \text {. }\end{array}$ & $\begin{array}{l}\text { Wash } 3 \text { times by adding } 20 \\
\text { ml water to } 9.365 \mathrm{~g} \text { slurry; } \\
\text { mix; centrifiuge; take } 0.2 \mathrm{~mL} \\
\text { sample; mix } 1 \text { hr at } 100^{\circ} \mathrm{C} \text {; } \\
\text { cool; centrifuge; decant; * } \\
\text { add } 30 \mathrm{~mL} 0.01 \mathrm{M} \mathrm{NaOH} \text {; } \\
\text { mix } 1 \mathrm{hr} \text { at } 100^{\circ} \mathrm{C} \text {; cool; } \\
\text { centrifuge; decant; repeat } \\
\text { from * with solids portion. }\end{array}$ & $\begin{array}{l}\text { In untreated } \\
\text { sludge- } \\
160215 \mathrm{ug} / \mathrm{g} \\
\text { wet sludge. }\end{array}$ & - & $\begin{array}{l}141897 \text { ug. } \\
9 \% \text { Al in } \\
\text { initial wash } \\
\text { step before } \\
\text { leaching. }\end{array}$ & - & - & $\begin{array}{l}\text { Using solids from wash step, add } \\
5.5 \mathrm{~mL} 10 \mathrm{M} \mathrm{NaOH} \text { and } 13.5 \mathrm{~mL} \\
\text { water; mix } 5 \mathrm{hr} \text { at } 100^{\circ} \mathrm{C} \text {; coopl; } \\
\text { centrifuge; take } 0.1 \mathrm{~mL} \text { sample; add } \\
0.53 \mathrm{~mL} 10 \mathrm{M} \mathrm{NaOH} \text {; mix } 5 \mathrm{hr} \text { at } \\
100^{\circ} \mathrm{C} \text {; cool; centrifuge; take } 0.5 \\
\mathrm{~mL} \text { supernate and } 0.1 \mathrm{~mL} \text { aliquot; } \\
\text { mix } 133 \mathrm{hr} \text { at } 1000^{\circ} \mathrm{C} \text {; cool; } \\
\text { centrifuge; decant. }\end{array}$ \\
\hline
\end{tabular}


Table A1-1. SSTs and DSTs Wash and Leach Information for Aluminum, Part 1. (38 sheets)

\begin{tabular}{|c|c|c|c|c|c|c|c|c|c|c|c|c|c|}
\hline Tank & $\begin{array}{c}\text { Lab Study } \\
\text { Reference(s) }\end{array}$ & $\begin{array}{l}\text { Year of } \\
\text { Sampling } \\
\text { Event }\end{array}$ & Test type & $\begin{array}{l}\text { Sample } \\
\text { color }\end{array}$ & Waste Sources & Sample ID & $\mathrm{NaOH}$ Wash Procedure & $\begin{array}{l}\text { Initial Al in } \\
\text { Untreated } \\
\text { Solids }\end{array}$ & $\begin{array}{l}\text { Final Al in } \\
\text { Washed } \\
\text { solids }\end{array}$ & $\begin{array}{l}\text { Al measured in } \\
\text { Wash Solution }\end{array}$ & $\begin{array}{c}\% \\
\text { recovery }\end{array}$ & comments & Caustic Leach \#1 Procedure \\
\hline 241-SX-101 & $\begin{array}{l}\text { PNWD- } \\
3512 \text { (WTP- } \\
\text { RPT-117) }\end{array}$ & $\begin{array}{l}\text { Sample } \\
\text { shipped } \\
\text { to } \\
\text { PNWD } \\
\text { in July } \\
2003\end{array}$ & $\begin{array}{l}\text { Lab scale } \\
\text { caustic } \\
\text { leaching }\end{array}$ & $\begin{array}{l}\text { Composit } \\
\text { e of black } \\
\text { slurry } \\
\text { sludge- } \\
\text { containing } \\
\text { segments }\end{array}$ & $\begin{array}{l}\text { Dilute Complexed } \\
\text { Waste. }{ }^{1}\end{array}$ & $\begin{array}{l}\text { Approximately } 75 \mathrm{~g} \\
\text { from Core 225, } \\
\text { Sample ID } \\
\text { S03T001373, Jar } \\
13998 \text {. }\end{array}$ & $\begin{array}{l}\text { Transfer portion into } \\
\text { separate } 50 \mathrm{~mL} \\
\text { centrifugation cone. Wash } \\
2 \text { fimes with } 0.01 \mathrm{M} \mathrm{NaOH} \\
\text { so total volume was } 50 \mathrm{~mL} \text {. } \\
\text { Centrifuge and separate } \\
\text { solid/liquid. Transfer solid } \\
\text { to } 50 \text { mL centrifuge botles } \\
\text { and contact approximately } 5 \\
\text { times (until bulk of color } \\
\text { removed) with fresh } 0.01 \mathrm{M} \\
\mathrm{NaOH} \text { and a solution-to- } \\
\text { solid ratio of approximately } \\
5: 1 \text {. Decant supernate after } \\
\text { each contact and discard. } \\
\text { Prepare final slurry, adding } \\
\text { a portion of } 0.01 \mathrm{M} \mathrm{NaOH} \\
\text { to washed, centrifuged } \\
\text { solids. Remove two } \\
\text { weighed aliquots of stirred } \\
\text { suspension and dry to } \\
\text { constant weight at } 105^{\circ} \mathrm{C} \text {. } \\
\end{array}$ & - & $\begin{array}{l}229000 \mathrm{ug} / \mathrm{g} \\
\text { dried solid. }\end{array}$ & - & - & $\begin{array}{l}\text { Could not find } \\
\text { initial analyses of } \\
\text { elements in } \\
\text { untreated sample. }\end{array}$ & 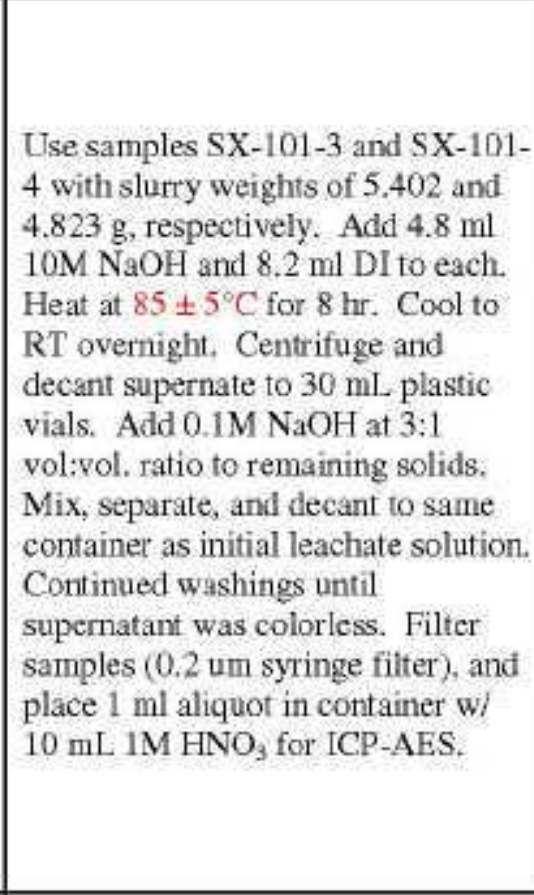 \\
\hline 241-SX-108 & $\begin{array}{l}\text { PNNL- } \\
11278\end{array}$ & FY96 & $\begin{array}{l}\text { Lab scale } \\
\text { caustic } \\
\text { leaching }\end{array}$ & - & $\begin{array}{l}\text { Primarily high-level } \\
\text { REDOX process waste: } \\
\text { Non-Complexed Waste. } \\
\text { Primarily high level } \\
\text { REDOX }^{2}\end{array}$ & $\begin{array}{l}\text { Auger Sample: } \\
\text { Upper half of auger } \\
\text { sample (222-S } \\
\text { LABCORE sample } \\
\text { \#S95T002574). }\end{array}$ & $\begin{array}{l}\text { Mix } 23.47 \mathrm{~g} \text { sample with } \\
47.32 \mathrm{~g} \text { water. With } 4.55 \mathrm{~g} \\
\text { slurry add } 10 \mathrm{~mL} 0.01 \mathrm{M} \\
\mathrm{NaOH} \text {. Mix at } 100^{\circ} \mathrm{C} \text { for } 1 \\
\text { hr, cool, centrifuge, and } \\
\text { decant. Repeat for a total of } \\
3 \text { washes. Dry solids after } \\
\text { washes at } 105^{\circ} \mathrm{C} \text {. } 1.506 \mathrm{~g} \\
\text { sludge initially in sample, } \\
0.618 \mathrm{~g} \text { washed solids and } \\
41 \text { w } \% \text { insoluble solids in } \\
\text { sludge. }\end{array}$ & $\begin{array}{l}9 \mathrm{wt} \% \\
\frac{\text { Summation: }}{1.3 \mathrm{E}+5 \mathrm{ug} / \mathrm{g} ;} \\
\frac{\text { Direct: }}{9.02 \mathrm{E}+4 \mathrm{ug} / \mathrm{g}}\end{array}$ & $\begin{array}{l}287000 \\
\text { ug/g; } \\
177366 \text { ug. } \\
\text { (94 wt\%) }\end{array}$ & $\begin{array}{l}372 \mathrm{ug} / \mathrm{mL} ; \\
11830 \mathrm{ug}(6 \\
\mathrm{wt}(\%)\end{array}$ & $\begin{array}{l}6 \% \\
\text { removed; } \\
\text { Total } \\
\text { mass = } \\
189196 \\
\text { ug; } 144 \% \\
\text { recovery. }\end{array}$ & - & $\begin{array}{l}\text { Wt. Sludge Solids in sample was } \\
20.22 \mathrm{~g} \text {. Add } 39 \mathrm{~mL} 10 \mathrm{M} \mathrm{NaOH} \\
\text { and water to a total volume of } 151 \\
\mathrm{~mL} \text { ( } 4.1 \text { wt } 15 \text { solids), mix at } 100^{\circ} \mathrm{C} \\
\text { for } 5 \mathrm{hr} \text {, cool, measure settling, and } \\
\text { decant. } 97 \mathrm{~mL} \text { decanted liquid, } 46 \\
\mathrm{~mL} \text { interstitial liquid, and } 2.7 \mathrm{M} \\
\mathrm{OH} \text {. }\end{array}$ \\
\hline
\end{tabular}


Table A1-1. SSTs and DSTs Wash and Leach Information for Aluminum, Part 1. (38 sheets)

\begin{tabular}{|c|c|c|c|c|c|c|c|c|c|c|c|c|c|}
\hline Tank & $\begin{array}{l}\text { Lab Study } \\
\text { Reference(s) }\end{array}$ & $\begin{array}{c}\text { Year of } \\
\text { Sampling } \\
\text { Event }\end{array}$ & Test type & $\begin{array}{l}\text { Sample } \\
\text { color }\end{array}$ & Waste Sources & Sample ID & $\mathrm{NaOH}$ Wash Procedure & $\begin{array}{l}\text { Initial } \mathrm{Al} \text { in } \\
\text { Untreated } \\
\text { Solids }\end{array}$ & $\begin{array}{l}\text { Final Al in } \\
\text { Washed } \\
\text { solids }\end{array}$ & $\begin{array}{l}\text { Al measured in } \\
\text { Wash Solution }\end{array}$ & $\begin{array}{c}\% \\
\text { tecovery }\end{array}$ & comments & Caustic Leach \#1 Procedure \\
\hline 241-SX-108 & $\begin{array}{l}\text { PNNL- } \\
11908\end{array}$ & FY98 & $\begin{array}{l}\text { Lab scale } \\
\text { caustic } \\
\text { plus } \\
\text { oxidant } \\
\text { leaching }\end{array}$ & - & $\begin{array}{l}\text { SOWRT Group IV. } \\
\text { Primary waste type is } \\
\text { high-level REDOX } \\
\text { process waste. } \\
\text { Non-Complexed Waste. } \\
\begin{array}{l}\text { Primary waste type was } \\
\text { high-level REDOX } \\
\text { waste. }\end{array}\end{array}$ & - & 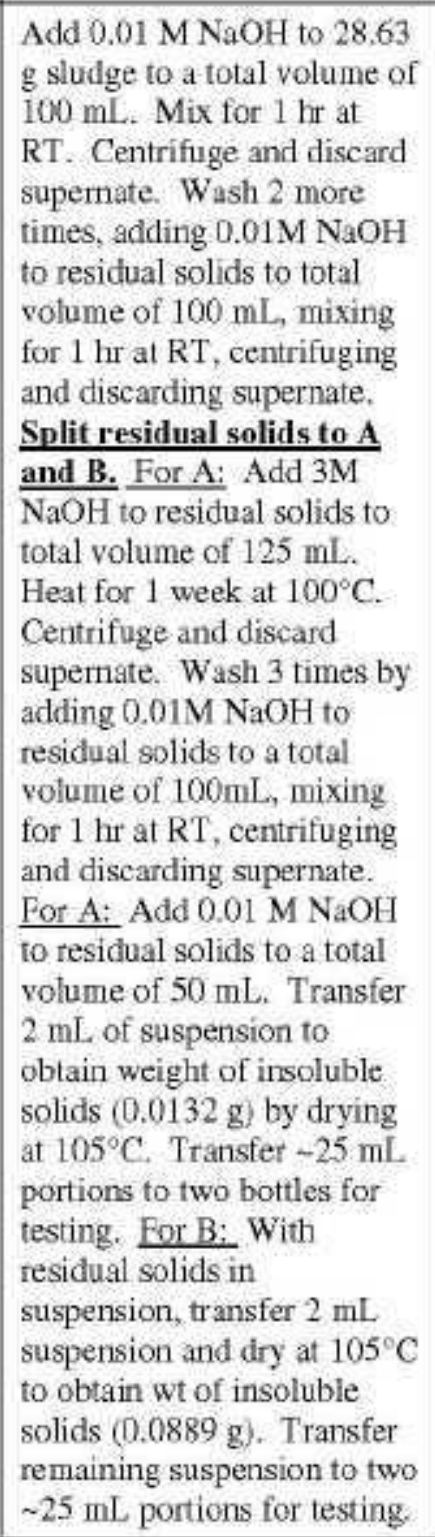 & - & $\begin{array}{l}270000 \text { ug } \\
\mathrm{Al} / \mathrm{g} \text { dry } \\
\text { solids }\end{array}$ & - & - & - & 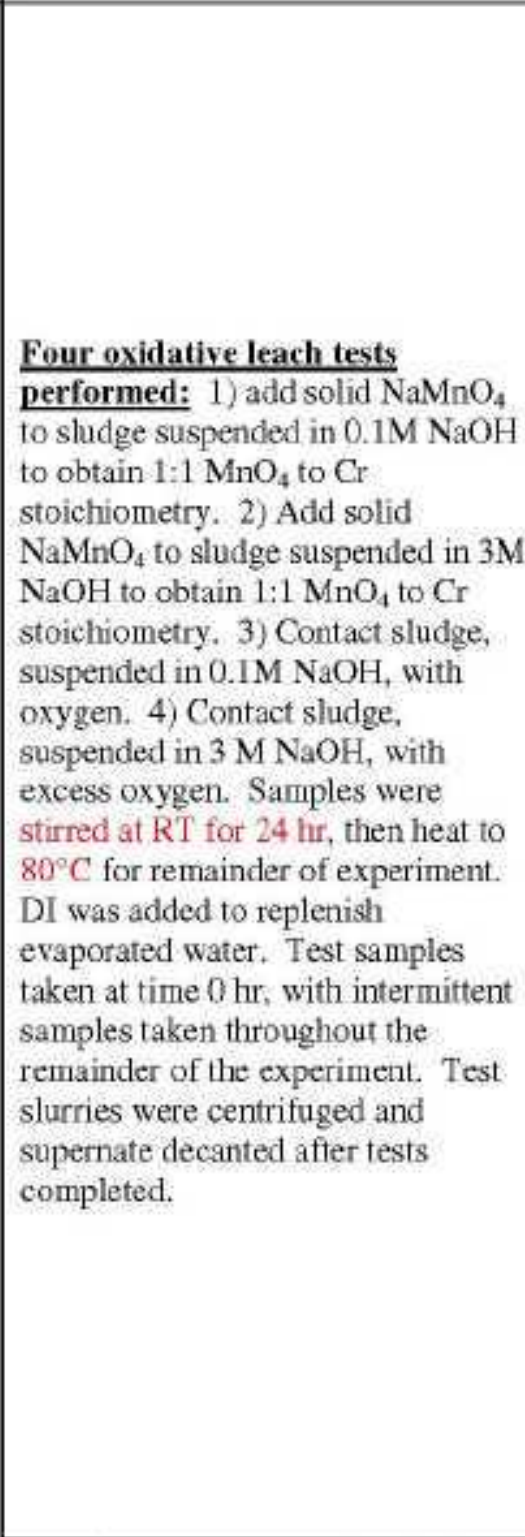 \\
\hline 241-SX-113 & $\begin{array}{l}\text { LAUR 97- } \\
2889\end{array}$ & FY97 & $\begin{array}{l}\text { Lab scale } \\
\text { caustic } \\
\text { leaching }\end{array}$ & $\begin{array}{l}\text { As- } \\
\text { received: } \\
\text { opaque } \\
\text { and tan in } \\
\text { color; } \\
\text { treated: } \\
\text { translucen } \\
\text { t and red } \\
\text { in color. }\end{array}$ & $\begin{array}{l}\text { Non-Complexed Waste. } \\
\text { Primary: High level } \\
\text { REDOX, Secondary: } \\
\text { Diatomaceous earth. }\end{array}$ & $\begin{array}{l}\text { 222-S Lab ID } \\
\text { S967001524, jar } \\
\text { \#9146 }\end{array}$ & $\begin{array}{l}\text { Mix } 5.81 \mathrm{~g} \text { wet sludge with } \\
11.6 \mathrm{~mL} \text { water, then use } 4.8 \\
\mathrm{~g} \text { sample for wash. Wash } 3 \\
\text { times by adding } 10 \mathrm{~mL} \\
0.01 \mathrm{M} \mathrm{NaOH} \text {; mix } 1 \mathrm{hr} \text { at } \\
100^{\circ} \mathrm{C} ; \text { cool; centrifuge; } \\
\text { decant. Had } 29.8 \mathrm{~mL} \\
\text { supernate and } 48.5 \text { wt\% } \\
\text { insoluble solids after dry at } \\
105^{\circ} \mathrm{C} \text {. }\end{array}$ & $2.1 \mathrm{w} \%$ & $\begin{array}{l}21419 \mathrm{ug} / \mathrm{g}, \\
16135 \mathrm{ug}\end{array}$ & $\begin{array}{l}2.1 \mathrm{ug} / \mathrm{mL}, \\
62.58 \mathrm{ug}\end{array}$ & \begin{tabular}{|l} 
Sum: \\
2.74E-2: \\
Direct: \\
$2.12 \mathrm{E}-2$; \\
$129.3 \%$ \\
mass \\
recovery.
\end{tabular} & - & 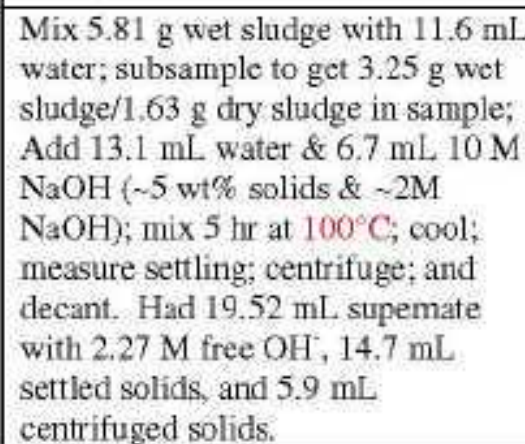 \\
\hline
\end{tabular}


Table A1-1. SSTs and DSTs Wash and Leach Information for Aluminum, Part 1. (38 sheets)

\begin{tabular}{|c|c|c|c|c|c|c|c|c|c|c|c|c|c|}
\hline Tank & $\begin{array}{c}\text { Lab Study } \\
\text { Reference(s) }\end{array}$ & $\begin{array}{l}\text { Year of } \\
\text { Sampling } \\
\text { Event }\end{array}$ & Test type & $\begin{array}{l}\text { Sample } \\
\text { color }\end{array}$ & Waste Sources & Sample ID & $\mathrm{NaOH}$ Wash Procedure & $\begin{array}{l}\text { Initial Al in } \\
\text { Untreated } \\
\text { Solids }\end{array}$ & $\begin{array}{l}\text { Final Al in } \\
\text { Washed } \\
\text { solids }\end{array}$ & $\begin{array}{l}\mathrm{Al} \text { measured in } \\
\text { Wash Solution }\end{array}$ & $\begin{array}{c}\% \\
\text { recovery }\end{array}$ & comments & Caustic Leach \#1 Procedure \\
\hline 241-SX-113 & $\begin{array}{l}\text { ORNL/TM- } \\
13500\end{array}$ & 1998 & $\begin{array}{l}\text { Lab scale } \\
\text { caustic } \\
\text { leaching: } \\
\text { two leach } \\
\text { tests at } 70 \\
\text { and } 80^{\circ} \mathrm{C} \\
\text { and } 6.33 \\
\text { M NaOH. }\end{array}$ & - & $\begin{array}{l}\text { REDOX waste } \\
\text { characterized as a low } \\
\text { Al, low Cr-bearing } \\
\text { sludge. } \\
\text { Non-Complexed Waste. }\end{array}$ & - & - & $\begin{array}{l}\text { l66000 ug/g; } \\
\text { Original } \\
\text { Sludge: } 7320 \\
\text { uggg (14000 } \\
\text { ug/g Table } \\
4.23 \text { ) }\end{array}$ & - & - & - & - & $\begin{array}{l}\text { Two leach tests performed: } 1) 1.46 \\
\text { g sludge; } 6.33 \mathrm{M} \text { (15 mL) NaOH; } \\
70^{\circ} \mathrm{C} ; 2 \mathrm{hr} ; 47.9 \mathrm{wr} \% \text { moisture; } 20 \\
\mathrm{~mL} \text { liquid/g golid. } \\
2 \text { 2) } 1.32 \mathrm{~g} \text { sludge; } 6.33 \mathrm{M}(30 \mathrm{~mL} \text { ) } \\
\mathrm{NaOH} ; 0^{\circ} \mathrm{C} ; 126 \mathrm{hr} ; 47.9 \mathrm{wt} \% \\
\text { moisture; } 44 \mathrm{~mL} \text { liquid/g solid. }\end{array}$ \\
\hline 241-T-104 & PNL-10712 & FY95 & $\begin{array}{l}\text { Lab scale } \\
\text { caustic } \\
\text { leaching }\end{array}$ & - & $\begin{array}{l}\text { Non-Complexed Waste. } \\
\text { Primary waste was } 1 C^{2,5}\end{array}$ & $\begin{array}{l}\text { Composite of } \\
\text { segments } 2 \text { through } \\
4 \text { from core } 46 \\
(222-\mathrm{S} \mathrm{Jar} \# 6555)\end{array}$ & $\begin{array}{l}\text { Add } 39.1 \mathrm{~g} \text { water to } 13.4 \mathrm{~g} \\
\text { T104-1, mix, and sample. } \\
\text { Using } 1.07 \mathrm{~g} \text { slurry, wash } 3 \\
\text { times by adding } 5 \text { ml } 0.01 \\
\mathrm{M} \mathrm{NaOH} / 0.01 \mathrm{M} \mathrm{NaNO} \text { to } \\
\text { slurry; mix } 0.5 \mathrm{hr} \text { at RT; } \\
\text { centrifuge; decant; repeat } \\
\text { with solids portion. Dry } \\
\text { remaining solids at } 80^{\circ} \mathrm{C} \\
(0.035 \mathrm{~g} \text { solid remained). }\end{array}$ & - & - & - & $=$ & $\begin{array}{l}\text { Retrieval Wash } \\
\text { prior to caustic } \\
\text { leach: add } 37.2 \mathrm{~g} \\
\text { water and } 0.97 \mathrm{~g} \\
1 \mathrm{M} \mathrm{NaOH} 1 \mathrm{M} \\
\mathrm{NaNO}_{\text {, to }} 4.18 \mathrm{~g} \\
\text { dry solids in slurry. } \\
\text { Mix } 1 \text { hr at } 100^{\circ} \mathrm{C} \text {, } \\
\text { cool, measure } \\
\text { settling, centrifuge, } \\
\text { decant. }\end{array}$ & $\begin{array}{l}\text { Add } 5.7 \mathrm{~g} \text { water and } 3 \mathrm{gg} 10 \mathrm{M} \mathrm{NaOH} \\
\text { to } 7.3 \mathrm{~mL} \text { centrifuged solids; mix } 5 \\
\text { hr at } 100^{\circ} \mathrm{C} \text {; cool; measure settling; } \\
\text { centrifuge; and decant. }\end{array}$ \\
\hline 241-T-104 & $\begin{array}{l}\text { PNNL- } \\
11089\end{array}$ & FY95 & $\begin{array}{l}\text { Sludge } \\
\text { washing } \\
\text { and } \\
\text { caustic } \\
\text { leaching }\end{array}$ & - & Primarily $1 C$ waste. & Core \#46 & $\begin{array}{l}\text { Add water to sludge sample } \\
\text { to get } \sim 2.3 \text { wt } \% \text { solids, as } \\
\text { well as inhibited water } \\
\text { (NaOH/NaNO} \text { ) to } 0.01 \mathrm{M} \text {. } \\
\text { Mix at } 100^{\circ} \mathrm{C} \text { for } 1 \mathrm{hr} \text {. } \\
\text { Cool, centrifuge, and } \\
\text { decant. }\end{array}$ & - & - & $\begin{array}{l}\text { uncorrected/ } \\
\text { corrected } \\
\text { recovery = } \\
1 / 1 \% \text { \% }\end{array}$ & - & - & $\begin{array}{l}\text { Caustic leach of solids: Add } \mathrm{NaOH} \\
\text { to get } \sim 8 \text { wt } \% \text { solids and a final } \\
\mathrm{NaOH} \text { of } 0.3 \mathrm{M} \text {. Mix at } 100^{\circ} \mathrm{C} \text { for } 5 \\
\text { hr. Cool, centrifuge, and decant. }\end{array}$ \\
\hline 241-T-104 & $\begin{array}{l}\text { ORNLTM- } \\
13660\end{array}$ & $\begin{array}{l}\text { Report } \\
\text { issued in } \\
1998\end{array}$ & $\begin{array}{l}\text { Sludge } \\
\text { washing } \\
\text { and } \\
\text { caustic } \\
\text { leaching } \\
\text { at RT } \\
\left(-22^{\circ} \mathrm{C}\right) \\
-60^{\circ} \mathrm{C} \\
\text { and } ~ \\
5^{\circ} \mathrm{C}\end{array}$ & - & - & - & $\begin{array}{l}\text { Add } 10 \text { mL inhibited water } \\
\text { to initial sludge sample } \\
66.09 \mathrm{~g} \text { for } \mathrm{RT} ; 4.4 \mathrm{~g} \text { for } \\
\left.60^{\circ} \mathrm{C} \text {, and } 6.18 \mathrm{~g} \text { for } 95^{\circ} \mathrm{C}\right) \\
\text { and place on shaker at } \\
\text { appropriate temperaturc. } \\
\text { Mix for } 65 \text { min and } \\
\text { centrifuge for } 8 \text { min. }\end{array}$ & $\begin{array}{l}1.82 \mathrm{ug} \mathrm{Al} / \mathrm{g} \\
\text { solids }\end{array}$ & - & $\begin{array}{l}\text { T }=\text { RT: } 11.5 \\
\text { ug/mL: } \\
\mathrm{T}=60^{\circ} \mathrm{C}: 18.5 \\
\mathrm{ug} / \mathrm{mL} \text {. } \\
\mathrm{T}=95^{\circ} \mathrm{C}: \\
19.3 \mathrm{ug} / \mathrm{mL} .\end{array}$ & - & $\begin{array}{l}\text { Solids from wash } \\
\text { observed to have } \\
\text { three distinct layers } \\
\text { and a volume of }-4 \\
\text { mL. Liquid was } \\
\text { yellow. Solution } \\
\text { was filtered } \\
\text { through a } 0.45 \text { um } \\
\text { filter, sealed, and } \\
\text { stored overnight. }\end{array}$ & $\begin{array}{l}\text { Add } \sim 10 \mathrm{~mL}, 3 \mathrm{M} \mathrm{NaOH} \text { to samples. } \\
\text { Mix } 4 \text { hr } 46 \text { min. Cool slightly and } \\
\text { centrifuge. Decant liquid and filter } \\
\text { through } 0.45 \text { um filter. Solids } \\
\text { volume for samples } 1(\mathrm{RT})=-3.5 \\
\text { mL; sample } 2\left(60^{\circ} \mathrm{C}\right)=\sim 3 \mathrm{~mL} \text {; and } \\
\text { sample } 3\left(95^{\circ} \mathrm{C}\right)=-3 \mathrm{~mL} \text {. } \\
\text { Samples were sealed and stored } \\
\text { overnight. }\end{array}$ \\
\hline
\end{tabular}


Table A1-1. SSTs and DSTs Wash and Leach Information for Aluminum, Part 1. (38 sheets)

\begin{tabular}{|c|c|c|c|c|c|c|c|c|c|c|c|c|c|}
\hline Tank & $\begin{array}{l}\text { Lab Study } \\
\text { Reference(s) }\end{array}$ & $\begin{array}{l}\text { Year of } \\
\text { Sampling } \\
\text { Event }\end{array}$ & Test type & $\begin{array}{l}\text { Sample } \\
\text { color }\end{array}$ & Waste Sources & Sample ID & $\mathrm{NaOH}$ Wash Procedure & $\begin{array}{l}\text { Initial } \mathrm{Al} \text { in } \\
\text { Untreated } \\
\text { Solids }\end{array}$ & $\begin{array}{l}\text { Final Al in } \\
\text { Washed } \\
\text { solids }\end{array}$ & $\begin{array}{l}\mathrm{Al} \text { measured in } \\
\text { Wash Solution }\end{array}$ & $\begin{array}{c}\% \\
\text { recovery }\end{array}$ & comments & Caustic Leach \#1 Procedure \\
\hline $241-T-104$ & $\begin{array}{l}\text { LAUR 95- } \\
2070\end{array}$ & 1995 & $\begin{array}{l}\text { Lab scale } \\
\text { caustic } \\
\text { leaching }\end{array}$ & $\begin{array}{l}\text { Untreated: } \\
\text { yellowish } \\
\text { in color } \\
\text { and } \\
\text { showed } \\
\text { both } \\
\text { yellow } \\
\text { and white } \\
\text { particles } \\
\text { under a } \\
\text { stereoscop } \\
\text { e; Treated: } \\
\text { brownish } \\
\text { solution. }\end{array}$ & $\begin{array}{l}\text { 1C waste } \\
\text { Non-Complexed Waste. }{ }^{1}\end{array}$ & - & $\begin{array}{l}\text { Add } \mathrm{NaOH} / \mathrm{NaNO}_{2} \text { to } \\
0.01 \mathrm{M} \text { solution, mix at } \\
100^{\circ} \mathrm{C} \text { for } 1 \mathrm{hr} \text {, settle and } \\
\text { decant. }\end{array}$ & - & $\begin{array}{l}29 \mathrm{ug} / \mathrm{mL} \\
(0.94 \%)\end{array}$ & - & - & $\begin{array}{l}\text { Detection limit for } \\
\text { sludge was } 3.137 \\
\text { ug/g. }\end{array}$ & $\begin{array}{l}\text { Add } \mathrm{NaOH} \text { to } 3.2 \mathrm{M} \text {, mix at } 100^{\circ} \mathrm{C} \\
\text { for } 5 \mathrm{hr} \text {, settle and decant }\end{array}$ \\
\hline $241-T-107$ & $\begin{array}{l}\text { LAUR 95- } \\
2070\end{array}$ & 1995 & $\begin{array}{l}\text { Lab scale } \\
\text { caustic } \\
\text { leaching }\end{array}$ & $\begin{array}{l}\text { As } \\
\text { received } \\
\text { sample } \\
\text { was } \\
\text { completel } \\
\text { y dry and } \\
\text { composed } \\
\text { of dark } \\
\text { material } \\
\text { and white } \\
\text { granules. } \\
\text { Both } \\
\text { treated } \\
\text { and as- } \\
\text { recived } \\
\text { samples } \\
\text { contained } \\
\text { reddish/ } \\
\text { reddish- } \\
\text { brown } \\
\text { solutions } \\
\text { with } \\
\text { settleable } \\
\text { particles. }\end{array}$ & $\begin{array}{l}\text { Three significant types } \\
\text { of waste: } 1 \text { C, PUREX } \\
\text { CW, and TBP from the } \\
\text { TBP U-extraction } \\
\text { process at U Plant. } \\
\text { Non-Complexed Waste. }{ }^{1} \\
\text { Primary: } 1 \text { C. Secondarv: } \\
\text { CW. }^{2} \\
\text { Primary: TBP/FeCN- } \\
\text { scavenged waste. } \\
\text { Secondary. } 1 \text {. Tertiary: } \\
\text { CW. Other: IX. }\end{array}$ & - & $\begin{array}{l}\text { Add } \mathrm{NaOH} / \mathrm{NaNO}_{2} \text { to } \\
0.01 \mathrm{M} \text {, mix at } 100^{\circ} \mathrm{C} \text { for } 1 \\
\mathrm{hr} \text {, settle and decant. }\end{array}$ & - & $\begin{array}{l}150 \mathrm{ug} / \mathrm{mL} \\
(3.56 \%)\end{array}$ & - & - & - & $\begin{array}{l}\text { To solids, add } \mathrm{NaOH} \text { to } 3.2 \mathrm{M} \text {, mix } \\
\text { at } 100^{\circ} \mathrm{C} \text { for } 5 \mathrm{hr} \text {, settle and decant: }\end{array}$ \\
\hline
\end{tabular}


Table A1-1. SSTs and DSTs Wash and Leach Information for Aluminum, Part 1. (38 sheets)

\begin{tabular}{|c|c|c|c|c|c|c|c|c|c|c|c|c|c|}
\hline Tank & $\begin{array}{l}\text { Lab Study } \\
\text { Reference(s) }\end{array}$ & $\begin{array}{l}\text { Year of } \\
\text { Sampling } \\
\text { Event }\end{array}$ & Test type & $\begin{array}{l}\text { Sample } \\
\text { color }\end{array}$ & Waste Sources & Sample ID & $\mathrm{NaOH}$ Wash Procedure & $\begin{array}{l}\text { Initial } \mathrm{Al} \text { in } \\
\text { Untreated } \\
\text { Solids }\end{array}$ & $\begin{array}{l}\text { Final Al in } \\
\text { Washed } \\
\text { solids }\end{array}$ & $\begin{array}{l}\text { Al measured in } \\
\text { Wash Solution }\end{array}$ & $\begin{array}{c}\% \\
\text { recovery }\end{array}$ & comments & Caustic Leach \#1 Procedure \\
\hline 241-T-110 & $\begin{array}{l}\text { PNNL- } \\
13956\end{array}$ & FY02 & $\begin{array}{l}\text { Lab scale } \\
\text { caustic } \\
\text { leaching: } \\
\text { nine leach } \\
\text { tests at } \\
\text { three } \\
\text { temperatu } \\
\text { res }(60 \text {, } \\
80, \& \\
\left.100^{\circ} \mathrm{C}\right) \\
\text { and three } \\
{[\mathrm{OH}](-1,} \\
-3, \text { and } \\
-4 \mathrm{M}) \text { with } \\
\text { samples } \\
\text { taken over } \\
\text { time. }\end{array}$ & - & $\begin{array}{l}\text { } \\
\text { Non-Complexed Waste. } \\
\text { Primary: 2C. Secondary. } \\
\text { lanthanum fluoride } \\
\text { decontamination waste. }\end{array}$ & 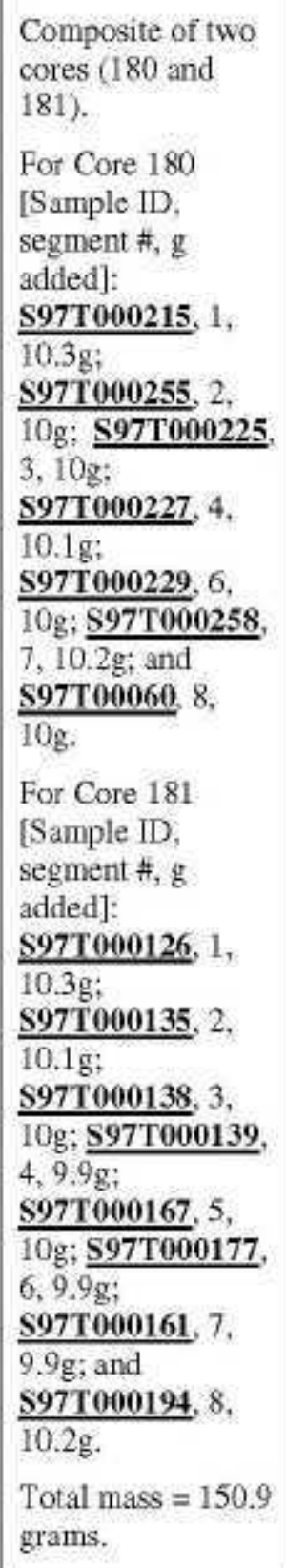 & 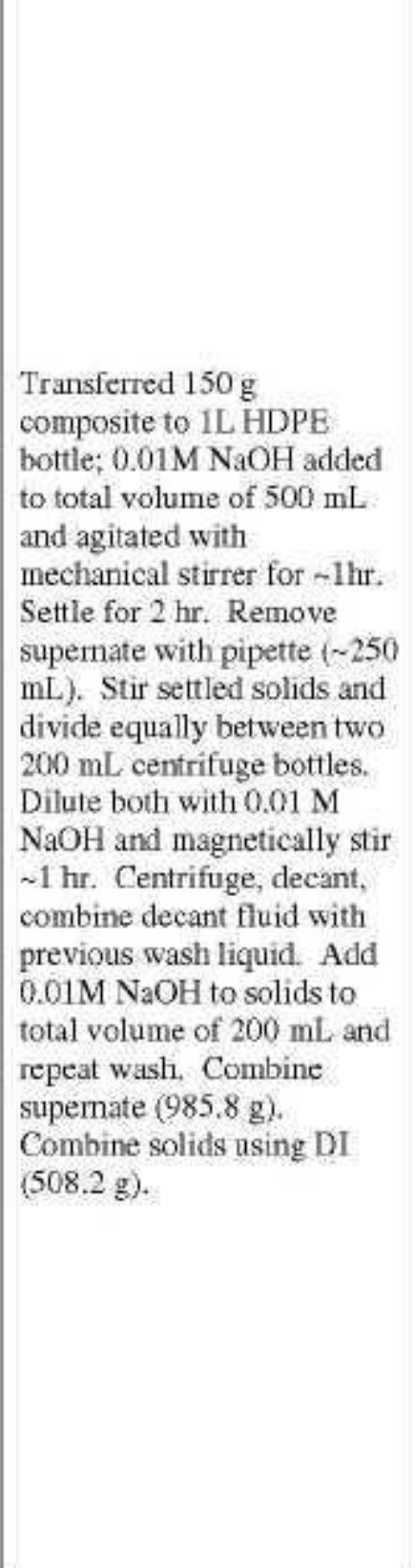 & - & $\begin{array}{l}1048 \mathrm{ug} / \mathrm{g} ; \\
44226 \mathrm{ug} \mathrm{Al}\end{array}$ & 0 & - & - & 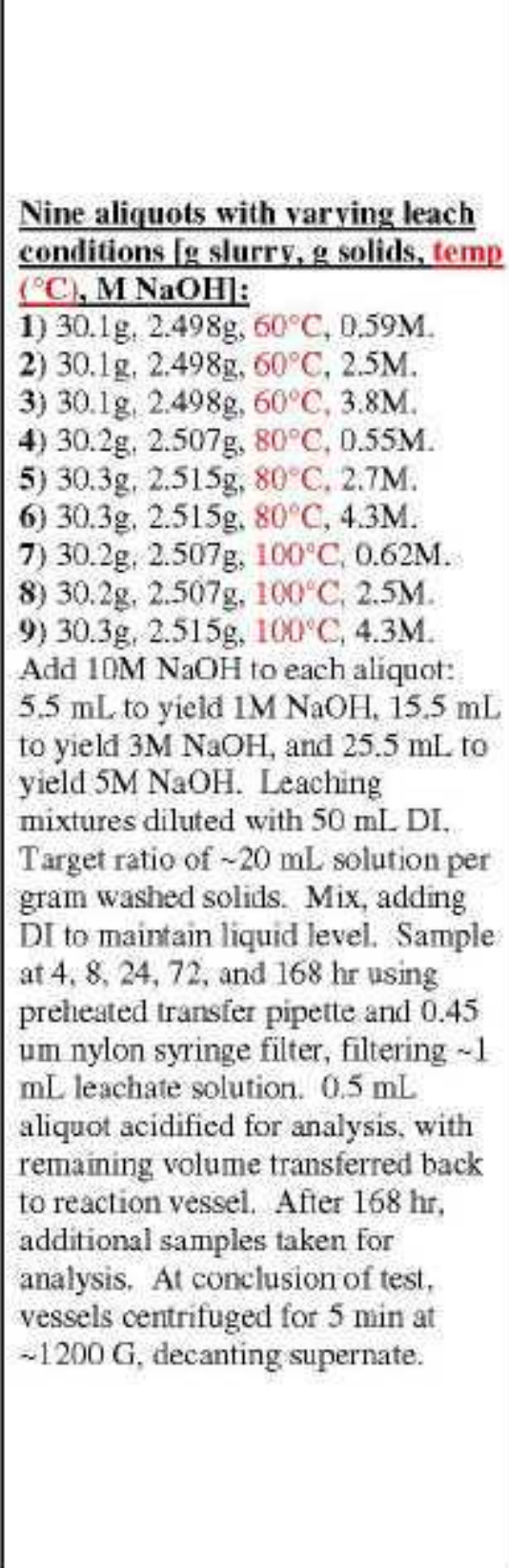 \\
\hline
\end{tabular}


Table A1-1. SSTs and DSTs Wash and Leach Information for Aluminum, Part 1. (38 sheets)

\begin{tabular}{|c|c|c|c|c|c|c|c|c|c|c|c|c|c|}
\hline Tank & $\begin{array}{l}\text { Lab Study } \\
\text { Reference(s) }\end{array}$ & $\begin{array}{l}\text { Year of } \\
\text { Sampling } \\
\text { Event }\end{array}$ & Test type & $\begin{array}{l}\text { Sample } \\
\text { color }\end{array}$ & Waste Sources & Sample ID & $\mathrm{NaOH}$ Wash Procedure & $\begin{array}{l}\text { Initial } \mathrm{Al} \text { in } \\
\text { Untreated } \\
\text { Solids }\end{array}$ & $\begin{array}{l}\text { Final Al in } \\
\text { Washed } \\
\text { solids }\end{array}$ & $\begin{array}{l}\text { Al measured in } \\
\text { Wash Solution }\end{array}$ & $\begin{array}{c}\% \\
\text { recovery }\end{array}$ & comments & Caustic Leach \#1 Procedure \\
\hline $241-\mathrm{T}-111$ & PNL-10712 & FY95 & $\begin{array}{l}\text { Lab scale } \\
\text { caustic } \\
\text { leaching }\end{array}$ & - & $\begin{array}{l}\text { Non-Complexed Waste. } \\
\text { Primary: } 2 \text { C, Secondary: } \\
\text { Lanthanum fluoride } \\
\text { decontamination } \\
\text { waste., }\end{array}$ & $\begin{array}{l}\text { Composite of } \\
\text { segments } 1 \text { and } 3 \\
\text { from core } 33 \text { (222- } \\
\text { S Jar \#6591). }\end{array}$ & 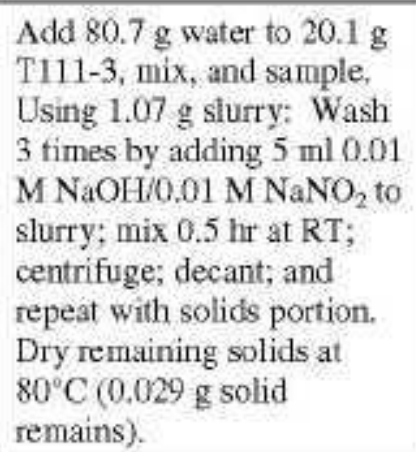 & - & - & - & - & $\begin{array}{l}\text { Retrieval Wash } \\
\text { prior to caustic } \\
\text { leach: add } 33.9 \mathrm{~g} \\
\text { water and } 1.2 \mathrm{~g} 1 \mathrm{M} \\
\text { NaOH/1 } 1 . \mathrm{MaNO} \\
\text { to } 4.27 \mathrm{gdry} \text { solids } \\
\text { in slurry. Mix } 1 \mathrm{~h} \\
\text { at } 100^{\circ} \mathrm{C} \text {, cool, } \\
\text { measure settling, } \\
\text { centrifuge, and } \\
\text { decant. }\end{array}$ & $\begin{array}{l}\text { Add } 7.8 \mathrm{~g} 10 \mathrm{M} \mathrm{NaOH} \text { to } 14 \mathrm{~mL} \\
\text { centrifuged solids; mix } 5 \mathrm{hr} \text { at } \\
100^{\circ} \text {; cool; measure settling; } \\
\text { centrifuge; and decant. }\end{array}$ \\
\hline 241-T-111 & $\begin{array}{l}\text { PNNL- } \\
11089\end{array}$ & FY95 & $\begin{array}{l}\text { Sludge } \\
\text { washing } \\
\text { and } \\
\text { caustic } \\
\text { leaching }\end{array}$ & - & $\begin{array}{l}\text { Primarily } 2 \mathrm{C} \text {, with } \\
\text { secondary waste from } \\
\text { lanthanum fluotide } \\
\text { decontamination waste } \\
\text { from the } \mathrm{BiPO}_{4} \text { process. }\end{array}$ & Core \# 33 & $\begin{array}{l}\text { Add water to sludge sample } \\
\text { to get } \sim 2.3 \text { wt } \% \text { solids, as } \\
\text { well as inhibited water } \\
\text { (NaOH/NaNO } \\
\text { Mix at } 100^{\circ} \mathrm{C} \text { for } 1 \mathrm{hr} \text { h. } \\
\mathrm{Cool} \text {, centrifuge, and } \\
\text { decant. }\end{array}$ & - & - & - & - & - & $\begin{array}{l}\text { Caustic leach of solids: Add } \mathrm{NaOH} \\
\text { to get } \sim 8 \mathrm{wt} \% \text { solids and a final } \\
\mathrm{NaOH} \text { of } 3.5 \mathrm{M} \text {. Mix at } 100^{\circ} \mathrm{C} \text { for } 5 \\
\text { hr. Cool, centrifuge, and decant. }\end{array}$ \\
\hline 241-TY-104 & $\begin{array}{l}\text { LAUR 96- } \\
2839\end{array}$ & FY95 & $\begin{array}{l}\text { Lab scale } \\
\text { caustic } \\
\text { leaching }\end{array}$ & $\begin{array}{l}\text { wet } \\
\text { reddish- } \\
\text { brown } \\
\text { paste }\end{array}$ & 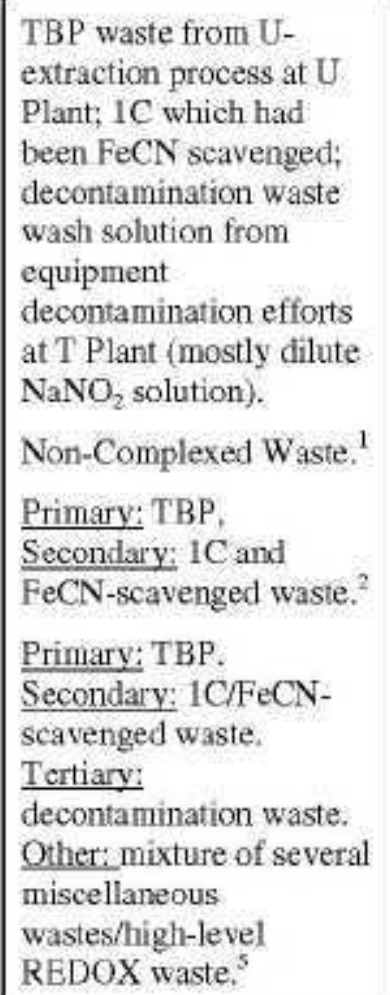 & - & $\begin{array}{l}\text { Mix } 11.4065 \mathrm{~g} \text { wet sludge } \\
\text { with } 45.6 \mathrm{~mL} \text { water; with } \\
\text { ImL of this sample, wash } 3 \\
\text { times by adding } 5 \mathrm{~mL} \text {. } 0.01 \\
\mathrm{M} \mathrm{NaOH/0.01} \mathrm{M} \mathrm{NaNO} \text {; } \\
\text { mix for } 0.5 \mathrm{hr} \text { at RT; } \\
\text { centrifuge, decant; and } \\
\text { repeat with solids. Dry } \\
\text { solids at } 80^{\circ} \mathrm{C} \text { to get } 0.0723 \\
\mathrm{~g} \text { or } 33.6 \mathrm{wt} \% \text { insoluble } \\
\text { solids. }\end{array}$ & $4.3 \mathrm{wt} \% \mathrm{Al}$ & - & - & $\begin{array}{l}9 \% \\
\text { removed } \\
\text { by simple } \\
\text { wash. }\end{array}$ & \begin{tabular}{|l} 
Mix $11.4441 \mathrm{~g}$ wet \\
sludge with $45 \mathrm{~mL}$ \\
water $(4.8916 \mathrm{~g}$ \\
dry). Retrieval \\
wash adding 32.4 \\
$\mathrm{ml}$ water and 0.78 \\
$\mathrm{~mL} 0.01 \mathrm{M}$ \\
$\mathrm{NaOH} / \mathrm{NaNO}_{2}$, mix \\
1 hr at $100 \mathrm{C}^{\circ}$, cool, \\
measure settling, \\
centrifuge, decant \\
and send solids to \\
caustic leach 1 .
\end{tabular} & $\begin{array}{l}\text { Add } 4 \mathrm{~mL} 10 \mathrm{M} \mathrm{NaOH} \text { and } 3.9 \mathrm{~mL} \text {. } \\
\text { water to solids (18 mL settled } \\
\text { solidss } 4.7 \mathrm{~mL} \text { centrifuged solids); } \\
\text { mix } 5 \mathrm{hr} \text { at } 100^{\circ} \mathrm{C} \text {; cool; measure } \\
\text { settling; centrifuge; and decant. }\end{array}$ \\
\hline
\end{tabular}


Table A1-1. SSTs and DSTs Wash and Leach Information for Aluminum, Part 1. (38 sheets)

\begin{tabular}{|c|c|c|c|c|c|c|c|c|c|c|c|c|c|}
\hline Tank & $\begin{array}{c}\text { Lab Study } \\
\text { Reference(s) }\end{array}$ & $\begin{array}{l}\text { Year of } \\
\text { Sampling } \\
\text { Event }\end{array}$ & Test type & $\begin{array}{l}\text { Sample } \\
\text { color }\end{array}$ & Waste Sources & Sample ID & $\mathrm{NaOH}$ Wash Procedure & $\begin{array}{l}\text { Initial } \mathrm{Al} \text { in } \\
\text { Untreated } \\
\text { Solids }\end{array}$ & $\begin{array}{l}\text { Final Al in } \\
\text { Washed } \\
\text { solids }\end{array}$ & $\begin{array}{l}\text { Al measured in } \\
\text { Wash Solution }\end{array}$ & $\begin{array}{c}\% \\
\text { recovery }\end{array}$ & comments & Caustic Leach \#1 Procedure \\
\hline 241-U-108 & $\begin{array}{l}\text { PNNL- } \\
11908\end{array}$ & FY98 & $\begin{array}{l}\text { Lab scale } \\
\text { caustic } \\
\text { plus } \\
\text { oxidant } \\
\text { leaching. }\end{array}$ & - & $\begin{array}{l}\text { SOWRT Group VI. } \\
\text { Primary waste type is } \\
\text { EB, with secondary } \\
\text { waste type being CW. } \\
\text { Non-Complexed Waste. } \\
\text { Primary: EB. Secondary: } \\
\begin{array}{l}\text { CW. Tertiary: mixture of } \\
\text { several miscellaneous } \\
\text { wastes. }\end{array}\end{array}$ & - & $\begin{array}{l}\text { Add } 0.01 \mathrm{M} \mathrm{NaOH} \text { to } 50 \mathrm{~g} \\
\text { sludge to a total volume of } \\
250 \mathrm{~mL} \text {. Mix for } 1 \mathrm{hr} \text { at } \\
\text { RT. Centrifuge and discard } \\
\text { supermate. Add } 0.01 \mathrm{M} \\
\text { NaOH to residual solids to a } \\
\text { total volume of } 150 \mathrm{~mL} \text {. } \\
\text { Mix for } 1 \mathrm{hr} \text { at RT. } \\
\text { Centrifuge and diseard } \\
\text { supenate. Addd } 0.01 \mathrm{M} \\
\mathrm{NaOH} \text { to residual solids to } \\
\text { total volume of } 150 \mathrm{~mL} \text {. } \\
\text { Mix for } 1 \mathrm{hr} \text { at RT. } \\
\text { Centrifuge and discard } \\
\text { supernate. Add } 0.01 \mathrm{M} \\
\text { NaOH to residual solids to } \\
\text { total volume of } 50 \mathrm{~mL} \text {. } \\
\text { Transfer } 2 \mathrm{~mL} \text { and dry at } \\
105^{\circ} \mathrm{C} \text { to obtain dry } \\
\text { insoluble solids weight } \\
60.5007 \mathrm{~g} \text {. Transfer }-4.5 \\
\mathrm{~mL} \text { portions to } 6 \text { bottles for } \\
\text { further testing. }\end{array}$ & - & $\begin{array}{l}42600 \text { ug } \\
\text { Algdry } \\
\text { solids }\end{array}$ & - & - & - & 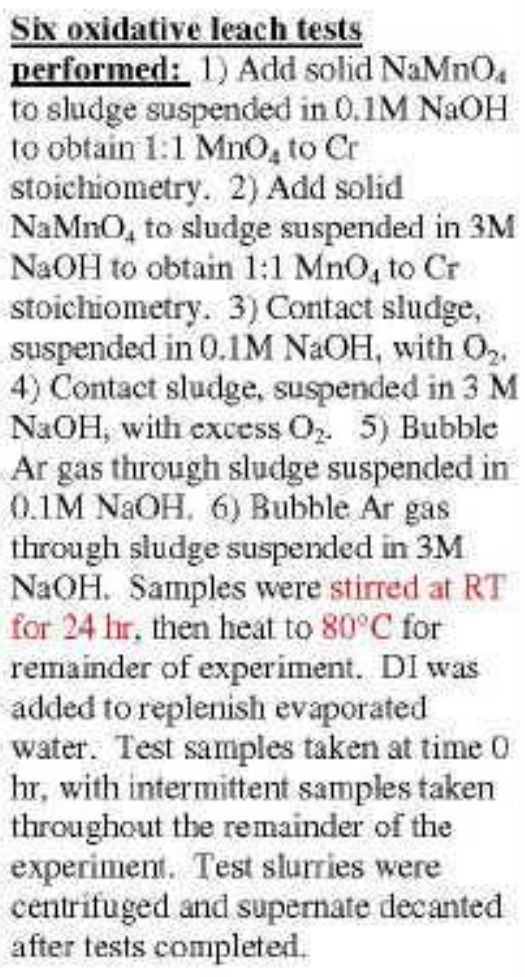 \\
\hline 241-U-108 & $\begin{array}{l}\text { PNNL- } \\
14019\end{array}$ & 2001 & $\begin{array}{l}\text { Labscale } \\
\text { caustic } \\
\text { plus } \\
\text { oxidant } \\
\text { leaching }\end{array}$ & - & $\begin{array}{l}\text { Tank waste group } 17 . \\
\text { Non-Complexed Waste. }{ }^{1}\end{array}$ & 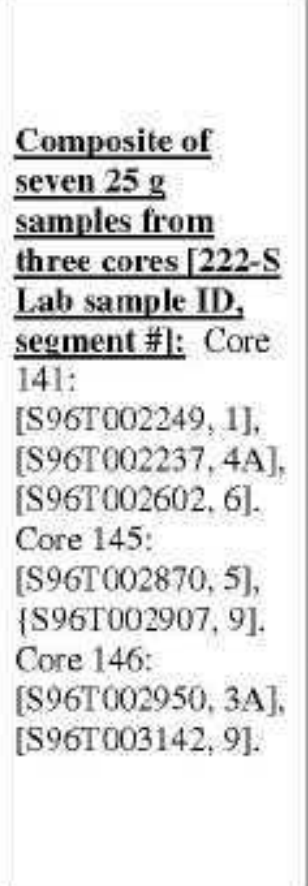 & $\begin{array}{l}\text { Transfer compositc to } 200 \\
\mathrm{~mL} \text { HDPE centrifuge bottles } \\
\text { and contacted } 4 \text { times with } \\
\text { fresh } 0.01 \mathrm{M} \mathrm{NaOH} \text { at initial } \\
\text { solution: solid ratio of } 3: 1 \text { to } \\
4: 1 \text {. After each contact, } \\
\text { decant and discard } \\
\text { supernate. After final wash, } \\
\text { prepared slurry by adding } \\
\text { final portion of } 0.01 \mathrm{M} \\
\mathrm{NaOH} \text { to washed solids. } \\
\text { Three weighed aliquots of } \\
\text { well-stirred suspension } \\
\text { removed and dried to } \\
\text { constant weight at } 105^{\circ} \mathrm{C} \text {. } \\
47.9 \mathrm{~g} \text { slurry remained. }\end{array}$ & - & $\begin{array}{l}3.63 \mathrm{~g}(7.75 \\
\text { wt } \%) \\
\text { insoluble } \\
\text { solids: } \\
126440 \mathrm{ug} \\
\mathrm{Al} / \mathrm{g} \text { dried } \\
\text { solids }\end{array}$ & - & - & - & 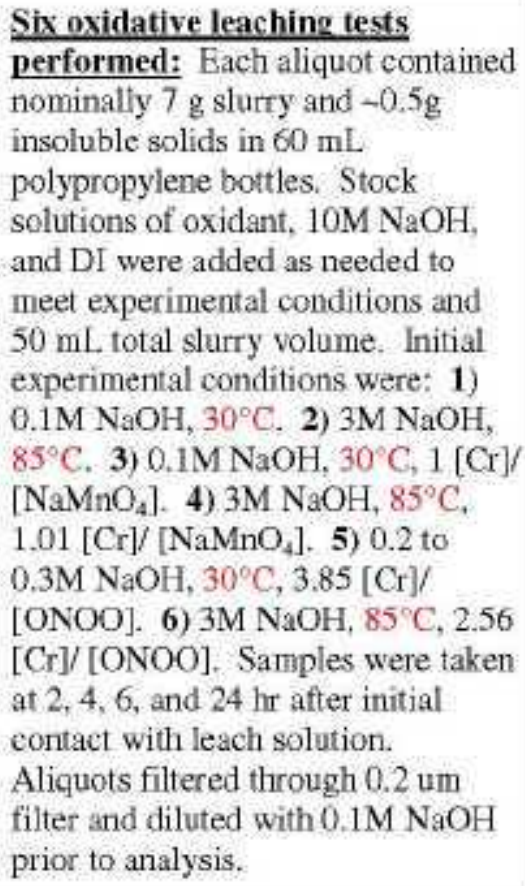 \\
\hline
\end{tabular}


Table A1-1. SSTs and DSTs Wash and Leach Information for Aluminum, Part 1. (38 sheets)

\begin{tabular}{|c|c|c|c|c|c|c|c|c|c|c|c|c|c|}
\hline Tank & $\begin{array}{c}\text { Lab Study } \\
\text { Reference(s) }\end{array}$ & $\begin{array}{l}\text { Year of } \\
\text { Sampling } \\
\text { Event }\end{array}$ & Test type & $\begin{array}{l}\text { Sample } \\
\text { color }\end{array}$ & Waste Sources & Sample ID & $\mathrm{NaOH}$ Wash Procedure & $\begin{array}{l}\text { Initial Al in } \\
\text { Untreated } \\
\text { Solids }\end{array}$ & $\begin{array}{l}\text { Final Al in } \\
\text { Washed } \\
\text { solids }\end{array}$ & $\begin{array}{l}\mathrm{Al} \text { measured in } \\
\text { Wash Solution }\end{array}$ & $\begin{array}{c}\% \\
\text { recovery }\end{array}$ & comments & Caustic Leach \#1 Procedure \\
\hline 241-U-109 & $\begin{array}{l}\text { PNNL- } \\
11908\end{array}$ & FY98 & $\begin{array}{l}\text { Lab scale } \\
\text { caustic } \\
\text { plus } \\
\text { oxidant } \\
\text { leaching. }\end{array}$ & - & $\begin{array}{l}\text { SOWRT Group VI. } \\
\text { Primary waste type is } \\
\text { EB, with secondary } \\
\text { waste type being CW. } \\
\text { Non-Complexed Waste. } \\
\text { Primary: EB. Secondary: } \\
\text { CW. Tertiarv: } \\
\text { RED igh-level } \\
\text { REX waste. }\end{array}$ & - & $\begin{array}{l}\text { Add } 0.01 \mathrm{M} \mathrm{NaOH} \text { to } 50 \mathrm{~g} \\
\text { sludge to a total volume of } \\
250 \mathrm{~mL} \text {. Mix for } 1 \mathrm{hr} \text { at } \\
\text { RT. Centrifuge and discard } \\
\text { supernate. Add } 0.01 \mathrm{M} \\
\text { NaOH to residual solids to a } \\
\text { total volume of } 150 \mathrm{~mL} \text {. } \\
\text { Mix for } 1 \mathrm{hr} \text { at RT. } \\
\text { Centrifuge and discard } \\
\text { supernate. Add } 0.01 \mathrm{M} \\
\text { NaOH to residual solids to } \\
\text { total volume of } 150 \mathrm{~mL} \text {. } \\
\text { Mix for } 1 \mathrm{hr} \text { at } \mathrm{RT} \text {. } \\
\text { Centrifuge and discard } \\
\text { supernate. Add } 0.01 \mathrm{M} \\
\text { NaOH to residual solids to } \\
\text { total volume of } 50 \mathrm{~mL} \text {. } \\
\text { Transter } 2 \mathrm{~mL} \text { and dry at } \\
105^{\circ} \mathrm{C} \text { to obtain dry } \\
\text { insoluble solids weight } \\
0.3163 \mathrm{~g} \text {. Transfer }-7 \mathrm{~mL} \text {. } \\
\text { portions to } 6 \text { bottles for } \\
\text { further testing }\end{array}$ & - & $\begin{array}{l}75100 \text { ug } \\
\text { Al/g dry } \\
\text { solids. }\end{array}$ & - & - & - & 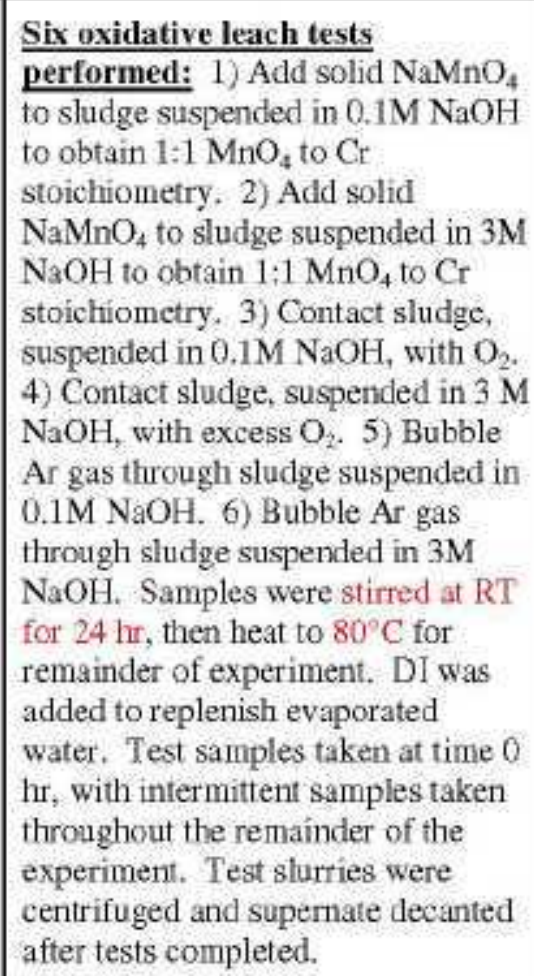 \\
\hline $241-\mathrm{U}-110$ & PNL-9387 & FY93 & $\begin{array}{l}\text { Lab scale } \\
\text { caustic } \\
\text { leaching }\end{array}$ & $\begin{array}{l}\text { Slurry of } \\
2.038 \mathrm{~g} \\
\text { sample }+ \\
5.306 \mathrm{~g} \\
0.1 \mathrm{M} \\
\mathrm{NaOH}\end{array}$ & $\begin{array}{l}\text { Non-Complexed Waste. } \\
\text { Primary: 1C. Secondary: } \\
\text { CW. }^{2} \\
\text { Primary: 1C. Secondary: } \\
\text { CW. Tertiary, high-level } \\
\text { REDOX waste. Other: } \\
\text { Laboratory waste from } \\
\text { 222-S building. }\end{array}$ & - & - & $\begin{array}{l}1.8 \mathrm{~g} \mathrm{Al} / \mathrm{g} \\
\text { sludge. }\end{array}$ & - & - & - & - & $\begin{array}{l}\text { Mix } 2.038 \mathrm{~g} \text { dry tank sludge with } \\
5.306 \mathrm{~g} 0.1 \mathrm{M} \mathrm{NaOH} \text {. using } 1.189 \mathrm{~g} \\
\text { for leach procedure. Add } 0.5 \mathrm{~mL} \text {. } \\
\text { water \& } 1.5 \mathrm{~mL} 10 \mathrm{M} \mathrm{NaOH} ; \text { stir \& } \\
\text { heat for } 1 \mathrm{hr} \text { at } 100^{\circ} \mathrm{C} \text {; cool: } \\
\text { centrifuge; decant; add } 3 \mathrm{~mL} .5 \mathrm{M} \\
\text { NaOH; stir \& heat } 5.5 \mathrm{hr} \text { at } 100^{\circ} \mathrm{C} \text {; } \\
\text { cool; centrifuge; and decant. }\end{array}$ \\
\hline 241-U-110 & PNL-10078 & FY94 & $\begin{array}{l}\text { Lab scale } \\
\text { caustic } \\
\text { leaching }\end{array}$ & - & \begin{tabular}{|l|} 
Neutralized $1 \mathrm{C}$ waste. \\
Other wastes include \\
REDOX process HLW, \\
CW, and lab waste from \\
the 222 -S building \\
(waste has high Al \\
content). \\
Non-Complexed Waste. \\
\end{tabular} & Core 14 & $\begin{array}{l}\text { Add } 2 \mathrm{~mL} 0.1 \mathrm{M} \mathrm{NaOH} \text { to } \\
0.57 \mathrm{~g} \text { dry siudge, mix at } \mathrm{RT} \\
\text { for } 1 \mathrm{hr} \text {, centrifuge, decant; } \\
\text { add } 2 \mathrm{~mL} \text { water to solids, } \\
\text { mix at } \mathrm{RT} \text { for } 0.5 \mathrm{hr} \text {, } \\
\text { centrifuge, and decant. }\end{array}$ & $\begin{array}{l}-20 \mathrm{wt} \% \\
2.1 \mathrm{E}-1 \mathrm{~g} \mathrm{Al} / \mathrm{g} \\
\text { dry sludge }\end{array}$ & - & $1 \%$ & - & $\begin{array}{l}\text { Dissolution of } \\
\text { solids using nitric } \\
\text { acid done after } \\
\text { final wash } \\
\text { following second } \\
\text { leach. }\end{array}$ & $\begin{array}{l}\text { Add } 4 \mathrm{~mL} 3 \mathrm{M} \mathrm{NaOH} / 2 \mathrm{M} \mathrm{Na}_{2} \mathrm{CO}_{3} \text { to } \\
\text { solids, mix at } 100^{\circ} \mathrm{C} \text { for } 5 \mathrm{hr} \text {, } \\
\text { centrifuge, and decant. }\end{array}$ \\
\hline
\end{tabular}


Table A1-1. SSTs and DSTs Wash and Leach Information for Aluminum, Part 1. (38 sheets)

\begin{tabular}{|c|c|c|c|c|c|c|c|c|c|c|c|c|c|}
\hline Tank & $\begin{array}{c}\text { Lab Study } \\
\text { Reference(s) }\end{array}$ & $\begin{array}{l}\text { Year of } \\
\text { Sampling } \\
\text { Event }\end{array}$ & Test type & $\begin{array}{l}\text { Sample } \\
\text { color }\end{array}$ & Waste Sources & Sample ID & $\mathrm{NaOH}$ Wash Procedure & $\begin{array}{l}\text { Initial Al in } \\
\text { Untreated } \\
\text { Solids }\end{array}$ & $\begin{array}{l}\text { Final Al in } \\
\text { Washed } \\
\text { solids }\end{array}$ & $\begin{array}{l}\text { Al measured in } \\
\text { Wash Solution }\end{array}$ & $\begin{array}{c}\% \\
\text { recovery }\end{array}$ & comments & Caustic Leach \#1 Procedure \\
\hline $241-\mathrm{U}-110$ & $\begin{array}{l}\text { PNNL- } \\
11779\end{array}$ & FY97 & $\begin{array}{l}\text { Lab scale } \\
\text { caustic } \\
\text { leaching }\end{array}$ & - & Non-Complexed Waste. ${ }^{1}$ & $\begin{array}{l}\text { Composite of } \\
\text { material taken from } \\
\text { seven different } \\
\text { core samplings of } \\
\text { the tank. }\end{array}$ & $\begin{array}{l}\text { Thorough wash using } \\
0.01 \mathrm{M} \text { NaOH to remove any } \\
\text { water-soluble components. } \\
\text { A sample was dried to } \\
\text { constant weight at } 105^{\circ} \mathrm{C} \text { for } \\
\text { analysis. }\end{array}$ & - & $\begin{array}{l}273000 \text { ug } \\
\text { Al/g dry } \\
\text { sludge } \\
\text { solids. }\end{array}$ & - & - & - & $\begin{array}{l}\text { Using } 12.858 \mathrm{~g} \text { slurry }(1.12 \mathrm{~g} \\
\text { selids), add } 10 \mathrm{~mL} 10 \mathrm{M} \mathrm{NaOH} \text { and } \\
5 \mathrm{~mL} \text { water. Mix at } 100^{\circ} \mathrm{C} \text { for } 85 \\
\text { hr. Cool, centrifuge, and decant. }\end{array}$ \\
\hline $\begin{array}{l}\text { Group 1 } \\
\text { Bismuth } \\
\text { Phosphate } \\
\text { Sludge (1C } \\
\text { and 2C) }\end{array}$ & $\begin{array}{l}\text { PNNL- } \\
17992 \\
\text { (WTP-RPT- } \\
\text { 166) }\end{array}$ & $\begin{array}{l}\text { N/A } \\
2006- \\
2009\end{array}$ & - & $\begin{array}{l}\text { As- } \\
\text { received } \\
\text { sample } \\
\text { colors: dry } \\
\text { white } \\
\text { crystals, } \\
\text { grey } \\
\text { pastes, } \\
\text { brownish } \\
\text { yellow } \\
\text { sludge, } \\
\text { yellow } \\
\text { supernate. }\end{array}$ & $\begin{array}{l}\text { Composite of } 31 \\
\text { samples. } 93 \% \text { from B- } \\
104,4 \% \text { from BX-112, } \\
\text { and } 3 \% \text { from T-104. } \\
\text { Solids passed through } \\
3.2 \mathrm{~mm} \text { sieve. } \\
\text { Additional } 742.97 \mathrm{~g} \text { DI } \\
\text { added during } \\
\text { compositing. }\end{array}$ & - & $\begin{array}{l}\text { Wash } 3 \text { times by mixing } \\
60 \mathrm{~mL} \text { aliquots of } 0.01 \mathrm{M} \\
\text { NaOH with olids for } 15 \\
\text { minutes, centrifuging at } \\
-1200 \mathrm{G} \text { for } 15 \text { min, and } \\
\text { decanting supernate } \\
\text { between subsequent washes. } \\
\text { After each wash step, the } \\
\text { centrifuged solids were } \\
\text { stratified in three layers: } \\
\text { white dense layer, darker } \\
\text { middle layer, and light } \\
\text { brown or tan top layer. }\end{array}$ & - & $\begin{array}{l}\text { By KOH } \\
\text { Fusion: } \\
\text { 26350 ug } \\
\text { Al/g. By } \\
\text { Acid } \\
\text { Digestion: } \\
28450 \text { ug } \\
\text { Al/g. }\end{array}$ & $\begin{array}{l}\text { By Acid } \\
\text { Digestion: } \\
\text { Supernatant = } \\
<3.77 \text { ug } / \mathrm{mL} \text {. } \\
\text { Wash } \\
\text { Composite } \\
<3.75 \text { ug } / \mathrm{mL} \text {. }\end{array}$ & - & $\begin{array}{l}\text { Washed solids } \\
\text { subdivided into } \\
\text { eight } 9.9 \mathrm{~g} \\
\text { washed samples for } \\
\text { caustic leaching } \\
\text { (-0.95 } \mathrm{g} \\
\text { undissolved } \\
\text { solids). Washed } \\
\text { and centrifuged } \\
\text { solids mixed with } \\
60 \mathrm{~mL} \text { DI (10g } \\
\text { solids in } 87 \mathrm{~g} \\
\text { slurry or } 11.5 \mathrm{wt} \% \\
\text { undissolved } \\
\text { solids). }\end{array}$ & $\begin{array}{l}\text { A total of } 8 \text { samples were leached at } \\
\text { different free hydroxide } \\
\text { concentrations (I to } 3 \mathrm{M} \mathrm{NaOH}) \\
\left.\text { and temperatures }(40,60 \mathrm{and}) 80^{\circ} \mathrm{C}\right) \text { ). } \\
\text { Solution samples were obtained at } \\
0,1,2,4,8 \text {, and } 24 \text { hours, with } \\
\text { obtained leachate filtered through a } \\
0.45 \text { um syringe filter. After final } \\
\text { samples removed, the slurries were } \\
\text { removed from heat and allowed to } \\
\text { cool to ambient temperature } \\
\left(-22^{\circ} \mathrm{C} \text {. Slurries were centrifuged }\right. \\
\text { and half the leachate was decanted. }\end{array}$ \\
\hline $\begin{array}{l}\text { Group 2 } \\
\text { Bismuth } \\
\text { Phosphate } \\
\text { Salteake } \\
\text { (BY and T } \\
\text { salteake) }\end{array}$ & $\begin{array}{l}\text { PNNL- } \\
\text { 17992 } \\
\text { (WTP-RPT- } \\
166 \text { ) }\end{array}$ & $\begin{array}{l}\text { N/A } \\
2006- \\
2009\end{array}$ & - & - & 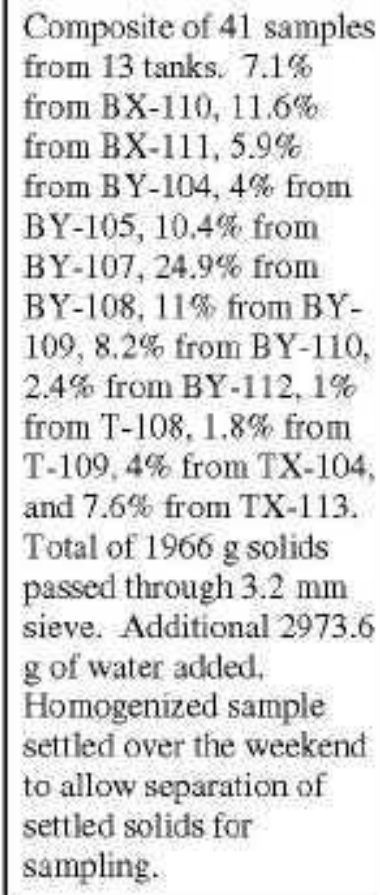 & - & $\begin{array}{l}\text { Remove a } 34.5 \mathrm{~g} \text { aliquot of } \\
\text { homogenized slurry and } \\
\text { transfer to } 200 \mathrm{~mL} \\
\text { centrifuge botlle }(0.29 \mathrm{~g} \text { dry } \\
\text { watet-insoluble solids/g } \\
\text { slurry). Centrifuge at } \\
-1200 \mathrm{G} \text { for } 15 \mathrm{~min} \text {. Wash } 3 \\
\text { times by mixing } 45 \mathrm{~mL} \\
\text { aliquots of } 0.01 \mathrm{M} \mathrm{NaOH} \\
\text { with solids for } 15 \mathrm{minutes} \\
\text { with an overhead mixer, } \\
\text { centrifuge at } \sim 1200 \mathrm{G} \text { for } 15 \\
\text { min, and decanting. } \\
\text { supernate between } \\
\text { subsequent washes. }\end{array}$ & - & $\begin{array}{l}\text { By KOH } \\
\text { Fusion: } \\
112500 \\
\text { ug/g. By } \\
\text { Acid } \\
\text { Digcstion: } \\
122500 \\
\text { ug/g. }\end{array}$ & $\begin{array}{l}\text { By Acid } \\
\text { Digestion: } \\
\text { Supernatant = } \\
2030 \text { ug/mL. } \\
\text { Wash } \\
\text { Composite } 322 \\
\text { ug/mL. }\end{array}$ & $\begin{array}{l}13.1 \% \text { in } \\
\text { supernate, } \\
1.1 \% \text { in } \\
\text { wash } \\
\text { solution, } \\
\text { and } 85.8 \% \\
\text { remained } \\
\text { with } \\
\text { solids. }\end{array}$ & $\begin{array}{l}\text { Add } 100 \mathrm{~mL} \text { DI to } \\
\text { washed solids and } \\
\text { subdivide into } \\
\text { seven } \sim 14 \mathrm{~g} \\
\text { washed samples for } \\
\text { caustic leaching } \\
(\sim 1 \text { g undissolved } \\
\text { solids). Average } \\
\text { Al in solids prior to } \\
\text { leaching = } 149500 \\
\text { ug/g. }\end{array}$ & $\begin{array}{l}\text { A total of } 7 \text { samples were leached at } \\
\text { different free hydroxide } \\
\text { concentrations ( } 1 \text { to } 5 \mathrm{M} \mathrm{NaOH}) \\
\text { and termperatures }(60,80 \text {, and } \\
100^{\circ} \mathrm{C} \text {. Solution samples were } \\
\text { obtained at } 0,1,2,4,8 \text {, and } 24 \\
\text { hours, with obtained leachate } \\
\text { fittered through a } 0.45 \text { um syringe } \\
\text { filter. After final samples removed, } \\
\text { the slurries were removed from heat } \\
\text { and allowed to cool to ambient } \\
\text { temperature }\left(-22^{\circ} \mathrm{C}\right) \text {. Slurries were } \\
\text { centififuged and half the leachate } \\
\text { was decanted. }\end{array}$ \\
\hline
\end{tabular}


Table A1-1. SSTs and DSTs Wash and Leach Information for Aluminum, Part 1. (38 sheets)

\begin{tabular}{|c|c|c|c|c|c|c|c|c|c|c|c|c|c|}
\hline Tank & $\begin{array}{l}\text { Lab Study } \\
\text { Reference(s) }\end{array}$ & $\begin{array}{l}\text { Year of } \\
\text { Sampling } \\
\text { Event }\end{array}$ & Test type & $\begin{array}{l}\text { Sample } \\
\text { color }\end{array}$ & Waste Sources & Sample ID & $\mathrm{NaOH}$ Wash Procedure & $\begin{array}{l}\text { Initial } \mathrm{Al} \text { in } \\
\text { Untreated } \\
\text { Solids }\end{array}$ & $\begin{array}{l}\text { Final Al in } \\
\text { Washed } \\
\text { solids }\end{array}$ & $\begin{array}{l}\mathrm{Al} \text { measured in } \\
\text { Wash Solution }\end{array}$ & $\begin{array}{c}\% \\
\text { recovery }\end{array}$ & comments & Caustic Leach \#1 Procedure \\
\hline $\begin{array}{l}\text { Group } 3 \\
\text { PUREX } \\
\text { Cladding } \\
\text { Waste } \\
\text { Sludge }\end{array}$ & $\begin{array}{l}\text { PNNL- } \\
18054 \\
\text { (WTP-RPT- } \\
\text { 167) }\end{array}$ & $\begin{array}{l}\text { N/A } \\
2006- \\
2009\end{array}$ & $\begin{array}{l}1: 100 \\
\text { solids to } \\
\text { solution } \\
\text { ratio } \\
\text { caustic } \\
\text { leaching } \\
\text { at various } \\
\text { T, [OH] } \\
\text { and } \\
{\left[\mathrm{NaNO}_{3}\right]} \\
\text { with } \\
\text { periodic } \\
\text { sampling } \\
\text { fromi } 0 \text { to } \\
48 \text { hours. }\end{array}$ & $\begin{array}{l}\text { Dark and } \\
\text { viscous } \\
\text { consistenc } \\
\text { y }\end{array}$ & $\begin{array}{l}15 \text { samples from } 6 \text { tanks: } \\
241-\mathrm{C}-103(14.06 \mathrm{~g} \\
3 \%), 241-\mathrm{B}-109(54.09 \\
\mathrm{g}, 11 \%), 241-\mathrm{C}-105 \\
(72.09 \mathrm{~g}, 14 \%), 241-\mathrm{B}- \\
108(57.39 \mathrm{~g}, 11 \%), 241- \\
\mathrm{C}-104(52.47 \mathrm{~g}, 10 \%), \\
241-\mathrm{BY}-109(259.14 \mathrm{~g} . \\
51 \%) \text {. Added Water = } \\
438 \mathrm{~g} \text {. }\end{array}$ & $\begin{array}{l}\text { 222-S IDs } \\
\text { available: } 6440, \\
11486,9765 \\
19798,11505 \\
11506,11507 \\
11244,11245 \\
11356,11366, \\
13043,13044, \\
19098, \text { and } 19134 \text {. }\end{array}$ & $\begin{array}{l}\text { Composite sieved through } \\
\text { stainless steel strainer and } \\
\text { mixed at } 29^{\circ} \mathrm{C} \text { for one hr } \\
\text { using overhead mixer to } \\
\text { homogenize. Wash } 3 \text { times } \\
\text { using approximately } 17.4 \\
\text { mL } 0.01 \mathrm{M} \text { NaOH. } \\
\text { Centrifuge and decant } \\
\text { supernate to composite } \\
\text { wash collection. }\end{array}$ & $\begin{array}{l}98.1 \text { wt\% Al. } \\
\text { In initial } \\
\text { slurry there } \\
\text { were } 311500 \\
\text { ug Al/g solids } \\
\text { and } 2035 \text { ug } \\
\text { Al/mL } \\
\text { aqueous } \\
\text { phase. }\end{array}$ & $\begin{array}{l}297500 \\
\text { ug/g } \\
\text { (Fusion), } \\
325500 \mathrm{ug} / \mathrm{g} \\
\text { (Acid } \\
\text { Digest). } \\
\text { Water } \\
\text { washed } \\
\text { insoluble } \\
\text { solids were } \\
-30 \text { wt\% Al. }\end{array}$ & $\begin{array}{l}\text { Supernate = } \\
2035 \text { ug } / \mathrm{mL} \text {. } \\
\text { Wash } \\
\text { composite = } \\
136 \text { ug } / \mathrm{mL} \text {. }\end{array}$ & - & $\begin{array}{l}\text { Wash prior to } \\
\text { caustic leach: } \\
\text { Centrifuge slurry } \\
\text { sample and remove } \\
\text { supernate to get } \\
\sim 15 \mathrm{~mL} \text { solids. } \\
\text { Add } 45 \mathrm{~mL} \\
0.01 \mathrm{M} \mathrm{NaOH} \text { and } \\
\text { shake on vortex } \\
\text { mixer for } 15 \\
\text { minutes. } \\
\text { Centrifuge for } 30 \\
\text { min and remove } \\
\text { supernate. Repeat } \\
\text { for a total of } 3 \\
\text { washes, } \\
\text { centrifuging the } \\
\text { last washed solids } \\
\text { for only } 20 \\
\text { minutes. }\end{array}$ & 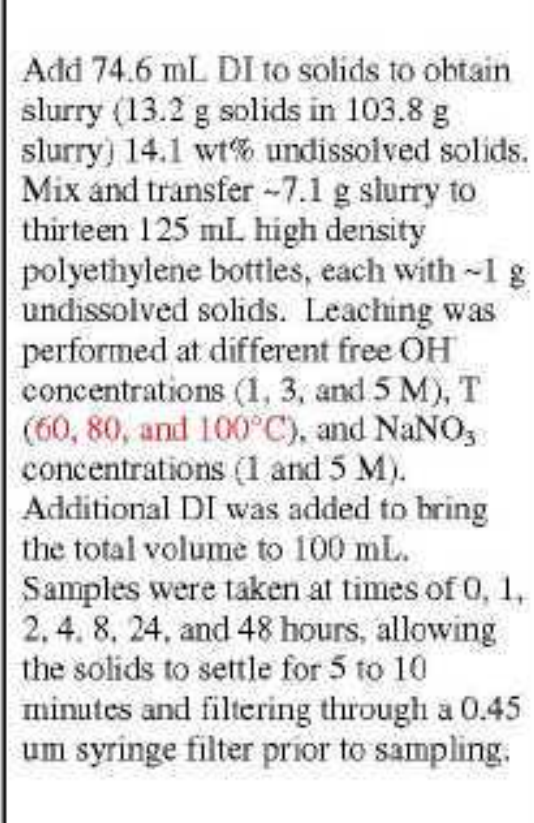 \\
\hline $\begin{array}{l}\text { Group 4 } \\
\text { REDOX } \\
\text { Cladding } \\
\text { Waste } \\
\text { Sludge }\end{array}$ & $\begin{array}{l}\text { PNNL- } \\
\text { 18054 } \\
\text { (WTP-RPT- } \\
\text { 167) }\end{array}$ & $\begin{array}{l}\text { N/A } \\
2006- \\
2009\end{array}$ & $\begin{array}{l}1: 100 \\
\text { solids to } \\
\text { solution } \\
\text { ratio } \\
\text { caustic } \\
\text { leaching } \\
\text { at various } \\
\mathrm{T} \text {, [OH] } \\
\text { and } \\
{[\mathrm{NaNO}} \\
\text { with } \\
\text { with } \\
\text { periodic } \\
\text { sampling } \\
\text { from 0 to } \\
48 \text { hours. }\end{array}$ & $\begin{array}{l}\text { Dry white } \\
\text { powders, } \\
\text { gray } \\
\text { solids, and } \\
\text { dark tar- } \\
\text { like } \\
\text { sludge } \\
\text { samples } \\
\text { with } \\
\text { yellow } \\
\text { supernate } \\
\text { liquid on } \\
\text { some } \\
\text { samples }\end{array}$ & $\begin{array}{l}14 \text { samples from } 5 \text { tanks: } \\
241-\mathrm{U}-105(191.68 \mathrm{~g} \\
34 \%), 241-\mathrm{U}-201(75.04 \\
\mathrm{g}, 13 \%), 241-\mathrm{U}-202 \\
(185.37 \mathrm{~g}, 32 \%), 241-\mathrm{U}- \\
203(24.24 \mathrm{~g}, 4 \%) \text {, and } \\
241-\mathrm{U}-204(95.69 \mathrm{~g}, \\
17 \%) \text {. Added Water = } \\
94 \mathrm{~g} .\end{array}$ & $\begin{array}{l}\text { 222-S IDs } \\
\text { available: } 19476 \text {, } \\
9702,9711,16961 \text {, } \\
6882,13462 \text {, } \\
19154,6911,6916 \text {, } \\
13486,15011 \text {, } \\
19169,13072 \text {, and } \\
15020 \text {. }\end{array}$ & $\begin{array}{l}\text { Composite sieved through } \\
\text { stainless steel strainer and } \\
\text { mixed at } 29^{\circ} \mathrm{C} \text { for one hr } \\
\text { using overhead mixer to } \\
\text { homogenize. Wash } 3 \text { times } \\
\text { using between } 10.2 \text { and } 14.2 \\
\text { mL 0.01M NaOH. } \\
\text { Centrifuge and decant } \\
\text { supernate to composite } \\
\text { wash collection. } 2 \text { samples } \\
\text { washed }=67.5 \mathrm{~mL} \text { wash } \\
\text { collection. }\end{array}$ & $\begin{array}{l}\text { In initial } \\
\text { slurry there } \\
\text { were } 320000 \\
\text { ug Al/g solids } \\
\text { and } 505 \text { ug } \\
\text { Al/mL } \\
\text { aqueous } \\
\text { phase. }\end{array}$ & $\begin{array}{l}296500 \mathrm{ug} / \mathrm{g} \\
\text { (Fusion), } \\
343500 \mathrm{ug} / \mathrm{g} \\
\text { (Acid } \\
\text { Digest). } \\
\text { Water } \\
\text { washed } \\
\text { insoluble } \\
\text { solids were } \\
\text { made up of } \\
\sim 32 \mathrm{wt} \% \text { Al. } \\
\text { Solids had } \\
99.6 \mathrm{wt} \% \text { of } \\
\text { Al. }\end{array}$ & $\begin{array}{l}\text { Supernate }= \\
505 \mathrm{ug} / \mathrm{mL} \text {. } \\
(0.29 \mathrm{wt} \%) . \\
\text { Wash } \\
\text { composite }= \\
60.2 \mathrm{ug} / \mathrm{mL} \\
(0.13 \mathrm{wt} \%) .\end{array}$ & - & $\begin{array}{l}\text { Wash prior to } \\
\text { caustic leach: } \\
\text { Centrifugc slurry } \\
\text { sample and remove } \\
\text { supernate to get } \\
-15 \mathrm{~mL} \text { solids. } \\
\text { Add }-45 \mathrm{~mL} \\
0.01 \mathrm{M} \text {. } \\
\text { shäl and and } \\
\text { share on vortex } \\
\text { mixer for } 15 \\
\text { minutes. } \\
\text { Centrifuge for } 30 \\
\text { min and remove } \\
\text { supernate. Repeat } \\
\text { for a total of } 3 \\
\text { washes, } \\
\text { centrifuging the } \\
\text { last washed solids } \\
\text { for only } 20 \\
\text { minutes. }\end{array}$ & 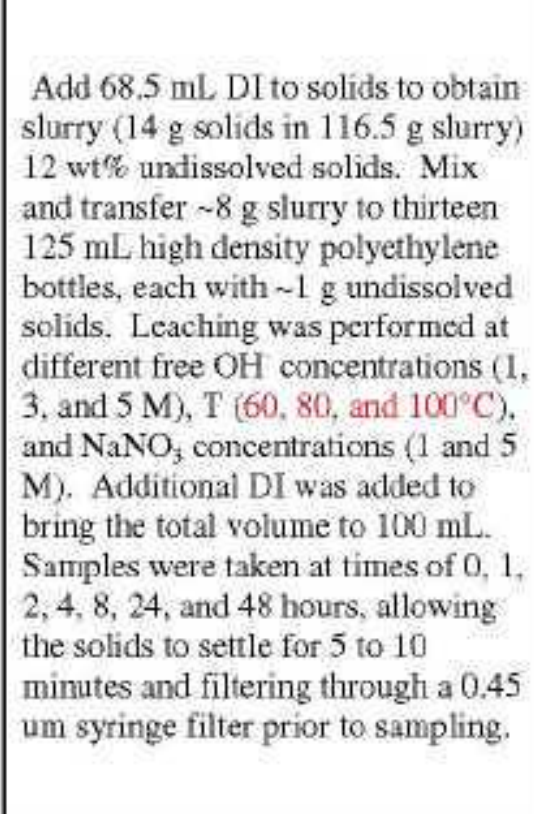 \\
\hline
\end{tabular}


Table A1-1. SSTs and DSTs Wash and Leach Information for Aluminum, Part 1. (38 sheets

\begin{tabular}{|c|c|c|c|c|c|c|c|c|c|c|c|c|c|}
\hline Tank & $\begin{array}{c}\text { Lab Study } \\
\text { Reference(s) }\end{array}$ & $\begin{array}{l}\text { Year of } \\
\text { Sampling } \\
\text { Event }\end{array}$ & Test type & $\begin{array}{l}\text { Sample } \\
\text { color }\end{array}$ & Waste Sources & Sample ID & $\mathrm{NaOH}$ Wash Procedure & $\begin{array}{l}\text { Initial Al in } \\
\text { Untreated } \\
\text { Solids }\end{array}$ & $\begin{array}{l}\text { Final Al in } \\
\text { Washed } \\
\text { solids }\end{array}$ & $\begin{array}{l}\text { Al measured in } \\
\text { Wash Solution }\end{array}$ & $\begin{array}{c}\% \\
\text { recovery }\end{array}$ & comments & Caustic Leach \#1 Procedure \\
\hline $\begin{array}{l}\text { Group } 5 \\
\text { REDOX }\end{array}$ & $\begin{array}{l}\text { PNNL- } \\
\text { 17368 } \\
\text { (WTP-RPT- } \\
\text { 157) }\end{array}$ & $\begin{array}{l}\text { N/A } \\
2006- \\
2008\end{array}$ & $\begin{array}{l}\text { Caustic } \\
\text { leach of } \\
\text { homogeni } \\
\text { zed } \\
\text { sample }\end{array}$ & $\begin{array}{l}\text { gray } \\
\text { solids } \\
\text { with } \\
\text { yellow } \\
\text { supernate }\end{array}$ & $\begin{array}{l}1283 \mathrm{~g} \text { from } 241-\mathrm{S}-101 \text {, } \\
397 \mathrm{~g} \text { from } 241-\mathrm{S}-110 \text {, } \\
213 \mathrm{~g} \text { from } 241-\mathrm{S}-107, \\
\text { and } 78 \mathrm{~g} \text { from } 241-\mathrm{SX}- \\
103 \text {. Composite of } 39 \\
\text { different archived } \\
\text { samples. Solids passed } \\
\text { through } 1 / 8 \text { inch } \\
\text { stainless steel sieve, with } \\
\sim 4 \mathrm{~L} \text { DI added to solids } \\
\text { to homogenize. }\end{array}$ & - & $\begin{array}{l}\text { Wash } 3 \text { times with } \sim 10 \mathrm{~mL} \\
0.01 \mathrm{M} \mathrm{NaOH} \text {, centrifuge } \\
\text { and decant supemate } \\
\text { between subsequent washes. }\end{array}$ & $388250 \mathrm{ug} / \mathrm{g}$ & $\begin{array}{l}326500 \mathrm{ug} / \mathrm{g} \\
(97 \%)\end{array}$ & $\begin{array}{l}2595 \mathrm{ug} / \mathrm{mL} \\
(3 \%) \text { in } \\
\text { supernatant } \\
\text { and } 704 \mathrm{ug} / \mathrm{mL} \\
(0.02 \%) \text { in } \\
\text { wash } \\
\text { composite. }\end{array}$ & - & - & $\begin{array}{l}\text { A total of } 13 \text { samples were leached } \\
\text { at different free } \mathrm{OH} \text { concentrations } \\
(1 \text { to } 5 \mathrm{M} \mathrm{NaOH} \text { ). T } 80,90 \text {, and } \\
\left.1000^{\circ} \mathrm{C}\right) \text {, and } \mathrm{NaNO}_{3} \text { concentrations } \\
\left(1 \text { to } 5 \mathrm{M} \mathrm{NaNO}_{3} \text {. Solution }\right. \\
\text { samples were obtained at } 1,4,8,24 \text {, } \\
72 \text {, and } 170 \text { hours. }\end{array}$ \\
\hline $\begin{array}{l}\text { Group 6S- } \\
\text { Saltcake }\end{array}$ & $\begin{array}{l}\text { PNNL- } \\
\text { 17368 } \\
\text { (WTP-RPT- } \\
\text { 157) }\end{array}$ & $\begin{array}{l}\text { N/A } \\
2006- \\
2008\end{array}$ & $\begin{array}{l}\text { Caustic } \\
\text { leach of } \\
\text { homogeni } \\
\text { zed } \\
\text { sample. }\end{array}$ & $\begin{array}{l}\text { dark } \\
\text { brown } \\
\text { settled } \\
\text { solids. } \\
\text { bright } \\
\text { yellow } \\
\text { supernate. }\end{array}$ & $\begin{array}{l}965.3 \mathrm{~g} \text { from } 241-\mathrm{SX}- \\
106,227.3 \mathrm{~g} \text { from } 241- \\
\mathrm{SY}-103,151.7 \mathrm{~g} \text { from } \\
241-\mathrm{SX}-105,131.5 \mathrm{~g} \\
\text { from } 241-\mathrm{SX}-102,76.2 \mathrm{~g} \\
\text { from } 241 \mathrm{~S}-111,55.1 \mathrm{~g} \\
\text { from } 241-\mathrm{U}-108,53.4 \mathrm{~g} \\
\text { from } 241-\mathrm{U}-103,30.3 \mathrm{~g} \\
\text { from } 241-\mathrm{S}-106 . \\
\text { Composite of } 28 \\
\text { different archived } \\
\text { samples. Solids passed } \\
\text { through } 1 / 8 \text { inch } \\
\text { stainless steel sieve to } \\
\text { remove large particles (- } \\
5.7 \mathrm{~g} \text {. Added DI to } \\
\text { make total volume - } 3 \mathrm{~L} \text {. }\end{array}$ & - & $\begin{array}{l}\text { Wash } 3 \text { times with }-5 \mathrm{~mL} \\
0.01 \mathrm{M} \mathrm{NaOH} \text {, centrifuge } \\
\text { and decant supernate } \\
\text { between subsequent washes. }\end{array}$ & - & $\begin{array}{l}187000 \mathrm{ug} / \mathrm{g} \\
(605)\end{array}$ & \begin{tabular}{|l|}
$7590 \mathrm{ug} / \mathrm{mL}$ \\
$(38 \%)$ in \\
supernatant \\
and 1580 \\
ug/mL $(2 \%)$ in \\
wash \\
composite
\end{tabular} & - & $\begin{array}{l}\text { Initial washing of } \\
\text { solids prior to } \\
\text { eaustic leaching: } \\
\text { With } \sim 35 \mathrm{~mL} \\
\text { centrifuged solids, } \\
\text { add } \sim 105 \mathrm{~mL} \\
0.01 \mathrm{M} \mathrm{NaOH} \text {. Mix } \\
5 \text { minutes by } \\
\text { shaking. Centrifuge } \\
\text { at } \sim 610 \mathrm{G} \text { for } 15 \\
\text { min and decant } \\
\text { supernate. Repeat } \\
\text { for a total of } 3 \\
\text { washes. }\end{array}$ & $\begin{array}{l}\text { Add } 100 \mathrm{~mL} 3 \mathrm{M} \mathrm{NaOH} \text { to washed } \\
\text { solids and allow leaching at } \sim 100^{\circ} \mathrm{C} \\
\text { for } 8 \text { hours. Solids were suspended } \\
\text { using a magnetic stir bar. After } 8 \\
\text { hours, removed slurry from heat and } \\
\text { cooled to ambient temperatures } \\
\text { (overnight). Centrifuge slurry at } \\
-610 \mathrm{G} \text { for } 15 \text { min and remove } \\
\text { leachate. }\end{array}$ \\
\hline
\end{tabular}


Table A1-1. SSTs and DSTs Wash and Leach Information for Aluminum, Part 1. (38 sheets)

\begin{tabular}{|c|c|c|c|c|c|c|c|c|c|c|c|c|c|}
\hline Tank & $\begin{array}{l}\text { Lab Study } \\
\text { Reference(s) }\end{array}$ & $\begin{array}{c}\text { Year of } \\
\text { Sampling } \\
\text { Event }\end{array}$ & Test type & $\begin{array}{l}\text { Sample } \\
\text { color }\end{array}$ & Waste Sources & Sample ID & $\mathrm{NaOH}$ Wash Procedure & $\begin{array}{l}\text { Initial Al in } \\
\text { Untreated } \\
\text { Solids }\end{array}$ & $\begin{array}{l}\text { Final Al in } \\
\text { Washed } \\
\text { solids }\end{array}$ & $\begin{array}{l}\mathrm{Al} \text { measured in } \\
\text { Wash Solution }\end{array}$ & $\begin{array}{c}\% \\
\text { recovery }\end{array}$ & comments & Caustic Leach \#1 Procedure \\
\hline $\begin{array}{l}\text { Group } 7 \\
\text { TBP Waste } \\
\text { Sludge }\end{array}$ & $\begin{array}{l}\text { PNNL- } \\
18119 \\
\text { (WTP-RPT- } \\
\text { 169) }\end{array}$ & $\begin{array}{l}\text { N/A } \\
2007- \\
2009\end{array}$ & - & \begin{tabular}{|l|} 
Centrifuge \\
$\mathrm{d}$ solids \\
were dark \\
brown-red \\
with pale \\
yellow \\
supernate.
\end{tabular} & $\begin{array}{l}\text { Composite of } 33 \text { samples } \\
\text { from } 2 \text { tanks. } 92 \% \text { ( } 30 \\
\text { samples) from BX-109 } \\
\text { and } 8 \% \text { (3 samples) from } \\
\text { B-106. Total of } 1479.16 \\
\mathrm{~g} \text { from original samples } \\
\text { transferred to composite. } \\
\text { A total of } 779.7 \mathrm{~g} \mathrm{DI} \\
\text { added during } \\
\text { compositing. This was } \\
\text { passed through a } \\
\text { stainless steel strainer to } \\
\text { ensure no large pieces of } \\
\text { foreign material in } \\
\text { composite. }\end{array}$ & $\begin{array}{l}\text { 222-S sample IDs } \\
\text { from 241-B-106: } \\
7417,7424, \text { and } \\
\text { 16913. } 222 . S \\
\text { sample IDs from } \\
241-\mathrm{BX}-109 \text { : } \\
6907,6921,6922, \\
6927,6930,6931 \text {, } \\
6932,6933,6934, \\
6935,7153,7154, \\
7157,7158,7372, \\
7378,9334,9346, \\
10116,11840 \text {, } \\
13092,13445, \\
13473,13515 \text {, } \\
13516,13517 \\
13522,13523, \\
16916, \text { and } 19302 .\end{array}$ & $\begin{array}{l}\text { Wash two samples } 3 \text { times } \\
\text { with } 20 \text { to } 22 \mathrm{~mL} 0.01 \mathrm{M} \\
\mathrm{NaOH} \text {. Centrifuge and } \\
\text { decant supernate between } \\
\text { subsequent washes. }\end{array}$ & - & $\begin{array}{l}16000 \mathrm{ug} / \mathrm{g} \\
\text { (Fusion), } \\
18550 \text { ug/g } \\
\text { (Acid } \\
\text { Digest). } \\
\text { Water } \\
\text { washed } \\
\text { insoluble } \\
\text { sclids were } \\
\text { made up of } \\
\sim 97 \text { wt } \% \text { of } \\
\text { the total Al. }\end{array}$ & $\begin{array}{l}\text { Supernate }= \\
<0.73 \text { ug/mL } \\
(<0.01 \mathrm{wt} \%) \\
\text { Al. } \\
\text { Wash } 1=8.96 \\
\text { ug/mL Al. } \\
\text { Wash } 2=28 \\
\text { ug/mL Al. } \\
\text { Wash } 3 \\
\text { (filtered) }= \\
46.3 \text { ug/mL } \\
\text { Al. } \\
\text { Combined } \\
\text { wash solution } \\
\text { contained } 3.4 \\
\text { wt } \% \text { of the } \\
\text { total Al. }\end{array}$ & - & 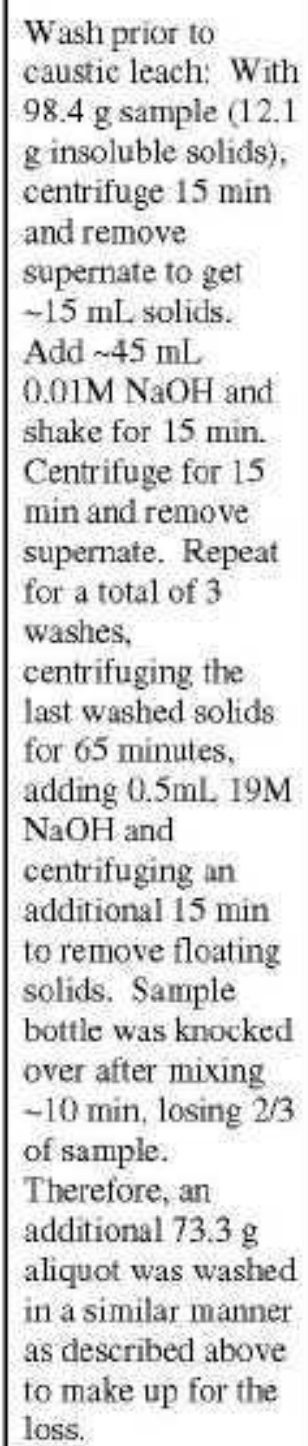 & $\begin{array}{l}\text { Additional DI water was added to } \\
\text { thin samples }(-84 \mathrm{~g} \text { DI). A total of } \\
10 \text { samples }(\sim 9.4 \mathrm{~g} \text { slurry in } 125 \mathrm{~mL} \mathrm{~L} \\
\mathrm{HDPE} \text { bottles with } \sim 1 \mathrm{~g} \text { UDS) were } \\
\text { leached at different free } \mathrm{OH} \\
\text { concentrations }(0.25,1 \text {, and } 3 \mathrm{M} \\
\mathrm{NaOH}) \text { and } \mathrm{T}\left(40,60 \text {, and } 80^{\circ} \mathrm{C}\right) \text {. } \\
\text { Solution samples were obtained at } \\
1,4,8 \text {, sand } 24 \text { hours, allowing the } \\
\text { samples to settle for } \sim 5 \text { to } 10 \text { min } \\
\text { before sampling. }\end{array}$ \\
\hline
\end{tabular}


Table A1-1. SSTs and DSTs Wash and Leach Information for Aluminum, Part 1. (38 sheets)

\begin{tabular}{|c|c|c|c|c|c|c|c|c|c|c|c|c|c|}
\hline Tank & $\begin{array}{c}\text { Lab Study } \\
\text { Reference(s) }\end{array}$ & $\begin{array}{l}\text { Year of } \\
\text { Sampling } \\
\text { Event }\end{array}$ & Test type & $\begin{array}{l}\text { Sample } \\
\text { color }\end{array}$ & Waste Sources & Sample ID & $\mathrm{NaOH}$ Wash Procedure & $\begin{array}{l}\text { Initial } \mathrm{Al} \text { in } \\
\text { Untreated } \\
\text { Solids }\end{array}$ & $\begin{array}{l}\text { Final Al in } \\
\text { Washed } \\
\text { solids }\end{array}$ & $\begin{array}{l}\mathrm{Al} \text { measured in } \\
\text { Wash Solution }\end{array}$ & $\begin{array}{c}\% \\
\text { recovery }\end{array}$ & comments & Caustic Leach \#1 Procedure \\
\hline $\begin{array}{l}\text { Group } 8 \\
\text { FeCN Waste } \\
\text { Sludge }\end{array}$ & $\begin{array}{l}\text { PNNL- } \\
18120 \\
\text { (WTP-RPT- } \\
170 \text { ) }\end{array}$ & $\begin{array}{l}\text { N/A } \\
2007- \\
2009\end{array}$ & $\begin{array}{l}\text { Cell Unit } \\
\text { Filtration, } \\
\text { caustic } \\
\text { leaching } \\
\text { and wash } \\
\text { of Group } \\
8 \text { archived } \\
\text { solids. }\end{array}$ & $\begin{array}{l}\text { Solids } \\
\text { ranged } \\
\text { from gray } \\
\text { dry } \\
\text { crystals, } \\
\text { gray } \\
\text { pastes, } \\
\text { white } \\
\text { solids, and } \\
\text { brown } \\
\text { sludge. } \\
\text { Aqueous } \\
\text { phase was } \\
\text { yellowish. }\end{array}$ & $\begin{array}{l}\text { Composite of } 30 \text { samples } \\
\text { from } 5 \text { tanks. } 45 \% \text { from } \\
\text { BY- } 110,22 \% \text { from BY- } \\
108,21 \% \text { from BY-105, } \\
10 \% \text { from BY-104, and } \\
2 \% \text { from BY-106. Total } \\
\text { of } 1358.8 \mathrm{~g} \text { from original } \\
\text { samples transferred to } \\
\text { composite. A total of } \\
-960 \mathrm{~g} \text { DI added during } \\
\text { compositing. This was } \\
\text { passed through a sieve } \\
\text { (collect objects }>3 \mathrm{~mm} \\
\text { diameter) to ensure no } \\
\text { large pieces of foreign } \\
\text { material in composite. } \\
\text { No significant sample } \\
\text { was trapped in sieve. }\end{array}$ & - & $\begin{array}{l}\text { Samples were composited } \\
\text { and sieved through a } 3 \mathrm{~mm} \\
\text { sieve. An additional } ~ 960 \mathrm{~g} \\
\text { DI was added during } \\
\text { compositing, with the } \\
\text { solution mixed lhr } 5 \mathrm{~min} \\
\text { before sub-sampling. Wash } \\
\text { two samples } 3 \text { times with } \\
6.6 \text { to } 9.1 \mathrm{~mL} \text {. } 0.01 \mathrm{M} \\
\text { NaOH. Centrifuge and } \\
\text { decant supernate between } \\
\text { subsequent washes and } \\
\text { composite the decanted } \\
\text { wash solution ( } 46.8 \mathrm{~mL} \\
\text { total). }\end{array}$ & - & $\begin{array}{l}88350 \mathrm{ug} / \mathrm{g} \\
\text { (Fusion), } \\
90950 \mathrm{ug} / \mathrm{g} \\
\text { (Acid } \\
\text { Digest). } \\
\text { Water } \\
\text { washed } \\
\text { insoluble } \\
\text { solids were } \\
\text { made up of } \\
9 \mathrm{wt} \% \text { Al; } \\
\text { with } 89.4 \\
\text { wt } \% \text { of the } \\
\text { total Al } \\
\text { remaining } \\
\text { after wash. }\end{array}$ & $\begin{array}{l}\text { Supernate } \\
\text { (acid digest) }= \\
1430 \text { ug/mL } \\
(8.5 \text { wt\%) Al. } \\
\text { Composite } \\
\text { wash (acid } \\
\text { digest) }=159 \\
\text { ug/mL }(2.1 \\
\text { wt } \% \text { ) Al. }\end{array}$ & - & $\begin{array}{l}\text { High-Solids slurry } \\
\text { composition (After } \\
\text { filtration using acid } \\
\text { digestion): Dry } \\
\text { solids }=39000 \text { ug } \\
\text { Al/g. Supernate = } \\
1470 \text { ug Al/mL. } \\
\text { Dry solids = } 85000 \\
\text { ug Al/g. }\end{array}$ & $\begin{array}{l}\text { Caustic leach after filtration to } 13 \\
\text { wt } \% \text { undissolved solids. Slurry had } \\
\text { an initial mass of } 3.9 \mathrm{~kg} \text {. } \\
\text { undissolved soliss concentration of } \\
230 \mathrm{~g} \text {, and initial volume of } 3.1 \mathrm{~L} \text {. } \\
\text { Caustie was added to the tank prior } \\
\text { to beginning of heat and mix. } \\
\text { Heating of slury from } 26 \text { to } 60^{\circ} \mathrm{C} \\
\text { took } 2.5 \mathrm{hr} \text {. Leaching was } \\
\text { performed at } 60^{\circ} \mathrm{C} \text { for } 8 \mathrm{hr} \text { in } 3.7 \mathrm{M} \\
\mathrm{OH} \text {, with samples taken at times- } \\
1.5 \mathrm{hr}, 0 \mathrm{hr}, 2 \mathrm{hr}, 4 \mathrm{hr}, 8 \mathrm{hr} \text {, and } \\
\text { final. }\end{array}$ \\
\hline $\begin{array}{l}\text { Mixture of } \\
\text { Group } 5 \text { and } \\
\text { Group } 6 \\
\text { waste. } 31.6 \\
\text { wt\% Group } \\
5 \text { Slurry + } \\
16.8 \text { wt \% } \\
\text { Group } 6 \\
\text { slurry }+51.6 \\
\text { w1\% Group } \\
6 \\
\text { supernatant }\end{array}$ & $\begin{array}{l}\text { PNNL- } \\
18007 \\
\text { (WTP-RPT- } \\
171 \text { ) }\end{array}$ & $\begin{array}{l}\text { N/A } \\
2007 . \\
2009\end{array}$ & $\begin{array}{l}\text { Only } \\
\text { initial } \\
\text { cuustic } \\
\text { leach of } \\
\text { mixed } \\
\text { Group } 5 \\
\text { and } 6 \\
\text { composite }\end{array}$ & & $\begin{array}{l}\text { Group } 5 \text { solids contained } \\
33 \text { wt } \% \text { Al, with }-90 \\
\text { wt\% Al in the form of } \\
\text { boehmite. The } \\
\text { individual tank waste } \\
\text { material sources, } \\
\text { compositions, and } \\
\text { physical properties are } \\
\text { described in PNNL- } \\
17368 \text { (WTP-RPT-157) } \\
\text { above. }\end{array}$ & - & - & $\begin{array}{l}\text { Group } 5 \\
\text { supernate had } \\
2595 \text { ug } \\
\text { Al/mL with } \\
326500 \text { ug } \\
\text { Alg washed } \\
\text { solids on a } \\
\text { dry mass } \\
\text { basis. Group } \\
6 \text { supernate } \\
\text { had } 7590 \text { ug } \\
\text { Al/g with } \\
187000 \text { ug } \\
\text { Al/g washed } \\
\text { solids on a } \\
\text { dry mass } \\
\text { basis. Al in } \\
\text { initial solids } \\
=312500 \\
\text { uggg. Al in } \\
\text { initial } \\
\text { supernate }= \\
5246 \text { ug } / \mathrm{mL} \text {. }\end{array}$ & - & - & - & $\begin{array}{l}\text { From total slurry, } \\
16 \mathrm{~g} \text { was removed } \\
\text { for analysis, with } \\
\text { the remainder } \\
\text { centrifuged. The } \\
\text { supernate ( } 106 \mathrm{~g}) \\
\text { was also removed. }\end{array}$ & $\begin{array}{l}\text { The remaining concentrated sludge } \\
(-18 \text { wt } \% \text { undissolved solids) was } \\
\text { combined with } 84 \text { mL of } 9.5 \mathrm{M} \\
\mathrm{NaOH} \text {, stirred and heated to }-100^{\circ} \mathrm{C} \\
\text { for } 12 \text { hr. Water was added to } \\
\text { account for evaporation. After } \\
\text { leaching, the solution was cooled to } \\
\text { ambient temperature. }\end{array}$ \\
\hline
\end{tabular}


Table A1-1. SSTs and DSTs Wash and Leach Information for Aluminum, Part 1. (38 sheets)

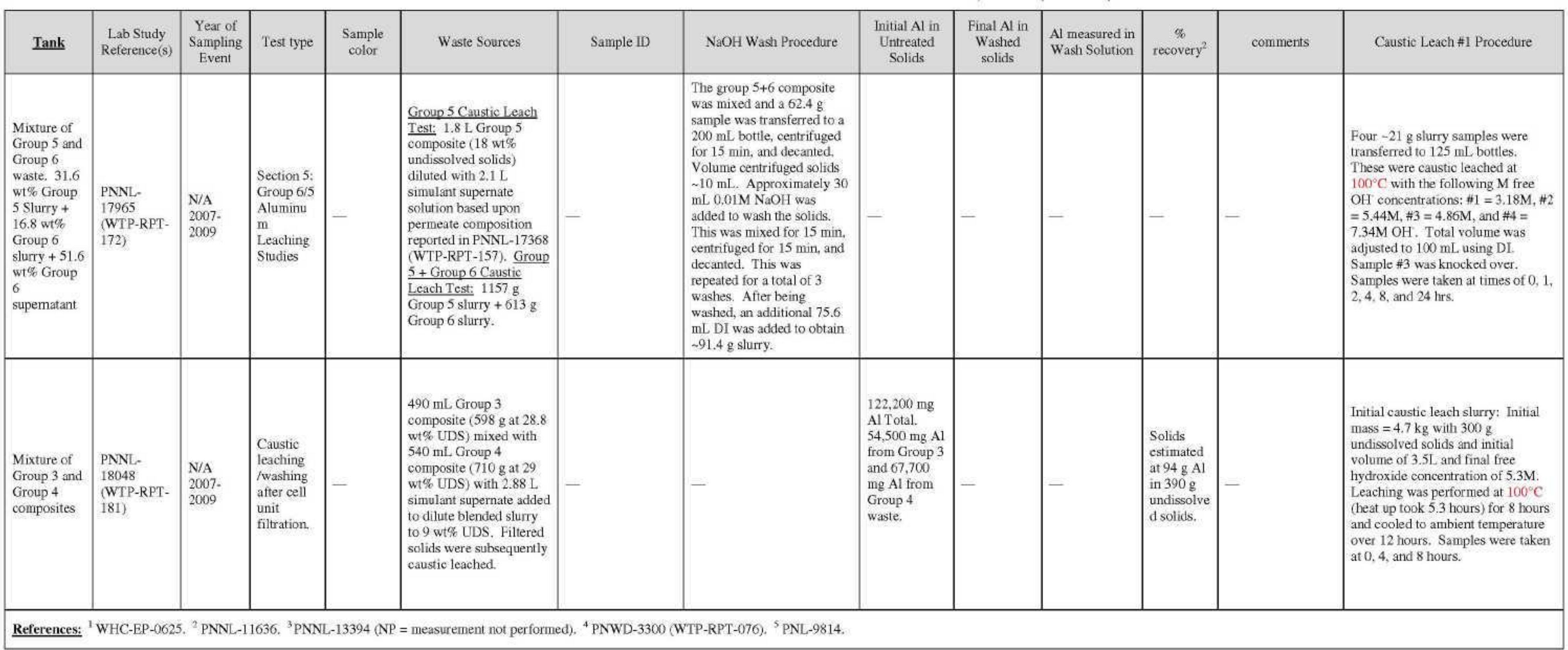


Table A1-2. SSTs and DSTs Wash and Leach Information for Aluminum, Part 2. (27 sheets)

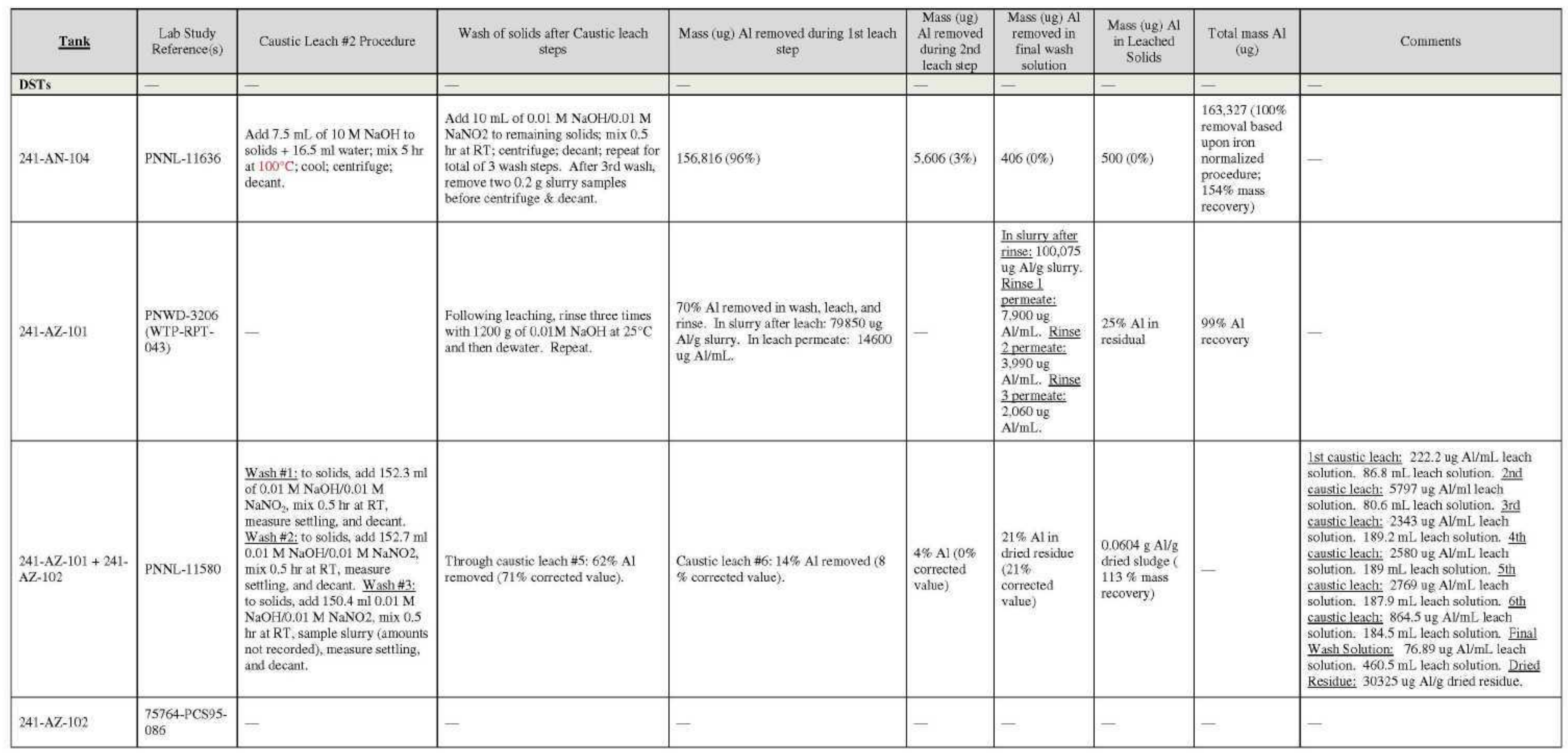


Table A1-2. SSTs and DSTs Wash and Leach Information for Aluminum, Part 2. (27 sheets)

\begin{tabular}{|c|c|c|c|c|c|c|c|c|c|}
\hline$\underline{\text { Tank }}$ & $\begin{array}{l}\text { Lab Study } \\
\text { Reference(s) }\end{array}$ & Caustic Leach $\# 2$ Procedure & $\begin{array}{l}\text { Wash of solids after Caustic leach } \\
\text { steps }\end{array}$ & $\begin{array}{l}\text { Mass (ug) Al removed during 1st leach } \\
\text { step }\end{array}$ & \begin{tabular}{|c|} 
Mass (ug) \\
Al removed \\
during 2nd \\
leach step \\
\end{tabular} & $\begin{array}{l}\text { Mass (ug) Al } \\
\text { removed in } \\
\text { final wash } \\
\text { solution } \\
\end{array}$ & \begin{tabular}{|c}
$\begin{array}{c}\text { Mass (ug) Al } \\
\text { in Leached } \\
\text { Solids }\end{array}$ \\
\end{tabular} & \begin{tabular}{|c|} 
Total mass $\mathrm{Al}$ \\
(ug)
\end{tabular} & Comments \\
\hline 241-AZ-102 & $\begin{array}{l}\text { PNWD-3045 } \\
\text { (BNFL-RPT- } \\
038 \text { ) }\end{array}$ & - & $\begin{array}{l}\text { Following leaching, rinse twice with } \\
0.01 \mathrm{M} \mathrm{NaOH}(1,390 \mathrm{~g} \text { and } 1,400 \mathrm{~g} \text { for } \\
\text { leach } 1 \text { and } 2) \text { for } 8 \mathrm{hr} \text { at } 85^{\circ} \mathrm{C} \text { and } \\
\text { then dewater. Repeat. }\end{array}$ & $\begin{array}{l}1.04 \mathrm{E}+4 \mathrm{ug} \mathrm{Al} / \mathrm{mL} \text {. Leach efficiency = } \\
59.3 \% \text {. }\end{array}$ & $\begin{array}{l}\text { Intermediate } \\
\text { sample: } \\
1.01 \mathrm{E}+5 \text { ug } \\
\mathrm{A} / \mathrm{g} \text { dry } \\
\text { solids }\end{array}$ & $\begin{array}{l}\text { Wash } \\
\text { permeate } 41 \text { : } \\
4.91 \mathrm{E}+3 \mathrm{ug} \\
\mathrm{Al} / \mathrm{mL}, 1.9 \% \\
\text { efficiency: } \\
\text { Wash } \\
\text { permeate } \# 2: \\
2.18 \mathrm{E}+3 \mathrm{ug} \\
\mathrm{Al} / \mathrm{mL} \\
<0.003 \% \\
\text { efficiency. }\end{array}$ & $\begin{array}{l}1.07 \mathrm{E}+5 \text { ug } \\
\mathrm{Al} \text { g dry } \\
\text { solids. } 36.3 \% \\
\text { Al in residue. }\end{array}$ & $\begin{array}{l}120 \% \mathrm{Al} \\
\text { recovery }\end{array}$ & - \\
\hline 241-SY-102 & $\begin{array}{l}\text { PNWD-3512 } \\
\text { (WTP-RPT- } \\
117 \text { ) }\end{array}$ & $\begin{array}{l}\text { 3. M NaOH leach of samples } \$ \mathrm{Y}- \\
102-5 \text { and } \mathrm{SY}-102-6 \text { (slurry } \\
\text { weights of } 15.46 \text { and } 16.174 \mathrm{~g} \text {, } \\
\text { tespectively). Add } 4.8 \mathrm{ml} 10 \mathrm{M} \\
\mathrm{NaOH} \text { and } 8.2 \mathrm{ml} \text { DI to each. } \\
\text { Heat at } 85 \pm 5^{\circ} \mathrm{C} \text {. for } 8 \mathrm{hr} \text {. Cool } \\
\text { to } \mathrm{RT} \text { overnight. Centrifuge and } \\
\text { decant supernate to } 30 \mathrm{~mL} \text {. } \\
\text { plastic vials. Add } 0.1 \mathrm{M} \mathrm{NaOH} \\
\text { at } 3: 1 \text { volivol ratio to remaining } \\
\text { solids. Mix, separate, and } \\
\text { decant to same container as } \\
\text { initial leachate solution. } \\
\text { Continue washings until } \\
\text { supernate was colorless. Filter } \\
\text { samples }(0.2 \text { um syringe filter), } \\
\text { and place } 1 \text { ml aliquot in } \\
\text { container w/ } 10 \mathrm{~mL} \text { of } 1 \mathrm{M} \\
\mathrm{HNO}_{3} \text { for ICP-AES. }\end{array}$ & 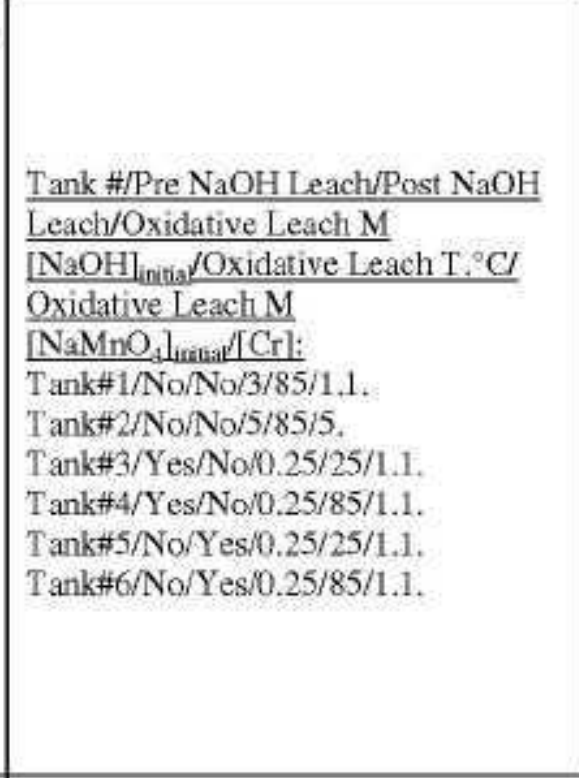 & - & - & no data & no data & $\begin{array}{l}\text { \% removal } \\
\text { values for } 6 \\
\text { bottles range } \\
\text { from } 76-94 \% \\
\text { Al removal ( } 86 \text {, } \\
88,76,78,94, \\
89 \% \text { removal } \\
\text { for bottles \#1-6, } \\
\text { respectively) }\end{array}$ & 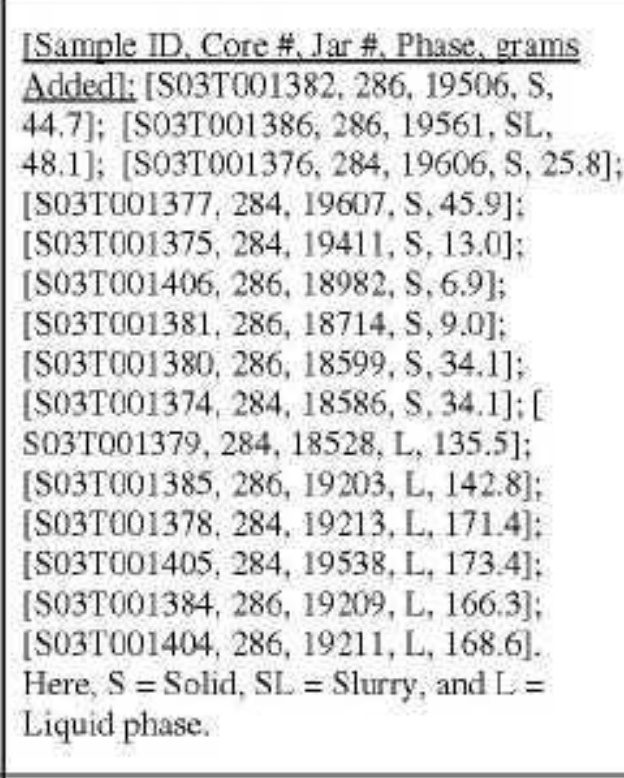 \\
\hline 241-SY-103 & PNL-10712 & $\begin{array}{l}\text { Solids did not settle. Add } 4.1 \mathrm{~g} \\
3 \mathrm{M} \mathrm{NaOH} \text { to } 1.1 \mathrm{~mL} \text { centrifuged } \\
\text { solids; mix } 5 \mathrm{hr} \text { at } 100^{\circ} \mathrm{C} ; \mathrm{cool} \text { : } \\
\text { measure settling; centrifuge; and } \\
\text { decant. }\end{array}$ & 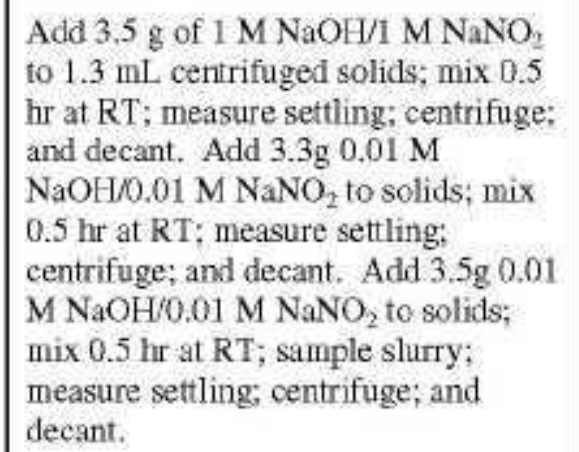 & $\begin{array}{l}48 \% \text { Al found, } 43350 \text { ug Al/mL } \\
\text { measured. }\end{array}$ & $\begin{array}{l}25 \% \mathrm{Al} \\
\text { found, } \\
15300 \text { ug } \\
\mathrm{Al} / \mathrm{mL} \\
\text { measured. }\end{array}$ & $\begin{array}{l}7 \% \text { Al found, } \\
1615 \text { ug } \\
\text { A1/mL } \\
\text { measured. }\end{array}$ & $\begin{array}{l}\text { Measured an } \\
\text { Al } \\
\text { concentration } \\
\text { of } 83,000 \\
\text { ugg; } 10 \% \text { Al } \\
\text { in residue. }\end{array}$ & $\begin{array}{l}\text { Direct analysis: } \\
4.7 \mathrm{E}-02 \mathrm{~g} \mathrm{Al} / \mathrm{g} \\
\text { sludge; } \\
\text { Summation } \\
\text { method: } 3.84 \mathrm{E}- \\
02 \mathrm{~g} \text { Al/g } \\
\text { sludge; } 82 \% \mathrm{Al} \\
\text { recovery: }\end{array}$ & - \\
\hline
\end{tabular}


Table A1-2. SSTs and DSTs Wash and Leach Information for Aluminum, Part 2. (27 sheets)

\begin{tabular}{|c|c|c|c|c|c|c|c|c|c|}
\hline Tank & $\begin{array}{l}\text { Lab Study } \\
\text { Reference (8) }\end{array}$ & Caustic Leach $\# 2$ Procedure & $\begin{array}{c}\text { Wash of solids after Caustic leach } \\
\text { steps }\end{array}$ & $\begin{array}{l}\text { Mass (ug) Al removed during 1st leach } \\
\text { step }\end{array}$ & \begin{tabular}{|c|} 
Mass (ug) \\
Al removed \\
during 2nd \\
leach step \\
\end{tabular} & $\begin{array}{l}\text { Mass (ug) Al } \\
\text { removed in } \\
\text { final wash } \\
\text { solution } \\
\end{array}$ & $\begin{array}{l}\text { Mass (ug) Al } \\
\text { in Leached } \\
\text { Solids }\end{array}$ & \begin{tabular}{|c|} 
Total mass $\mathrm{Al}$ \\
(ug)
\end{tabular} & Comment: \\
\hline 241-SY-103 & PNNL-11089 & $\begin{array}{l}\text { Add } \mathrm{NaOH} \text { to get } \sim 8 \mathrm{wt} \% \text { solids } \\
\text { and a final } \mathrm{NaOH} \text { of } 2.8 \mathrm{M} \text {. Mix } \\
\text { at } 100^{\circ} \mathrm{C} \text { for } 5 \mathrm{hr} \text {. Cool, } \\
\text { centrifuge, and decant. }\end{array}$ & $\begin{array}{l}\text { Add inhibited water. Mix at RT for } \\
0.5 \mathrm{hr} \text { (target } 8 \text { wt } \% \text { solids). } \\
\text { Centrifuge, decant, and repeat twice } \\
\text { more. }\end{array}$ & $\begin{array}{l}\text { uncorrected// corrected recovery }= \\
48 / 78 \% \mathrm{Al} \text {. }\end{array}$ & $\begin{array}{l}\text { Uncorrected } / \\
\text { corrected Al } \\
\text { recovery }= \\
25 / 2 \% \text {. }\end{array}$ & $\begin{array}{l}\text { Uncorrected/ } \\
\text { corrected Al } \\
\text { recovery = } \\
7 / 1 \% \text {. }\end{array}$ & \begin{tabular}{|l|} 
Total wt $\% \mathrm{Al}=$ \\
$4.7 ;$ Total $\% \mathrm{Al}$ \\
removed $=$ \\
$90 \% ;$ percent \\
undissolved $\mathrm{Al}$ \\
in residue = \\
$10 \%$. \\
\end{tabular} & - & - \\
\hline SSTs & - & - & - & - & - & - & - & - & - \\
\hline 241-B-101 & PNNL-12026 & $\begin{array}{l}\text { After leach test, remove vessel } \\
\text { from heating block and cool to } \\
\text { RT; centrifuge } 15 \text { min; decant; } \\
\text { wash solids } 3 \text { times with } 10 \mathrm{~mL} \\
0.01 \mathrm{M} \mathrm{NaOH} / \mathrm{NaNO}_{2} \text {; dry at } \\
105^{\circ} \mathrm{C} \text {. }\end{array}$ & - & $\begin{array}{l}\text { At } 60^{\circ} \mathrm{C}: 1 \mathrm{M} \mathrm{NaOH} \text { removed } 42 \% \mathrm{Al} \\
\text { (Fe normalized }=43 \% \mathrm{Al} \text {; } 3 \mathrm{M} \mathrm{NaOH} \\
\text { removed } 51 \% \mathrm{Al} \text { (Fe normalized = } \\
51 \% \mathrm{Al}) . \mathrm{At} 100^{\circ} \mathrm{C}: 1 \mathrm{M} \mathrm{NaOH} \\
\text { removed } 46 \% \mathrm{Al} \text { (Fe normalized }= \\
49 \% \mathrm{Al}) ; 3 \mathrm{M} \mathrm{NaOH} \text { removed } 50 \% \mathrm{Al} \\
\text { (Fe normalized }=43 \% \mathrm{Al} \text { ). } \\
\end{array}$ & - & - & - & - & - \\
\hline 241-B-104 & $\begin{array}{l}\text { L.AUR 96- } \\
2839\end{array}$ & $\begin{array}{l}\text { Add } 25.2 \mathrm{~mL} \text { of } 10 \mathrm{M} \mathrm{NaOH} \text { to } \\
\text { solids and } \sim 68.1 \mathrm{ml} \text { water to get } \\
\sim 1 \mathrm{wt} \% \text { solids and final } \mathrm{NaOH} \\
\text { of }-3 \mathrm{M} ; 11.2 \mathrm{~mL} \text { settled solids/ } \\
4.8 \mathrm{~mL} \text { centrifuged solids; mix } 5 \\
\text { hr at } 100^{\circ} \mathrm{C} \text {; oool; measure } \\
\text { settling; centrifuge; and decant. }\end{array}$ & $\begin{array}{l}\text { Add } 18.2 \mathrm{~mL} \text { inhibited water to solids } \\
\text { (12 mL settled solids/ } 3.7 \mathrm{~mL} \\
\text { centrifuged solids); mix } 0.5 \mathrm{hr} \text { at } \mathrm{RT} \text {; } \\
\text { measure settling; centrifuge; decant. } \\
\text { Repeat } 2 \text { times with solids portion, } \\
\text { sampling slurry (0.64 mL) before } \\
\text { measuring settling in wash } \# 3 \text {. Dry } \\
\text { solids at } 105^{\circ} \mathrm{C} \text {. }\end{array}$ & $\begin{array}{l}5.27 \mathrm{ug} \mathrm{Al} / \mathrm{mL} ; 73.62 \text { ug } \mathrm{Al}(0.5 \% \mathrm{Al} \\
\text { removed). }\end{array}$ & $\begin{array}{l}97.79 \text { ug } \\
\mathrm{Al} / \mathrm{mL} ; \\
8045.17 \mathrm{ug} \\
\mathrm{Al}(56.4 \% \\
\mathrm{Al} \\
\text { removed). }\end{array}$ & $\begin{array}{l}15.45 \mathrm{ug} \\
\mathrm{Al} / \mathrm{mL} ; 808.5 \\
\text { ug Al (5.7\% } \\
\text { Al removed). }\end{array}$ & $\begin{array}{l}\text { 10210.13 ug } \\
\text { Al/g; } 5327.64 \\
\text { ug Al (37.4\% } \\
\text { Al removed; } \\
63 \% \text { [Table } \\
\text { S.1] Al } \\
\text { removed } \\
\text { during sludge } \\
\text { wash//asustic } \\
\text { leach). } \\
\end{array}$ & $\begin{array}{l}\text { Direct analysis: } \\
2.47 \mathrm{E}-3 \mathrm{~g} \mathrm{Al} / \mathrm{g} \\
\text { sludge; } \\
\text { Summation } \\
\text { method: } 2.53 \mathrm{E}- \\
3 \mathrm{~g} \mathrm{Al} \text { - } \\
97.7 \mathrm{gldge} \text {. } \\
\text { recovery. }\end{array}$ & - \\
\hline $241-B-106$ & $\begin{array}{l}\text { LAUR 97- } \\
2889\end{array}$ & $\begin{array}{l}\text { Add } 43.1 \mathrm{~mL} \text { water and } 18.9 \mathrm{~mL} \\
10 \mathrm{M} \mathrm{NaOH} \text { to get }-1 \text { wt } \% \text { solids } \\
\&-3 \mathrm{M} \mathrm{NaOH} \text {. Mix } 5 \mathrm{hr} \text { at } \\
100^{\circ} \mathrm{C} \text {; cool; measure settling; } \\
\text { centrifuge; deeant. Supernate: } \\
59.88 \mathrm{~mL} \text { with } 3.26 \mathrm{M} \text { free } \mathrm{OH} \text {, } \\
9.58 \mathrm{~mL} \text { settled solids, \& } 4.11 \\
\mathrm{~mL} \text { centrifuged solids. }\end{array}$ & $\begin{array}{l}\text { Wash } 3 \text { times: With solids, add } 13.22 \\
\text { mL inhibited water; mix } 0.5 \mathrm{hr} \text { at RT; } \\
\text { measure settling; centrifuge; decant. } \\
\text { Sample slurry }(0.6 \mathrm{~g}) \text { after } 3 \mathrm{rd} \text { mix. } \\
\text { Dry solids at } 105^{\circ} \mathrm{C} \text { after } 3 \mathrm{~d} \text { decant to } \\
\text { obtain } 0.5 \mathrm{~g} \text { solids. }\end{array}$ & $\begin{array}{l}744.9 \text { ug Al/mL, } 19426.99 \text { ug } \mathrm{Al} \text { ( } 74 \% \\
\text { Al removed). }\end{array}$ & $\begin{array}{l}49.4 \mathrm{ug} \\
\mathrm{Al} / \mathrm{mL}, \\
2958.07 \mathrm{ug} \\
\mathrm{Al}(11.3 \% \\
\mathrm{Al} \\
\text { removed). }\end{array}$ & $\begin{array}{l}5.5 \mathrm{ug} \mathrm{Al} / \mathrm{mL} \text {, } \\
215.88 \mathrm{ug} \mathrm{Al} \\
(0.8 \% \cdot \mathrm{Al} \\
\text { removed). }\end{array}$ & $\begin{array}{l}7304.117 \mathrm{ug} \\
\mathrm{Al} / \mathrm{g}, 3652.05 \\
\mathrm{ug} \mathrm{Al}(13.9 \% \\
\text { Al removed). }\end{array}$ & $\begin{array}{l}\text { Summation: } \\
7.45 \mathrm{E}-3 \mathrm{~g} \mathrm{Al} / \mathrm{g} \text { : } \\
\text { Direct: 7.29E-3 } \\
\text { g Alg; } 102.1 \% \\
\text { Al mass } \\
\text { recovery; } 26253 \\
\text { ug Al; } 86 \% \mathrm{Al} \\
\text { removed. }\end{array}$ & - \\
\hline 241-B-110 & PNL-9387 & $\begin{array}{l}\mathrm{ACID} \text { LEACH: Add } 3 \mathrm{~mL} 2 \mathrm{M} \\
\mathrm{HNO}_{3} \& 0.2 \mathrm{~mL} 10 \mathrm{M} \mathrm{HF} \text { to } \\
\text { solids; mix } 1 \mathrm{hr} \text { at } 100^{\circ} \mathrm{C} \text {; } \\
\text { analyze solution - all solids } \\
\text { dissolved. }\end{array}$ & - & - & $\begin{array}{l}\text { Al in caustic } \\
\text { leach was } \\
\text { less than or } \\
\text { equal to } \\
26 \% \\
\end{array}$ & - & \begin{tabular}{|l|} 
Leached \\
sludge had \\
greater than or \\
equal to $47 \%$ \\
Al. \\
\end{tabular} & - & - \\
\hline $241-B-110$ & $\begin{array}{l}\text { Internal Letter } \\
9404238\end{array}$ & $\begin{array}{l}\text { Add } 11.8 \mathrm{~mL} \text { water and } 5 \mathrm{~mL} \text { of } \\
10 \mathrm{M} \mathrm{NaOH} \text { to solids. Mix, } \\
\text { using a magnetic stirrer, for } 5 \mathrm{hr} \\
\text { at } 100^{\circ} \mathrm{C} \text {. Cool, then settle and } \\
\text { decant liguid. }\end{array}$ & $\begin{array}{l}\text { Wash } 5 \text { times with water for } 0.5 \mathrm{hr} \text { at } \\
\text { RT }(12 \mathrm{~mL} ; 15 \mathrm{~mL} ; 13 \mathrm{~mL} ; 13 \mathrm{~mL} \text {; } \\
\text { and } 12 \mathrm{~mL}) \text {. Settle solids and decant } \\
\text { liquid portion between washes. }\end{array}$ & - & $\begin{array}{l}\text { Total of } 18- \\
19 \% \mathrm{Al} \\
\text { removed } \\
\text { during } \\
\text { caustic leach } \\
\text { and final } \\
\text { wash steps. }\end{array}$ & - & - & - & - \\
\hline
\end{tabular}


Table A1-2. SSTs and DSTs Wash and Leach Information for Aluminum, Part 2. (27 sheets)

\begin{tabular}{|c|c|c|c|c|c|c|c|c|c|}
\hline$\underline{\text { Tank }}$ & $\begin{array}{l}\text { Lab Study } \\
\text { Reference(s) }\end{array}$ & Caustic Leach $\# 2$ Procedure & $\begin{array}{l}\text { Wash of solids after Caustic leach } \\
\text { steps }\end{array}$ & $\begin{array}{l}\text { Mass (ug) Al removed during 1st leach } \\
\text { step }\end{array}$ & \begin{tabular}{|c|} 
Mass (ug) \\
Al removed \\
during 2nd \\
leach step \\
\end{tabular} & $\begin{array}{l}\text { Mass (ug) Al } \\
\text { removed in } \\
\text { final wash } \\
\text { solution } \\
\end{array}$ & $\begin{array}{l}\text { Mass (ug) Al } \\
\text { in Leached } \\
\text { Solids }\end{array}$ & $\begin{array}{c}\text { Total mass Al } \\
\text { (ug) }\end{array}$ & Comments \\
\hline 241-B-111 & PNL-10712 & $\begin{array}{l}\text { Add } 1.44 \mathrm{~g} \text { of } 3 \mathrm{M} \mathrm{NaOH} \text { to } 5.2 \\
\mathrm{~mL} \text { centrifuged solids; mix } 5 \mathrm{hr} \\
\text { at } 100^{\circ} \mathrm{C} \text {; cool; measure settling; } \\
\text { centrifuge; and decant. }\end{array}$ & 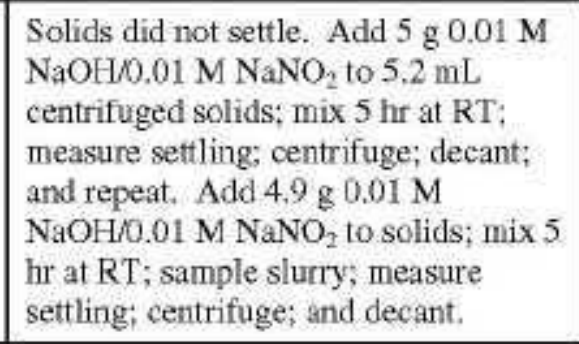 & $\begin{array}{l}99 \% \text { Al removed (1\% Al found, } 17.85 \\
\text { ug AVL). }\end{array}$ & $\begin{array}{l}\text { Remaining } \\
1 \% \mathrm{Al}(16 \\
\text { ug } \mathrm{Al} / \mathrm{mL} \\
\text { detected). }\end{array}$ & $\begin{array}{l}99 \% \mathrm{Al} \\
\text { removed } \\
\text { (measured } 8.5 \\
\text { ug A//mL). }\end{array}$ & $\begin{array}{l}\text { Measured } \\
\text { concentration } \\
\text { of } 18000 \text { ug } \\
\text { Al/g. }\end{array}$ & $\begin{array}{l}\text { Direct analysis: } \\
\text { 3E-03g Al/g } \\
\text { sludge; } \\
\text { Summation } \\
\text { method: } 4.11 \mathrm{E}- \\
03 \mathrm{~g} \text { Al/g } \\
\text { sluge; } 137 \% \\
\text { Al recovered. } \\
\end{array}$ & - \\
\hline 241-B-111 & PNNL- 11089 & $\begin{array}{l}\text { Add } \mathrm{NaOH} \text { to get }-8 \mathrm{wt} \% \text { solids } \\
\text { and a final } \mathrm{NaOH} \text { of } 4.3 \mathrm{M} \text {. Mix } \\
\text { at } 100^{\circ} \mathrm{C} \text { for } 5 \mathrm{hr} \text {. Cool, } \\
\text { centrifuge, and decant. }\end{array}$ & $\begin{array}{l}\text { Add inhibited water. Mix at RT for } \\
0.5 \mathrm{hr} \text { (target } 8 \mathrm{wt} \% \text { solids). } \\
\text { Centrifuge, decant, and repeat twice } \\
\text { more. }\end{array}$ & $1 \%$ Al removed. & - & $\begin{array}{l}1 \% \mathrm{Al} \\
\text { removed. }\end{array}$ & $\begin{array}{l}\text { Total wt } \% \text { Al } \\
=0.3 ; \text { Total \% } \\
\text { Al removed = } \\
2 \% ; 98 \% \mathrm{Al} \\
\text { undissolved. }\end{array}$ & - & - \\
\hline 241-B-201 & PNL-10078 & $\begin{array}{l}\text { Drv cores: Add } 5 \mathrm{~mL} \text { of } 1 \mathrm{M} \\
\mathrm{K}_{2} \mathrm{CO}_{3} \text { to solids, mix at } 100^{\circ} \mathrm{C} \\
\text { for } 5 \mathrm{hr} \text {, centrifuge, decant. Add } \\
5 \mathrm{~mL} 1 \mathrm{M} \mathrm{K} \mathrm{CO}_{3} \text { to solids, mix at } \\
100^{\circ} \mathrm{C} \text { for } 5 \mathrm{hr} \text {, centrifuge, } \\
\text { decant. Wet core 26: Add } 1 \mathrm{~mL} \\
1 \mathrm{M} \mathrm{K}_{2} \mathrm{CO}_{3} \text { to solids, mix at } \\
100^{\circ} \mathrm{C} \text { for } 5 \mathrm{hr} \text {, centrifuge, } \\
\text { decant and repeat. }\end{array}$ & $\begin{array}{l}\text { Add } 5 \mathrm{~mL} \text { water to solids, mix at RT } \\
\text { for } 0.5 \mathrm{hr} \text {, centrifuge, decant; dry at } \\
80^{\circ} \mathrm{C} \text {. }\end{array}$ & $\begin{array}{l}\text { Core 26: } 16 \% \text { Al dissolved Core 27: } \\
38-39 \% \mathrm{Al} \text { dissolved. }\end{array}$ & $\begin{array}{l}\text { Core 26: } 0- \\
\text { 1\% Al } \\
\text { dissolved. } \\
\text { Core 27: } \\
5 \% \text { Al } \\
\text { dissolved. }\end{array}$ & - & $\begin{array}{l}\text { Core 26: } 82- \\
84 \% \mathrm{Al} \\
\text { dissolved. } \\
\text { Core 27: } 55- \\
56 \% \mathrm{Al} \\
\text { dissolved. }\end{array}$ & 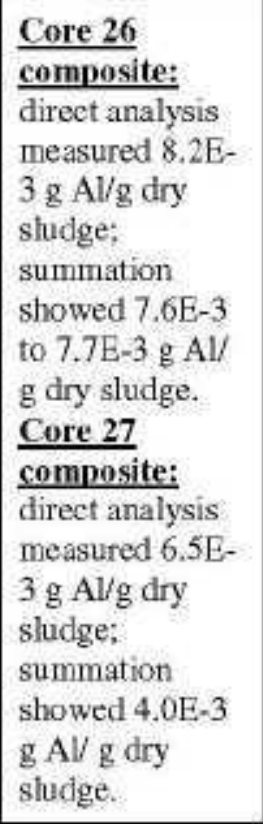 & - \\
\hline $241-B-202$ & $\begin{array}{l}\text { LAUR 95- } \\
2070\end{array}$ & $\begin{array}{l}\text { Add } 2.5 \mathrm{~mL} 3 \mathrm{M} \mathrm{NaOH} \text { to solids, } \\
\text { mix at } 100^{\circ} \mathrm{C} \text { for } 5 \mathrm{hr} \text {, cool, } \\
\text { measure settling, centrifuge, } \\
\text { decant (Vol. settled solids } \\
-6.5 \mathrm{~mL} \text { : Vol. centrifuged solids } \\
\sim 4.6 \mathrm{~mL}) \text {. }\end{array}$ & $\begin{array}{l}\text { Wash } 3 \text { times by adding } 11.5 \mathrm{~mL} \text {. } \\
\text { inhibited water to solids and mix at } \\
\text { RT for } 0.5 \mathrm{hr} \text {, measure settling, } \\
\text { centrifuge and decant. For wash } \# 3 \text {, } \\
\text { sample slurry }(0.9 \mathrm{~mL}) \text { affer mix } \\
\text { phase. Dry solids a } 80^{\circ} \mathrm{C} \text {. }\end{array}$ & $102 \mathrm{ug} \mathrm{Al} / \mathrm{mL}$. (5.24\% Al removed). & $\begin{array}{l}119 \mathrm{ug} \\
\mathrm{Al} / \mathrm{mL} \\
\text { (5.47\% } \mathrm{Al} \\
\text { removed). }\end{array}$ & $\begin{array}{l}8.5 \mathrm{ug} \mathrm{Al} / \mathrm{mL} \\
(5.48 \% \mathrm{Al} \\
\text { removed). }\end{array}$ & $\begin{array}{l}3874 \text { ug } \mathrm{Al} / \mathrm{g} \\
\text { sludge } \\
(80.92 \% \mathrm{Al} \\
\text { remaining. }\end{array}$ & $\begin{array}{l}\text { Direct Analysis: } \\
2.6 \mathrm{E}-3 \mathrm{~g} \mathrm{Al} / \mathrm{g} \\
\text { sludge; } \\
\text { Summation: } \\
2.4 \mathrm{E}-3 \mathrm{~g} \mathrm{Al} / \mathrm{g} \\
\text { siugde (92.14\% } \\
\text { Al recovery). }\end{array}$ & - \\
\hline
\end{tabular}


Table A1-2. SSTs and DSTs Wash and Leach Information for Aluminum, Part 2. (27 sheets)

\begin{tabular}{|c|c|c|c|c|c|c|c|c|c|}
\hline$\underline{\text { Tank }}$ & $\begin{array}{l}\text { Lab Study } \\
\text { Reference(s) }\end{array}$ & Caustic Leach \#2 Procedure & $\begin{array}{c}\text { Wash of solids after Caustic leach } \\
\text { steps }\end{array}$ & $\begin{array}{l}\text { Mass (ug) Al removed during 1st leach } \\
\text { step }\end{array}$ & $\begin{array}{l}\text { Mass (ug) } \\
\text { Al removed } \\
\text { during 2nd } \\
\text { leach step } \\
\end{array}$ & $\begin{array}{l}\text { Mass (ug) Al } \\
\text { removed in } \\
\text { final wash } \\
\text { solution } \\
\end{array}$ & $\begin{array}{l}\text { Mass (ug) Al } \\
\text { in Leached } \\
\text { Solids }\end{array}$ & $\begin{array}{l}\text { Total mass Al } \\
\text { (ug) }\end{array}$ & Comments \\
\hline 241-BX-103 & $\begin{array}{l}\text { LAUR 97- } \\
2889\end{array}$ & $\begin{array}{l}\text { Add } 155.2 \mathrm{~mL} \text { water } \& 67.5 \mathrm{~mL} \\
10 \mathrm{M} \mathrm{NaOH} \text { to achieve } 1.7 \mathrm{wt} \% \\
\text { solids \& }-3 \mathrm{M} \mathrm{NaOH} \text {, Mix } 5 \mathrm{hr} \\
\text { at } 100^{\circ} \mathrm{C} \text {; cool; measure settling; } \\
\text { centrifuge; ;nd decant. } \\
\text { Supernate: } 212.44 \mathrm{~mL} \text { with } 3.17 \\
\text { M free } \mathrm{OH}, 5.2 \mathrm{~mL} \text { settled } \\
\text { solids, } 4 \mathrm{~mL} \text { centrifuged solids }\end{array}$ & $\begin{array}{l}\text { Wash } 3 \text { times by adding } 72 \mathrm{~mL} \\
\text { inhibited water to solids; mix } 0.5 \mathrm{hr} \text { at } \\
\text { RT; measure settling; centrifuge; and } \\
\text { decant. Subsample ( } 0.61 \mathrm{~g} \text { ) after mix } \\
\text { of wash \#3. Dry solids at } 105^{\circ} \mathrm{C} \text {. } \\
\text { (0.855 g solids \& } 212.44 \mathrm{~mL} \\
\text { supernate). }\end{array}$ & $\begin{array}{l}17082 \mathrm{ug} \mathrm{Al} / \mathrm{mL}, 1136294.6 \text { ug } \mathrm{Al} \\
\text { (93.126. Al removed). }\end{array}$ & $\begin{array}{l}365.3 \text { ug } \\
\mathrm{Al} / \mathrm{mL}, \\
77604.33 \text { ug } \\
\mathrm{Al}(6.36 \% \\
\mathrm{Al} \\
\text { removed). }\end{array}$ & $\begin{array}{l}11.66 \text { ug } \\
\mathrm{A} / \mathrm{mL} \text {, } \\
2471.69 \text { ug Al } \\
(0.2 \% \mathrm{Al} \\
\text { removed). }\end{array}$ & $\begin{array}{l}4542.526 \mathrm{ug} \\
\mathrm{Al} / \mathrm{g}, 3882.04 \\
\text { ug Al }(0.32 \% \\
\text { Al remaining) }\end{array}$ & $\begin{array}{l}\text { Concentration } \\
\text { in initial solids; } \\
\text { Summation = } \\
2.33 \mathrm{E}-1 \mathrm{~g} \mathrm{Al} / \mathrm{g} \text {, } \\
\text { Direct analysis } \\
=2.1 \mathrm{E}-1 \mathrm{~g} \mathrm{Al} / \mathrm{g} . \\
110.916 \% \mathrm{Al} \\
\text { mass recovery. } \\
1220252.7 \mathrm{ug} \\
\mathrm{AL} .\end{array}$ & - \\
\hline $241-\mathrm{BX}-105$ & $\begin{array}{l}\text { LAUR 95- } \\
2070\end{array}$ & $\begin{array}{l}\text { Add } 47.5 \mathrm{~mL} \text { of } 3 \mathrm{M} \mathrm{NaOH} \text {, mix } \\
5 \mathrm{hr} \text { at } 100^{\circ} \mathrm{C} \text {, cool, measure } \\
\text { settling, centrifuge, and decant. } \\
\text { Volume settled solids }<1 \mathrm{~mL} \text {; } \\
\text { Volume centrifuged solids }<1 \\
\mathrm{~mL}, 47.16 \mathrm{~mL} \text { liquid from } \\
\text { decant. }\end{array}$ & $\begin{array}{l}\text { Wash } 3 \text { times by adding } 50.5 \mathrm{~mL} \\
\text { inhibited water to solids and mix at } \\
\text { RT for } 0.5 \mathrm{hr} \text {, measure settling, } \\
\text { centrifuge, and decant. For wash } \# 3 \text {, } \\
\text { sample slurry }(0.3 \mathrm{~mL}) \text { ) after mix } \\
\text { phase. Dry solids at } 80^{\circ} \mathrm{C}(0.0352 \mathrm{~g}) \text {. }\end{array}$ & 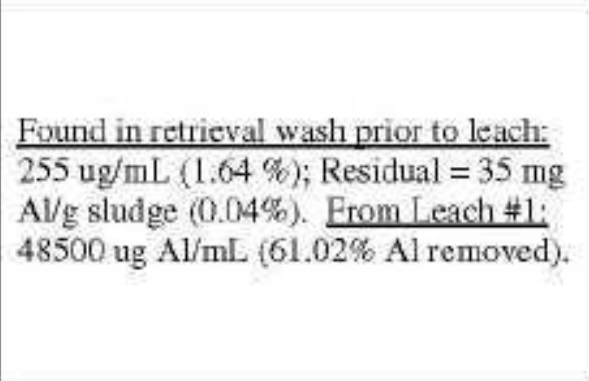 & $\begin{array}{l}23800 \text { ug } \\
\mathrm{Al} / \mathrm{mL} \\
\text { (35.21\% } \mathrm{Al} \\
\text { removed). }\end{array}$ & $\begin{array}{l}442 \mathrm{ug} \mathrm{Al} / \mathrm{mL} \\
(2.09 \% \mathrm{Al} \\
\text { removed). }\end{array}$ & $\begin{array}{l}34632 \text { ug } \mathrm{Al} / \mathrm{g} \\
\text { sludge. }\end{array}$ & $\begin{array}{l}\text { Direct analysis: } \\
3.05 \mathrm{E}-01 \mathrm{~g} \mathrm{Al} / \mathrm{g} \\
\text { sludge; } \\
\text { Summation } \\
\text { method: } 3.41 \mathrm{E}- \\
01 \mathrm{~g} \text { - Al/g } \\
\text { sludge; } \\
111.71 \% \text { Al } \\
\text { recovery. }\end{array}$ & - \\
\hline 241-BX-107 & PNL-10712 & $\begin{array}{l}\text { Add } 10.5 \mathrm{~g} \text { of } 3 \mathrm{M} \mathrm{NaOH} \text { to } 6.1 \\
\text { mL centrifuged solids; mix } 5 \mathrm{htr} \\
\text { at } 100^{\circ} \mathrm{C} \text {; cool; measure settling: } \\
\text { centrifuge; and decant. }\end{array}$ & $\begin{array}{l}\text { Add } 10.9 \mathrm{~g} 0.01 \mathrm{M} \mathrm{NaOH} / 0.01 \mathrm{M} \\
\mathrm{NaNO}{ }_{2} \text { to } 4.0 \mathrm{~mL} \text { centrifuged solids; } \\
\text { mix } 0.5 \mathrm{hr} \text { at } \mathrm{RT} \text {; measure settling; } \\
\text { centrifuge; and decant. Add } 11.6 \mathrm{~g} \\
0.01 \mathrm{M} \mathrm{NaOH} / 0.01 \mathrm{M} \mathrm{NaNO} \mathrm{N}_{2} \text { to } \\
\text { solids; mix } 0.5 \mathrm{hr} \text { at } \mathrm{RT} \text {; measure } \\
\text { settling; centrifuge; and decant. Add } \\
11.7 \mathrm{~g} 0.01 \mathrm{M} \mathrm{NaOH} / 0.01 \mathrm{M} \mathrm{NaNO}{ }_{2} \\
\text { to solids; mix } 5 \mathrm{hr} \text { at RT; sample } \\
\text { slurry; measure settling; centrifuge; } \\
\text { and decant. }\end{array}$ & $49 \% \mathrm{Al}, 7225$ ug $\mathrm{Al} / \mathrm{mL}$ & $\begin{array}{l}11 \% \mathrm{Al}, \\
2720 \text { ug } \\
\mathrm{Al} / \mathrm{mL} \text {. }\end{array}$ & $\begin{array}{l}7 \% \mathrm{Al}, 272 \mathrm{ug} \\
\mathrm{Al} / \mathrm{mL} .\end{array}$ & $\begin{array}{l}41000 \mathrm{ug} \mathrm{Al} / \mathrm{g}, \\
32 \% \text { Al in } \\
\text { residue. }\end{array}$ & $\begin{array}{l}\text { Direct analysis: } \\
3.5 \mathrm{E}-02 \mathrm{~g} \mathrm{Al} / \mathrm{g} \\
\text { sludge; } \\
\text { Summation } \\
\text { method: } 2.83 \mathrm{E}- \\
02 \mathrm{~g} \mathrm{Al} / \mathrm{g} \\
\text { sludge; } 81 \% \mathrm{Al} \\
\text { recovery. }\end{array}$ & - \\
\hline $241-\mathrm{BX}-107$ & PNNL-11089 & $\begin{array}{l}\text { Add } \mathrm{NaOH} \text { to get }-8 \text { wto solids } \\
\text { and a final } \mathrm{NaOH} \text { of } 3.5 \mathrm{M} \text {. Mix } \\
\text { at } 100^{\circ} \mathrm{C} \text { for } 5 \mathrm{hr} \text {. Cool, } \\
\text { centrifuge, and decant. }\end{array}$ & $\begin{array}{l}\text { Add inhibited water. Mix at RT for } \\
0.5 \mathrm{hr} \text { (target } 8 \mathrm{wt} \% \text { solids). } \\
\text { Centrifuge, decant, and repeat two } \\
\text { more times. }\end{array}$ & $\begin{array}{l}49 \% \text { Al uncorrected; } 75 \% \mathrm{Al} \text { corrected } \\
\text { value. }\end{array}$ & $\begin{array}{l}11 \% \mathrm{Al} \\
\text { uncorrected; } \\
-5 \% \mathrm{Al} \\
\text { corrected } \\
\text { value. }\end{array}$ & $\begin{array}{l}7 \% \mathrm{Al} \\
\text { uncorrected; - } \\
2 \% \mathrm{Al} \\
\text { corrected } \\
\text { value. }\end{array}$ & $\begin{array}{l}\text { Total wt\% } \mathrm{Al} \\
=3.5 ; \text { Total \% } \\
\text { Al removed = } \\
68 \% ; \mathrm{Al} \text { in } \\
\text { undissolved } \\
\text { residue }=32 \% \\
\text { (31\% } \\
\text { corrected } \\
\text { value). }\end{array}$ & - & - \\
\hline
\end{tabular}


Table A1-2. SSTs and DSTs Wash and Leach Information for Aluminum, Part 2. (27 sheets)

\begin{tabular}{|c|c|c|c|c|c|c|c|c|c|}
\hline$\underline{\text { Tank }}$ & $\begin{array}{l}\text { Lab Study } \\
\text { Reference (8) }\end{array}$ & Caustic Leach $\# 2$ Procedure & $\begin{array}{l}\text { Wash of solids after Caustic leach } \\
\text { steps }\end{array}$ & $\begin{array}{l}\text { Mass (ug) Al removed during 1st leach } \\
\text { step }\end{array}$ & \begin{tabular}{|l|} 
Mass (ug) \\
Al removed \\
during 2nd \\
leach step \\
\end{tabular} & $\begin{array}{c}\begin{array}{c}\text { Mass (ug) Al } \\
\text { removed in } \\
\text { final wash } \\
\text { solution }\end{array} \\
\end{array}$ & $\begin{array}{l}\text { Mass (ug) Al } \\
\text { in Leached } \\
\text { Solids }\end{array}$ & \begin{tabular}{|c|} 
Total mass Al \\
(ug)
\end{tabular} & Comments \\
\hline $241-\mathrm{BX}-109$ & $\begin{array}{l}\text { LAUR 96- } \\
2839\end{array}$ & $\begin{array}{l}\text { Add } 11 \mathrm{~mL} 10 \mathrm{M} \mathrm{NaOH} \text { and } \\
-23 \mathrm{ml} \text { water to solids to get }-1 \\
\text { wt } \% \text { solids and final } \mathrm{NaOH} \text { of } \\
-3 \mathrm{M}(6.2 \mathrm{~mL} \text { settled solids; } 1.5 \\
\mathrm{mL} \text {, centrifuged solids). Mix } 5 \\
\text { hr at } 100^{\circ} \mathrm{C} \text {, coofl; measure } \\
\text { settling; centrifuge; and decant. }\end{array}$ & $\begin{array}{l}\text { Add } 7.1 \mathrm{~mL} \text {. inhibited water to solids } \\
\text { (3.9 mL, settled solids } / 1.6 \mathrm{~mL} \\
\text { centrifuged solids); mix } 0.5 \mathrm{hr} \text { at } \mathrm{RT} \text {; } \\
\text { measure settling, centrifuge; decant. } \\
\text { Repeat } 2 \text { times with solids portion, } \\
\text { sampling slurry ( } 0.7 \mathrm{~mL} \text { ) before } \\
\text { measuring settling in wash } \# 3 \text {. Dry } \\
\text { solids at } 105^{\circ} \mathrm{C} \text {. }\end{array}$ & $\begin{array}{l}148.413 \text { ug Al/mL; } 1959.44 \text { ug Al: } \\
74.7 \% \text { Al. }\end{array}$ & $\begin{array}{l}17.423 \text { ug } \\
\text { Al/mL; } \\
569.55 \text { ug } \\
\text { Al; } 21.6 \% \\
\text { Al. }\end{array}$ & $\begin{array}{l}1.387 \text { ug } \\
\text { Al/mL; } 27.91 \\
\text { ug Al; } 1.0 \% \\
\text { Al. }\end{array}$ & $\begin{array}{l}20875 \text { ug Al/g; } \\
7031 \text { ug Al; } \\
2.7 \% \mathrm{Al} \text { in } \\
\text { solids; } 97 \% \mathrm{Al} \\
\text { removed. }\end{array}$ & $\begin{array}{l}\text { Direct analysis: } \\
1.11 \mathrm{E}-3 \mathrm{~g} \mathrm{Al} / \mathrm{g} \\
\text { sludge: } \\
\text { Summation } \\
\text { method: } 1.38 \mathrm{E}- \\
3 \mathrm{~g} \mathrm{Al} / \mathrm{g} \text { sludge. } \\
80.6 \% \mathrm{Al} \\
\text { recovery: }\end{array}$ & - \\
\hline $241-\mathrm{BX}-110$ & PNNL-12026 & $\begin{array}{l}\text { After leach test, remove vessel } \\
\text { from heating block and } \\
\text { centrifuge } 5 \text { min. Replace on } \\
\text { heating block \& transfer solution } \\
\text { to clean bottle. Wash solids } 3 \\
\text { times with } 15 \mathrm{~mL} 0.01 \mathrm{M} \\
\mathrm{NaOH} \text {, stirring } 30 \text { min } \\
\text { (minimum), centrifuge, and } \\
\text { decant. Dry at } 105^{\circ} \mathrm{C} \text {. }\end{array}$ & $\begin{array}{l}\text { For } 60^{\circ} \mathrm{C} \text { studies: } 95 \% \text { Al removal at } \\
1 \mathrm{M} \mathrm{NaOH}(94 \% \% \text { Fe normalized), } \\
99 \% \mathrm{Al} \text { removal at } 3 \mathrm{M} \mathrm{NaOH}(99 \% \\
\text { Fe normalized). } \\
\text { For } 80^{\circ} \mathrm{C} \text { studies: } 98 \% \mathrm{Al} \text { removal at } \\
1 \mathrm{M} \mathrm{NaOH}(97 \% \% \text { Fe normalized), } \\
97 \% \mathrm{Al} \text { removal at } 3 \mathrm{M} \mathrm{NaOH}(96 \% \\
\text { Fe normalized). }\end{array}$ & $\begin{array}{l}\text { For } 95^{\circ} \mathrm{C} \text { studies: } 99 \% \text { Al removal at } 1 \\
\text { M NaOH ( } 99 \% \mathrm{Fe} \text { normalized), } 99 \% \\
\text { Al removal at } 3 \mathrm{M} \mathrm{NaOH}(98 \% \mathrm{Fe} \\
\text { normalized). }\end{array}$ & - & - & - & - & - \\
\hline $241-\mathrm{BX}-112$ & PNNL-12026 & $\begin{array}{l}\text { After leach test, leachate } \\
\text { solution transferred from settled } \\
\text { solids. Settled solids centrifuged } \\
\text { \& supernate decanted \& } \\
\text { combined w/ leachate solution. } \\
\text { Wash solids } 3 \text { times with } 15 \mathrm{~mL} \\
0.01 \mathrm{M} \mathrm{NaOH} \text { stirring } 30 \mathrm{~min} \\
\text { (minimum), centrifuge, and } \\
\text { decant. Dry at } 105^{\circ} \mathrm{C} \text {. }\end{array}$ & $\begin{array}{l}\text { For } 60^{\circ} \mathrm{C} \text { studies: } 62 \% \text { Al removal at } \\
1 \mathrm{M} \mathrm{NaOH} \text { (64\% Fe normalized), } \\
68 \% \text { Al removal at } 3 \mathrm{M} \mathrm{NaOH}(75 \% \\
\text { Fe normalized). } \\
\text { For } 80^{\circ} \mathrm{C} \text { cstudies: } 54 \% \text { Al removal at } \\
1 \mathrm{M} \mathrm{NaOH} \mathrm{(59 \%} \text { Fe normalized), } \\
63 \% \text { Al removal at } 3 \mathrm{M} \mathrm{NaOH} 69 \% \\
\text { Fe normalized). }\end{array}$ & $\begin{array}{l}\text { For } 100^{\circ} \mathrm{C} \text { studies: } 51 \% \text { Al removal at } \\
1 \mathrm{M} \mathrm{NaOH}(57 \% \mathrm{Fe} \text { normalized), } 59 \% \\
\text { Al removal at } 3 \mathrm{M} \mathrm{NaOH}(59 \% \mathrm{Fe} \\
\text { normalized). }\end{array}$ & - & - & - & - & - \\
\hline 241-BY-104 & PNNL-11278 & $\begin{array}{l}\text { Add } 10.3 \mathrm{~mL}, 10 \mathrm{M} \mathrm{NaOH} \text { and } \\
\text { water to a total volume of } 32 \mathrm{~mL} \\
(2 \text { wt'\% solids) to solids, mix at } \\
100^{\circ} \mathrm{C} \text { for } 5 \mathrm{hr} \text {, cool, measure } \\
\text { settling, centrifuge, and decant } \\
631.5 \mathrm{~mL} \text { decanted liquid, } 1.0 \\
\mathrm{~mL} \text { interstitial liquid, } 3.3 \mathrm{M} \mathrm{OH} \\
\text { 3. }\end{array}$ & 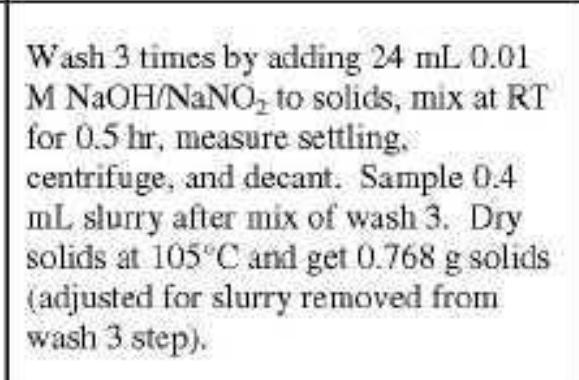 & $\begin{array}{l}10595 \text { ug Al/mL; } 225674 \text { ug Al; } 32 \% \\
\text { Al. }\end{array}$ & $\begin{array}{l}606 \mathrm{ug} \\
\mathrm{AV} / \mathrm{mL} ; 4862 \\
\mathrm{ug} \mathrm{Al} ; 196 \\
\mathrm{Al} .\end{array}$ & $\begin{array}{l}14.6 \mathrm{ug} \\
\mathrm{Al} / \mathrm{mL} ; 426 \mathrm{ug} \\
\mathrm{Al} ; 0 \% \mathrm{Al}\end{array}$ & $\begin{array}{l}14900 \mathrm{ug} \\
\mathrm{Al} / \mathrm{mL} ; 11443 \\
\text { ug Al; } 2 \% \mathrm{AI} .\end{array}$ & $\begin{array}{l}695880 \text { ug Al; } \\
\text { Summation: } \\
1.75 \mathrm{E}+4 \text { ug } \\
\text { Alyg sludge; } \\
\text { Direct: } \\
1.88 \mathrm{E}+4 \text { ug } \\
\text { Al/g sludge; } \\
\text { Mass Recovery: } \\
93 \% \text { Al. }\end{array}$ & $\begin{array}{l}98 \% \text { removed by leaching } 2 \text { times with } \\
\text { caustic and washing } 3 \text { times with } 0.01 \mathrm{M} \\
\mathrm{NaOH} / \mathrm{NaNO}_{2} \text { solution. Initial Wash } \\
\text { Solution: } 2425 \text { ug Al/mL; } 453475 \text { ug Al; } \\
65 \% \text {. }\end{array}$ \\
\hline 241-BY-108 & PNNL-11636 & $\begin{array}{l}\text { Add } 46.85 \mathrm{~mL}, 10 \mathrm{M} \mathrm{NaOH} \text { and } \\
\text { water to solids to total volume of } \\
\sim 170 \mathrm{~mL} \text {; mix } 5 \mathrm{hr} \text { at } 100^{\circ} \mathrm{C} \text {; } \\
\text { cool; gravity settle; decant. }\end{array}$ & 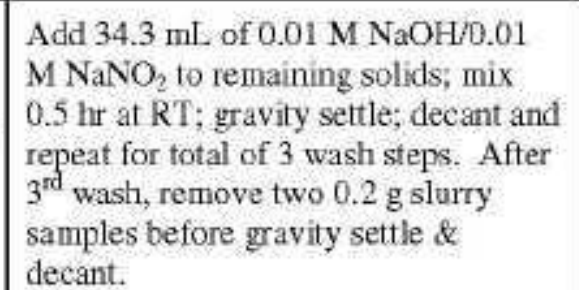 & 40222 ug Al $(30 \% \mathrm{Al})$. & $\begin{array}{l}50276 \mathrm{ug} \mathrm{Al} \\
(37 \% \mathrm{Al})\end{array}$ & $\begin{array}{l}6290 \mathrm{ug} \mathrm{Al} \\
\text { (5\% Al). }\end{array}$ & $\begin{array}{l}39336 \text { ug Al } \\
(29 \% \mathrm{Al}) \text {. }\end{array}$ & $\begin{array}{l}136124 \text { ug Al } \\
(126 \% \mathrm{Al} \\
\text { recovery). }\end{array}$ & - \\
\hline
\end{tabular}


Table A1-2. SSTs and DSTs Wash and Leach Information for Aluminum, Part 2. (27 sheets)

\begin{tabular}{|c|c|c|c|c|c|c|c|c|c|}
\hline$\underline{\text { Tank }}$ & $\begin{array}{l}\text { Lab Study } \\
\text { Reference(s) }\end{array}$ & Caustic Leach \#2 Procedure & $\begin{array}{c}\text { Wash of solids after Caustic leach } \\
\text { steps }\end{array}$ & $\begin{array}{l}\text { Mass (ug) Al removed during 1st leach } \\
\text { step }\end{array}$ & \begin{tabular}{|c|} 
Mass (ug) \\
Al removed \\
during 2nd \\
leach step \\
\end{tabular} & $\begin{array}{l}\text { Mass (ug) Al } \\
\text { removed in } \\
\text { final wash } \\
\text { solution } \\
\end{array}$ & \begin{tabular}{|c} 
Mass (ug) Al \\
in Leached \\
Solids \\
\end{tabular} & \begin{tabular}{|c|} 
Total mass $\mathrm{Al}$ \\
(ug)
\end{tabular} & Comments \\
\hline $241-B Y-110$ & PNNL-11278 & $\begin{array}{l}\text { Add } 13 \mathrm{~mL} .10 \mathrm{M} \mathrm{NaOH} \text { and } \\
\text { water to a total volume of } 43 \mathrm{~mL} \\
(1.1 \text { wt's solids). To solids, mix } \\
\text { at } 100^{\circ} \mathrm{C} \text { for } 5 \mathrm{hr} \text {, cool, measurc } \\
\text { settling, centrifuge, and decant } \\
(39.3 \mathrm{~mL} \text { decanted liquid, } 2.0 \\
\mathrm{mL} \text { interstitial liquid, } 3.2 \mathrm{M} \mathrm{OH} \\
\text { ). }\end{array}$ & $\begin{array}{l}\text { Wash } 3 \text { times by adding } 9 \mathrm{~mL}, 0.01 \mathrm{M} \\
\mathrm{NaOH} / \mathrm{NaNO} \mathrm{O}_{2} \text { to solids, mix at RT for } \\
0.5 \mathrm{hr} \text {, measure settling, centrifuge, } \\
\text { and decant. Sample } 0.4 \mathrm{~mL} \text { slurry } \\
\text { after mix of wash } 3 \text {. Dry solids at } \\
105^{\circ} \mathrm{C}=0.548 \mathrm{~g} \text { solids (adjusted for } \\
\text { slurry removed from wash } 3 \text { step); }\end{array}$ & $\begin{array}{l}9048 \mathrm{ug} \mathrm{Al} / \mathrm{mL} ; 164674 \text { ug Al; } 94 \% \\
\text { Al. }\end{array}$ & $\begin{array}{l}454 \mathrm{ug} \\
\mathrm{Al} / \mathrm{mL} ; 3414 \\
\text { ug Al; } 2 \% \\
\mathrm{AL} .\end{array}$ & $\begin{array}{l}38.6 \mathrm{ug} \\
\mathrm{Al} / \mathrm{mL}: 177 \text { ug } \\
\mathrm{Al} ; 0 \% \mathrm{Al}\end{array}$ & $\begin{array}{l}13850 \text { ug } \\
\mathrm{Al} / \mathrm{mL} ; 7590 \\
\text { ug Al; } 4 \% \mathrm{Al} .\end{array}$ & \begin{tabular}{|l}
175854 ug Al; \\
Summation: \\
$4.39 \mathrm{E}+4$ ug \\
Al/g sludge. \\
Direct: \\
$3.37 \mathrm{E}+4$ ug \\
Al/g sludge. \\
Mass Recoverv: \\
$130 \%$ Al. Total \\
of $96 \% \mathrm{Al}$ \\
removed by \\
leaching 2 times \\
with canstic and \\
washing 3 tines \\
with $0.01 \mathrm{M}$ \\
$\mathrm{NaOH} / \mathrm{NaNO}_{2}$ \\
solution.
\end{tabular} & - \\
\hline 241-C-102 & PNNL-12026 & $\begin{array}{l}\text { Remove reaction vessel and } \\
\text { allow to cool to RT, centrifuge } \\
15 \text { minutes, decant liquid, wash } \\
\text { solids } 3 \text { times with } 10 \mathrm{~mL} 0.01 \mathrm{M} \\
\mathrm{NaOH} / \mathrm{NaNO}_{2} \text {, dry at } 105^{\circ} \mathrm{C} \text {. }\end{array}$ & $\begin{array}{l}\text { At time of } 5 \mathrm{hr}: \# 1=5185 \mathrm{ug} \mathrm{Al} / \mathrm{mL} \text {; } \\
\# 2=8524 \text { ug Al} / \mathrm{mL} ; \# 3=12030 \text { ug } \\
\mathrm{Al} / \mathrm{mL} ; \# 4=24160 \mathrm{ug} \mathrm{Al} / \mathrm{mL}\end{array}$ & $\begin{array}{l}\text { At time of } 72 \mathrm{hr}: \# 1=6273 \mathrm{ug} \mathrm{Al} / \mathrm{mL} \text {, } \\
115419 \text { ug } \mathrm{Al} ; \# 2=9406 \text { ug Al} / \mathrm{mL} \text {, } \\
115694 \text { ug Al; } \# 3=16260 \mathrm{ug} \mathrm{Al} / \mathrm{mL} \text {, } \\
635766 \text { ug Al; } \$ 4=30620 \text { ug Al} / \mathrm{mL} \text {, } \\
532788 \text { ug Al. }\end{array}$ & $\begin{array}{l}\# 1=745 \text { ug } \\
\mathrm{Al} / \mathrm{mL}, \\
22344 \text { ug } \\
\mathrm{Al} ; \# 2=535 \\
\text { ug } \mathrm{Al} / \mathrm{mL}, \\
16059 \text { ug } \\
\mathrm{Al} ; \# 3=2066 \\
\text { ug Al/mL, } \\
62393 \mathrm{ug} \\
\mathrm{Al} \# \# 4=3356 \\
\text { ug } \mathrm{Al} / \mathrm{mL}, \\
101026 \text { ug } \\
\mathrm{Al}\end{array}$ & $\begin{array}{l}\# 1=139345 \text { ug } \\
\mathrm{Al} / \mathrm{g}, 373891 \\
\text { ug Al: } \\
\# 2=180464 \text { ug } \\
\mathrm{Al} / \mathrm{g}, 530202 \\
\text { ug Al; } \\
\# 3=26042 \text { ug } \\
\mathrm{Al} / \mathrm{g}, 36321 \mathrm{ug} \\
\mathrm{Al} ; \# 4=27600 \\
\text { ug Al/g, } 34949 \\
\text { ug Al. }\end{array}$ & $\begin{array}{l}\text { Mass } \\
\text { recovery: } \\
\# 1=84 \% \mathrm{Al} ; \\
\# 2=99 \% \mathrm{Al} ; \\
\# 3=118 \% \mathrm{Al} ; \\
\# 4=117 \% \mathrm{Al} \text {. } \\
\text { Summation } \\
\text { method: } \% \mathrm{Al} \\
\text { removed by } \\
\text { washing + } \\
\text { leaching: } \\
\# 1=27, \neq 2=20 \text {, } \\
\# 3=95 \text {, and } \\
\# 4=95 \text {. } \\
\end{array}$ & - & - \\
\hline $241-\mathrm{C}-103$ & PNL-10712 & $\begin{array}{l}\text { Add } 7.98 \mathrm{~g} 3 \mathrm{M} \mathrm{NaOH} \text { to } 6.4 \\
\mathrm{~mL} \text { centrifuged solids; mix } 5 \mathrm{hr} \\
\text { at } 100^{\circ} \mathrm{C} \text {; cool; measure settling; } \\
\text { centrifuge; and decant. }\end{array}$ & $\begin{array}{l}\text { Add } 6.5 \mathrm{~g} 0.01 \mathrm{M} \mathrm{NaOH} / 0.01 \mathrm{M} \\
\mathrm{NaNO}_{2} \text { to } 7.0 \mathrm{~mL} \text { centrifuged solids; } \\
\text { mix } 0.5 \mathrm{hr} \text { at RT; measure settling; } \\
\text { centrifuge; and decant. Add } 6.6 \mathrm{~g} \\
0.01 \mathrm{M} \mathrm{NaOH} / 0.01 \mathrm{M} \mathrm{NaNO}_{2} \text { to } \\
\text { solids; mix } 0.5 \mathrm{hr} \text { at RT; measure } \\
\text { settling; centrifuge; and decant. Add } \\
6.5 \mathrm{~g} 0.01 \mathrm{M} \mathrm{NaOH} / 0.01 \mathrm{M} \mathrm{NaNO} \mathrm{Na}_{2} \text { to } \\
\text { solids; mix } 5 \mathrm{hr} \text { at RT; sample slurry; } \\
\text { measure settling; centrifuge; and } \\
\text { decant. }\end{array}$ & $\begin{array}{l}16 \% \text { Al found, } 25500 \mathrm{ug} \mathrm{Al} / \mathrm{mL} \\
\text { measured. }\end{array}$ & $\begin{array}{l}23 \% \mathrm{Al} \\
\text { found, } \\
30600 \text { ug } \\
\text { Al/mL } \\
\text { measured. }\end{array}$ & $\begin{array}{l}9 \% \text { Al found, } \\
4505 \text { ug } \\
\mathrm{Al} / \mathrm{mL} \\
\text { messured. }\end{array}$ & $\begin{array}{l}\text { Measured } \\
\text { concentration } \\
\text { of } 105,000 \text { ug } \\
\text { Alyg, } 52 \% \text { Al } \\
\text { in residue. }\end{array}$ & $\begin{array}{l}\text { Direct analysis: } \\
1.4 \mathrm{E}-01 \mathrm{~g} \text {. Al/g } \\
\text { sludge; } \\
\text { Summation } \\
\text { method: } 1.58 \mathrm{E}- \\
\text { 01 g Al/g } \\
\text { sludge; } 113 \% \\
\text { Al recovery. }\end{array}$ & - \\
\hline
\end{tabular}


Table A1-2. SSTs and DSTs Wash and Leach Information for Aluminum, Part 2. (27 sheets)

\begin{tabular}{|c|c|c|c|c|c|c|c|c|c|}
\hline$\underline{\text { Tank }}$ & $\begin{array}{l}\text { Lab Study } \\
\text { Reference (s) }\end{array}$ & Caustic Leach \$2 Procedure & $\begin{array}{c}\text { Wash of solids after Caustic leach } \\
\text { steps }\end{array}$ & $\begin{array}{l}\text { Mass (ug) Al removed during 1st leach } \\
\text { step }\end{array}$ & \begin{tabular}{|l|} 
Mass (ug) \\
Al removed \\
during 2nd \\
leach step \\
\end{tabular} & $\begin{array}{l}\text { Mass (ug) Al } \\
\text { removed in } \\
\text { final wash } \\
\text { solution } \\
\end{array}$ & $\begin{array}{l}\text { Mass (ug) Al } \\
\text { in Leached } \\
\text { Solids }\end{array}$ & $\begin{array}{l}\text { Total mass Al } \\
\text { (ug) }\end{array}$ & Comments \\
\hline 241-C-103 & PNNL-11089 & $\begin{array}{l}\text { Add } \mathrm{NaOH} \text { to get } \sim 8 \text { wts solids } \\
\text { and a final } \mathrm{NaOH} \text { of } 1.0 \mathrm{M} \text {. Mix } \\
\text { at } 100^{\circ} \mathrm{C} \text { for } 5 \mathrm{hr} \text {. Cool, } \\
\text { centrifuge, and decant. }\end{array}$ & $\begin{array}{l}\text { Add inhibited water. Mix at RT for } \\
0.5 \mathrm{hr} \text { (target } 8 \text { wts solids). } \\
\text { Centrifuge, decant, and repeat twice } \\
\text { more. }\end{array}$ & $\begin{array}{l}\text { Uncorrected/corrected recovery = } \\
16 / 48 \% \mathrm{Al} \text {. }\end{array}$ & $\begin{array}{l}\text { Uncorrected/ } \\
\text { corrected } \\
\text { recovery }= \\
23 / 29 \% \text { Al. }\end{array}$ & $\begin{array}{l}\text { Uncorrected/ } \\
\text { corrected } \\
\text { recovery }=9 /- \\
3 \% \mathrm{Al} \text {. }\end{array}$ & \begin{tabular}{|l|} 
Total wt\% $\mathrm{Al}$ \\
$=14 ;$ Total $\%$ \\
Al removed $=$ \\
$48 \% ;$ \\
Undissolved \\
residue \\
(uncorrected/c \\
orrected) $=$ \\
$52 / 26 \% \mathrm{Al}$.
\end{tabular} & - & - \\
\hline 241-C-104 & $\begin{array}{l}\text { LAUR 97- } \\
2889\end{array}$ & $\begin{array}{l}\text { Add } 154.1 \mathrm{~mL} \text { water and } 66.8 \\
\mathrm{~mL} 10 \mathrm{M} \mathrm{NaOH} \text { to solids to get } \\
-1 \text { wt\% solids \& }-3 \mathrm{M} \mathrm{NaOH} \text {. } \\
\text { Mix } 5 \mathrm{hr} \text { at } 100^{\circ} \mathrm{C} \text {; cool; } \\
\text { measure settling; centrifuge; and } \\
\text { decant. Supernate: } 211.3 \mathrm{~mL} \\
\text { with } 3.16 \mathrm{M} \text { free } \mathrm{OH} ; 13.89 \mathrm{~mL} \\
\text { settled solids, and } 7.12 \mathrm{~mL} \\
\text { centrifuged solids. }\end{array}$ & $\begin{array}{l}\text { Wash } 3 \text { times by adding } 45.3 \mathrm{~mL} \\
\text { inhibited water to solids, mixing for } \\
0.5 \mathrm{hr} \text { at RT; measure settling; } \\
\text { centrifuge; and decant. Sample slurry } \\
\left(0.59 \mathrm{~g} \text { ) after } 3^{\text {te }} \text { mix cycle. Dry solids }\right. \\
\text { at } 105^{\circ} \mathrm{C}(0.5 \mathrm{~g}) \text {. }\end{array}$ & $\begin{array}{l}6068.4 \mathrm{ug} \mathrm{Al} / \mathrm{mL}, 221132.5 \mathrm{ug} \mathrm{Al} \\
(75.5 \% \mathrm{Al})\end{array}$ & $\begin{array}{l}288.6 \text { ug } \\
\mathrm{Al} / \mathrm{mL}, \\
60981.18 \text { ug } \\
\mathrm{Al}(20.8 \% \\
\mathrm{Al}) .\end{array}$ & $\begin{array}{l}17.16 \text { ug } \\
\text { Al/mL, } \\
22242.98 \text { ug } \\
\mathrm{Al}(0.8 \% \text { Al). }\end{array}$ & $\begin{array}{l}5000 \mathrm{ug} \mathrm{Al} / \mathrm{g}, \\
8548 \mathrm{ug} \mathrm{Al} \\
(2.9 \% \mathrm{Al}) .\end{array}$ & $\begin{array}{l}292904.66 \mathrm{ug} \\
\text { Al; Summation } \\
\text { method; } 6.95 \mathrm{E}- \\
2 \mathrm{~g} \mathrm{Al} / \mathrm{g} \text {; Direct } \\
\text { analysis: } 6.32 \mathrm{E}- \\
2 \mathrm{~g} \mathrm{Al} / \mathrm{g} ; 110 \% \\
\text { Al mass } \\
\text { recovery. }\end{array}$ & - \\
\hline $241-C-104$ & $\begin{array}{l}\text { PNWD-3027 } \\
\text { (BNFL-RPT- } \\
\text { 021) }\end{array}$ & - & $\begin{array}{l}\text { Transfer most of filtered solids from } \\
\text { leach back into HDPE bottle using } \\
\text { spatula and } 0.01 \mathrm{M} \mathrm{N} \text { aOH. Total } \\
\text { slurry volume made to } \sim 100 \mathrm{~mL} \text { using } \\
\text { additional } 0.01 \mathrm{M} \mathrm{NaOH} \text { (total slurry } \\
\text { wt } \sim 123 \mathrm{~g} \text {. Heat \& stir at } 85^{\circ} \mathrm{C} \text { for } 21 \\
\mathrm{hr} \text {. Filter hot, yielding } 92.45 \mathrm{~g} \\
\text { washing solution and } 33.35 \mathrm{~g} \text { wet } \\
\text { solids, Repeat wash of wet solids, } \\
\text { heating at } 85^{\circ} \mathrm{C} \text { for } 22.5 \mathrm{hr} \text {. Yield } \\
88.31 \mathrm{~g} \text { washing liquid and } 33.92 \mathrm{~g} \\
\text { wet solids. Antalyze composite of two } \\
\text { wash solutions. Filtered solids } \\
\text { evaporated at } 80^{\circ} \mathrm{C} \text {, then dried } \\
\text { overnight at } 105^{\circ} \mathrm{C} \text { to yield } 7.6051 \mathrm{~g} \\
\text { dried leached solids. }\end{array}$ & $\begin{array}{l}\text { Direct }=45900 \text { ug Al/g; Adjusted due } \\
\text { to } 0.06 \% \text { loss from evaporation }= \\
45,873 \text { ug Al/g; Amount }=453,3915 \text { ug } \\
\text { Al. }\end{array}$ & - & $\begin{array}{l}\text { Direct }=2065 \\
\text { uz Al/g; } \\
\text { Adjusted due } \\
\text { to } 0.23 \% \text { loss } \\
\text { from } \\
\text { evaporation }= \\
2060 \text { ug Al/g; } \\
\text { Amount }= \\
372,430 \text { ug Al. }\end{array}$ & \begin{tabular}{|l} 
Mean from \\
KOH Fusion \\
analysis = \\
32600 ug $\mathrm{Al} / \mathrm{g}$ \\
dry solids; \\
mean from \\
$\mathrm{Na}_{2} \mathrm{O}_{2}$ Fusion \\
analysis $=$ \\
35,900 ug Al/g \\
dry solids; \\
Amount $=$ \\
260,475 ug Al.
\end{tabular} & $\begin{array}{l}\text { From original } \\
\text { sample, assume } \\
127,892 \text { ug Al; } \\
\text { with } 34,250 \text { ug } \\
\text { Al remaining in } \\
\text { leached solids. } \\
95 \% \text { Al } \\
\text { removed. }\end{array}$ & $\begin{array}{l}\text { Solubility versus temperature study done. } \\
\text { with temperatures of } 30^{\circ} \mathrm{C} \text { for } 18 \mathrm{hr} \text {, } \\
\text { increased to } 40^{\circ} \mathrm{C} \text { and held for } 24 \mathrm{hr} \text {, then } \\
\text { increased to } 50^{\circ} \mathrm{C} \text { and stirred for } 21 \text { hours. } \\
\text { Samples taken after each time. Used } 0.1 \mathrm{M} \\
\text { NaOH to fluidize sample. }\end{array}$ \\
\hline 241-C-104 & $\begin{array}{l}\text { PNWD-3024 } \\
\text { (BNFL-RPT- } \\
\text { 030) }\end{array}$ & - & $\begin{array}{l}\text { Wash twice using } 1405.22 \mathrm{~g} \text { and } \\
1512.18 \mathrm{~g} 0.01 \mathrm{M} \mathrm{NaOH} \text { for } 8 \text { hrs at } \\
85^{\circ} \mathrm{C}(0.9 \text { and } 0.3 \mathrm{M} \mathrm{NaOH} \\
\text { concentrations). De-water by } \\
\text { filtration. }\end{array}$ & $\begin{array}{l}\text { Caustic leach permeate: } 15600 \text { ug } \\
\text { Al/mL }(4.3 \mathrm{E}+7 \text { ug Al). Caustic leach } \\
\text { permeate } \# 1: 7770 \text { ug Al/mL }(1.2 \mathrm{E}+7 \\
\text { ug Al). Permeate \#2:2650 ug Al/mL } \\
(3.8 \mathrm{E}+6 \text { ug Al }) .90 .8 \% \mathrm{Al} \text { removed. }\end{array}$ & - & $\begin{array}{l}\text { Wash \#1: } \\
<0.003 \% \mathrm{Al} \\
\text { removed. } \\
\text { Wash \#2: } \\
<0.0006 \% \mathrm{Al} \\
\text { removed }\end{array}$ & $\begin{array}{l}\text { Final slurry } \\
\text { sample: } 36700 \\
\text { ug AlmL } \\
\text { (7.7E } \mathrm{E} 6 \mathrm{ug} \\
\text { Al). } 6.8 \% \mathrm{Al} \\
\text { in residue. }\end{array}$ & $\begin{array}{l}98 \% \mathrm{Al} \\
\text { recovery }\end{array}$ & - \\
\hline
\end{tabular}


Table A1-2. SSTs and DSTs Wash and Leach Information for Aluminum, Part 2. (27 sheets)

\begin{tabular}{|c|c|c|c|c|c|c|c|c|c|}
\hline$\underline{\text { Tank }}$ & $\begin{array}{l}\text { Lab Study } \\
\text { Reference(s) }\end{array}$ & Caustic Leach $¥ 2$ Procedure & $\begin{array}{c}\text { Wash of solids after Caustic leach } \\
\text { steps }\end{array}$ & $\begin{array}{l}\text { Mass (ug) Al removed during 1st leach } \\
\text { step }\end{array}$ & \begin{tabular}{|c|} 
Mass (ug) \\
Al removed \\
during 2nd \\
leach step \\
\end{tabular} & $\begin{array}{l}\text { Mass (ug) Al } \\
\text { removed in } \\
\text { final wash } \\
\text { solution }\end{array}$ & $\begin{array}{l}\text { Mass (ug) Al } \\
\text { in Leached } \\
\text { Solids }\end{array}$ & $\begin{array}{c}\text { Total mass Al } \\
\text { (ug) }\end{array}$ & Comments \\
\hline 241-C-104 & $\begin{array}{l}\text { ORNL TM- } \\
13500\end{array}$ & - & $\begin{array}{l}\text { After mixing, the samples were } \\
\text { centrifuged for } 15-20 \mathrm{~min} \text { at } 2500 \\
\text { ppm. The wet sludge was rinsed three } \\
\text { times with inhibited water (0.01 M } \\
\text { sodium hydroxide and } 0.01 \mathrm{M} \text { sodium } \\
\text { nitrate), using } ~ 15 \mathrm{~mL} \text { for each rinse. }\end{array}$ & $\frac{\text { Leachate: } 1) 441 \mathrm{ug} \mathrm{Al} / \mathrm{g} ; 2) 559 \mathrm{ug}}{\mathrm{Al} / \mathrm{g} ; 3) 2440 \mathrm{ug} \mathrm{Al} / \mathrm{g}}$ & - & $\begin{array}{l}\text { Rinse: 1) } 17.3 \\
\text { ug Al/g; } 2) \\
16.5 \text { ug Al/g: } \\
\text { 3) } 83.4 \text { ug Al/g }\end{array}$ & $\begin{array}{l}\text { Residual: 1) } \\
\text { 22800 ug Al/g: } \\
\text { 2) } 19900 \mathrm{ug} \\
\mathrm{Al} / \mathrm{g} ; 3) 3920 \\
\text { ug Al/g }\end{array}$ & 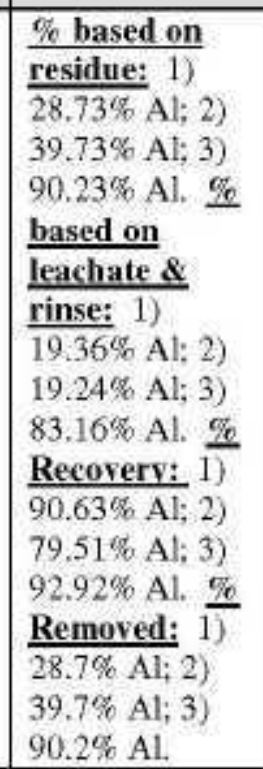 & - \\
\hline $241-C-105$ & $\begin{array}{l}\text { LAUR 97- } \\
2889\end{array}$ & $\begin{array}{l}\text { Add } 82.6 \mathrm{~mL} \text { water and } 38 \mathrm{~mL} \\
10 \mathrm{M} \mathrm{NaOH} \text { to solids ( } 1.6 \mathrm{wt} \% \\
\text { solids and } 3 \mathrm{M} \mathrm{NaOH} \text { in final } \\
\text { solution); mix } 5 \mathrm{hr} \text { at } 100^{\circ} \mathrm{C} ; \\
\text { cool; measure settling; } \\
\text { centrifuge; decant. Supernate; } \\
114.9 \mathrm{~mL} \text { w/ } 3.13 \mathrm{M} \text { free } \mathrm{OH} \text {, } \\
1.57 \mathrm{~mL} \text { settled solids, and } 1.36 \\
\text { mL centrifuged solids. }\end{array}$ & $\begin{array}{l}\text { Wash } 3 \text { times by adding } 39 \mathrm{~mL} \\
\text { inhibited water to solids; mix } 0.5 \mathrm{hr} \text { at } \\
\text { RT; measure settling; centrifuge; } \\
\text { decant. Sample } 0.6 \mathrm{~g} \text { slurry after mix } \\
\text { of wash } 3 \text {. Dry solids at } 105^{\circ} \mathrm{C}(0.175 \\
\mathrm{g} \text { solids, } 114.5 \mathrm{~mL} \text { supernate from } \\
\text { decant). }\end{array}$ & $\begin{array}{l}19410.3 \mathrm{ug} \mathrm{Al} / \mathrm{mL}, 681301.53 \mathrm{ug} \mathrm{Al} ; \\
83.7 \% \mathrm{Al} \text {. }\end{array}$ & $\begin{array}{l}1066 \text { ug } \\
\text { Al/mL, } \\
122440.76 \\
\text { ug Al; } 15 \% \\
\text { Al. }\end{array}$ & \begin{tabular}{|l}
14.63 ug \\
$\mathrm{Al} / \mathrm{mL}$, \\
1674.99 ug Al; \\
$0.2 \% \mathrm{AL}$.
\end{tabular} & $\begin{array}{l}48192.771 \text { ug } \\
\text { Alg, } 5414.45 \\
\text { ug Al; } 1 \% \text { Al. }\end{array}$ & $\begin{array}{l}813831.74 \text { ug } \\
\text { Al. }\end{array}$ & - \\
\hline $241-\mathrm{C}-105$ & $\begin{array}{l}\text { ORNL TM- } \\
13500\end{array}$ & - & $\begin{array}{l}\text { After mixing, the samples were } \\
\text { centrifuged for } 15-20 \text { min at } 2500 \\
\text { rpm. The wet sludge was rinsed three } \\
\text { times with inhibited water }(0.01 \mathrm{M} \\
\mathrm{NaOH} \text { and } 0.01 \mathrm{M} \mathrm{NaNO} \text { ), using } \sim 15 \\
\mathrm{~mL} \text { for each rinse. }\end{array}$ & Leachate: 1) $18100 \mathrm{ug} \mathrm{Al} / \mathrm{g}$. & - & $\frac{\text { Rinse: }}{\text { ug Al/g. }} 1160$ & $\frac{\text { Residual: }}{18700 \text { ug Alg. }}$ & 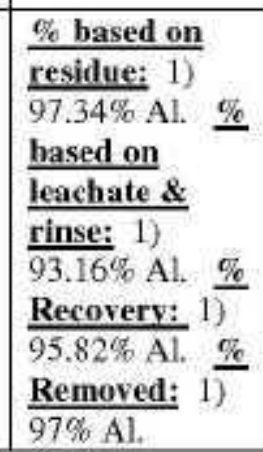 & - \\
\hline
\end{tabular}


Table A1-2. SSTs and DSTs Wash and Leach Information for Aluminum, Part 2. (27 sheets)

\begin{tabular}{|c|c|c|c|c|c|c|c|c|c|}
\hline$\underline{\text { Tank }}$ & $\begin{array}{l}\text { Lab Study } \\
\text { Reference(s) }\end{array}$ & Caustic Leach \#2 Procedure & $\begin{array}{l}\text { Wash of solids after Caustic leach } \\
\text { steps }\end{array}$ & $\begin{array}{l}\text { Mass (ug) Al removed during 1st leach } \\
\text { step }\end{array}$ & \begin{tabular}{|c|} 
Mass (ug) \\
Al removed \\
during 2nd \\
leach step \\
\end{tabular} & $\begin{array}{l}\text { Mass (ug) Al } \\
\text { removed in } \\
\text { final wash } \\
\text { solution }\end{array}$ & \begin{tabular}{|c}
$\begin{array}{c}\text { Mass (ug) Al } \\
\text { in Leached } \\
\text { Solids }\end{array}$ \\
\end{tabular} & \begin{tabular}{|c|} 
Total mass Al \\
(ug)
\end{tabular} & Comments \\
\hline $241-C-106$ & PNNL-11381 & $\begin{array}{l}\text { With solids, add } 8 \mathrm{~mL} 10 \mathrm{M} \\
\text { NaOH and water to a total } \\
\text { volume of } 35 \mathrm{~mL}(42 \mathrm{~g} \text { slurny; } \\
3.9 \mathrm{wt} \% \text { solids). Mix for } 5 \mathrm{hr} \text { at } \\
100^{\circ} \mathrm{C} \text {. Cool, settle, and decant } \\
\text { off liquid. Liquid: } 27.6 \mathrm{~mL} \\
\text { with } 3 \mathrm{M} \mathrm{OH} \text {. Solids: } 13.5 \mathrm{~g} \\
\text { slurry with } 10.5 \mathrm{~mL} \text { interstitial } \\
\text { liquid. }\end{array}$ & $\begin{array}{l}\text { With solids after } 2^{\text {th }} \text { caustic leach, } \\
\text { wash } 3 \text { times by addding } 45 \mathrm{~mL} 0.01 \mathrm{M} \\
\text { NaOH/NaNO } \text { to solids portion. Mix } \\
0.5 \mathrm{hr} \text { at RT. Settle. Decant off } \\
\text { liquid. Take a sample (1.02 } \mathrm{g} \text { ) of the } \\
\text { slurry after mixing during } 3 \mathrm{rd} \text { wash } \\
\text { step. Total liquid: } 133 \mathrm{~mL} \text {. Total } \\
\text { solids: } 10.3 \mathrm{~g} \text { wet. Dry solids at } \\
105^{\circ} \mathrm{C} \text { to obtain dry solids wt of } 1.651 \\
\mathrm{~g} \text { (adjusted for samples removed from } \\
3^{\text {rd }} \text { wash step). }\end{array}$ & $2048 \mathrm{ug} \mathrm{Al} / \mathrm{mL} ; 84378 \mathrm{ug} \mathrm{Al}(3956 \mathrm{Al})$. & $\begin{array}{l}845 \text { ug } \\
\mathrm{Al} / \mathrm{mL} ; \\
12534 \text { ug Al } \\
(6 \% \mathrm{Al}) \text {. }\end{array}$ & $\begin{array}{l}86.8 \mathrm{ug} \\
\mathrm{Al} / \mathrm{mL} ; 2672 \\
\text { ug Al (1\% Al). }\end{array}$ & $\begin{array}{l}69100 \mathrm{ug} \mathrm{Al} / \mathrm{g} \text { : } \\
114084 \mathrm{ug} \mathrm{Al} \\
(53 \% \mathrm{Al})\end{array}$ & $\begin{array}{l}213667 \text { ug } \mathrm{Al} \text {. } \\
\text { Concentration } \\
\text { in initial sludge } \\
\text { solids as } \\
\text { determined in } \\
\text { caustic leaching } \\
\text { test: } \\
\text { Summation: } \\
4.49 \mathrm{E}+04 \text { ug } \\
\text { Allg. Direct: } \\
4.85 \mathrm{E}+04 \text { ug } \\
\text { Al/g. Percent } \\
\text { recovery }=93 \% \\
\text { Al. }\end{array}$ & - \\
\hline 241-C-106 & PNNL-1 1432 & 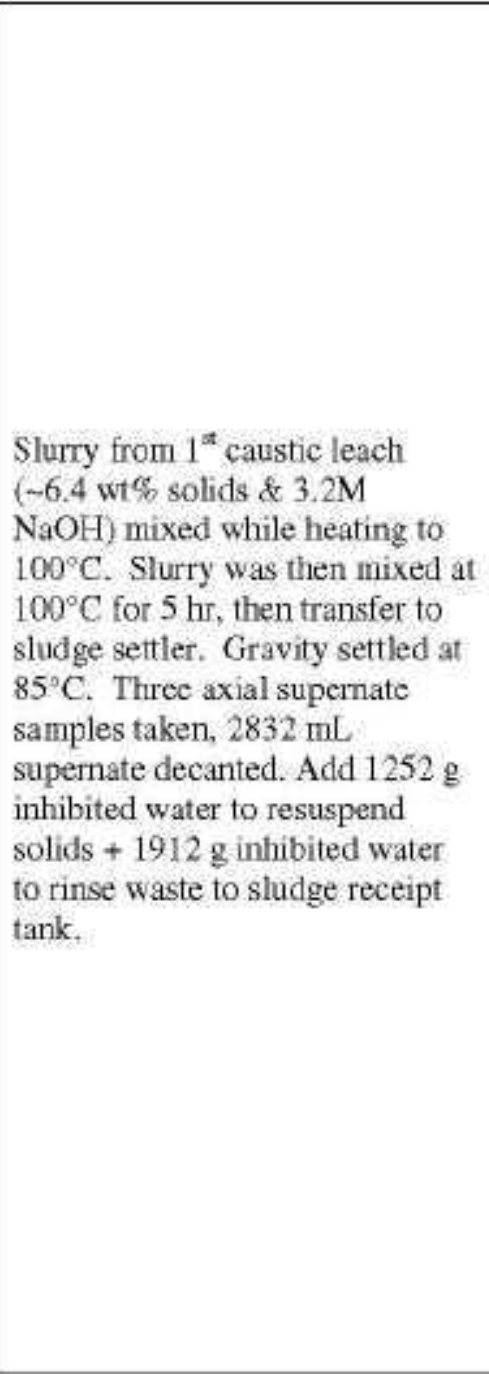 & 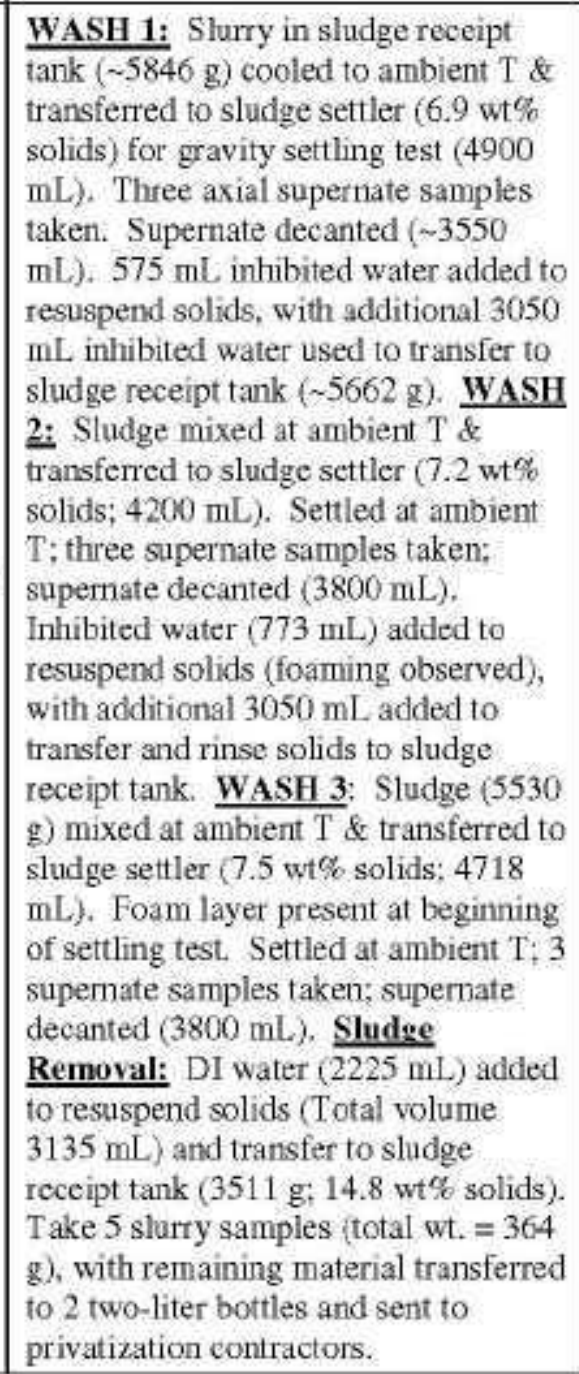 & $\begin{array}{l}30 \% \text { removed; } 3.1 \mathrm{E}+3 \mathrm{ug} / \mathrm{mL} ; 1.44 \mathrm{E}+7 \\
\text { ug } \mathrm{AL}\end{array}$ & $\begin{array}{l}<0.1 \% \\
\text { removed; } \\
1388 \text { ugimL; } \\
335024 \text { ug } \\
\text { Al. }\end{array}$ & $\begin{array}{l}\text { First Water } \\
\text { Wash: } 438 \\
\text { ug/mL; } 0 \text { ug } \\
\text { Al. Second } \\
\text { Water Wash: } \\
150 \text { ug/mL; } \\
1.5 \mathrm{E}+5 \text { ug Al. } \\
\text { Third Water } \\
\text { Wash: } 55 \\
\mathrm{ug} / \mathrm{mL} ; 1.1 \mathrm{E}+5 \\
\text { ug Al. }\end{array}$ & $\begin{array}{l}4.91 \mathrm{E}+4 \mathrm{ug} / \mathrm{g} ; \\
2.64 \mathrm{E}+7 \mathrm{ug} \mathrm{Al} \\
(-69 \% \mathrm{Al} \text { in } \\
\text { residue). }\end{array}$ & $\begin{array}{l}\text { Mass Recovery: } \\
\text { Direct Analysis; } \\
4.78 \mathrm{E}+7 \text { ug; } \\
\text { Summation } \\
\text { method: } \\
4.64 \mathrm{E}+7 \text { ug; } \\
97 \% \text { recovery. }\end{array}$ & - \\
\hline
\end{tabular}


Table A1-2. SSTs and DSTs Wash and Leach Information for Aluminum, Part 2. (27 sheets)

\begin{tabular}{|c|c|c|c|c|c|c|c|c|c|}
\hline$\underline{\text { Tank }}$ & $\begin{array}{l}\text { Lab Study } \\
\text { Reference (8) }\end{array}$ & Caustic Leach \#2 Procedure & $\begin{array}{l}\text { Wash of solids after Caustic leach } \\
\text { steps }\end{array}$ & $\begin{array}{l}\text { Mass (ug) Al removed during 1st leach } \\
\text { step }\end{array}$ & \begin{tabular}{|l|} 
Mass (ug) \\
Al removed \\
during 2nd \\
leach step \\
\end{tabular} & $\begin{array}{c}\begin{array}{c}\text { Mass (ug) Al } \\
\text { removed in } \\
\text { final wash } \\
\text { solution }\end{array} \\
\end{array}$ & $\begin{array}{l}\text { Mass (ug) Al } \\
\text { in Leached } \\
\text { Solids }\end{array}$ & $\begin{array}{l}\text { Total mass Al } \\
\text { (ug) }\end{array}$ & Comments \\
\hline 241-C-106 & $\begin{array}{l}\text { PNWD-3013 } \\
\text { (BNFL-RPT- } \\
017 \text { ) }\end{array}$ & - & 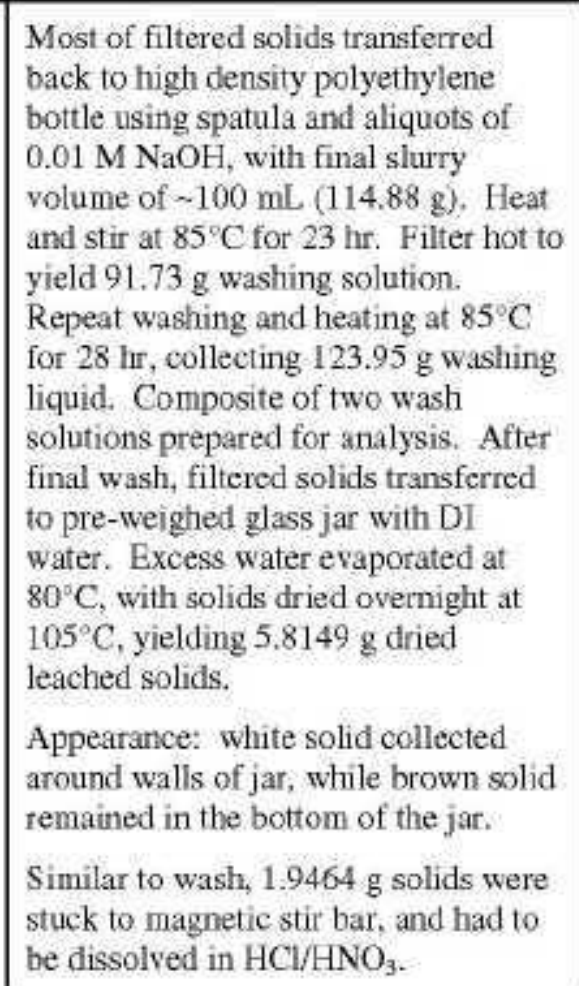 & $22 \%$ removed by leaching. & - & - & - & - & 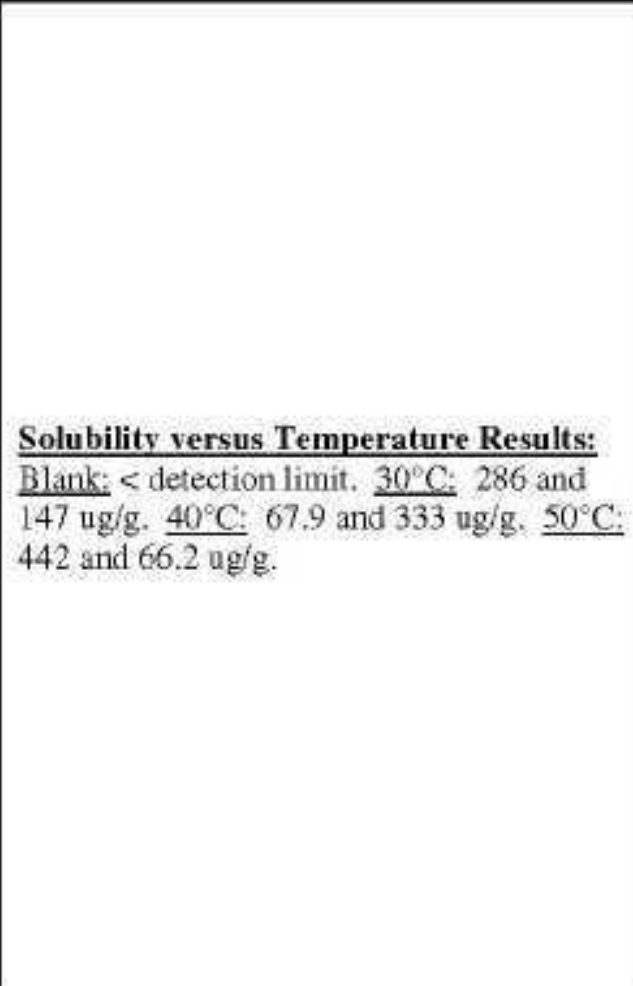 \\
\hline 241-C-107 & $\begin{array}{l}\text { LAUR 96- } \\
2839\end{array}$ & $\begin{array}{l}\text { Add } 9.8 \mathrm{~mL}, 3 \mathrm{M} \mathrm{NaOH} \text { to solids } \\
\text { (no settled solids } / 4.5 \mathrm{~mL} \\
\text { centrifuged solids); mix } 5 \mathrm{hr} \text { at } \\
100^{\circ} \mathrm{C} \text {; cool; measure settling; } \\
\text { centrifuge; decant. }\end{array}$ & $\begin{array}{l}\text { Add } 9.6 \mathrm{~mL} \text { inhibited water to solids } \\
\text { (no settled solids } 4.7 \mathrm{~mL} \text { centrifuged } \\
\text { solids); mix } 0.5 \mathrm{hr} \text { at RT; measure } \\
\text { settling; centrifuge; decant; repeat } \\
\text { twice with solids portion, sampling } \\
\text { slurry (0.3 mL) before measuring } \\
\text { settling in wash \#3. Dry solids at } \\
80^{\circ} \mathrm{C} \text {. }\end{array}$ & $25500 \mathrm{ug} / \mathrm{mL}(45.51 \%)$. & $\begin{array}{l}9350 \mathrm{ug} / \mathrm{mL} . \\
(21.11 \%)\end{array}$ & $\begin{array}{l}61020 \mathrm{ug} / \mathrm{mL} \\
(6.91 \%)\end{array}$ & $\begin{array}{l}61307.9 \mathrm{ug} / \mathrm{g} \\
\text { (24.28 \%\% in } \\
\text { residue; } 76 \% \\
\text { removed } \\
\text { during sludge } \\
\text { wash and } \\
\text { caustic leach). }\end{array}$ & $\begin{array}{l}\text { Direct analysis: } \\
1.05 \mathrm{E}-1 \mathrm{~g} / \mathrm{g} \\
\text { sludge; Sum } \\
\text { method: } 9.03 \mathrm{E}- \\
2 \mathrm{~g} / \mathrm{g} \text { sludge. } \\
86.32 \% \\
\text { recovery. }\end{array}$ & - \\
\hline 241-C-107 & PNNL-11278 & $\begin{array}{l}\text { Add } 22.8 \mathrm{~g} 3 \mathrm{M} \mathrm{NaOH} \text { to solids, } \\
\text { mix at } 100^{\circ} \mathrm{C} \text { for } 5 \mathrm{hr} \text {, cool, } \\
\text { measure settling, centrifuge, } \\
\text { decant }(19.5 \mathrm{~mL} \text { decanted liquid, } \\
2.8 \mathrm{~mL} \text { interstitial liquid, and } \\
2.96 \mathrm{M} \mathrm{OH}) \text {. }\end{array}$ & $\begin{array}{l}\text { Wash } 3 \text { times: } 1^{\text {st }} \text { wash: add } 24.2 \mathrm{~g} \\
0.01 \mathrm{M} \mathrm{NaOH} / \mathrm{NaNO} \mathrm{O}_{2} \text { to solids, mix } \\
\text { at RT for } 0.5 \mathrm{hr} \text {, measure settling, } \\
\text { centrifuge, and decant. } 2^{\text {st }} \text { and } 3^{\text {rid }} \\
\text { wash: Add } 19.1 \mathrm{~g} 0.01 \mathrm{M} \\
\mathrm{NaOH} / \mathrm{NaNO} \mathrm{N}_{2} \text { to solids, mix at RT for } \\
0.5 \mathrm{hr} \text {, measure settling, centrifuge, } \\
\text { and decant. Sample } 0.22 \mathrm{~g} \text { slurry after } \\
\text { mix of wash } 3 \text {. Dry solids at } 80^{\circ} \mathrm{C}= \\
2.17 \mathrm{~g} \text { solids (adjusted for slurry } \\
\text { removed from wash } 3 \text { step). }\end{array}$ & $12852 \mathrm{ug} / \mathrm{mL} ; 253000 \mathrm{ug} ; 64 \%$. & $\begin{array}{l}2997 \mathrm{ug} / \mathrm{mL} ; \\
28271 \mathrm{ug} ; \\
7 \% .\end{array}$ & $\begin{array}{l}550 \mathrm{ug} / \mathrm{mL} ; \\
21930 \mathrm{ug} ; 6 \%\end{array}$ & $\begin{array}{l}41061 \mathrm{ug} / \mathrm{mL} ; \\
89102 \text { ug: } \\
22 \% \text {. }\end{array}$ & $\begin{array}{l}397543 \text { ug; } \\
\text { Summation: } \\
8.68 \mathrm{E}-2 \mathrm{~g} \mathrm{~A} \mathrm{~A} / \mathrm{g} \\
\text { sludge; } \mathrm{Direct:} \\
9.48 \mathrm{E}-2 \mathrm{~g} \mathrm{Al} / \mathrm{g} \\
\text { sludge; } \text { Mass } \\
\text { Recovery: } \\
109 \% \text {. }\end{array}$ & 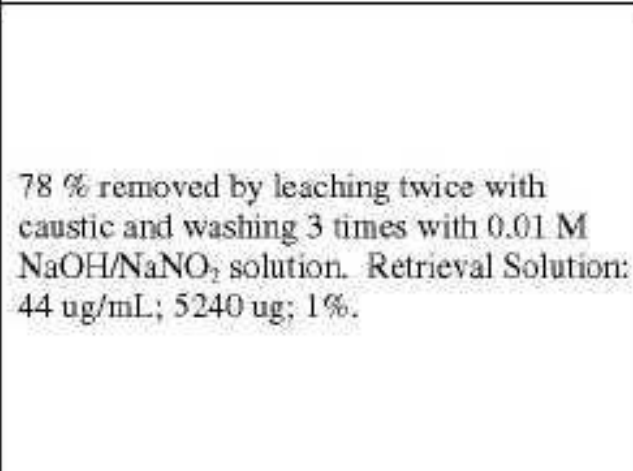 \\
\hline
\end{tabular}


Table A1-2. SSTs and DSTs Wash and Leach Information for Aluminum, Part 2. (27 sheets)

\begin{tabular}{|c|c|c|c|c|c|c|c|c|c|}
\hline$\underline{\text { Tank }}$ & $\begin{array}{l}\text { Lab Study } \\
\text { Reference(s) }\end{array}$ & Caustic Leach $\# 2$ Procedure & $\begin{array}{l}\text { Wash of solids after Caustic leach } \\
\text { steps }\end{array}$ & $\begin{array}{l}\text { Mass (ug) Al removed during 1st leach } \\
\text { step }\end{array}$ & \begin{tabular}{|l} 
Mass (ug) \\
Al removed \\
during 2nd \\
leach step \\
\end{tabular} & $\begin{array}{l}\text { Mass (ug) Al } \\
\text { removed in } \\
\text { final wash } \\
\text { solution }\end{array}$ & \begin{tabular}{|c|c|} 
Mass (ug) Al \\
in Leached \\
Solids
\end{tabular} & \begin{tabular}{|c|} 
Total mass $\mathrm{Al}$ \\
(ug)
\end{tabular} & Comments \\
\hline 241-C-107 & $\begin{array}{l}\text { ORNL TM- } \\
13500\end{array}$ & - & $\begin{array}{l}\text { After mixing, the samples were } \\
\text { centrifuged for } 15-20 \text { min at } 2500 \\
\text { pm. The wet sludge was rinsed three } \\
\text { times with inhibited water }(0.01 \mathrm{M} \\
\text { sodium hydroxide and } 0.01 \mathrm{M} \text { sodium } \\
\text { nitrate), using } ~ 15 \mathrm{~mL} \text { inhibited water } \\
\text { for each rinse. } \\
\end{array}$ & Leachate: $7060 \mathrm{ug} / \mathrm{g}$. & - & $\begin{array}{l}\text { Rinse: } 319 \\
\text { ug/g. }\end{array}$ & $\begin{array}{l}\text { Residual: } \\
20900 \text { ug/g. }\end{array}$ & $\begin{array}{l}82 \% \text { based on } \\
\text { residue; } 79.71 \% \\
\text { based on } \\
\text { leachate } \& \\
\text { rinse; } 97.71 \% \\
\text { recovery; } 82 \% \\
\text { removed. } \\
\end{array}$ & - \\
\hline $241-\mathrm{C}-108$ & $\begin{array}{l}\text { LAUR 95- } \\
2070\end{array}$ & $\begin{array}{l}\text { Add } 16.1 \mathrm{~mL} 3 \mathrm{M} \mathrm{NaOH} \text {, mix } 5 \\
\text { hr at } 100^{\circ} \mathrm{C}, \text { cool, measure } \\
\text { settling, centrifuge, and decant. } \\
\text { Volume settled solids } \sim 20.8 \mathrm{~mL} \text {, } \\
\text { volume centrifuged solids }-6.2 \\
\mathrm{~mL}, 15.78 \mathrm{~mL} \text { liquid from } \\
\text { decant. }\end{array}$ & $\begin{array}{l}\text { Wash } 3 \text { times by adding } 15.9 \mathrm{~mL} \\
\text { inhilited water to solids and mixing at } \\
\text { RT for } 0.5 \mathrm{hr} \text {, measure settling, } \\
\text { centrifuge, and decant. For wash } \# 3 \text {, } \\
\text { sample slurry (0.3 mL) after mix } \\
\text { phase. Dry solids at } 80^{\circ} \mathrm{C}(1.1869 \mathrm{~g}) \text {. }\end{array}$ & $39100 \mathrm{ug} / \mathrm{mL}(49.51 \%)$ & $\begin{array}{l}21250 \\
\mathrm{u} / \mathrm{mL} \\
(28.14 \%)\end{array}$ & $\begin{array}{l}3230 \mathrm{ug} / \mathrm{mL} \\
(12.87 \%) .\end{array}$ & $\begin{array}{l}\begin{array}{l}64779 \text { ug Al/g } \\
\text { sludge. }\end{array} \\
\text { lat }\end{array}$ & \begin{tabular}{|l} 
Direct analysis: \\
$1.51 \mathrm{E}-01 \mathrm{~g} / \mathrm{g}$ \\
sludge; \\
Summation \\
method: $1.8 \mathrm{E}-$ \\
$01 \mathrm{~g} / \mathrm{g}$ sludge; \\
$119.1 \%$ \\
recovery. \\
\end{tabular} & $\begin{array}{l}\text { Found in retrieval wash prior to leach: } \\
280.5 \text { ug } \mathrm{mL}(3.03 \%) ; \text { Residual }=65 \mathrm{mg} \\
\mathrm{Al} / \mathrm{g} \text { sludge }(6.45 \%) \text {. }\end{array}$ \\
\hline $241-C-109$ & PNL-9387 & - & - & - & $\begin{array}{l}73 \% \text { from } \\
\text { leach. }\end{array}$ & - & $\begin{array}{l}19 \% \text { in } \\
\text { leached } \\
\text { sludge. }\end{array}$ & - & - \\
\hline $241-C-112$ & PNL-9387 & - & - & - & $\begin{array}{l}51 \% \text { found } \\
\text { in leach } \\
\text { solution. } \\
\end{array}$ & - & \begin{tabular}{|l|}
$15 \%$ in \\
leached \\
sludge.
\end{tabular} & - & - \\
\hline $241-S-101$ & PNNL-12026 & N/A & - & - & - & \begin{tabular}{|l} 
Summation \\
Method [leach \\
(wash + \\
leach)]: 1) \\
$66 \%(70 \%) ; 2)$ \\
$87 \%(88 \%) ; 3)$ \\
$59 \%(63 \%) ; 4)$ \\
$89 \%(90 \%)$. \\
Fe \\
Normalized: \\
1) $71 \% \%$ \\
2) $75 \% ;$ \\
3) $57 \% ;$ \\
4) $70 \%$.
\end{tabular} & - & - & 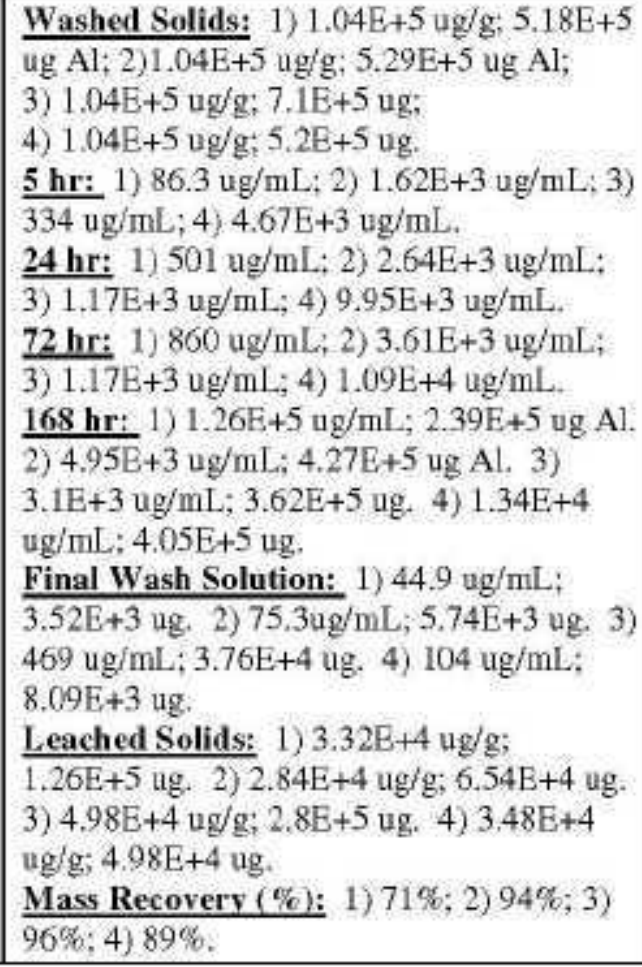 \\
\hline
\end{tabular}


Table A1-2. SSTs and DSTs Wash and Leach Information for Aluminum, Part 2. (27 sheets)

\begin{tabular}{|c|c|c|c|c|c|c|c|c|c|}
\hline$\underline{\text { Tank }}$ & $\begin{array}{l}\text { Lab Study } \\
\text { Reference( } 8 \text { ) }\end{array}$ & Caustic Leach \#2 Procedure & $\begin{array}{c}\text { Wash of solids after Caustic leach } \\
\text { steps }\end{array}$ & $\begin{array}{l}\text { Mass (ug) Al removed during 1st leach } \\
\text { step }\end{array}$ & $\begin{array}{l}\text { Mass (ug) } \\
\text { Al removed } \\
\text { during 2nd } \\
\text { leach step } \\
\end{array}$ & $\begin{array}{l}\text { Mass (ug) Al } \\
\text { removed in } \\
\text { final wash } \\
\text { solution } \\
\end{array}$ & $\begin{array}{l}\text { Mass (ug) Al } \\
\text { in Leached } \\
\text { Solids }\end{array}$ & $\begin{array}{l}\text { Total mass Al } \\
\text { (ug) }\end{array}$ & Comments \\
\hline 241-S-101 & PNNL-11636 & $\begin{array}{l}\text { Add } 34 \mathrm{~mL}, 10 \mathrm{M} \mathrm{NaOH} \text { and } 80 \\
\text { mL water to solidssludge from } \\
\text { previous step; mix } 5 \mathrm{hr} \text { at } 100^{\circ} \mathrm{C} \text {; } \\
\text { cool; gravity settle; take a } 1 \mathrm{ml} \\
\text { sample; mix } 95 \mathrm{hr} \text { at } 100^{\circ} \mathrm{C} \text {; } \\
\text { cool; settle; and decant. }\end{array}$ & $\begin{array}{l}\text { Add } 24 \mathrm{~mL} \text { of } 0.01 \mathrm{M} \mathrm{NaOH} / 0.01 \mathrm{M} \\
\mathrm{NaNO}_{2} \text { to remaining solids; mix } 0.5 \mathrm{hr} \\
\text { at RT; centrifuge; decant; and repeat } \\
\text { for total of } 3 \text { wash steps. After } 3^{\text {rd }} \\
\text { wash, remove two } 0.194 \mathrm{~g} \text { slurry } \\
\text { samples before centrifuge \& decant. }\end{array}$ & $72742(11 \%)$ & $\begin{array}{l}488485 \\
(76 \%)\end{array}$ & $54553(9 \%)$. & $25645(4 \%)$. & $\begin{array}{l}641425(109 \% \\
\text { recovery })\end{array}$ & - \\
\hline 241-S-101 & $\begin{array}{l}\text { ORNL TM- } \\
13500\end{array}$ & - & $\begin{array}{l}\text { After mixing, the samples were } \\
\text { centrifuged for } 15-20 \text { min at } 2500 \\
\text { pmin. The wet sludge was rinsed three } \\
\text { times with inhibited water }(0.01 \mathrm{M} \\
\mathrm{NaOH} \text { and } 0.01 \mathrm{M} \mathrm{NaNO}_{2} \text {, using } ~ 15 \\
\mathrm{~mL} \text { inhibited water for each rinse. }\end{array}$ & Leachate: $7050 \mathrm{ug} / \mathrm{mL}$. & - & $\begin{array}{l}\text { Rinse: } 106 \\
\text { ug/mL. }\end{array}$ & $\begin{array}{l}\text { Residual: } 8760 \\
\text { ug/g. }\end{array}$ & $\begin{array}{l}97.76 \% \text { based } \\
\text { on residue: } \\
97.29 \% \text { based } \\
\text { on leachate \& } \\
\text { rinse; } 99.54 \% \\
\text { Recovery; } 98 \% \\
\text { Removed. }\end{array}$ & - \\
\hline 241-S-101 & $\begin{array}{l}\text { ORNL TM- } \\
13655\end{array}$ & - & $\begin{array}{l}\text { Centrifuge leached solids for } 20 \mathrm{~min} \\
\text { at } 4500 \mathrm{rpm} \text {, decant liquid, and weigh } \\
\text { solids. Wash } 3 \text { times with } 25 \mathrm{~mL} \\
\text { inhibited water. Place sample in } \\
\text { vortex mixer until all solids suspended } \\
\text { in solution, weigh sample, centrifuge } \\
\text { for } 5 \text { min at } 4500 \text { rpm, decant liquid, } \\
\text { and repeat twice. After last wash, } \\
\text { sample centrifuged for } 20 \text { min at } 4500 \\
\text { rpm. Weights of solids and combined } \\
\text { water washes were determined. }\end{array}$ & 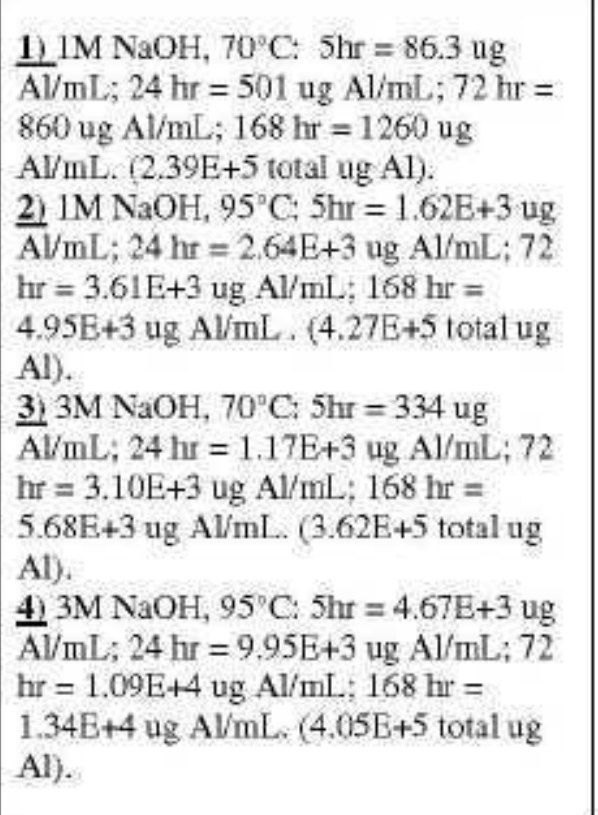 & 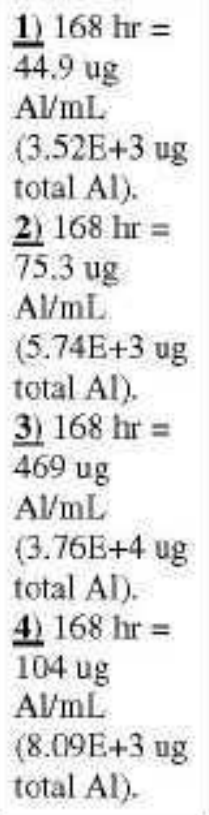 & 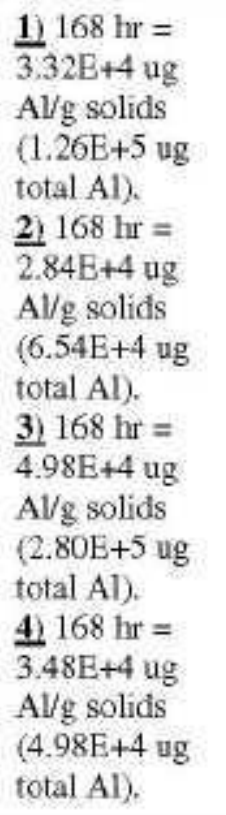 & - & - & $\begin{array}{l}\text { The water content in sample of washed } \\
\text { sludge was } 72.8 \% \text {. This was assumed to be } \\
\text { the same for each aliquot used for leaching. } \\
\text { The density of the composite wash solution } \\
\text { (26.043 } \mathrm{g} / 25 \mathrm{~mL} \text { filtered composite) was } \\
\text { used to determine that the original sludge } \\
\text { sample contained } 33.4 \mathrm{~g}(30 \mathrm{wt} \% \text { ) water- } \\
\text { soluble solids, } 23.17(21 \mathrm{w} \%) \text { water- } \\
\text { insoluble solids, and } 54.04 \mathrm{~g} \text { ( } 49 \mathrm{wt} \%) \\
\text { water. }\end{array}$ \\
\hline
\end{tabular}


Table A1-2. SSTs and DSTs Wash and Leach Information for Aluminum, Part 2. (27 sheets)

\begin{tabular}{|c|c|c|c|c|c|c|c|c|c|}
\hline$\underline{\text { Tank }}$ & $\begin{array}{l}\text { Lab Study } \\
\text { Reference(s) }\end{array}$ & Caustic Leach $\# 2$ Procedure & $\begin{array}{c}\text { Wash of solids after Caustic leach } \\
\text { steps }\end{array}$ & $\begin{array}{l}\text { Mass (ug) Al removed during 1st leach } \\
\text { step }\end{array}$ & \begin{tabular}{|c|} 
Mass (ug) \\
Al removed \\
during 2nd \\
leach step \\
\end{tabular} & $\begin{array}{l}\text { Mass (ug) Al } \\
\text { removed in } \\
\text { final wash } \\
\text { solution } \\
\end{array}$ & $\begin{array}{l}\text { Mass (ug) Al } \\
\text { in Leached } \\
\text { Solids }\end{array}$ & \begin{tabular}{|c|} 
Total mass $\mathrm{Al}$ \\
(ug)
\end{tabular} & Comments \\
\hline $241-S-104$ & PNL-10712 & $\begin{array}{l}\text { Solids did not settle. Add } 13.6 \mathrm{~g} \\
3 \mathrm{M} \mathrm{NaOH} \text { to } 10 \mathrm{~mL} \text { centrifuged } \\
\text { solids; mix } 5 \mathrm{hr} \text { at } 100^{\circ} \mathrm{C} \text {; cool; } \\
\text { measure settling; centrifuge; and } \\
\text { decant. }\end{array}$ & 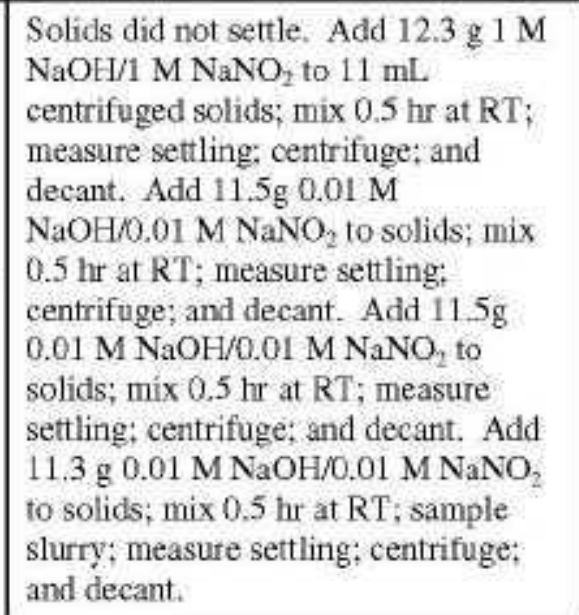 & $10 \%$ found, $18700 \mathrm{ug} / \mathrm{mL}$ measured. & $\begin{array}{l}12 \% \text { found, } \\
13600 \\
\mathrm{ug} / \mathrm{mL} \\
\text { measured. }\end{array}$ & $\begin{array}{l}13 \% \text { found, } \\
3060 \mathrm{ug} / \mathrm{mL} \\
\text { measured. }\end{array}$ & $\begin{array}{l}\text { Measured } \\
\text { concentration } \\
\text { of } 330000 \\
\text { ug/g, } 62 \% \text { in } \\
\text { residue: }\end{array}$ & $\begin{array}{l}\text { Direct analysis: } \\
1.5 \mathrm{E}-01 \text { g/g } \\
\text { sludge; } \\
\text { Summation } \\
\text { method: } 1.77 \mathrm{E}- \\
01 \mathrm{~g} / \mathrm{g} \text { sludge; } \\
118 \% \text { recovery. }\end{array}$ & - \\
\hline $241-\mathrm{S}-104$ & $\begin{array}{l}\text { L.AUR 95- } \\
2070\end{array}$ & $\begin{array}{l}\text { Add } 6.6 \mathrm{~mL}, 3 \mathrm{M} \mathrm{NaOH} \text {, mix } 5 \mathrm{hir} \\
\text { at } 100^{\circ} \mathrm{C} \text {, cool, measure settling, } \\
\text { centrifuge, and decant. Volume } \\
\text { settled solids } \sim 16.1 \mathrm{~mL} \text {; volume } \\
\text { centrifuged solids } \sim 9.1 \mathrm{~mL} \text {. }\end{array}$ & $\begin{array}{l}\text { Wash } 3 \text { times by adding } 7.8 \mathrm{~mL} \\
\text { inhibited water to solids and mix at } \\
\text { RT for } 0.5 \mathrm{hr} \text {, measure settling. } \\
\text { centrifuge, and decant. For wash \#3, } \\
\text { sample slurry } 0.3 \mathrm{~mL} \text { ) after mix } \\
\text { phase. Dry solids at } 80^{\circ} \mathrm{C}(2.0985 \mathrm{~g} \text {. }\end{array}$ & $16150 \mathrm{ug} / \mathrm{mL}(9.04 \%)$. & $\begin{array}{l}17850 \\
\text { ug/mL } \\
(10.39 \%)\end{array}$ & $\begin{array}{l}4590 \mathrm{ug} / \mathrm{mL} \\
(9.67 \%)\end{array}$ & $\begin{array}{l}328947 \text { ug } \\
\mathrm{Al} / \mathrm{g} \text { sludge. }\end{array}$ & $\begin{array}{l}\text { Direct analysis: } \\
\text { 1.67E-01 g/g } \\
\text { sludge; } \\
\text { Summation } \\
\text { method: } 1.86 \mathrm{E}- \\
01 \mathrm{~g} / \mathrm{g} \text { sludge; } \\
111.46 \% \\
\text { recovery. }\end{array}$ & $\begin{array}{l}\text { Found in retrieval wash prior to leach: } 417 \\
\text { ug/mL }(3.7 \%) \text {; Residual }=329 \mathrm{mg} \mathrm{Al} / \mathrm{g} \\
\text { sludge }(67.2 \%) \text {. }\end{array}$ \\
\hline 241-S-104 & PNNL-11089 & $\begin{array}{l}\text { Add } \mathrm{NaOH} \text { to get }-8 \mathrm{wt} \% \text { solids } \\
\text { and a final } \mathrm{NaOH} \text { of } 3.0 \mathrm{M} \text {. Mix } \\
\text { at } 100^{\circ} \mathrm{C} \text { for } 5 \mathrm{hr} \text {. Cool, } \\
\text { centrifuge, and decant. }\end{array}$ & $\begin{array}{l}\text { Add inthibited water. Mix at RT for } \\
0.5 \mathrm{hr} \text { (target } 8 \text { wts solids). } \\
\text { Centrifuge, decant, and repeat twice } \\
\text { more. }\end{array}$ & $\begin{array}{l}\text { Uncorrected/corrected recovery }= \\
10 / 25 \% \text {. }\end{array}$ & $\begin{array}{l}\text { Uncorrected/ } \\
\text { corrected } \\
\text { recovery }= \\
12 / 10 \% \text {. }\end{array}$ & $\begin{array}{l}\text { Uncorrected/ } \\
\text { corrected } \\
\text { recovery = } \\
13 / 2 \% \text {. }\end{array}$ & \begin{tabular}{|l|} 
Total of \\
$15 \mathrm{wt} \%$ Al; \\
Total of $38 \%$ \\
Al removed; \\
percent \\
undissolved \\
residue \\
(uncorrected/c \\
orrected) = \\
$62 / 59 \%$.
\end{tabular} & - & - \\
\hline 241-S-104 & PNNL-11636 & $\begin{array}{l}\text { Add } 12 \mathrm{ml} 10 \mathrm{M} \mathrm{NaOH} \text { and } 10 \\
\text { ml water to give } 121 \mathrm{~g} \text { slurry; } \\
\text { mix } 76 \text { hr at } 100^{\circ} \mathrm{C} \text {; cool; } \\
\text { centrifuge; and decant. }\end{array}$ & $\begin{array}{l}\text { Add } 50 \mathrm{~mL} \text { of } 0.01 \mathrm{M} \mathrm{NaOH} / 0.01 \mathrm{M} \\
\mathrm{NaNO}_{2} \text { to remaining solids; mix } 0.5 \mathrm{hr} \\
\text { at } \mathrm{RT} \text {; centrifuge; decant; and repeat } \\
\text { for total of } 3 \text { wash steps. After } 3^{\text {tid }} \\
\text { wash, remove two }(0.501 \mathrm{~g} \text { and } 0.201 \\
\text { g) slurry samples before centrifuge \& } \\
\text { decant. }\end{array}$ & $\begin{array}{l}539570 \text { ug ( } 95 \% \text { removal; mass value } \\
\text { corrected for mass of material present } \\
\text { in samples taken at } 5 \& 75 \mathrm{hr} \text {; no } \\
\text { change between } 75 \& 211 \mathrm{hr} \text { ). }\end{array}$ & $\begin{array}{l}15429 \text { ug } \\
(3 \% \\
\text { removal). }\end{array}$ & $\begin{array}{l}9654 \text { ug (2\% } \\
\text { removal). }\end{array}$ & $5530 \mathrm{ug}(1 \%)$. & 570183 ug. & - \\
\hline
\end{tabular}


Table A1-2. SSTs and DSTs Wash and Leach Information for Aluminum, Part 2. (27 sheets)

\begin{tabular}{|c|c|c|c|c|c|c|c|c|c|}
\hline$\underline{\text { Tank }}$ & $\begin{array}{l}\text { Lab Study } \\
\text { Reference (8) }\end{array}$ & Caustic Leach \#2 Procedure & $\begin{array}{c}\text { Wash of solids after Caustic leach } \\
\text { steps }\end{array}$ & $\begin{array}{l}\text { Mass (ug) Al removed during 1st leach } \\
\text { step }\end{array}$ & $\begin{array}{l}\text { Mass (ug) } \\
\text { Al removed } \\
\text { during 2nd } \\
\text { leach step } \\
\end{array}$ & $\begin{array}{l}\text { Mass (ug) Al } \\
\text { removed in } \\
\text { final wash } \\
\text { solution } \\
\end{array}$ & $\begin{array}{l}\text { Mass (ug) Al } \\
\text { in Leached } \\
\text { Solids }\end{array}$ & $\begin{array}{l}\text { Total mass Al } \\
\text { (ug) }\end{array}$ & Comments \\
\hline 241-S-104 & $\begin{array}{l}\text { ORNL TM- } \\
13500\end{array}$ & - & $\begin{array}{l}\text { After mixing, the samples were } \\
\text { centrifuged for } 15-20 \text { min at } 2500 \\
\text { rpm. The wet sludge was rinsed three } \\
\text { times with inhibited water }(0.01 \mathrm{M} \\
\left.\mathrm{NaOH} \text { and } 0.01 \mathrm{M} \mathrm{NaNO}_{2}\right) \text {, using } \sim 15 \\
\mathrm{~mL} \text { for each rinse. }\end{array}$ & $\begin{array}{l}\text { Leachate: } \\
\text { 1) } 1200 \mathrm{ug} / \mathrm{mL} \text {; } \\
\text { 2) } 1870 \mathrm{ug} / \mathrm{mL} ; \\
\text { 3) } 2750 \mathrm{ug} / \mathrm{mL} ; \\
\text { 4) } 1620 \mathrm{ug} / \mathrm{mL} ; \\
\text { 5) } 1340 \mathrm{ug} / \mathrm{g} ; \\
\text { 6) } 3830 \mathrm{ug} / \mathrm{g} \text {. }\end{array}$ & - & $\begin{array}{l}\text { Rinse: } \\
\text { 1) } 162 \mathrm{ug} / \mathrm{mL} ; \\
\text { 2) } 292 \mathrm{ug} / \mathrm{mL} ; \\
\text { 3) } 68.8 \mathrm{ug} / \mathrm{mL} ; \\
\text { 4) } 33.8 \mathrm{ug} / \mathrm{mL} ; \\
\text { 5) } 46.7 \mathrm{ug} / \mathrm{g} ; \\
\text { 6) } 45.5 \mathrm{ug} / \mathrm{g} \text {. }\end{array}$ & $\begin{array}{l}\text { Residual: } \\
\text { 1) } 77300 \mathrm{ug} / \mathrm{g} ; \\
\text { 2) } 63900 \mathrm{ug} / \mathrm{g} \text {; } \\
\text { 3) } 50000 \mathrm{ug} / \mathrm{g} ; \\
\text { 4) } 40100 \mathrm{ug} / \mathrm{g} ; \\
\text { 5) } 66600 \mathrm{ug} / \mathrm{g} \text {; } \\
\text { 6) } 12600 \mathrm{ug} / \mathrm{g} \text {. }\end{array}$ & 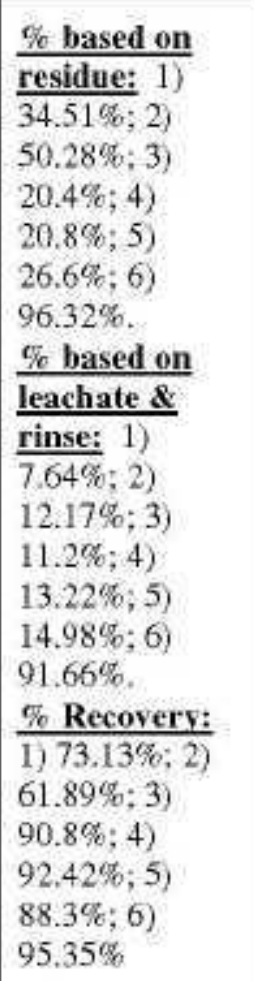 & - \\
\hline $241-S-107$ & PNNL-11278 & $\begin{array}{l}\text { Add } 55 \mathrm{~mL} 10 \mathrm{M} \mathrm{NaOH} \text { and } \\
\text { water to solids for a total volume } \\
\text { of } 185 \mathrm{~mL} \text { ( } 0.35 \mathrm{wt} \text { ts solids). } \\
\text { Mix at } 100^{\circ} \mathrm{C} \text { for } 5 \mathrm{hr} \text {, cool, } \\
\text { measure settling, centrifuge, and } \\
\text { decant. Obtain } 171 \mathrm{~mL} \text { decanted } \\
\text { liquid, } 5.9 \mathrm{~mL} \text { interstitial liquid, } \\
\text { and } 2.8 \mathrm{M} \mathrm{OH} \text {. }\end{array}$ & $\begin{array}{l}\text { Wash } 3 \text { times by adding } 37 \mathrm{~mL} 0.01 \\
\mathrm{M} \mathrm{NaOH} / \mathrm{NaNO}_{2} \text { to solids, mix at RT } \\
\text { for } 0.5 \mathrm{hr} \text {, measure setlling, } \\
\text { centrifuge, and decant. Sample } 0.4 \\
\mathrm{~mL} \text { slurry after mix of wash } 3 \text {. Dry } \\
\text { solids at } 105^{\circ} \mathrm{C} \text { to get } 0.702 \mathrm{~g} \text { solids } \\
\text { (adjusted for slurry removed from } \\
\text { wash } 3 \text { step). }\end{array}$ & $11725 \mathrm{ug} / \mathrm{mL} ; 398650 \mathrm{ug}: 56 \%$. & $\begin{array}{l}949 \mathrm{ug} / \mathrm{mL} \\
118728 \mathrm{ug} \\
17 \%\end{array}$ & $\begin{array}{l}83.2 \mathrm{ug} / \mathrm{mL} ; \\
3220 \mathrm{ug} ; 0 \%\end{array}$ & $\begin{array}{l}272000 \\
\text { ug/mL; } \\
190944 \text { ug; } \\
27 \%\end{array}$ & $\begin{array}{l}711542 \text { ug: } \\
\text { Summation: } \\
2.09 \mathrm{E}+5 \mathrm{ug} / \mathrm{g} ; \\
\text { Direct: } 2.05 \mathrm{E}+5 \\
\text { ugg: } \\
\text { Mass Recovery: } \\
102 \%\end{array}$ & $\begin{array}{l}73 \% \text { removed by leaching twice with } \\
\text { caustic and washing } 3 \text { times with } 0.01 \mathrm{M} \\
\mathrm{NaOH} / \mathrm{NaNO}_{2} \text { solution. }\end{array}$ \\
\hline
\end{tabular}


Table A1-2. SSTs and DSTs Wash and Leach Information for Aluminum, Part 2. (27 sheets)

\begin{tabular}{|c|c|c|c|c|c|c|c|c|c|}
\hline$\underline{\text { Tank }}$ & $\begin{array}{l}\text { Lab Study } \\
\text { Reference(s) }\end{array}$ & Caustic Leach $¥ 2$ Procedure & $\begin{array}{l}\text { Wash of solids after Caustic leach } \\
\text { steps }\end{array}$ & $\begin{array}{l}\text { Mass (ug) Al removed during 1st leach } \\
\text { step }\end{array}$ & $\begin{array}{l}\text { Mass (ug) } \\
\text { Al removed } \\
\text { during 2nd } \\
\text { leach step } \\
\end{array}$ & $\begin{array}{l}\text { Mass (ug) Al } \\
\text { removed in } \\
\text { final wash } \\
\text { solution } \\
\end{array}$ & $\begin{array}{c}\text { Mass (ug) Al } \\
\text { in Leached } \\
\text { Solids }\end{array}$ & $\begin{array}{l}\text { Total mass Al } \\
\text { (ug) }\end{array}$ & Comments \\
\hline $241-5-107$ & PNNL-12010 & $\begin{array}{l}\text { Add } 3670 \mathrm{~g} 3 \mathrm{M} \mathrm{NaOH} \text { (target } \\
3 \mathrm{M} \mathrm{NaOH} \text { final concentration \& } \\
5 \text { wt } \% \text { insoluble solids) and } 540 \\
\mathrm{~g} \text { DI to sludge. Heat at } 100^{\circ} \mathrm{C} \text { for } \\
5.75 \text { hr (take slurry samples; free } \\
\mathrm{OH}-2.86 \mathrm{M} \text { ). Mix } 5 \text { min at } \\
80^{\circ} \mathrm{C} \text { and transfer to settler (lost } \\
603 \mathrm{~g} \text { due to evaporation). Add } \\
437 \mathrm{~g} \text { Di for settling tests ( } 7.13 \\
\mathrm{~cm} / \mathrm{hr} \text { at } 7.2 \mathrm{wt} \% \text { solids } \& 80^{\circ} \mathrm{C} \text {. } \\
\mathrm{Lost} 599 \mathrm{~g} \text { due to evaporation, } \\
47 \mathrm{~g} \text { for } 3 \text { liquid samples, and } \\
\text { decanted } 4343 \text { g supernate). }\end{array}$ & 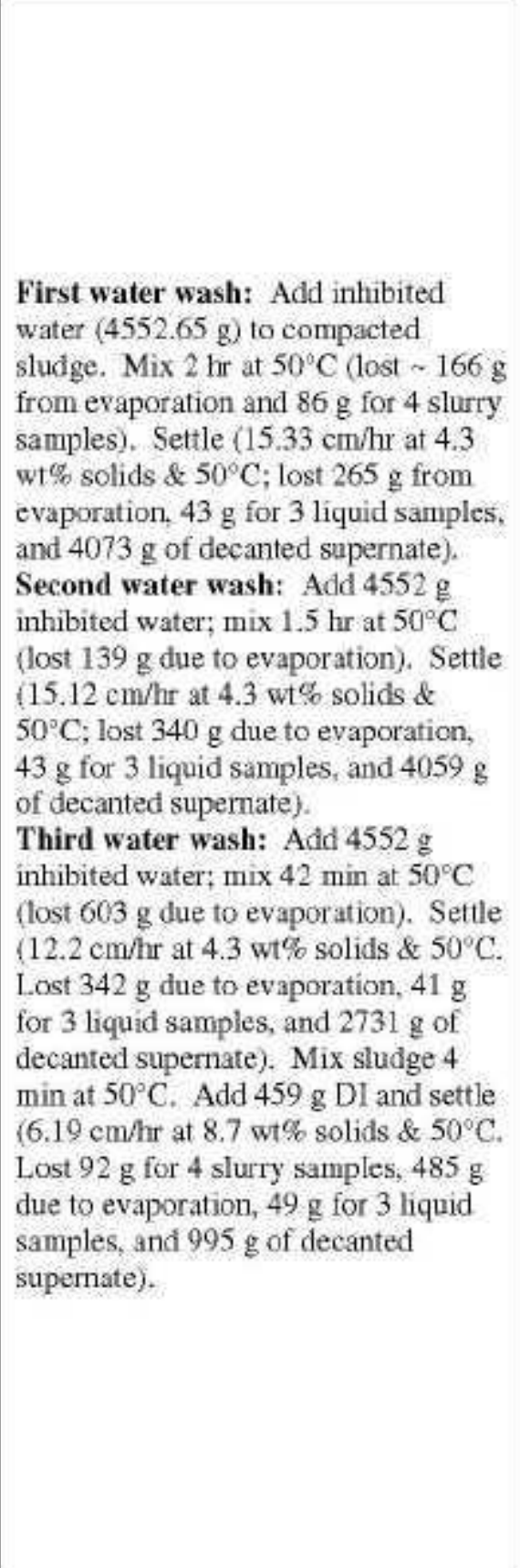 & $\begin{array}{l}1.34 \mathrm{E}+4 \mathrm{ug} / \mathrm{g} ; \\
4.12 \mathrm{E}+7 \text { ug } \mathrm{Al}(21.8 \%) .\end{array}$ & $\begin{array}{l}8.65 \mathrm{E}+3 \\
\mathrm{ug} / \mathrm{z} \\
3.34 \mathrm{E}+7 \mathrm{ug} \\
\mathrm{Al}(17.6 \%) .\end{array}$ & $\begin{array}{l}\text { First water } \\
\text { wash: } \\
2.64 \mathrm{E}+3 \text { ug/g, } \\
1.19 \mathrm{E}+7 \text { ug Al } \\
(6.3 \%) \text {. } \\
\text { Second water } \\
\text { wash: } 724 \\
\text { ug/g, } 9.09 \mathrm{E}+4 \\
\text { ug Al } \\
(0.048 \%) \text {. } \\
\text { Third water } \\
\text { wash: } 199 \\
\text { ug/g, } 5.49 \mathrm{E}+4 \\
\text { ug Al } \\
(0.029 \%)\end{array}$ & $\begin{array}{l}3.14 \mathrm{E}+5 \mathrm{ug} / \mathrm{g}, \\
9.81 \mathrm{E}+7 \mathrm{ug} \text { Al } \\
\text { (51.8\% left in } \\
\text { residue). }\end{array}$ & $\begin{array}{l}\text { Direct: } 2 \mathrm{E}+8 \\
\text { ug. } \\
\text { Summation: } \\
1.89 \mathrm{E}+8.95 \% \\
\text { recovery. }\end{array}$ & 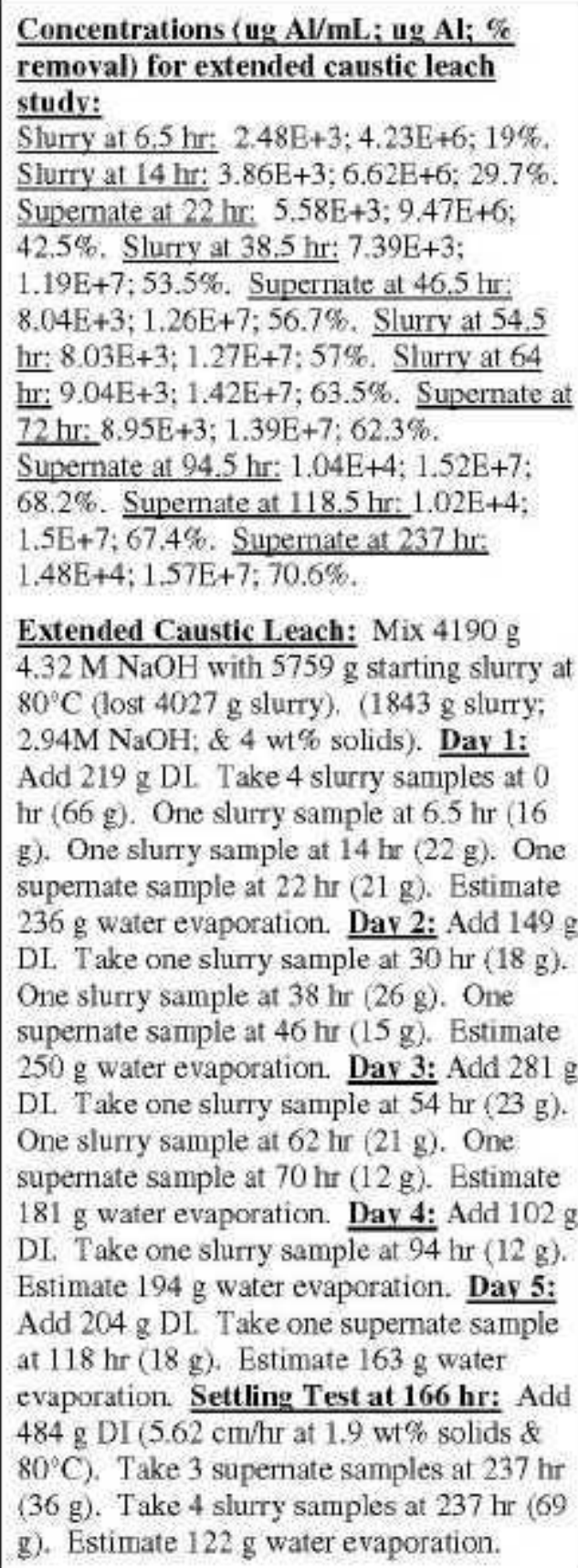 \\
\hline
\end{tabular}


Table A1-2. SSTs and DSTs Wash and Leach Information for Aluminum, Part 2. (27 sheets)

\begin{tabular}{|c|c|c|c|c|c|c|c|c|c|}
\hline$\underline{\text { Tank }}$ & $\begin{array}{l}\text { Lab Study } \\
\text { Reference(s) }\end{array}$ & Caustic Leach \#2 Procedure & $\begin{array}{c}\text { Wash of solids after Caustic leach } \\
\text { steps }\end{array}$ & $\begin{array}{l}\text { Mass (ug) Al removed during 1st leach } \\
\text { step }\end{array}$ & $\begin{array}{l}\text { Mass (ug) } \\
\text { Al removed } \\
\text { during 2nd } \\
\text { leach step } \\
\end{array}$ & $\begin{array}{l}\text { Mass (ug) Al } \\
\text { removed in } \\
\text { final wash } \\
\text { solution } \\
\end{array}$ & $\begin{array}{l}\text { Mass (ug) Al } \\
\text { in Leached } \\
\text { Solids }\end{array}$ & $\begin{array}{l}\text { Total mass Al } \\
\text { (ug) }\end{array}$ & Comments \\
\hline 241-S-110 & PNNL-13702 & - & - & 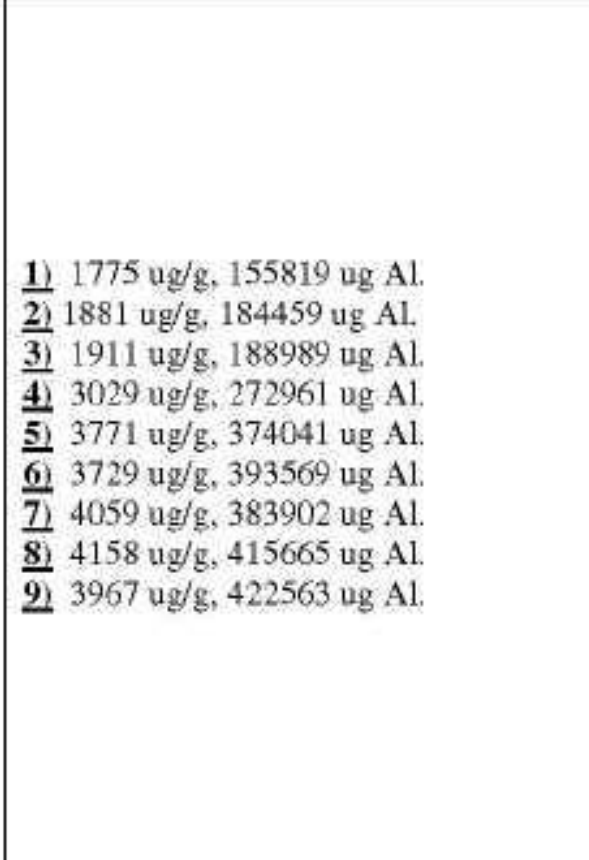 & $\begin{array}{l}\frac{\text { Fo Al }}{\text { removed at }} \\
168 \mathrm{hr}: \\
1) 39 \% \\
\text { 2) } 47 \% \\
\text { 3) } 50 \% \\
\text { 4) } 69 \% \\
\text { 5) } 91 \% \\
\text { 6) } 96 \% \\
\text { 7) } 91 \% \text {. } \\
\text { 8) } 100 \% \\
\text { 9) } 100 \%\end{array}$ & 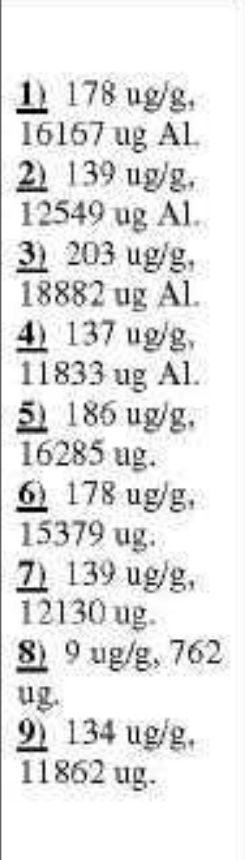 & 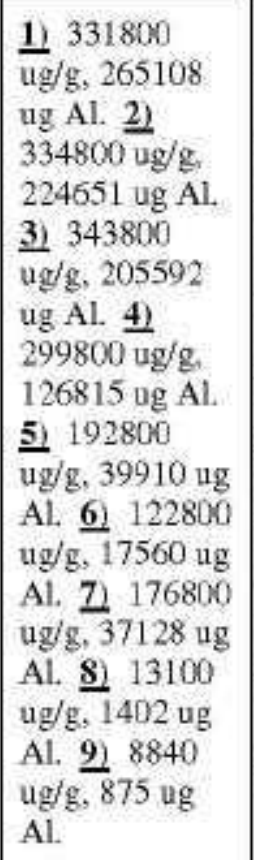 & 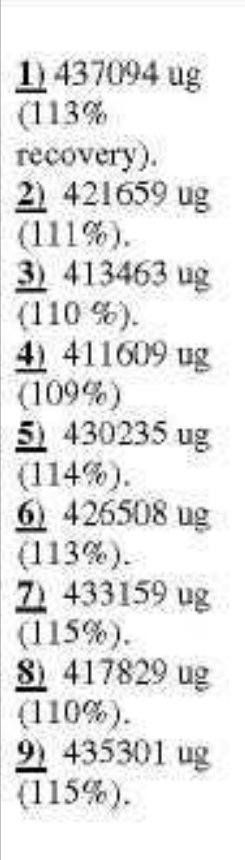 & 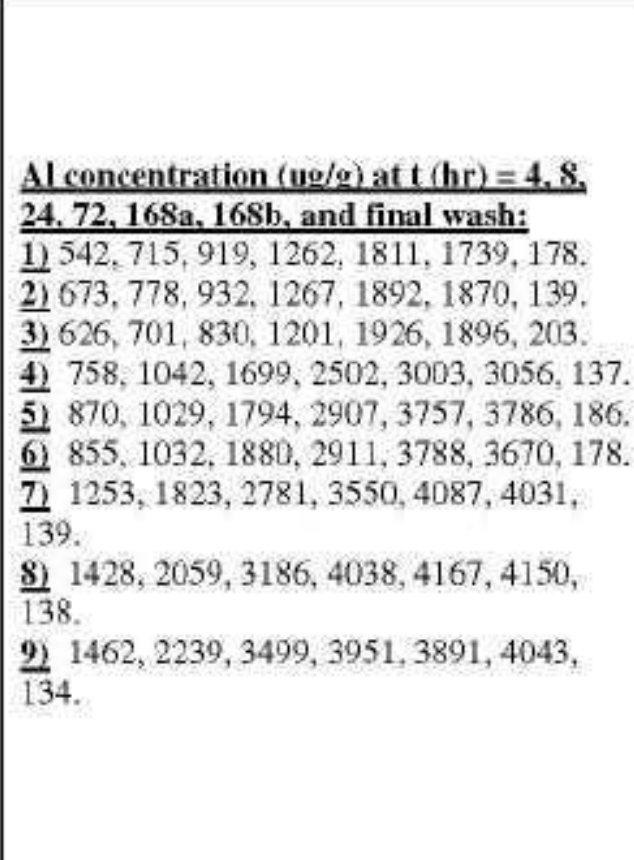 \\
\hline 241-S-110 & PNNL-14018 & - & $\begin{array}{l}\text { Centrifuge leached slurries at } 3000 \\
\text { tpm for a minimum of } 5 \text { min. Decant } \\
\text { supernate from solids. Wash solids } 3 \\
\text { times with } 0.1 \mathrm{MNaOH} \text {. Centrifuge } \\
\text { samples after each wash, with } \\
\text { supernate combined with final } \\
\text { leachate. Residual solids dried to a } \\
\text { constant weight at } 105^{\circ} \mathrm{C} \text {. }\end{array}$ & 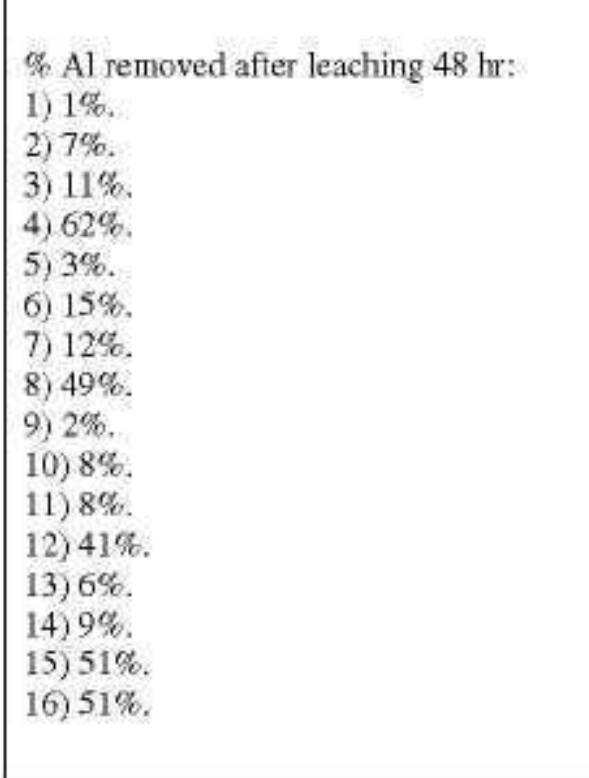 & - & - & $\begin{array}{l}\text { ug Alg } \\
\text { leached } \\
\text { sludge: } \\
\text { 1) } 350000 \text {. } \\
\text { 2) } 333000 \text {. } \\
\text { 3) } 310000 \\
\text { 4) } 332000 \\
\text { 5) } 332000 \\
\text { 6) } 349000 \\
\text { 7) } 330000 \text {. } \\
\text { 8) } 293000 \text {. } \\
\text { 9) } 338000 \\
\text { 10) } 355000 . \\
\text { 11) } 358000 \text {. } \\
\text { 12) } 333000 \text {. } \\
\text { 13) } 305000 \text {. } \\
\text { 14) } 304000 \\
\text { 155 } 273000 \\
\text { 16) } 281000 \text {. }\end{array}$ & - & - \\
\hline 241-S-111 & PNNL-11636 & $\begin{array}{l}\text { Add } 28 \mathrm{~mL} 10 \mathrm{M} \mathrm{NaOH} \text { and } 64 \\
\mathrm{~mL} \text { water to solids from } \\
\text { previous leach step; mix } 60 \mathrm{hr} \text { at } \\
100^{\circ} \mathrm{C} \text {; cool; gravity settle; and } \\
\text { decant. }\end{array}$ & $\begin{array}{l}\text { Add } 19 \mathrm{~mL} \text { of } 0.01 \mathrm{M} \mathrm{NaOH} / 0.01 \mathrm{M} \\
\text { NaNO2 to remaining solids; mix } 0.5 \\
\text { hr at RT; centrifuge; decant; repeat for } \\
\text { total of } 3 \text { wash steps. After } 3 \text { rd wash, } \\
\text { remove two }(0.50 \mathrm{~g} \text { and } 0.20 \mathrm{~g} \text { ) slurry } \\
\text { samples before centrifuge } \& \text { decant. }\end{array}$ & $717046(48 \%)$ & $\begin{array}{l}541896 \text { ug } \\
(36 \%)\end{array}$ & 96448 ug $(6 \%)$ & 3131 ug $(0 \%)$ & 1500417 & - \\
\hline
\end{tabular}


Table A1-2. SSTs and DSTs Wash and Leach Information for Aluminum, Part 2. (27 sheets)

\begin{tabular}{|c|c|c|c|c|c|c|c|c|c|}
\hline$\underline{\text { Tank }}$ & $\begin{array}{l}\text { Lab Study } \\
\text { Reference(s) }\end{array}$ & Caustic Leach $\# 2$ Procedure & $\begin{array}{l}\text { Wash of solids after Caustic leach } \\
\text { steps }\end{array}$ & $\begin{array}{l}\text { Mass (ug) Al removed during 1st leach } \\
\text { step }\end{array}$ & \begin{tabular}{|c|} 
Mass (ug) \\
Al removed \\
during 2nd \\
leach step \\
\end{tabular} & $\begin{array}{l}\text { Mass (ug) Al } \\
\text { removed in } \\
\text { final wash } \\
\text { solution } \\
\end{array}$ & $\begin{array}{l}\text { Mass (ug) Al } \\
\text { in Leached } \\
\text { Solids }\end{array}$ & $\begin{array}{l}\text { Total mass Al } \\
\text { (ug) }\end{array}$ & Comments \\
\hline 241-SX-101 & $\begin{array}{l}\text { PNWD-3512 } \\
\text { (WTP-RPT- } \\
117 \text { ) }\end{array}$ & $\begin{array}{l}3 \mathrm{M} \mathrm{NaOH} \text { leach of samples } \mathrm{SX} \text { - } \\
101-5 \text { and } \mathrm{SX}-101-6 \text { [slurry } \\
\text { weights of } 3.851 \mathrm{~g} \text { and } \\
(1.301 / 9.963) \mathrm{g} \text {, respectively } \\
\text { (see Table } 2.6 \text { in reference)]. } \\
\text { Add } 4.8 \mathrm{ml} 10 \mathrm{M} \mathrm{NaOH} \text { and } 8.2 \\
\mathrm{ml} \mathrm{DI} \text { to each. Heat at } 85 \pm 5^{\circ} \mathrm{C} \\
\text { for } 8 \mathrm{hr} \text {. Cool to RT overnight. } \\
\mathrm{Centrifuge} \mathrm{and} \mathrm{decant} \mathrm{supernate} \\
\text { to } 30 \mathrm{~mL} \text { plastic vials. Add } \\
0.1 \mathrm{M} \mathrm{NaOH} \text { at } 3: 1 \text { volvol ratio } \\
\text { to remaining solids. Mix, } \\
\text { separate, and decant to same } \\
\text { container as initial leachate } \\
\text { solution. Continue washings } \\
\text { until supernate was colorless. } \\
\text { Filter samples }(0.2 \text { um syringe } \\
\text { filter), and place } 1 \text { ml aliquot in } \\
\text { container w/ } 10 \mathrm{~mL} \text { of } 1 \mathrm{M} \\
\mathrm{HNO}_{3} \text { for ICP-AES. }\end{array}$ & 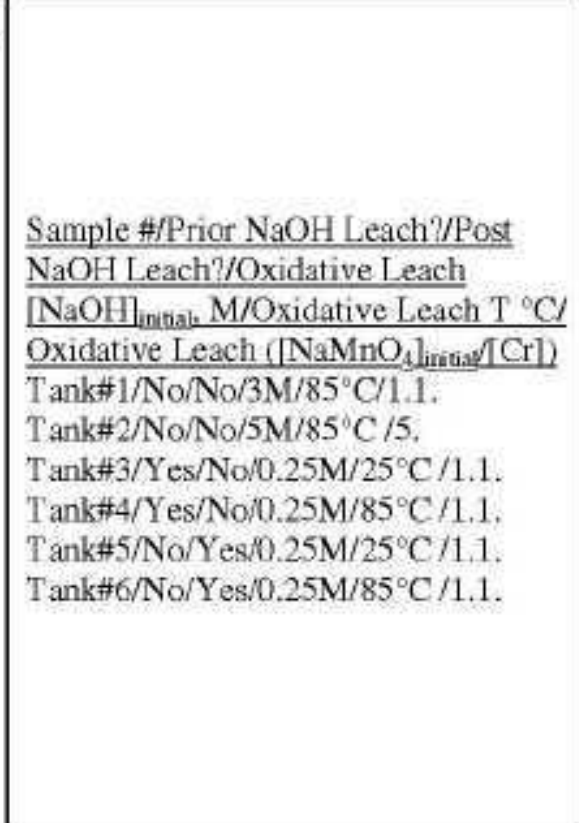 & - & - & - & - & $\begin{array}{l}\text { \% removal } \\
\text { values for } 6 \\
\text { bottles range } \\
\text { from } 83-97 \% \\
\text { Al removal } \\
1=96 \% \\
2=96 \% \\
3=83 \% \\
4=89 \% \\
5=97 \% \\
6=97 \%\end{array}$ & - \\
\hline $241-S X-108$ & PNNL-11278 & $\begin{array}{l}\text { Add } 60 \mathrm{~mL} 10 \mathrm{M} \mathrm{NaOH} \text { and } \\
\text { water to a total volume of } 240 \\
\mathrm{~mL}(2.5 \mathrm{wt} \% \text { solids) to solids, } \\
\text { mix at } 100^{\circ} \mathrm{C} \text { for } 5 \mathrm{hr} \text {, cool, } \\
\text { measure settling, and decant } \\
\text { resulting in } 191 \mathrm{~mL} \text { decanted } \\
\text { liquid, } 51 \mathrm{~mL} \text { interstitial liquid, } \\
\text { and } 2.9 \mathrm{M} \mathrm{OH} \text {. }\end{array}$ & $\begin{array}{l}\text { Wash } 3 \text { times by adding } 151 \mathrm{~mL} 0.01 \\
\mathrm{M} \mathrm{NaOH} \text { OHaNO } \text { to olids, mix at RT } \\
\text { for } 0.5 \mathrm{hr} \text {, measure settling, } \\
\text { centrifuge, and decant. Sample } 0.4 \\
\mathrm{~mL} \text { slurry after mix of wash } 3 \text {. Dry } \\
\text { solids at } 105^{\circ} \mathrm{C}=7.19 \mathrm{~g} \text { solids } \\
\text { (adjusted for slurry removed from } \\
\text { wash } 3 \text { step). }\end{array}$ & $5369 \mathrm{ug} / \mathrm{mL} ; 767767 \mathrm{ug}+28 \%$. & $\begin{array}{l}1080 \mathrm{ug} / \mathrm{mL} ; \\
14386 \mathrm{ug} ; \\
1 \%\end{array}$ & $\begin{array}{l}104 \mathrm{ug} / \mathrm{mL} ; 0 \\
\mathrm{ug} ; 0 \% .\end{array}$ & $\begin{array}{l}270000 \\
\text { ug/mL; } \\
194130 \text { ug } \\
\text { (table shows } \\
1941300 \text { ug, } \\
\text { which may be } \\
\text { incorrect); } \\
71 \% \text {. }\end{array}$ & $\begin{array}{l}2723453 \text { ug; } \\
\text { Summation: } \\
1.34 \mathrm{E}+5 \mathrm{ug} / \mathrm{g} ; \\
\text { Direct: } 9.02 \mathrm{E}+4 \\
\mathrm{ug} / \mathrm{g} ; \\
\text { Mass Recovery: } \\
149 \%\end{array}$ & $\begin{array}{l}29 \% \text { removed by leaching twice with } \\
\text { caustic and washing } 3 \text { times with } 0.01 \mathrm{M} \\
\mathrm{NaOH} / \mathrm{NaNO}_{2} \text { solution. }\end{array}$ \\
\hline 241-SX-108 & PNNL-11908 & - & $\begin{array}{l}\text { Wash solids } 3 \text { times with } 0.1 \mathrm{M} \mathrm{NaOH} \text {. } \\
\text { After each wash, centrifuge and } \\
\text { transfer supernate to final leachate } \\
\text { container. The washed residual solids } \\
\text { were dried to a constant wt at } 105^{\circ} \mathrm{C} \text {. }\end{array}$ & 1) $70 \%$. 2) $93 \%$. 3) $63 \%$. 4) $89 \%$. & - & - & $\begin{array}{l}\text { 0.1M oxidative } \\
\text { leached solids } \\
\text { - initial: } \\
\text { 22900 ug Al/g } \\
\text { solids. } \\
\text { 3M oxidative } \\
\text { leached solids } \\
\text { - initial: } \\
235000 \text { ug } \\
\text { Alg solids. } \\
\text { 1) } 13300 \mathrm{ug} / \mathrm{g} \text {. } \\
\text { 2) } 505000 \mathrm{ug} / \mathrm{g} \text {. } \\
\text { 3) } 10900 \mathrm{ug} / \mathrm{g} \text {. } \\
\text { 4) } 116000 \mathrm{ug} / \mathrm{g} \\
\text { residual } \mathrm{Al} \text {. }\end{array}$ & - & - \\
\hline
\end{tabular}


Table A1-2. SSTs and DSTs Wash and Leach Information for Aluminum, Part 2. (27 sheets)

\begin{tabular}{|c|c|c|c|c|c|c|c|c|c|}
\hline$\underline{\text { Tank }}$ & $\begin{array}{l}\text { Lab Study } \\
\text { Reference (8) }\end{array}$ & Caustic Leach $\# 2$ Procedure & $\begin{array}{c}\text { Wash of solids after Caustic leach } \\
\text { steps }\end{array}$ & $\begin{array}{l}\text { Mass (ug) Al removed during 1st leach } \\
\text { step }\end{array}$ & \begin{tabular}{|c|} 
Mass (ug) \\
Al removed \\
during 2nd \\
leach step \\
\end{tabular} & 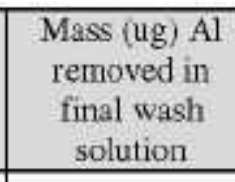 & $\begin{array}{c}\text { Mass (ug) Al } \\
\text { in Leached } \\
\text { Solids }\end{array}$ & \begin{tabular}{|c|} 
Total mass $\mathrm{Al}$ \\
(ug)
\end{tabular} & Comments \\
\hline 241-SX-113 & $\begin{array}{l}\text { LAUR 97- } \\
2889\end{array}$ & $\begin{array}{l}\text { Add } 93 \mathrm{~mL} \text { water \& } 40.4 \mathrm{~mL} 10 \\
\mathrm{M} \mathrm{NaOH}(\sim 1 \text { wt } \% \text { solids \& } 3 \mathrm{M} \\
\mathrm{NaOH}) \text {; mix } 5 \mathrm{hr} \text { at } 100^{\circ} \mathrm{C} ; \text { cool; } \\
\text { measure settling; centrifuge; and } \\
\text { decant. Final solution had } 126.4 \\
\mathrm{~mL} \text { supernate with } 2.99 \mathrm{M} \text { free } \\
\mathrm{OH}, 17.3 \text { mLL settled solids, \& } 6 \\
\mathrm{~mL} \text { centrifuged solids. }\end{array}$ & $\begin{array}{l}\text { Wash } 3 \text { times by adding } 27.5 \mathrm{~mL} \\
\text { inhibited water to solids; mix } 0.5 \mathrm{hr} \text { at } \\
\text { RT; measure settling; centrifuge; and } \\
\text { decant. Subsample after mixing for } \\
\text { wash } \$ 3 \text { ( } 0.62 \mathrm{~g} \text { ). Dry solids at } 105^{\circ} \mathrm{C} \text {. }\end{array}$ & $623.35 \mathrm{ug} / \mathrm{mL} .12167 .79 \mathrm{ug}(27.3 \%)$. & $\begin{array}{l}208 \mathrm{ug} / \mathrm{mL} \text {. } \\
26289.12 \mathrm{ug} \\
(58.9 \%)\end{array}$ & $\begin{array}{l}17.05 \mathrm{ug} / \mathrm{mL} \text {. } \\
1371.67 \mathrm{ug} \\
(3.1 \%) .\end{array}$ & $\begin{array}{l}30438 \mathrm{ug} / \mathrm{g} . \\
4812.21 \mathrm{ug} \\
(10.8 \%)\end{array}$ & 44641 ug. & - \\
\hline 241-SX-113 & $\begin{array}{l}\text { ORNL TM- } \\
13500\end{array}$ & - & $\begin{array}{l}\text { After mixing, the samples were } \\
\text { centrifuged for } 15-20 \text { min at } 2500 \\
\text { rpm. The wet sludge was rinsed three } \\
\text { times with inhibited water }\{0.01 \mathrm{M} \\
\text { NaOH and } 0.01 \mathrm{MNaNO} \text {, using } \rightarrow 15 \\
\text { mL inhibited water for each rinse. }\end{array}$ & Leachate: 1) $307 \mathrm{ug} / \mathrm{g} ; 2) 215 \mathrm{ug} / \mathrm{g}$. & - & $\begin{array}{l}\text { Rinse: 1) } 10 \\
\mathrm{ug} / \mathrm{g} ; 2) 6.61 \\
\mathrm{ug} / \mathrm{g} .\end{array}$ & $\begin{array}{l}\text { Residual: } 1) \\
5080 \mathrm{ug} / \mathrm{g} ; 2) \\
2820 \mathrm{ug} / \mathrm{g} .\end{array}$ & $\begin{array}{l}\frac{\% \text { based on }}{\text { residue: } 1)} \\
\frac{51.04 \% ; 2)}{78.69 \%} \\
\frac{\% \text { based on }}{\text { leachate \& }} \\
\frac{\text { rinse: } 1)}{62.12 \% ; 2)} \\
81.75 \% . \\
\frac{\% \text { Recooverv: }}{1) 111.08 \% ; 2)} \\
103.06 \% \% \\
\frac{\% \text { Removed: }}{1,51 \% \% 2)} \\
78.7 \% \text {. }\end{array}$ & - \\
\hline $241-\mathrm{T}-104$ & PNL-10712 & $\begin{array}{l}\text { Add } 11.4 \mathrm{~g} 3 \mathrm{M} \mathrm{NaOH} \text { to } 4.8 \\
\mathrm{~mL} \text { centrifuged solids; mix } 5 \mathrm{hr} \\
\text { at } 100^{\circ} \mathrm{C} \text {; cool; measure settling; } \\
\text { centrifuge; and decant. }\end{array}$ & 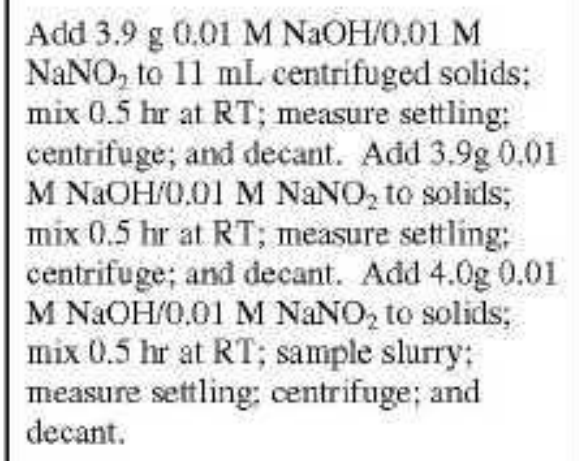 & $24 \%, 5015 \mathrm{ug} / \mathrm{mL}$ measured. & $\begin{array}{l}26 \%, 4845 \\
\text { ug/mL } \\
\text { measured. }\end{array}$ & $\begin{array}{l}11 \%, 1615 \\
\text { ug/mL } \\
\text { measured. }\end{array}$ & $\begin{array}{l}\text { Measured } \\
\text { concentration } \\
\text { of } 33000 \text { ug/g; } \\
38 \% \text { Al in } \\
\text { residue. }\end{array}$ & $\begin{array}{l}\text { Direct analysis: } \\
4.7 \mathrm{E}-02 \mathrm{~g} / \mathrm{g} \\
\text { sludge; } \\
\text { Summation } \\
\text { method: } 4.29 \mathrm{E}- \\
02 \text { g/g sludge; } \\
91 \% \text { recovery. }\end{array}$ & - \\
\hline $241-\mathrm{T}-104$ & PNNL-11089 & $\begin{array}{l}\text { Add } \mathrm{NaOH} \text { to get }-8 \mathrm{wt} \% \text { solids } \\
\text { and a final } \mathrm{NaOH} \text { of } 2.2 \mathrm{M} \text {. Mix } \\
\text { at } 100^{\circ} \mathrm{C} \text { for } 5 \mathrm{hr} \text {. Cool, } \\
\text { centrifuge, and decant. }\end{array}$ & $\begin{array}{l}\text { Add inhibited water. Mix at RT for } \\
0.5 \mathrm{hr} \text { (target } 8 \text { wt } \% \text { solids). } \\
\text { Centrifuge, decant, and repeat twice } \\
\text { morc. }\end{array}$ & $\begin{array}{l}\text { Uncorrected/corrected recovery }= \\
24 / 37 \% \text {. }\end{array}$ & $\begin{array}{l}\text { Uncorrected } \\
\text { corrected } \\
\text { recovery = } \\
26 / 24 \%\end{array}$ & $\begin{array}{l}\text { Uncorrected/c } \\
\text { orrected } \\
\text { recovery }= \\
11 / 2 \% \text {. }\end{array}$ & $\begin{array}{l}\text { Total wt\% } \mathrm{Al} \\
=4.7 \% ; \\
\text { Total \% } \mathrm{Al} \\
\text { removed = } \\
62 \% ; \\
\text { Percent } \\
\text { undissolved } \\
\text { residue } \\
\text { (uncorrected/ } \\
\text { corrected) }= \\
38 / 35 \% \text {. }\end{array}$ & - & - \\
\hline
\end{tabular}


Table A1-2. SSTs and DSTs Wash and Leach Information for Aluminum, Part 2. (27 sheets)

\begin{tabular}{|c|c|c|c|c|c|c|c|c|c|}
\hline$\underline{\text { Tank }}$ & $\begin{array}{l}\text { Lab Study } \\
\text { Reference (8) }\end{array}$ & Caustic Leach $\# 2$ Procedure & $\begin{array}{c}\text { Wash of solids after Caustic leach } \\
\text { steps }\end{array}$ & $\begin{array}{l}\text { Mass (ug) Al removed during 1st leach } \\
\text { step }\end{array}$ & \begin{tabular}{|c|} 
Mass (ug) \\
Al removed \\
during 2nd \\
leach step \\
\end{tabular} & $\begin{array}{l}\text { Mass (ug) Al } \\
\text { removed in } \\
\text { final wash } \\
\text { solution } \\
\end{array}$ & $\begin{array}{l}\text { Mass (ug) Al } \\
\text { in Leached } \\
\text { Solids }\end{array}$ & $\begin{array}{l}\text { Total mass Al } \\
\text { (ug) }\end{array}$ & Comments \\
\hline 241-T-104 & $\begin{array}{l}\text { ORNL TM- } \\
13660\end{array}$ & $\begin{array}{l}\text { Add }-10 \mathrm{~mL} \text { of } 3 \mathrm{M} \mathrm{NaOH} \text { to } \\
\text { samples. Mix for } 5 \mathrm{hr} 30 \mathrm{~min} \text {. } \\
\text { Cool. Centrifuge for } \sim 10 \mathrm{~min} \text {. } \\
\text { Decant and filter. }\end{array}$ & $\begin{array}{l}\text { Wash } 3 \text { times by adding inhibited } \\
\text { water and place on shaker at RT for } 30 \\
\text { min. Remove samples from shaker, } \\
\text { centrifuge, decant liquid and filter. } \\
\text { Repeat process. }\end{array}$ & $\begin{array}{l}\text { RT: } 7320 \mathrm{ug} / \mathrm{mL} . \quad \mathrm{T}=60: 5160 \mathrm{ug} / \mathrm{mL} . \\
\mathrm{T}=95: 5870 \mathrm{ug} / \mathrm{mL} .\end{array}$ & $\begin{array}{l}\text { RT: } 1720 \\
\text { ug/mL. } \\
\mathrm{T}=60: 1040 \\
\mathrm{ug} / \mathrm{mL} . \\
\mathrm{T}=95: 1380 \\
\mathrm{ug} / \mathrm{mL} .\end{array}$ & $\begin{array}{l}\text { RT: } 220,52.8, \\
\text { and } 24.4 \\
\text { ug/mL. T=60: } \\
106,18.4, \text { and } \\
8.48 \mathrm{ug} / \mathrm{mL} . \\
\mathrm{T}=95: 174, \\
28.4, \text { and } 10.7 \\
\text { ug/mL. }\end{array}$ & - & - & - \\
\hline 241-T-107 & $\begin{array}{l}\text { LAUR 95- } \\
2070\end{array}$ & $\begin{array}{l}\text { To solids, add } 3 \mathrm{M} \mathrm{NaOH} \text {, mix at } \\
100^{\circ} \mathrm{C} \text { for } 5 \mathrm{hr} \text {, settle and decant. }\end{array}$ & $\begin{array}{l}\text { Wash solids } 3 \text { times with inhibited } \\
\text { water at RT for } 0.5 \text { hrr, settle and } \\
\text { decant. After wash, centrifuge, } \\
\text { decant, and dry solids at } 80^{\circ} \mathrm{C} \text {. }\end{array}$ & $14000 \mathrm{ug} / \mathrm{mL}(62.71 \%)$. & $\begin{array}{l}1900 \mathrm{ug} / \mathrm{mL} \\
(10.22 \%)\end{array}$ & $\begin{array}{l}78 \mathrm{ug} / \mathrm{mL} \\
(1.46 \%)\end{array}$ & $\begin{array}{l}50691 \mathrm{ug} / \mathrm{g} \text { in } \\
\text { leached sludge } \\
\text { (22.06\% in } \\
\text { residue). }\end{array}$ & \begin{tabular}{|l} 
Direct analysis: \\
$5.67 \mathrm{E}-2 \mathrm{~g} \mathrm{Al} / \mathrm{g}$ \\
sludge; \\
Summation: \\
$6.17 \mathrm{E}-2 \mathrm{~g} \mathrm{Al} / \mathrm{g}$ \\
sludge \\
(108.79\% \\
recovery).
\end{tabular} & Residual: $51 \mathrm{mg} \mathrm{Al/g} \mathrm{sludge}(22 \%)$. \\
\hline $241-\mathrm{T}-110$ & PNNL-13956 & - & $\begin{array}{l}\text { Leached solids were washed } 3 \text { times } \\
\text { with } 30 \mathrm{~mL} \text { portions of } 0.01 \mathrm{M} \mathrm{NaOH} \text {. } \\
\text { and then dried at } 105^{\circ} \mathrm{C} \text {. }\end{array}$ & 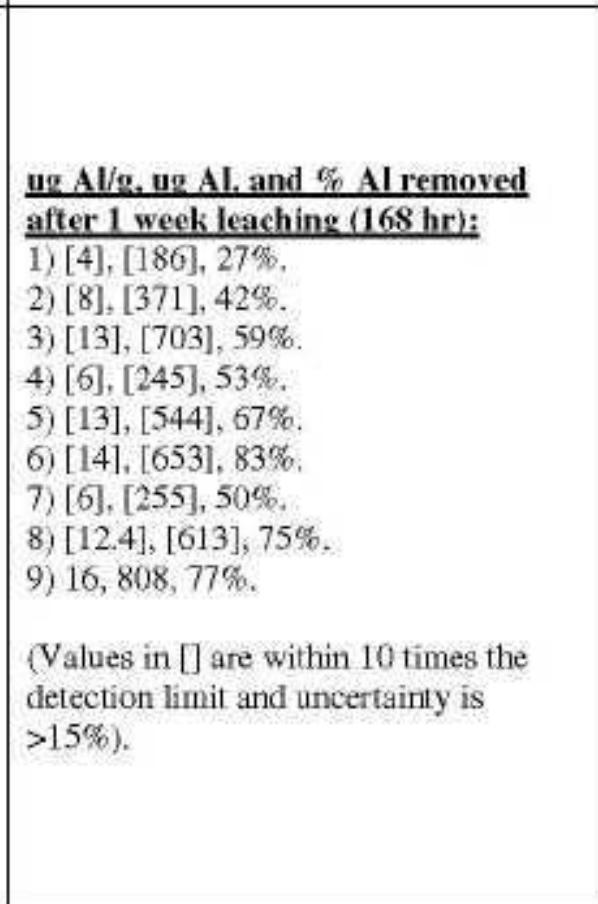 & - & 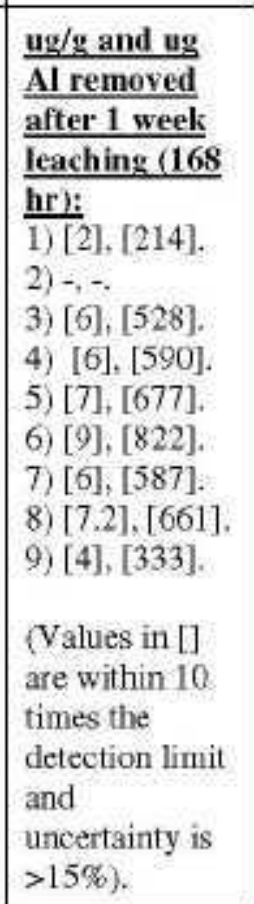 & 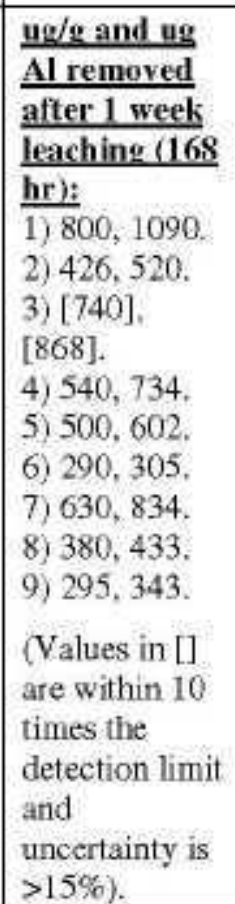 & $\begin{array}{l}\text { 1) } 1490 \text { ug Al. } \\
\text { 2) } 890 \text { ug. } \\
\text { 3) [2098] ug. } \\
\text { 4) } 1569 \text { ug. } \\
\text { 5) [1823] ug. } \\
\text { 6) [1781] ug. } \\
\text { 7) } 1676 \text { ug. } \\
\text { 8) } 1707 \text { ug. } \\
\text { 9) } 1484 \text { ug. } \\
\text { (Values in } \square \text { are } \\
\text { within } 10 \text { times } \\
\text { the detection } \\
\text { limit and } \\
\text { uncertainty is } \\
>15 \% \text {. }\end{array}$ & 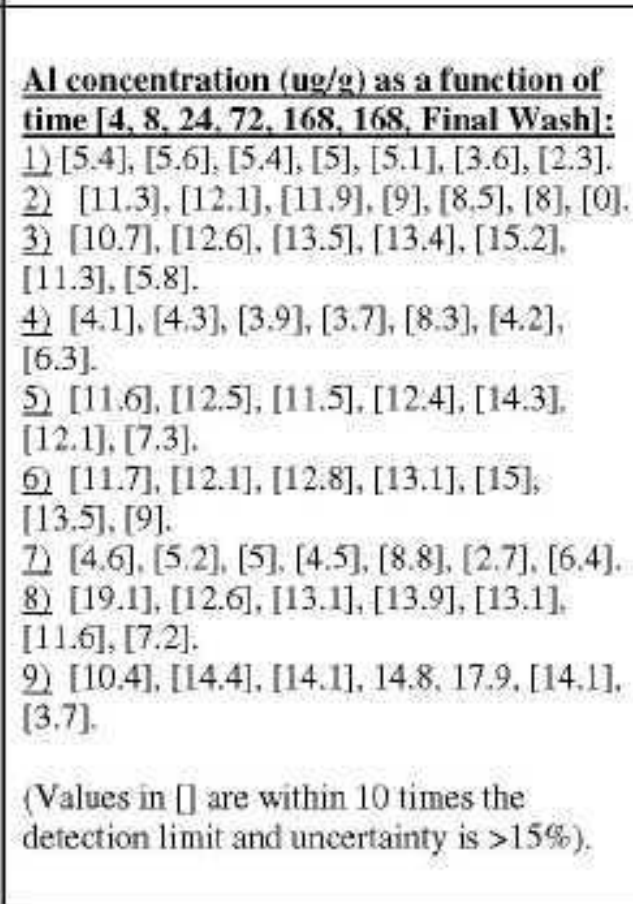 \\
\hline
\end{tabular}


Table A1-2. SSTs and DSTs Wash and Leach Information for Aluminum, Part 2. (27 sheets)

\begin{tabular}{|c|c|c|c|c|c|c|c|c|c|}
\hline$\underline{\text { Tank }}$ & $\begin{array}{l}\text { Lab Study } \\
\text { Reference (8) }\end{array}$ & Caustic Leach \#2 Procedure & $\begin{array}{c}\text { Wash of solids after Caustic leach } \\
\text { steps }\end{array}$ & $\begin{array}{l}\text { Mass (ug) Al removed during 1st leach } \\
\text { step }\end{array}$ & \begin{tabular}{c|} 
Mass (ug) \\
Al removed \\
during 2nd \\
leach step \\
\end{tabular} & $\begin{array}{l}\text { Mass (ug) Al } \\
\text { removed in } \\
\text { final wash } \\
\text { solution } \\
\end{array}$ & $\begin{array}{l}\text { Mass (ug) Al } \\
\text { in Leached } \\
\text { Solids }\end{array}$ & $\begin{array}{l}\text { Total mass Al } \\
\text { (ug) }\end{array}$ & Comments \\
\hline 241-T-111 & PNL-10712 & $\begin{array}{l}\text { Solids did not settle. Add } 11.7 \mathrm{~g} \\
3 \mathrm{M} \text { NaOH to } 8.5 \mathrm{~mL} \\
\text { centrifuged solids; mix } 5 \mathrm{hr} \text { at } \\
100^{\circ} \mathrm{C} \text {; cool; measure settling: } \\
\text { centrifuge; and decant. }\end{array}$ & $\begin{array}{l}\text { Solids did not settle. Add } 12.7 \mathrm{~g} \\
0.01 \mathrm{M} \mathrm{NaOH} / 0.01 \mathrm{M} \mathrm{NaNO} \mathrm{N}_{2} \text { to } 6.2 \\
\text { mL centrifuged solids; mix } 0.5 \mathrm{hr} \text { at } \\
\text { RT; measure settling; centrifuge; and } \\
\text { decant. Add } 13 \mathrm{~g} 0.01 \mathrm{M} \mathrm{NaOH} / 0.01 \mathrm{M} \\
\mathrm{NaNO}_{2} \text { to solids; mix } 0.5 \mathrm{hr} \text { at } \mathrm{RT} \text {; } \\
\text { measure settling; centrifuge; and } \\
\text { decant. Add } 12.9 \mathrm{~g} 0.01 \mathrm{M} \\
\mathrm{NaOH} / 0.01 \mathrm{M} \text { NaNO } \\
0.5 \mathrm{hr} \text { to solids; mix } \\
\text { settling; centrifupe; slurry; measure } \\
\text { secant. }\end{array}$ & $4 \%: 79.9 \mathrm{ug} / \mathrm{mL}$ measured. & $\begin{array}{l}5 \% ; 78.2 \\
\text { ug/mL } \\
\text { measured. }\end{array}$ & $\begin{array}{l}4 \% ; 17 \mathrm{ug} / \mathrm{mL} \\
\text { measured. }\end{array}$ & $\begin{array}{l}\text { Measured } \\
\text { concentration } \\
\text { of } 5700 \mathrm{ug} / \mathrm{g} \text { : } \\
87 \% \text { in } \\
\text { residue. }\end{array}$ & $\begin{array}{l}\text { Direct analysis: } \\
4.9 \mathrm{E}-103 \mathrm{~g} / \mathrm{g} \\
\text { sludge. } \\
\text { Summation } \\
\text { method: } 3.68 \mathrm{E}- \\
03 \mathrm{~g} / \mathrm{g} \text { sludge. } \\
75 \% \text { recovery. }\end{array}$ & - \\
\hline 241-T-111 & PNNL-1 1089 & $\begin{array}{l}\text { Add } \mathrm{NaOH} \text { to get }-8 \mathrm{wt} \% \text { solids } \\
\text { and a final } \mathrm{NaOH} \text { of } 3.4 \mathrm{M} \text {. Mix } \\
\text { at } 100^{\circ} \mathrm{C} \text { for } 5 \mathrm{hr} \text {. Cool, } \\
\text { centrifuge, and decant. }\end{array}$ & $\begin{array}{l}\text { Add inhibited water. Mix at RT for } \\
0.5 \text { hr (target } 8 \text { wt } \$ \text { solids). } \\
\text { Centrifuge, decant, and repeat twice } \\
\text { more. }\end{array}$ & $\begin{array}{l}\text { Uncorrected/ corrected recovery = } \\
4 / 8 \% \text {. }\end{array}$ & $\begin{array}{l}\text { Uncorrected } \\
\text { corrected } \\
\text { recovery = } \\
5 / 5 \% \text {. }\end{array}$ & $\begin{array}{l}\text { Uncorrected/ } \\
\text { corrected } \\
\text { recovery }= \\
4 / 1 \% \text {. }\end{array}$ & $\begin{array}{l}\text { Total wt } \% \text { Al } \\
=0.49 \text {. } \\
\text { Total \% Al } \\
\text { removed = } \\
13 \% \text {. } \\
\text { Percent } \\
\text { undissolved } \\
\text { residue } \\
\text { (uncorrected/ } \\
\text { corrected) }= \\
87 / 86 \%\end{array}$ & - & - \\
\hline 241-TY-104 & $\begin{array}{l}\text { LAUR 96- } \\
2839\end{array}$ & $\begin{array}{l}\text { Add } 12.6 \mathrm{~mL}, 3 \mathrm{M} \mathrm{NaOH} \text { to } \\
\text { solids (no settled solids/ } 4.6 \mathrm{~mL} \\
\text { centrifuged solids); mix } 5 \mathrm{hr} \text { at } \\
100^{\circ} \mathrm{C} \text {; cool; measure settling; } \\
\text { centrifuge; and decant. }\end{array}$ & $\begin{array}{l}\text { Add } 8.1 \mathrm{~mL} \text { inhibited water to solids } \\
\text { (no settled solids } 4.5 \mathrm{~mL} \text { centrifugged } \\
\text { solids); mix } 0.5 \mathrm{hr} \text { at } \mathrm{RT} \text {; measure } \\
\text { settling; centrifuge; decant; and repeat } \\
\text { twice with solids portion, sampling } \\
\text { slurry }(0.3 \mathrm{~mL} \text { ) before settling in wash } \\
\# 3 \text {. Dry solids at } 80^{\circ} \mathrm{C} \text {. }\end{array}$ & $7480 \mathrm{ug} / \mathrm{mL}(27.67 \%)$ & $\begin{array}{l}2890 \mathrm{ug} / \mathrm{mL} \\
(18.23 \%)\end{array}$ & $\begin{array}{l}688.5 \mathrm{ug} / \mathrm{mL} \\
(8.23 \%)\end{array}$ & $\begin{array}{l}42857.14 \text { ug/g. } \\
36.74 \% \text { Al in } \\
\text { residue; } 63 \% \\
\text { Al removed } \\
\text { during sludge } \\
\text { wash/caustic } \\
\text { leach. }\end{array}$ & $\begin{array}{l}\text { Direct analysis: } \\
4.28 \mathrm{E}-2 \mathrm{~g} / \mathrm{g} \\
\text { sludge: } \mathrm{Sum} \\
\text { method: } 4.03 \mathrm{E}- \\
2 \mathrm{~g} / \mathrm{g} \text { sludge. } \\
94.90 \% \\
\text { recovery. }\end{array}$ & - \\
\hline $241-U-108$ & PNNL-11908 & - & $\begin{array}{l}\text { Wash solids } 3 \text { times with } 0.1 \mathrm{M} \mathrm{NaOH} \text {. } \\
\text { After each wash, centrifuge and } \\
\text { transfer supernate to final leachate } \\
\text { container. The washed residual solids } \\
\text { were dried to a constant wt at } 105^{\circ} \mathrm{C} \text {. }\end{array}$ & $\begin{array}{l}\text { 1) } 6.5 \% \text {. 2) } 96.4 \% \text {. 3) } 12 \% .4) 94.7 \% \text {. } \\
\text { 5) } 10 \% .6,81 \% \text {. }\end{array}$ & - & - & $\begin{array}{l}202000 \text { ug } \\
\text { Alg initial } \\
\text { sludge. } \\
\text { ug Alg final } \\
\text { leached } \\
\text { sludge: } \\
1,183500 . \\
\text { 2) } 18100 . \\
\text { 3) } 149000 \\
\text { 4) } 66500 \\
\text { 5) } 17900 . \\
\text { 6) } 81500 .\end{array}$ & - & - \\
\hline
\end{tabular}


Table A1-2. SSTs and DSTs Wash and Leach Information for Aluminum, Part 2. (27 sheets)

\begin{tabular}{|c|c|c|c|c|c|c|c|c|c|}
\hline$\underline{\text { Tank }}$ & $\begin{array}{l}\text { Lab Study } \\
\text { Reference(s) }\end{array}$ & Caustic Leach \#2 Procedure & $\begin{array}{l}\text { Wash of solids after Caustic leach } \\
\text { steps }\end{array}$ & $\begin{array}{l}\text { Mass (ug) Al removed during 1st leach } \\
\text { step }\end{array}$ & \begin{tabular}{|l|} 
Mass (ug) \\
Al removed \\
during 2nd \\
leach step \\
\end{tabular} & $\begin{array}{l}\text { Mass (ug) Al } \\
\text { removed in } \\
\text { final wash } \\
\text { solution } \\
\end{array}$ & $\begin{array}{l}\text { Mass (ug) Al } \\
\text { in Leached } \\
\text { Solids }\end{array}$ & $\begin{array}{l}\text { Total mass Al } \\
\text { (ug) }\end{array}$ & Comments \\
\hline 241-U-108 & PNNL-14019 & - & $\begin{array}{l}\text { After } 24 \text { lir, centrifuge slurries at } 3000 \\
\text { rpm for } 5 \text { min (minimum), Decant } \\
\text { supernate. Wash residual solids } 2-3 \\
\text { times with } 0.01 \mathrm{M} \text { NaOH. After each } \\
\text { washh, centrifuge samples and combine } \\
\text { supernate with final leachate. Final } \\
\text { leachate was passed through a } 0.2 \text { um } \\
\text { syringe filter prior to analysis. } \\
\text { Washed residual solids were dried to } \\
\text { constant weight at } 105^{\circ} \mathrm{C} \text { and } \\
\text { analyzed by KOH fusion in Ni } \\
\text { crucible followed by dissolution into } \\
\text { nitric acid. }\end{array}$ & $\begin{array}{l}\frac{u g / g \text { Al in treated sludge, } \% \text {. Al }}{\text { removed from oxidative alkaline }} \\
\text { leaching of washed sludge solids: } \\
\text { 1) } 102630,11 \% \text {. } \\
\text { 2) } 51300,73 \% \text {. } \\
\text { 3) } 92830,25 \% \text {. } \\
\text { 4) } 23630,86 \% \text {. } \\
\text { 5) } 140630,17 \% \text {. } \\
\text { 6) } 63330,75 \% \text {. }\end{array}$ & - & - & - & - & - \\
\hline 241-U-109 & PNNL-- 11908 & - & $\begin{array}{l}\text { Wash solids } 3 \text { times with } 0.1 \mathrm{M} \mathrm{NaOH} \text {. } \\
\text { After each wash, centrifuge and } \\
\text { transfer supernate to final leachate } \\
\text { container. The washed residual solids } \\
\text { were dried to a constant wt at } 105^{\circ} \mathrm{C} \text {. }\end{array}$ & $\begin{array}{l}\text { 1) } 9.2 \% \text {. } \\
\text { 2) } 77 \% \text {, } \\
\text { 3) } 17 \% \text {. } \\
\text { 4) } 80 \% \\
\text { 5) } 24 \% \\
\text { 6) } 54 \%\end{array}$ & - & - & $\begin{array}{l}86800 \text { ug Al/g } \\
\text { initial sludge. } \\
\text { ug Al/g final } \\
\text { leached } \\
\text { sludge: } \\
1776600 \\
\text { 2)222300. } \\
\text { 3) } 90200 \\
\text { 4) } 82900 . \\
\text { 5) } 77150 . \\
\text { 6) } 49500\end{array}$ & - & - \\
\hline $241-\mathrm{U}-110$ & PNL-9387 & $\begin{array}{l}\text { Residual sludge was washed } \\
\text { with } 2 \mathrm{~mL} 1 \mathrm{M} \mathrm{K} \mathrm{KO}_{3} \text {; mix } \\
\text { sludge with } \mathrm{K}_{2} \mathrm{CO}_{3} \text { for } 2 \mathrm{hr} \text { at } \\
\text { RT; centrifuge; decant; and } \\
\text { repeat. Add } 2 \mathrm{~mL} 1 \mathrm{M} \mathrm{K}_{2} \mathrm{CO}_{3} \text { to } \\
\text { sludge; heat } 5 \mathrm{hr} \text { at } 100^{\circ} \mathrm{C}_{;} \text {cool; } \\
\text { centrifuge; decant; and repeat. } \\
\text { Wash sludge with } 5 \mathrm{~mL} \text { water at } \\
\text { RT. }\end{array}$ & $\begin{array}{l}\text { Acid leaching done after } \mathrm{K}_{2} \mathrm{CO}_{3} \\
\text { digestion - not described here. }\end{array}$ & - & $\begin{array}{l}\text { Total } 79 \% \\
\mathrm{Al} \text { in } \mathrm{NaOH} \\
\text { leach. }\end{array}$ & $\begin{array}{l}\text { Total } 4 \% \mathrm{Al} \text { in } \\
\mathrm{K}_{2} \mathrm{CO}_{3} \text { digest. }\end{array}$ & $\begin{array}{l}12 \% \mathrm{Al} \text { in } \\
\text { leached } \\
\text { sludge. }\end{array}$ & - & - \\
\hline $241-\mathrm{U}-110$ & PNL-10078 & $\begin{array}{l}\text { Add } 4 \mathrm{~mL} 3 \mathrm{M} \mathrm{NaOH} / 2 \mathrm{M} \\
\mathrm{Na}_{2} \mathrm{CO}_{3} \text { to solids, mix at } 100^{\circ} \mathrm{C} \\
\text { for } 5 \mathrm{hr} \text {, remove samples of } \\
\text { liquid and solid, continue to mix } \\
\text { at } \sim 20^{\circ} \mathrm{C} \text { for } 5 \mathrm{hr} \text { with } \\
\text { sonication, centrifuge, and } \\
\text { decant when finished. }\end{array}$ & $\begin{array}{l}\text { Add } 3 \mathrm{~mL} \text { water to solids, mix at RT } \\
\text { for } 0.5 \mathrm{lr} \text {, centrifuge, decant, and } \\
\text { repeat with solids. }\end{array}$ & $37 \%$. & $\begin{array}{l}46 \%(0.38 \mathrm{M} \\
\text { Al before } \\
\text { sonication; } \\
0.42 \mathrm{M} \mathrm{Al} \\
\text { after } \\
\text { sonication). }\end{array}$ & - & - & - & - \\
\hline
\end{tabular}


Table A1-2. SSTs and DSTs Wash and Leach Information for Aluminum, Part 2. (27 sheets)

\begin{tabular}{|c|c|c|c|c|c|c|c|c|c|}
\hline$\underline{\text { Tank }}$ & $\begin{array}{l}\text { Lab Study } \\
\text { Reference(s) }\end{array}$ & Caustic Leach $\# 2$ Procedure & $\begin{array}{l}\text { Wash of solids after Caustic leach } \\
\text { steps }\end{array}$ & $\begin{array}{l}\text { Mass (ug) Al removed during 1st leach } \\
\text { step }\end{array}$ & $\begin{array}{l}\text { Mass (ug) } \\
\text { Al removed } \\
\text { during } 2 \text { nd } \\
\text { leach step } \\
\end{array}$ & $\begin{array}{l}\text { Mass (ug) Al } \\
\text { removed in } \\
\text { final wash } \\
\text { solution } \\
\end{array}$ & \begin{tabular}{|c|c|}
$\begin{array}{c}\text { Mass (ug) } \mathrm{Al} \\
\text { in Leached } \\
\text { Solids }\end{array}$ \\
\end{tabular} & \begin{tabular}{|c|} 
Total mass $\mathrm{Al}$ \\
(ug)
\end{tabular} & Comments \\
\hline $241-\mathrm{U}-110$ & PNNL-11779 & $\begin{array}{l}\text { Add } 23 \mathrm{~mL} 3.2 \mathrm{M} \mathrm{NaOH} \text { to } \\
\text { solids. Mix at } 100^{\circ} \mathrm{C} \text { for } 62 \mathrm{hr} \text {. } \\
\text { Cool. centrifuge, and decant. }\end{array}$ & $\begin{array}{l}\text { Wash solids } 3 \text { times using } 20 \mathrm{~mL} \\
0.1 \mathrm{M} \text { NaOH. Mix } 0.25 \mathrm{hr} \text { at RT, } \\
\text { centrifuge, decant, and repeat with } \\
\text { solids portion. All supernate from } \\
\text { washtleach mixed together for } \\
\text { analysis. }\end{array}$ & - & - & - & $\begin{array}{l}96 \% \text { Al } \\
\text { removed from } \\
\text { caustic leach + } \\
\text { wash. } \\
3 \% \text { removed } \\
\text { from first } \\
\text { Tiron leach + } \\
\text { wash. } \\
1 \% \text { removed } \\
\text { from second } \\
\text { Tiron leach. } \\
0.2 \% \text { removed } \\
\text { from third } \\
\text { Tiron leach + } \\
\text { wash. } \\
0 \% \text { in solid } \\
\text { residue. }\end{array}$ & - & 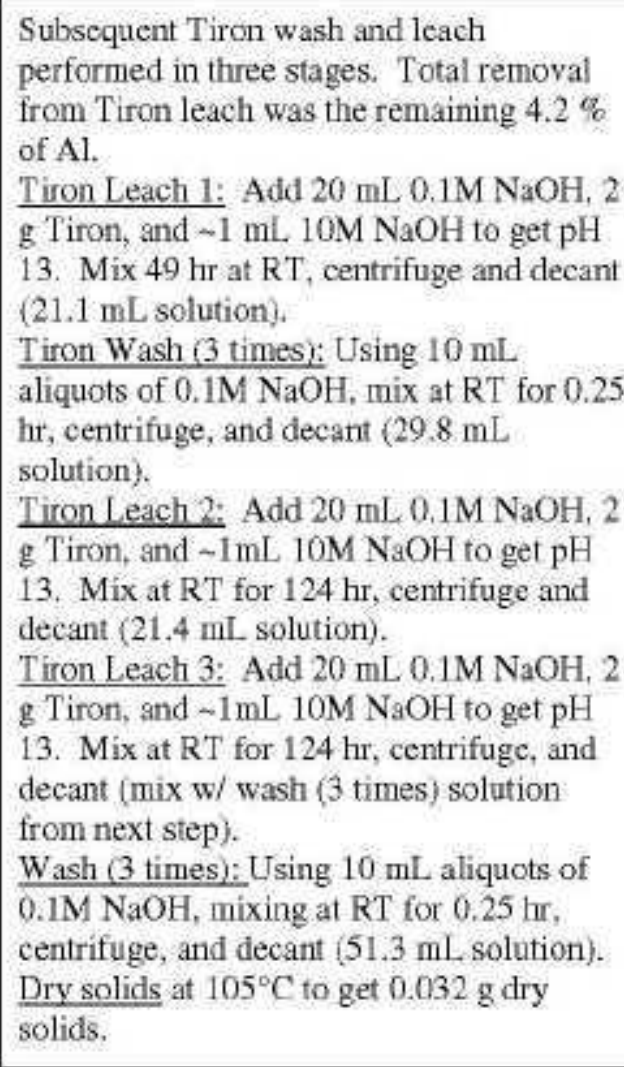 \\
\hline $\begin{array}{l}\text { Group } 1: \mathrm{BiPO}_{4} \\
\text { Sludge }(1 \mathrm{C} \text { and } \\
2 \mathrm{C} \text {. }\end{array}$ & $\begin{array}{l}\text { PNNL-17992 } \\
\text { (WTP-RPT- } \\
\text { 166) }\end{array}$ & - & $\begin{array}{l}\text { Three samples (G1-40-3a, }-3 \mathrm{~b} \text {, and - } \\
3 \mathrm{c} \text {, leached at } 40^{\circ} \mathrm{C} \text { and } 3 \mathrm{M} \mathrm{NaOH} \text { ) } \\
\text { analyzed. Slurry one of the samples } \\
\text { with } 15 \mathrm{~mL} 0.01 \mathrm{MNaOH} \text { and divide } \\
\text { between remaining two samples. } \\
\text { Rinse leaching bottle with } 10 \mathrm{~mL} \\
0.01 \mathrm{M} \mathrm{NaOH} \text { and split wash solution } \\
\text { between two samples. Mix solits on } \\
\text { shaker table for } 15 \text { min and centrifuge } \\
\text { for } 5 \text { min. Remove supernate. Wash } \\
\text { both again for a total of } 3 \text { washes. } \\
\text { Slurry remaining solids in }-2 \mathrm{~mL} \text {. DI } \\
\text { and sub-divide for analysis. }\end{array}$ & 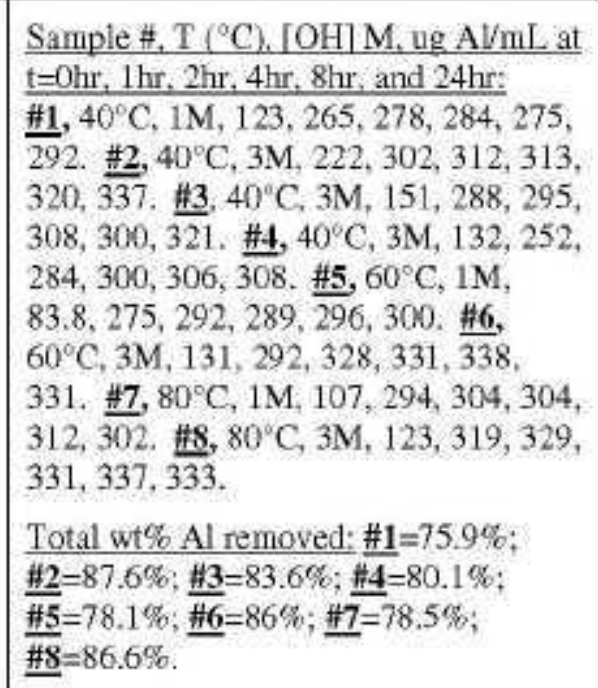 & - & $4.73 \mathrm{ug} / \mathrm{mL}$. & $-11500 \mathrm{ug} / \mathrm{g}$ & - & - \\
\hline
\end{tabular}


Table A1-2. SSTs and DSTs Wash and Leach Information for Aluminum, Part 2. (27 sheets)

\begin{tabular}{|c|c|c|c|c|c|c|c|c|c|}
\hline$\underline{\text { Tank }}$ & $\begin{array}{l}\text { Lab Study } \\
\text { Reference(s) }\end{array}$ & Caustic Leach $\# 2$ Procedure & $\begin{array}{l}\text { Wash of solids after Caustic leach } \\
\text { steps }\end{array}$ & $\begin{array}{l}\text { Mass (ug) Al removed during 1st leach } \\
\text { step }\end{array}$ & $\begin{array}{l}\text { Mass (ug) } \\
\text { Al removed } \\
\text { during 2nd } \\
\text { leach step } \\
\end{array}$ & $\begin{array}{l}\text { Mass (ug) Al } \\
\text { removed in } \\
\text { final wash } \\
\text { solution } \\
\end{array}$ & $\begin{array}{c}\text { Mass (ug) Al } \\
\text { in Leached } \\
\text { Solids }\end{array}$ & \begin{tabular}{|c|} 
Total mass $\mathrm{Al}$ \\
(ug)
\end{tabular} & Comments \\
\hline $\begin{array}{l}\text { Group 2: } \mathrm{BiPO}_{4} \\
\text { Saltcake (BY and T } \\
\text { saltcake). }\end{array}$ & $\begin{array}{l}\text { PNNL-17992 } \\
\text { (WTP-RPT- } \\
\text { 165) }\end{array}$ & - & $\begin{array}{l}\text { Three samples ( } \mathrm{G} 2-80-3 \mathrm{a},-3 \mathrm{~b} \text {, and - } \\
\left.3 \mathrm{c} \text {, leached at } 80^{\circ} \mathrm{C} \text { and } 3 \mathrm{M} \mathrm{NaOH}\right) \\
\text { analyzed. Slurry all of the solids into } \\
\text { one sample. Wash } 3 \text { times with } \\
0.01 \mathrm{M} \mathrm{NaOH} \text {. Centrifuge and decant } \\
\text { between washes. }\end{array}$ & 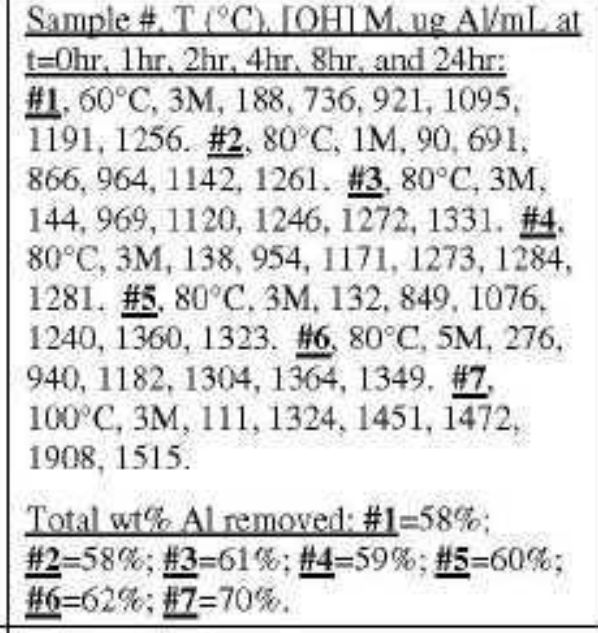 & - & $103.5 \mathrm{ug} / \mathrm{Ml}$ & $91450 \mathrm{ug} / \mathrm{g}$. & - & - \\
\hline $\begin{array}{l}\text { Group 3: PUREX } \\
\text { CW sludge. }\end{array}$ & $\begin{array}{l}\text { PNNL-18054 } \\
\text { (WTP-RPT- } \\
\text { 167) }\end{array}$ & 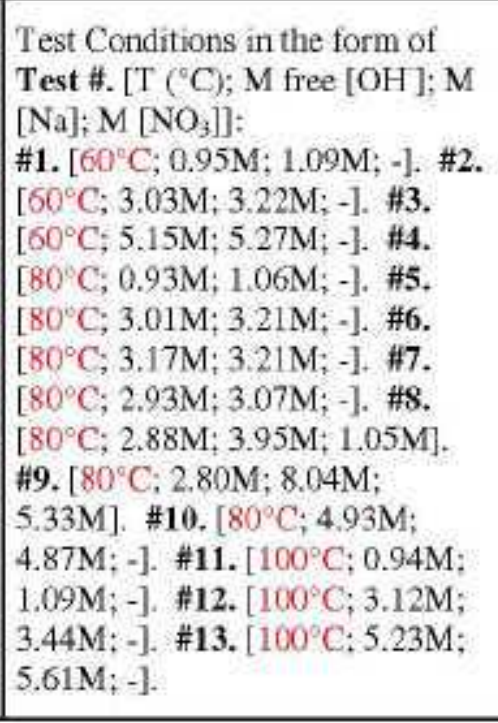 & $\begin{array}{l}\text { Three samples leached at } 80^{\circ} \mathrm{C} \text { in } 3 \mathrm{M} \\
\mathrm{NaOH} \text { were composited into two } \\
\text { samples using } 15 \mathrm{~mL} \text {. } 0.01 \mathrm{M} \mathrm{NaOH} \\
\text { and splitting between two remaining } \\
\text { samples. The two samples were then } \\
\text { washed with } 15 \mathrm{~mL} \text { aliquots of } 0.01 \mathrm{M} \\
\mathrm{NaOH} \text { three times, shaking for } 15 \\
\text { minutes prior to centrifuging and } \\
\text { decanting supernate. }\end{array}$ & 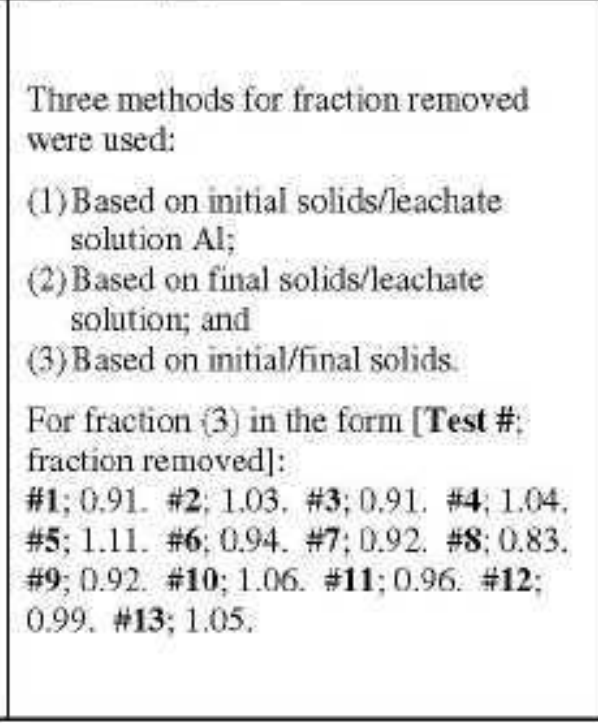 & - & $111.1 \mathrm{ug} / \mathrm{mL}$. & $\begin{array}{l}\text { Average } \mathrm{Al} \text { in } \\
\text { solids before } \\
\text { leaching = } \\
299500 \mathrm{ug} / \mathrm{g} \\
\text { Average } \mathrm{Al} \text { in } \\
\text { solids after } \\
\text { leaching }= \\
27100 \mathrm{ug} / \mathrm{g} \text {. }\end{array}$ & $\begin{array}{l}\text { Average initial } \\
\text { Al in solids = } \\
297500 \mathrm{ug} / \mathrm{g}\end{array}$ & 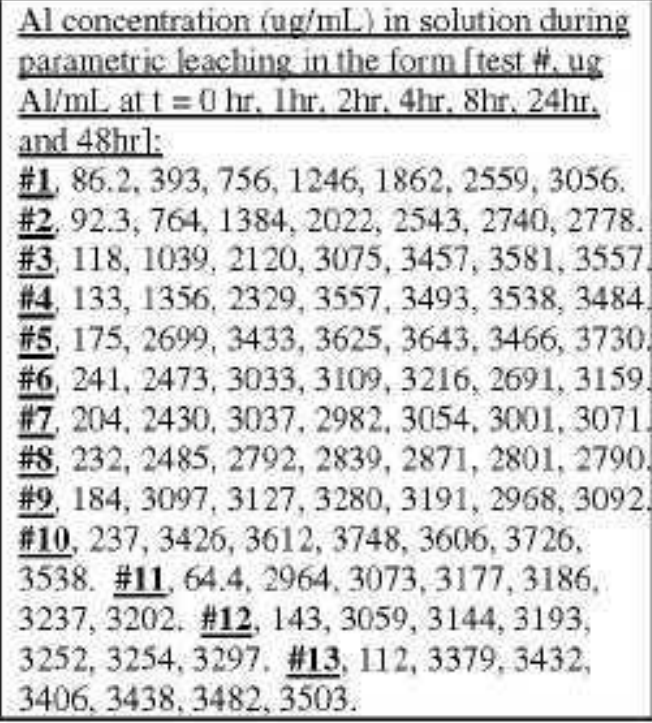 \\
\hline
\end{tabular}


Table A1-2. SSTs and DSTs Wash and Leach Information for Aluminum, Part 2. (27 sheets)

\begin{tabular}{|c|c|c|c|c|c|c|c|c|c|}
\hline$\underline{\text { Tank }}$ & $\begin{array}{l}\text { Lab Study } \\
\text { Reference(8) }\end{array}$ & Caustic Leach $\# 2$ Procedure & $\begin{array}{l}\text { Wash of solids after Caustic leach } \\
\text { steps }\end{array}$ & $\begin{array}{l}\text { Mass (ug) Al removed during 1st leach } \\
\text { step }\end{array}$ & \begin{tabular}{|c|} 
Mass (ug) \\
Al removed \\
during 2 nd \\
leach step \\
\end{tabular} & $\begin{array}{l}\text { Mass (ug) Al } \\
\text { removed in } \\
\text { final wash } \\
\text { solution } \\
\end{array}$ & $\begin{array}{c}\text { Mass (ug) Al } \\
\text { in Leached } \\
\text { Solids }\end{array}$ & $\begin{array}{l}\text { Total mass Al } \\
\text { (ug) }\end{array}$ & Comments \\
\hline $\begin{array}{l}\text { Group 4: REDOX } \\
\text { CW Sludge, }\end{array}$ & $\begin{array}{l}\text { PNNL-18054 } \\
\text { (WTP-RPT. } \\
\text { 167) }\end{array}$ & 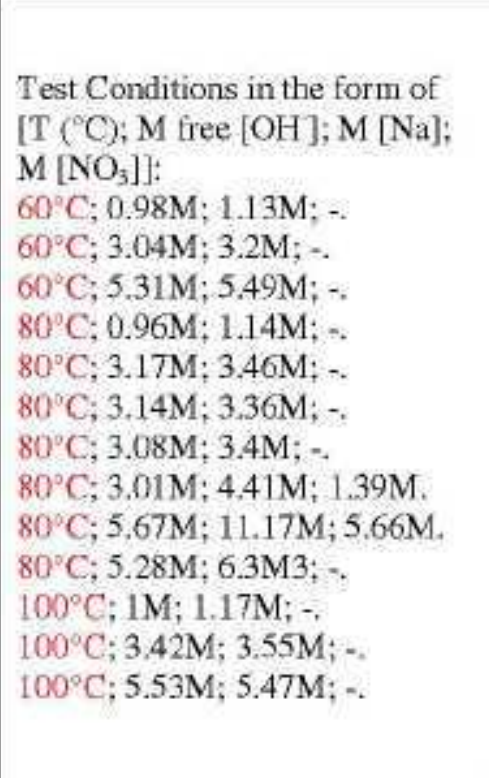 & $\begin{array}{l}\text { Three samples leached at } 80^{\circ} \mathrm{C} \text { in } 3 \mathrm{M} \\
\mathrm{NaOH} \text { were composited into two } \\
\text { samples using } 15 \mathrm{~mL} \text {. } 0.01 \mathrm{M} \mathrm{NaOH} \\
\text { and splitting between two remaining } \\
\text { samples. The two samples were then } \\
\text { washed with } 15 \mathrm{~mL} \text { aliquots of } 0.01 \mathrm{M} \\
\text { NaOH three times, shaking for } 15 \\
\text { minutes prior to centrifuging and } \\
\text { decanting supernate. }\end{array}$ & 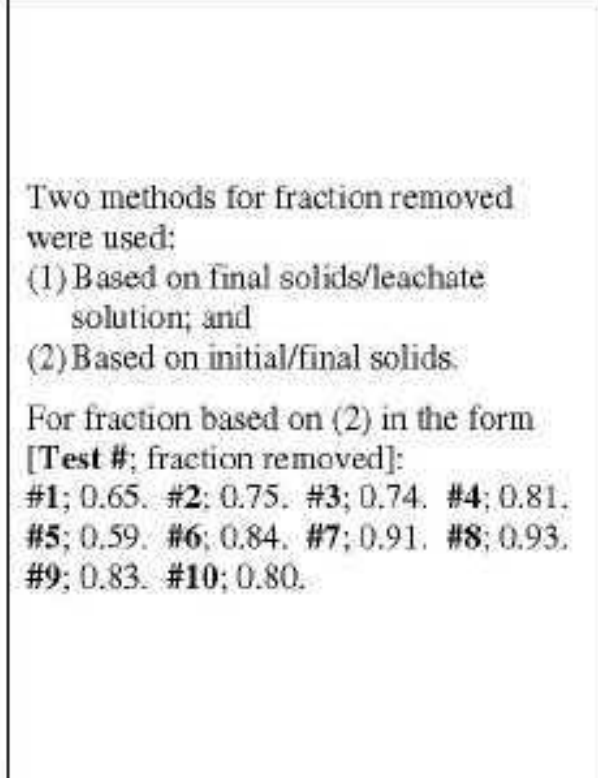 & - & $180.3 \mathrm{ug} / \mathrm{mL}$. & $\begin{array}{l}\text { Average } \mathrm{Al} \text { in } \\
\text { solids before } \\
\text { leaching }= \\
332500 \mathrm{ug} / \mathrm{g} \text {. } \\
\text { Average Al in } \\
\text { solids sfter } \\
\text { leaching }= \\
{[38000] \mathrm{ug} / \mathrm{g} \text {. }}\end{array}$ & $\begin{array}{l}\text { Average initial } \\
\text { Al in solids = } \\
296500 \mathrm{ug} / \mathrm{g} \text {. }\end{array}$ & 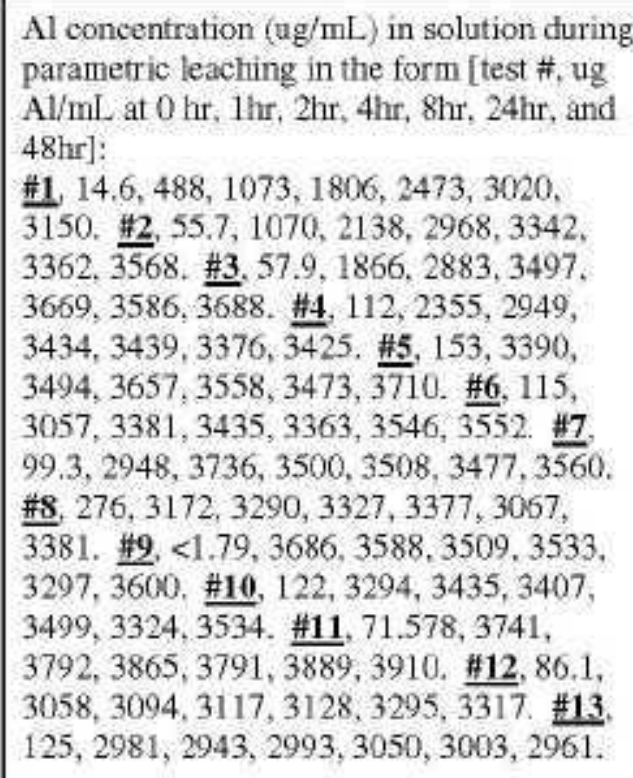 \\
\hline Group 5: REDOX. & $\begin{array}{l}\text { PNNL-17368 } \\
\text { (WTP-RPT. } \\
\text { 157) }\end{array}$ & 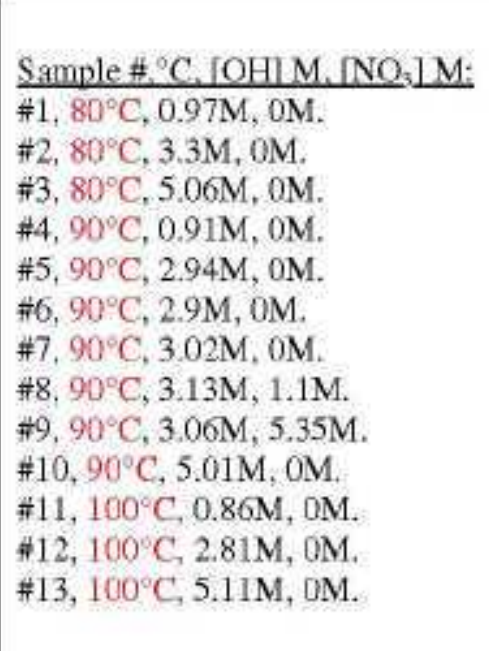 & $\begin{array}{l}\text { Three samples ( } \mathrm{G} 5-90-3 \mathrm{a},-3 \mathrm{~b} \text {, and - } \\
3 \mathrm{c} \text { ) leached at } 90^{\circ} \mathrm{C} \text { and } 3 \mathrm{M} \mathrm{NaOH} \\
\text { with } \sim 0.2 \mathrm{~mL} \text { centrifuged solids each. } \\
\text { Slurry one of the samples with } 0.01 \mathrm{M} \\
\mathrm{NaOH} \text { and divide between remaining } \\
\text { two samples. Wash both } 3 \text { times by } \\
\text { adding } ~ \\
\text { mixing in vortex mixer for } 5 \text { minutes. } \\
\text { Centrifuge slurries for } 5 \text { minutes and } \\
\text { remove supernate. Combine two } \\
\text { samples using additional } 0.01 \mathrm{M} \\
\mathrm{NaOH} \text {. Centrifuge and remove } \\
\text { aqueous phase. Slurry solids with } \sim 4 \\
\mathrm{~mL} \text { DI and sub-divide for analysis. }\end{array}$ & 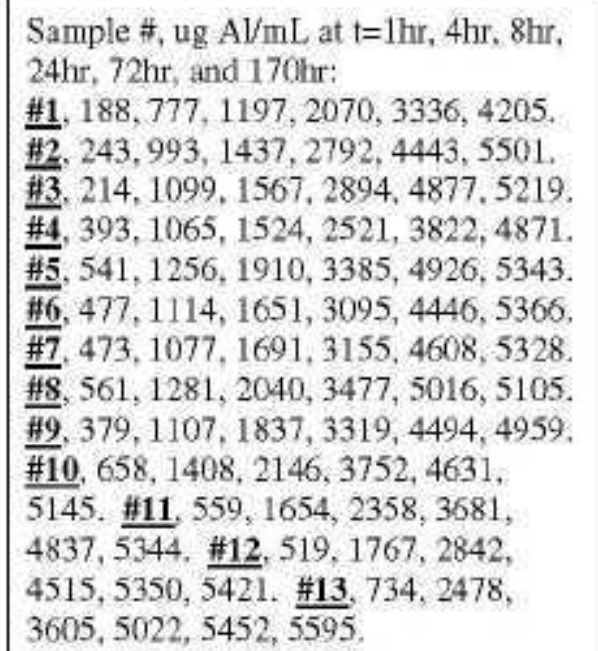 & - & - & $83675 \mathrm{ug} / \mathrm{g}$. & $\begin{array}{l}\text { Sample } \#= \\
\text { total wt } \% \\
\text { removed: } \\
\# 1=77 \% . \\
\# 2=101 \% . \\
\# 3=96 \% . \\
\# 4=89 \% . \\
\# 5=98 \% . \\
\# 6=98 \% . \\
\# 7=98 \% . \\
\# 8=94 \% . \\
\# 9=91 \% . \\
\# 10=94 \% . \\
\$ 11=98 \% . \\
\# 12=100 \% . \\
\# 13=103 \% .\end{array}$ & - \\
\hline
\end{tabular}


Table A1-2. SSTs and DSTs Wash and Leach Information for Aluminum, Part 2. (27 sheets)

\begin{tabular}{|c|c|c|c|c|c|c|c|c|c|}
\hline$\underline{\text { Tank }}$ & $\begin{array}{l}\text { Lab Study } \\
\text { Reference(s) }\end{array}$ & Caustic Leach $¥ 2$ Procedure & $\begin{array}{l}\text { Wash of solids after Caustic leach } \\
\text { steps }\end{array}$ & $\begin{array}{l}\text { Mass (ug) Al removed during 1st leach } \\
\text { step }\end{array}$ & $\begin{array}{l}\text { Mass (ug) } \\
\text { Al removed } \\
\text { during } 2 \text { nd } \\
\text { leach step } \\
\end{array}$ & $\begin{array}{l}\text { Mass (ug) Al } \\
\text { removed in } \\
\text { final wash } \\
\text { solution } \\
\end{array}$ & $\begin{array}{l}\text { Mass (ug) Al } \\
\text { in Leached } \\
\text { Solids }\end{array}$ & $\begin{array}{l}\text { Total mass Al } \\
\text { (ug) }\end{array}$ & Comments \\
\hline $\begin{array}{l}\text { Group 6: S- } \\
\text { Saltcake. }\end{array}$ & $\begin{array}{l}\text { PNNL-17368 } \\
\text { (WTP-RPT. } \\
\text { 157) }\end{array}$ & - & $\begin{array}{l}\text { Add } 50 \mathrm{~mL} 0.01 \mathrm{M} \mathrm{NaOH} \text { to leached } \\
\text { centrifuged solids. Mix for } 5 \text { min by } \\
\text { shaking. Centrifuge at } \sim 610 \mathrm{G} \text { for } 15 \\
\text { min and remove supernate. Repeat for } \\
\text { a total of three washes. Note: } \\
\text { Oxidative leaching performed on } \\
\text { final caustic leached solids. This } \\
\text { information is not included here. } \\
\text { They performed } 17 \text { tests analyzing } \\
\text { the effects of temperature, M }[\mathrm{OH}] \text {, } \\
\text { and } \mathrm{Mn} / \mathrm{Cr} \text { mole ratio on leaching } \\
\text { of Cr. }\end{array}$ & - & - & - & $\begin{array}{l}\sim 20 \mathrm{~mL} \\
113500 \mathrm{ug} / \mathrm{g}\end{array}$ & $\begin{array}{l}\text { Total of 59\% Al } \\
\text { leached from } \\
\text { the solids by } \\
\text { caustic leaching } \\
\text { (with } 46 \% \\
\text { removed due to } \\
\text { oxidative } \\
\text { leaching). }\end{array}$ & - \\
\hline $\begin{array}{l}\text { Group 7: TBP } \\
\text { Waste Sludge. }\end{array}$ & $\begin{array}{l}\text { PNNL-18119 } \\
\text { (WTP-RPT- } \\
\text { 169) }\end{array}$ & $\begin{array}{l}\text { Sample } \#, T\left({ }^{\circ} \mathrm{C}\right),[\mathrm{OH}] \mathrm{M}: \\
\# 1,40^{\circ} \mathrm{C}, 1.02 \mathrm{M} \text {. } \\
\# 2,40^{\circ} \mathrm{C}, 3.12 \mathrm{M} \text {. } \\
\# 3,40^{\circ} \mathrm{C}, 3 \mathrm{M} \text {. } \\
\# 4,40^{\circ} \mathrm{C}, 3.19 \mathrm{M} \text {. } \\
\# 5,60^{\circ} \mathrm{C}, 0.24 \mathrm{M} \text {. } \\
\# 6,60^{\circ} \mathrm{C}, 1.01 \mathrm{M} \text {. } \\
\# 7,60^{\circ} \mathrm{C}, 3.02 \mathrm{M} \text {. } \\
\# 8,80^{\circ} \mathrm{C}, 0.25 \mathrm{M} \text {. } \\
\#, 80^{\circ} \mathrm{C}, 1.05 \mathrm{M} \text {. } \\
\# 10,80^{\circ} \mathrm{C}, 3.05 \mathrm{M} .\end{array}$ & $\begin{array}{l}\text { Slurries were removed from heat and } \\
\text { cooled to ambient temperature. They } \\
\text { were then centrifuged and decanted. } \\
\text { Triplicate solids (samples } \# 2,3 \text {, and } \\
4 \text { ) were used for wash study after } \\
\text { caustic leach. One of the samples was } \\
\text { slurried in } \sim 15 \mathrm{~mL} 0.01 \mathrm{MNaOH} \text { and } \\
\text { divided between the other two } \\
\text { samples. Solids werc mixed for } 15 \\
\text { minn, centrifuged for } 5 \text { min, and } \\
\text { decanted. A total of } 3 \text { washes were } \\
\text { performed using this technique. } \\
\text { Additional } 0.01 \mathrm{M} \mathrm{NaOH} \text { was used to } \\
\text { transfer solids from one bottle to } \\
\text { combine all solids in one bottle. This } \\
\text { was centrifuged for } 5 \text { min and } \\
\text { decanted. }\end{array}$ & - & - & $\begin{array}{l}\text { Wash solution } \\
\text { Al } \\
\text { composition = } \\
5.45 \text { ug/mL. }\end{array}$ & $\begin{array}{l}\text { Average } \mathrm{Al} \text { in } \\
\text { solids before } \\
\text { leaching }= \\
15100 \text { ug/g. } \\
\text { Average Al in } \\
\text { solids after } \\
\text { leaching }= \\
{[6550] \text { ug/g. }}\end{array}$ & $\begin{array}{l}\text { Average initial } \\
\text { Al in solids = } \\
16000 \mathrm{ug} / \mathrm{g} \text {. }\end{array}$ & 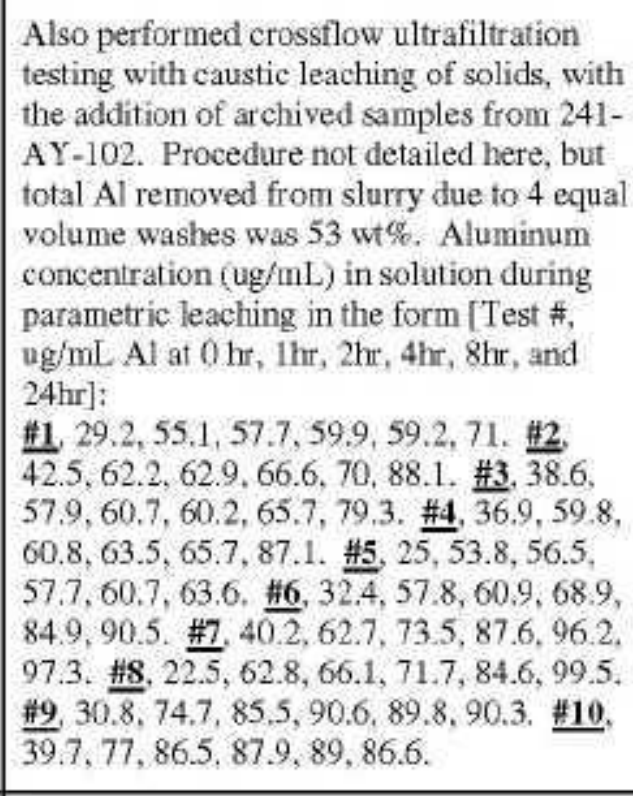 \\
\hline $\begin{array}{l}\text { Group 8: FeCN } \\
\text { Waste Sludge. }\end{array}$ & $\begin{array}{l}\text { PNNL-18120 } \\
\text { (WTP-RPT- } \\
\text { 170) }\end{array}$ & - & $\begin{array}{l}\text { Wash } 4 \text { times after dewatering leached } \\
\text { slurry. Concentration of } \mathrm{NaOH} \text { added } \\
\text { for wash } \# 1 \text { was } 0.47 \mathrm{M} \mathrm{NaOH} \text {, wash } \\
\# 2 \text { was } 0.16 \mathrm{M} \mathrm{NaOH} \text {, wash } \# 3 \text { was } \\
0.049 \mathrm{M} \mathrm{NaOH} \text {, and wash } \# 4 \text { was } \\
0.014 \mathrm{M} \mathrm{NaOH} \text {, with a total wolume of } \\
1.2 \mathrm{~L} \text { wash solution and mass of } 1.2 \\
\mathrm{~kg} \text {. }\end{array}$ & $\begin{array}{l}\text { Concentration of } \mathrm{Al} \text { (ug } / \mathrm{mL} \text { ) in } \\
\text { supernate calculated based on mass } \\
\text { balance was } 697 \text { ug } \mathrm{Al} / \mathrm{mL} \text {. At }-1.5 \mathrm{hr} \\
\text { (during heat-up after addition of } \\
\text { caustic; } 40^{\circ} \mathrm{C} \text { ) had } 4580 \mathrm{ug} \mathrm{Al} / \mathrm{mL} \text {. At } \\
\text { times of } 0,2,4, \text { and } 8 \mathrm{hr} \text { (during mix } \\
\text { after reaching leaching temperature of } \\
60^{\circ} \mathrm{C} \text { ), had } 4600,4520,4600 \text {, and } 4650 \\
\text { ug } \mathrm{A} / \mathrm{mL} \text { respectively. The final } \mathrm{Al} \\
\text { concentration (after cool down to } 25^{\circ} \mathrm{C} \text { ) } \\
\text { was } 4570 \text { ug Al/mL. Total of } 60 \mathrm{wt} \% \\
\text { Al removed. }\end{array}$ & - & - & - & $\begin{array}{l}\text { Initial } \\
\text { Composition: } \\
\text { Supernate had } \\
1430 \text { ug/mL Al, } \\
\text { Washed Solids } \\
\text { had } 89650 \text { ug/g } \\
\text { Al. } \\
\text { Final } \\
\text { composition of } \\
\text { leached and } \\
\text { washed solids } \\
\text { had } 50000 \text { ug/g } \\
\text { Al. }\end{array}$ & - \\
\hline
\end{tabular}


Table A1-2. SSTs and DSTs Wash and Leach Information for Aluminum, Part 2. (27 sheets)

\begin{tabular}{|c|c|c|c|c|c|c|c|c|c|}
\hline$\underline{\text { Tank }}$ & $\begin{array}{c}\text { Lab Study } \\
\text { Reference (s) }\end{array}$ & Caustic Leach \#2 Procedure & $\begin{array}{l}\text { Wash of solids after Caustic leach } \\
\text { steps }\end{array}$ & $\begin{array}{l}\text { Mass (ug) Al removed during 1st leach } \\
\text { step }\end{array}$ & \begin{tabular}{|l} 
Mass (ug) \\
Al removed \\
during 2nd \\
leach step \\
\end{tabular} & $\begin{array}{l}\text { Mass (ug) Al } \\
\text { removed in } \\
\text { final wash } \\
\text { solution } \\
\end{array}$ & $\begin{array}{c}\text { Mass (ug) } \mathrm{Al} \\
\text { in Leached } \\
\text { Solids } \\
\end{array}$ & $\begin{array}{l}\text { Total mass Al } \\
\text { (ug) }\end{array}$ & Comments \\
\hline $\begin{array}{l}\text { Mixture of Group } 5 \\
\text { and Group } 6 \text { waste. } \\
31.6 \text { wt } \% \text { Group } 5 \\
\text { Slurry }+16.8 \text { wt } \$ \\
\text { Group } 6 \text { slurry }+ \\
51.6 \text { wt } \% \text { Group } 6 \\
\text { supernatant }\end{array}$ & $\begin{array}{l}\text { PNNL-18007 } \\
\text { (WTP-RPT. } \\
\text { 171) }\end{array}$ & - & $\begin{array}{l}\text { After decanting leachate }(81.7 \mathrm{~g} \text { ), the } \\
\text { solids were washed six times with } 40 \\
\mathrm{~mL} 0.01 \mathrm{M} \mathrm{NaOH} \text {. Wash solution was } \\
\text { added to the solids and mixed for } 15 \\
\text { min, centrifuged for } 10 \text { min, and } \\
\text { decanted. }\end{array}$ & $\begin{array}{l}\text { Caustic leach solution }=15180 \mathrm{ug} \\
\mathrm{A} / \mathrm{mL} \text {. }\end{array}$ & - & $\begin{array}{l}\text { Combined } \\
\text { wash solution } \\
=2540 \text { ug } \\
\text { Al/mL. } \\
\text { Final contact } \\
\text { solution }=280 \\
\text { ug Al/mL. }\end{array}$ & $\begin{array}{l}\text { Caustic } \\
\text { leached and } \\
\text { washed solids } \\
=288000 \mathrm{ug} \\
\mathrm{Al} / \mathrm{g} \text {. }\end{array}$ & $38 \%$ leached. & - \\
\hline $\begin{array}{l}\text { Mixture of Group } 5 \\
\text { and Group } 6 \text { waste. } \\
31.6 \text { wt } \% \text { Group } 5 \\
\text { Slurry }+16.8 \text { wt } \% \\
\text { Group } 6 \text { slurry }+ \\
51.6 \text { wt } \% \text { Group } 6 \\
\text { supernatant }\end{array}$ & $\begin{array}{l}\text { PNNL-17965 } \\
\text { (WTP-RPT- } \\
172 \text { ) }\end{array}$ & - & $\begin{array}{l}\text { The solids from sample } \$ 2 \text { was } \\
\text { washed } 3 \text { times by adding } 15 \mathrm{~mL} \\
0.01 \mathrm{M} \mathrm{NaOH} \text {, mixed on a shaker table } \\
\text { for } 15 \mathrm{~min} \text {, centrifuged } 5 \text { min, and } \\
\text { decanted. The solids after the third } \\
\text { wash were slurried in } ~ 2 \mathrm{~mL} \text { DI for } \\
\text { analysis. }\end{array}$ & $\begin{array}{l}\text { Initial } \mathrm{Al} \text { in sample was } 0.61 \mathrm{~g} \text {. Total } \\
\text { dissolution of } \mathrm{Al} \text { in samples were: } \\
\# 1=0.4 \mathrm{~g} \text {. } \\
\# 2=0.42 \mathrm{~g} \text {, and } \\
\# 4=0.59 \mathrm{~g} \mathrm{Al} \text { removed. }\end{array}$ & - & $164.1 \mathrm{ug} / \mathrm{mL}$. & $\begin{array}{l}\text { Sample \#2: } \\
\text { Average Al } \\
\text { before } \\
\text { leaching = } \\
612,393 \text { ug. } \\
\text { Average Al } \\
\text { after leaching } \\
24 \mathrm{hr}= \\
193,907 \text { ug. }\end{array}$ & $\begin{array}{l}\text { Total wt } \% \text { Al } \\
\text { removed: } \\
\text { Sample } \$ 1=65 \\
\text { wt } \% ; \\
\# 2=68 \text { wt } \% \\
\text { and } \\
\# 4=97 \mathrm{wt} \% \text {. }\end{array}$ & 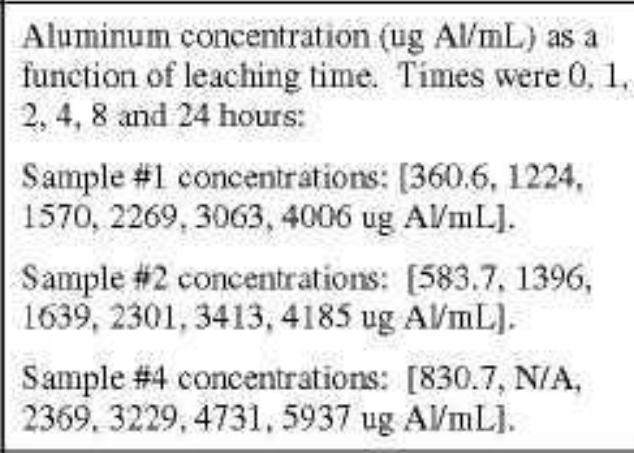 \\
\hline $\begin{array}{l}\text { Mixture of Group } 3 \\
\text { and Group } 4 \\
\text { composites }\end{array}$ & $\begin{array}{l}\text { PNNL-18048 } \\
\text { (WTP-RPT- } \\
\text { 181) }\end{array}$ & - & $\begin{array}{l}\text { After caustic leach and cool down, } \\
\text { slurry was dewatered, removing }-1.9 \mathrm{~L} \\
\text { slurry supemate. } \\
\text { After fittration testing (leach and } \\
\text { dewatering), the slurry was washed } 3 \\
\text { times with } 1.2 \mathrm{~L} \text { caustic at } \\
\text { concentrations of } 1.78 \mathrm{M} \mathrm{NaOH} \text {, } \\
0.78 \mathrm{M} \mathrm{NaOH} \text {, and } 0.3 \mathrm{M} \mathrm{NaOH} \text {. It } \\
\text { was re-circulated in the filter for } ~ 30 \\
\text { min and then dewatered after each } \\
\text { wash. Final dewatering was stopped } \\
\text { at } 1 \mathrm{~L} \text { due to cavitation. }\end{array}$ & $\begin{array}{l}\text { By the end of the heat ramp to } 100^{\circ} \mathrm{C} \text {, } \\
94 \% \text { of the Aluminum had been } \\
\text { leached into solution. } \\
\text { Aluminum concentration in the } \\
\text { aqueous phase at start of heat up }\left(32^{\circ} \mathrm{C}\right) \\
=650 \text { ug } / \mathrm{mL} \text {. } \\
\text { At } 1 \mathrm{hr} \text { heat up }\left(38^{\circ} \mathrm{C}\right)=7800 \mathrm{ug} / \mathrm{mL} \text {, } \\
\text { At } 3 \mathrm{hr} \text { heat up }\left(7^{\circ} \mathrm{C}\right)=24000 \mathrm{ug} / \mathrm{mL} \text {. } \\
\text { At } 0 \mathrm{hr} \text { leach }\left(90^{\circ} \mathrm{C}\right)=25000 \mathrm{ug} / \mathrm{mL} \text {. } \\
\text { At } 4 \mathrm{hr} \text { leach }\left(93^{\circ} \mathrm{C}\right)=25000 \mathrm{ug} / \mathrm{mL} \text {. } \\
\text { At } 8 \mathrm{hr} \text { leach }\left(95^{\circ} \mathrm{C}\right)=25000 \mathrm{ug} / \mathrm{mL} \text {. }\end{array}$ & - & - & $\begin{array}{l}\text { Al in caustic } \\
\text { leached, } \\
\text { dewatered } \\
\text { slurry was } 40 \\
\text { g. } 38 \mathrm{~g} \text { were } \\
\text { in the liquid, } \\
\text { with } 1.7 \mathrm{~g} \text { in } \\
\text { the solids } \\
\text { fraction. }\end{array}$ & $\begin{array}{l}\text { Pre-leached } \\
\text { solids were } 98 \\
\text { wt\% Al. } \\
\text { Caustic } \\
\text { leaching } \\
\text { removed } 94 \\
\text { wt\% Al. After } \\
\text { washing } 75 \\
\text { wt\% Al was } \\
\text { removed. }\end{array}$ & - \\
\hline
\end{tabular}


RPP-RPT-46791

Revision 0

\section{A2.0 SINGLE-AND DOUBLE-SHELL TANKS WITHOUT LABORATORY WASH AND LEACH INFORMATION}

A total of 108 SSTs and 23 DSTs were found to have no information concerning wash and leach of aluminum from tank solids. Information was available concerning the waste types found in these tanks. as well as two papers which had information on the potential or known aluminum solid species found in the tank sludge. This information is provided in Table A2-1 below.

Table A2-1. SSTs and DSTs with no wash and leach information available for Aluminum. (8 sheets)

\begin{tabular}{|c|c|}
\hline Tank & Waste Sources \\
\hline \multicolumn{2}{|l|}{ DSTs } \\
\hline 241-AN-101 & $\begin{array}{l}\text { Dilute Non-Complexed Waste. } \\
\text { A1-SltCk (liquid and solid) and NA (liquid and solid). }{ }^{2}\end{array}$ \\
\hline 241-AN-102 & $\begin{array}{l}\text { CC. } \\
\text { A2-SitSlr (solid) and NA (liquid), } \\
\text { Potential Al solid species in washed solids: boehmite, Zeolite, Na aluminate, diaspore. }\end{array}$ \\
\hline 241-AN-103 & $\begin{array}{l}\text { Double Shell Slurry. } \\
\text { A2-SitSlr (liquid and solid). }\end{array}$ \\
\hline $241-\mathrm{AN}-105$ & $\begin{array}{l}\text { Double Shell Slurry Feed. } \\
\text { A2-SltSlr (liquid and solid), }\end{array}$ \\
\hline 241-AN-106 & $\begin{array}{l}\text { Concentrated Phosphate Waste. } \\
\text { NA (liquid, sludge, and SltCk). }\end{array}$ \\
\hline 241-AN-107 & $\begin{array}{l}\text { CC. }{ }^{1} \\
\text { A2-SitSlr (liquid and solid). }\end{array}$ \\
\hline 241-AP-101 & $\begin{array}{l}\text { Dilute Non-Complexed Waste. } \\
\text { NA (liquid). }\end{array}$ \\
\hline 241-AP-102 & $\begin{array}{l}\text { Dilute Non-Complexed Waste. } \\
\text { NA (liquid and sludge). }\end{array}$ \\
\hline 241-AP-103 & $\begin{array}{l}\text { Dilute Non-Complexed Waste. } \\
\text { NA (liquid and SltCk). }\end{array}$ \\
\hline 241-AP-104 & $\begin{array}{l}\text { Dilute Non-Complexed Waste. } \\
\text { A2-SltS1r (solid) and NA (liquid). }\end{array}$ \\
\hline 241-AP-105 & $\begin{array}{l}\text { Double Shell Slurry Feed. } \\
\text { A2-SltSlr (solid) and NA (liquid). }\end{array}$ \\
\hline 241-AP-106 & $\begin{array}{l}\text { Dilute Non-Complexed Waste.' } \\
\text { NA (liquid). }\end{array}$ \\
\hline 241-AP-107 & $\begin{array}{l}\text { Dilute Non-Complexed Waste. } \\
\text { NA (liquid). }\end{array}$ \\
\hline 241-AP-108 & $\begin{array}{l}\text { Dilute Non-Complexed Waste. } \\
\text { NA (liquid and SltCk). }\end{array}$ \\
\hline 241-AW-101 & $\begin{array}{l}\text { Double Shell Slurry Feed. } \\
\text { A2-SltSlr (liquid and solid). }\end{array}$ \\
\hline $241-\mathrm{AW}-102$ & $\begin{array}{l}\text { Dilute Non-Complexed Waste. } \\
\text { NA (liquid and sludge). }\end{array}$ \\
\hline 241-AW-103 & $\begin{array}{l}\text { PUREX Neutralized Cladding Removal Waste (TRU). } \\
\text { Al-SltCk (liquid and solid). CWZr2 (solid), and NA (liquid). }\end{array}$ \\
\hline 241-AW-104 & $\begin{array}{l}\text { Dilute Non-Complexed Waste. } \\
\text { A2-Silslr (liquid and solid), PL2 (liquid and solid), and NA (liquid). }\end{array}$ \\
\hline
\end{tabular}


RPP-RPT-46791

Revision 0

Table A2-1. SSTs and DSTs with no wash and leach information available for Aluminum. ( 8 sheets)

\begin{tabular}{|c|c|}
\hline Tank & Waste Sources \\
\hline 241-AW-105 & $\begin{array}{l}\text { DN/PD. }{ }^{1} \\
\text { CWZ } 2 \text { (solid), PL2 (solid), and NA (liquid). } \\
\text { Aluminum containing phases identified in sludge: gibbsite and crystalline AISi in as-received; } \\
\quad \text { crystalline AISi in leached sludge (determined by TEM/SEM/XRD). }\end{array}$ \\
\hline 241-AW-106 & $\begin{array}{l}\text { Dilute Non-Complexed Waste. } \\
\text { A2-SltSlr (solid) and NA (liquid). }\end{array}$ \\
\hline 241-AY-101 & $\begin{array}{l}\text { Dilute Complexed Waste, } \\
\text { NA (liquid and sludge), NA Lower (liquid), and NA Upper (liquid). }\end{array}$ \\
\hline $241-\mathrm{AY}-102$ & $\begin{array}{l}\text { Dilute Non-Complexed Waste, } \\
\text { BL (liquid and solid) and NA (liquid and sludge). }\end{array}$ \\
\hline 241-SY-101 & $\begin{array}{l}\mathrm{CC}{ }^{\mathrm{I}} \\
\mathrm{S} 2-\mathrm{SitSir} \text { (solid) and NA (liquid). } \\
\text { Aluminum containing phases identified in sludge: } \mathrm{NaAlO}_{2} \text { and amorphous gibbsite in as- } \\
\text { received; none in leached sludge (determined by TEM/SEM/XRD). }\end{array}$ \\
\hline \multicolumn{2}{|l|}{ SSTs } \\
\hline 241-A-101 & $\begin{array}{l}\text { Double Shell Slurry Feed. } \\
\text { A1-SitCk (liquid and solid) and P2 (solid). }{ }^{2} \\
\text { Primary: double-shell slurry feed. Secondary; non-complexed waste. Tertiary; evaporator feed. }\end{array}$ \\
\hline 241-A-102 & $\begin{array}{l}\text { Double Shell Slurry Feed. } \\
\text { Al-SltCk (liquid and solid). } \\
\text { Primary: double-shell slurry feed, Secondary: non-complexed waste. Tertiary: evaporator feed, }\end{array}$ \\
\hline 241-A-103 & $\begin{array}{l}\text { Double Shell Slurry Feed. } \\
\text { Al-SltCk (liquid and solid) and AR (solid) }{ }^{2} \\
\text { Primary: double-shell slurry feed. Secondary: non-complexed waste. Tertiary: evaporator feed. }\end{array}$ \\
\hline 241-A-104 & $\begin{array}{l}\text { Non-Complexed Waste. } \\
\text { AR (solid) and P1 (solid). } \\
\text { Primary: SLUTCE. Secondary: neutralized acid waste. Tertiary: } \mathrm{H}_{2} \mathrm{O} \text {. Other: high-level waste } \\
\quad \text { from waste fractionization process at B plant starting in } 1967^{5}\end{array}$ \\
\hline 241-A-105 & $\begin{array}{l}\text { Non-Complexed Waste. } \\
\text { P2 (solid), } \\
\text { Primary: neutralized acid waste. Secondary: } \mathrm{IX}^{5}\end{array}$ \\
\hline 241-A-106 & $\begin{array}{l}\text { Double Shell Slurry Feed. } \\
\text { A1-SltCk (solid), AR (solid), and SRR (solid). }{ }^{2} \\
\text { Primary: CC. Secondary: non-complexed waste. Tertiary: evaporator feed. Other: high-level } \\
\text { waste from waste fractionization process at B plant starting in } 1967 \text {. }\end{array}$ \\
\hline 241-AX-101 & $\begin{array}{l}\text { Double Shell Slurry Feed. } \\
\text { Al-SitCk (liquid and solid) and SRR (solid). } \\
\text { Primary: double-shell slurry feed. Secondary: non-complexed waste. Tertiary; evaporator feed, }\end{array}$ \\
\hline 241-AX-102 & $\begin{array}{l}\text { Complexant Concentrate Waste, } \\
\text { A1-SltCk (solid) and B (solid), } \\
\text { Primary: CC. Secondary: double-shell shurry feed. Tertiary: evaporator feed. }\end{array}$ \\
\hline 241-AX-103 & $\begin{array}{l}\text { Complexant Concentrate Waste. } \\
\text { A1-SltCk (liquid and solid) and P2 (solid). }{ }^{2} \\
\text { Primary; CC. Secondary; double-shell slurry feed. Tertiary: evaporator feed. }\end{array}$ \\
\hline $241-\mathrm{AX}-104$ & $\begin{array}{l}\text { Non-Complexed Waste. } \\
\text { P2 (solid), } \\
\text { Primary: evaporator feed. Secondary: non-complexed waste. Tertiary: neutralized acid waste. }{ }^{5}\end{array}$ \\
\hline 241-B-102 & $\begin{array}{l}\text { Non-Complexed Waste. } \\
\text { B-SitCk (liquid and solid). } \\
\text { Primary: CW. Secondary: EB. Tertiary: B Plant low-level waste. Other: IX waste. }\end{array}$ \\
\hline
\end{tabular}


RPP-RPT-46791

Revision 0

Table A2-1. SSTs and DSTs with no wash and leach information available for Aluminum. (8 sheets)

\begin{tabular}{|c|c|}
\hline Tank & Waste Sources \\
\hline $241-B-103$ & $\begin{array}{l}\text { Non-Complexed Waste. } \\
\text { B-SltCk (liquid and solid) and MW (solid), } \\
\text { Primary: CW. Secondary: EB. Tertiary: IX waste. Other: mixture of several miscellaneous } \\
\text { wastes. }\end{array}$ \\
\hline 241-B-105 & $\begin{array}{l}\text { Non-Complexed Waste, } \\
\text { B-SitCk (liquid and solid), } 1 \mathrm{C} \text { (solid), and } 2 \mathrm{C} \text { (solid). } \\
\text { Primary: EB. Secondary: } 1 \mathrm{C}^{5}\end{array}$ \\
\hline $241-B-107$ & $\begin{array}{l}\text { Non-Complexed Waste. } \\
\text { B-SitCk (liquid and solid), 1C (solid), and CWP2 (solid). }{ }^{2} \\
\text { Primary: } 1 \text { C. Secondary: EB. Tertiary: CW. Other: TBP. }\end{array}$ \\
\hline 241-B-108 & $\begin{array}{l}\text { Non-Complexed Waste. } \\
\text { B-SitCk (liquid and solid) and CWP2 (solid). } \\
\text { Primary: 1C. Secondary: EB. Tertiary: CW. Other: LX/TBP. }\end{array}$ \\
\hline 241-B-109 & $\begin{array}{l}\text { Non-Complexed Waste. } \\
\text { B-SltCk (liquid and solid) and CWP2 (solid), } \\
\text { Primary: 1C. Secondary: EB. Tertiary: CW. Other: IX }\end{array}$ \\
\hline $241-B-112$ & $\begin{array}{l}\text { BY-SltCk (liquid and solid) and } 2 \mathrm{C} \text { (solid). } \\
\text { Primary: } 2 \mathrm{C} \text {. Secondary: high-level B Plant waste. Tertiary: Fission Products Waste. Other: } \\
\text { EB/in-tank solidification. }\end{array}$ \\
\hline 241-B-203 & $\begin{array}{l}224-2 \text { (solid) and NA (liquid) }{ }^{2} \\
\text { Primary: lanthanum fluoride decontamination waste. }\end{array}$ \\
\hline 241-B-204 & $\begin{array}{l}224-2 \text { (solid) and NA (liquid). } \\
\text { Primary: lanthanum fluoride decontamination waste. }\end{array}$ \\
\hline 241-BX-101 & $\begin{array}{l}\text { Non-Complexed Waste. } \\
\text { BL (solid), CWP2 (solid), and TBP (solid), }{ }^{2} \\
\text { Primary: TBP. Secondary; CW. Tertiary; B plant low level waste, Other: IX. }\end{array}$ \\
\hline $241-B X-102$ & $\begin{array}{l}\text { Non-Complexed Waste. } \\
\text { CWP2 (solid), DE (solid), and TBP (solid). } \\
\text { Primary; TBP. Secondary: CW. Tertiary: B plant low level waste. Other: diatomaceous earth. }\end{array}$ \\
\hline 241-BX-104 & $\begin{array}{l}\text { Non-Complexed Waste, } \\
\text { CWP2 (solid), CWR1 (liquid and solid), MW1 (solid), and TBP (solid). }{ }^{2} \\
\text { Primary: TBP. Secondary: CW. Tertiary: IX. Other: high-level REDOX waste. }\end{array}$ \\
\hline 241-BX-106 & $\begin{array}{l}\text { Non-Complexed Waste. } \\
\text { BY-SltCk (liquid and solid), CWP2 (solid), and TBP (solid). } \\
\text { Primary: TBP. Secondary: CW. Tertiary: EB/LX. Other: B plant low level waste. }\end{array}$ \\
\hline 241-BX-108 & $\begin{array}{l}\text { IC (solid) and TBP (solid) }{ }^{2} \\
\text { Primary: TBE. Secondary: } \mathrm{CW} \text {. Tertiary: IC. Other: } \mathrm{IX}^{5}\end{array}$ \\
\hline $241-B X-111$ & $\begin{array}{l}\text { Non-Complexed Waste. } \\
\text { BY-SltCk (liquid and solid) and } 1 \mathrm{C} \text { (solid) }{ }^{2} \\
\text { Primary: } 1 \mathrm{C} \text {. Secondary; EB/in-tank solidification. Tertiary: CW. Other: } \mathrm{IX}^{\mathrm{S}}\end{array}$ \\
\hline 241-BY-101 & $\begin{array}{l}\text { Non-Complexed Waste. } \\
\text { BY-SltCk (liquid and solid) and PFeCN (solid), }{ }^{2} \\
\text { Primary: TBP/FeCN-scavenged waste. Secondary: } \mathrm{EB} / \text { in-tank solidification. Tertiary: } \mathrm{CW} \text {. } \\
\quad \text { Other; } 1 \mathrm{C}^{5}\end{array}$ \\
\hline 241-BY-102 & $\begin{array}{l}\text { Non-Complexed Waste. } \\
\text { BY-SltCk (liquid and solid). } \\
\text { Primary; TBP. Secondary: EB/in-tank solidification. Tertiary; CW. Other: } 1 \mathrm{C}^{5}\end{array}$ \\
\hline $241-B Y-103$ & $\begin{array}{l}\text { Non-Complexed Waste. } \\
\text { BY-SltCk (liquid and solid) and CWP2 (solid). } \\
\text { Primary: TBP/FeCN-scavenged waste. Secondary: EB/in-tank solidification. Tertiary: } \\
\quad \text { neutralized acid waste, Other: CW/OWW. }\end{array}$ \\
\hline
\end{tabular}


RPP-RPT-46791

Revision 0

Table A2-1. SSTs and DSTs with no wash and leach information available for Aluminum. ( 8 sheets)

\begin{tabular}{|c|c|}
\hline Tank & Waste Sources \\
\hline $241-B Y-105$ & $\begin{array}{l}\text { Non-Complexed Waste. } \\
\text { BY-SltCk (liquid and solid), PFeCN (solid), and Portland Cement (solid). }{ }^{2} \\
\text { Primary: TBP/FeCN-scavenged waste. Secondary: EB/in-tank solidification. Tertiary: } \mathrm{CW}^{5}\end{array}$ \\
\hline 241-BY-106 & $\begin{array}{l}\text { Non-Complexed Waste. } \\
\text { BY-SltCk (liquid and solid) and PFeCN (solid). }{ }^{2} \\
\text { Primary: TBP/FeCN-scavenged waste. Secondary: } \mathrm{EB} / \mathrm{in} \text {-tank solidification. Tertiary: } \mathrm{CW}^{5}\end{array}$ \\
\hline 241-BY-107 & $\begin{array}{l}\text { Non-Complexed Waste. } \\
\text { BY-SltCk (liquid and solid) and PFeCN (solid), } \\
\text { Primary: TBP/FeCN-scavenged waste. Secondary: EB/in-tank solidification. Tertiary: CW. }\end{array}$ \\
\hline $241-B Y-109$ & $\begin{array}{l}\text { Non-Complexed Waste. } \\
\text { BY-SltCk (liquid and solid) and CWP2 (solid), }{ }^{2} \\
\text { Primary: TBP. Secondary: EB/in-tank solidification. Tertiary: CW. Other: metal waste. }\end{array}$ \\
\hline 241-BY-111 & $\begin{array}{l}\text { Non-Complexed Waste. } \\
\text { BY-SltCk (liquid and solid). }{ }^{2} \\
\text { Primary: TBP/FeCN-scavenged waste. Secondary: EB/in-tank solidification. Tertiary; OWW, } \\
\quad \text { Other; } \mathrm{CW}^{5}\end{array}$ \\
\hline $241-\mathrm{BY}-112$ & $\begin{array}{l}\text { Non-Complexed Waste. } \\
\text { BY-SltCk (liquid and solid) and MW2 (solid). }{ }^{2} \\
\text { Primary: TBP/FeCN-scavenged waste. Secondary: BB/in-tank solidification. Tertiary: } \mathrm{CW}^{5}\end{array}$ \\
\hline 241-C-101 & $\begin{array}{l}\text { Non-Complexed Waste. } \\
\text { CWP1 (solid) and TBP (solid), }{ }^{2} \\
\text { Primary: TBP. Secondary: CW. Tertiary: neutralized acid waste. Other: OWW. }\end{array}$ \\
\hline $241-C-110$ & $\begin{array}{l}\text { Dilute Complexed Waste. } \\
\text { 1C (solid). } \\
\text { Primary: } 1 \text { C. Secondary: TBP. Tertiary: OWW. Other: EB/IX. }\end{array}$ \\
\hline $241-C-111$ & $\begin{array}{l}\text { Non-Complexed Waste, } \\
\text { 1C (solid), CWP1 (solid), HS (solid), and TFeCN (solid). }{ }^{2} \\
\text { Primary: TBP/FeCN-scavenged waste. Secondary: 1C. Tertiary: CW. Other: hot semiworks } \\
\text { waste. }\end{array}$ \\
\hline $241-C-201$ & $\begin{array}{l}\text { HS (solid) and NA (liquid), } \\
\text { Primarily hot semiworks waste. }\end{array}$ \\
\hline $241-C-202$ & $\begin{array}{l}\text { HS (solid) and NA (liquid). } \\
\text { Primarily hot semiworks waste. }\end{array}$ \\
\hline $241-C-203$ & $\begin{array}{l}\text { HS (solid) and NA (liquid)? } \\
\text { Primarily hot semiworks waste. }\end{array}$ \\
\hline $241-C-204$ & $\begin{array}{l}\text { HS (solid) and NA (liquid), } \\
\text { Primarily hot semiworks waste. }\end{array}$ \\
\hline $241-5-102$ & $\begin{array}{l}\text { Double Shell Slurry Feed. }{ }^{1} \\
\text { NA (SltCk) and R1 (solid). } \\
\text { Primary: High-level REDOX waste. Secondary; EB. Tertiary; double-shell slurry feed, }\end{array}$ \\
\hline $241-S-103$ & $\begin{array}{l}\text { Double Shell Slurry Feed. } \\
\text { S1-SitCk (liquid and solid), S2-SltSlr (liquid and solid), and R1 (solid). } \\
\text { Primary: High-level REDOX waste. Secondary: EB. Tertiary: double-shell slurry feed. }\end{array}$ \\
\hline 241-S-105 & $\begin{array}{l}\text { Non-Complexed Waste. } \\
\text { S1-SltCk (liquid and solid) and RL (solid)? } \\
\text { Primary: High-level REDOX waste. Secondary: EB. }\end{array}$ \\
\hline $241-S-106$ & $\begin{array}{l}\text { Non-Complexed Waste }{ }_{+}^{1} \\
\text { S1-SitCk (liquid and solid) }{ }^{2} \\
\text { Primary: High-level REDOX waste. Secondary: EB. }\end{array}$ \\
\hline
\end{tabular}


RPP-RPT-46791

Revision 0

Table A2-1. SSTs and DSTs with no wash and leach information available for Aluminum. ( 8 sheets)

\begin{tabular}{|c|c|}
\hline Tank & Waste Sources \\
\hline $241-S-108$ & $\begin{array}{l}\text { Non-Complexed Waste. } \\
\text { S1-SitCk (liquid and solid) and R1 (solid). } \\
\text { Primary: High-level REDOX waste. Secondary: EB. }\end{array}$ \\
\hline $241-S-109$ & $\begin{array}{l}\text { Non-Complexed Waste. } \\
\text { S1-SltCk (liquid and solid) and R1 (solid). } \\
\text { Primary: High-level REDOX waste. Secondary: EB. }\end{array}$ \\
\hline $241-S-112$ & $\begin{array}{l}\text { Non-Complexed Waste. } \\
\text { R1 (solid) and NA (liquid), } \\
\text { Primary: High-level REDOX waste. Secondary: } \mathrm{EB}^{2}\end{array}$ \\
\hline $241-S X-102$ & $\begin{array}{l}\text { Double Shcll Slurry Feed. } \\
\text { S1-SitCk (liquid and solid, S2-SitSlr (liquid and solid), and R1 (solid), }{ }^{2} \\
\text { Primary: High-level REDOX waste. Secondary: EB. Tertiary: REDOX IX. }\end{array}$ \\
\hline 241-SX-103 & $\begin{array}{l}\text { Non-Complexed Waste. } \\
\text { R-SItCk (liquid and solid), S1-SltCk (liquid and solid), and R1 (solid). } \\
\text { Primary: High-level REDOX waste. Secondary: EB. Tertiary: CW, Other: OWW. }\end{array}$ \\
\hline 241-SX-104 & $\begin{array}{l}\text { Double Shell Slurry Feed. } \\
\text { R-SltCk (liquid and solid), S1-SltCk (liquid and solid), and R1 (solid). } \\
\text { Primary: High-level REDOX waste. Secondary: EB. Tertiary: REDOX IX. }\end{array}$ \\
\hline 241-SX-105 & $\begin{array}{l}\text { Double Shell Slurry Feed. }{ }^{1} \\
\text { S1-SitCk (liquid and solid), R1 (solid), and R2 (solid), } \\
\text { Primary: High-level REDOX waste. Secondary: EB. Tertiary: REDOX IX. Other waste type: } \\
\text { Hanford Laboratory Operations. }\end{array}$ \\
\hline 241-SX-106 & $\begin{array}{l}\text { Non-Complexed Waste. } \\
\text { S1-SItCk (liquid and solid) and S2-SitSir (liquid and solid). } \\
\text { Primary: High-level REDOX waste. Secondary: EB. Tertiary: REDOX IX. Other: Laboratory } \\
\text { waste from the } 300 \text { area and mixed waste. }\end{array}$ \\
\hline 241-SX-107 & $\begin{array}{l}\text { Non-Complexed Waste. } \\
\text { R1 (solid) and R2 (solid). } \\
\text { Primarily high-level REDOX waste. }\end{array}$ \\
\hline 241-SX-109 & $\begin{array}{l}\text { Non-Complexed Waste. } \\
\text { R-SltCk (solid), R1 (solid), and R2 (solid)? } \\
\text { Primarily high-level REDOX waste, }\end{array}$ \\
\hline 241-SX-110 & $\begin{array}{l}\text { Non-Complexed Waste. } \\
\text { R-SltCk (solid) and R2 (solid). } \\
\text { Primary: high-level REDOX waste. Secondary: REDOX LX. Tertiary: mixture of several } \\
\text { miscellaneous wastes. }\end{array}$ \\
\hline 241-SX-111 & $\begin{array}{l}\text { Non-Complexed Waste. } \\
\text { R-SirCk (solid), R1 (solid), and R2 (solid). } \\
\text { Primary: high-level REDOX waste. Secondary: REDOX IX. }\end{array}$ \\
\hline $241-S X-112$ & $\begin{array}{l}\text { Non-Complexed Waste. } \\
\text { R1 (solid) and R2 (solid). } \\
\text { Primarily high-level REDOX waste. }\end{array}$ \\
\hline 241-SX-114 & $\begin{array}{l}\text { Non-Complexed Waste, } \\
\text { R-SitCk (liquid and solid), R1 (solid), and R2 (solid). } \\
\text { Primary: high-level REDOX waste, Secondary: REDOX IX. Tertiary: EB. }\end{array}$ \\
\hline 241-SX-115 & $\begin{array}{l}\text { Non-Complexed Waste. } \\
\text { R2 (solid). } \\
\text { Primarily high-level REDOX waste. }\end{array}$ \\
\hline
\end{tabular}


RPP-RPT-46791

Revision 0

Table A2-1. SSTs and DSTs with no wash and leach information available for Aluminum. (8 sheets)

\begin{tabular}{|c|c|}
\hline Tank & Waste Sources \\
\hline $241-T-101$ & $\begin{array}{l}\text { Non-Complexed Waste. } \\
\text { T2-SltCk (liquid and solid) and CWR2 (solid) }{ }^{2} \\
\text { Primary: CW. Secondary: mixture of several miscellaneous wastes. Tertiary: TBP/FeCN- } \\
\quad \text { scavenged waste. Other: evaporator feed. }\end{array}$ \\
\hline $241-T-102$ & $\begin{array}{l}\text { Non-Complexed Waste. } \\
\text { CWP2 (solid), MW2 (solid), and CSR (liquid). } \\
\text { Primary: CW, Secondary: mixture of several miscellaneous wastes. Tertiary: IX. }\end{array}$ \\
\hline $241-T-103$ & $\begin{array}{l}\text { CWP2 (solid), CWR1 (solid), MW2 (solid), and CSR (liquid) }{ }^{2} \\
\text { Primary: CW Secondary; mixture of several miscellaneous wastes. }\end{array}$ \\
\hline $241-T-105$ & $\begin{array}{l}\text { Non-Complexed Waste. } \\
\text { IC (solid), } 2 \mathrm{C} \text { (solid), and CWR1 (solid), }{ }^{2} \\
\text { Primary: 1C. Secondary: CW. Tertiary: } 2 \text { C. Other: B Plant low-level waste/IX. }{ }^{5}\end{array}$ \\
\hline 241-T-106 & $\begin{array}{l}\text { Non-Complexed Waste, } \\
\text { 1C (solid), CWR1 (solid), and CWR2 (solid) }{ }^{2} \\
\text { Primary: } 1 \text { C. Secondary: CW. Tertiary: } 2 \text { C. Other: mixture of several miscellaneous wastes, }\end{array}$ \\
\hline $241-T-108$ & $\begin{array}{l}\text { Non-Complexed Waste. } \\
\text { T1-SitCk (liquid and solid) and } 1 \mathrm{C} \text { (solid), }{ }^{2} \\
\text { Primary: 1C. Secondary: TBP. Tertiary: EB. Other: Hanford Laboratory Operations. }\end{array}$ \\
\hline $241-\mathrm{T}-109$ & $\begin{array}{l}\text { Non-Complexed Waste. } \\
\text { T1-SltCk (liquid and solid), } \\
\text { Primary: TBP. Secondary: EB. Tertiary: mixture of several miscellaneous wastes. }\end{array}$ \\
\hline $241-\mathrm{T}-112$ & $\begin{array}{l}224-2 \text { (solid), } 2 \text { C (solid), CSR (liquid), and DW (liquid). }{ }^{2} \\
\text { Primary: } 2 \mathrm{C} \text {. Secondary: Lanthanum fluoride decontamination waste. Tertiary: } \\
\text { decontamination waste. Other: mixture of several miscellaneous wastes. }\end{array}$ \\
\hline $241-T-201$ & $\begin{array}{l}\text { 224-1 (liquid and solid). } \\
\text { Primarily lanthanum fluoride decontamination waste. }\end{array}$ \\
\hline $241-T-202$ & $\begin{array}{l}224-2 \text { (solid). } \\
\text { Primarily lanthanum fluoride decontamination waste. }\end{array}$ \\
\hline $241-T-203$ & $\begin{array}{l}224-2 \text { (sclid), } \\
\text { Primarily lanthanum fluoride decontamination waste, }\end{array}$ \\
\hline 241-T-204 & $\begin{array}{l}224-2(\text { solid })^{2} \\
\text { Primarily lanthanum fluoride decontamination waste. }\end{array}$ \\
\hline 241-TX-101 & $\begin{array}{l}\text { Non-Complexed Waste. } \\
\text { T2-Slick (liquid and solid), MW2 (solid), R1 (solid), and Z (solid). } \\
\text { Primary: high-level REDOX waste. Secondary: mixture of several miscellaneous wastes, } \\
\text { Tertiary: mixture of several miscellaneous wastes, }\end{array}$ \\
\hline $241-\mathrm{TX}-102$ & $\begin{array}{l}\text { Non-Complexed Waste, } \\
\text { T2-SltCk (liquid and solid) and MW2 (solid) }{ }^{2} \\
\text { Primary: High-level REDOX waste. Secondary: EB. Tertiary: mixture of several } \\
\text { miscellaneous wastes. }\end{array}$ \\
\hline 241-TX-103 & $\begin{array}{l}\text { Non-Complexed Waste. } \\
\text { T1-SitCk (liquid and solid) and T2-SltCk (liquid and solid). }{ }^{2} \\
\text { Primary: TBP. Secondary: EB. }\end{array}$ \\
\hline 241-TX-104 & $\begin{array}{l}\text { Non-Complexed Waste. } \\
\text { T2-SitCk (liquid and solid), and R1 (solid). }{ }^{2} \\
\text { Primary: High-level REDOX waste. Secondary: EB. Tertiary; mixture of several } \\
\text { miscellaneous wastes. }\end{array}$ \\
\hline 241-TX-105 & $\begin{array}{l}\text { Non-Complexed Waste. } \\
\text { T2-SitCk (liquid and solid) and MW2 (solid). } \\
\text { Primary: High-level REDOX waste. Secondary: EB. Tertiary: mixture of several } \\
\text { miscellaneous wastes. }\end{array}$ \\
\hline
\end{tabular}


RPP-RPT-46791

Revision 0

Table A2-1. SSTs and DSTs with no wash and leach information available for Aluminum. (8 sheets)

\begin{tabular}{|c|c|}
\hline Tank & Waste Sources \\
\hline 241-TX-106 & $\begin{array}{l}\text { Non-Complexed Waste. } \\
\text { T2-SltCk (liquid and solid), MW2 (solid), and R1 (solid) }{ }^{2} \\
\text { Primary: High-level REDOX waste. Secondary: EB. Tertiary: mixture of several } \\
\text { miscellaneous wastes. }\end{array}$ \\
\hline 241-TX-107 & $\begin{array}{l}\text { Non-Complexed Waste. } \\
\text { R-SltCk (liquid and solid) and T2-SitCk (liquid and solid). }{ }^{2} \\
\text { Primary: High-level REDOX waste, Secondary: EB. }\end{array}$ \\
\hline 241-TX-108 & $\begin{array}{l}\text { Non-Complexed Waste. } \\
\text { T2-SltCk (liquid and solid), MW2 (solid), and TBP (solid) }{ }^{2} \\
\text { Primary: EB. Secondary: TBP. Tertiary: high-level REDOX waste. }\end{array}$ \\
\hline 241-TX-109 & $\begin{array}{l}\text { Non-Complexed Waste. } \\
\text { IC (solid). } \\
\text { Primary: EB. Secondary: 1C. Tertiary: TBP. }\end{array}$ \\
\hline $241-\mathrm{TX}-110$ & $\begin{array}{l}\text { Non-Complexed Waste. } \\
\text { T2-SltCk (liquid and solid) and 1C (solid). }{ }^{2} \\
\text { Primary: EB. Secondary: 1C. Tertiary: TBP. }\end{array}$ \\
\hline 241-TX-111 & $\begin{array}{l}\text { Non-Complexed Waste, } \\
\text { T2-SitCk (liquid and solid) and IC (solid), } \\
\text { Primary: EB. Secondary: 1C. Tertiary: TBP. }\end{array}$ \\
\hline $241-\mathrm{TX}-112$ & $\begin{array}{l}\text { Non-Complexed Waste. } \\
\text { T1-SltCk (liquid and solid) and T2-SitCk (liquid and solid). }{ }^{2} \\
\text { Primary: EB. Secondary: } 1 C^{5}\end{array}$ \\
\hline $241-\mathrm{TX}-113$ & $\begin{array}{l}\text { Non-Complexed Waste. } \\
\text { T2-SitCk (liquid and solid) and } 1 \mathrm{C} \text { (solid). } \\
\text { Primary: EB. Secondary: } 1 \mathrm{C}^{5}\end{array}$ \\
\hline 241-TX-114 & $\begin{array}{l}\text { Non-Complexed Waste } \\
\text { T1-SltCk (liquid and solid), T2-SltCk (liquid and solid), and IC (solid), } \\
\text { Primary: EB. Secondary: IC. }\end{array}$ \\
\hline $241-\mathrm{TX}-115$ & $\begin{array}{l}\text { Non-Complexed Waste. } \\
\text { T2-SitCk (liquid and solid) and TBP (solid), }{ }^{2} \\
\text { Primary: EB. Secondary: high-level REDOX waste. Tertiary: CW. Other: decontamination } \\
\text { waste. }\end{array}$ \\
\hline $241-\mathrm{TX}-116$ & $\begin{array}{l}\text { Non-Complexed Waste. } \\
\text { T1-SitCk (liquid and solid), T2-SitCk (solid), and DE (solid). } \\
\text { Primary: EB. Secondary: iC. }\end{array}$ \\
\hline 241-TX-117 & $\begin{array}{l}\text { Non-Complexed Waste. } \\
\text { T1-S1tCk (liquid and solid), T2-SltCk (solid), and DE (solid). } \\
\text { Primary: EB. Secondary: 1C. }\end{array}$ \\
\hline 241-TX-118 & $\begin{array}{l}\text { Non-Complexed Waste. } \\
\text { T2-SltCk (liquid and solid) and NA (SItCk). }{ }^{2} \\
\text { Primary: EB. Secondary: TBP. Tertiary: CW. Other: } 1 C^{\text {s. }}\end{array}$ \\
\hline 241-TY-101 & $\begin{array}{l}\text { Non-Complexed Waste. } \\
\text { T1-SltCk (liquid and solid) and } 1 \mathrm{CFeCN} \text { (solid). }{ }^{2} \\
\text { Primary: } 1 \mathrm{C} / \mathrm{FeCN} \text {-scavenged waste. Secondary: EB. Tertiary; TBP. Other: high-level } \\
\text { REDOX waste. }\end{array}$ \\
\hline 241-TY-102 & $\begin{array}{l}\text { Non-Complexed Waste. } \\
\text { T1-SitCk (liquid and solid) and T2-S1tCk (liquid and solid). } \\
\text { Primary: EB. Secondary: 1C. Tertiary: mixture of several miscellaneous wastes. }\end{array}$ \\
\hline
\end{tabular}


RPP-RPT-46791

Revision 0

Table A2-1. SSTs and DSTs with no wash and leach information available for Aluminum. ( 8 sheets)

\begin{tabular}{|c|c|}
\hline Tank & Waste Sources \\
\hline 241-TY-103 & $\begin{array}{l}\text { Non-Complexed Waste. } \\
\text { T2-SltCk (liquid and solid), } 1 \mathrm{CFeCN} \text { (solid), and TBP (solid) }{ }^{2} \\
\text { Primary: TBP. Secondary: } 1 \mathrm{C} / \mathrm{FeCN} \text {-scavenged waste. Tertiary: CW. Other: high-level } \\
\quad \text { RBDOX waste/ mixture of several miscellaneous wastes. }\end{array}$ \\
\hline 241-TY-105 & $\begin{array}{l}\text { Non-Complexed Waste. } \\
\text { TBP (solid). } \\
\text { Primarily TBP? }\end{array}$ \\
\hline 241-TY-106 & $\begin{array}{l}\text { Non-Complexed Waste. } \\
\text { DE (solid) and TBP (solid). } \\
\text { Primarily TBP. }\end{array}$ \\
\hline 241-U-101 & $\begin{array}{l}\text { Non-Complexed Waste. } \\
\text { R1 (solid). } \\
\text { Primarily high-level REDOX waste. }\end{array}$ \\
\hline $241-\mathrm{U}-102$ & $\begin{array}{l}\text { Non-Complexed Waste. } \\
\text { T2-SltCk (liquid and solid), \$2-SltSir (solid), and R1 (solid). }{ }^{2} \\
\text { Primary: EB. Secondary: high-level REDOX waste, }\end{array}$ \\
\hline $241-\mathrm{U}-103$ & $\begin{array}{l}\text { Non-Complexed Waste. } \\
\text { SI-SitCk (liquid and solid), S2-SitSlr (solid), and R1 (solid). }{ }^{2} \\
\text { Primary: EB. Secondary: high-level REDOX waste. Tertiary: mixture of several miscellaneous } \\
\text { wastes. }\end{array}$ \\
\hline 241-U-104 & $\begin{array}{l}\text { Non-Complexed Waste. } \\
\text { DE (solid) and MW2 (solid). } \\
\text { Primary: high-level REDOX waste. Secondary: diatomaceous earth. }\end{array}$ \\
\hline 241-U-105 & $\begin{array}{l}\text { Non-Complexed Waste. } \\
\text { S2-SitSir (liquid and solid), T2-SltCk (liquid and solid), and CWR1 (solid). } \\
\text { Primary: EB. Secondary: CW. Tertiary: high-level REDOX waste. }\end{array}$ \\
\hline $241-\mathrm{U}-106$ & $\begin{array}{l}\text { Non-Complexed Waste }{ }^{1} \\
\text { S1-SitCk (liquid and solid) }{ }^{2} \\
\text { Primary: EB. Secondary: high-level REDOX waste. Tertiary: B Plant low-level waste. Other: } \\
\quad \text { Low-level waste from PUREX plant. }\end{array}$ \\
\hline $241-\mathrm{U}-107$ & $\begin{array}{l}\text { Double Shell Slurry Feed. } \\
\text { S2-SltSlr (liquid and solid), T2-StiCk (liquid and solid), and CWR1 (solid), } \\
\text { Primary: EB. Secondary: CW. Tertiary: mixture of several miscellaneous wastes, }{ }^{5}\end{array}$ \\
\hline 241-U-111 & $\begin{array}{l}\text { Double Shell Slurry Feed. }{ }^{1} \\
\text { SI-SitCk (liquid and solido, S2-SitSir (liquid and solid), 1C (solid), and RI (solid). }{ }^{2} \\
\text { Primary: EB. Secondary: high-level REDOX waste. Tertiary: } 1 \mathrm{C}^{3}\end{array}$ \\
\hline $241-\mathrm{U}-112$ & $\begin{array}{l}\text { Non-Complexed Waste. } \\
\text { IC (solid), CWR1 (solid), and R1 (solid). } \\
\text { Primary waste type is unknown. }\end{array}$ \\
\hline 241-U-201 & $\begin{array}{l}\text { CWR1 (liquid and solid). } \\
\text { Primarily CW: }\end{array}$ \\
\hline $241-\mathrm{L}-202$ & $\begin{array}{l}\text { CWR1 (liquid and solid). } \\
\text { Primarily } \mathrm{CW}^{2}\end{array}$ \\
\hline $241-\mathrm{U}-203$ & $\begin{array}{l}\text { CWR1 (liquid and solid) }{ }^{2} \\
\text { Primarily CW. }\end{array}$ \\
\hline $241-\mathrm{U}-204$ & $\begin{array}{l}\text { CWR1 (liquid and solid) }{ }^{2} \\
\text { Primary: high-level REDOX waste, Secondary: } 2 \text { C. Tertiary: } \mathrm{CW}^{5}\end{array}$ \\
\hline \multicolumn{2}{|c|}{$\begin{array}{l}\text { Superscript References: }{ }^{1} \text { WHC-EP-0625. }{ }^{2} \text { WRPS, } 2010 .{ }^{3} \text { PNNL-13394. }{ }^{4} \text { PNWD-3300 (WTP-RPT-076). } \\
{ }^{5} \text { PNL-9814. }\end{array}$} \\
\hline
\end{tabular}


RPP-RPT-46791

Revision 0

\section{A3,0 SINGLE- AND DOUBLE-SHELL TANK LABORATORY CONCENTRATION VERSUS TIME INFORMATION.}

Concentration versus time data was compiled from four laboratory studies (see Table A3-1) on seven separate SSTs. This information was then ploted (see Figure A3-1 through Figure A3-14) to observe correlations in how the percent removal of aluminun was affected due to changes in the solution caustic concentration, leaching temperature, and/or time for the various tanks. Much variation occurred between the different tank samples analyzed, with all of the analyses showing a positive amount of aluninum removal. 
Table A3-1. Caustic Leaching Aluminum Concentration versus Time Data (2 sheets).

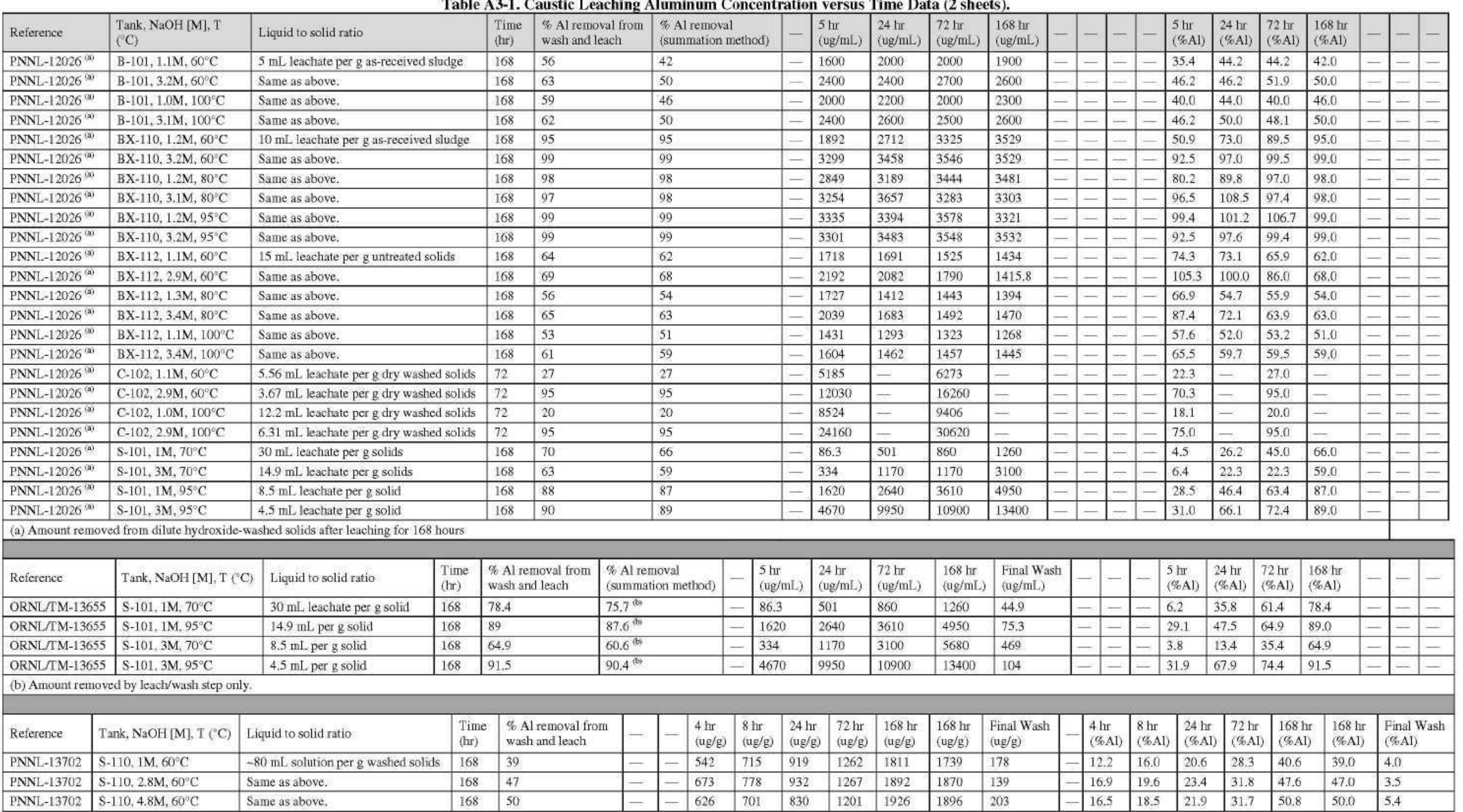


Table A3-1. Caustic Leaching Aluminum Concentration versus Time Data (2 sheets)

\begin{tabular}{|c|c|c|c|c|c|c|c|c|c|c|c|c|c|c|c|c|c|c|c|c|c|}
\hline PNNL-13702 & S- $110,0.9 \mathrm{M}, 80^{\circ} \mathrm{C}$ & Same as above. & 168 & 69 & - & - & 758 & 1042 & 1699 & 2502 & 3003 & 3056 & 137 & - & 17.1 & 23.5 & 38.4 & 56.5 & 67.8 & 69.0 & 3.1 \\
\hline PNNL-13702 & $\mathrm{S}-110,2.7 \mathrm{M}, 80^{\circ} \mathrm{C}$ & Same as above. & 168 & 91 & - & - & 870 & 1029 & 1794 & 2907 & 3757 & 3786 & 186 & - & 20.9 & 24.7 & 43.1 & 69.9 & 90.3 & 91.0 & 4.5 \\
\hline PNNL-13702 & S- $110,4.6 \mathrm{M}, 80^{\circ} \mathrm{C}$ & Same as above. & 168 & 96 & - & - & 855 & 1032 & 1880 & 2911 & 3788 & 3670 & 178 & - & 22.4 & 27.0 & 49.2 & 76.1 & 99.1 & 96.0 & 4.7 \\
\hline PNNL-13702 & S- $110,0.8 \mathrm{M}, 100^{\circ} \mathrm{C}$ & Same as above. & 168 & 91 & - & - & 1253 & 1823 & 2781 & 3550 & 4087 & 4031 & 139 & - & 28.3 & 41.2 & 62.8 & 80.1 & 92.3 & 91.0 & 3.1 \\
\hline PNNL-13702 & $\mathrm{S}-110,2.7 \mathrm{M}, 100^{\circ} \mathrm{C}$ & Same as above, & 168 & 100 & - & - & 1428 & 2059 & 3186 & 4038 & 4167 & 4150 & 138 & - & 34.4 & 49.6 & 76.8 & 97.3 & 100.4 & 100.0 & 3.3 \\
\hline PNNL-13702 & $\mathrm{S}-110,4.6 \mathrm{M}, 100^{\circ} \mathrm{C}$ & Same as above. & 168 & 100 & - & - & 1462 & 2239 & 3499 & 3951 & 3891 & 4043 & 134 & - & 36.2 & 55.4 & 86.5 & 97.7 & 96.2 & 100.0 & 3.3 \\
\hline & & & & & & & & & & & & & & & & & & & & & \\
\hline Reference & Tank, $\mathrm{NaOH}[\mathrm{M}], \mathrm{T}\left({ }^{\circ} \mathrm{C}\right)$ & Liquid to solid ratio & $\begin{array}{l}\text { Time } \\
\text { (hr) }\end{array}$ & $\begin{array}{l}\text { \% Al removal from } \\
\text { wash and leach }\end{array}$ & - & - & $\begin{array}{l}4 \mathrm{hr} \\
\text { (ug/g) }\end{array}$ & $\begin{array}{l}8 \mathrm{hr} \\
\text { (ug/g) }\end{array}$ & $\begin{array}{l}24 \mathrm{hr} \\
\text { (ug/g) }\end{array}$ & $\begin{array}{l}72 \mathrm{hr} \\
(\mathrm{ug} / \mathrm{g})\end{array}$ & $\begin{array}{l}168 \mathrm{hr} \\
(\mathrm{ug} / \mathrm{g})\end{array}$ & $\begin{array}{l}168 \mathrm{hr} \\
(\mathrm{ug} / \mathrm{g})\end{array}$ & \begin{tabular}{|l}
$\begin{array}{l}\text { Final Wash } \\
\text { (ug/g) }\end{array}$ \\
\end{tabular} & - & \begin{tabular}{|l|}
$4 \mathrm{hr}$ \\
$(\% \mathrm{Al})$
\end{tabular} & $\begin{array}{l}8 \mathrm{hr} \\
(\% \mathrm{Al})\end{array}$ & $\begin{array}{l}24 \mathrm{hr} \\
(\% \mathrm{Al})\end{array}$ & $\begin{array}{l}72 \mathrm{hr} \\
(\% \mathrm{Al}) \\
\end{array}$ & $\begin{array}{l}168 \mathrm{hr} \\
(\% \mathrm{Al})\end{array}$ & $\begin{array}{l}168 \mathrm{hr} \\
(\% \mathrm{Al}) \\
\end{array}$ & \begin{tabular}{|l}
$\begin{array}{l}\text { Final Wash } \\
(\% \mathrm{Al})\end{array}$ \\
\end{tabular} \\
\hline PNNL-13956 & $\mathrm{T}-110,0.6 \mathrm{M}, 60^{\circ} \mathrm{C}$ & $-20 \mathrm{~mL}$ solution per $\mathrm{g}$ washed solids & 168 & 27 & - & - & 5.4 & 5.6 & 5.4 & 5 & 5.1 & 3.6 & 2.3 & - & 28.6 & 29.6 & 28.6 & 26.5 & 27.0 & 19.1 & 12.2 \\
\hline PNNL-13956 & T-110, $2.5 \mathrm{M}, 60^{\circ} \mathrm{C}$ & Same as above. & 168 & 42 & - & - & 11.3 & 12.1 & 11.9 & 9 & 8.5 & 8 & 0 & - & 55.8 & 59.8 & 58.8 & 44.5 & 42.0 & 39.5 & 0.0 \\
\hline PNNL-13956 & $\mathrm{T}-110,3,8 \mathrm{M}, 60^{\circ} \mathrm{C}$ & Same as above. & 168 & 59 & - & - & 10.7 & 12.6 & 13,5 & 13,4 & 15.2 & 11.3 & 5.8 & - & 41.5 & 48.9 & 52.4 & 52.0 & 59,0 & 43,9 & 22.5 \\
\hline PNNL-13956 & $\mathrm{T}-110,0.6 \mathrm{M}, 80^{\circ} \mathrm{C}$ & Same as above. & 168 & 53 & - & - & 4.1 & 4.3 & 3.9 & 3.7 & 8.3 & 4.2 & 6.3 & - & 26.2 & 27.5 & 24.9 & 23.6 & 53.0 & 26.8 & 40.2 \\
\hline PNNL-13956 & T- $110,2.7 \mathrm{M}, 80^{\circ} \mathrm{C}$ & Same as above. & 168 & 67 & - & - & 11.6 & 12.5 & 11.5 & 12.4 & 14.3 & 12.1 & 7.3 & - & 54.3 & 58.6 & 53.9 & 58.1 & 67.0 & 56,7 & 34.2 \\
\hline PNNL-13956 & T- $110,4.2 \mathrm{M}, 80^{\circ} \mathrm{C}$ & Same as above. & 168 & 83 & - & - & 11.7 & 12.1 & 12.8 & 13.1 & 15 & 13.5 & 9 & - & 64.7 & 67.0 & 70.8 & 72.5 & 83.0 & 74.7 & 49.8 \\
\hline PNNL-13956 & $\mathrm{T}-110,0.6 \mathrm{M}, 100^{\circ} \mathrm{C}$ & Same as above. & 168 & 50 & - & - & 4.6 & 5.2 & 5 & 4.5 & 8.8 & 2.7 & 6.4 & - & 26.1 & 29.5 & 28.4 & 25.6 & 50.0 & 15.3 & 36.4 \\
\hline PNNL-13956 & $\mathrm{T}-110,2.5 \mathrm{M}, 100^{\circ} \mathrm{C}$ & Same as above. & 168 & 75 & - & - & 19.1 & 12.6 & 13.1 & 13.9 & 13.1 & 11.6 & 7.2 & - & 109.4 & 72.1 & 75.0 & 79.6 & 75.0 & 66.4 & 41.2 \\
\hline PNNL-13956 & $\mathrm{T}-110,4.3 \mathrm{M}, 100^{\circ} \mathrm{C}$ & Same as above. & 168 & 77 & - & - & 10.4 & 14.4 & 14.1 & 14.8 & 17.9 & 14.1 & 3.7 & - & 44.7 & 61.9 & 60.7 & 63.7 & 77.0 & 60.7 & 15.9 \\
\hline
\end{tabular}


Figure A3-1. Compilation of Laboratory Data for Percent Aluminum Removal versus Leaching Time

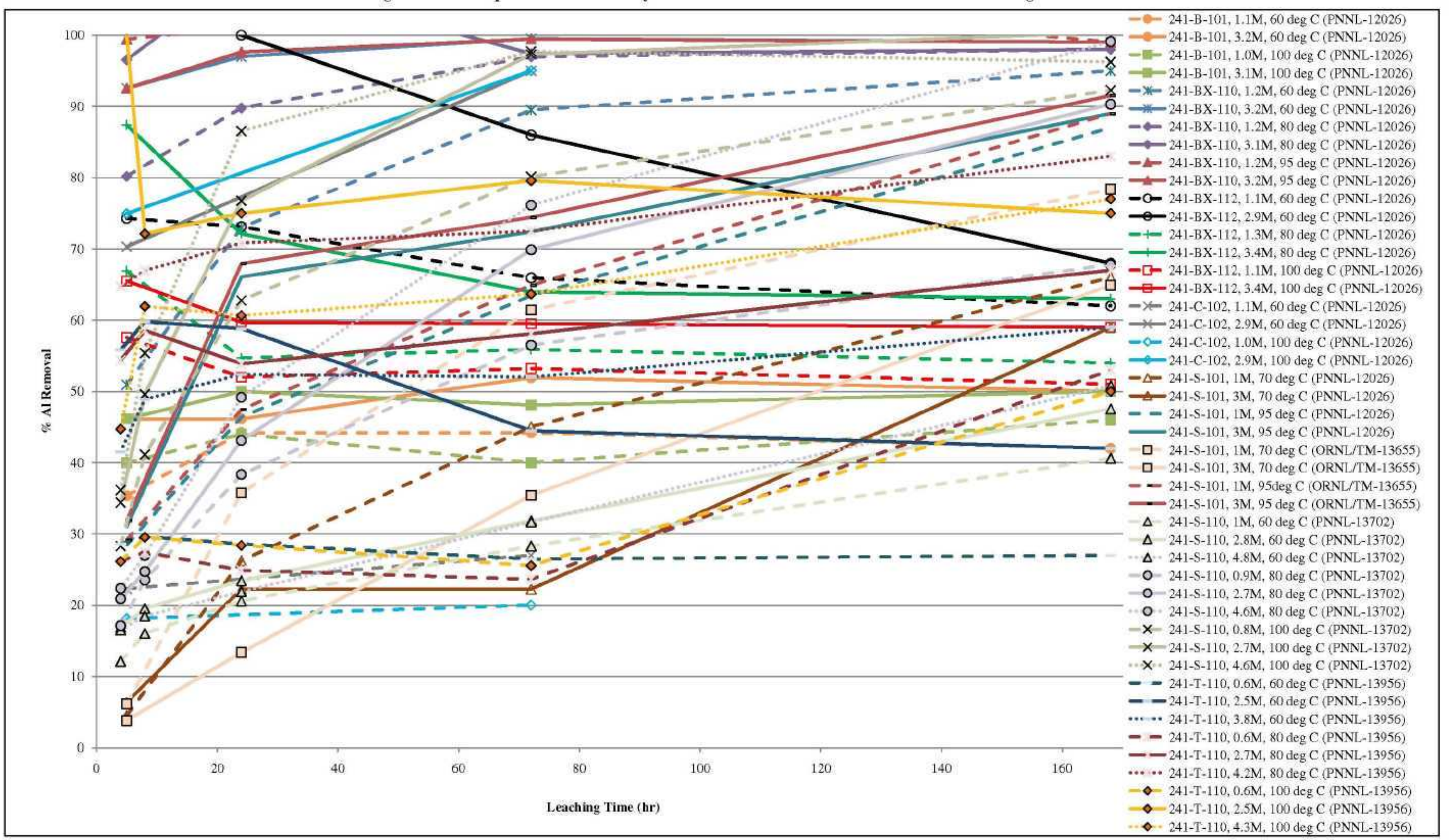


Figure A3-2. All caustic leaching analyses for Tank 241-B-101.

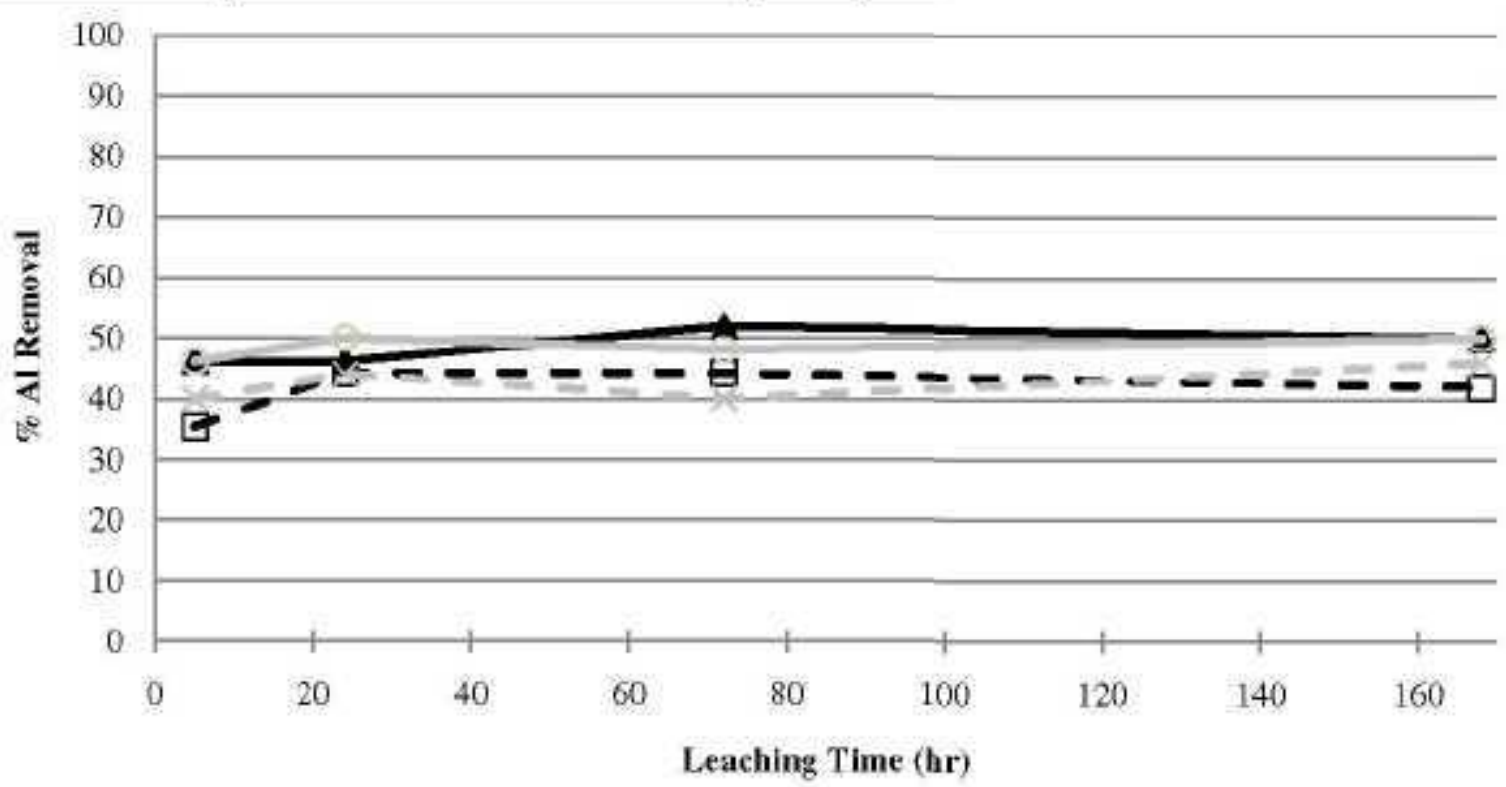

-[- 241-B-101, 1.1M, 60 deg C (PNNL-12026) - $241-\mathrm{B}-101,3.2 \mathrm{M}, 60 \mathrm{deg}$ C (PNNL-12026)

$-\mathrm{X}-241-\mathrm{B}-101,1.0 \mathrm{M}, 100 \mathrm{deg} \mathrm{C}$ (PNNL-12026) - 241-B-101, 3.1M, $100 \mathrm{deg} \mathrm{C}$ (PNNL-12026)

Figure A3-3. All caustic leaching analyses for Tank 241-BX-110.

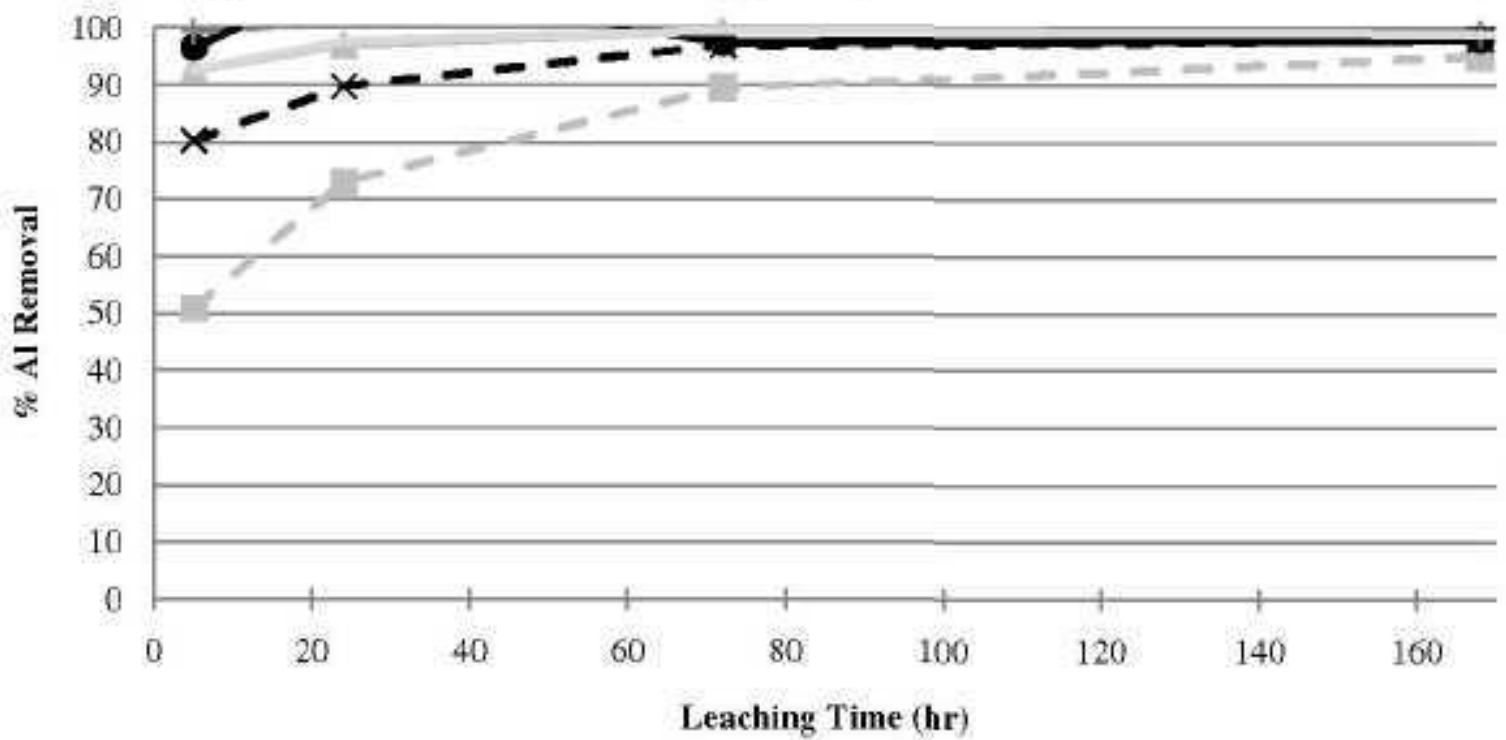

- $241-\mathrm{BX}-110,1.2 \mathrm{M}, 60 \operatorname{deg} \mathrm{C}$ (PNNL-12026) 241-BX-110, 3.2M, 60 deg C (PNNL-12026)

$->241-\mathrm{BX}-110,1.2 \mathrm{M}, 80 \operatorname{deg} \mathrm{C}(\mathrm{PNNL}-12026)$

$-241-\mathrm{BX}-110,3.1 \mathrm{M}, 80 \mathrm{deg} \mathrm{C}$ (PNNL-12026)

$=+-241-\mathrm{BX}-110,1.2 \mathrm{M}, 95 \mathrm{deg} \mathrm{C}$ (PNNL-12026) 241-BX-110,3,2M,95 deg C (PNNL-12026) 
Figure A3-4. All caustic leaching analyses for Tank 241-BX-112.

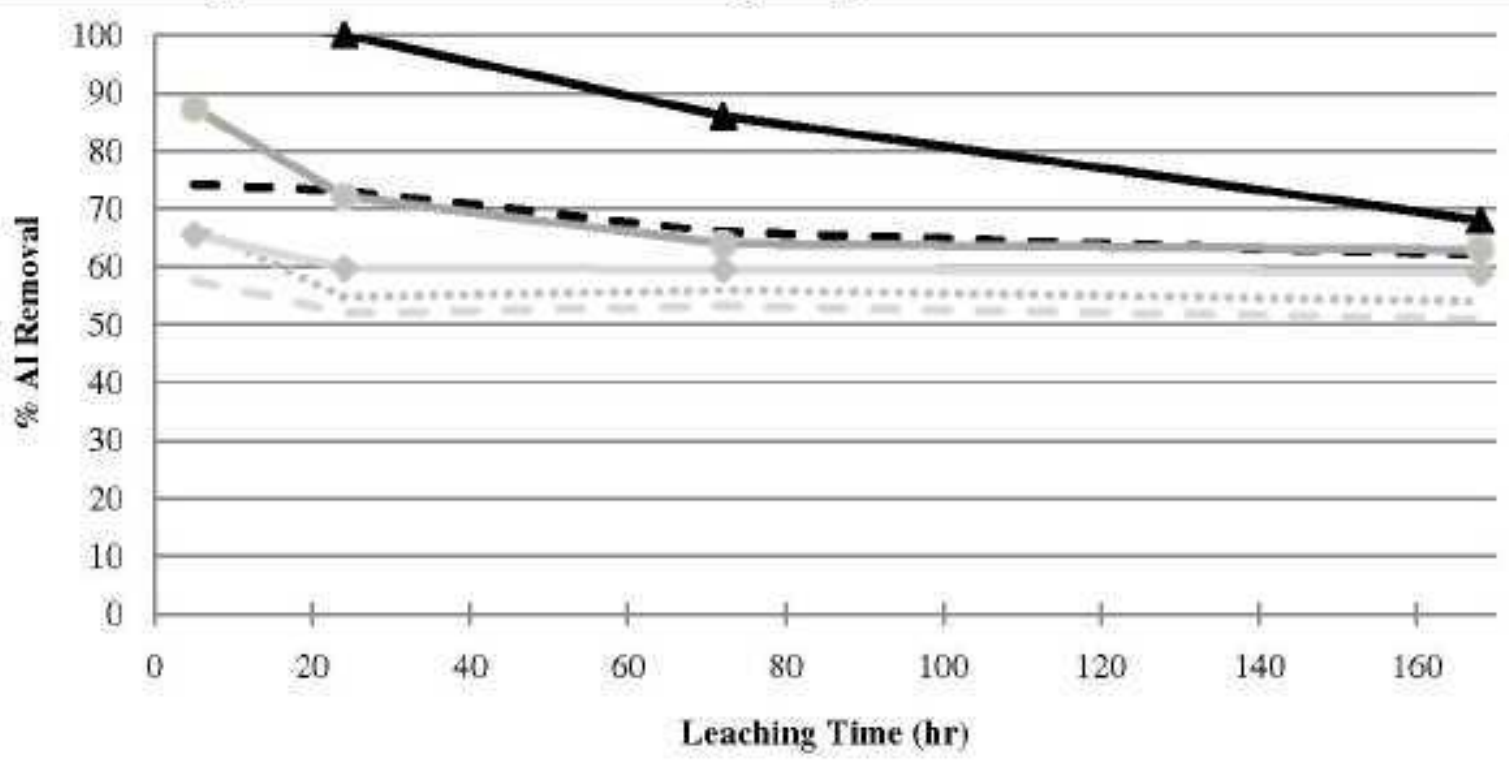

- - 241-BX-112, 1.1M, 60 deg C (PNNL-12026) - 241-BX-112, 2.9M, 60 deg C (PNNL-12026)

….. 241-BX-112, 1.3M, 80 deg C (PNNL-12026) - $241-\mathrm{BX}-112,3.4 \mathrm{M}, 80 \mathrm{deg} \mathrm{C}$ (PNNL-12026)

$-241-\mathrm{BX}-112,1,1 \mathrm{M}, 100 \mathrm{deg} \mathrm{C}$ (PNNL-12026) $\longrightarrow 241-\mathrm{BX}-112,3.4 \mathrm{M}, 100 \mathrm{deg} \mathrm{C}$ (PNNL-12026)

Figure A3-5. All caustic leaching analyses for Tank 241-C-102.

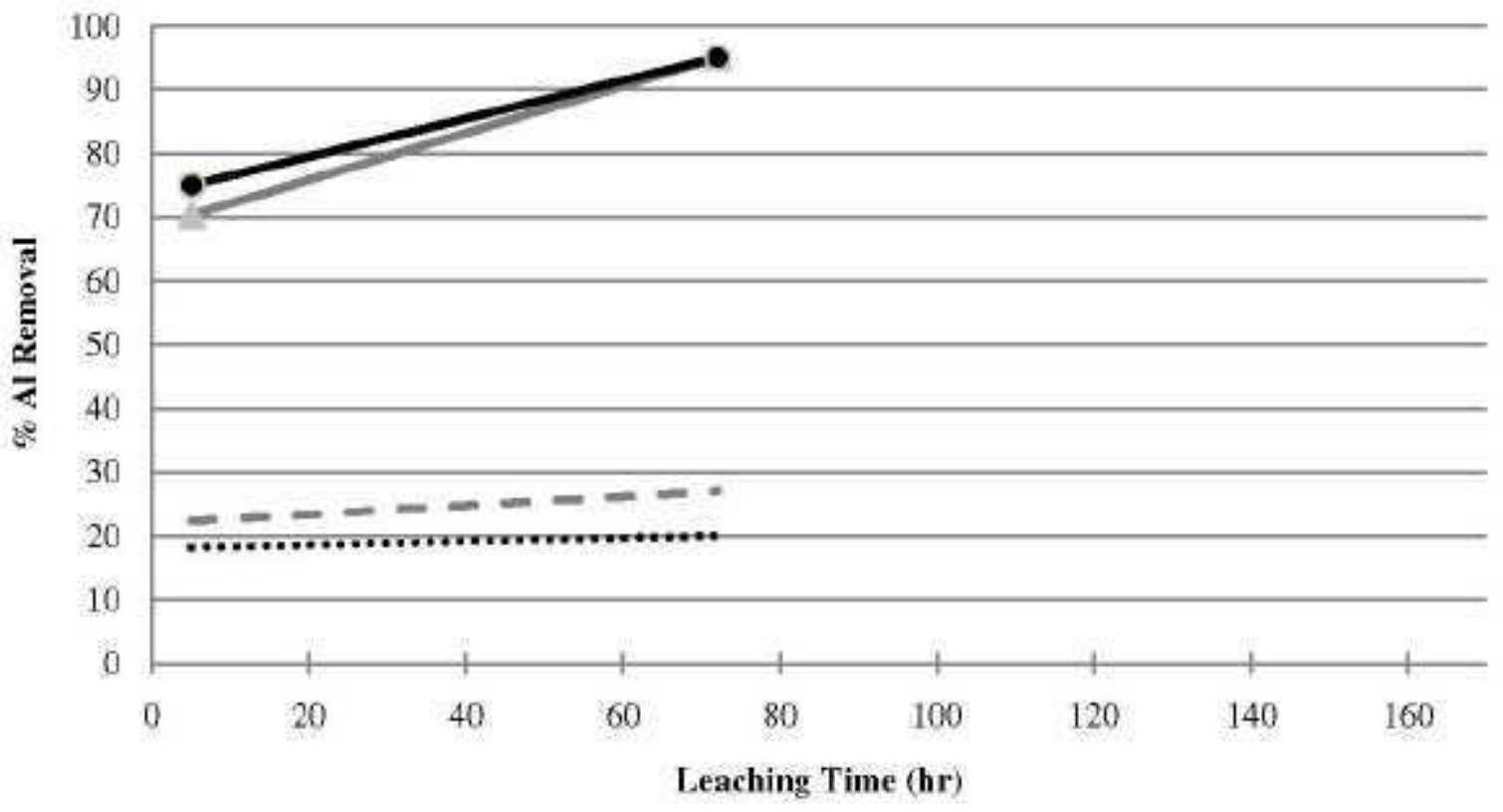

- 241-C-102, 1.1M, 60 deg C (PNNL-12026) -i-241-C-102, 2.9M, 60 deg C (PNNL-12026)

*... 241-C-102, 1.0M, $100 \mathrm{deg}$ C (PNNL-12026) - - 241-C-102, 2.9M, $100 \mathrm{deg}$ C (PNNL-12026) 
Figure A3-6. All caustic leaching analyses for Tank 241-S-101.

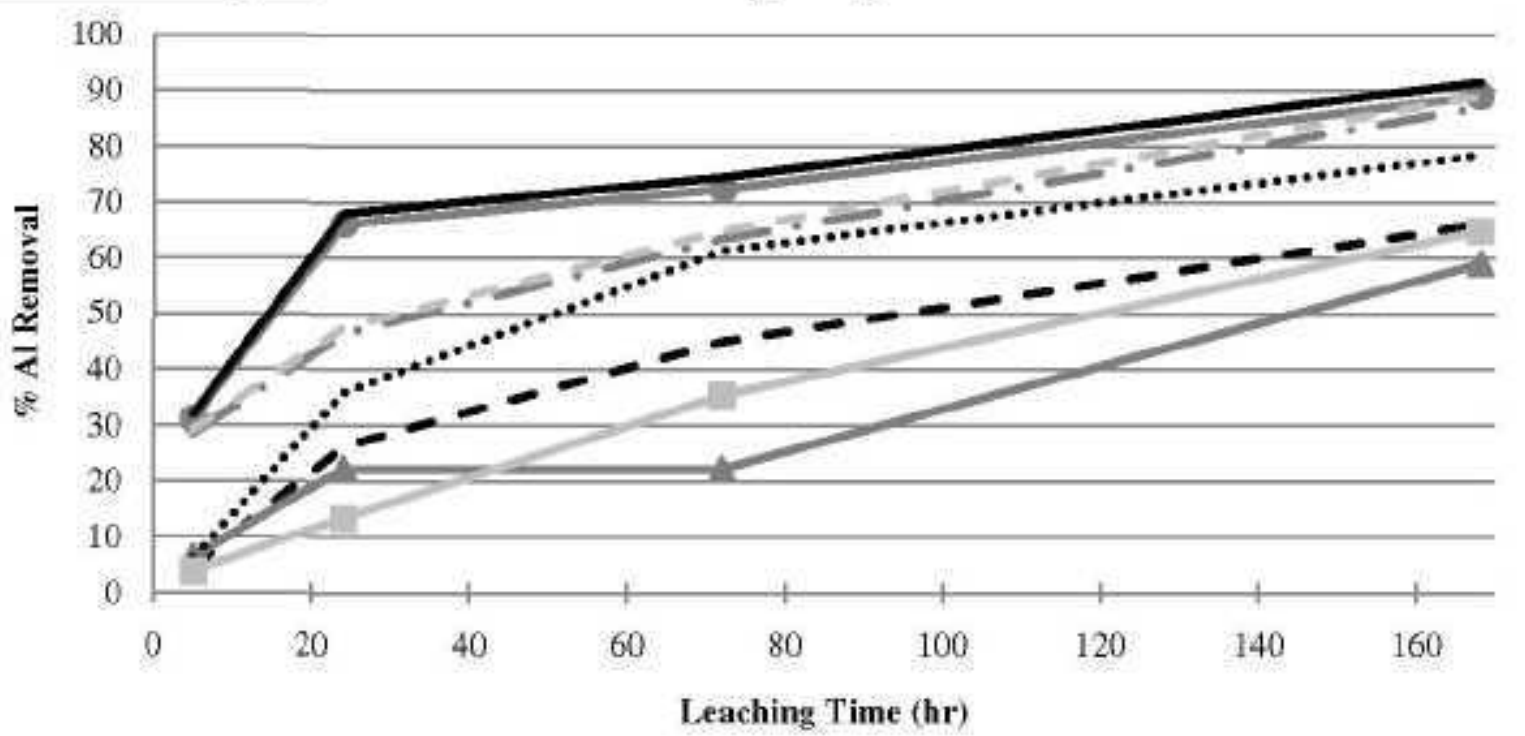

- - 241-S-101, 1M, 70 deg C (PNNL-12026)

- 241-S-101, 3M, 70 deg C (PNNL-12026)

- 241-S-101, 1M, $95 \operatorname{deg} \mathrm{C}$ (PNNL-12026)

-241-S-101, 3M, 95 deg C (PNNL-12026)

241-S-101, 1M, $70 \mathrm{deg}$ C (ORNL/TM-13655)

-n-241-S-101, 3M, 70 deg C (ORNL/TM-13655)

- - 241-S-101, 1M, 95deg C (ORNL/TM-13655)

241-S-101, 3M, 95 deg C (ORNL/TM-13655)

Figure A3-7. All caustic leaching analyses for Tank 241-S-110 .

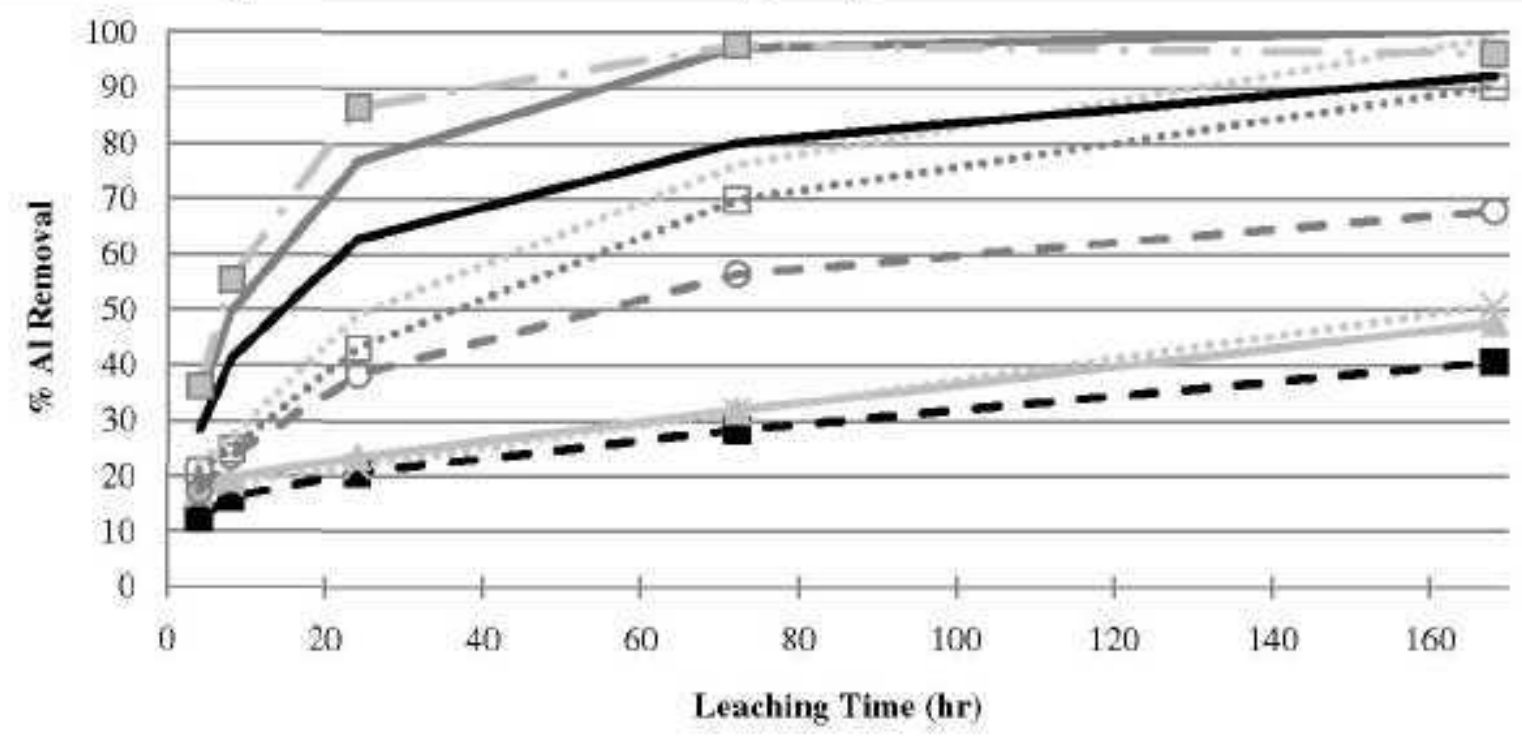

-E 241-S-110, 1M, 60 deg C (PNNL-13702)

$\cdots \times 241-\mathrm{S}-110,4.8 \mathrm{M}, 60 \mathrm{deg} \mathrm{C}$ (PNNL-13702)

*[․ 241-S-110, 2.7M, $80 \operatorname{deg} \mathrm{C}$ (PNNL-13702)

-241-S-110,0.8M, 100 deg C (PNNL-13702)

- $241-\mathrm{S}-110,4.6 \mathrm{M}, 100 \mathrm{deg} \mathrm{C}$ (PNNL-13702)
$=241-\mathrm{S}-110,2.8 \mathrm{M}, 60 \mathrm{deg} \mathrm{C}$ (PNNL-13702)

$=\mathrm{O}-241-\mathrm{S}-110,0.9 \mathrm{M}, 80 \mathrm{deg} \mathrm{C}$ (PNNL-13702)

**.. 241-S-110, 4,6M, 80 deg C (PNNL-13702)

$-241-\mathrm{S}-110,2.7 \mathrm{M}, 100 \operatorname{deg} \mathrm{C}$ (PNNL-13702) 
Figure A3-8. All caustic leaching analyses for Tank 241-T-110.

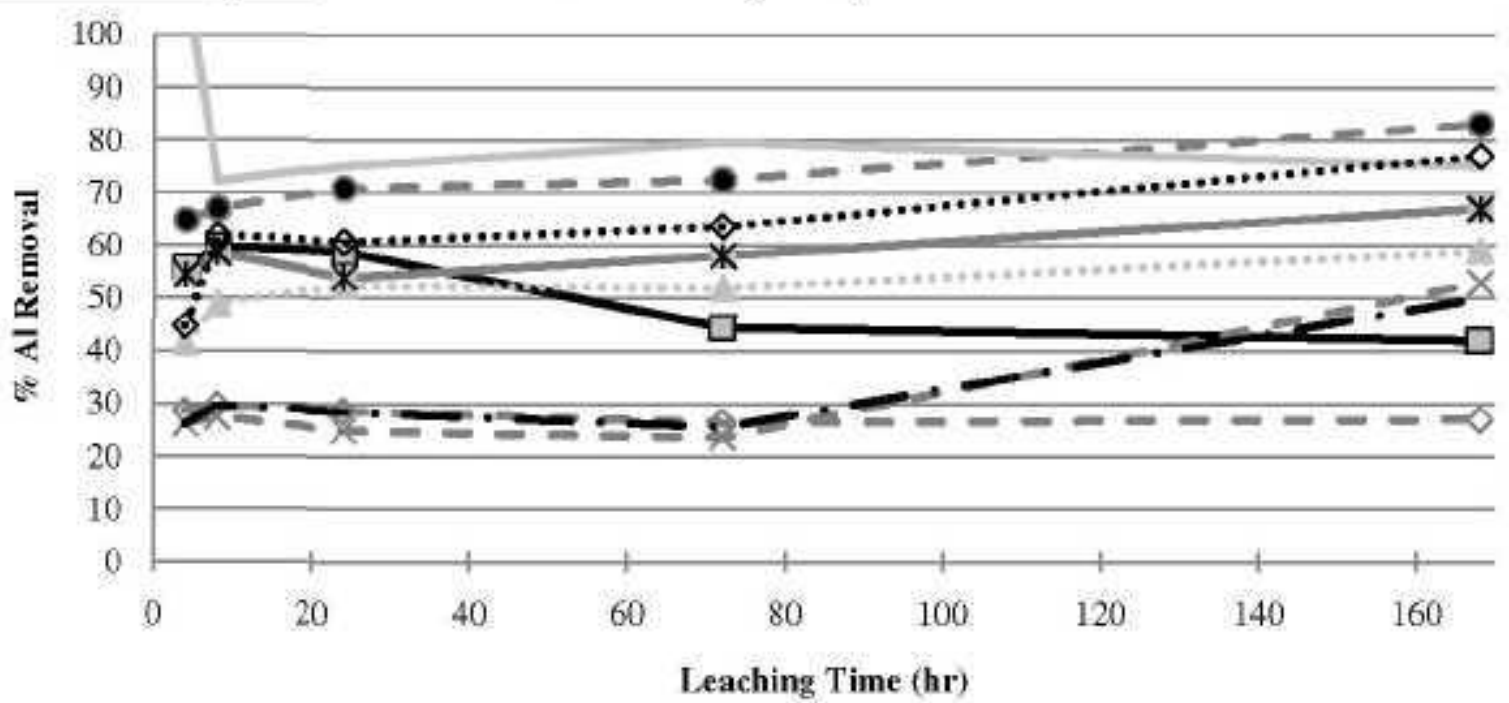

$-\measuredangle 241-\mathrm{T}-110,0.6 \mathrm{M}, 60 \mathrm{deg} \mathrm{C}$ (PNNL-13956)

$\cdots$ Av 241-T-110, 3.8M, 60 deg C (PNNL-13956)

- - 241-T-110, 2.7M, 80 deg C (PNNL-13956)

- 241-T-110, 0.6M, 100 deg C (PNNL-13956)

..2. 241-T-110,4.3M, 100 deg C (PNNL-13956)
$-\square-241-\mathrm{T}-110,2.5 \mathrm{M}, 60 \mathrm{deg} \mathrm{C}$ (PNNL-13956) $-x$ 241-T-110,0.6M, $80 \mathrm{deg}$ C (PNNL-13956)

- -241 - T-110,4.2M, 80 deg C (PNNL-13956) 241-T-110,2.5M, $100 \operatorname{deg}$ C (PNNL-13956)

Figure A3-9. Caustic leaching using $1 \mathrm{M} \mathrm{NaOH}$ at $60^{\circ} \mathrm{C}$.

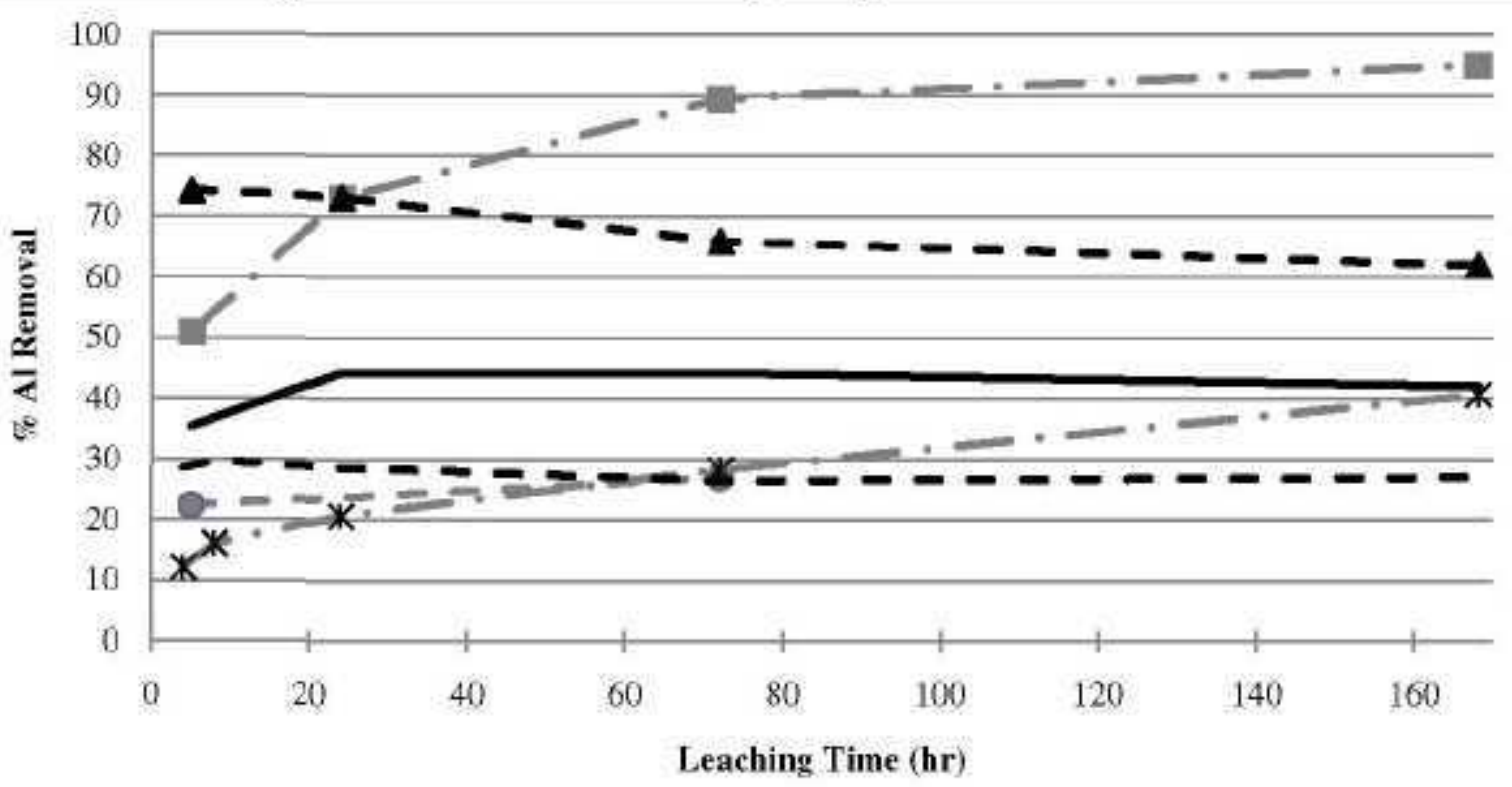

241-B-101, 1.1M, 60 deg C (PNNL-12026)

-n - 241-BX-110, 1.2M, $60 \mathrm{deg}$ C (PNNL-12026)

- $\mathbf{A}$ - 241-BX-112, 1.1M, 60 deg C (PNNL-12026)

- O- 241-C-102, 1.1M, $60 \operatorname{deg} \mathrm{C}$ (PNNL-12026)

* * 241-S-110,1M, 60 deg C (PNNL-13702)

- -241-T-110, 0.6M, 60 deg C (PNNL-13956) 
Figure A3-10. Caustic leaching using $3 \mathrm{M} \mathrm{NaOH}$ at $60^{\circ} \mathrm{C}$.

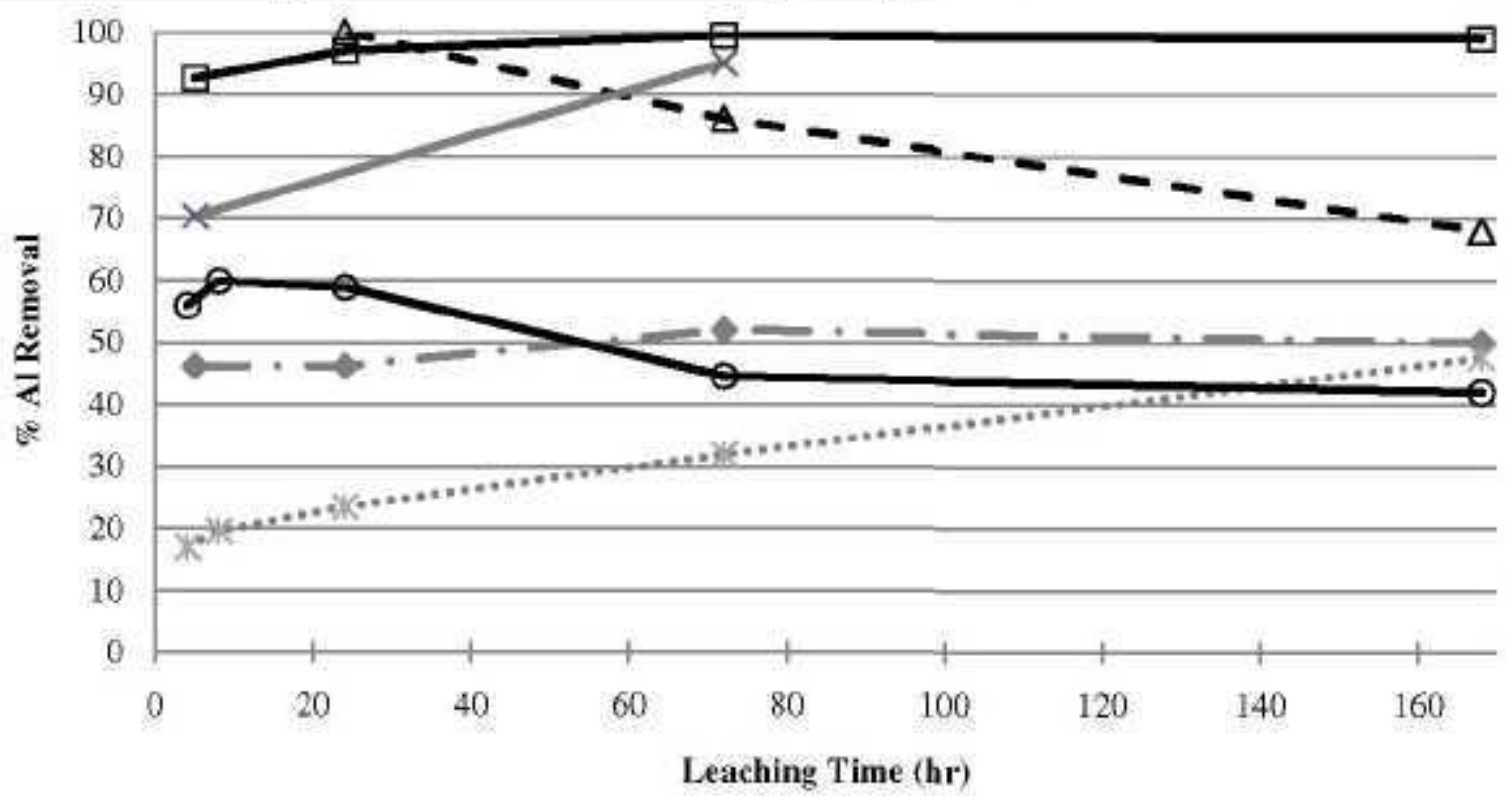

$\Rightarrow$ 241-B-101, 3.2M, $60 \mathrm{deg}$ C (PNNL-12026)

$-\Delta-241-\mathrm{BX}-112,2.9 \mathrm{M}, 60 \mathrm{deg} \mathrm{C}$ (PNNL-12026)

$\cdots *(*$ 241-S-110, 2.8M, 60 deg C (PNNL-13702)
- 241-BX-110,3.2M, 60 deg C (PNNL-12026) $\rightarrow$ 241-C-102, 2.9M, 60 deg C (PNNL-12026) -241-T-110, 2.5M. $60 \mathrm{deg}$ C (PNNL-13956)

Figure A3-11. Caustic leaching using $1 \mathrm{M} \mathrm{NaOH}$ at $80^{\circ} \mathrm{C}$.

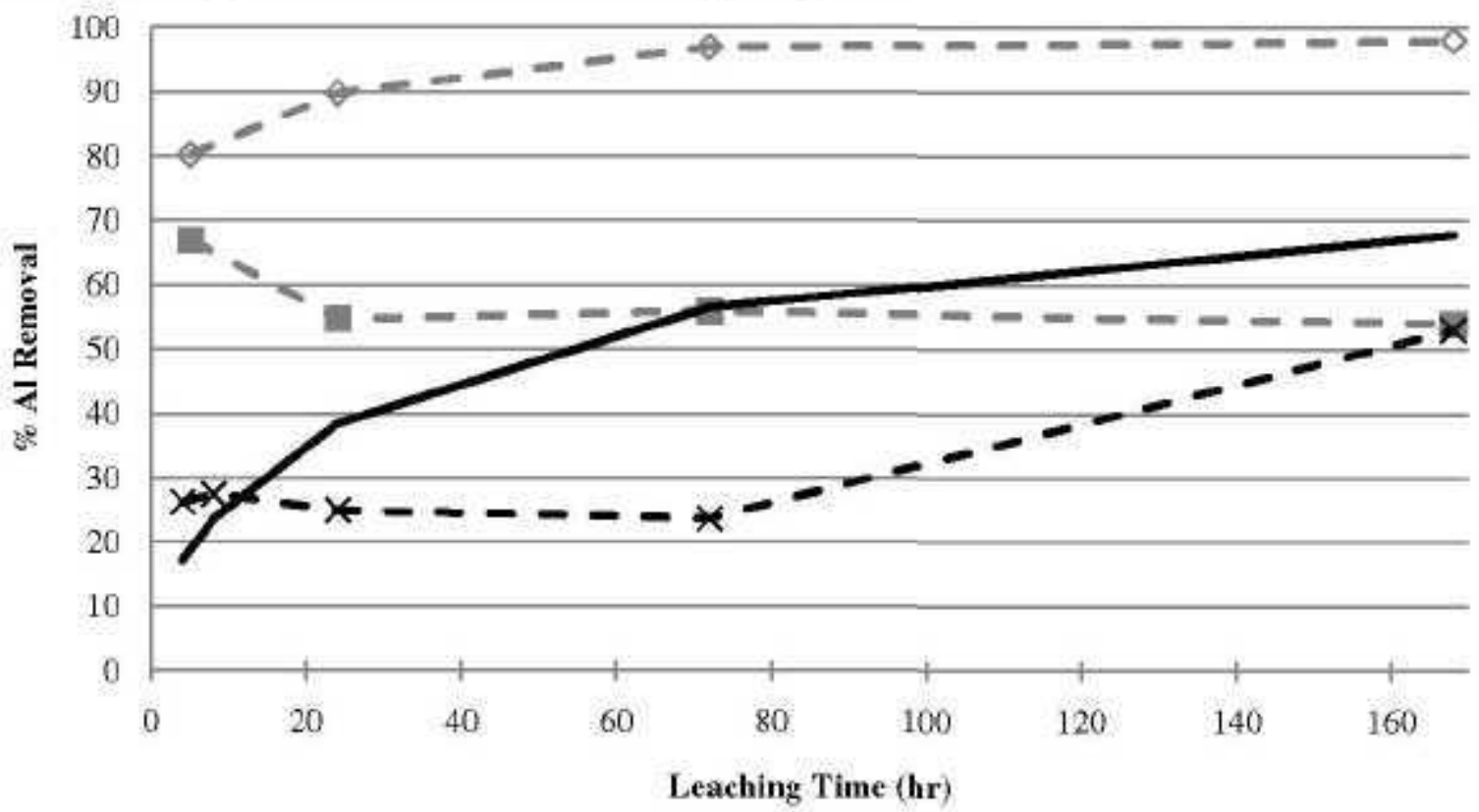

$=\infty$ 241-BX-110, 1.2M, $80 \mathrm{deg}$ C (PNNL-12026) $=-1-241-\mathrm{BX}-112,1.3 \mathrm{M}, 80 \mathrm{deg}$ C (PNNL-12026)

241-S-110.0.9M. $80 \operatorname{deg}$ C (PNNL-13702) $\quad-\times-241-\mathrm{T}-110,0.6 \mathrm{M}, 80 \mathrm{deg}$ C (PNNL-13956) 
Figure A3-12. Caustic leaching using $3 \mathrm{M} \mathrm{NaOH}$ at $80^{\circ} \mathrm{C}$.

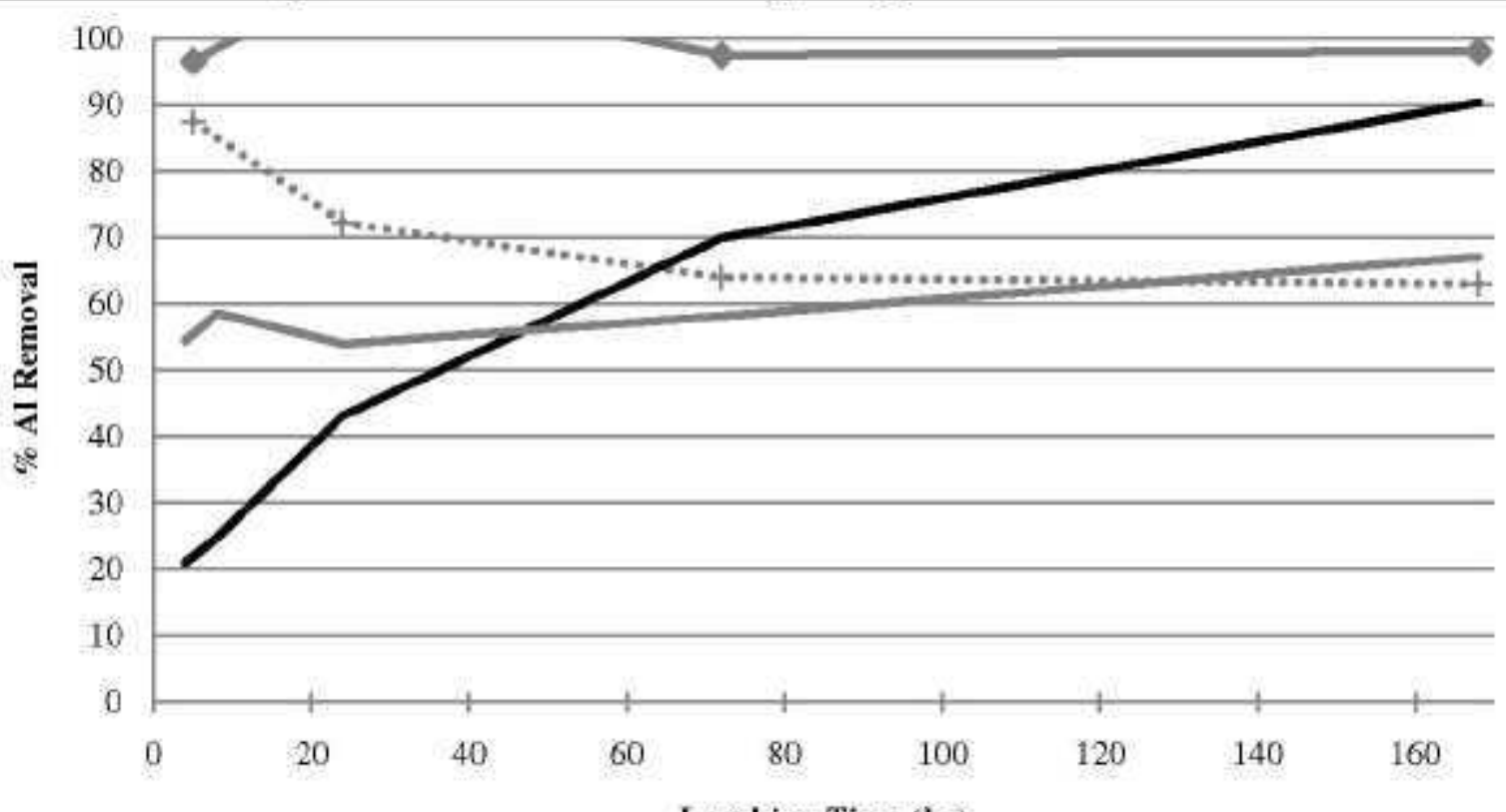

Leaching Time (hr)

- 241-BX-110,3.1M, $80 \operatorname{deg}$ C (PNNL-12026) ‥* 241-BX-112, 3.4M, 80 deg C (PNNL-12026)

-241-S-110, 2.7M, $80 \operatorname{deg} \mathrm{C}$ (PNNL-13702) 241-T-110, 2.7M, 80 deg C (PNNL-13956)

Figure A3-13. Caustic leaching using $1 \mathrm{M} \mathrm{NaOH}$ at $100^{\circ} \mathrm{C}$.

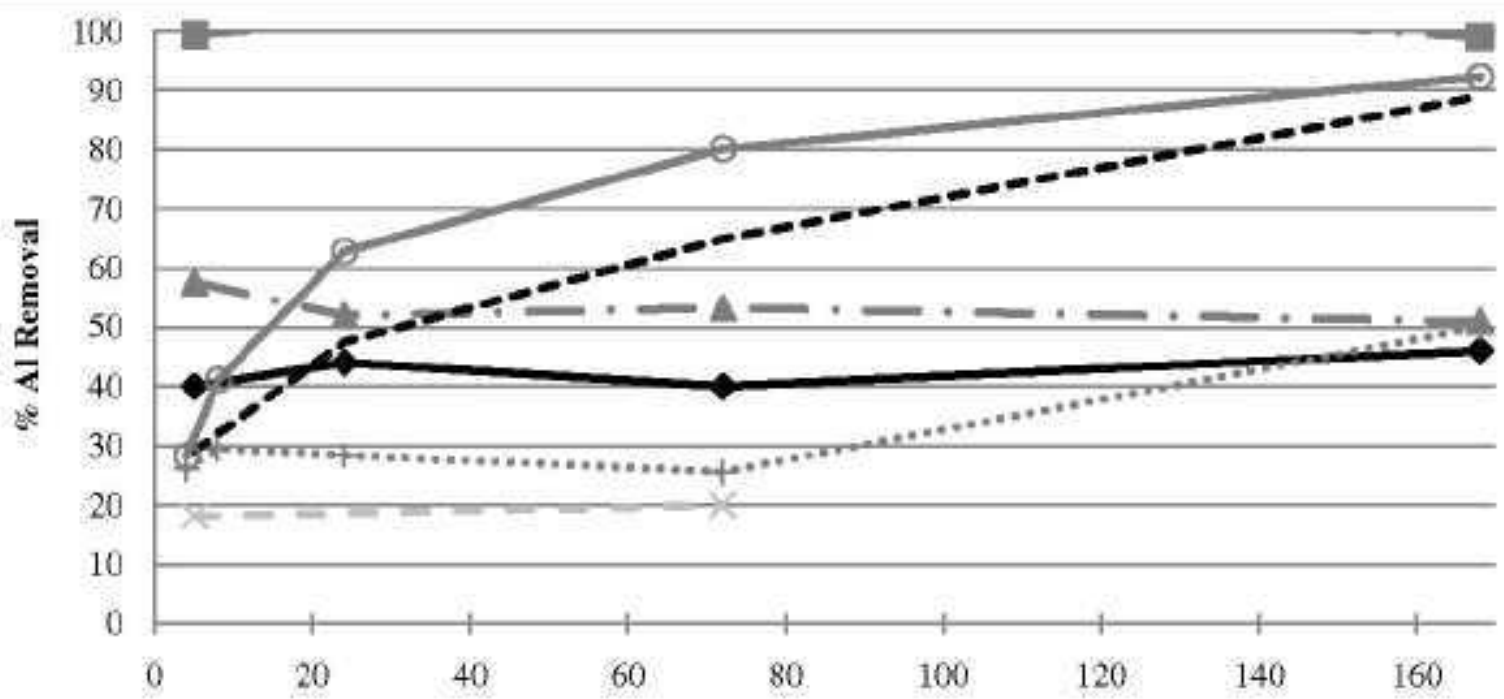

Leaching Time (hr)

\footnotetext{
-241-B-101, 1.0M, 100 deg C (PNNL-12026)

-241-BX-110, 1.2M, 95 deg C (PNNL-12026)

- $241-\mathrm{BX}-112,1.1 \mathrm{M}, 100 \mathrm{deg}$ C (PNNL-12026)

- - 241-S-101, 1M, 95deg C (ORNLITM-13655)

$-241-\mathrm{S}-110,0.8 \mathrm{M}, 100 \operatorname{deg} \mathrm{C}$ (PNNL-13702)

m+* 241-T-110, 0.6M, 100 deg C (PNNL-13956)
} 
Figure $\mathrm{A3}-14$. Caustic leaching using $3 \mathrm{M} \mathrm{NaOH}$ at $100^{\circ} \mathrm{C}$.

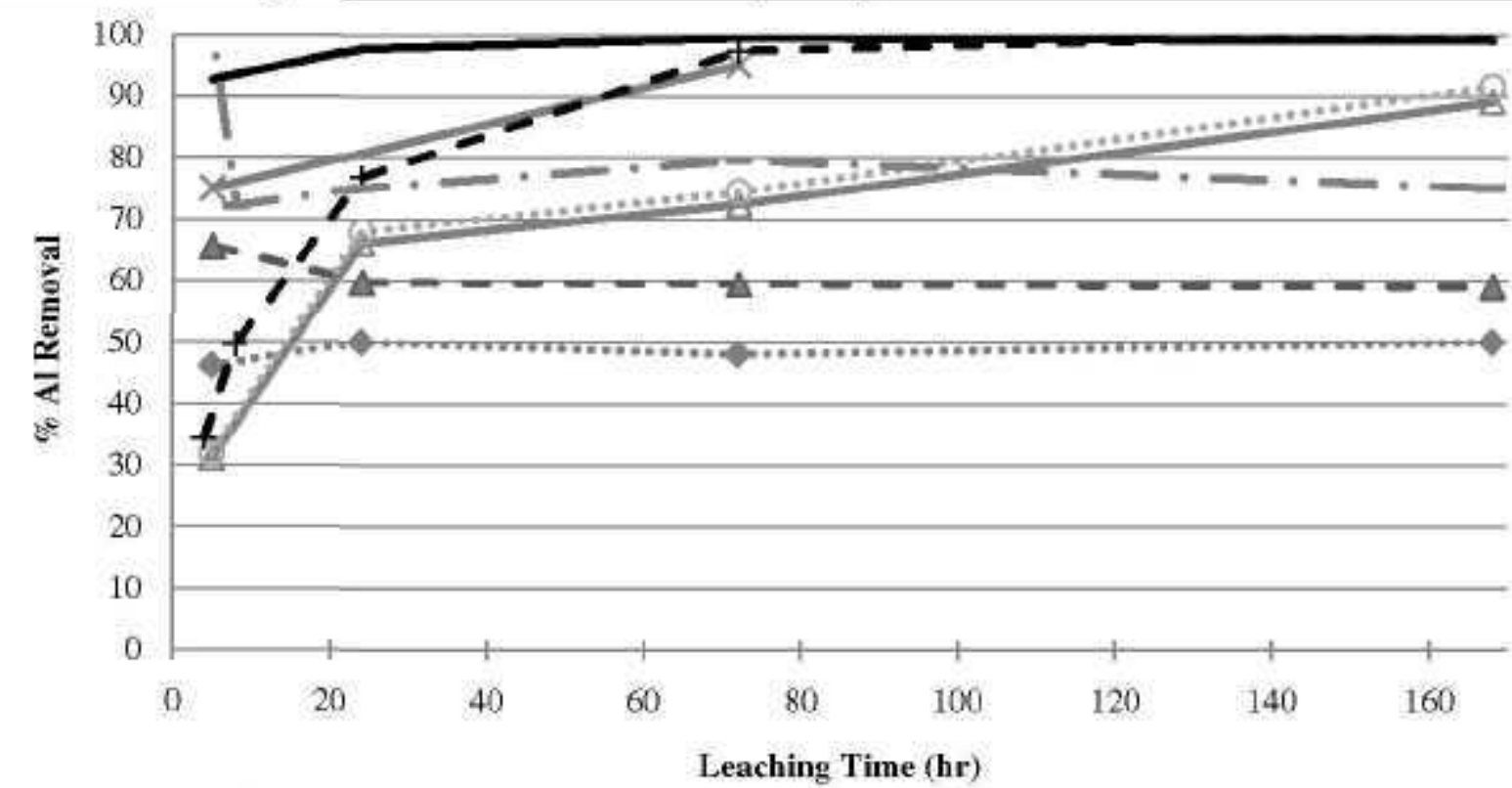

** 241-B-101, 3.1M, 100 deg C (PNNL-12026) - 241-BX-110, 3.2M, 95 deg C (PNNL-12026)

$-\triangle 241-\mathrm{BX}-112,3.4 \mathrm{M}, 100 \operatorname{deg} \mathrm{C}$ (PNNL-12026) $\rightarrow 241-\mathrm{C}-102,2.9 \mathrm{M}, 100 \operatorname{deg} \mathrm{C}$ (PNNL-12026)

$-241-\mathrm{S}-101,3 \mathrm{M}, 95 \mathrm{deg}$ C (PNNL-12026) *. 241-S-101,3M, $95 \mathrm{deg}$ C (ORNL/TM-13655)

-+ 241-S-110, 2.7M, 100 deg C (PNNL-13702) - -241-T-110, 2.5M, 100 deg C (PNNL-13956) 
RPP-RPT-46791

Revision 0

\section{A4,0 APPENDIX REFERENCES}

9404238. 1994, "Transmittal of Third Quarterly Report for Sludge Treatment Extraction Task Entitled "Sludge Treatment and Extraction Technology Development: Radionuclide Separations"." (intemal letter from L. K. Holton to K. A. Gasper. June 28), Battelle. Pacific Northwest Division, Richland, Washington.

LAUR 95-2070, 1995, Studge Washing and Alkaline Leaching Tests on Actual Hanford Tank Sludge: A Status Report, Los Alamos National Laboratory, Los Alamos, New Mexico.

LAUR 96-2839, 1996, Shudge. Washing and Alkaline Leaching Tests on Actual Hanford Tank Siludge: FY 1996 Results. Los Alamos National Laboratory. Los Alamos, New Mexico.

LAUR 97-2889, 1997, Sladge Washing and Alkaline Leaching Tests on Actual Hanford Tank Situdge: FY 1997 Results, Los Alamos National Laboratory, Los Alamos, New Mexico.

ORNLTM-13500, 1998. Caustic Leaching of Sludges from Selected Hanford Tanks, Oak Ridge National Laboratory, Oak Ridge, Tennessee.

ORNLTM-13655, 1998, Water Washes and Caustic Leaches of Studge from Hanford Tank S. 101 and Water Washes of Sludge from Hanford Tonk C-103. Oak Ridge National Laboratory, Oak Ridge, Tennessee.

ORNLTM-13660, 1998, Status Report on Solid Control in Leachates, Oak Ridge National Laboratory, Oak Ridge, Tennessee.

PNL-9387. 1994. Studge Treatment and Extraction Technology Development: Results of FY 1993 Studies, Pacific Northwest Laboratory. Richland, Washington.

PNL-9814, 1994, The Sort On Radioactive Waste Type Model: A Method to Sirt Single-Shell Tanks lno Characteristic Groups, Pacific Northwest Laboratory, Richland, Washington.

PNL-10078. 1994. Washing and Alkatine Leaching of Hanford Tank Sludges: A Status Report. Pacific Northwest Laboratory, Richland, Washington.

PNL-10712, 1995, Washing and Caustic Leaching of Hatford Tank SItudges: Results of FY 1995 Studies, Pacific Northwest Laboratory, Richland, Washington.

PNNL-1 1089, 1996, The Chemistry of Sludge Washing and Caustic Leaching Processes for Selected Hanfford Tank Wastes, Pacific Northwest National Laboratory, Richland, Washington.

PNNL-1 1278, 1996, Washing and Caustic Leaching of Hanford Tant Shdges: Results of FY 1996 Studies, Pacific Northwest National Laboratory, Richland, Washington.

PNNL-1 1381, 1996, Washing and Caustic Leaching of Hanford Tank C-166 Sludge, Pacific Northwest National Laboratory, Richland. Washington.

PNNL-1 1432, 1997, Bench-Scale Enhanced Sludge Washing and Gravity Settling of Hanford Tank C-106 Sludge, Pacific Northwest National Laboratory, Richland, Washington. 
RPP-RPT-46791

Revision 0

PNNL-11580, 1997, Caustic Leaching of Composite AZ-101/AZ-102 Hanford Tank Siudge, Pacific Northwest National Laboratory, Richland, Washington.

PNNL-1 1636. 1997. Washing and Caustic Leaching of Hanford Tank Sludge: Results of FY 1997 Studies, Pacific Northwest National Laboratory, Richland, Washington.

PNNL-11779, 1997, Leaching of Iron from Hanford Tank Studge: Results of FY 1997 Studies. Pacific Northwest National Laboratory, Richland, Washington.

PNNL-1 1908, 1998, Oxidative Alkaline Dissolution of Chromium from Hanford Tank Sludges: Results of FY 98 Studies, Pacific Northwest National Laboratory, Rictland, Washington.

PNNL-12010, 1998, Bench-Scale Enhanced Sludge Washing and Gravity Settling of Hanford Tank S. 167 Studge, Pacific Northwest National Laboratory. Richland, Wastington.

PNNL-12026, 1998, Washing and Caustic Leaching of Hanford Tant Shdge: Results of FY 1998 Studies, Pacific Northwest National Laboratory, Richland, Washington.

PNNL-13394, 2000, Status Report on Phase Identification in Hanford Tank Studges. Pacific Northwest National Laboratory, Richland. Washington.

PNNL-13702, 2001, Caustic Leaching of Hanford Tank S-110 Sludge. Pacific Northwest National Laboratory, Richland, Washington.

PNNL-13956, 2002, Caustic Leaching of Hanford Tank T.110 Shudge. Pacific Northwest National Laboratory, Richland, Washinglon.

PNNL-14018, 2002, Alkaline Leaching of Key, Nont-Radicactive Contponents from Simulants and Hanford Tank Sludge 241-S-110: Results of FY01 Studies, Pacific Northwest National Laboratory, Richland, Washington.

PNNL-14019, 2002. Selective Leaching of Chrominm from Hanford Tank Sludge 241.U.108, Pacific Northwest National Laboratory, Richland, Washington.

PNNL-17368 (WTP-RPT-157), 2008, Characterization and Leach Testing for REDOX Situdge. and S-Saltcake Actual Waste Sample Composites, Pacific Northwest National Laboratory, Richland, Washington.

PNNL-1 7965 (WTP-RPT-172), 2009, Filtration and Leach Testing for REDOX Sludge and SSaltcake Actual Waste Sample Composites, Pacific Northwest National Laboratory, Richland, Washington.

PNNL-17992 (WTP-RPT-166), 2009, Characterization, Leaching, and Filtration Testing for Bismuth Phosphate Sludge (Group 1) and Bisnuth Phosphate Saltcake (Group 2) Actual Waste Sample Composites, Pacific Nortbwest National Laboratory, Richland, Washington.

PNNL-18007 (WTP-RPT-171), 2009. Laboratory Demonstration of the Pretreatment Process with Caustic and Oxidative Leaching Using Actual Hanford Tank Waste. Pacific Northwest National Laboratory, Richland. Washington.

PNNL-18048 (WTP-RPT-181), 2009, Filtration and Leach Testing for PUREX Cladding Sludge and REDOX Cladding Studge Actual Waste Sample Composites, Pacific Northwest National Laboratory, Richland, Washington. 
RPP-RPT-46791

Revision 0

PNNL-18054 (WTP-RPT-167), 2009, Characterization and Leach Testing for PUREX Cladding Waste Sludge (Group 3) and REDOX Cladding Waste Sludge (Group 4) Actual Waste Sample Composites, Pacific Northwest National Laboratory, Richland, Washington.

PNNL-18119 (WTP-RPT-169), 2009, Characterization, Leaching, and Filtration Testing, for Tributyl Phosphate (TBP, Group 7) Actual Waste Sample Composites, Pacific Northwest National Laboratory, Richland, Washington.

PNNL-18120 (WTP-RPT-170), 2009, Characterization, Leaching, and Filtration Testing of Ferrocyanide Tank Sludge (Group 8) Actual Waste Composite, Pacific Northwest National Laboratory, Richland, Washington.

PNWD-3013 (BNFL-RPT-017), 2000, C.106 High-Level Waste Solids: Washing/Leaching and Solubility Versus Temperature Studies, Battelle, Richland, Washington.

PNWD-3024 (BNFL-RPT-030), 2000, Characterization, Washing, Leaching, and Filtration of C-104 Sludge, Battelle, Richland, Washington.

PNWD-3027 (BNFL-RPT-021), 2000, C-104 High-Level Waste Solids: Washing/Leaching and Solubility Versus Temperature Studies, Battelle, Richland, Washington.

PNWD-3045 (BNFL-RPT-038), 2000, Characterization, Washing, Leaching, and Filtration of AZ-102 Sludge, Battelle, Richland, Washington.

PNWD-3206 (WTP-RPT-043), 2003, Filtration, Washing, and Caustic Leaching of Hanford Tank AZ-101 Sludge, Battelle, Richland, Washington.

PNWD-3300 (WTP-RPT-076), 2003, Identification of Washed Solids from Hanford Tanks 241. AN-102 and 241-AZ-101 with X-Ray Diffraction, Scanning Electron Microscopy, and Light-Scattering Particle Analysis, Battelle, Richland, Washington.

PNWD-3512 (WTP-RPT-117), 2004, Oxidative-Alkaline Leaching of Washed 241-SY-102 and 241-SX-101 Tank Slidges, Battelle, Richland, Washington.

WHC-EP-0625, 1993, Hanford Site Waste Storage Tank Information Notebook, Westinghouse Hanford Company, Richland, Washington.

WRPS, 2010, Tank Waste Information Network System (TWINS), Queried 06/2010 (FY10 Q3), [Data, Data source selection forms, Best Basis Inventory, Best Basis Inventory Calculation Detail, All tanks, Analyte $=\mathrm{Al}$, waste phase $=$ sludge $\mathbf{J}$, Internet at http://twins.pnl.gov/twinsdata/forms/BuildQuery.aspx?SourceName=bb published.dbo.p calc detail\& whatsnew=Best+Basis+Inventory, Washington River Protection Solutions, LLC, Richland, Washington. 